\title{
Lecture Abstracts
}

\section{Pre-Congress Meeting: Better Understanding of HCC Tumor Biology for Cure}

\section{PCM-1 \\ Critical Reappraisal of HCC Diagnosis Based on Imaging Alone}

Do Young Kim

Yonsei University, Seoul, South Korea

The classical imaging appearance of hepatocellular carcinoma (HCC) on contrast-enhanced computed tomography (CT) or magnetic resonance imaging (MRI) is directly attributable to its pathogenesis. In particular, HCC is supplied by abnormal hepatic arteries in distinction to normal hepatocytes whose supply is primarily via the portal venous system. Resulting imaging characteristics are of a lesion that enhances during the late arterial phase and demonstrates both progressive washout of contrast relative to normal liver parenchyma and a peripheral rim of enhancement (pseudocapsule) on either portal venous or 3-5 minute delayed phase imaging. Lesions meeting these criteria over a predefined size threshold are categorized as definitely HCC by imaging per both by Liver Imaging Reporting and Data System (LI-RADS) (LR-5) and United Network for Organ Sharing-Organ Procurement and Transplantation Network (UNOS-OPTN) (Class 5) criteria.

Most international guidelines for HCC diagnosis solely depends on the findings of imaging such as contrast-enhanced liver CT or MRI. Addition of traditional biomarker for HCC, alpha-fetoprotein (AFP), to imaging is no longer permitted due to its low sensitivity. However, there are many examples with uncertain diagnosis when we use only imaging feature to characterize hepatic lesions. For instance, it is not always easy to differentiate HCC from intrahepatic cholangiocarcinoma (CC) or combined HCC-CC. Moreover, sometimes the imaging features of benign lesions including angiomyolipoma (AML) mimick those of HCC.

In other solid cancers except for HCC, confirmation of cancer cell is the mainstay for not only diagnosis, but also selection of appropriate therapy based on genomic analysis.

Based on this, the reappraisal of imaging based HCC diagnosis is necessary to encourage searching novel biomarkers for HCC and gene-based individual therapy. 


\section{PCM-2 \\ Role of Tissue Biopsy to Predict Biological Behavior and Prognosis in HCC}

\section{Hirohisa Yano}

Kurume University, Kurume, Japan

There are 2 types of liver biopsy, i.e., tumor biopsy and non-tumor biopsy, but liver tumor biopsy is definitely more useful to predict biological behavior and prognosis in liver tumors, such as hepatocellular carcinoma (HCC). However, nowadays, the number of liver tumor biopsy performed per year is decreasing in number. The number of liver tumor biopsy performed in recent 5 years (20122016) in our University Hospital is about $40 \%$ of that performed about 20 years ago (1994-1999). This is because tumor biopsy is recommended only in cases where radiology is not diagnostic according to the current diagnostic algorithm for suspected HCCs, proposed by Liver Cancer Study Group of Japan and AASLD. As a result, biopsy of rare liver tumors, e.g., combined hepatocellular and cholangiocarcinoma (cHCC-CCA) and intrahepatic cholangiocarcinoma (iCCA), showing atypical imaging features relatively increased in number in recent 5 years. In this regard, such liver tumor biopsy has high significance because the treatment and prognosis of cHCC-CCA and iCCA are different from those of HCC. Even in the case of HCCs, histological examination for subtype classification gives important information to predict biological behavior and prognosis. For examples, patients with poorly differentiated HCC, small cell carcinoma, sarcomatoid HCC, or HCC with neuroendocrine differentiation have the worse prognosis. Poorly differentiated HCC is often associated with microvascular invasion (MVI) that is an important factor to influence the prognosis in HCC. Recently, Ziol et al. (Hepatology, 2018) reported macrotrabecular-massive subtype of HCC, consisting at least $50 \%$ of the tumor component of macrotrabecular architectural pattern, shows poor prognosis with frequent MVI.

Several studies proposed molecular subclassifications and their relationship to tumor histology, biological behavior, and patient prognosis mainly based on gene expression profiling data. For example, Hoshida et al. (Cancer Res, 2009) defined three HCC subclasses (termed S1, S2, and S3), and found that S1 (WNT signaling pathway activation) and S2 (MYC/AKT activation) were associated with poor prognosis. Although gene expression profiling analysis of HCC provides valuable information, it is not realistic to apply this analysis to small tumor biopsy specimen. Recently, Tsujikawa et al. (Human Pathol, 2016) identified 3 HCC subgroups based on immunohistochemical molecular analysis and found that B/S subgroup expressing biliary/stem cell markers, e.g., cytokeratin 19 (CK19), sal-like protein 4 (SALL4), and epithelial cell adhesion molecule (EpCAM), exhibited aggressive features and poor prognosis as compared with the other groups. In addition, p53-positive HCCs showed more aggressive features. Immunohistochemical analysis of these molecules on tumor biopsy specimens may provide useful information to evaluate biological features and prognosis of HCC. However, compared with surgical specimen, biopsy specimen may be too small in amount to investigate molecular expression, so the results should be interpreted with caution. One successful study was reported by Tsuchiya et al (Oncology, 2011) who examined Keratin19 (synonym for CK19) expression in tumor biopsy specimens taken just prior to the radiofrequency 
The 9th Asia-Pacific Primary Liver Cancer Expert Meeting (APPLE 2018)

ablation (RFA) therapy and successfully found Keratin 19 expression of HCC in biopsy specimens is related to high recurrence of HCC after RFA.

In conclusion, morphological observation and immunohistochemical molecular analysis of liver tumor biopsy may provide useful information on the biological behavior and prognosis in HCC. However, it may be difficult because tumor tissue obtained by biopsy is mostly small in amount. Especially, molecular analysis of tumor biopsy specimen may be challenging. 


\author{
PCM-3 \\ Clinical Implication of Molecular Classifications in HCC \\ Irene Oi-Lin $\mathrm{Ng}$ \\ The University of Hong Kong, Hong Kong SAR, China
}

Our increasing understanding of HCC biology holds promise for personalized care and in the development of molecular drugs. The identification of new or important molecular targets can be translated into clinical practice. The ultimate goal of precision medicine is to utilize molecular information to assign patients to the most effective treatment. However, the molecular alterations in advanced HCCs are quite diverse. Classifications of different HCC subtypes combining histology and gene mutations have been proposed. These may provide guidance for future consolidation but currently consensus for integrative morphological-molecular classification has not yet reached. In this presentation, recent data will be discussed to demonstrate how genomic/genetic analysis of patients' HCC samples can help identify important molecular targets and possibly molecular signature of HCC for personalized treatment. We investigated the mutational landscape of mammalian target of rapamycin (mTOR) signaling cascade in HCCs with chronic HBV background, aiming to evaluate and delineate mutation-dependent mechanism of mTOR hyperactivation in hepatocarcinogenesis. Next-generation sequencing was performed on human HCC samples and HCC cell line panel. Systematic mutational screening of mTOR pathway-related genes was undertaken and mutant genes were evaluated based on their recurrence. We identified and confirmed multiple mTOR components as recurrently mutated in HBV-associated HCCs. Of significance, we detected frequent $16.2 \%$, $\mathrm{n}=18 / 111$ ) mutations of TSC 1 and TSC2 genes in the HCC samples. The spectrum of TSC1/2 mutations likely disrupts the endogenous gene functions in suppressing the downstream mTOR activity through different mechanisms and leads to more aggressive tumor behaviour. Mutational disruption of TSC1 and TSC2 was also observed in HCC cell lines and our HCC patient-derived tumor xenografts (PDTX) models. TSC-mutant cells exhibited reduced colony-forming ability on rapamycin treatment. With the use of biologically relevant TSC2-mutant PDTXs, we demonstrated the therapeutic benefits of the hypersensitivity towards rapamycin treatment. Our findings suggest the significance of previously undocumented mutation dependent mTOR hyperactivation and frequent TSC1/2 mutations in HBV-associated HCCs. They define a molecular subset of HCC having genetic aberrations in mTOR signalling, with potential significance of effective specific drug therapy. On the other hand, fibrolamellar HCC (FL-HCC) is a subtype of HCC occurring mostly in young patients. A segmental deletion resulting in DNAJB1PRKACA gene fusion is now recognized as the signature genetic event of FL-HCC. DNAJB1-PRKACA fusion kinase is an oncogenic driver and candidate drug target for FL-HCC. Identification of these specific molecular targets can be useful in novel clinical treatment. 


\section{PCM-4 \\ Immunologic Microenvironment and Tumor Immunology in HCC}

Bruno Sangro

The University of Navarra, Pamplona, Spain

The treatment of patients with hepatocellular carcinoma remains a great challenge. Effective systemic therapies are scarce and they have been tested in the advanced stage. After decades of failures of immune therapies, immune checkpoint inhibitors have emerged as potentially effective treatments for patients with hepatocellular carcinoma in the advanced stage. Immune checkpoints, including human cancer, cytotoxic T-lymphocyte protein 4 (CTLA-4) and programmed cell death protein 1 (PD-1), are surface proteins expressed in a variety of immune cells, and mostly provide immunosuppressive signals. Monoclonal antibodies able to block these molecules have shown antitumor activity against a wide spectrum of human cancers. Clinical experience with checkpoint inhibitors in hepatocellular carcinoma includes early trials with the anti-CTLA-4 agent tremelimumab, and a large phase 2 trial with the anti-PD- 1 agent nivolumab. The latter has shown strong activity particularly as second-line therapy, both in terms of tumor response and patient survival. A large phase 3 trial comparing nivolumab vs. standard of care sorafenib will report results soon. If positive, the landscape will change and new strategies should be tested.

First, the activity of checkpoint inhibitors should be tested in patients at less advanced stages, including the adjuvant treatment of patients with resectable or ablatable tumors. Second, combination therapies should be tested for enhanced efficacy, particularly those combinations with targeted and locoregional therapies that may have a synergistic effect or act upon mechanisms of primary or acquired resistance to checkpoint inhibitors. Finally, the identification of clinical features and serum or tissue biomarkers that would allow a better patient selection for individual treatments is an unmet need. 


\section{Session 1: Update of HCC Guidelines}

\section{S1-1 \\ 2018 AASLD Guidelines}

Lewis R. Roberts

Mayo Clinic, Rochester, USA

The AASLD has transitioned to a process of guideline development aimed at producing trustworthy clinical practice guidelines for major liver diseases. The process is based on a systematic review of the existing evidence. Based on this core data, the guideline is then developed by a knowledgeable, multidisciplinary panel of experts. The panels consider important patient subgroups and patient preferences, as appropriate. The new process is explicit and transparent and minimizes distortions, biases, and conflicts of interest. It provides a clear explanation of the logical relationships between alternative care options and health outcomes, and produces ratings of both the quality of evidence and the strength of recommendations. The guidelines are reviewed and revised as appropriate when new evidence warrants modifications.

For the 2018 HCC guidelines, key questions were structured in terms of answerable and actionable recommendations, focusing on clinically relevant outcomes and using the PICO format: $\mathrm{P}=$ population; $\mathrm{I}=$ intervention; $\mathrm{C}=$ comparator; $\mathrm{O}=$ outcome. Systematic reviews of literature were performed with application of the GRADE - Grading of Recommendations, Assessment, Development and Evaluations system for rating the quality of the evidence.

\section{Key questions addressed in the $\mathbf{2 0 1 8}$ guidelines included:}

Should adults with cirrhosis undergo surveillance for HCC? If so, which surveillance test is best?

Should adults with cirrhosis and suspected HCC undergo diagnostic evaluation with multiphasic CT or multiphasic MRI?

Should adults with cirrhosis and an indeterminate hepatic nodule undergo a biopsy, repeated imaging, or alternative imaging?

Should adults with Child's class A cirrhosis and early-stage HCC (T1 or T2) be treated with resection or locoregional therapy?

Should adults with cirrhosis and HCC that has been resected or ablated successfully undergo adjuvant therapy?

Should adults with cirrhosis awaiting liver transplantation and 1-2 cm HCC (Organ Procurement and Transplantation Network [OPTN] T1) be treated or undergo observation Should adults with cirrhosis awaiting liver transplantation and HCC (OPTN T2) undergo transplant alone or transplant with bridging therapy while waiting?

Should adults with cirrhosis awaiting liver transplantation and 1-2 cm HCC (Organ Procurement and Transplantation Network [OPTN] T1) be treated or undergo observation?

Should adults with cirrhosis awaiting liver transplantation and HCC beyond Milan criteria (T3) undergo transplantation after being down-staged to within Milan criteria? Should adults with cirrhosis and HCC (T2 or T3, no vascular involvement) who are not candidates for resection or transplantation be treated with transarterial chemoembolization, transarterial radioembolization, or external radiation?

Should adults with Child-Pugh Class A/B cirrhosis and advanced HCC with macrovascular invasion and/or metastatic disease be treated with systemic or locoregional therapies or no therapy?

The evidence, rationale and recommendations will be reviewed and discussed. 


\section{S1-2 \\ EASL Clinical Practice Guidelines: Management of HCC - Update}

\section{Peter R. Galle}

Johannes Gutenberg University Mainz, Mainz, Germany

In 2012, the previous guideline for the management of hepatocellular carcinoma (HCC) was published as a result of a joint effort by the European Association for the Study of the Liver (EASL) and the European Organisation for Research and Treatment of Cancer (EORTC). Since then several clinical and scientific advances have been achieved. Thus, an updated version of the document is needed. For this purpose EASL established a guideline development group composed of international experts in the field of HCC, comprising the areas of hepatology (Peter R. Galle, Alejandro Forner, Josep Llovet, Fabio Psicaglia), surgery (Vincenzo Mazzaferro), radiology (Valerie Vilgrain), oncology (Jean-Luc Raoul) and pathology (Peter Schirmacher). The process was moderated by a methodologist (Markus Follmann).

In a first step the panel identified, prioritised and selected relevant topics and agreed on key questions to be answered. These questions were clustered and distributed according to the defined working groups, which are reflected in the different chapters.

According to the key questions, a literature search was performed. The studies identified and included were assessed and assigned to categories related to study design and strength of evidence according to endpoints. Based on this evidence, the drafts for recommendation and chapters were created.

Consent was provided for all recommendations during the consensus conference. Formal consensus methodology (nominal group technique) was used to agree upon the recommendations. All expert panel members were entitled to vote on the recommendations. The consensus conference was performed as a personal meeting over two days (in June and September 2017). When evaluating the evidence, the balance of benefits and harms, and the quality of the evidence were taken into consideration. Expert opinion and experience was included, particularly, if the body of evidence was insufficient and if further aspects such as time and costs, additional side effects, quality of life, resource use, etc. had to be considered.

The presentation will give an overview and focus on changes compared to the previous version. 
The 9th Asia-Pacific Primary Liver Cancer Expert Meeting (APPLE 2018)

\section{S1-3}

\section{Asia-Pacific Guidelines}

\section{Ann-Lii Cheng}

National Taiwan University, Taipei, Taiwan

HCC is unique in that tumor stage often not align well with simple treatment choice. Many treatment algorisms, particularly those from Asian countries, are therefore characterized by multiple treatment options under each individual stage. The latter practice is often criticized by Western experts whose practice generally follows BCLC guideline. HKLC is one of the few Asia-pacific guidelines that attempt to align stage with simple treatment option. However, significant discrepancies are observed between BCLC and HKLC, as the latter tends to be relatively more aggressive in adopting surgical resection and loco-regional treatment.

Standalone treatment guidelines i.e. not attemping to formulate stages, such as JSH and APASL which simply advise therapeutic options for a certain grouping of clinicopathologic features, are also often adapted in Asia-pacific regions. A detailed analysis and comparison of all these different systems will be discussed. 


\section{S1-4 \\ KLCA-NCC Korea Guidelines \\ Joong-Won Park \\ National Cancer Center Korea, Goyang, South Korea}

The Korean guidelines for the management of hepatocellular carcinoma (HCC) were first developed in 2003, and were revised in 2009 and 2014 by the Korean Liver Cancer Study Group (KLCSG) and the National Cancer Center (NCC), Korea. The KLCSG is the predecessor of the Korean Liver Cancer Association (KLCA). Because of an increasing number of studies and publications, the KLCA-NCC decided to update the 2014 guidelines to reflect the recent state of evidence.

The ultimate goal of treatment for patients with HCC may vary depending on the patient's cancer stage, liver function, and performance status, but generally, improvement in the overall survival is the most important and quality of life should also be considered.

For this purpose, it is necessary to establish a multidisciplinary treatment plan involving hepatologists, oncologists, surgeons, imaging radiologists, interventional radiologists, radiation oncologists, pathologists, and other specialists.

The choice of treatment modality should be as evidence-based as possible, and the highest degree of and best evidence should be based on a randomized controlled trial (RCT) or meta-analyses of prospective cohort study. Although these studies are gradually increasing in number, the best evidence, such as RCTs of treatment for HCC, is still lacking, and much of the treatment planning is based on moderate evidence. Therefore, great understanding and care is needed to administer treatment to a patient with HCC in practice.

The reason for the difficulty in establishing a balanced multidisciplinary treatmentplaninclinical practiceisthatthereisalackof objectivityintheindications and outcomes claimed byeach department that directly conductspatient treatment. Therefore, to propose a recommendation in the guidelines, a more objective assessment is needed through discussion among the multidisciplinary group.

The best treatment provided in these guidelines is the result of evidencebased medicine, which requires the patient to be in the best condition provided with a substantial facility and trained personnel to mobilize all possible treatments, and the patient's economic condition and adherence among patients and their caregivers should also be supported. Therefore, in these guidelines, the best and alternative treatments according to the modified Union for International Cancer Control stage was first proposed in 2014 and the same approach was adopted in this revision. However, the best treatment for HCC could not be simply recommended because it is not possible to enumerate all cases depending on not only the stage, but also liver function, co-morbidities, and performance status. Therapy for various cases of HCC has been selected in consideration of medical evidence and expert opinion, and is detailed in each treatment method chapter.

In the treatment overview, a schematic approach is suggested in a patient with HCC who had excellent liver function (Child-Pugh score A), no portal hypertension complications, and excellent performance (Eastern Cooperative Oncology Group performance $0-1$ ) for understanding these guidelines. These guidelines deal with the second-line treatment separately for the first time in this revision, and the treatment overview here only involves first-line treatment. Second-line treatments for residual tumors, recurrence, or progression after the first treatment are described separately. 


\title{
Session 2: HCC Surveillance and Detection of Early-Stage HCC
}

\author{
S2-5 \\ HCC Surveillance with Ultrasonography Alone vs. \\ Ultrasonography plus Tumor Markers
}

Morris Sherman

University Health Network, Toronto, Canada

Screening for hepatocellular carcinoma is commonly practiced. patients at risk for cancer are offered to be screened every 6 months. However, the methods for screening remain controversial. Western guidelines suggest that alphafetoprotein and other biomarker testing is not essential, whereas guidelines from Asia include biomarkers testing as routine. There is universal agreement that ultrasound is an essential component of screening, but the value of adding biomarkers has not been rigorously tested.

As a result, a randomized controlled study was designed comparing HCC screening with ultrasound alone to ultrasound with the addition of three biomarkers, AFP, AFP-L3 and DCP. The endpoint was rate of first detection of cancer. Recruitment is nearly complete. The follow-up period is due to end later this year, but may be extended because the incidence of HCC has been lower than expected because of the advent of effective hepatitis $B$ and hepatitis $C$ treatment that has reduced the likelihood of patients with cirrhosis due to those infections developing HCC.

The current status of the trial will be presented. 


\section{S2-6 \\ MRI as a Tool for HCC Surveillance: Efficacy and Cost-Effectiveness}

Young-Suk Lim

University of Ulsan, Seoul, South Korea

The prognosis of patients with HCC is extremely poor with the 5-year survival rate below $20 \%$. The prognosis largely depends on tumor stage, and curative treatments are available only for patients diagnosed when the cancer is at an early stage. Even for patients with early stage HCC, the chance of liver transplantation is often limited due to donor shortage, and surgical resection is seldom possible because of significant portal hypertension. Thus, the only curative treatment option is local ablation in many cases, which highlights the importance of surveillance to detect HCCs at a very early stage (a single lesion $<2 \mathrm{~cm}$ ).

Currently, ultrasonography (US) at 6-month intervals is recommended for the surveillance of patients at risk to detect HCC at an early stage. However, the sensitivity of US is suboptimal. Paradoxically, the sensitivity of US may particularly be impaired in those at highest risk of developing HCC because of the very nodular liver [1-4].

In diagnostic setting, magnetic resonance imaging (MRI) using a liverspecific contrast agent, gadoxetic acid, has been shown to be superior to dynamic CT or MRI enhanced by other types of contrast agents for detecting and characterizing liver lesions in the intrahepatic staging workup of HCC [5].

Our recent prospective study showed that MRI with liver-specific contrast is more sensitive than US to detect early stage HCC in high-risk patients with cirrhosis [6]. For very early stage HCC (single lesion $<2 \mathrm{~cm}$ ), MRI screening yielded a detection rate of $84.8 \%$, significantly higher than the $27.3 \%$ detected by US. MRI also showed a significantly lower false positive results and a significantly higher positive predictive value than US. The proportion of patients with very early stage and early stage HCC was strikingly high in our study patients $(97.7 \%)$. Consistent with that finding, the patients in our study group also had a high chance of receiving curative treatments $(67.4 \%)$ and a favorable rate of survival. The 3-year survival rate of $86 \%$ in our study patients with HCC was obtained despite the severely limited access to liver transplantation.

MRI surveillance is limited by its higher cost than US. Nonetheless, in general, the cost-effectiveness (CE) of cancer screening program greatly relies on the incidence of the cancer and the sensitivity of the screening test [7]. Therefore, the use of an expensive but highly sensitive test (e.g. MRI) for cancer screening may be justified in a population with high risk for cancer $[8,9]$.

In another recent study, we have demonstrated that MRI surveillance for HCC can be cost-effective in high-risk patients with compensated cirrhosis. When the annual HCC incidence was higher than $4 \%$, MRI surveillance with liverspecific contrast was preferred than US surveillance despite the higher test cost of MRI, with cost-effectiveness threshold of $\$ 20,000$ per QALY. With higher CE thresholds, MRI surveillance was acceptable as a cost-effective option at lower HCC incidence rate and higher MRI/US cost ratio.

Based on our data, recent HCC practice guidance of American Association for the Study of Liver (AASLD) stated that, in select patients with a high likelihood of having an inadequate US or if ultrasound is attempted but inadequate, MRI may be utilized for the surveillance of HCC [10]. 


\section{References}

1. Del Poggio P, Olmi S, Ciccarese F, et al. Factors that affect efficacy of ultrasound surveillance for early stage hepatocellular carcinoma in patients with cirrhosis. Clin Gastroenterol Hepatol 2014;12:1927-1933 e1922.

2. Singal AG, Nehra M, Adams-Huet B, et al. Detection of hepatocellular carcinoma at advanced stages among patients in the HALT-C trial: where did surveillance fail? Am J Gastroenterol 2013;108:425-432.

3. Yu NC, Chaudhari V, Raman SS, et al. CT and MRI improve detection of hepatocellular carcinoma, compared with ultrasound alone, in patients with cirrhosis. Clin Gastroenterol Hepatol 2011;9:161-167.

4. Liu WC, Lim JH, Park CK, et al. Poor sensitivity of sonography in detection of hepatocellular carcinoma in advanced liver cirrhosis: accuracy of pretransplantation sonography in 118 patients. Eur Radiol 2003;13:1693-1698.

5. Kim HD, Lim YS, Han S, et al. Evaluation of Early Stage Hepatocellular Carcinoma by Magnetic Resonance Imaging With Gadoxetic Acid Detects Additional Lesions and Increases Overall Survival. Gastroenterology 2015.

6. Global Burden of Disease Cancer Collaboration. Global, regional, and national cancer incidence, mortality, years of life lost, years lived with disability, and disability-adjusted life-years for 32 cancer groups, 1990 to 2015: a systematic analysis for the global burden of disease study. JAMA Oncol 2017;3:524-548.

7. Yang JD, Mannalithara A, Piscitello AJ, et al. Impact of Surveillance for Hepatocellular Carcinoma on Survival in Patients with Compensated Cirrhosis. Hepatology 2017:ePub ahead of print.

8. Arguedas MR, Chen VK, Eloubeidi MA, et al. Screening for hepatocellular carcinoma in patients with hepatitis C cirrhosis: a cost-utility analysis. Am J Gastroenterol 2003;98:679-690.

9. Goossens N, Singal AG, King LY, et al. Cost-Effectiveness of Risk Score-Stratified Hepatocellular Carcinoma Screening in Patients with Cirrhosis. Clin Transl Gastroenterol 2017;8:e101.

10. Marrero JA, Kulik LM, Sirlin C, et al. Diagnosis, Staging and Management of Hepatocellular Carcinoma: 2018 Practice Guidance by the American Association for the Study of Liver Diseases. Hepatology 2018. 


\title{
S2-7
}

\section{Dynamic MR Imaging Using Hepatocyte Specific Contrast Agents: Advantages and Pitfalls}

\author{
Jeong Min Lee
}

Seoul National University, Seoul, South Korea

With the recent dramatic advances in diagnostic modalities, the diagnosis of hepatocellular carcinoma (HCC) is primarily based on imaging. Ultrasound (US) plays a crucial role in HCC surveillance. Dynamic multiphasic multidetectorrow CT (MDCT), and magnetic resonance imaging (MRI) using extracellular gadolinium based contrast agents are the standard diagnostic methods for the noninvasive diagnosis of HCC, which can be made based on hemodynamic features (arterial phase hyperenhancement and portal /delayed washout). Besides assessing the hemodynamic or perfusion features of HCC using dynamic CT or MRI, new advances in MRI can provide a cellular information of HCC. Hepatocyte specific contrast agents, such as gadoxetic acid, give information regarding hepatocellular function or defect of the lesion, which improves detection of focal liver malignancies. The signal intensity of lesions relative to the liver in the hepatobiliary phase mainly depends on OATP8 expression. Thus, most hepatocellular carcinomas including many early HCCs, and some highgrade dysplastic nodules, appear hypointense in the hepatobiliary phase, and therefore, the hepatobiliary phase can increase HCC conspicuity and delineation. Another benefit is in the differentiation of hypervascular HCCs from hypervascular pseudolesions such as focal perfusion alterations due to arterioportal shunts, a frequent source of diagnostic confusion using extracellular agents. However, gadoxetic acid has several pitfalls, including absence of true interstitial phase which may cause false positive diagnosis of hemangioma or cholangiocarcinoma as HCC, and transient dyspnea developments resuting in subdiagnostic quality arterial phase imaging. In this lecture, I would like to address advantages and pitfalls of liver MRI using hepatocyte-specific contrast media for evaluation of liver nodules in patients with liver cirrhosis. 


\title{
S2-8 \\ Role of Contrast-Enhanced Ultrasound in Diagnosis of Early-Stage HCC
}

\author{
Masatoshi Kudo
}

Kindai University, Osaka, Japan

The basic concept of contrast-enhanced ultrasonography (CEUS) has been confined to the hepatic nodule, which is detected by B-mode ultrasound (US). This is applicable to contrast agents such as SonoVue ${ }^{\circledR}$. However, the use of Sonazoid ${ }^{\circledR}$ in combination with a technique termed defect reperfusion US imaging has changed the management of hepatocellular carcinoma (HCC) drastically. Sonazoid ${ }^{\circledR}$ has three favorable properties: it allowsreal-time vascular imaging, stable Kupffer phase imaging lasting up to 60 minutes, and its use is tolerable for multiple scanning. Defect reperfusion US imaging, which is based on reinjection at the Kupffer phase, enables the detection of B-mode ill-defined nodules and locally recurring nodules. In addition, it facilitates the correct diagnosis of nodules detected on screening/surveillance or the detection of additional nodules for staging before treatment. This is a breakthrough technique that will change the clinical practice pattern of HCC management.

The detection of small nodular lesions in coarse liver parenchyma is difficult by B-mode US alone, although dynamic CT or dynamic MRI can detect arterial enhancing nodules with venous washout. Approximately $10 \%$ of HCC nodules that are not detected by B-mode US can be clearly identified by defect reperfusion imaging with Sonazoid ${ }^{\circledR}$. The false positive rate increases when the technique is confined to Kupffer phase scanning. In addition to the Kupffer defect, information on arterial vascularity, i.e., the reinjection method, increases the diagnostic accuracy to $100 \%$ even in deeply seated nodules.

This breakthrough method allows the detection of nodules that cannot be visualized by B-mode US, as defects on Kupffer images in the stable Kupffer phase. The presence of arterial blood flow in nodules with Kupffer defects is subsequently determined by the reinjection technique, making this method a breakthrough in diagnostic imaging. CEUS with Sonazoid ${ }^{\circledR}$ reinjection requires no special apparatus or analysis, and is the result of a change in the way of thinking regarding CEUS. For the typical CT image (so-called early enhancement with late washout nodules), defects are easily detected in the Kupffer phase, and arterial perfusion within the defect is subsequently demonstrated by the reinjection test (visualization of staining within the Kupffer defects, which is the reverse phenomenon of early enhancement with late washout). The introduction of this technique has allowed almost $100 \%$ accuracy in the detection of lesions observed on CT images that are not visualized on B-mode US images. If the reinjection test shows no enhancement of a Kupffer defect, this defect differs from the nodule detected by CT. This method then serves to guide needle insertion. In surveillance, this procedure facilitates screening because Sonazoid ${ }^{\circledR}$ US can be performed in the setting of a routine examination. In addition, operators only need to concentrate on the delineation of Kupffer defects in the Kupffer phase in contrast to routine B-mode US, in which regenerative nodules or dysplastic nodules may mimic malignant ones. If defects are detected, HCC can be confirmed by Sonazoid ${ }^{\circledR}$ reinjection, which provides information on both Kupffer cell function and arterial blood flow on the same cross-sectional image. This dual phase fusion imaging allows detection and definitive diagnosis of HCC 
The 9th Asia-Pacific Primary Liver Cancer Expert Meeting (APPLE 2018)

with $100 \%$ confidence. As a result, Sonazoid ${ }^{\circledR}$ has markedly improved the efficiency of HCC detection. In the past, CEUS was considered only for nodules previously depicted by B-mode US and was not used as a screening tool. However, this concept changed with the introduction of defect reperfusion imaging using Sonazoid ${ }^{\circledR}$. With this method, defection of early-stage HCC is easily possible. 


\title{
Session 3: Pros and Cons: Anti-Viral Therapy and HCC Risk
}

\author{
S3-9 \\ Pros: Treating CHB Patients in Immune-Tolerant Phase \\ Jeong-Hoon Lee \\ Seoul National University, Seoul, South Korea
}

Most of the medical decisions are drawn based on the comparison between risk and benefit or between cost and benefit. ${ }^{1}$ Current international guidelines on the management of chronic hepatitis B recommend no antiviral treatment for immune-tolerant chronic hepatitis B patients except for those with advanced fibrosis. $^{2-4}$ Those recommendations can be explained by the insufficient data on both efficacy and safety of a life-long anti-hepatitis B virus (HBV) treatment. It has been still uncertain whether antiviral treatment in immune-tolerant phase can reduce the risk of cirrhosis and hepatocellular carcinoma more profoundly compared to antiviral treatment in active hepatitis phase. Thus, at this time, it is clear that there is few evidence supporting the early antiviral treatment for immune-tolerant phase chronic hepatitis B patients.

However, this issue might have to be re-evaluated in near future. During the recent two decades, highly potent antivirals (i.e., entecavir and tenofovir) were developed and consequently the long-term risk related to antiviral resistance was dramatically reduced. In addition, several evidences showed that the immune-tolerant phase of chronic hepatitis B is not a completely safe status. HBV can directly induce HCC without advanced fibrosis and this direct carcinogenic effect with viral DNA integration to host genome followed by clonal hepatocyte expansion already started from immune-tolerant phase. ${ }^{5,6}$ In a recent Korean study, untreated patients in immune-tolearnt phase showed significantly higher risk of HCC compared to patients who received antivirals for immune-active hepatitis. ${ }^{7}$ Another Korean retrospective multicenter study reported that patients who underwent an early antiviral treatment from immune-tolerant phase was significantly associated with lower risk of HCC occurrence compared to patients who started antiviral treatment according to current guidelines. ${ }^{8}$ On the other hand, chronic HBV infection was reportedly related to non-liver cancers such as a lymphoma, pancreatic cancer, and stomach cancer, and a study using big data reported that antiviral treatment was associated with attenuation of the HBV-related increased risk of extrahepatic malignancies. ${ }^{9}$ Of course, these potential benefit of antiviral treatment from immunetolerant phase should be studied further and validated with well-designed prospective studies.

In conclusion, current evidence-based guidelines do not recommend antiviral treatment in immune-tolerant phase of chronic hepatitis B. However, there are several recent data that favor antiviral treatment for immunetolerant chronic hepatitis B. Further studies may give us chance to reevaluate the risk-benefit of antiviral treatment for immune-tolerant patients in near future.

\section{References}

1. Pauker SG, Kassirer JP. Therapeutic decision making: a cost-benefit analysis. N Engl J Med. 1975;293:229-234. 
2. European Association for the Study of the Liver. EASL 2017 Clinical Practice Guidelines on the management of hepatitis B virus infection. J Hepatol. 2017;67:370-398.

3. Terrault NA, Lok ASF, McMahon BJ, et al. Update on prevention, diagnosis, and treatment of chronic hepatitis B: AASLD 2018 hepatitis B guidance. Hepatology. 2018;67:1560-1599.

4. Sarin SK, Kumar M, Lau GK, et al. Asian-Pacific clinical practice guidelines on the management of hepatitis B: a 2015 update. Hepatol Int. 2016;10:1-98.

5. Kew MC. Hepatitis B and C viruses and hepatocellular carcinoma. Clin Lab Med. 1996;16:395-406.

6. Mason WS, Gill US, Litwin S, et al. HBV DNA Integration and Clonal Hepatocyte Expansion in Chronic Hepatitis B Patients Considered Immune Tolerant. Gastroenterology. 2016;151:986-998 e984.

7. Kim GA, Lim YS, Han S, et al. High risk of hepatocellular carcinoma and death in patients with immune-tolerant-phase chronic hepatitis B. Gut. 2018;67:945952.

8. Chang Y, Choe WH, Sinn DH, et al. Nucleos(t)ide Analogue Treatment for Patients With Hepatitis B Virus (HBV) e Antigen-Positive Chronic HBV Genotype C Infection: A Nationwide, Multicenter, Retrospective Study. J Infect Dis. 2017;216:1407-1414.

9. Lee D, Lee JH, Cho Y, et al. Nucleos(t)ide Analogues against Hepatitis B Virus Reduces the Risk of Various Major Malignancies: a Nationwide Cohort Study Based on the Health Insurance Review and Assessment Service Database. Hepatology 2015;62(1 suppl):334A. 


\section{S3-10}

\section{Antiviral Therapy in Immune Tolerant Chronic Hepatitis B -} Cons

Jeong Won Jang

The Catholic University of Korea, Seoul, South Korea

The current practice guidelines do not recommend anti-HBV therapy but close monitoring with serial aminotransferases and HBV DNA measurements for chronic hepatitis B (CHB) patients in the immune tolerant phase. Such recommendation is based on the previous observations that the risk of disease progression and the chance of a sustained response with anti-HBV agents are low among patients in the immune tolerant phase. However, only monitoring instead of treating immune tolerant patients can raise a concern especially when managing elderly patients or those with a family history of hepatocellular carcinoma (HCC). In fact, some of them often reportedly have advanced fibrosis or are at higher risk of advanced liver diseases. Continued high HBV replication in such patients could increase the risk of disease progression or development of HCC. Emerging data show that treating during the immune tolerant phase of CHB can reduce disease progression or the risk of HCC. Thus, the optimal management of patients in the immune tolerant phase can often be individualized according to age, family history or other relevant factors. A liver biopsy and/or non-invasive assessment of fibrosis may be helpful to determine the therapeutic strategy when patients' age is over 40 or there is clinical suspicion of advanced liver disease. Recent studies suggest that $\mathrm{T}$ cell immunity against HBV is relatively preserved especially in young patients. Thus, it is urgent that definitive criteria for immune tolerant phase of chronic HBV infection should be introduced before we develop the optimal management strategy in this setting.

Keywords: Hepatitis B, natural history, immune tolerant phase, antiviral therapy 


\section{S3-11 \\ Does Direct-Acting Antiviral Therapy Increase HCC Risk? - Yes}

Jung Hyun Kwon

The Catholic University of Korea, Incheon, South Korea

\section{Introduction}

Patients with SVR after pegylated interferon (Peg-IFN)-based treatment had a marked reduction in death/liver transplantation and in liver related morbidity/mortality [1-3]. Since the introduction of oral direct acting antiviral (DAA) therapy, an SVR rate was markedly increased over $95 \%$ and the rate of adverse events was low. It is being expected to lead to all-cause mortality benefits, as seen with Peg-IFN based treatment. However, an unexpected high rate of HCC recurrence/occurrence after DAA therapy were reported in the short-term observational studies [4,5]. After these reports, there are so many studies refuting these findings have been published. Most of studies suggested that more cirrhotic background and old age in the oral DAAs groups may play a role in increasing HCC risk [6-8].

Here, I focus on the initial reports to emerging the new issue about the early development of de novo HCC and recurrence of previous treated HCC after DAA treatment. In addition, I review the recently reported studies about the risk factor for HCC occurrence and recurrence after DAA treatment.

\section{De novo HCC occurrence after DAA treatment}

In the early report of 344 consecutive cirrhotic patients, HCC was detected in 9 of 285 patients without previous HCC after 24-week follow up [5]. This study firstly suggested that the occurrence of liver cancer was not reduced even in DAA treated cirrhotic patients. Child-Pugh Class B, more severe liver fibrosis, lower platelet count, and previous HCC were significantly associated with HCC development [5].

In the immune study, 10 patients who developed de novo HCC within 18 months after DAA treatment had significantly higher levels of 12 immune mediator (cytokines, growth factors, and apoptosis markers) before treatment compared with controls [9]. A panel of 9 cytokines, measured in serum before treatment (MIG, IL22, TRAIL, APRIL, VEGF, IL3, TWEAK, SCF, IL21), identified patients who developed de novo HCC with an area under the receiver operating characteristic curve value higher than 0.8 . In another micro-environmental study, liver angiopoietin 2 is a key predictor of de novo HCC after DAA treatment [10].

\section{HCC recurrence after DAA treatment}

Reig et al. firstly reported unexpected high rate of early tumor recurrence in patients with HCV-related HCC undergoing DAAs [4]. Among 58 patients with prior treated HCC received DAA after a median follow-up of 5.7 months, 16 developed radiologic tumor recurrence $(27.6 \%)$. Another study also reported an incidence of $28 \%$ HCC recurrence 24 weeks after DAA treatment of CHC [5].

In Japan report, the recurrence rate increased in accordance with treatment times in the past [11]. One-year recurrence rates were 18.1, 28.2, and 60.2\%, and 2 -year rates were $22.1,41.6$, and $74.5 \%$, respectively $(\mathrm{P}<0.0001)$ in patients with one time, two or three times and four or more times of HCC therapy. 


\section{Conclusions}

In 2016, the World Health Organization (WHO) drafted a strategy for combating viral hepatitis and set a goal for the elimination of viral hepatitis by 2030. Investigating the risk factors to identify high-risk patients for the surveillance of HCC is essential. Conventional or novel biomarkers may provide insights into risk stratification for successfully treated patients. In addition to the glycolmarker, host genetic variants would be useful for implementing a personalized surveillance of HCC in patients with HCV infection. Until now, it is important to remind the clinicians and inform the patients that there are still risk of HCC even after successful DAA treatment.

\section{References}

1. Morgan TR, Ghany MG, Kim HY, Snow KK, Shiffman ML, De Santo JL, et al. Outcome of sustained virological responders with histologically advanced chronic hepatitis C. Hepatology 2010;52:833-44.

2. Morgan RL, Baack B, Smith BD, Yartel A, Pitasi M, Falck-Ytter Y. Eradication of hepatitis $C$ virus infection and the development of hepatocellular carcinoma: a meta-analysis of observational studies. Ann Intern Med 2013;158:329-37.

3. Nahon P, Bourcier V, Layese R, Audureau E, Cagnot C, Marcellin P, et al. Eradication of Hepatitis C Virus Infection in Patients With Cirrhosis Reduces Risk of Liver and Non-Liver Complications. Gastroenterology 2017;152:142-56 e2.

4. Reig M, Marino Z, Perello C, Inarrairaegui M, Ribeiro A, Lens S, et al. Unexpected high rate of early tumor recurrence in patients with HCV-related HCC undergoing interferon-free therapy. J Hepatol 2016;65:719-726.

5. Conti F, Buonfiglioli F, Scuteri A, Crespi C, Bolondi L, Caraceni P, et al. Early occurrence and recurrence of hepatocellular carcinoma in HCV-related cirrhosis treated with direct-acting antivirals. J Hepatol 2016;65:727-733.

6. Romano A, Angeli P, Piovesan S, Noventa F, Anastassopoulos G, Chemello L, et al. "Newly diagnosed Hepatocellular Carcinoma in patients with advanced hepatitis C treated with DAAs: a prospective population study". J Hepatol 2018.

7. Ogawa E, Furusyo N, Nomura H, Dohmen K, Higashi N, Takahashi K, et al. Shortterm risk of hepatocellular carcinoma after hepatitis $\mathrm{C}$ virus eradication following direct-acting anti-viral treatment. Aliment Pharmacol Ther 2018;47:104-113.

8. Waziry R, Hajarizadeh B, Grebely J, Amin J, Law M, Danta M, et al. Hepatocellular carcinoma risk following direct-acting antiviral HCV therapy: A systematic review, meta-analyses, and meta-regression. J Hepatol 2017;67:1204-1212.

9. Debes JD, van Tilborg M, Groothuismink ZMA, Hansen BE, Schulze Zur Wiesch J, von Felden J, et al. Levels of Cytokines in Serum Associate With Development of Hepatocellular Carcinoma in Patients With HCV Infection Treated With DirectActing Antivirals. Gastroenterology 2018;154:515-517.e3.

10. Faillaci F, Marzi L, Critelli R, Milosa F, Schepis F, Turola E, et al. Liver Angiopoietin-2 is a key predictor of de novo or recurrent hepatocellular cancer after HCV direct-acting antivirals. Hepatology 2018.

11. Ikeda K, Kawamura Y, Kobayashi M, Kominami Y, Fujiyama S, Sezaki H, et al. Direct-Acting Antivirals Decreased Tumor Recurrence After Initial Treatment of Hepatitis C Virus-Related Hepatocellular Carcinoma. Dig Dis Sci 2017;62:29322942. 
S3-12

Does Direct-Acting Antiviral Therapy Increase HCC Risk? No

Kieron Lim

National University of Singapore, Singapore

The advent of direct acting antiviral agents (DAA) have revolutionized the treatment paradigm for patients with chronic hepatitis C. DAAs have shown SVR or cure rates far superior compared to interferon-based therapy with significantly less toxicities, which have in turn resulted in fewer patients requiring liver transplantation for HCV-related decompensated cirrhosis. Over the past few years, some centres have observed a higher than expected HCC recurrence rate in HCV-infected patients treated with DAA. This led to many centres reporting on their experience. This lecture will review the latest data on this controversial and important topic on whether DAA therapy increases HCC risk. 


\section{Session 4: Selection of Treatment Modality in Early-Stage HCC}

\section{S4-13}

Resection vs. Transplantation

Dong-Sik Kim

Korea University, Seoul, South Korea

Both resection and liver transplantation (LT) are comprising main stream of curative treatment for early stage hepatocellular carcinoma (HCC). Although LT is considered to provide lower recurrence rate with better long-term survival, higher morbidity and mortality risk as well as scarcity of organ donor limit its wider application. Intention-to-treat analyses in patients with well compensated cirrhosis patients have reported conflicting results in long-term outcomes and selection of optimal treatment has been a difficult issue. Selection between these two options should be based on multiple factors including but not limited to underlying liver function, overall performance status including cardiopulmonary function, social status, compliance, and local organ availability including living donor.

Usage of salvage strategy for recurrent tumor after liver resection can be a useful option depending on region.

More detailed discussion about pros and cons of each treatment and issues to be considered in decision making will be presented during the session. 
DOI 10.1159/000490877

The 9th Asia-Pacific Primary Liver Cancer Expert Meeting (APPLE 2018)

\section{S4-14}

\section{Aggressive Tumor Recurrence after Radiofrequency} Ablation: How to Prevent It?

Tae Wook Kang

Sungkyunkwan University, Seoul, South Korea

Image-guided radiofrequency ablation (RFA) for early stage hepatocellular carcinoma (HCC) is an accepted non-surgical treatment that provides excellent local tumor control and favorable survival benefit. RFA offers significant advantages as it is less invasive than surgery and carries a low risk of major complications. However, serious complications, including aggressive tumor recurrence, may be observed during follow-up, and recently, mechanical or thermal damage during RFA has been proposed to be one of the causes of this kind of recurrence. Although the exact mechanism of this still remains unclear, physicians should be familiar with the imaging features of aggressive tumor recurrence after RFA for HCC and its risk factors. In addition, in order to prevent or minimize this newly recognized tumor recurrence, a modified RFA technique, combined RFA treatments with transarterial chemoembolization, and cryoablation can be used as alternative treatments. Ultimately, combining an understanding of this potential complication of RFA with an understanding of the possible risk factors for aggressive tumor recurrence and choosing alternative treatments are crucial to optimize clinical outcomes in each patient with HCC. 
DOI 10.1159/000490877

The 9th Asia-Pacific Primary Liver Cancer Expert Meeting (APPLE 2018)

S4-15

New Locoregional Ablative Techniques (Microwave Ablation, Cryotherapy, Irreversible Electroporation, etc.)

Edward Wolfgang Lee

University of California, Los Angeles (UCLA), Los Angeles, USA

As the trend continues toward the minimally invasive and image-guided treatment of tumors, percutaneous ablations have begun to partake a big role in therapeutic oncology. The benefits of percutaneous ablations are improved complication profiles, lower morbidities, decreased healthcare cost with shorter hospital stay and notably its ability to treat non-surgical cancer patients with comparable treatment outcomes. Currently, a percutaneous tumor ablation for an early-stage HCC is considered curative.

In this presentation, the latest advancement of some of the newest locoregional ablative techniques including microwave ablation and irreversible electroporation will be presented in detail including clinical cases. This presentation will also discuss some of the important advantages and disadvantages of each ablation technique and future directions in the field of ablation therapy. 


\section{S4-16 \\ Ablative Radiotherapy in the Treatment of Early HCC Tomoki Kimura}

Hiroshima University, Hiroshima, Japan

Ablative radiotherapy, so called stereotactic body radiotherapy (SBRT) or stereotactic ablative radiotherapy (SABR), has been recognized as one of the treatment modalities for small hepatocellular carcinoma (HCC) because of its excellent local control (90\%-100\% in 3-year) without an increase in severe adverse effects in patients with Child-Pugh score of $\leq 7$ for small tumors $(\leq 3 \mathrm{~cm})$. However, according to several guidelines, such as the updated Barcelona Clinic Liver Cancer Classification (BCLC) and Japanese guidelines, SBRT can be considered alternative treatment modalities for patients with HCC who were ineligible for resection or ablation therapies.

Recently, although there have not been prospective randomized study, several investigators reported the comparison of SBRT and other modalities, such as transcatheter arterial chemoembolization (TACE), radiofrequency ablation (RFA), and resection, using propensity score matching (PSM) methods.

1) TACE: Sapir E, et al compared SBRT (158 patients) with TACE (112 patients). Although local control of SBRT was significantly better than that of TACE with low toxicities, there was no difference in overall survival between patients treated with TACE or SBRT (IJROBP100: 122, 2018). Huo YR, et al. conducted meta-analysis radiation therapy including SBRT plus TACE vs TACE alone, and concluded the pooled median survival for TACE plus RT was significantly better than for TACE alone (22.7 months vs 13.5 months; $p<0.001$, respectively) and the benefit of TACE plus RT continuously increased for 2-, 3-, 4-, and 5-year survival. (JAMA Oncol 6: 756, 2015).

2) RFA: A retrospective study comparing SBRT (63 patients) with RFA (163 patients) suggested a 2-year overall survival and local progression-free survival of about $50 \%$ and $80 \%$ for both modalities. For tumors $\geq 2 \mathrm{~cm}$, there was decreased local progression-free survival for FA compared with SBRT ( $p=$ 0.025). The author concluded that both RFA and SBRT were effective local treatment options for inoperable HCC. (JCO 34: 452, 2016). On the other hand, according to the comparison of RFA (3684 pts) and SBRT (296 pts) from National Cancer Database for stage I- II HCC using PSM, 5 -year overall survival was $29.8 \%$ in the RFA group vs $19.3 \%$ in the SBRT group ( $\mathrm{p}=0.001$ ). The authors suggests that treatment with RFA yields superior survival compared with SBRT for nonsurgically managed patients with stage I or II HCC. (JCO 36: 600, 2018).

3) Resection: Su TS et al. reported the comparison of SBRT and resection, the 5-year overall survival and progression-free survival was 74.3\%, 43.9\% in SBRT, $69.3 \%, 35.9 \%$ in resection ( $p=0.405,0.945)$ with similar toxicities, respectively. (IJROBP 98: 639, 2017). The authors concluded that SBRT has an advantage over resection in being less invasive. On the other hand, according to our institutional ccomparison of resection (54 pts) and SBRT (27 pts) using PSM, 5-overall survival was $75.2 \%$ in resection vs $47.8 \%$ in SBRT ( $p=0.0149)$. The authors concluded that although SBRT may be an effective alternative treatment for inoperable patients with early HCC, resection should be considered as the first-line treatment for patients deemed eligible for surgery. (unpublished data).

These comparisons are retrospective nature, further prospective trials, especially comparison of overall survival between SBRT and other modalities for untreated HCC patients, are needed before SBRT can become a true competitor of the other modalities, such as TACE, RFA and resection. 


\section{Session 5: Challenges in the Treatment of Intermediate Stage HCC}

\section{S5-17}

Subclassification of BCLC Stage B and Treatment Options

Su Jong Yu

Seoul National University, Seoul, South Korea

Barcelona-Clinic Liver Cancer (BCLC)-B, intermediate-stage hepatocellular carcinoma (HCC) includes a heterogeneous population of patients with varying tumor burdens [tumor size ( $>3 \mathrm{~cm} \sim$ over $10 \mathrm{~cm}$ ), tumor number ( $4 \sim$ over 20 )], liver function (Child-Pugh score 5-9) and disease etiology. Bolondi's subclassification of BCLC B stage is feasible and other groups have tried to modify the Bolondi's Subclassification; however, there are several weak points. Therefore, we have evaluated the prognostic performance of a Model to Estimate Survival In Ambulatory Patients with HCC (MESIAH) and to compare it with original BCLC $B$ subclassification and modified models. The new prognostication model MESIAH accurately stratified heterogeneous BCLC stage B and would be an effective method for facilitating per-subclass-based treatment options. However, current guidelines recommend transarterial chemoembolization (TACE) as the standard treatment of all patients with intermediate-stage HCC. Not all patients with intermediate-stage HCC may derive similar benefit from TACE, and some patients may benefit from other treatment options, which are currently approved or being explored. These include different TACE modalities, such as selective TACE or drug-eluting beads TACE and radioembolization. The introduction of immunotherapies or molecular targeted agents in the therapeutic armamentarium for HCC has provided a new therapeutic option for the treatment of BCLC-B patients who are unsuitable to TACE or in whom TACE resulted in unacceptable toxicity. In addition, clinical trials aimed at investigating the potential role of immunotherapies or molecular targeted agents in the treatment of patients with intermediate-stage HCC within combination therapeutic regimens are ongoing. 
The 9th Asia-Pacific Primary Liver Cancer Expert Meeting (APPLE 2018)

\section{S5-18 \\ CTACE vS DEB TACE}

Hojong Chun

The Catholic University of Korea, Seoul, South Korea

Transarterial chemoembolization (TACE) is the standard of care for patients with intermediate HCC, due to positive results of two randomized trial using conventional TACE (CTACE) with either doxorubicin or cisplatin. TACE has level 1 evidence and grade 1 recommendation for treatment of intermediate stage HCC with a Child-Pugh score up to B7 [1].

Lipiodol is used for CTACE, and is emulsified with chemotherapeutic drug because Lipiodol demonstrated some specific property such as selectivity for tumor, pharmacokinetic benefit, unique tagging capacity of tumor, and some degree of embolization of the arterial feeders as well as peritumor portal venules $[2,3]$. The embolic effect of Lipiodol is not sufficient, so it should be followed by particle embolization to increase rate of tumor necrosis as well as overall survival $[4,5]$. Gelatin particles are most commonly used due to their resorbabilty allowing for subsequent treatment.

Drug-eluting beads (DEB) have demonstrated reproducible loading and eluting capacity in vitro with doxorubicin, epirubicin and irinotecan. Sustained drug release and high tumor exposure have been demonstrated in an animal model $[6,7]$, and lower systemic concentration of drug than cTACE has been demonstrated in clinical studies $[8,9]$. DEB are available in various size ranging from 75 to 700 micrometer, with a tendency for smaller bead being used in clinical practice.

For HCC treatment, two randomized trial compared cTACE with DEB-TACE with either tumor response at 6 months or 2-year survival as a primary endpoint $[12,13]$. No significant superiority of DEB-TACE has been demonstrated for these two endpoints. Toxicity was significantly lower for DEB-TACE in one study, while post-procedure pain was more frequent and severe after cTACE in the other study. In contrast, biliary necrosis has been reported to be significantly higher with DEB-TACE than with cTACE [14]. This is probably due to aggressive embolization with high drug concentration in healthy liver close to the peribiliary plexus.

For further comparison between two TACE modalities for intermediate stage HCC, larger scale clinical trials with more standardized emulsion method for Lipiodol and more stratified selection for patients should be necessary. In addition, technological advances for DEB-TACE, including resorbable drug eluting beads and new loading capability for more active drugs against HCC, may influence the choice of TACE modalities for intermediate stage HCC in the future.

\section{References}

1. Llovet J, Ducreux M, Lencioni R, et al. EASL-EORTC clinical practice guidelines: management of hepatocellular carcinoma. Eur J Cancer 2012; 48:599-641.

2. Raoul JL, Heresbach D, Bretagne JF, et al. Chemoembolization of hepatocellular carcinomas. A study of the biodistribution and pharmacokinetics of doxorubicin. Cancer 1992; 70:585-90.

3. Choi JW, Cho HJ, Park JH, et al. Comparison of drug release and pharmacokinetics after transarterial chemoembolization using diverse lipiodol emulsions and drug-eluting beads. PLoS One 2014; 9:e115898.

4. Takayasu K, Shima Y, Muramatsu Y, et al. Hepatocellular carcinoma: Treatment with intra arterial iodized oil with and without chemotherapeutic agents. Radiology 1987; 162:345-51. 
5. Takayasu K, Arii S, Ikai I, et al. Overall survival after transarterial lipiodol infusion chemotherapy with or without embolization for unresectable hepatocellular carcinoma: propensity score analysis. AJR Am J Roentgenol 2010; 194:830-7.

6. Hong K, Khwaja A, Liapi E, Torbenson MS, Georgiades CS, Geschwind JF. New intra-arterial drug delivery system for the treatment of liver cancer: preclinical assessment in a rabbit model of liver cancer. Clin Cancer Res 2006; 12:2563-7.

7. Rao PP, Pascale F, Seck A, et al. Irinotecan loaded in eluting beads: preclinical assessment in a rabbit VX2 liver tumor model. Cardiovasc Intervent Radiol 2012; 35:1448-59.

8. Malagari K, Pomoni M, Moschouris H, et al. Chemoembolization of hepatocellular carcinoma with HepaSphere 30-60 mum. Safety and efficacy study. Cardiovasc Intervent Radiol 2014; 37:165-75.

9. Varela M, Real MI, Burrel M, et al. Chemoembolization of hepatocellular carcinoma with drug eluting beads: efficacy and doxorubicin pharmacokinetics. J Hepatol 2007; 46:474-81.

10. Deipolyi AR, Oklu R, Al-Ansari S, Zhu AX, Goyal L, Ganguli S. Safety and Efficacy of 70-150 mum and 100-300 mum Drug-Eluting Bead Transarterial Chemoembolization for Hepatocellular Carcinoma. J Vasc Interv Radiol 2015; 26:516-22.

11. Malagari K, Pomoni M, Kelekis A, et al. Prospective randomized comparison of chemoembolization with doxorubicin-eluting beads and bland embolization with BeadBlock for hepatocellular carcinoma. Cardiovasc Intervent Radiol 2010; 33:541-51.

12. Lammer J, Malagari K, Vogl T, et al. Prospective randomized study of doxorubicin-eluting-bead embolization in the treatment of hepatocellular carcinoma: results of the PRECISION V study. Cardiovasc Intervent Radiol 2010; 33:41-52.

13. Golfieri R, Giampalma E, Renzulli M, et al. Randomised controlled trial of doxorubicin-eluting beads vs conventional chemoembolisation for hepatocellular carcinoma. Br J Cancer 2014; 111:255-64.

14. Guiu B, Deschamps F, Aho S, et al. Liver/biliary injuries following chemoembolisation of endocrine tumours and hepatocellular carcinoma: lipiodol vs. drugeluting beads. J Hepatol 2012; 56:609-17. 
TACE Refractoriness: Definition and Treatment Options

Masatoshi Kudo

Kindai University, Osaka, Japan

In the 2010 version of the JSH consensus-based treatment algorithm, TACE failure/ refractoriness was defined assuming the use of superselective lipiodol TACE - which has been widely used worldwide and particularly in Japan-and areas with lipiodol deposition were considered to be necrotic. However, this concept is not well accepted internationally. Furthermore, following the approval in Japan in February 2014 of embolic drug-eluting beads, an embolic material that does not use lipiodol, the phrase needed to be changed from "lipiodol deposition" to "necrotic lesion or viable lesion." Accordingly, the section was revised to define TACE failure as an ineffective response after two or more consecutive TACE procedures that is evident on response evaluation CT or MRI after 1-3 months, even after chemotherapeutic agents are changed and/or the feeding artery is reanalyzed. In addition, the appearance of more lesions in the liver than the number of lesions recorded at the previous TACE procedure (other than the nodule being treated) was added definition of TACE failure/refractoriness. Following discussion of the other issues concerning continuous elevation of tumor markers, vascular invasion, and extrahepatic spread, descriptions similar to those in the previous version were approved (table 1). The revision of these TACE failure definitions were approved by more than $85 \%$ of HCC expert. 


\section{S5-20 \\ When to Switch to Systemic Therapy: Early or as Late as Possible?}

\section{Peter R. Galle}

Johannes Gutenberg University Mainz, Mainz, Germany

Patients with HCC are treated according to the stage of their disease. If diagnosed in early stage and treated in curative intent disease recurrence rates are high, requiring further downstream lines of therapy in many patients. For patients in intermediate stage TACE is standard of care followed by systemic therapy upon progress. As more systemic options become available including first, second and even third lines of therapy a timely switch from loco-regional to systemic therapy is evolving as an important challenge in the optimized treatment of patients with HCC.

Guidelines such as the updated EASL clinical practice guidelines on the management of HCC recommend systemic therapy for patients in advanced stage, typically defined by macroscopic vascular invasion and/or extra-hepatic spread or for patients still in intermediate stage but not or no longer responding to TACE, a situation described as treatment stage migration for TACE-refractory cases.

In clinical reality there are several obstacles resulting in an under-usage of systemic therapies.

1. Observational trials such as "BRIDGE" or "OPTIMIS" have demonstrated an excessive use of TACE beyond guideline recommendations and in neglect of contraindications.

Table 1. Definition of TACE Failure/Refractoriness (LCSGJ)

(1) Intrahepatic lesion

Two or more consecutive ineffective responses seen within the treated tumors (viable lesion $>50 \%$ ), even after changing the chemotherapeut ic agents and/or reanalysis of feeding artery, on response evaluation CT/MRI after 1-3 months following adequately performed selective TACE

Two or more consecutive progressions in the liver (including an increase in the number of tumors compared to that before the previous TACE procedure), even after changing the chemo therapeutic agents and/or reanalysis of feeding artery, on response evaluation CT/M RI after 1-3 months following adequately performed selective TACE

(2) Tumor marker Continuous elevation of tumor markers right after TACE even though transient minor reduc tion is observed

(3) Appearance of vascular invasion

(4) Appearance of extrahepatic spread 
The 9th Asia-Pacific Primary Liver Cancer Expert Meeting (APPLE 2018)

2. The definition of TACE failure may be difficult and patients may receive overtreatment with TACE.

3. TACE can impair liver function, particularly when applied in a nonselective way, as a result of damage of the hepatic artery. This may result in a patient condition unfit for systemic therapy

Thus, in order to give the patient a chance to benefit from systemic therapy it is important:

1. To restrict TACE to patients in intermediate stage.

2. To avoid TACE in patients with contraindications.

3. To discontinue TACE in patients with SD or PD after not more than two procedures.

4. To perform TACE as selective as possible.

\section{Conclusion:}

1. If a patient presenting with HCC in intermediate stage is well controlled (CR and PR) by TACE a late switch to systemic therapy upon progression is appropriate.

2. If, during TACE, a patient is not sufficiently responding (SD) after two procedures or progresses (PD) to advanced stage an immediate (early) switch to systemic therapy is suggested. 


\section{Session 6: Optimal Treatment Options in Locally-Advanced HCC}

\section{S6-21 \\ Selection of Good Candidates for Resection and Transplantation}

Kwang-Woong Lee

Seoul National University, Seoul, South Korea

Liver resection and liver transplantation are the curative treatments for early stage HCC. However, the treatment for locally advanced HCC has been controversial for a long time. According to Barcelona Clinic Liver Cancer (BCLC) system, transaterial chemoembolization (TACE) is recommended and liver resection is not suitable for Stage B. However, tumor situation in stage B HCC is widely ranged, researchers believe that some patients can still benefit from aggressive surgical approach. Retrospective studies demonstrated that curative resection provided better survival than TACE.

What are potentially good indication for surgical treatments in locally advanced HCC. Surgical resection would be better than TACE or RFA in selective situations such as large tumor with good biological behavior, de novo multifocal HCC confined to uni-lobar, uni-lobar intrahepatic recurrence after resection with low tumor markers. Of course, liver function should be good for resection. Liver transplantation would be better than TACE, RFA or even resection in selective situation such as de novo multifocal HCC with good biological behavior, bi-lobar intrahepatic recurrence after resection with good biological behavior regardless of liver function. Therefore, biological markers such as AFP, PIVKA, PET scan, growing speed, and so on are important to select optimal indication for surgical treatments.

Furthermore, in cases of locally advanced HCC with unknown biology, combined treatment such as TACE, TARE followed by resection or LT can be a better option than surgery only. 

Thrombosis

\author{
Jason Cheng
}

National Taiwan University, Taipei, Taiwan

Hepatocellular Carcinoma (HCC) with portal vein tumor thrombosis (PVTT), has been a challenging obstacle for multi-modality treatments. Given targeted drugs as the evidence-based treatment, the unsatisfactory response and dismal prognosis remain a barrier to disease control. The patterns of progression in HCC patients with PVTT include bulky and/or multiple intrahepatic tumors and high risk of extrahepatic metastasis, frequently with intrahepatic disease load and compromised functional reserve of liver as the survival threat. Therefore, the key to improved outcome relies on the effective control in both PVTT and intrahepatic tumors, and demands the combined treatment strategy.

Radiotherapy (RT) uses external high-energy radiation targeting on the localized tumor by inducing DNA damage and cell apoptosis, as well as the microenvironmental inflammatory reactions. RT, with its focal high intensity, has been selectively used in HCC patients with limited number of liver tumor(s) refractory to ablation and embolization. Recent Korean randomized controlled trial (RCT) compared HCC patients with macrovascular invasion treated by chemoembolization followed by RT to PVTT and liver tumor, with sorafenib as the standard treatment. The encouraging data demonstrated significantly better response and superior survival outcomes. The other ongoing RCT (RTOG1112) with similar study design is expected to confirm the outcome advantage by the combination strategy. In contrast, both trials using selective internal radiation therapy as the single modality failed to show survival benefit when compared with sorafenib. The difference between combined modalities and single treatment using radiation (external or internal) might imply the added effect of RT for bulky tumor and PVTT to the combined treatment for multiple intrahepatic disease. Similarly with the optimistic results by immunotherapy for advanced/metastatic HCC, the addition of RT for bulky tumor(s) may potentially improve the disease control and be worthy of investigation. 


\section{S6-24}

\section{Systemic and Targeted Therapies}

Yoon Jun Kim

Seoul National University, Seoul, South Korea

The global incidence of primary liver cancer has been increased during recent decades. Among the primary liver malignancies, hepatocellular carcinoma (HCC) is recognized as the most prevalent and aggressive. The locally advanced HCC was defined as the disease not amenable to surgical resection or immediate liver transplantation, and the disease should be locally advanced as defined by BCLC (B) intermediate stage or BCLC (C) advanced stage without extrahepatic spread except regional lymph node involvement. For advanced HCC, systemic therapy is frequently adopted as an important palliative method. In recent years, clinical studies and observations have often reported about systemic anti-cancer therapy of advanced HCC, including molecular target therapy, systemic chemotherapy and immunotherapy. In this lecture, these treatment modalities will be reviewed. 


\section{Session 7: Strategies to Improve Outcome after Downstaging of HCC}

\section{S7-25}

The Role of Transarterial Treatment in Downstaging of HCC Gyoung Min Kim

Yonsei University, Seoul, South Korea

Liver transplantation is the treatment of choice for cirrhotic patients with unresectable hepatocellular carcinoma within Milan criteria. Because of the organ shortage, allocation policies such as MELD score have been developed and applied to prioritize recipients based on the risk of death or severity of illness. And liver-directed therapy (LDT) is recommended if the waiting list exceeds six months to minimize drop-out by disease progression.

Unfortunately, still majority of the HCC patients is above Milan criteria at initial presentation. Thus, another application of LDT is 'downstaging'. Options for downstaging include radiofrequency ablation, transarterial chemoembolization (TACE), transarterial radioembolization (TARE), stereotactic body radiation, or a combination of therapies. By conducting downstaging treatment, we can expect decrease of tumor burden and have time to identify aggressiveness of tumor biology.

Both TACE and TARE are most commonly used LDT worldwide for downstaging. Though there is no well-designed randomized controlled study comparing treatment modalities for LDT, published articles report the success rates of downstaging range $24-77 \%$ after TACE and $11-58 \%$ after TARE. In some meta-analysis, the success rate showed no significant difference between TACE and TARE.

The patients underwent liver transplantation after successful downstaging show similar survival outcome compared with that of patients within Milan criteria initially. 


\title{
S7-26
}

\section{External Beam Radiotherapy for HCC Patients Awaiting Liver Transplantation}

\author{
Sang Min Yoon \\ University of Ulsan, Seoul, South Korea
}

Liver transplantation (LT) is the best treatment modality for patients with selected hepatocellular carcinoma (HCC) because it usually offers removal of both HCC and underlying a cirrhotic liver. However, drop-out from the waiting list due to progression can be occurred because of the shortage of donor organs. According to the American Association for the Study of Liver Diseases guidelines for the management of HCC, bridging therapies has been recommended if the expected waiting time exceeds 6 months. Therefore, the primary aim of bridging therapy is to minimize the risk of HCC progression while the patients is awaiting LT and to reduce the potential risk of post-transplant HCC recurrence. Various modalities of locoregional therapies have been employed, including transarterial chemoembolization, transarterial radioembolization, radiofrequency ablation, percutaneous ethanol injection, external beam radiotherapy (EBRT), and/or the combination of these locoregional therapies. The choice of the use of these modalities may be influenced by tumor size/location/number, background hepatic function, and the preferences of the individual center.

Another purpose of the locoregional therapies before LT is a reduction in tumor burden to predefined criteria (known as down-staging). In the living donor liver transplantation (LDLT), more flexible and expanded selection criteria for LT for HCC, in terms of tumor size and/or number, have been introduced and some large-volume medical centers have reported comparable overall survival and/or recurrence-free survival rates after LDLT according to those expanded selection criteria. Moreover, locoregional therapies attempted to reduce the size and/or number of HCCs which exceeded conventional selection criteria in several studies. The patients who achieved successful down-staging showed also comparable overall survival and/or recurrence-free survival rates.

With remarkable recent technological advances in EBRT, including fourdimensional computed tomography, intensity-modulated radiation therapy, image-guided radiation therapy, and respiratory-gated delivery, EBRT has been widely used for patients with HCCs. Here, we summarize previous studies and recent updates and discuss the future perspectives of EBRT for HCC patients awaiting LT. 


\section{S7-28}

\section{Liver Transplantation after Downstaging of HCC beyond} Milan Criteria

\section{Shin Hwang}

University of Ulsan, Seoul, South Korea

Unresectable hepatocellular carcinoma (HCC) is a common indication for liver transplantation (LT). The Milan criteria became standard criteria but expansion beyond the Milan criteria (tumor size and number) have resulted in similar post-transplant outcomes, thus suggesting LT is a viable treatment option for HCC presenting beyond the Milan criteria. There have been conflicting opinions on the best downstaging protocols, criteria for downstaging eligibility and for assessment of response. Pooled analyses have suggested success in down staging in about half of patients treated, but with higher recurrence rates than patients initially within the Milan criteria. Studies with strict inclusion criteria and mandatory waiting time before transplantation reported survival equivalent to patients who did not require downstaging. In carefully selected patients, there is a role for down staging to provide the chance of transplantation and cure, with acceptable outcomes. Patients can be successfully transplanted with HCC beyond Milan criteria, or patients beyond Milan criteria can be downstaged to within Milan criteria and achieve successful post-liver transplant outcomes. The current reliance on tumor burden (size and number) alone ignores the mounting data supporting the prognostic use of additional surrogates of tumor biology in identifying appropriate candidates. With cautious patient selection criteria and the use of locoregional therapy before LT, good results can be obtained for patients beyond Milan criteria who had no better chance other than LT. However, so far, downstaging criteria should be stated within transplantation programs and relevant decisions should be discussed by multidisciplinary teams. 


\section{Session 8: Evidence-Based Medicine in the Treatment of Advanced HCC}

\section{S8-29}

1st Line Molecular Targeted Therapy: What Is Key to Success Ann-Lii Cheng

National Taiwan University, Taipei, Taiwan

Sorafenib was the only drug available for 1st line treatment of HCC before 2017. In 2018, lenvatinib, following its approval in Japan will probably become the second drug available for 1st line treatment of HCC in the world. The reasons for failure of other pivotal drugs including sunitinib, linifanib, brivanib, and erlotinib have already been well discussed in several reviews and editorials. In summary, among all drugs, lenvatinib appears to have the most robust phase II results which suggested promising efficacy, as well as meticulous phase I results which led to a HCC-specific dosing schedule and lower toxicity.

At this point, no other phase III studies are ongoing for single-agent molecular targeted therapy in 1st treatment of HCC. Instead, a large-scale phase III study for IO-MTT (atezolizumab-bevacizumab) combination was recently activated. It is likely that MTT not only works on driver signal transduction of cancer cells, but also on pivotal signal transduction pathways of environmental immune cells, thereby modulates host immune response against cancers. This novel use of MTT, particularly in combination with IO agents, are being enthiusiastically pursued. 


\section{S8-30 \\ Second Line Molecular Targeted Therapy: Currently Available Options}

\section{Ghassan Abou-Alfa}

Memorial Sloan Kettering Cancer Center, New York, USA

Several efforts evaluating different tyrosine kinase inhibitors in the second line setting in HCC failed to show an improvement in survival. These included brivanib, ramucirumab, everolimus, add to metabolomic ADI-PEG20.

The stalled efforts were resurrected by the RESORCE trial a randomized, double-blind, global phase III trial evaluating regorafenib versus placebo in adults with HCC who had tolerated sorafenib but progressed on it. Regorafenib demonstrated improved overall survival of 10.6 months compared with 7.8 months for placebo. This already led to the approval of regorafenib for advanced HCC post progression on sorafenib.

Despite the negative outcome of the ramucirumab phase III study, in a prespecified subgroup of patients with a baseline $\alpha$-fetoprotein (AFP) concentration of $\geq 400 \mathrm{ng} / \mathrm{mL}$, median $\mathrm{OS}$ was 7.8 versus 4.2 months, respectively (HR $0.67 ; p=0.006$ ). On such basis, the follow-up REACH-2 study was launched for patients with advanced HCC with baseline AFP $>400 \mathrm{ng} / \mathrm{mL}$ with progression during or after sorafenib. A press release reported this study was a positive one. The data is yet to be reported.

$\mathrm{C}$-met has been a target of much interest in HCC. Tivantinib was studied in a phase 2 study where c-met overexpression was noted to be associated with an increased response to tivantinib. However, the phase III METIV-HCC study of tivantinib versus placebo., which was limited to patients with high c-met expression was negative. In a disagreement about the necessity of c-met expression, another c-met inhibitor cabozantinib, which targets c-met, in addition to RET, VEGFR2, AXL-1, and TIE-2 was reported in a phase III trial versus placebo and showed a median OS of 10.2 for cabozantinib vs 8.0 months for placebo.

Other than tyrosine kinase inhibitors, checkpoint inhibitors including nivolumab and pembrolizumab have shown response in the $15-20 \%$ range in limited phase II trials. Nivolumab was granted a conditional approval pending a randomized phase III trial versus sorafenib in the first line setting. 


\author{
S8-31 \\ TKI-Based Combination Therapy: The More the Better? \\ Masatoshi Kudo \\ Kindai University, Osaka, Japan
}

Hepatic arterial infusion chemotherapy plus sorafenib in phase 2 trials has shown favourable tumour control and a manageable safety profile in patients with advanced, unresectable hepatocellular carcinoma. However, no randomised phase 3 trial has tested the combination of sorafenib with continuous arterial infusion chemotherapy. We aimed to compare continuous hepatic arterial infusion chemotherapy plus sorafenib with sorafenib alone in patients with advanced, unresectable hepatocellular carcinoma.

We did an open-label, randomised, phase 3 trial (SILIUS) at 31 sites in Japan. Eligible patients were aged 20 years or older, with advanced hepatocellular carcinoma not suitable for resection, local ablation, or transarterial chemoembolisation; Eastern Cooperative Oncology Group (ECOG) performance status 0-1; Child-Pugh score 7 or lower; and adequate bone marrow, liver, and renal function. Patients were randomly assigned (1:1) via an interactive web response system with a computer-generated sequence to receive $400 \mathrm{mg}$ sorafenib orally twice daily or $400 \mathrm{mg}$ sorafenib orally twice daily plus hepatic arterial infusion chemotherapy (cisplatin $20 \mathrm{mg} / \mathrm{m}^{2}$ on days 1 and 8 and fluorouracil $330 \mathrm{mg} / \mathrm{m}^{2}$ continuously on days 1-5 and 8-12 of every 28-day cycle via an implanted catheter system). The primary endpoint was overall survival. The primary efficacy analysis comprised all randomised patients (the intention-to-treat population), and the safety analysis comprised all randomised patients who received at least one dose of study treatment. This trial is registered with ClinicalTrials. gov, number NCT01214343.

Between Nov 4, 2010, and June 10, 2014, 206 patients were randomly assigned (103 to the sorafenib group, 103 to the sorafenib plus hepatic arterial infusion chemotherapy group). One patient in the sorafenib plus hepatic arterial infusion chemotherapy group withdrew after randomisation. Median overall survival was similar in the sorafenib plus hepatic arterial infusion chemotherapy $(\mathrm{n}=102)$ and sorafenib monotherapy $(\mathrm{n}=103)$ groups $(11.8$ months [95\% CI 9.1-14.5] vs 11.5 months [8.2-14.8]; hazard ratio 1.009 [95\% CI $0.743-1.371$ ]; $p=0.955$ ). Grade 3-4 adverse events that were more frequent in the sorafenib plus hepatic arterial infusion chemotherapy group than in the sorafenib monotherapy group included anaemia (15 [17\%] of 88 vs six [6\%] of 102), neutropenia (15 [17\%] vs one [1\%]), thrombocytopenia (30 [34\%] vs 12 [12\%]), and anorexia (12 [14\%] vs six [6\%]).

Addition of hepatic arterial infusion chemotherapy to sorafenib did not significantly improve overall survival in patients with advanced hepatocellular carcinoma. 


\author{
S8-32 \\ New Promising Targets under Clinical Trials \\ Baek-Yeol Ryoo \\ University of Ulsan, Seoul, South Korea
}

Hepatocellular carcinoma (HCC) develops in the condition of uncontrolled growth of hepatic cells caused by the genetic alterations in complex signaling cascades. Therefore, we can expect the effect of targeted agents attacking important targets in carcinogenic pathways along with minimizing general adverse effects. However, we have failed to find the dominant signal pathways in hepatocarcinogenesis, yet.

Sorafenib is a multikinase inhibitor especially inhibiting serine/threonine kinases Raf-1/B-Raf, tyrosine kinases VEGFR-2/-3 and PDGFR- $\beta$, which suppresses cancer progression and angiogenesis. Sorafenib is the first systemic therapy prolonged survival significantly in HCC patients. After that sorafenib not only established a proof of concept for the use of multi-kinase inhibitors strategy for the treatment of HCC, but also is the reference standard for systemic therapy for HCC patients.

Nevertheless, subsequent clinical trials using targeted agents have failed in succession for about 10 years. The agents which failed to prove their activity in HCC were sunitinib, linifanib, brivanib, nintedanib in the first-line treatment and axitinib, ramucirumab, brivanib, erlotinib, everolimus, tivantinib, ADI-PEG20 in the second-line treatment after sorafenib failure.

Recently, lenvatinib, another angiogenesis inhibitor, showed non-inferior overall survival in the 1st-line treatment compared with sorafenib in the REFLECT trial. Lenvatinib also showed rather superior response rate, progression free survival, time to progression and preferable toxicity profiles.

For the patients who failed sorafenib treatment, regorafenib and cabozantinib, mainly angiogenesis inhibitors overcame placebo control in RESORCE and CELESTIAL trials.

In addition, nivolumab showed meaningful results for HCC in phase II CheckMate 040 trial. We have now 2 options in the 1 st-line and 3 options after sorafenib failure in HCC, with angiogenesis inhibitor or immune checkpoint inhibitor. However, the current effects of systemic treatments for HCC are far from satisfaction as ever. By looking at the trials currently conducted for HCC, we are discussing ways to improve the prognosis of HCC patients. 


\section{Session 10: New Kids on the Block: Immuno-Oncology in HCC}

\section{S10-33 \\ Basic Concepts of Immune Cells and Immunologic Targets in HCC}

Eui-Cheol Shin

Korea Advanced Institute of Science and Technology (KAIST), Daejeon, South Korea

During immune responses, antigen-specific T cells are regulated by various mechanisms including inhibitory receptors and regulatory $\mathrm{T}$ cells to avoid excessive and persistent immune responses. These regulatory mechanisms, called 'immune checkpoint', suppress T cell responses particularly in chronic viral infection and cancer, in which viral antigens or tumor antigens persist for a longtime, and lead to $\mathrm{T}$ cell exhaustion in patients with chronic viral infection or cancer. Among them, cytotoxic T lymphocyte-associated protein 4 (CTLA-4) and programmed cell death 1 (PD-1) are the most well-known receptors and have been targeted for drug development. As a result, anti-CTLA-4 and anti-PD-1 (or anti-PD-L1) blocking antibodies were developed for cancer treatment and known as 'immune checkpoint inhibitors'. However, anti-CTLA-4 and anti-PD-1 (or anti-PD-L1) blocking antibodies fail to control tumors in a significant proportion of cancer patients. Therefore, it is an important question how the coverage of immune checkpoint inhibitors can be extended to the majority of cancer patients who do not have control or regression of their cancer. In this lecture, basic principles of $\mathrm{T}$ cell exhaustion and reinvigoration and the present and future of immuno-oncology will be discussed. 


\section{S10-34 \\ Mechanisms of Immune Escape}

\section{Tim Greten}

National Cancer Institute, Bethesda, USA

Immunotherapy has gained a lot of interest in the context of HCC. The basic idea of currently employed immune based treatment approaches is to activate tumor specific immune responses using immune checkpoint inhibitors. However, this approach dismisses multiple mechanism, which tumors have developed to escape immunosurveillance and to suppress anti-tumor immunity. There are different mechanism inducing immune escape mechanism: (1) Tumor-dependent (intrinsic) mechanisms, (2) local factors in the tumor microenvironment such as stress dependent mechanism or diet induced mechanisms (NASH) and (3) treatment induced mechanism (checkpoint inhibitors or CIK cells). I will present data from our preclinical research studies providing examples for all three mechanisms: CD4+ regulatory T cells are present in HCC and suppress adaptive immune responses [1]. Myeloid derived suppressor cells are increased in HCC, suppress T cell and NK cell responses [2, 3]. We found that the effect of stress induced MDSC in the liver have different effects on immunosurveillance dependent on tumor stage [4].

Recently we studied the effect of immune based approaches such as the use of immune checkpoint inhibitors and CIK therapy on escape mechanisms. Here we noticed an IFN-gamma dependent upregulation of ID01 in response to antiCTLA4 and anti-PD1 therapy. IDO blockade dramatically increased the effect of single agent checkpoint blockade. CIK therapy of established tumors led to accumulation of MDSC, which impair the efficacy of CIK cells. Using a PGE5 inhibitor we were able to show that it is not only possible to specifically target MDSC in HCC, but also improve the anti-tumor efficacy of CIK therapy.

\section{References}

1. Ormandy LA, Hillemann T, Wedemeyer H, Manns MP, Greten TF, Korangy F. Increased populations of regulatory T cells in peripheral blood of patients with hepatocellular carcinoma. Cancer Res. 2005;65(6):2457-2464.

2. Hoechst B, Voigtlaender T, Ormandy L, et al. Myeloid derived suppressor cells inhibit natural killer cells in patients with hepatocellular carcinoma via the NKp30 receptor. Hepatology. 2009;50(3):799-807.

3. Hoechst B, Ormandy LA, Ballmaier M, et al. A new population of myeloid-derived suppressor cells in hepatocellular carcinoma patients induces CD4(+)CD25(+) Foxp3(+) T cells. Gastroenterology. 2008;135(1):234-243.

4. Eggert T, Wolter K, Ji J, et al. Distinct Functions of Senescence-Associated Immune Responses in Liver Tumor Surveillance and Tumor Progression. Cancer Cell. 2016;30(4):533-547. 


\section{S10-35}

\section{Current Role of Immune Checkpoint Inhibitors in HCC}

\section{Namiki Izumi}

Musashino Red Cross Hospital, Tokyo, Japan

Currently, efficacy and safety of immune checkpoint inhibitors are evaluated in clinical trials for advanced stage of hepatocellular carcinoma (HCC), Among them, nivolumab showed objective response of $20 \%$ and pembrolizumab achieved progression-free survival of 4.8 months, and overall survival for 6 months was $77.9 \%$. However, response rate of immune-checkpoint inhibitor mono-therapy is not satisfactory. Combination with anti-PD-1 of durvalumab plus tremelimumab was reported and the response was slightly weak. Recently, tyrosine kinase inhibitor (TKI) has been suggested to induce down regulation of immune inhibitory receptors such as PD-1 or Lag3, and immune stimulatory cytokines such as IFN $\gamma$ and IL12 are up-regulated. Based on this mechanism, antitumor activity with PD-1 and PD-L1 inhibitors is improved. Thus, TKI has been suggested to cause immune stimulating tumor microenviroment. Promissing results with lenvatinib and pembrolizumab was reported. Moreover, PD-L1 of atezolizmab pus anti-VEGF of bevacizumab combination phase $1 \mathrm{~b}$ study showed promising results. In the patients who showed PR, serum AFP level has dropped within three months. Combination with IO and VEGFR has shown slightly weak results and HCC-specific immune microenviroment and treatment are under exporation. 


\section{S10-36 \\ Ongoing Clinical Trials of Immuno-Oncology in HCC}

Tae-You Kim

Seoul National University, Seoul, South Korea

Hepatocellular cancer (HCC) develop through the sequence of chronic inflammation, regeneration, and fibrosis regardless of its etiology. Deregulation of immunological network causes immune tolerance and suppression in tumor microenvironments during the carcinogenesis. The promise of immunotherapy seen in other tumors is also observed in HCC. For example, nivolumab, a programmed cell death protein-1 (PD-1) immune checkpoint inhibitor, has shown promising anti-tumor activity with ORR of $18.6 \%$ and mOS of 15.6 months in advanced HCC who had been treated with sorafenib (CheckMate 040). These results have led to the design of a phase III clinical trial directly comparing sorafenib and nivolumab and its results will be available by the end of this year (CheckMate 459). Anti-PD-1 antibody pembrolizumab also showed similar efficacy with ORR of 16.3\% (KEYNOTE 394). On Sep 2017, US FDA granted accelerated approval to nivolumab as a second line therapy after sorafenib failure.

Based on these promising clinical trials, 3 strategies of clinical investigation are currently being tested to evaluate the utility of immune checkpoint inhibitor as combination therapy.

First, various phase II trials are underway to see the combination of PD1/ PD-L1 blockade with other immunotherapies. Inhibition of PD-1 and PD-L1 only cannot stimulate cancer immunity in the absence of CD8+ T cells. Concurrent use of anti-CTLA-4 antibody results in CD8+ T cell proliferation, thus enhancing antitumor effects. In phase II trial, combined anti-PD-L1 antibody durvalumab and anti-CTLA-4 antibody tremelimumab in HCC showed ORR of $25 \%$, and phase III trial of this combination as a first line therapy is currently ongoing.

Secondly, PD1/PD-L1 blockade is being tested with targeted agents such as bevacizumab or sorafenib. Anti-PD-L1 antibody atezolizumab can restore anticancer immunity, through T-cell mediated cancer cell killing, and this is further enhanced through bevacizumab's VEGF-mediated immunomodulatory effects. In G030140 phase IB trial, combination of atezolizumab and bevacizumab showed promising early efficacy in patients with advanced HCC with ORR of $61 \%$, and phase III trial comparing atezolizumab and bevacizumab versus sorafenib as a first line therapy is currently ongoing.

Third, combination with loco-regional therapies are also being investigated since TACE or RFA can activate tumor immunogenicity by releasing tumor-associated antigen and by inducing T-cell migration to metastasis. It is expected that subsequent administration of anti-PD-1 antibody may control small intrahepatic metastatic nodules.

Immunotherapy is a rapidly evolving field in HCC. The results of ongoing clinical trials with combination strategies may drastically change the treatment landscape for HCC. In the era of precision medicine, the identification of clinical and immune biomarkers for immunotherapy is also crucial for precise immunotherapy. 


\title{
Session 11: Emerging Innovative Approaches in HCC Management
}

\author{
S11-37 \\ Clinical Results and Future Direction of Particle Therapy for \\ HCC
}

Testuo Akimoto

National Cancer Center Hospital East, Kashiwa, Japan

The standard treatment option for hepatocellular carcinoma (HCC) are consisted from surgery, transcatheter arterial embolization (TAE), radiofrequency ablation (RFA) and hepatic transplantation according to the stages, liver function and tumor locations. Photon radiation therapy (RT), especially stereotactic body RT, is also a treatment option although indication is limited due to excessive dose to the normal liver. Proton beam therapy (PBT) enables us to deliver more conformal radiation to the target due to its unique physical character called as Bragg Peak. The results of the phase II study in National Cancer Center Hospital East (NCCHE) demonstrated that a 2-tear local control rate of $96 \%$ in treatment of $76 \mathrm{GyE}$ in 20 fractions, and a favorable liver function reserve that was determined by the ICG R15 was related to good overall survival. The similar treatment outcomes have been reported from the Tsukuba University. Main advantage of PBT compared with surgery is non-invasive nature of PBT, indicating that PBT can be applied for patients with older ages or who are not suited for surgery due to commodities. In Japan, more than 4000 new patients were treated with PBT in 2017, and about $20 \%$ of all patients were HCC. Hence, particle therapy including PBT is considered to be an established treatment approach for HCC, although there have been no prospective trials evaluated the efficacy or local control compared with that after surgery. From these backgrounds, we are now conducting the multi-institutional study that compared survival rate between PBT and surgery in the patients with resectable HCC (Non-randomized controlled study comparing proton beam therapy and hepatectomy for resectable hepatocellular carcinoma (JCOG1315C: SPRING study). The main eligibility criteria are as follows: 1) primary, solitary, and nodular resectable HCC which is diagnosed by dynamic CT/MRI or biopsy, 2) no metastatic lesions (cN0M0) (7th UICC classification), 3) maximum diameter of tumor; $3 \mathrm{~cm}-12 \mathrm{~cm}, 4) 2 \mathrm{~cm}$ or more distant from intestines 5) no ascites and hepatic encephalopathy with Child-Pugh score of 5, 6) 20 to 79 years of age, 7) ECOG performance status 0 or 1, 8) no prior treatment for HCC, 9) adequate organ functions, 10) written informed consent. Treatment modality (surgical resection or PBT) is selected based on pt's preference, and the inverse probability of treatment weighting method using propensity score is applied to adjust for selection bias between treatment modalities. The primary endpoint is overall survival, and the secondary endpoints include progression-free survival, incidence of acute and late adverse events (grade>=3), non-reduction rate of QOL by EQ-5D, qualityadjusted life year (QALY), and incremental cost-effectiveness ratio (ICER). We assumed a 5-year survival both in surgical resection and PBT of 55\%. The sample size was calculated as a total of 290 (expected ratio of the number of pts between SR and PBT is 2.5:1) by setting non-inferiority margin of hazard ratio of 1.50 , with a one-sided alpha of $5 \%$ and power of $75 \%$; planned accrual 
The 9th Asia-Pacific Primary Liver Cancer Expert Meeting (APPLE 2018)

period and follow-up period are 6 years and 5 years. Primary analysis will be conducted using propensity score analysis. Accrual has started from Jun 2017 and 2 pts are enrolled as of Feb 2018.

In this symposium, we will present advantages and effectiveness of PBT for patients with HCC, and also we summarize the current status of PBT in the treatment of HCC. 


\section{S11-38}

\section{Novel Immunotherapy Strategies: Immune Cell Therapy in} HCC

Jung-Hwan Yoon

Seoul National University, Seoul, South Korea

Hepatocellular carcinoma (HCC) is an aggressive cancer and can recur even when tumors are completely removed. Effective treatments for advanced stage HCC are still lacking. Cell-based immunotherapy is an attractive therapy for HCC with few toxicities. Several strategies including adoptive transfer of autologous activated $\mathrm{T}$ lymphocytes, natural killer (NK) cells, autologous tumor-pulsed dendritic cells, and cytokine-induced killer (CIK) cells have been tested in human clinical trials. A recent randomized controlled trial demonstrated that adjuvant immunotherapy with CIK cells prolonged both recurrence-free and overall survivals of patients with HCC receiving curative treatment. Moreover, significant gain in recurrence-free and overall survival by adjuvant CIK cell immunotherapy was maintained for over 5 years without boosting. However, tumor response rates are still limited because immunosuppressive factors or cells like myeloid-derived suppressor cells (MDSCs) interfere with the effects of immunotherapy. The combination of therapeutic regimens that both amplify tumorspecific immunity and counteract these immunosuppressive mechanisms are expected to strongly improve clinical outcomes of cell-based immunotherapy for HCC patients. 


\section{S11-39 \\ Targeting HCC with Cancer Vaccine \\ Seung Kew Yoon \\ The Catholic University of Korea, Seoul, South Korea}

Recently, immunotherapy for cancer has been developing remarkably through various approaches. In particular, therapeutic cancer vaccines, which are designed to induce robust tumor-specific T-cell immune responses, have the potential to cover the mainstream of refractory cancer treatment in the future. The reason for the rapid development of these cancer vaccines in recent years is the systemic discovery of tumor-specific neoantigens through high-throughput technologies such as next-generation sequencing (NGS) and novel bioinformatics. Tumor antigens, which are produced in tumor cells can trigger an immune response in the host, can be useful tumor biomarker and potential target for cancer vaccine. Tumor antigens are classified into two types according to their expression pattern: tumor-associated antigens (TAA), which are overexpressed in tumor cells but also expressed in some normal cells and tumor-specific neoantigens arising from mutations that alter amino acid coding sequences are expressed only on tumor cells but not in other normal tissues and thus can be ideal target for T-cell based immunotherapy. However, tumor-specific neoepitopes are different between individuals, personalized cancer vaccine strategies are required.

There are four components to generate a successful cancer vaccine: tumorspecific neoantigens, formulations, immune adjuvants and efficient delivery system. Recent clinical trials of cancer vaccines using neoantigen in different tumors have been actively initiated. These include peptide-based vaccines, RNAor DNA-based vaccines and a peptide-loaded DC vaccine.

Hepatocellular carcinoma (HCC) is the third leading cause of death from cancer globally. Hepatocarcinogenesis is very complex and occurs through a multistep biological process during malignant transformation of normal hepatocytes in which various factors, including genetic and epigenetic alterations, are involved. Specifically, recent advances in NGS technologies have facilitated a more profound understanding of the molecular mechanisms of HCC, which have contributed to the development of targeted therapies for cancers by identifying genes and associated signaling involved in carcinogenesis and tumor progression. Despite these advances, it remains difficult to effectively treat advanced HCC because most advanced cases are accompanied by poor liver function and liver cirrhosis. Surgical approaches, including resection and liver transplantation, are not available in these cases, so molecular targeted therapy combined with immunotherapy has become an alternative strategy to prolong patient' survival. Recent data suggest that the microenvironment of HCC has immunosuppressive features due to chronic nature of the disease and tolerogenic liver properties. The factors involved in the immune tolerance of HCC include exhausted T-cell dysfunction, up-regulated function of $\mathrm{T}$ regulatory cells (T-reg), abundance of inhibitory myeloid cells or myeloid-derived suppressor cells (MDSC) and liver resident macrophage or Kupffer cells. Among various immunotherapy for HCC, the strategy that inhibit immune suppressive microenvironment of HCC may be promising. To date, several clinical trials of therapeutic vaccine for HCC have been conducted worldwide. These cancer vaccines are designed to target TAA which are overexpressed in cancer cells of HCC and thus the targets include 
The 9th Asia-Pacific Primary Liver Cancer Expert Meeting (APPLE 2018)

alpha-fetoprotein (AFP), glypican-3 (GPC3), melanoma-associated antigen (MAGE), NY-ESO-1, carcinoembryonic antigen, heat shock protein gp90 and human TERT. However, unlike to melanoma, the clinical efficacy of cancer vaccine in HCC has been limited even though it is safe and well tolerated. These unsatisfactory clinical outcomes may be due to the failure to identify a tumorspecific and immunogenic neoantigens in HCC. Also, immunosuppresive microenvironment of underlying liver disease may have contributed to an unsuccessful outcome of different clinical trials. Therefore, in order to achieve maximize the clinical effectiveness of therapeutic cancer vaccine for HCC, several key components that should be considered, include discovery of novel tumor-specific neoantigens, development of efficient neoantigen delivery system and combination with immune checkpoint inhibitors. Thus, the personalized cancer vaccine as one of immunotherapeutic approaches for advanced HCC is just the beginning, this approach has evolved to open up a new era of anti-cancer therapy in the near future. 


\title{
Parallel Session 1: Lifestyles, NAFLD, and HCC
}

\author{
PS1-1 \\ Global Burden of NAFLD and Its Association with Liver \\ Cancer
}

Vincent Wong

The Chinese University of Hong Kong, Hong Kong SAR, China

Nonalcoholic fatty liver disease (NAFLD) is currently the most common chronic liver disease, affecting at least $25 \%$ of the general adult population globally. In the United States, NAFLD has already become the third leading cause of end-stage liver disease and hepatocellular carcinoma (HCC). By 2030, the incidence of liver decompensation and HCC secondary to NAFLD is projected to increase by $168 \%$ and $137 \%$, respectively. As treatments for chronic viral hepatitis become readily available, the relative importance of NAFLD is expected to increase over time.

There is substantial difference in disease burden and severity across countries. This is likely due to a mixture of genetic and environmental factors. For instance, the minor allele frequency of PNPLA3 rs 430397 (patatin-like phospholipase domain-containing protein 3) is highest in Mexicans and Hispanics and lowest in African Americans, mirroring the prevalence and severity of NAFLD among these ethnic groups. Interestingly, this gene polymorphism is more common in East Asians than Caucasians, which may explain the relatively high prevalence of NAFLD in East Asians despite a lower metabolic burden. Asians also develop NAFLD and metabolic complications at a lower body mass index, thus explaining the condition of non-obese NAFLD in this population. It is also important to recognize that different regions and ethnic groups have different dietary habits, physical activity levels and socioeconomic environments, all interacting to shape the local epidemiology and disease burden.

At present, NAFLD-associated HCC remains uncommon in Asia. Other than metabolic risk factors, one important factor is the duration of disease. As many Asians only develop NAFLD and obesity later in life, they may not have sufficient time to progress to cirrhosis and HCC. Nonetheless, with childhood obesity starting to affect Asia, the natural history of NAFLD in this region will likely change in the coming decades.

Compared with other etiologies, NAFLD-associated HCC appears to occur more often in non-cirrhotic patients. This is partly due to shared risk factors between NAFLD and HCC. This may also be due to misclassification of some cases as cryptogenic cirrhosis and the different progression rate between NAFLD and other liver diseases. The bottom line is we need to develop better tools to predict and detect NAFLD-associated HCC in the non-cirrhotic population.

In Asia, chronic hepatitis B remains the leading cause of HCC. Longitudinal studies have shown that obesity and diabetes are important risk factors of cirrhosis and HCC in patients with chronic hepatitis B. Chronic hepatitis B patients with obesity and diabetes are also less likely to have reversal of cirrhosis after antiviral therapy. This suggests that metabolic factors and possibly fatty liver play an important role in shaping the natural history of other liver diseases. The role of metabolic management in such patients deserves further evaluation. 


\section{PS1-2 \\ Clinical Patterns and Surveillance Strategy for NAFLD-Related HCC}

\section{Etsuko Hashimoto}

Seibu Railway Company Health Support Center, Tokyo, Japan

The obesity pandemic has caused a sharp rise in the incidences of NAFLD worldwide. Thus, NAFLD has become the most rapidly increasing cause of cirrhosis and HCC. Epidemiological studies on the etiology of HCC in Japan showed that NAFLD accounts for $2-20 \%$ of all cases of HCC and is the most rapidly increasing cause of HCC in both the percentage and number of cases. Changes in the percentage of underlying chronic liver disease in cirrhosis and HCC have also been largely affected by the introduction of effective therapy against hepatitis $C$ and hepatitis $B$ virus infection. As with other liver diseases, cirrhosis, advanced age and male sex are the most important risk factors for HCC in NAFLD. In addition, obesity, diabetes, and patatin-like phospholipase domaincontaining-3 gene polymorphism (which is associated with the development and progression of NAFLD/NASH) increase the risk for HCC.

NAFLD is associated with an increase in the standardized mortality ratio compared with the general population due to an increased rate of cardiovascular and liver-related mortality. The most common causes of death in patients with NAFLD are cardiovascular disease or malignancy, followed by liver-related disease. Overall, NAFLD appears to be slowly progressive, and development of HCC and liver-related mortality are minor among patients with NAFLD or NASH with mild fibrosis. However, the population-attributable fraction of the burden of NAFLD-related HCC is substantial, because a quarter of the global population is affected by NAFLD.

However, in cirrhotic NAFLD patients, the situation is completely different. The most common cause of death is liver failure and development of HCC is the most important risk factor for mortality. Previous studies have reported that patients with cirrhotic NASH showed survival rates similar to those of patients with hepatitis $\mathrm{C}$ virus-related cirrhosis, although the rate of development of HCC was lower (5-year HCC development rate: about $10 \%$ in cirrhotic NASH, 35\% in hepatitis $\mathrm{C}$ virus-related cirrhosis).

There have been several reports described HCC arising in patients with NAFLD/NASH. NAFLD-related HCC has mostly been detected by outside surveillance, and was larger and showed a more infiltrative pattern at the time of diagnosis. The mean age at diagnosis was around 70 years, with male predominance. Compared to those with HCC of other etiology, more patients were complicated by obesity, diabetes, hypertension, and cardiovascular disease, and had lower levels of transaminases. Non-cirrhotic NASH accounts for $10-75 \%$ of NASHrelated HCC. However, further studies are required to clarify the true incidence of HCC arising from non-cirrhotic NASH because the diagnosis of NASH is based on histology and can lead to selection bias. Moreover, end-stage cirrhotic NASH cannot be diagnosed due to its "burned out" histology. One-third of patients with NAFLD-related HCC showed elevation of alpha-fetoprotein levels and more than half of those with NAFLD-related HCC showed elevation of des-gamma-carboxy prothrombin levels. These tumor marker elevation patterns are opposite to those in HCC of other etiology. Histologically, moderately differentiated HCC with trabecular type was the most common pattern. NAFLD-related HCC may be of 
The 9th Asia-Pacific Primary Liver Cancer Expert Meeting (APPLE 2018)

multicentric origin, similar to HCC based on viral hepatitis. These patients had similar outcomes compared to those with other etiology (5-year recurrence rate; $69 \%$ and 5-year survival rate; 55\%).

Interestingly some NAFLD-related HCC showed peculiar features. Steatohepatitic HCC (SH-HCC) and some patients showed benign outcomes, even if they had large tumors. SH-HCC is a newly proposed concept, with histological features of steatohepatitis in the HCC lesion, and is strongly associated with metabolic syndrome and steatosis/steatohepatitis in noncancerous lesions. Prevalence of SH-HCC is significantly higher in NAFLD-related HCC than in HCC of other etiology. A total of 108 biopsy-proven NAFLD-related HCC patients were diagnosed at our university hospital (male, 65\%; median age, 71 years). Prevalence of SH-HCC was $20 \%$. Among these patients, 6 were extremely obese and developed HCC at age less than 50 years; 4 of the 6 had cirrhosis and 4 had benign outcomes after curative treatment, despite having large HCCs.

There are no special treatments for NAFLD -related HCC. Ultrasonography every 4-6 months is the most practical screening modality, although diagnosis with ultrasonography in obese patients and those with fatty liver is difficult.

In conclusion, regular screening for HCC is extremely important in patients with cirrhotic NAFLD patients and patients with HCC even after curative treatment. There are no special treatments for HCC based on NAFLD. However, patients should be checked for cardiovascular complications due to high complication rates. Outcomes are similar to those in HCC of other etiologies. Interestingly, some NAFLD-related HCC cases show characteristic features of HCC, i.e., SH-HCC and benign outcomes. There is an urgent need to elucidate the pathogenesis and clinical features of NAFLD-related HCC. 


\section{PS1-3 \\ Carcinogenesis and Therapeutic Strategy for NASH-Induced HCC}

Kenichi Ikejima

Juntendo University, Tokyo, Japan

The epidemiological surveys have revealed that the proportion of hepatocellular carcinoma (HCC) based on non-viral chronic liver diseases gradually increases, although the majority of HCC develops in patients of chronic viral hepatitis especially in Asian countries including Japan. Non-alcoholic fatty liver disease (NAFLD) is now the most prevalent chronic liver disease, especially in the developed countries worldwide. Nonalcoholic steatohepatitis (NASH), a progressive form of NAFLD, also develops HCC in the advanced phase. Nowadays, we sometimes encounter patients with NASH bearing large size HCCs, due to lack of proper screening strategies. Since metabolic syndrome-related NASH appears to be increasing, it would be predictable that NASH takes the place of viral hepatitis in terms of the major cause of HCC even in Asian countries including Japan in future. From the viewpoint of pathophysiology, the mechanisms underlying the hepatic carcinogenesis in steatohepatitis most likely involve metabolic abnormalities including altered reaction to insulin, excessive cellular stress/ damage responses, and impaired immune surveillance. Susceptibility to fatty liver disease is genetically determined by polymorphisms of lipid metabolizing molecules such as PNPLA3 and TM6SF2. Lipotoxicity and cellular stress responses involving ER stress, oxidative stress, and abnormalities in autophagy contribute to hepatocellular injury/cell death. Innate immune responses triggered by gutderived pathogen-associated molecular patterns (PAMPs) and damage-associated molecular patterns (DAMPs) elicits release of cytokines and reactive oxygen species (ROS) from macrophages/leukocytes, leading to extended tissue damages and fibrogenesis. Hepatocellular carcinogenesis in steatohepatitis is closely related to the damage of hepatocellular organelle/chromosome caused by excessive oxidative stress. Further, hyperinsulinemia and altered adipokine balance most likely facilitate tumor growth and angiogenesis. Moreover, impairment of hepatic macrophages and innate lymphoid cells in steatohepatitis lead to dysregulation in immune surveillance of tumors. Therefore, the potential targets for chemoprevention of NASH-induced HCC include a wide variety of pathophysiological basis. 


\section{PS1-4}

Toward Risk Reduction in Liver Cancer: Lifestyles, Coffee, Statins, Metformin, Aspirin, and NSAIDs

\section{Jin-Wook Kim}

Seoul National University, Seongnam, South Korea

Identification of risk factors is crucial for prevention, surveillance and early diagnosis of hepatocellular carcinoma (HCC). In addition to etiological factors such as liver cirrhosis, alcohol, and chronic viral hepatitis, and demographic factors such as age and sex, non-conventional risk factors are being reported. Genetic, environmental, occupational, lifestyle related and healthcare associated factors affect the risk of HCC [1]. Metabolic risk factors including obesity and diabetes increasingly contribute to the risk worldwide [2-4]. Risk factors may interact with each other, and antiviral treatment may modify the risks. Multivariate analysis with risk-measuring nomogram may incorporate multiple risk factors into single risk stratification score. Risk-based personalized approach may enhance the efficiency of surveillance and improve the prognosis of HCC.

\section{References}

1. Abdel-Hamid NM. Recent insights on risk factors of hepatocellular carcinoma. World J Hepatol 2009;1:3-7.

2. Chen CL, Yang HI, Yang WS, et al. Metabolic factors and risk of hepatocellular carcinoma by chronic hepatitis B/C infection: a follow-up study in Taiwan. Gastroenterology 2008;135:111-21.

3. Marrero JA, Fontana RJ, Fu S, et al. Alcohol, tobacco and obesity are synergistic risk factors for hepatocellular carcinoma. J Hepatol 2005;42:218-24.

4. Streba LA, Vere CC, Rogoveanu I, et al. Nonalcoholic fatty liver disease, metabolic risk factors, and hepatocellular carcinoma: an open question. World J Gastroenterol 2015;21:4103-10. 


\section{Parallel Session 2: Recent Progress in HCC Resection and Transplantation}

\section{PS2-5 \\ Laparoscopic Living Donor Hepatectomy}

Gyu-seong Choi

Sungkyunkwan University, Seoul, South Korea

Living donor liver transplantation (LDLT) became an accepted treatment modality for end-stage liver disease and hepatocellular carcinoma. By shortening the gap between demand and organ availability, it not only offered a survival benefit, but also reduced waiting time for recipients. Despite advancement in surgical technique, donor morbidity still remains significant and acts as the greatest barriers. Moreover, cosmetic and functional demand of donor is increasing. In this regard, minimally invasive surgical techniques such as laparoscopic surgery have been proposed to lessen physical and psychological burden.

The first report on pure laparoscopic donor hepatectomy in liver transplantation was performed for an adult to pediatric LDLT in 2002. Afterwards, consistent reports on pure laparoscopic left lateral sectinectomy have demonstrated safety and similar graft results compared to open technique. However, right lobe grafting is preferred over the left lobe grafting in adult-to-adult LDLT because the size of graft acts as one of the most important factors in the recipient's outcome.

The pure laparoscopic living donor right hepatectomy (LDRH) program at our institution began in 2013. From May 2013 to G.S. Choi and C.D. Kwon have performed consecutive 91 cases of pure LDRH and 197 cases of open donor right hepatectomy (ODRH). Procedure of pure LDRH requires a significant experience in both LDLT and laparoscopic liver surgery, and has not been widely performed even in large volume centers. Therefore, pure LDRH is not yet standardized owing to limited reports on clinical outcome and surgical risk factor. To the best of our knowledge, this is the first study to compare, using propensity score matching analysis, the incidence and severity of complications, and graft result of pure LDRH to ODRH.

The aims of this study were 1) to describe our institutional technique of pure LDRH, 2) to compare clinical outcomes of pure LDRH and ODRH in various aspects, and evaluate clinical feasibility of LDRH, 3) to analyze surgical risk factors of pure LDRH. This is the first study not only to compare clinical outcome but also to evaluate risk factors which are particular to pure LDRH. 


\section{PS2-6 \\ Robotic Donor Hepatectomy \\ Gi Hong Choi \\ Yonsei University, Seoul, South Korea}

Living donor right hepatectomy is one of the complex liver resections because it requires the meticulous dissection of the liver hilum and the preservation of V5 and V8. Laparoscopic donor right hepatectomy has been performed in a few centers by expert surgeons. However, it is still a challenging procedure to many donor surgeons. Compared to laparoscopic hepatectomy, the robotic hepatectomy has the advantages in the meticulous dissection of the liver hilum, the posterior side of the right liver and the inferior vena cava.

However, because of limitations of current available instruments, parenchymal transection is a challenging procedure. To facilitate robotic parenchymal transection, we have established unique techniques for parenchymal transection, named rubber band traction method. The elastic power of rubber band can automatically expose parenchymal transection plane. In addition, all three robotic instruments can be used during parenchymal transection. During parenchymal transection, Harmonic scalpel is mounted on the left hand and Maryland forceps on the right hand. These traction and transection method facilitate parenchymal transection with current available robotic instruments.

Fluorescence was incorporated into the Da Vinci HD System in 2010. Now, the robotic "firefly" system is approved for vascular and biliary imaging. But, there are other several applications of ICG such as assessment of tissue and organ perfusion, LN mapping and real-time identification of lesions. During living donor hepatectomy, ICG is used for fluorescence cholangiography and identification of the border of the left and right liver. $5 \mathrm{mg}$ ICG is injected into a peripheral vein just after temporary occlusion of the right hepatic artery and portal vein and ICG is immediately accumulated in the left liver, which provide a clear border between the right and left liver. In 2-3 hours later, ICG cholangiography is available because it is secreted into the bile, allowing visualization of the biliary anatomy approximately 45-60 min.

From March 2016 to January 2018, thirty liver donors received robotic hepatectomy (29 right grafts and one left lateral graft) in our institute. Clinical characteristics and perioperative outcomes are analyzed in 29 patients who underwent robotic donor right hepatectomy. The median age was 22 years (range, 17-50) and 13 donors (44.8\%) were female. The median BMI of all donors was 21.7 (range, 18.6-26.8). The median total liver volume and graft volume were $1130 \mathrm{ml}$ (range, 936-1480) and $695 \mathrm{~mL}$ (range, 517-919), respectively. The median operative time and blood loss were 506 min (range, 353-717) and $100 \mathrm{ml}$ (range, 30-320 m), respectively. The median warm ischemic demarcation time was 13 mins (range, 7-24). There was no perioperative transfusion. The first case was converted to mini-laparotomy (3.4\%) due to injury to the left bile duct and Roux-en-Y hepaticojejunostomy was performed. Postoperative complications (biloma $=1$, fever $=2$, voiding difficulty $=1$ and ileus $=3$ ) occurred in seven patients $(24.1 \%)$ (grade I = 3 and grade II $=4$ ). The median hospital stay was 9 days (range, 7-14 days).

In conclusion, robotic living donor right hepatectomy is feasible and can be safely performed in experienced hands. 


\section{PS2-7}

\section{Living Donor Liver Transplantation}

\section{Kenneth Chock}

The University of Hong Kong, Hong Kong SAR, China

Living donor liver transplantation (LDLT) is considered an effective alternative to deceased donor liver transplantation (DDLT). It has evolved significantly over the past two decades, especially in places where deceased organ donation is scarce.

Better understanding of the pathophysiology and technical advancement have contributed to marked improvement in outcomes in patients with extended criteria for hepatocellular carcinoma. Nonetheless, new indications for liver transplant are emerging (e.g. unresectable colorectal liver metastases), and the results are pending. Widening of the living donor pool by accepting more ABOincompatible donors and strategic use of donor interchange could potentially benefit more patients worldwide. Minimally invasive donor hepatectomy (especially right or left donor hepatectomy) is gaining popularity, but the technique is not fully mastered at most experienced centers. More data from this part of the world is warranted.

Methods to manage small-for-size syndrome are of utmost importance in partial graft transplantation. Dual graft can definitely solve part of the problem, but risking two donors in order to help one recipient invites hot debate from a bioethical point of view. In summary, we have reached the consolidated phase of the procedure and further innovative procedures will make this seemingly "impossible" operation continue to flourish in the future. 
The 9th Asia-Pacific Primary Liver Cancer Expert Meeting (APPLE 2018)

\title{
PS2-8
}

\section{Associated Liver Partition and Portal Vein Ligation for Staged Hepatectomy (ALPPS)}

\author{
Albert Chan \\ The University of Hong Kong, Hong Kong SAR, China
}

Since the initial experience described by Schnizbauer and co-workers on the application for ALPPS for a series of patients with non-cirrhotic liver tumors in 2012 [1, 2], there has been a surge in the interest on this novel procedure globally. Our ALPPS program was started in December 2013 in a 62-year old patient with hepatitis-B related hepatocellular carcinoma. It was a $13 \mathrm{~cm}$ right lobe HCC with a left liver measuring $26 \%$ of the estimated standard liver volume (ESLV). After the ALPPS procedure, the left liver hypertrophied to $33.5 \%$ ESLV and an extended right hepatectomy was performed 10 days after the stage I procedure [3]. The patient remained well until this day with no evidence of tumor recurrence. The indications for ALPPS in our center are as follow: 1) hepatocellular carcinoma or colorectal liver metastasis, 2) preoperative future liver remnant (FLR) volume $<30 \%$ ESLV, 3) central-locating tumor in right liver with sizeable non-tumorous liver to be sacrificed in a right hepatectomy, 4) Child A cirrhosis, 5) indocyanine green clearance rate $<20 \%$ at 15 minutes, 6 ) platelet count $\geq 100 \times 10^{9} / \mathrm{L}, 7$ ) absence of complete right portal vein (RPV) thrombosis (although partial RPV thrombosis is regarded as a good indication for ALPPS as portal vein embolization in this situation would have a chance to induce thrombus dislodgement into the left/main portal vein upon catheterization of the RPV). Since the success of the first adult case in our center, the ALPPS program has expanded to include 42 patients. Among them, 38 patients (90\%) had HCC (hepatitis $B=36$, hepatitis $C=1$, fatty liver $=1$ ) and all of them underwent both stage I \& II operation. Our initial report indicated that the FLR gained volume by $48.7 \%$ to induce an increment of FLR/ESLV ratio from $24.2 \%$ to $38.5 \%$ over a median of 6 days [4]. The 90-day mortality rate was $7.1 \%(n=3)$ and the cause of death was liver failure. When compared with portal vein embolization (PVE), the benefits of ALPPS are several folds:

\section{Clinical}

1) A complete portal flow diversion to contralateral liver is guaranteed surgically and avoids the chance of incomplete occlusion that could be encountered in PVE. As such, the chance of FLR augmentation is optimised

2) A direct visual assessment of the quality of liver parenchyma in stage I procedure accompanied by a liver biopsy for histological grading of cirrhosis became possible

3) Measurement of portal hemodynamics in stage I to predict the risk of post-hepatectomy liver failure and flow modulation by splenic artery ligation if necessary

4) The entire treatment procedure is completed within one hospitalization in a timely manner. 


\section{Patient}

1) Shorter duration of patient anxiety and psychological stress while waiting for FLR hypertrophy

\section{Logistics}

1) Surgeon-led service and relieve the burden on interventional radiology service

Although the degree of FLR hypertrophy in fibrotic/cirrhotic liver is somewhat less substantial than that in non-cirrhotic, non-cholestatic livers $[5,6]$ probably due to the quality of the liver parenchyma, our experience showed that the ALPPS procedure remained an effective approach for FLR augmentation in patients with hepatitis-related HCC.

\section{References}

1. Schnitzbauer AA, Lang SA, Goessmann H, Nadalin S, Baumgart J, Farkas SA, et al. Right portal vein ligation combined with in situ splitting induces rapid left lateral liver lobe hypertrophy enabling 2-staged extended right hepatic resection in small-for-size settings. Annals of surgery. 2012;255(3):405-14.

2. de Santibanes E, Clavien PA. Playing Play-Doh to prevent postoperative liver failure: the "ALPPS" approach. Annals of surgery. 2012;255(3):415-7.

3. Chan AC, Pang R, Poon RT. Simplifying the ALPPS procedure by the anterior approach. Annals of surgery. 2014;260(2):e3.

4. Chan AC, Poon RT, Chan C, Lo CM. Safety of ALPPS Procedure by the Anterior Approach for Hepatocellular Carcinoma. Annals of surgery. 2016;263(2):e14-6.

5. Alvarez FA, Ardiles V, de Santibanes M, Pekolj J, de Santibanes E. Associating Liver Partition and Portal Vein Ligation for Staged Hepatectomy Offers High Oncological Feasibility With Adequate Patient Safety: A Prospective Study at a Single Center. Annals of surgery. 2014.

6. Schadde E, Ardiles V, Robles-Campos R, Malago M, Machado M, HernandezAlejandro R, et al. Early survival and safety of ALPPS: first report of the International ALPPS Registry. Annals of surgery. 2014;260(5):829-36; discussion 36-8. 


\title{
Parallel Session 3: Updates on Cholangiocarcinoma
}

\author{
PS3-9 \\ Pathological Classification of Cholangiocarcinomas
}

Yoh Zen

Kobe University, Kobe, Japan

Recent large-scale molecular studies using next-generation sequencing protocols provided a lot of genetic features of bile duct cancers. However, how the obtained results relate to histopathological features remain unclear, requiring close molecular-histology correlation studies.

We recently proposed a novel two-group classification scheme of intrahepatic cholangiocarcinomas. One group is histologically identical to perihilar cholangiocarcinomas (large duct type), while the other is characterized by anastomosing tubular architecture (small duct type). Among the 47 cases examined, 26 (55\%) were classified as the large-duct type, while 21 (45\%) were the small-duct type. The former had higher $\mathrm{pT}$ stages and more frequently showed a periductalinfiltrating gross appearance and microscopic perineural infiltration than the latter. The presence of low-grade biliary intraepithelial neoplasia in the adjacent bile ducts was only found in large-duct intrahepatic cholangiocarcinomas (6/21, 29\%). The immunophenotype also differed between the two types with MUC5AC and MUC6 being more commonly expressed in the large-duct type. One third of large-duct cholangiocarcinomas lacked the expression of SMAD4, suggesting SMAD4 mutations, while the loss of BAP1 expression and IDH1 mutations were almost restricted to the small-duct type (35\% and $15 \%$, respectively). Patients with large-duct cholangiocarcinoma had worse overall survival than those with small-duct cancer $(p=0.027)$. A multivariate analysis identified the histologic classification as an independent prognostic factor ( $p=0.005$, HR $=3.638$ ). Comparisons between intrahepatic and perihilar cholangiocarcinomas also revealed that the molecular features and prognosis of large-duct intrahepatic cholangiocarcinomas were very similar to those of perihilar cholangiocarcinomas. Those results indicate that this histology-based classification scheme of intrahepatic cholangiocarcinomas will be useful and clinically relevant because it represents different underlying molecular features and has an independent prognostic value.

We also recently identified frequent MDM2 amplification in cholangiocarcinomas. Interestingly, this molecular alteration appeared to be closely linked to anatomical location. MDM2 was amplified in 10-15\% of large-duct intrahepatic cholangiocarcinomas, perihilar bile duct cancers, and gallbladder cancers, while this molecular feature was never identified in small-duct intrahepatic cholangiocarcinomas and distal bile duct cancers. The frequency of TP53 mutations was similar between MDM2-amplified and non-amplified cases, while KRAS mutations were less frequent in MDM2-amplified cancers.

Although bile duct cancers have been classified into intrahepatic, perihilar and distal cholangiocarcinomas based on the predominant location for decades, a classification scheme better representing genetic features may be needed in the molecular-targeting therapy era. 
The 9th Asia-Pacific Primary Liver Cancer Expert Meeting (APPLE 2018)

\section{References}

1. Sawada R, Ku Y, Akita M, Otani K, Fujikura K, Itoh T, Ajiki T, Fukumoto T, Kakeji Y, Zen Y. IL-33 overexpression reflects less aggressive tumour features in largeduct type cholangiocarcinomas. Histopathology. [Epub ahead of print].

2. Kim SJ, Akita M, Sung YN, Fujikura K, Lee JH, Hwang S, Yu E, Otani K, Hong SM, Zen Y. MDM2 amplification in intrahepatic cholangiocarcinomas: Its relationship with large-duct type morphology and uncommon KRAS mutations. Am J Surg Pathol. 2018;42:512-21.

3. Akita M, Fujikura K, Ajiki T, Fukumoto T, Otani K, Azuma T, Itoh T, Ku Y, Zen Y. Dichotomy in intrahepatic cholangiocarcinomas based on histologic similarities to hilar cholangiocarcinomas. Mod Pathol. 2017;30:986-97. 


\section{PS3-11 \\ Extended Resection for Advanced Perihilar Cholangiocarcinoma}

\section{Tomoki Ebata}

Nagoya University, Nagoya, Japan

The goal of surgical therapy for perihilar cholangiocarcinoma is to achieve tumor-free surgical margin (namely, R0 resection). Bile duct resection used to be applied but not anymore. Instead, hemihepatectomy including caudatelobectomy has been standardized as a first-line approach for this disease, because this procedure enhances the probability of R0 resection, compared to bile duct resection alone. However, perihilar cholangiocarcinoma frequently exhibits an extensive infiltrating nature towards intrahepatic biliary tree (Bismuth type IV), intrapancreatic bile duct (diffuse cholangiocarcinoma), major vasculatures, or these combinations. For such advanced disease, extended surgical approach including hepatic trisectionectomy, hepatopancreatoduodenectomy (HPD), and combined vascular resection are required for curative-intended resection, although technically demanding.

Between 2006 and 2016, 728 consecutive patients with perihilar cholangiocarcinoma were treated at Nagoya University Hospital. According to Bismuth classification, there were 123 (17\%) patients with type I/II, 247 (34\%) patients with type III, and 358 (49\%) patients with type IV. According to JarnaginBlumgart system, the cohort involved 191 (26\%) patients with T1, 94 (13\%) patients with T2, and $443(61 \%)$ patients with T3 tumor.

Of the 728, 587 were surgically explored and 512 patients underwent resection, giving a resectability rate of $70 \%$. The main causes of irresectability were distant metastatic disease $(n=107,50 \%)$, locally advanced disease $(n=63$, $29 \%)$, poor functional liver reserve $(\mathrm{n}=25,12 \%)$, poor general condition $(\mathrm{n}=$ $18,25 \%)$, and refusal of surgery ( $\mathrm{n}=3,1 \%)$. Only $9(2 \%)$ patients underwent bile duct resection alone; the remaining 503 (98\%) patients underwent hepatectomy including caudate lobectomy. Right hepatectomy $(n=159,31 \%)$ was the most common, followed by left hepatectomy $(\mathrm{n}=148,29 \%)$, left trisectionectomy $(\mathrm{n}=$ $138,27 \%)$, right trisecctionectomy $(\mathrm{n}=52,10 \%)$, and central bisegmentectomy $(\mathrm{n}=6,1 \%)$. Resection of the portal vein and hepatic artery was performed in 174 (34.0\%) and 107 (20.9\%) patients, respectively; HPD was performed in 67 (9\%) patients. Overall, the extended procedures accounted for $63 \%(n=323)$ among all resections. Median blood loss and operating time were $1189 \mathrm{~mL}$ and 585 minutes, respectively. Complications $\geq$ Clavien 3 occurred in 217 (30\%) patients; 90-day mortality was $2 \%(n=8)$. Hospital stay after surgery was 28 days in median. Pathologically, nodal and distant metastases were found in 234 (46\%) and 53 (10\%), respectively; margin status was R0 in 408 (80\%), R1 in 95 (19\%), and R2 in 9 (2\%) patients.

Overall survival rate for all 728 patients was $43.7 \%$ at 3 years, $32.4 \%$ at 5 years, and $25.5 \%$ at 10 years, with a median survival time (MST) of 27.3 months; 131 patients actually survived over 5 years after surgery. Survival for 216 unresected patients was poor with a 3-year survival rate of 5\% and an MST of only 11 months, although chemotherapy was given as much as possible. In contrast, patients who received standard surgery had a favorable survival with an MST of 81 months; those who underwent extended surgery had a worse survival than standard surgery group with an MST of 41 months $(p=0.001)$. However, the 
The 9th Asia-Pacific Primary Liver Cancer Expert Meeting (APPLE 2018)

latter survival was significantly better than that for unresected patients $(p<$ $0.001)$.

In conclusion, extended resectional procedures represented by hepatic trisectionectomy, HPD, and vascular resection are needed to expand surgical indication for advanced perihilar cholangiocarcinoma otherwise unresectable, thereby upgrading the overall survival probability of this devastating disease. 


\section{PS3-12}

\section{Systemic Therapy for Biliary Tract Carcinoma}

\section{Chiun Hsu}

National Taiwan University, Taipei, Taiwan

The vast clinical and molecular heterogeneity accounts for the difficulty in developing novel systemic therapies for biliary tract carcinoma (BTC). Cytotoxic chemotherapy is still the standard treatment for patients with advanced BTC. In this presentation the current management guidelines will be reviewed. The experience and challenges of developing new treatment for different stages of BTC will be discussed.

\section{References:}

1. Jusakul A, Cutcutache I, Yong $\mathrm{CH}$, et al. Whole-Genome and epigenomic landscapes of etiologically distinct subtypes of cholangiocarcinoma. Cancer Discov 2017; 7: 1116-1135.

2. Ueno M, Morizane C, Okusaja T, et al. Randomized phase III study of gemcitabine plus $\mathrm{S}-1$ combination therapy versus gemcitabine plus cisplatin combination in advanced biliary tract cancer (JCOG1113). J Clin Oncol 2018; 36 (suppl): abstr\#4014

3. Chen JS, Hsu C, Chiang NJ, et al. A KRAS mutation status-stratified randomized phase II trial of gemcitabine and oxaliplatin alone or in combination with cetuximab in advanced biliary tract cancer. Ann Oncol 2015; 26: 943-9

4. Hainsworth JD, Meric-Bernstam F, Swanton C, et al. Targeted therapy for advanced solid tumors on the basis of molecular profiles: results from MyPathway, an open-label, phase IIa multiple basket study. J Clin Oncol 2018; 36:536-542

5. Arkenau HT, Martin-Liberal J, Calvo E, et al. Ramucirumab plus pembrolizumab in patients with previously treated advanced or metastatic biliary tract cancer: nonrandomized, open-label, phase I trial (JVDF). Oncologist. 2018 May 31. pii: theoncologist.2018-0044. 


\title{
Basic \& Translational Forum 1: Novel Noninvasive Biomarkers for Liver Cancer: Liquid Biopsy
}

\author{
BTF1-1 \\ Discovery of Biomarkers for Clinical Response to \\ Radiotherapy in HCC via Deep Immunoprofiling of \\ Peripheral Blood \\ Valerie Chew ${ }^{1 *}$, Yun Hua Lee', Lu Pan ${ }^{1}$, Nurul J.M. Nasir', Chun Jye Lim 1 , \\ Camillus Chua', Liyun Lai', S. Nur Hazirah', Tony Kiat Hon Lim ${ }^{2,3}$,

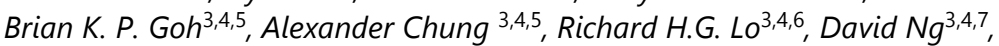 \\ Rene L.F. Filarca ${ }^{3,4,5}$, Salvatore Albani', Pierce K.H. Chow 3 3,4,5 \\ ${ }^{1}$ Translational Immunology Institute, SingHealth-DukeNUS Academic \\ Medical Centre, Singapore; ${ }^{2}$ Department of Pathology, Singapore General \\ Hospital, Singapore; ${ }^{3}$ Duke-NUS Medical School, Singapore; ${ }^{4}$ National \\ Cancer Centre, Singapore (NCCS); ${ }^{5}$ Department of Hepatopancreatobiliary \\ and Transplant Surgery, Singapore General Hospital, Singapore; \\ ${ }^{6}$ Department of Diagnostic Radiology, Singapore General Hospital, \\ Singapore; ${ }^{7}$ Department of Nuclear Medicine and Molecular Imaging, \\ Singapore General Hospital, Singapore
}

Yttrium-90 (Y90)-radioembolisation (RE) significantly regresses locally advanced hepatocellular carcinoma (HCC) and delays disease progression. However the mechanism of delayed and sustained clinical response was largely unknown. Here we used high-dimensional single-cell mass cytometry (CyTOF) and next-generation sequencing (NGS) to analyse the immune landscapes of tumour and peripheral blood of HCC patients before and after Y90-RE. We discovered that local immune microenvironment was activated after Y90-RE: higher expression of granzyme B (GB) and infiltration of CD8+ T cells, CD56+ NK cells and CD8+ CD56+ NKT cells. NGS confirmed the upregulation of genes involved in innate and adaptive immune activation in Y90-RE-treated tumours. Chemotactic pathways involving CCL5 and CXCL16 correlated with the recruitment of activated $\mathrm{GB}+\mathrm{CD} 8+\mathrm{T}$ cells to the Y90-RE-treated tumours. In peripheral blood, we observed an increase in activated CD8+ and CD4+ T cells as well as antigen-presenting cells upon Y90-RE, implying a systemic immune activation. Interestingly, a high percentage of PD-1+/Tim-3+CD8+ $T$ cells co-expressing the homing receptors CCR5 and CXCR6 in the peripheral blood denoted Y90-RE responders. A prediction model, based on the immune biomarkers from the pre-treatment peripheral blood, was built to identify sustained responders to Y90-RE. 


\author{
BTF1-2 \\ Clinical Applications of Exosomes and MicroRNA \\ Yong-Han Paik \\ Sungkyunkwan University, Seoul, South Korea
}

Exosomes are small membranous vesicles that originate from internal multivesicular bodies released by various types of cells. Exosomes have been found in body fluids such as plasma, urine, saliva, breast milk, and synovial fluid. Exosomes contain cell-specific protein, mRNA, and microRNA. Recent studies showed that exosomal microRNA is stable in blood because exosomes have a protective function against degradation from enzymes, such as RNAse. Exosomal proteins and microRNAs can be functional and regulate cell signaling resulting in pathophysiology in target cells. It has been understood increasingly important that exosome can mediate cell to cell communication by transfering cargos to regulate cell activities such as protein expression, cell proliferation or differentiation, and antiviral responses in the recipient cells. Additionally, exosomal microRNA has a potential role as a diagnostic biomarker in patients with cancer. This lecture summarizes recent exosome research in liver diseases mainly focusing on biomarkers of hepatocellular carcinoma (HCC).

We investigated the feasibility of using serum exosomal microRNAs as novel serologic biomarkers for HCC. ${ }^{1}$ We measured the expression levels of serum exosomal microRNAs in patients with hepatitis B virus (HBV)-related chronic hepatitis, liver cirrhosis (LC), and HCC. Serum exosomal microRNA was extracted from $500 \mu \mathrm{l}$ of serum using an Exosome RNA Isolation kit. The expression levels of microRNAs were quantified by real-time PCR. The expression levels of selected microRNAs were normalized to Caenorhabditis elegans microRNA (Cel-miR-39). The expression of serum exosomal microRNAs in HCC patients were compared with those of patients with chronic hepatitis B (CHB) or LC. The serum levels of exosomal miR-18a, miR-221, miR-222 and miR-224 were significantly higher in patients with HCC than those with CHB or liver cirrhosis $(p<0.05)$. Further, the serum levels of exosomal miR-101, miR-106b, miR-122, and miR-195 were lower in patients with HCC than in patients with CHB ( $p=$ $0.014, p<0.001, p<0.001$, and $p<0.001$, respectively). Additionally, the serum levels of circulating microRNAs showed a smaller difference between HCC and either CHB or LC. Wang etal, reported that the expression level of serum exosomal miR-21 was significantly higher in patients with HCC than those with CHB or healthy volunteers. ${ }^{2}$ Sugimachi et al. recently reported that expression of miR-718 is significantly decreased in the serum exosome of patients with HCC 
recurrence after LT. They identified HOXB8 as a potential target gene of miR-718, and its upregulation was associated with poor prognosis. ${ }^{3}$ Collectively, these data suggest that serum exosomal microRNAs may have a potential for novel serologic biomarkers for HCC.

\section{References}

1. Sohn W, Kim J, Kang SH, Yang SR, Cho JY, Cho HC, Shim SG, Paik YH. Serum exosomal microRNAs as novel biomarkers for hepatocellular carcinoma. Exp Mol Med. 2015 Sep 18;47:e184.

2. Wang H, Hou L, Li A, Duan Y, Gao H, Song X. Expression of serum exosomal microRNA-21 in human hepatocellular carcinoma. Biomed Res Int 2014;2014:864-894.

3. Sugimachi K, Matsumura T, Hirata H, Uchi R, Ueda M, Ueo H, et al. Identification of a bona fide microRNA biomarker in serum exosomes that predicts hepatocellular carcinoma recurrence after liver transplantation. BrJ Cancer 2015;112:532538. 


\section{BTF1-3 \\ Utilization of Circulating Tumor Cells in Hepatocellular Carcinoma}

Jian Zhou

Fudan University, Shanghai, China

Hepatocellular carcinoma (HCC) is the most prevalent malignancy in the world and ranks the second as the cause of cancer deaths. The lack of effective methods for timely diagnosis, prognosis prediction, monitoring anticancer treatment response is the main obstacle to further improving the prognosis of HCC patients. Accumulating evidences indicated that the spread of circulating tumor cells (CTCs) is an early event in tumor progression, and plays an important role in the initiation of tumor metastases and recurrence.

Based on the CellSearch system, 123 HCC patients were tested prior to resection and 1 month thereafter. The CTC s were present in $66.67 \%$ of patients, and the cell count measured in $7.5 \mathrm{ml}$ of blood ranged between $1-34$. Fifty-one patients had CTCs $\geq 2$ preoperatively, and these individuals developed tumor recurrence earlier than those with CTCs $<2$ CTC s $(p<0.001)$. A preoperative CTC $\geq 2$ was an independent prognostic factor for tumor recurrence $(p<0.001)$. Significant decrease of CTC-positive rates $(66.67 \%$ to $28.15 \%, p<0.05)$ and CTCs values $(2.60 \pm 4.73$ to $1.00 \pm 3.70, p<0.05)$ was observed 1 month after resection. Patients with postoperative CTCs dropping to $<2$ had lower recurrence rates than those with values consistently $\geq 2$ (43.33\% vs. 87.50\%, $p=0.043$ ). Furthermore, positive correlation was observed between microvascular invasion (MVI)-an independent risk factor of tumor recurrence-and CTC number, which gave rise to the hypothesis that preoperative CTC load might determine the surgical margin for HCC patients. Retrospectively, we discovered patients with surgical margin $\leq 1 \mathrm{~cm}$ showed significantly higher risk of postoperative recurrence than those with margin of 1 to $3 \mathrm{~cm}$ in preoperative CTC $\geq 2$ group, while no difference was detected in predicting recurrence in preoperative CTC $<2$ group by classifying different surgical margins. What's more, postoperative CTC number $\geq 3$ appeared to be the most significant factor for predicting extrahepatic metastasis (EHM) in HCC patients. The prognostic utility of postoperative CTC number was further evaluated within clinical subgroups. Recently, based on a prospective randomized controlled study on HBV-related HCC, we proved that adjuvant transarterial chemoembolization (TACE) significantly reduce tumor recurrence, improve recurrence-free survival and overall survival after curative resection. As aforementioned, we found patients with preoperative CTCs $\geq 2$ had a higher recurrence rate than those with CTCs $<2$. We evaluated the effect of adjuvant TACE in patients with preoperative CTCs $\geq 2$ and found it could significantly reduce tumor recurrence rates and prolong overall survival in a training set $(n=47)$ and a validation set $(n=52)$.

In order to overcome the shortcoming of CellSearch system for CTC detection, a novel optimal platform based on negative selection and qRT-PCR was constructed, and EpCAM mRNA-positive CTC detection by this platform was performed and its clinical significance was then investigated. We optimized the platform through negative enrichment (CD45 ${ }^{+}$cell depletion) and single marker qRT-PCR (EpCAM ${ }^{\text {mNA+ }}$ qRT-PCR), and the optimized platform showed high sensitivity, specificity, and reproducibility and exhibited $76.70 \%$ consistency with the CellSearch system ( $r=0.54, p<0.05$ ). Our new platform just need $2 \mathrm{ml}$ 
The 9th Asia-Pacific Primary Liver Cancer Expert Meeting (APPLE 2018)

plasma rather than $7.5 \mathrm{ml}$ in CellSearch, and is faster to have report (3-4 hours) with lower expense. As the analyses on the subpopulations of CTC with stem cell phenotypes might be more clinically relevant than on total CTC counts, and our recent studies and the results for other labs suggest that these CTCs subpopulations in HCC are phenotypically diversity. A multimarker diagnostic panel for HCC, targeting CTC subpopulation with stem-like phenotypes, was thereby constructed through a multicenter patient study with independent validation based on negative enrichment and multimarker qRT-PCR analysis. We further identified 4 markers (EpCAM, CD133, CD90, CK19) from 9 putative stem markers and confirmed the existence of these subpopulation in CTCs. With a cutpoint of 0.57 by ROC curve after logistic regression (training: $\mathrm{AUC}=0.88,95 \% \mathrm{CI} 0.84-$ 0.91 ; sensitivity, $72.5 \%$; specificity, $95.0 \%$; validation: $\mathrm{AUC}=0.92,95 \%$ CI 0.89 0.94; sensitivity, $82.1 \%$; specificity, $94.5 \%$ ). Results were similar for early-stage HCC (training: AUC $=0.87,95 \%$ CI $0.83-0.90$; sensitivity, $71.8 \%$; specificity, 95.0\%; validation: $\mathrm{AUC}=0.93,95 \%$ CI 0.89-0.95; sensitivity, $85.1 \%$; specificity, 94.5\%). Compared with all controls, the panel performed equally well in detecting $\alpha$-fetoprotein (AFP)-negative HCC (including early-stage tumors), also distinguishing HCC from CHB, LC and BHL. Using an optimal cutoff point of 0.80 , the prognostic significance of preoperative CTIC detection in predicting tumor recurrence after operation was further confirmed in two independent cohorts (all $p<0.001$ ), and its prognostic potential was also retained in AFP-negative and early-stage subgroups. More importantly, the CTIC load was decreased significantly after resection in $78 \%$ of HCC patients with blood sample collection 1 month after surgery, and the positive rate decreased from $76.00 \%$ to $34.00 \%$. Compared with CTC persistent negative patients, the patients with persistently high CTIC load showed high tumor recurrence rate after surgery $(70.6 \%$ vs. $13.3 \%)$.

In conclusion, the detection of CTCs showed great clinical significances in covering diagnosis, outcome prediction, and the evaluation of therapeutic response in HCC, whereas novel detection methods are warranted, and further basic and translational researches are obligatory. 


\section{BTF1-4 \\ Clinical Utility of Single Cell Genome Analysis in Human Cancers}

Woong-Yang Park

Samsung Genome Institute, Seoul, South Korea

Single-cell transcriptome analysis provides information on the characteristics of individual cells in heterogeneous tumor tissues. We isolated more than 500 unsorted single cells from 11 breast cancer tissues for RNA-seq. Based on the copy number variation inferred from RNA-seq data, we could separate tumor cells with variable copy numbers over the genome from non-cancer cells without apparent changes in copy numbers. At a single cell level, carcinoma cells showed common features within each breast cancer subtype, and also intra-tumoral genetic heterogeneity with mixed signature of different breast cancer subtypes. Non-cancer cells consisted of immune cells with three distinct populations of $\mathrm{T}$ lymphocytes, B lymphocytes, and macrophages. T lymphocytes and macrophages displayed immunosuppressive characteristics; regulatory or an exhausted phenotype. Single cell trascriptome profile could figure out the range of intratumoral heterogeneity, and also immune cell profiles within breast cancer tissue, which might determine the response to immunotherapy. 


\title{
Basic \& Translational Forum 2: New Emerging Concepts and Technologies in Oncology
}

\author{
BTF2-5 \\ Non-Coding RNA as Critical Regulators for \\ Hepatocarcinogenesis
}

Suk Woo Nam

The Catholic Univ of Korea, Seoul, South Korea

The classic view of the transcriptome landscape and its mRNA-centric paradigm for transcript annotation has undergone a fundamental change. The ENCODE project estimates that (mostly non-coding) transcripts cover $62-75 \%$ of our genome, and contribute greatly to the overall estimate of $80 \%$ potentially functional sequence in our DNA. Similarly, RNAseq studies show that transcripts from these non-coding regions dominate the population of non-ribosomal nonmitochondrial RNAs in a human cell. Non-coding RNAs (ncRNAs) have emerged as a major source of biomarkers, targets for therapeutics, and potential explanations for the function of non-coding GWAS variants. Constant expansion of the evidence for broad functionality of ncRNAs, in processes ranging from heritable epigenetic change to species-specific changes in cognition, may finally answer the long-standing question of the role of non-coding DNA in eukaryotic biology.

Long noncoding RNAs (lncRNAs) are emerging as important regulators of tissue physiology and disease processes including cancer. MicroRNAs (miRNAs) comprise species of short noncoding RNA that regulate gene expression posttranscriptionally. The emergence of high-throughput RNA-seq technology provides a revolutionary means for the systematic discovery of transcriptional units. Indeed, RNA-seq has led to a deeper appreciation of the intricate nature of transcription by identifying a milieu of IncRNAs, both located in intergenic 'gene deserts' and overlapping protein-coding loci. The aligned sequence data generated by RNA-seq experiments can be used to predict full-length transcripts in silico with ab initio transcriptome assembly. This presentation summarizes the basic concept of approaching strategy to identify early-stage driver and their regulatory mechanisms through navigating RNA landscape of multi-step hepatocarcinogenesis. 


\title{
BTF2-6 \\ Novel Approaches in Immunotherapy for HCC
}

\author{
Tim Greten
}

National Cancer Institute, Bethesda, USA

Treatment options for patients with hepatocellular carcinoma (HCC) are rapidly changing based on positive results from a number of clinical phase III trials evaluating targeted and immune-based treatment approaches in HCC. More agents targeting molecular pathways and immune checkpoints are currently under clinical evaluation. Some agents have already demonstrated improved outcome in HCC in the first- and second-line setting and are either awaiting FDA approval or have recently been approved. Based on these positive results investigators are trying a variety of different approaches to further improve the outcome for both targeted and immunotherapy in HCC. In our group we have been evaluating combination studies for a number of years, in which we combine locoregional therapies with immune checkpoint inhibitors. In our first study we combined tremelimumab (anti-CTLA4) with locoregional therapies and observed a response rate of approximately $20 \%$ [1]. We have been able to demonstrate a correlation between treatment response and tumor infiltrating $\mathrm{T}$ cells; however, this observation was made in biopsies from patients already on treatment and therefore does not function as a prognostic marker. In a more recent analysis of possible immune correlates in patients treated with antiCTLA4 we observed that patients with higher CD4+PD1+ T cell frequencies were more likely to respond to therapy. Currently, we are exploring murine HCC models to evaluate the best immune combination approach. As part of these studies we have also recently started looking at the effect of the gut microbiome on anti-tumor immunity in the liver. It is known that dysbiosis impairs the efficacy of immunotherapy including the immune checkpoint blockade anti-PD1 treatment in melanoma patients. However, the role of gut bacteria in anti-tumor surveillance in the liver is still poorly understood. Using a primary liver model, we found that altering commensal gut bacteria induced a liver-selective antitumor effect. A selective increase of hepatic CXCR6+ NKT cells was observed, independent of mouse strain, gender or presence of liver tumor. In vivo studies using both antibody-mediated cell depletion and NKT-deficient mice confirmed that NKT cells mediated inhibition of tumor growth in the liver. Further investigation showed that NKT cell accumulation was regulated by the expression of CXCL16, the solo ligand for CXCR6, on liver sinusoidal endothelial cells, which form the lining of liver capillaries. Primary bile acids increased CXCL16 
The 9th Asia-Pacific Primary Liver Cancer Expert Meeting (APPLE 2018)

expression, while secondary bile acids showed the opposite effect. Removing gram-positive bacteria, which contains the bacteria mediating primary-tosecondary bile acid conversion, by vancomycin was sufficient to induce hepatic NKT cell accumulation and decrease liver tumor growth. Feeding secondary bile acids or colonization of bile acid-metabolizing bacteria reversed both NKT cell accumulation and inhibition of liver tumor growth in mice with altered gut commensal bacteria. In non-tumor liver tissue from patients with primary liver cancer, primary bile acid CDCA levels correlated with CXCL16 expression, the opposite was found with secondary bile acid GLCA indicating the finding also applies to humans [2].

\section{References}

1. Duffy AG, Ulahannan SV, Makorova-Rusher O, et al. Tremelimumab in combination with ablation in patients with advanced hepatocellular carcinoma. Journal of Hepatology. 2017;66(3):545-551. doi:10.1016/j.jhep.2016.10.029.

2. Ma C, Han M, Heinrich B, et al. Gut microbiome-mediated bile acid metabolism regulates liver cancer via NKT cells. Science. 2018;360(6391):eaan5931-11. doi:10.1126/science.aan5931. 
BTF2-7

Targeting Mutant KRAS with CRISPR-Cas9 Controls Tumor Growth

Wonjoo Kim 1 , Sangeun Lee ${ }^{1,2}$, Han Sang Kim ${ }^{1,2,3}$, Minjung Song ${ }^{1,4}$, Yong Hoon Cha ${ }^{5}$, Young-Hoon Kim ${ }^{1,6}$, Jeonghong Shin ${ }^{1,2}$, Eun-Seo Lee ${ }^{1,7}$, Yeonsoo Joo ${ }^{8}$, Jae J. Song ${ }^{8}$, Eun Ju Choi ${ }^{9}$, Jae W Choi ${ }^{8}$, Jinu Lee ${ }^{9}$, Moonkyung Kang ${ }^{10}$, Jong In Yook ${ }^{5}$, Min Goo Lee ${ }^{1,2}$, Yeon-Soo Kim ${ }^{10}$, Soonmyung Paik ${ }^{8}$, Hyongbum (Henry) Kim ${ }^{1,2,6,8}$

${ }^{1}$ Department of Pharmacology, Yonsei University College of Medicine, Seoul, 03722, South Korea; ${ }^{2}$ Brain Korea 21 Plus Project for Medical Sciences, Yonsei University College of Medicine, Seoul, 03722, South Korea; ${ }^{3}$ Division of Medical Oncology, Department of Internal Medicine, Yonsei University College of Medicine, Seoul, 03722, South Korea; ${ }^{4}$ Department of Food Biotechnology, Division of Bioindustry, College of Medical and Life Sciences, Silla University, Busan, 46958, South Korea; ${ }^{5}$ Department of Oral Pathology, Oral Cancer Research Institute, College of Dentistry, Yonsei University, Seoul 03722, Korea; ${ }^{6}$ Graduate Program of Nano Science and Technology, Yonsei University, Seoul, 03722, South Korea; ${ }^{7}$ School of Chemical and Biological Engineering, Seoul National University, Seoul, 08826, South Korea; ${ }^{8}$ Severance Biomedical Science Institute, Yonsei University College of Medicine, Seoul, 03722, South Korea; ${ }^{9}$ College of Pharmacy, Yonsei Institute of Pharmaceutical Sciences, Yonsei University, 21983, South Korea; ${ }^{10}$ Graduate School of New Drug Discovery \& Development, Chungnam National University, Daejeon 305763 , South Korea

KRAS is the most frequently mutated oncogene in human tumors and its activating mutations represents important therapeutic targets. The combination of Cas 9 and guide RNA from the CRISPR-Cas system recognizes a specific DNA sequence and makes a double-strand break, which enables editing of the relevant genes. Here we harnessed CRISPR to specifically target mutant KRAS alleles in cancer cells. We screened guide RNAs using a reporter system and validated them in cancer cells after lentiviral delivery of Cas 9 and guide RNA. The survival, proliferation, and tumorigenicity of cancer cells in vitro and the growth of tumors in vivo were determined after delivery of Cas 9 and guide RNA. We identified guide RNAs that efficiently target mutant KRAS without significant alterations of the wild-type allele. Doxycycline-inducible expression of this guide RNA in KRAS mutant cancer cells transduced with a lentiviral vector encoding Cas 9 disrupted the mutant KRAS gene, leading to inhibition of cancer cell proliferation both in vitro and in vivo. Intratumoral injection of lentivirus and adeno-associated virus expressing Cas9 and sgRNA suppressed tumor growth in vivo, albeit incompletely, in immunodeficient mice. Expression of Cas 9 and the guide RNA in cells containing wild-type KRAS did not alter cell survival or proliferation either in vitro and in vivo. Our study provides a proof-of-concept that CRISPR can be utilized to target driver mutations of cancers in vitro and in vivo. 
BTF2-8

Targeting Cancer Stem Cells in HCC

Irene Oi-Lin $\mathrm{Ng}$

The University of Hong Kong, Hong Kong SAR, China

Many lines of evidence have demonstrated that cancer stem cells (CSCs) or tumor-initiating cells residing within the bulk tumor are a subpopulation of tumor cells that possess the ability to self-renew and differentiate. They are also capable of maintaining tumor propagation and metastasis and confer chemoand radio-resistance. As a consequence, CSCs are believed to play a crucial role in tumor initiation and tumor relapse clinically; hence CSCs are considered a pivotal target for the eradication of cancers. Hence besides killing the bulk tumor, there is a need to also target this specific CSC subpopulation for novel treatment. In hepatocellular carcinoma (HCC), using in vitro and in vivo models, a number of liver CSC markers have been identified, including, among others, CD133, CD24, CD47, and EpCAM. Notably, liver CSCs can be enriched by chemotherapeutic agents. Using cell sorting approach from patients' HCC samples and HCC cell lines and lenti-viral based knockdown approach, HCC cells positive for these markers are more chemoresistant than the marker-negative cells. These cells also have the functional features of CSCs. Furthermore, they are functional liver CSC markers that drive hepatocarcinogenesis through specific signaling pathways such as the STAT3-mediated NANOG pathway. Translational work using antibodies targeting these markers has shown promising data in animal models. In addition, tumor microenvironment is now known to play an important role in regulating cancer stemness. The potential interaction of liver CSCs and the tumor microenvironment as exemplified by cancer-associated fibroblasts and other cellular components will also be presented. This presentation attempts to highlight the importance of CSCs implicated in the pathogenesis of hepatocellular carcinoma as well as potential targets for therapy. Detailed understanding of the molecular pathogenesis is crucial for the development of new therapeutic approaches against cancers. 


\section{Presidential Lecture}

\section{1}

Strategic Application of Radiotherapy for HCC

Jinsil Seong

Yonsei University, Seoul, Korea

External beam radiotherapy (EBRT) is a well-established cancer modality in most major solid cancers. In hepatocellular carcinoma (HCC), its role hasn't been recognized enough due to lack of high level evidence. However, its efficacy has been witnessed by soaring number of papers reporting beneficial clinical outcome as well as increasing level of evidence. Consequently major treatment guidelines start to either consider EBRT as one of the treatment options (NCCN guideline 2018) or just mention (AASLD guideline 2017) depending on disease status.

EBRT can be administered in various ways. For small tumors, high dose focal radiation can be given in short term, which is referred to stereotactic body radiotherapy (SBRT). It needs precision technology involving image-guided beam delivery (image guided radiotherapy) as well as motion control. For HCC beyond early stage, EBRT can be given using conventional or hypo-fractionation scheme, which is adopting preferably combination scheme with other modality, either as an additional treatment for HCCs showing incomplete result to TACE or as a combination of EBRT and vascular therapy for HCC accompanying vascular invasion.

In HCC with major vascular invasion as portal vein tumor thrombosis (PVTT), recent nationwide multicenter study reported oncologic outcome of EBRT for HCC patients with PVTT with response rate $51.8 \%$ and better survival in responders (15.2 months) than in non-responders (6.9 months). More recently, encouraging results have come showing better outcome in combination of EBRT with TACE than in sorafenib in a phase 3 trial. In another phase 2 trial, combination of EBRT with hepatic arterial infusion chemotherapy showed median survival time as high as 18 months.

In conclusion, strategic application of radiotherapy for HCC may bring local control of HCC, which can further be translated into improved oncologic outcome. 


\section{State-of-the-Art Lecture 1}

2

\section{Molecular Pathogenesis of HCC}

Irene Oi-Lin Ng

The University of Hong Kong, Hong Kong SAR, China

Hepatocarcinogenesis is a multistep process evolving from chronic liver diseases to hepatocellular carcinoma (HCC) and consists of progressive accumulation of molecular alterations pinpointing different molecular and cellular events. Recent advances in molecular methods have led to growing understanding of the underlying mechanisms of hepatocarcinogenesis. Chromosomal, genetic, and epigenetic abnormalities can lead to deregulation in many cell signaling pathways implicated in tumor proliferation, progression, and survival. Specific signaling cascades have consistently been found dysregulated in HCC. There is emerging evidence supporting the importance of cancer metabolism and tumor microenvironment in providing a favorable and supportive niche to expedite HCC development. Moreover, recent studies have identified distinct surface markers of cancer stem cell (CSC) in HCC. Significantly, the introduction of sophisticated genomics technologies has enhanced extensive research on its molecular pathogenesis. HCC has complicated and highly heterogeneous genomic, genetic and epigenetic profiles. Using different genomics analyses (whole genome, exome, and transcriptomic sequencing), recent remarkable advances have been made to provide comprehensive profiling data on mutational landscape. The molecular alterations in advanced HCCs are quite diverse. These mutations translate into different gene expression alterations as well as altered cell signaling pathways. Very recently, the cutting-edge technology of single cell analysis has provided important information of its intratumoral heterogeneity and is able to identify important sub-populations. Chronic HBV infection is a major etiological factor of HCC. HBV frequently integrates into the human genome, with TERT gene being the most frequent target of HBV integration in HBV-associated HCC. This presentation attempts to highlight the molecular mechanisms currently implicated in the pathogenesis of HCC. Dissection of hepatocarcinogenesis at multiple molecular levels potentially sheds light on alternative treatment options. Discoveries and insight into these complex pathways have created opportunities for targeted agents and new therapeutic approaches for this malignant disease. The ultimate goal of precision medicine is to utilize molecular information to assign patients to the most effective treatment. 


\section{State-of-the-Art Lecture 2}

\section{3}

\section{Advancements of Liver Resection and Transplantation for} HCC

Kyung-Suk Suh

Seoul National University, Seoul, South Korea

The rationale for anatomic resection is based on the assumption that the hepatocellular carcinoma (HCC) invades the nearby portal vein branches and makes daughter nodules. So the anatomic resection means the resection of the tumor-bearing portal territory.

There have been several reports showing the more favorable prognosis in patients underwent anatomic resection than those underwent non-anatomical resection. But still, there are controversies about this procedure.

There are several technical misconceptions in the anatomic resection. For example, in the anterior sectionectomy, some surgeons emphasize that the right hepatic vein and the middle hepatic vein should be fully visualized after resection. However, the concept of the anatomical resection is based on portal tributaries not on hepatic veins. And even smaller resection around the tumor would be done according to the concept of the anatomic resection.

Many patients with HCC have cirrhosis and so larger volume of resection sometimes causes poor prognosis. And rapid regeneration due to large volume of resection may be a risk factor for tumor growth.

Another consideration in HCC resection is the effect of hypoxia, congestion and inflammation of the liver on HCC. Several reports showed the hypoxia, congestion and inflammation are risk factors of tumor recurrence.

Therefore, in hepatic resection for HCC, anatomic resection is important but also the amount of resection, ischemia and congestion after resection should be considered.

Laparoscopic hepatic resection even in live donor hepatectomy has become more popular nowadays. The liver transplantation is regarded as most effective treatment in the early HCC. So whenever the hepatic resection is done in the patient with HCC, we have to consider the liver transplantation which may be done later. In this situation the hepatic resection would rather be simpler without much dissection of hilum and laparoscopic approach whenever possible.

Recurrence of transplant recipients with far advanced HCC including macrovascular invasion or huge HCC or numerous HCC is common and macrovascular invasion is considered as a contraindication of liver transplantation. A living donor is uniquely matched to a certain recipient and this special relationship between a donor and recipient can provide a patient with the opportunity to 
undergo liver transplantation even in suboptimal patients with advanced HCC. However, a high probability of tumor recurrence should evoke ethical issues concerning risk to the living donor. There are no accepted criteria for patients with tumors that lie outside the conventional criteria (e.g. Milan criteria).

To expand the Milan criteria, prognostic factors other than size and number of tumor may be necessary. Between November 1997 and December 2005, 104 cases of liver transplantation for patients with HCC were performed at our center. Twenty-four patients did not meet the Milan criteria preoperatively. Among these 24 patients, 19 had no major vascular invasion at the time of surgery. We analyzed the survival and prognostic factors of these 19 patients. The mean follow-up period was 33 months (range 6-89). Three-year survival rate in 19 patients was $67.4 \%$. Three-year survival rates were significantly higher when preoperative alpha-fetoprotein was less than $400 \mathrm{ng} / \mathrm{ml}$ (86.2 vs. $0 \%, p<0.001$ ) when Edmonson-Steiner's histological grade 1 or 2 (100 vs. $40 \%$, $p=0.036$ ) and when microvascular invasion was absent (78.6 vs. $30 \%, p=0.039$ ). Only AFP was a preoperative factor.

Positron emission tomography using F-18 fluoro-2-deoxy-d-glucose $\left({ }^{18} \mathrm{~F}-\mathrm{FDG}\right.$-PET) imaging is now well established as a noninvasive diagnostic tool for the detection of a variety of malignant tumors. However, in the case of HCC, several investigators have reported controversial conclusions and an inadequate sensitivity for PET (50\%-55\%). Nevertheless, a high positive rate of FDG accumulation has been reported in patients with high-grade HCC and in those with markedly elevated alpha fetoprotein (AFP) levels. When we analyzed the association between tumor factors and PET (+) (greater PET lesion uptake) in liver, preoperative AFP level and vascular invasion were found to be significantly associated with PET $(+)(p=0.003$ and $p<0.001$, respectively). The 2-yr recurrencefree survival rate (2-yr RFSR) of PET $(-)$ patients was significantly higher than that of PET $(+)$ patients ( $85.1 \%$ vs $46.1 \%)(p=0.0005)$. PET imaging could be a good preoperative tool for estimating the post-LT risk of tumor recurrence, because histological grade and vascular invasion cannot be determined preoperatively.

The retrospective data of 178 consecutive HCC patients who underwent LDLT from January 2003 to December 2009 in Seoul National University Hospital were collected. We analyzed 113 patients who were evaluated all 3 tests including 18F-FDG PET positivity, serum AFP level and serum PIVKA II level preoperatively. Multivariate analysis showed that serum AFP level, serum PIVKA II level and 18F-FDG PET positivity were statistically significant variables. HCC of all 3 patients with high risk of 3 biological factors and within Milan criteria were recurred. However, HCC of only 1 patient among the 9 patients beyond Milan criteria and with low risk of biological factors (AFP $<100 \mathrm{ng} / \mathrm{mL}$, PIVKA II $<100$ $\mathrm{mAU} / \mathrm{mL}$, PET negative) were recurred.

We did another study about far advanced HCC. 22 transplant recipients with HCC larger than $10 \mathrm{~cm}$ or more than 10 numbers or with macrovascular invasion preoperatively from January 2003 to October 2010 were included. 1-year disease free survival (DFS) and 2-year DFS were 40.9\% and 23.9\%, respectively. 1-year survival rates (SR) and 2-year SR were $71.6 \%$ and $59.7 \%$, respectively. 6 patients were without tumor recurrence and the median survival duration was 25.4 
The 9th Asia-Pacific Primary Liver Cancer Expert Meeting (APPLE 2018)

(13-85) months and the median survival duration of 16 patients with tumor recurrence was 12.37 (3-40) months. There were no statistical differences of DFS and SR according to macrovascular invasion (MVi) and 2-year SR were 68.2\%(no MVi) and 50.9\% (MVi). There were no significant different outcomes according to treatment modality. However, there was significant different $2 \mathrm{yr}$ DFS according to serum alpha-fetoprotein level, 54.5\% (AFP $<200 \mathrm{ng} / \mathrm{mL}$ ) and $0.00 \%$ (AFP > $200 \mathrm{ng} / \mathrm{mL}$ ) after $\operatorname{LDLT}(p=0.023$ ).

Conclusion: In hepatic resection for HCC, anatomic resection is important but also the amount of resection, ischemia and congestion after resection should be considered. And so "functional resection" rather than "anatomical resection" would be the better approach.

Tumor biological markers have the possibility to overcome the limitation of the Milan criteria. Preoperative serum AFP level, PIVKA II level and 18F-FDG PET positivity of the tumor predict the tumor recurrence better than Milan criteria in living donor liver transplantation for the patients with HCC. The patients with far advanced HCC have usually poor prognosis. However, the patients even with far advanced HCC can have better outcome after living donor liver transplantation if we select the candidates carefully by better selection criteria such as alphafetoprotein. 


\section{State-of-the-Art Lecture 3}

\section{4}

Multidisciplinary Approach in the Management of HCC

Kwang-Hyub Han

Yonsei University, Seoul, South Korea

Hepatocellular carcinoma (HCC) is one of the most common malignancies worldwide and there has been a marked increase in HCC-related annual death rates during the past two decades. At present, majority of patients with HCC are still diagnosed at advanced stage. Although management of HCC has been remarkably improved since 21 century, unsolved issues are still remained, and overall prognosis is still grave in advanced stage. Although there are guidelines for the management of HCC, still optimal care of HCC is not simple because of the complexity of managing HCC. Moreover, many specialists are needed to manage patients with HCC according to disease status. To optimize care and to improve quality of life and clinical outcomes, patients with advanced HCC are needed to be managed by a multidisciplinary team (MDT). A MDT is composed of specialists including hepatologist, oncologist (medical and radiation oncologist), surgeon, radiologist (diagnostic and interventional radiologist), pathologist and coordinator who meet on a regular basis to discuss individual patients and treatment approach.

PVTT is a quite common finding in advanced HCC with macrovascular invasion (MVI). HCC with PVTT is usually inoperable and is associated with poor prognosis. The recently updated AASLD guidelines point out that the selection of treatment may vary depending on the extent of MVI, but still no recommendation could be made. Despite guidelines recommending sorafenib as the standard treatment for HCC with any MVI, many studies suggested that alternative or more aggressive loco-regional treatment approaches could benefit selected patients. There are many positive trial reports for HCC with any MVI using hepatic arterial infusion chemotherapy (HAIC), external radiation therapy or selective internal radiation therapy (SIRT).

In our institute, for the MDT approach in patients with difficult management, we had established Yonsei Liver Cancer Special Clinic since 2003. We performed concurrent loco-regional chemo-radiotherapy (CCRT) in localized advanced HCC with MVI. After the localized CCRT, hepatic arterial infusional chemotherapy was followed every 4 weeks. By using MDT approach, we could improve survival outcome in advanced HCC with MVI.

At present, it is widely accepted that a coordinated MDT approach offers the best approach to individualize therapy achieving optimal outcomes. 


\section{State-of-the-Art Lecture 4}

\section{5}

Gd-EOB-DTPA Enhanced MR Imaging for HCC: A New Paradigm from Early Detection and Characterization to Personalized Medicine

Osamu Matsui

Kanazawa University, Kanazawa, Japan

Gd-EOB-DTPA (PRIMOVIST_Bayer Schering Pharma AG, Berlin, Germany) is a hepatocyte-specific MR contrast agent with a lipophilic modification of Gd-DTPA. Because around 50\% of Gd-EOB-DTPA (gadoxetic acid) is taken up by hepatocytes and then excreted into the bile ducts, the liver parenchyma is strongly enhanced in the hepatobiliary (HB) phase of Gd-EOB-DTPA enhanced MR imaging (EOB-MRI) taken around 20 minutes after intravenous contrast injection, resulting in clear visualization of mass lesions which commonly do not take up EOB-DTPA as definite hypointense nodules. It is now the most sensitive modality for the detection of hepatocellular carcinoma (HCC) including hypovascular pathological early HCC. On the other hand, around $10-15 \%$ of hypervascular classic HCC show iso or hyperintensity relative to the surrounding liver on HB phase (paradoxical enhancement, hyperintense HCC) indicating a peculiar subtype of HCC. These facts promoted the investigation of molecular/genetic mechanisms of EOB-MRI. In addition, EOB-MRI has been proved to be valuable in the differential diagnosis of various hepatocellular nodules.

\section{Molecular Mechanism}

By analyzing the differences of expression of membranous transporters between hypointense and hyperintense HCC, it has become clear that there is a significant correlation between signal intensity of HCC on HB phase of EOB-MRI and hepatocyte uptake transporter OATP8 (1B3) expression in HCC cells and HB phase of EOB-MRI is an extremely sensitive (indirect) molecular imaging for hepatocyte uptake transporter OATP1B3 expression in HCC cells.

\section{Visualization of Multistep Hepatocarcinogenesis and Pathological "early HCC"}

OATP1B3 expression significantly decreases from very early stage of multistep hepatocarcinogenesis and around 85\% of early HCCs already demonstrates decreased OATP1B3 expression relative to the surrounding liver parenchyma resulting in an extremely high sensitivity of EOB-MRI in the detection of pathological early HCC.

Biological and Molecular/Genetic Features of Hyperintense HCC

HCC showing hyperintensity on HB phase (hyperintense HCC) is a peculiar subtype of HCC with OATP1B3 overexpression, biologically less aggressive nature and molecular/genetic features of mature hepatocytes with $\beta$-catenin and hepatocyte nuclear factor (HNF) $4 \alpha$ activation. The interaction between Wnt/ $\beta$ catenin signaling and HNF $4 \alpha$ may play an important role in OATP1B3 expression and development of the less aggressive biological nature of hyperintense HCC. 


\section{Differential Diagnosis from Benign Hepatocellular Nodules and Molecular Background}

Focal nodular hyperplasia (FNH) commonly shows iso- or hyperintensity in the HB phase with equal or stronger OATP1B3 expression, with map-like distribution of glutamine synthetase (a target of Wnt/ $\beta$-catenin signaling) and OATP1B3 expression. Nodular regenerative nodule (NRH) shows doughnut-like enhancement with hypointensity in the central portion in the HB phase with OATP1B3 expression with intramodular portal perfusion. Majority of hepatocellular adenoma (HCA) show hypointensity in the HB phase, but $\beta$-cateninactivated HCA exclusively demonstrates iso- or hyperintensity with increased expression of nuclear $\beta$-catenin, glutamine synthetase, and OATP1B3.

\section{Summary}

HB phase of EOB-MRI is a sensitive indirect molecular imaging for OATP1B3 expression in HCC cells, and therefore, a powerful imaging biomarker for the early diagnosis, subtype identification and prognosis prediction of HCC, thereby contributing to the personalized medicine of HCCs.

\section{References}

Kitao A, Zen Y, Matsui O, et al. Hepatocellular carcinoma: signal intensity at gadoxetic acid-enhanced MR Imaging: correlation with molecular transporters and histopathologic features. Radiology 2010;256:817-826.

Kitao A, Matsui O, Yoneda N, et al. The uptake transporter OATP8 expression decreases during multistep hepatocarcinogenesis: correlation with gadoxetic acid enhanced MR imaging. Eur Radiol. 2011;21:2056-2066.

Kitao A, Matsui O, Yoneda N, et al. Hypervascular hepatocellular carcinoma: correlation between biologic features and signal intensity on gadoxetic acid-enhanced MR images. Radiology. 2012;265:780-789.

Yamashita T, Kitao A, Matsui O, et al. Gd-EOB-DTPA-enhanced magnetic resonance imaging and alpha-fetoprotein predict prognosis of early-stage hepatocellular carcinoma. Hepatology 2014;60:1674-1685.

Yoneda N, Matsui O, Kitao A, et al. Hypervascular hepatocellular carcinomas showing hyperintensity on hepatobiliary phase of gadoxetic acid-enhanced magnetic resonance imaging: a possible subtype with mature hepatocyte nature. Jpn J Radiol. 2013;31:480-490.

Kitao A, Matsui O, Yoneda N, et al. Hepatocellular carcinoma with $\beta$-Catenin mutation: imaging and pathologic characteristics. Radiology 2015;275:708-717.

Yoneda N, Matsui O, Kitao A, et al. Hepatocyte transporter expression in FNH and FNH-like nodule: correlation with signal intensity on gadoxetic acid enhanced magnetic resonance images. Jpn J Radiol. 2012;30:499-508.

Yoneda N, Matsui 0, Kitao A, et al. Beta-catenin-activated hepatocellular adenoma showing hyperintensity on hepatobiliary-phase gadoxetic-enhanced magnetic resonance imaging and overexpression of OATP8. Jpn J Radiol. 2012;30:777782.

Kitao A, Matsui O, Yoneda N, et al. Differentiation Between Hepatocellular Carcinoma Showing Hyperintensity on the Hepatobiliary Phase of Gadoxetic Acid-Enhanced MRI and Focal Nodular Hyperplasia by CT and MRI. AJR Am J Roentgenol. 2018;30:1-11.

Kitao A, Matsui O, Yoneda N, et al. Gadoxetic acid-enhanced magnetic resonance imaging reflects co-activation of $\beta$-catenin and hepatocyte nuclear factor $4 \alpha$ in hepatocellular carcinoma. Hepatol Res. 2018;48:205-216.

Yoneda N, Matsui 0, Kitao A, et al. Benign Hepatocellular Nodules: Hepatobiliary Phase of Gadoxetic Acid-enhanced MR Imaging Based on Molecular Background. Radiographics. 2016;36:2010-2027. 


\section{Oral Poster}

\section{Biomarkers / Cell Biology and Translational Research}

\author{
PO-01 \\ Proteomic Features of HCC Reveal Clinically \\ Distinct Subtypes Independent of Oncogenic \\ Mutations \\ Ju-Seog Lee ${ }^{1}$, Ji-Hyun Shin'1, Yun Sung Jeong ${ }^{1}$, \\ Bo Hwa Sohn', Jae-Jun Shim², Sun Young Yim ${ }^{3}$ \\ ${ }^{1}$ Department of Systems Biology, UT MD Anderson \\ Cancer Center, USA; ${ }^{2}$ Department of Internal Medicine, \\ Kyung Hee University Medical Center, Seoul, Korea; \\ ${ }^{3}$ Department of Internal Medicine, Korea University \\ Medical Center, Seoul, Korea
}

\footnotetext{
Aims: Recent massive sequencing studies of HCC genomes revealed many new genetic alterations that might be accountable for HCC development and provided comprehensive view of malignant disease. However, genomic profiling of tumors is limited by a loose correlation between genetic alterations and their functional products such as proteins and metabolites. To overcome such limitation, several approaches such as proteomics and metabolomics have been developed to add more functional information to genomic characteristics of tumors. Reverse-phase protein array (RPPA) is one of such approaches and allows us to simultaneously measure multiple protein features, such as expression, modification of proteins, and interaction with ligands from the samples. We aim to characterize proteomic characteristics of HCC. Methods: We generated proteomic data including expression, phosphorylation, and cleavage of proteins from $297 \mathrm{HCC}$ tumors by using RPPA platform. Supervised and unsupervised approaches were applied to analyze proteomic data and multiple genomic data such as somatic mutations, mRNA expression, miRNA expression, and copy number alterations were integrated with proteomic data to uncover most correlated genomic alterations with functional products. Clinical significance of identified key protein features were validated in multiple independent cohorts of HCC patients. Results: Unsupervised analysis shows three subtypes of HCC with substantial difference in proteomic patterns. The differentially expressed proteins among three subtypes include cell cycle regulators such as CCND1, CCNE1, and CHK2 as well as cell growth-regulating kinases such as RAF and VEGFR2. Interestingly, smallest
}

subtype of HCC is characterized by low expression of epithelial marker proteins such as $\beta$-catenin and E-cadherin. Based on proteomic characteristics, three subtypes were named to mesenchymal (MES), metabolically active (MA), and kinaseactive and genome stable (KAGS) subtypes. When assessed clinical relevance, the overall survival rate of patients in MES subtype was significantly worse than those in MA and KAGS subtype $(P=0.001)$. Poor clinical outcomes of MES subtype is further validated in three independent cohorts (NCI cohort, $n=113$; Korean cohort, $n=100$; TCGA cohort, $n=371$ ). Integration with genomic data identified genetic and epigenetic alterations correlated with MES subtype. Further analysis showed that proteomic subtype is more correlated with copy number alterations than somatic mutations. Functional validation with cell lines demonstrated that some of correlated genes are essential for growth and survival of HCC cells. Conclusions: HCC can be classified into distinct subtypes by proteomic features independent of mutation profile. Proteomic analysis has identified potential key biomarkers with prognostic importance that can be easily translated to clinics. Our study also demonstrated merit of integrated analysis of proteomic data with genomic data to uncover potential driver genes of HCC development. Keywords: Proteomics, Genomics, Prognostic subtypes, Biomarkers.
PO-02
Mammalian Target of Rapamycin (mTOR) and Autophagy in Hepatitis C Virus-Related Hepatocellular Carcinoma: Relation to Tumor Progression
Hoda El Aggan ${ }^{1}$, Myriam Helmy², Laila Younis ${ }^{3}$, Ehab Hassona ${ }^{1}$, Sameh Lashen ${ }^{1}$
${ }^{1}$ Department of Medicine (Hepatobiliary Unit), Faculty of Medicine, University of Alexandria, Alexandria, Egypt; ${ }^{2}$ Department of Clinical Pathology, Faculty of Medicine, University of Alexandria, Alexandria, Egypt; ${ }^{3}$ Department of Pathology, Faculty of Medicine, University of Alexandria, Alexandria, Egypt

Aims: Mammalian target of rapamycin (mTOR) is a key regulator of cell metabolism, growth and proliferation and an inhibitor of autophagy. The present study was designed to study the role of mTOR and autophagy in hepatitis $C$ virus (HCV)-related hepatocellular carcinoma (HCC). Methods: 58 patients with HCV-related liver disease (30 patients with 
chronic hepatitis C (CHC), 14 patients with cirrhosis and 14 patients with HCC who underwent surgical resection) and 15 healthy subjects, were included in the study. Measurement of serum mTOR protein was determined using enzyme-linked immunosorbant assay. Tumor staging was determined according to Barcelona Clinic Liver Cancer (BCLC) system. Tumor histologicalgrade was assessed according to Edmonson and Steiner's criteria. Immunohistochemical examination was performed using anti-human antibodies against mTOR and autophagy-related protein 5 (Atg5), as a marker for autophagy. Results: Serum mTOR levels were significantly higher in patients with HCV-related liver disease than in healthy subjects with a progressive increase in patients with CHC to cirrhotic patients without HCC to patients with HCC $(P<$ 0.001). By plotting ROC curve, the sensitivity and specificity of serum mTOR in the detection of HCC were $92.9 \%$ and $100 \%$ respectively at a cut-off value of $4.55 \mathrm{ng} / \mathrm{ml}(\mathrm{AUC}=0.970)$. HCC tissues showed a significant increase in mTOR expression and a significant decrease in Atg5 expression compared with non-cancerous liver tissues $(P=0.013$ and $P=0.017)$. Serum mTOR levels and intratumoral mTOR expression in patients with HCC were inversely correlated with Atg5 expression $(P=$ 0.005 and $P<0.001$ respectively) and were positively correlated with serum alpha fetoprotein levels $(P=0.003$ and $P=$ $0.003)$ respectively and tumor size $(P=0.018$ and $P=0.032$ respectively), stage ( $P=0.032$ and $P=0.002$ respectively) and histological grade $(P=0.003$ and $P=0.025$ respectively). Conclusions: Activation of mTOR pathway plays an important role in the pathogenesis of HCV-related HCC, possibly, through inhibition of autophagy. Serum mTOR levels may represent a potential, simple diagnostic biomarker for HCC in chronic HCV infection. Keywords: Mammalian target of rapamycin, Autophagy, Hepatocellular carcinoma, Hepatitis C virus.

\section{PO-03 The Diagnostic Efficacy of M2BPGi for Liver Fibrosis in HCC and NAFLD Patients}

Se Young Jang ${ }^{1}$, Won Young Tak', Soo Young Park', Young-Oh Kweon ${ }^{1}$, Yu Rim Lee 1 , Bina Jeong ${ }^{1}$, Sangkyung Seo ${ }^{1}$ Gyoun-Eun Kang ${ }^{1}$, Gyeonghwa Kim², Keun Hur', Heon Tak Ha ${ }^{3}$, Jae Min Chun', Young Seok Han ${ }^{3}$, Man-Hoon Han ${ }^{4}$, Won Gee Lee ${ }^{5}$, Jung Gil Park ${ }^{6}$

${ }^{1}$ Department of Internal Medicine, School of Medicine, Kyungpook National University, Kyungpook National University Hospital, Daegu, South Korea; ${ }^{2}$ Department of Biochemistry and Cell Biology, Cell and Matrix Research Institute, School of Medicine, Kyungpook National University, Daegu, South Korea; ${ }^{3}$ Department of Surgery, School of Medicine, Kyungpook National University, Kyungpook National University Hospital, Daegu, South Korea; ${ }^{4}$ Department of Pathology, School of Medicine, Kyungpook National University, Kyungpook National University Hospital, Daegu, South Korea; ${ }^{5}$ Biostatistics, Medical Research Collaboration Center in KNUH, School of Medicine, Kyungpook National University, Daegu, South Korea; ${ }^{6}$ Department of Internal Medicine, College of Medicine, Yeungnam University, Daegu, South Korea

Aims: Mac-2 binding protein glycan isomer (M2BPGi) is recently identified as a useful non-invasive biomarker for the diagnosis of liver fibrosis. This study aimed to evaluate the diagnostic efficacy of serum M2BPGi for liver fibrosis in hepatocellular carcinoma (HCC) and non-alcoholic fatty liver disease (NAFLD) patients. Methods: M2BPGi levels were analyzed in serum samples collected from biopsy-proven HCC $(n=135)$ and NAFLD $(n=113)$ patients. Fibrosis was graded histopathologically in non-tumorous portion of HCC and NAFLD. Serum M2BPGi levels were determined with an automated immunoassay analyzer. Spearman's correlation and Kruskal-Wallis test were used to evaluate the correlation and comparison among groups. Diagnostic efficacy for fibrosis was evaluated by the area under the receiver operating characteristic curve (AUC). Results: Median levels (range) of M2BPGi in HCC and NAFLD patients were 1.21 (0.12-14.33) cut-off index (COI) and $0.59(0.13-5.90)$ COI, respectively. In HCC patients, fibrosis stages were $0(n=22), 1(n=10), 2(n=$ 11), $3(n=16)$, and $4(n=76)$. The M2BPGi levels showed a significant positive correlation $(r=0.436, p<0.001)$ with fibrosis grade in HCC patients and yielded the lower AUC value, $0.787(\mathrm{p}<0.001)$ than transient elastography (TE), AUC value, $0.806(p=0.030)$ to predict advanced fibrosis $(F>2)$. In NAFLD patients, fibrosis stages were $0(n=22), 1(n=34), 2$ $(n=28), 3(n=19)$, and $4(n=10)$. The M2BPGi levels showed a significant positive correlation $(r=0.578, p<0.001)$ with fibrosis grade in NAFLD patients and yielded the higher AUC value, $0.824(\mathrm{p}<0.001)$ than TE, AUC value, $0.637(\mathrm{p}=0.035)$ to predict advanced fibrosis ( $\mathrm{F}>2$ ). Conclusions: Serum 
M2BPGi can be a useful non-invasive biomarker for predicting fibrosis in HCC and especially in NAFLD patients. Keywords: Hepatocellular carcinoma, Liver fibrosis, Non-alcoholic fatty liver disease, Biomarker.

\section{PO-04 \\ RPS6KA3 Mutations in HBV-Associated HCCs Represent Aggressive Tumor Behavior Underscored by Increased MAPK Signaling and Implicate Sensitivity to Sorafenib \\ Lo-Kong Chan ${ }^{1,2}$, Daniel Wai-Hung Ho ${ }^{1,2}$, Charles Shing Kam ${ }^{1,2}$, Elley Yung-Tuen Chiu ${ }^{1,2}$, Tan-To Cheung ${ }^{3}$, Chung N. Tang ${ }^{4}$, Victor W.L. Tang ${ }^{5}$, Irene L.O. Lo ${ }^{6}$, Derek T.W. Yau ${ }^{6}$, Chun-Ming Wong ${ }^{1,2}$, Irene Oi-Lin $\mathrm{Ng}^{1,2}$ \\ ${ }^{1}$ Department of Pathology, The University of Hong Kong, Hong Kong; ${ }^{2}$ State Key Laboratory for Liver Research, The University of Hong Kong, Hong Kong ${ }^{3}$ Department of Surgery, The University of Hong Kong, Hong Kong; ${ }^{4}$ Department of Surgery, Pamela Youde Hospital, Hong Kong; ${ }^{5}$ Department of Pathology, Pamela Youde Hospital, Hong Kong \\ ${ }^{6}$ Department of Surgery, Queen Elizabeth Hospital, Hong Kong}

Aims: Molecular classifications of HCC are less well defined. In this study, somatic mutations of RPS6KA3 gene in HBV-associated HCCs were identified and functionally characterized. Methods: HBV-associated HCCs were subjected to discovery whole exome-sequencing $(n=16)$ and validation targeted DNA-sequencing analysis $(\mathrm{n}=95)$. Novel gene mutation identification and functional characterization of RPS6KA3 were performed. Results: RPS6KA3 was found to be recurrently mutated $(7 / 111,6.3 \%)$. All RPS6KA3 mutations were somatic and affected by various mutation types including non-synonymous $(3 / 7=42.9 \%)$, non-sense $(2 / 7=28.6 \%)$, INDEL $(1 / 7=14.3 \%)$ and splicing-related $(1 / 7=14.3 \%)$. RPS6KA3 encodes RSK2 (p90-ribosomal S6 kinase 2), an ERK1/2 downstream target with two kinase domains. Most of the mutations affected either one of these kinase domains. Interestingly, RPS6KA3 mutations had low co-occurrence with known HCC driver mutations (CTNNB1, AXIN1, ARID1A and TSC1/2). Upon clinicopathological correlation, RPS6KA3 mutation was significantly associated with venous invasion and tended to be associated with larger tumor size and advanced tumor stage. To functionally characterize RPS6KA3, a RPS6KA3 rescue model was established in Hep3B cells, which intrinsically carry an exon 10 truncation at RPS6KA3 with no RSK2 protein expression. Restoration of RSK2 expression in Hep3B significantly suppressed cell proliferation and migration, together with a reduction in c-Raf-MEKERK signaling. Importantly, RSK2-expressing Hep3B cells were more resistant to Sorafenib (Raf-inhibitor suppressing
MAPK signaling). On the other hand, RPS6KA3 was frequently $(44 / 105,42 \%)$ upregulated in our HCCs. The upregulation was associated with more aggressive tumor behavior. Stable shRSK2 knockdown suppressed the tumorigenicity and metastatic abilities in animal models. Conclusions: Altogether, our findings suggest that RPS6KA3 dysregulation plays an oncogenic role in HCC. The somatic RPS6KA3 mutations may define an HCC subgroup with more aggressive tumor behavior, underscored by increased MAPK signaling and more prone to MAPK signaling suppression (e.g. with Sorafenib). This previously unrecognized finding signifies RSK2 mutation may reflect cellular dependency on MAPK signaling in HCC. Funding support: Hong Kong Research Grants Council Themebased Research Scheme (T12-704116-R) and RGC General Research Fund (17141416). Keywords: HCC, Next generation sequencing, HBV, Gene mutation, MAPK signaling, RPS6KA3, RSK2.

\section{PO-05 \\ HHLA2 Expression Associates with Intratumoral Imbalance of Cytotoxic and Regulatory T Cells and Predicts Survival in Intrahepatic Cholangiocarcinoma after Resection}

Chu-Yu Jing, Yi-Peng Fu, Yong Yi, Shuang-Jian Qiu, Bo-Heng Zhang

The Liver Cancer Institute, Zhongshan Hospital and Shanghai Medical School, Fudan University, Shanghai, P.R. China

Aims: Intrahepatic cholangiocarcinoma (ICC) is a highly mortal malignancy which poses a great therapeutic challenge. Recently, immunotherapies abrogating immune suppression via blocking B7/CD28 family members have become a promising treatment for various cancers. HHLA2, a newly identified member of B7 family, potentially promotes cancer progression via inhibiting the function of $\mathrm{T}$ cells. Therefore, it is imperative to investigate the expression pattern of HHLA2 to ascertain whether it could serve as a candidate target of immunotherapy for ICC. Methods: Immunohistochemistry was performed to examine the expression of HHLA2 in tissue microarrays derived from 218 ICC patients underwent resection, including 153 and 65 patients in training and validation cohort respectively. The clinical significance of HHLA2 and its association with infiltrating $\mathrm{T}$ cells including cytotoxic $\mathrm{T}$ cells (CTLs) and regulatory $\mathrm{T}$ cells (Tregs) were also analyzed. Results: The expression of HHLA2 was prevalent in ICC with high expression rate of $49.0 \%$ in training cohort and a higher rate of $67.7 \%$ in validation cohort, which comprised more patients with lymph node metastasis. HHLA2 significantly correlated with elevated CEA levels and was identified as an independent prognostic indicator for overall survival in both cohorts. High HHLA2 expression was associated with 


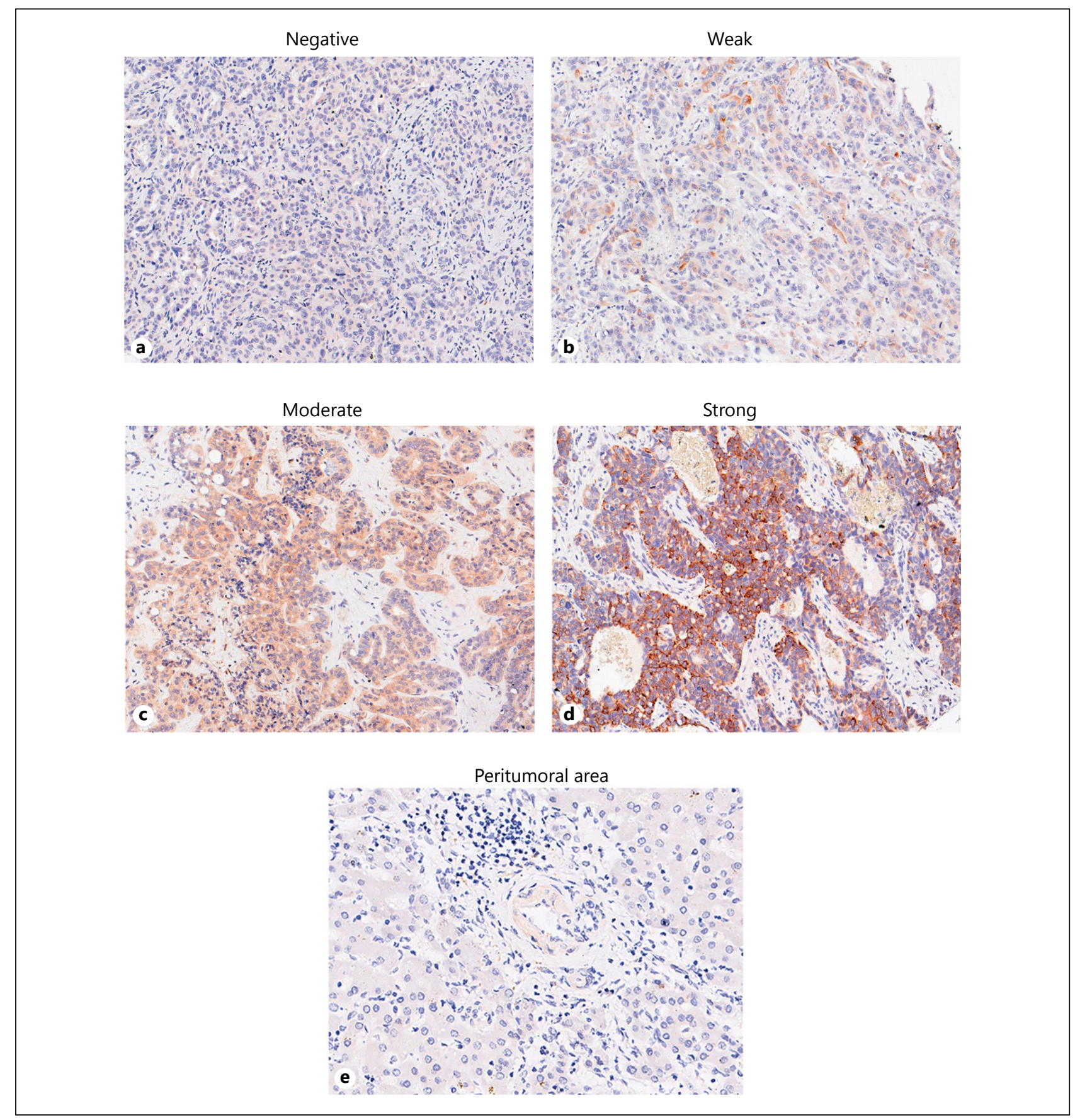

Fig. 1. (for Abstract no PO-05).

lower intra-tumor counts of T cells, CTLs and higher ratio of Tregs to CTLs. Conclusions: In ICC, HHLA2 is frequently expressed. High HHLA2 expression is an independent prognostic indicator and associates with less CTLs and higher proportion of Tregs, therefore conferring the rationale for targeting HHLA2 as an immunotherapy for ICC. Keywords:
Intrahepatic cholangiocarcinoma, HHLA2, Cytotoxic T cells, Regulatory T cells. 
The 9th Asia-Pacific Primary Liver Cancer Expert Meeting (APPLE 2018)

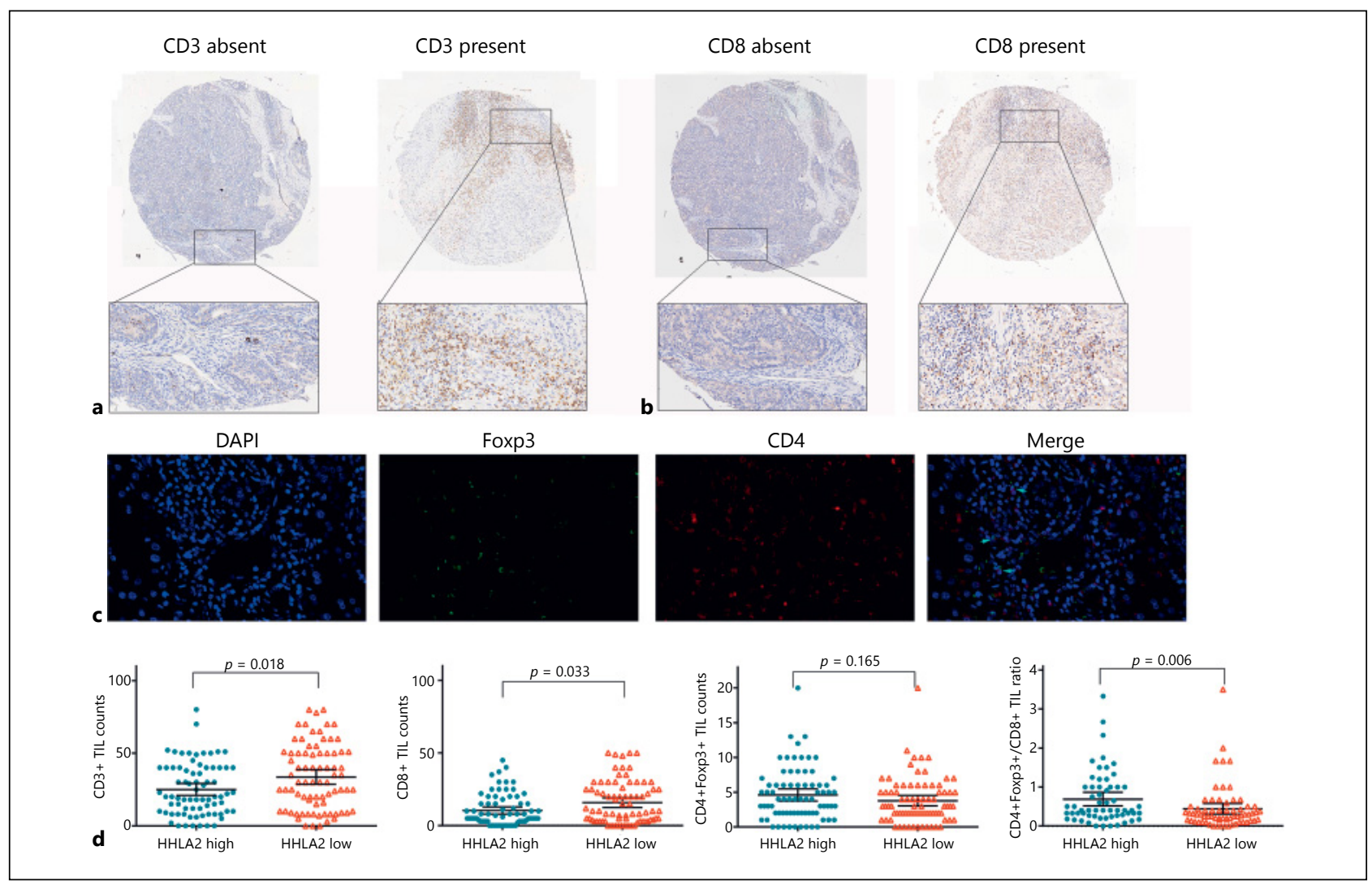

Fig. 2. (for Abstract no PO-05).

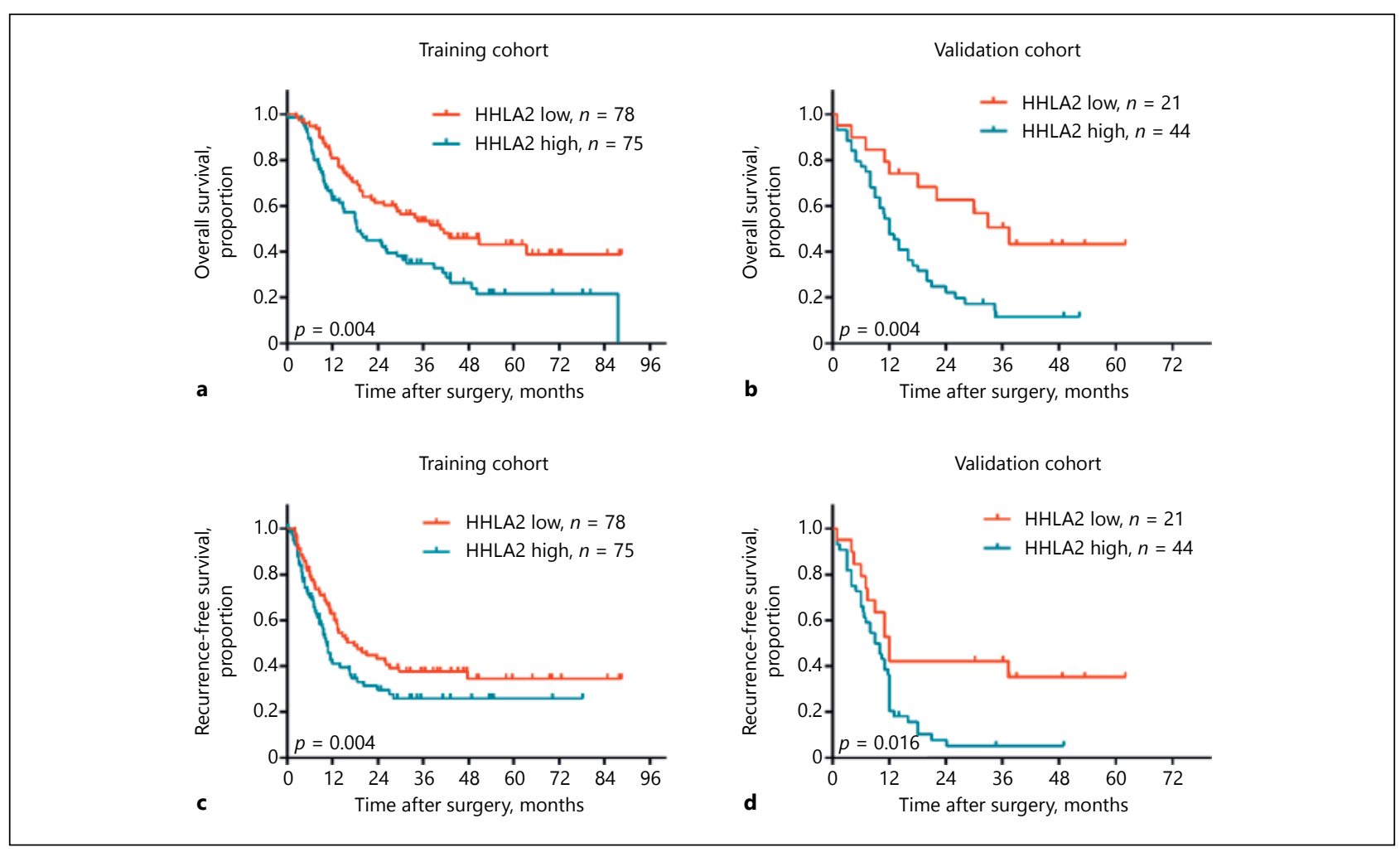

Fig. 3. (for Abstract no PO-05). 


\section{Viral Hepatitis / Cirrhosis and Related Complications}

\author{
PO-06 \\ Hepatic Progenitor Cells as Predictors of \\ Sustained Virologic Response to Antiviral \\ Therapy in Chronic Hepatitis C Viral Infection \\ Ali Kassem, Amr Abdul Fattah ${ }^{1}$, Ehab Moustafa', \\ Ali Ghweil', Eman Salah-Eldein ${ }^{3}$ \\ Internal Medicine, Tropical and Gastroenterology ${ }^{1}$ and \\ Pathology ${ }^{3}$ Departments, Sohag and Quna ${ }^{2}$ Faculties of \\ Medicine, Egypt
}

Aims: Primary therapeutic goal in chronic HCV infection is sustained virologic response (SVR), defined as undetectable HCV RNA at the end of a 24-week period after treatment completion. Hepatic progenitor cells (HPC) are small periportal cells capable of proliferation and differentiation into hepatocytes and bile ductular epithelium. Activation of these cells is histologically detectable only when hepatocyte proliferation is suppressed. We aim to detect predictors of sustained response to treatment in patients with HCV infection and to examine the correlation between hepatic progenitor cells expression and treatment response in these patients. Methods: We studied 483 patients ( 273 males and 210 females) with (HCV) infection, their ages ranged between (19-58 years). History, clinical and sonographic examinations and PCR for HCV were done to all patients. 438 liver biopsy samples were submitted to histological examination. 50 specimens of formalin-fixed paraffinembedded 5 micron tissue sections mounted on pre-cleaned coated slides were immunostained using peroxidase-labelled streptavidine-biotin technique to detect the expression of CK7 and CK19. Results: End treatment response was achieved in 231 (70\%), while 99 (30\%) patients were non-responders. Six months after the end of treatment, patients were categorized according to their PCR status into: patients who achieved SVR $(\mathrm{n}=132$ ) and patients who didn't achieve SVR \{including nonresponders $(\mathrm{n}=99)$ and relapsers $(\mathrm{n}=99)\}$. Sustained responders were significantly younger $(\mathrm{P}<0.004)$, with lower body weight $(\mathrm{P}<0.001)$, lower AST levels $(\mathrm{P}<0.000)$, lower AFP levels $(\mathrm{P}<0.000)$, lower fibrosis stages $(\mathrm{P}<0.000)$ and lower HPCs expression $(\mathrm{P}<0.000)$ Vs no responders. SVR was significantly higher in female Vs male patients $(\mathrm{P}<0.008)$. Conclusions: Patients with chronic HCV usually achieve SVR, if they are females, with younger age, lower body weight, lower AST and AFP levels, lower fibrosis stages and lower HPCs presentations. A highly significant inverse correlation between SVR and number of HPCs as assessed by CK19 expression was observed in HCV patients. Keywords: Hepatitis C virusinfection, Hepatic progenitor cells, Predictors of treatment response., Sustained Virologic response.

\section{PO-07 \\ Impact of Antiviral Therapy on Risk Prediction Models for Hepatocellular Carcinoma Development in Patients with Chronic Hepatitis B}

Hye Yeon Chon ${ }^{1}$, Tae Seop Lim', Mi Young Jeon', Hye Won Lee ${ }^{1,2,3}$, Beom Kyung Kim 1,2,3, Jun Yong Park ${ }^{1,2,3}$, Do Young Kim ${ }^{1,2,3}$, Sang Hoon Ahn 1,2,3, Kwang-Hyub Han 1,2,3, Seung Up Kim ${ }^{1,2,3}$

${ }^{1}$ Department of Internal Medicine, Yonsei University College of Medicine, Seoul, Republic of Korea; ${ }^{2}$ Institute of Gastroenterology, Yonsei University College of Medicine, Seoul, Republic of Korea; ${ }^{3}$ Yonsei Liver Center, Severance Hospital, Seoul, Republic of Korea

Aims: Risk prediction models for hepatocellular carcinoma (HCC) development have been proposed. However, the influence of antiviral therapy (AVT) on these models in patients with chronic hepatitis B (CHB) is unknown. Methods: We investigated the dynamics of risk prediction models during AVT and the association between on-treatment values from these models and the risk of HCC development. Results: Between 2005 and 2017, 6,098 patients with CHB (1,758 noncirrhotic, 4,340 cirrhotic) in whom AVT was initiated with entecavir $(n=3,818)$ or tenofovir $(n=2,280)$ were recruited. The mean age of the study population was 49.1 years and $61.4 \%(n=3,742)$ of the patients were male. The mean CU-HCC value was 12.7 at baseline in the entire study population; it was significantly lower (mean, 8.6) after 1-year of AVT ( $p<$ 0.001 ) and was maintained throughout 5-year of AVT (mean, 8.2-8.4; $p>0.05$ ). The proportion of high-risk patients (CU-HCC score $\geq 20$ ) was $28.9 \%$ at baseline, which significantly decreased after 1-year of AVT $(4.8 \% ; p<0.001)$ and was then maintained through 5-year of AVT $(2.6-3.5 \%$; $p>$ 0.05 ). The CU-HCC score after 1-year of AVT independently predicted the risk of HCC development (hazard ratio $=1.037$ ), together with age, male gender, liver cirrhosis, and platelet count (all $p<0.05$ ). Similar findings were obtained when the REACH-B criteria were used for non-cirrhotic patients. Conclusions: CU-HCC and REACH-B scores were significantly lower after 1-year of AVT and were maintained thereafter. CU-HCC and REACH-B scores after 1-year of AVT independently predicted the risk of HCC development in patients with CHB in whom AVT was initiated. Keywords: Antiviral therapy, Risk prediction, Hepatocellular carcinoma, Chronic hepatitis B. 


\section{PO-08 \\ Transient Elastography as a Predictor of De Novo Hepatocellular Carcinoma (HCC) Development in Cirrhotic Patients with Chronic Hepatitis C Treated with Direct Acting Antiviral Drugs (DAAs) \\ Mohamed Kohla, Mohamed Akl Rady, Maha Mohammad Elsabaawy, Mahmoud Helmy Allam, Abdelaleem Helal}

National Liver Institute, Menoufia University, Egypt

Aims: To evaluate Liver stiffness measurement by transient elastography as a predictor of HCC development after direct acting antiviral drugs (DAAs). Methods: All patients with HCV related fibrosis who did FibroScan ${ }^{\text {TM }}$ before treatment with DAAs were included retrospectively. Liver, renal function tests, CBC, INR, alpha-fetoprotien, abdominal ultrasonography, Triphasic CT, and FibroScan ${ }^{\mathrm{TM}}$ were performed at the National Liver Institute, Menofia University from January 2015 to December 2016. Patients who had a Transient Elastography measurement before treatment with DAAs were included and divided into two groups; Group (I) patients who developed De novo HCC and Group (II) are those who did not developed HCC after DAAs. Results: Patients who developed de novo HCC after DAAs (group I, thirty patients) had higher serum AST levels (68.3 \pm 38.2 vs. $48.7 \pm 32.4 \mathrm{U} / \mathrm{L}$ ), lower platelet count $\left(131.5 \pm 55.6\right.$ vs. $\left.179.5 \pm 69.8 \times 10^{3} / \mu \mathrm{L}\right)$

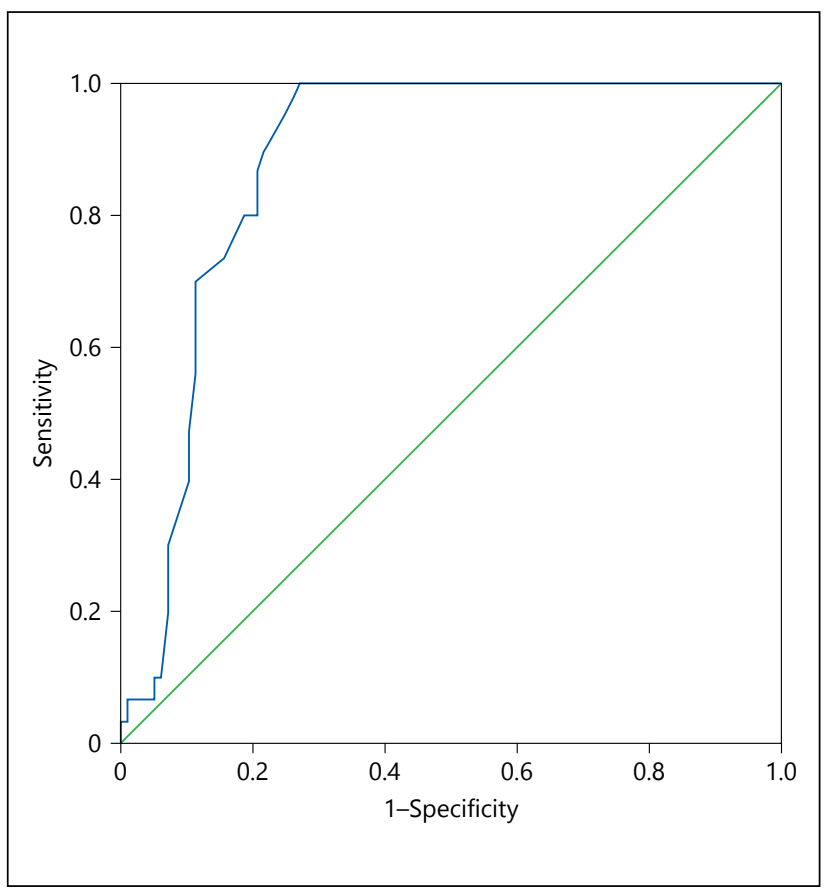

Fig. 1. Diagonal segments are produced by ties (for Abstract no PO-08). and older age ( $59.5 \pm 6.4$ vs. $51.3 \pm 10.5$ years) than patients who did not developed HCC (group II, ninety patients) with a $p$ value $<0.05$. Group I patients had a statistically significant higher liver stiffness measurement (LSM) by Fibroscan ${ }^{\mathrm{TM}}$ $(32.1 \pm 10.7$ vs $15.5 \pm 11.5 \mathrm{kPa}$, $\mathrm{p}$ value $=0.001)$ than patients who did not developed HCC. With a cutoff of $18.5 \mathrm{kPa}$ being the most predictive value of de novo HCC development after treatment of chronic HCV infection with DAAs $190.0 \%$ sensitivity, $80.0 \%$ specificity, $55.0 \%$ PPV, $97.3 \%$ NPV, $80.0 \%$ accuracy) as shown in figure 1 . Conclusions: The measurement of liver stiffness by FibroScan ${ }^{\mathrm{TM}}$ could be a reliable method for risk stratification and prediction of de novo HCC development after DAAs in cirrhotic patients. Keywords: Transient elastography, Hepatocellular carcinoma, Chronic hepatitis C, DAAs.

\section{PO-09 \\ Automatic Calculation of Muscle Mass of the Iliopsoas Muscle Using SYNAPSE 3D}

Chikara Ogawa ${ }^{1,2}$, Masahiro Morita ${ }^{1}$, Akina Omura ${ }^{1}$, Teruyo Noda ${ }^{1}$, Atsushi Kubo ${ }^{1}$, Toshihiro Matsunaka ${ }^{1}$, Hiroyuki Tamaki ${ }^{1}$, Mitsushige Shibatoge ${ }^{1}$,

Masatoshi Kudo 2

${ }^{1}$ Department of Gastroenterology and Hepatology, Takamatsu Red Cross Hospital 4-1-3 Ban-cho Takamatsu, Kagawa 760-0017, Japan; ${ }^{2}$ Department of Gastroenterology and Hepatology Kinki University Faculty of Medicine 377-2 Ohno-higashi Osakasayama, Osaka 589-8511, Japan

Aims: Many studies have reported on the importance of diagnosing sarcopenia in patients with liver disease. The guidelines for sarcopenia in Japan have defined the iliopsoas muscle mass measurement at L3 level as the criteria for diagnosis. In this study, the 3D image volume analyzer system SYNAPSE 3D was used to evaluate the correlation between the automatically calculated total volume of the iliopsoas muscle and the L3-level iliopsoas muscle area and the measurement results of the area by using a simple method, as well as test result reproducibility and measurement time of different operators. Methods: The subjects were 14 patients who were hospitalized for liver disease and who underwent plain computed tomography (CT) scan from the abdomen to the pelvis. The SYNAPSE 3D application 3D Fat Analysis was used to determine the difference in the psoas muscle regions by automatic calculation (PMRAC) and psoas muscle volume by automatic calculation (PMVAC) performed by a Synapse 3D expert and a beginner (test 1), the difference in the measurement times of the technicians performing the measurements (test 2), and the correlation among iliopsoas muscle area, PMRAC, and PMVAC with the simple method (test 3). Results: (Test 1) The L3-level PMRAC and PMVAC were $13.4 \pm 5.0 \mathrm{~cm}^{2}$ and $13.2 \pm 3.9 \mathrm{~cm}^{2}$ and $300.6 \pm 87.5 \mathrm{~cm}^{3}$ and $301.4 \pm 87.2 \mathrm{~cm}^{3}$, respectively. A very strong correlation 


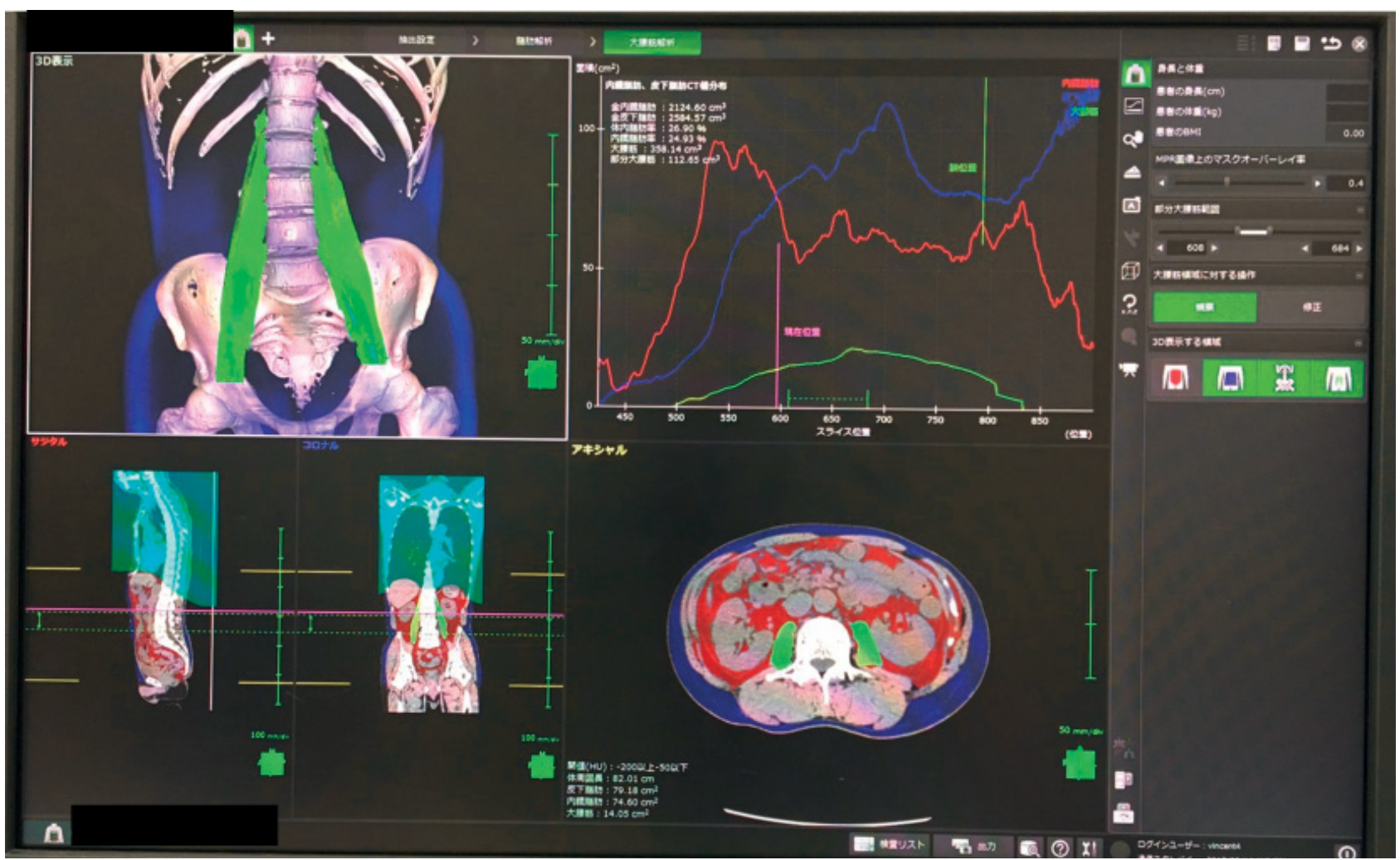

Fig. 1. (for Abstract no PO-09).

was observed, with correlation coefficients $\left(\mathrm{R}^{2}\right)$ of 0.8951 and 0.9987, respectively. (Test 2) The time until L3-level PMRAC and PMVAC were calculated was $96.4 \mathrm{~s}$ and $94.3 \mathrm{~s}$, respectively, with no significant difference observed ( $\mathrm{p}=0.7895)$. (Test 3) The correlation coefficients $\mathrm{R}^{2}$ between the L3-level simple method iliopsoas muscle volume and PMRAC, between the simple method and PMVAC, and between PMRAC and PMVAC were 0.837, 0.3647, and 0.5975, respectively, indicating strong correlations. Conclusions: PMRAC and PMVAC measurement using SYNAPSE 3D was convenient, reproducible, and useful because no differences were found with regard to the operators performing the measurements. Keywords: Sarcopenia, Iliopsoas, SYNAPSE 3D, VINCENT.

\section{PO-10 \\ Endothelial Dysfunction, Oxidative Stress and Inflammation in Patients with Hepatitis C Virus-Related Cirrhosis: Relation to Renal Function and Hemodynamics}

H. El Aggan ${ }^{1}$, S. Abodeya ${ }^{2}$, S. Mahmoud ${ }^{3}$

${ }^{1}$ Department of Medicine, Faculty of Medicine, University of Alexandria, Alexandria, Egypt; ${ }^{2}$ Department of Medical Biochemistry, Faculty of Medicine, University of Alexandria, Alexandria, Egypt

Aims: Endothelial dysfunction plays an important role in the pathogenesis of multiple diseases and may be related to oxidative stress and inflammation. The present work was designed to study markers of endothelial dysfunction, oxidative stress and inflammation in patients with hepatitis $C$ virus (HCV)-related cirrhosis in relation to renal function and hemodynamics. Methods: Thirty patients with HCV-related cirrhosis and 15 healthy subjects were included in the study. Endothelial dysfunction was evaluated by plasma von Willibrand factor (vWF) activity and serum angiotensin converting enzyme (ACE) levels. Serum levels of malondialdehyde (MDA) and high sensitivity C-reactive protein (hsCRP) were measured as markers for oxidative stress and systemic inflammation respectively. Glomerular injury was assessed by urinary 
albumin excretion rate (UAER) and tubular damage was determined by 24-hour urinary leucine aminopeptidase (LAP) levels. Renal hemodynamics were assessed using Duplexdoppler ultrasonography by calculating the diastolic/systolic renal flow velocity ratio $(\mathrm{d} / \mathrm{s})$, intrarenal resistive index (RI) and hilar renal blood flow (RBF). Results: Patients with HCVrelated cirrhosis showed significant increases in plasma vWF activity, serum levels of ACE, MDA and hsCRP, urinary LAP levels and RI and significant decreases in $\mathrm{d} / \mathrm{s}$ ratio and RBF compared with healthy subjects $(P<0.05)$. Plasma vWF activity and serum ACE levels were positively correlated with serum levels MDA and hsCRP, uAER, urinary LAP levels and RI and were inversely correlated with $\operatorname{RBF}(P<0.05)$. No statistically significant correlations were found between severity of liver disease and plasma vWF activity, serum ACE levels and urinary LAP levels in patients with cirrhosis $(P>0.05)$. Conclusions: Endothelial dysfunction, possibly due to oxidative stress and inflammation, plays an important role in the pathogenesis of renal dysfunction and increased renovascular impedence in patients with HCV-related cirrhosis. Pharmacological approaches to enhance endothelial function could improve renal function in these patients. Keywords: Endothelial dysfunction, Oxidative stress, Inflammation, Cirrhosis..

\section{Diagnosis and Liver Imaging / Epidemiology and Surveillance / Miscellaneous}

\section{PO-11 \\ Single Hepatocellular Carcinoma: Preoperative Diffusion-Weighted MRI for Predicting Early Recurrence after Curative Resection}

Sunyoung Lee ${ }^{1,2}$, Seong Hyun Kim¹, Jeong Ah Hwang ${ }^{1,3}$, Ji Eun Lee ${ }^{1,4}$, Sang Yun $\mathrm{Ha}^{5}$

${ }^{1}$ Department of Radiology and Center for Imaging Science, Samsung Medical Center, Sungkyunkwan University School of Medicine, ${ }^{2}$ Department of Radiology and Research Institute of Radiological Science, Severance Hospital, Yonsei University College of Medicine, ${ }^{3}$ Department of Radiology, Soonchunhyang University College of Medicine, Cheonan Hospital, ${ }^{4}$ Department of Radiology, Soonchunhyang University College of Medicine, Bucheon Hospital, ${ }^{5}$ Department of Pathology, Samsung Medical Center, Sungkyunkwan University School of Medicine, South Korea

Aims: To assess the value of preoperative diffusionweighted imaging (DWI) in predicting early recurrence $(<2$ years) and to determine the best predictive DWI parameters for early recurrence after surgery in single hepatocellular carcinoma (HCC). Methods: The study included 114 patients with surgically resected single HCC who underwent preoperative magnetic resonance imaging (MRI) with DWI. Radiologists evaluated the diffusion restriction of the tumors using qualitative assessment and the mean and minimum apparent diffusion coefficient values (ADCmean and ADCmin) of the tumors using quantitative measurement. Results: The optimal cutoff values of ADCmean and ADCmin for predicting early recurrence were $\leq 1.023$ and $\leq 0.773 \times 10^{-3} \mathrm{~mm}^{2} / \mathrm{s}$, respectively. The area under the receiver operating characteristic curve of ADCmin $(0.808,95 \%$ confidence interval [CI] 0.724 $0.876)$ was significantly greater than that of ADCmean $(0.698$, 95\% CI 0.605-0.781) ( $\mathrm{p}=0.022$ ). No significant difference was observed in early recurrence rates of HCCs between those with and those without diffusion restriction $(p=0.484)$. Early recurrence rates of HCCs with ADCmean and ADCmin values lower than the optimal cutoffs for these variables were significantly higher compared with those with values higher than the optimal cutoffs ( $p=0.001$ and $p<0.001$, respectively). Among the DWI parameters, ADCmin $\leq 0.773 \times 10^{-3} \mathrm{~mm}^{2} / \mathrm{sec}$ (hazard ratio 8.923, 95\% CI 2.358-33.769, $\mathrm{p}=0.001$ ) was the only significantly independent predictor of early HCC recurrence. Conclusions: DWI is a promising imaging tool in the prediction of early recurrence of HCC. Among qualitative and quantitative assessments of DWI, ADCmin is the best predictor of early recurrence after surgery in single HCC. Keywords: Hepatocellular carcinoma, Magnetic resonance imaging, Recurrence.

\section{PO-12 \\ The Clinical Application of Three- Dimensional Technology in Preoperative Planning of Centrally Located Liver Tumor \\ Rongfa Yuan, Yu wang, Linquan Wu, Jun Lei, Baolin Zhu, Kai Wang, Jianghua Shao \\ Department of Hepatopancreatobiliary Surgery, Second Affiliated Hospital of Nanchang University, Nanchang, China}

Aims: To explore the application value of 3D visualization technique in the preoperation planning of centrally located liver tumor. Methods: The clinical data of 28 patients with central liver tumor from January 2016 to November 2017 were analyzed retrospectively. Preoperative twodimensional CT images were obtained and imported into 3D visualization software. Image segmentation and 3D reconstruction were performed on liver, hepatic artery system, portal vein system, hepatic venous system and tumor respectively. According to the results of 3D reconstruction, the operation planning was carried out, and the rational operation path and method were selected. Results: The 3D visualization model was successfully established in all 28 patients. 3D tech- 

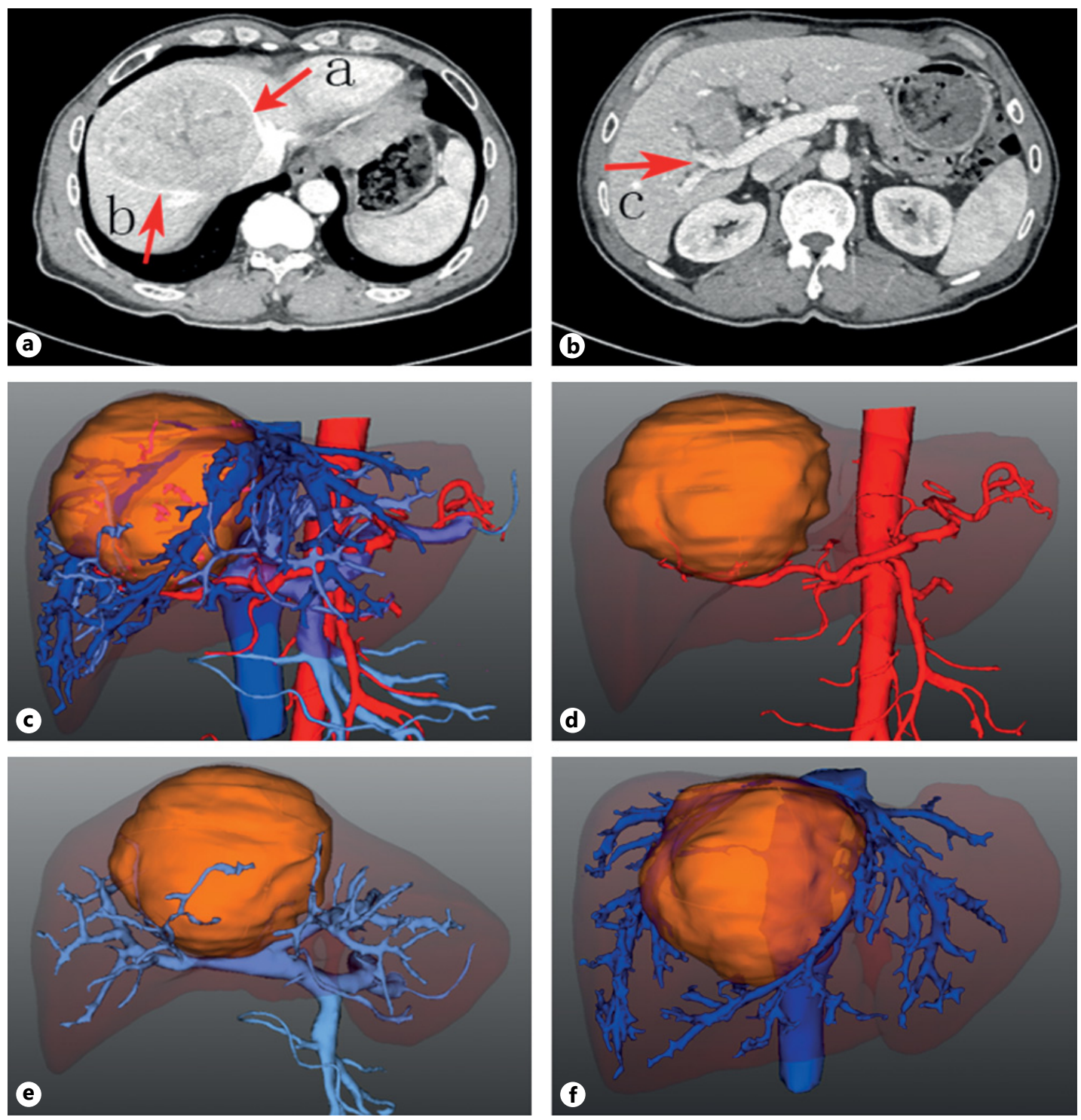

Fig. 1. 3D visualization analysis based on preoperative CT. a, b Upper abdomen enhanced CT scan (a indicates the middle hepatic vein, $\mathbf{b}$ indicates the right hepatic vein, and $\mathbf{c}$ indicates the right anterior portal vein). c 3D reconstruction results showing the relationship between tumor and peripheral blood vessels. d Spatial relationship between tumor and hepatic artery. e Spatial relationship between tumor and portal vein. $\mathbf{f}$ Spatial relationship between tumor and hepatic vein. (for Abstract no PO-12). nique can display the anatomy of each vascular system in the liver, the specific location, the size and the adjacent relationship of the tumor. It can also be used to perform individual liver segmentation and the centrally located liver tumor classification. The median volume of total liver was 1532 (10251864) $\mathrm{ml}$, the median volume of liver tumor was 466 (45-558) $\mathrm{ml}$, and the virtual resection liver volume was 595 (108-806) $\mathrm{ml}$. Radical resection of tumor was performed in 28 patients. The coincidence rate between the actual operation path and preoperative operation planning was $100 \%$ (28/28). The median of intraoperative bleeding and blood transfusion were
$700 \mathrm{ml}(100 \mathrm{ml}-2000 \mathrm{ml})$ and $600 \mathrm{ml}(0 \mathrm{ml}-1400 \mathrm{ml})$, the rate of blood transfusion was $71.42 \%(20 / 28)$, the incidence of complications was $28.57 \%(8 / 28)$ and the mortality rate was $0 \%$. Conclusions: $3 \mathrm{D}$ visualization technique can calculate the volume of the residual liver, and can improve the accuracy and safety of the preoperative planning of hepatectomy for centrally located liver tumor. Keywords: 3D technique, Centrally located liver tumor, Preoperative planning. 

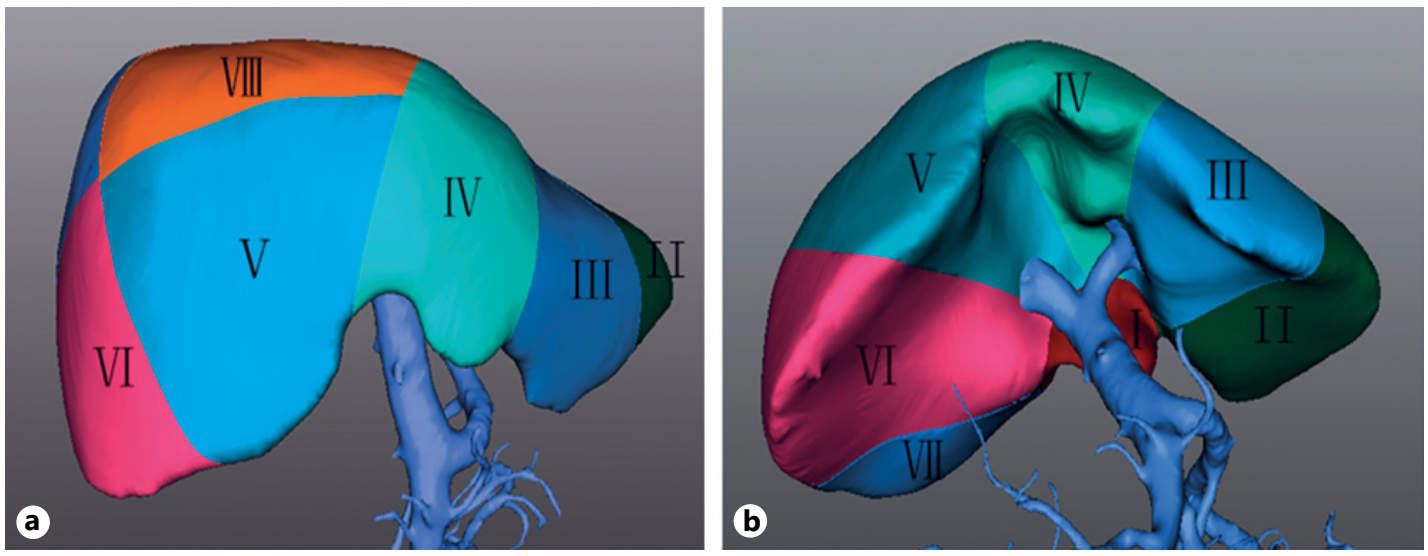

Fig. 2. 3D visualization of Couinaud liver segmentation. a The diaphragm surface of liver. $\mathbf{b}$ The visceral surface of liver (for Abstract no PO-12).

\section{PO-13}

\section{Unenhanced MRI versus Ultrasonography} for Hepatocellular Carcinoma Surveillance: Preliminary Results of a Prospective Randomized Trial (MIRACLE-HCC)

Hyungjin Rhee ${ }^{1}$, Chansik An ${ }^{1}$, Do-Young Kim², Jin-Young Choi ${ }^{1}$, Kwang-Hyub Han ${ }^{2}$, Myeong-Jin Kim ${ }^{1}$

${ }^{1}$ Department of Radiology, Severance Hospital, Yonsei University College of Medicine, Seoul, South Korea; ${ }^{2}$ Department of Internal Medicine, Severance Hospital, Yonsei University College of Medicine, Seoul, South Korea;

Aims: Guidelines recommend biannual ultrasonography (US) for hepatocellular carcinoma (HCC) surveillance in at-risk patients. However, there has been concern about the suboptimal performance of US. Our aim was to compare the performance of unenhanced magnetic resonance imaging (MRI) and US in the surveillance for HCC. Methods: For this prospective, nonblinded, balanced-randomized study (ClinicalTrials.gov identifier: NCT02514434), we have recruited patients with liver cirrhosis from a tertiary hospital in Korea. Patients were randomly assigned to biannual unenhanced MRI or US with alpha-fetoprotein (AFP) testing, for HCC surveillance. Our primary endpoints were detection rate of early-stage HCC, stage distribution at diagnosis, and false positive rate. Fisher's exact test was performed to compare the primary endpoints. Results: We performed a preliminary analysis of 377 patients who have received 1 to 6 examinations between May 2015 and March 2018. HCC incidence rate was 3.8\% per year. In 188 patients in the MRI group (504 examinations), 12 (6.4\% of patients) HCCs were detected at very early $(n=9)$ or early $(n=3)$ stage, with 2 ( $0.4 \%$ of exams) false referrals. In 189 patients in the US group (517 examinations), 10 (5.3\% of patients) HCCs were detected at very early $(n=3)$, early $(n=3)$, intermediate $(n=1)$, or advanced stage $(n=3)$ with $18(3.4 \%$ of exams) false referrals. The detection rate of very early or early HCC was higher in the
MRI group without statistical significance (6.4\% vs. 3.2\%; $P=$ $0.156)$. In the MRI group, the proportion of very early or early stage at diagnosis was higher (100\% vs. $60 \% ; P=0.029)$, and the false positive rate was lower $(0.4 \%$ vs. $3.4 \%$; $P<0.001)$, compared to the US group. Conclusions: The preliminary results demonstrate that unenhanced MRI may be a promising tool for HCC surveillance. Keywords: Hepatocellular carcinoma, Surveillance, Magnetic resonance imaging, Ultrasound.

\section{PO-14 \\ Comparison of Risk Prediction Model for Hepatocellular Carcinoma in Patients with Hepatitis B Virus-Related Cirrhosis Receiving Antiviral Therapy}

Sang Jun Suh ${ }^{1}$, Hyung Joon Yim ${ }^{1}$, Young-Sun Lee ${ }^{2}$, Han Ah Lee, , Tae Hyung Kim ${ }^{3}$, Sun Young Yim, Young Kul Jung ${ }^{1}$, Ji Hoon Kim², Yeon Seok Seo ${ }^{3}$, Jong Eun Yeon', Kwan Soo Byun', Soon Ho Um

${ }^{1}$ Department of Internal Medicine, Korea University Ansan Hospital, Ansan, South Korea; ${ }^{2}$ Department of Internal Medicine, Korea University Guro Hospital, Seoul, South Korea; ${ }^{3}$ Department of Internal Medicine, Korea University Anam Hospital, Seoul, South Korea

Aims: Various models for the prediction of hepatocellular carcinoma (HCC) in the patients with chronic hepatitis B (CHB) were suggested. The aim of study is to identify if the HCC risk scores are improved as antiviral therapy is prolonged in the patients with CHB-related liver cirrhosis. Methods: The patients with CHB who received entecavir (ETV) or tenofovir (TDF) were investigated retrospectively. Patients with liver cirrhosis patients diagnosed by sonography, CT or biopsy were enrolled. We calculated the HCC risk scores at pre-antiviral therapy, and each year from year 1 to 5 of post-antiviral therapy. The models were GAG-HCC, CU-HCC, REACH-B, modified REACH-B 
(mREACH-B), LSM-HCC, and PAGE-B. The primary endpoint was decrease of the risk scores after antiviral therapy. The secondary endpoint was finding the best model by AUROC after antiviral therapy. Results: A total of 362 patients were enrolled, and 198 and 164 patients were treated by ETV and TDF respectively. Child-Pugh scores were $5.7 \pm 1.3$ and MELD were $9.9 \pm 3$.8. Fiftysix patients (15.5\%) occurred HCC at median 1.6 years $(0.1-9.7$ years). Most HCC scores (GAG, CU-HCC, REACH-B) decreased at year 1 and plateaued from year 1 to 5. mREACH-B and LSM-HCC scores decreased until year 2 and plateaued after year 2. PAGE-B showed no decrease from pre to post-antiviral therapy. The AUROC of PAGE-B was largest at baseline (GAG-HCC 0.472, CU-HCC 0.753, REACH-B 0.633, mREACH-B 0.688, LSM-HCC 0.649 , and PAGE-B 0.760). After antiviral therapy, the AUROC changed.AUROCs of models employing HBVDNAlevelsincreased (GAG-HCC, REACH-B, and LSM-HCC), that of liver stiffness based models (mREACH-B and PAGE-B) were persistent, and that of models employing hepatic function (CU-HCC) decreased (GAG-HCC 0.582, CU-HCC 0.686, REACH-B 0.689, mREACH-B 0.689 , LSM-HCC 0.716, and PAGE-B 0.755 at 1 year). The decrease of scores from baseline to each years were not different between ETV and TDF (all p > 0.05). AUROC were largest in PAGE-B, however the scores were not changed after antiviral therapy. Second largest AUROC is that of LSM-HCC at year 1 and its AUROC became larger after antiviral therapy. Conclusions: In conclusion, HCC prediction models such as PAGE-B and LSMbased models worked well in patients with HBV-related cirrhosis and decrease of the scores was associated with effects of the antiviral therapy. Keywords: Hepatocellular carcinoma, Chronic hepatitis B, Liver cirrhosis, Prediction risk model.

\section{PO-15 \\ Effects of Influenza Vaccination on the Admission Outcomes of Liver Cancer: A Nationwide Matched Study}

Chien-Chang Liao ${ }^{1,2}$, Ta-Liang Chen ${ }^{1,2}$

${ }^{1}$ Department of Anesthesiology, Taipei Medical University Hospital, Taiwan; ${ }^{2}$ School of Medicine, Taipei Medical University, Taiwan

Aims: Limited information is available on the association between influenza vaccination (IV) and cancer outcomes. The purpose of this study is to evaluate the effects of IV on the outcomes of liver cancer. Methods: Using reimbursement claims data of National Health Insurance in Taiwan in 20082013 , we conducted a matched cohort study including 32330 patients aged $\geq 20$ years underwent inpatient care of liver cancer and 5841 of them received IV within previous one year. Using propensity score matching procedure, we selected 5621 patients with IV and 5621 patients without IV for comparison. Logistic regressions were used to calculate odds ratios (ORs) with 95\% confidence intervals (CIs) of IV associated with complications and in-hospital mortality during admission of liver cancer. Results: Patients with liver cancer receiving IV showed lower risks of pneumonia (OR 0.77, 95\% CI 0.620.96 ) and acute renal failure (OR 0.75, 95\% CI 0.57-0.97) compared with those without IV. In addition, IV was associated with decreased risk of in-hospital mortality during the cancer admission (OR, 0.57; 95\% CI, 0.44-0.73) after adjustment. Vaccinated patients with liver cancer also had shorter length of hospital stay $(\mathrm{p}<0.0001)$ than non-vaccinated patients. Conclusions: Among patients with admission of liver cancer, vaccinated patients showed lower risks of complications and in-hospital mortality compared with unvaccinated patients. Further studies are needed to explain how IV improves outcomes of liver cancer. Keywords: Liver cancer, Influenza vaccination, Outcomes, Admission.

\section{Molecular Pathogenesis and Pathology / Staging and Prognosis}

\section{PO-16 \\ Epithelial-Mesenchymal Transition Promoted by Activated Hepatic Stellate Cells through Transglutaminase 2-Induced Pseudohypoxia in Hepatocellular Carcinoma}

Hui Ma ${ }^{1}$, Liqi Xie ${ }^{2}$, Lan Zhang ${ }^{1}$, Xin Yin ${ }^{1}$, Hucong Jiang ${ }^{2}$, Xiaoying Xie ${ }^{1}$, Rongxin Chen ${ }^{1}$, Haojie L $\mathrm{Lu}^{2}$, Zhenggang Ren ${ }^{1}$

${ }^{1}$ Liver Cancer Institute, Zhongshan Hospital, Fudan University; ${ }^{2}$ Department of Chemistry and Institutes of Biomedical Sciences of Shanghai Medical School, Fudan University, China

Aims: Activation of hepatic stellate cells (HSCs) reportedly contributes to progression of hepatocellular carcinoma (HCC). However, crosstalk between HCC cells and HSCs pertaining to HSC activation and the promoting of HCC progression is still poorly understood. Methods: Quantitative proteomics were used an overview of dynamic changes promoted in HCC cells by activated HSCs and ingenuity pathway analysis was used to cluster functional data and further characterize roles of differentially expressed proteins. Transglutaminase 2 (TGM2) upregulation was confirmed by western blot, immunofluorescence and immunohistochemistry in the course of activated HSCs promoting epithelialmesenchymal transition (EMT) in HCC-derived cells in vivo and in vitro. Lentivirus-mediated micro-RNA was used to upregulate or downregulate TGM2 expression to investigate the significance of TGM2 in promoting HCC cells EMT in vivo and in vitro. Plasmid-mediated micro-RNA downregulating hypoxia inducible factor-1a (HIF-1a) expression and immunoprecipitation revealing protein-protein interactions were 


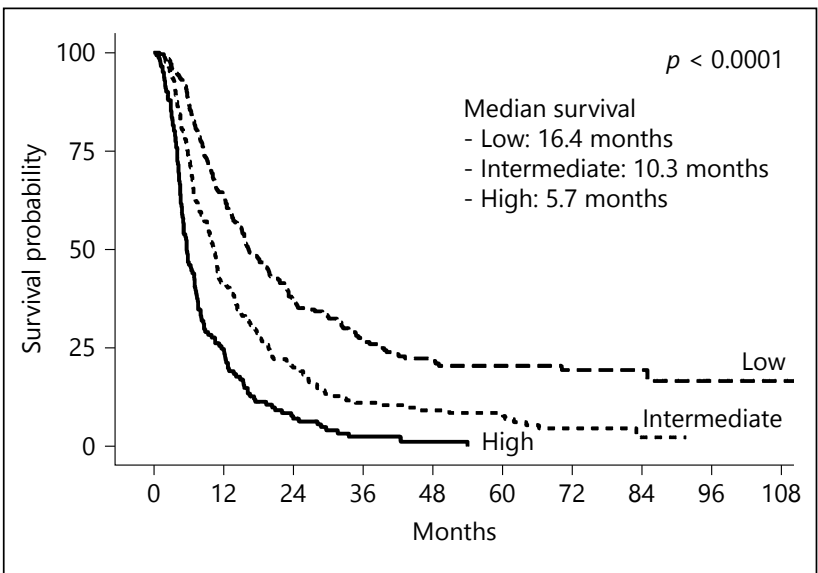

Fig. 1. (for Abstract no PO-16).

performed to study the mechanism. Expression levels of TGM2 were assessed using immunohistochemistry in tissue from 149 postoperative HCC patients, and the significance of TGM2 associated with overall survival and recurrence free survival were calculated with Kaplan Meier method. Results: TGM2 was upregulated in the course of activated HSCs promoting epithelial-mesenchymal transition (EMT) in HCCderived cells. Mechanistically, activated HSCs promoted TGM2 upregulation in HCC cells through inflammatory signaling; and TGM2 induced depletion of von Hippel-Lindau (VHL) protein, a key molecule in the degradation of HIF-1a under normoxia, then caused HIF-1a to accumulate, thereby producing a pseudohypoxic state that promotes EMT in HCC cells. After a median follow-up of 47 months, patients with high (vs low) TGM2 levels displayed significantly worse recurrence free $(P=0.006)$ and overall $(P=0.003)$ survival rates. Conclusions: We conclude that the promotion of EMT in HCC cells by activated HSCs is mediated by pseudohypoxia induced via TGM2/VHL/HIF-1a pathway. Keywords: Transglutaminase 2, Hepatocellular carcinoma, Epithelial-mesenchymal transition, Activated hepatic stellate cells.

\section{PO-17 \\ Single-Cell Transcriptome Sequencing Delineates Intra-Tumoral Heterogeneity and Reveals Rare Stemness-Related Cell Subpopulation in HCC}

Daniel Wai-Hung Ho ${ }^{1,2}$, Yu-Man Tsui ${ }^{1,2}$, Karen Man-Fong Sze ${ }^{1,2}$, Lo-Kong Chan ${ }^{1,2}$, Tan-To Cheung ${ }^{3}$, Lu Cheng ${ }^{1,2}$, Eva Lee ${ }^{1,2}$, Angela Ruohao $\mathrm{Wu}^{4}$, Pak-Chung Sham ${ }^{5}$, Stephen Kwok-Wing Tsui ${ }^{6}$, Terence Kin-Wah Lee ${ }^{7}$, Irene Oi-Lin $\mathrm{Ng}^{1,2}$

${ }^{1}$ Department of Pathology, University of Hong Kong, ${ }^{2}$ State Key Laboratory for Liver Research, University of Hong Kong, ${ }^{3}$ Department of Surgery, University of Hong Kong, ${ }^{4}$ Division of Life Science, Hong Kong University of Science and Technology, ${ }^{5}$ Department of Psychiatry, University of Hong Kong, ${ }^{6}$ School of Biomedical Sciences, Chinese University of Hong Kong, ${ }^{7}$ Department of Applied Biology and Chemical Technology, Hong Kong Polytechnic University

Background: Single-cell genomics has emerged as a powerful strategy to delineate the complex molecular landscapes of cancers, particularly HCC. It is a state-of-the-art technology which can unprecedentedly address intra-tumoral heterogeneity, instead of using the traditional bulk-cell methods which mask cell-level biological variations. Methods: We employed the perfect combination of Fluidigm C1 singlecell capturing system and next-generation sequencing to interrogate the intra-tumoral heterogeneity based on a patient-derived HCC tumor xenograft (PDTX) model. Upon the generated single-cell transcriptome sequencing data on 139 unselected HCC single cells, we applied unsupervised hierarchical clustering, dimension reduction analyses, and heatmap visualization to undertake discovery exploration. We confirmed the discovery findings on the same PDTX sample and other relevant HCC cell-lines, using fluorescence-assisted cell sorting, liver cancer stem cell (CSC) marker-sorted transcriptome sequencing, and sphere formation assays. Results: Single-cell transcriptomic landscape revealed an intratumoral heterogeneity pattern, which could be categorized into 2 major cell lineages by EPCAM expression. Moreover, we observed a negative correlation between EPCAM and CD13 expressions and identified a rare $\mathrm{CD} 24^{+} / \mathrm{CD} 44^{+}$-enriched cell subpopulation residing within the tumor-bulk. Upon dissecting the intricate inter-relationships among various liver CSC markers, we were able to classify HCC single cells into different liver CSC groups and define their enrichment of individual liver CSC markers. Most HCC cells displayed enriched expression of liver CSC markers, ranging from one to three markers. Finally, upon confirmation of the single-cell analysis findings, we demonstrated that $\mathrm{CD} 24^{+} / \mathrm{CD} 44^{+}$enriched HCC cells possess specific oncogenic gene expression signature and may represent a novel stemness-related subclone. Conclusions: Our proof-of-concept investigation 


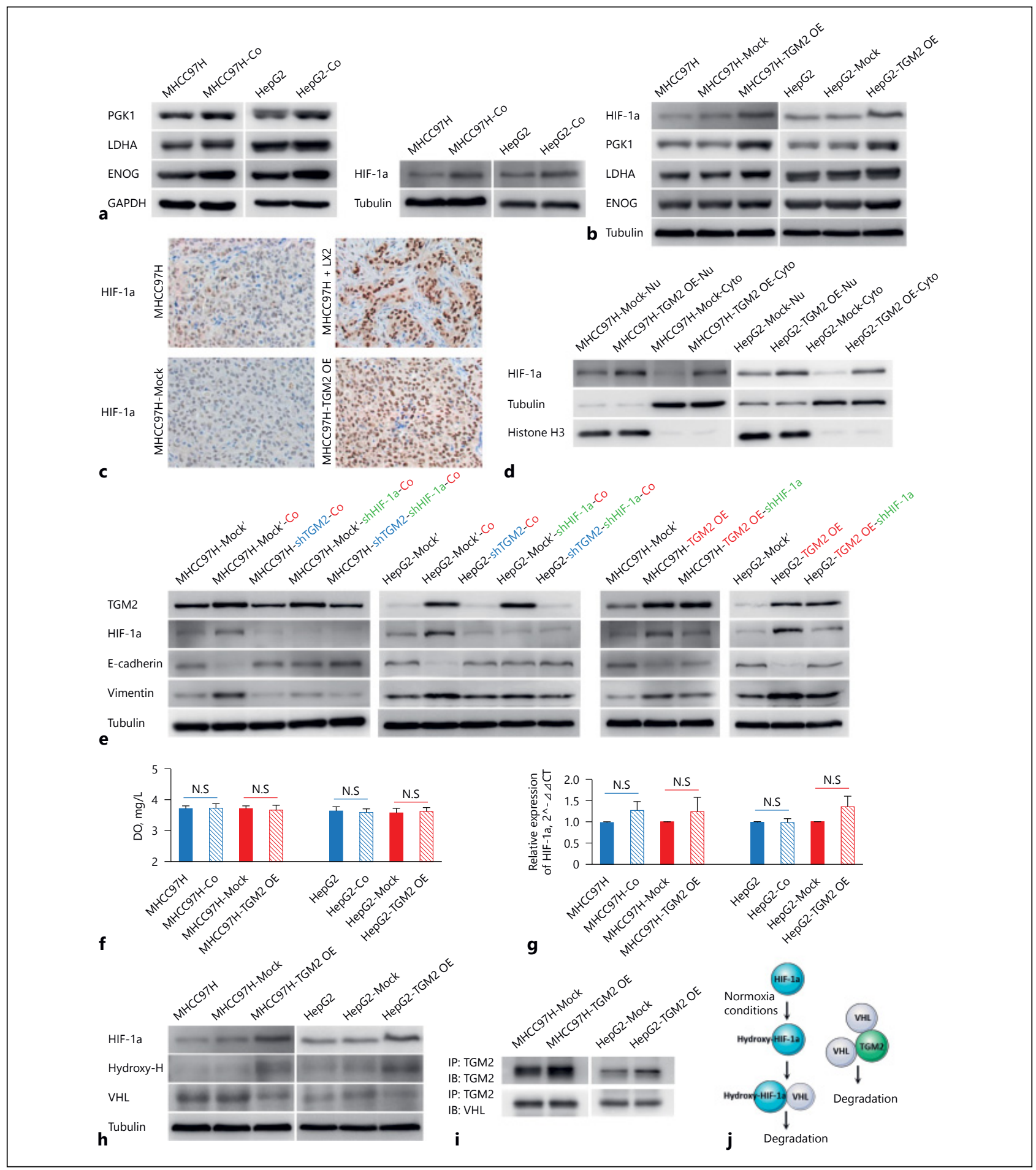

Fig. 2. (for Abstract no PO-16).

has provided essential evidence supporting the importance of single-cell genomics in dissecting the tumor biology of HCC and liver CSCs. We anticipate single-cell genomics will provide not only useful insight to HCC research but also pragmatic guidance on better precision medicine in the long run. Funding support: Hong Kong Research Grants Council Theme-based Research Scheme (T12-704116-R). Keywords: Single-cell RNA-seq, Intra-tumoral heterogeneity, Cancer stem cell, Patient-derived tumor xenograft, HCC. 

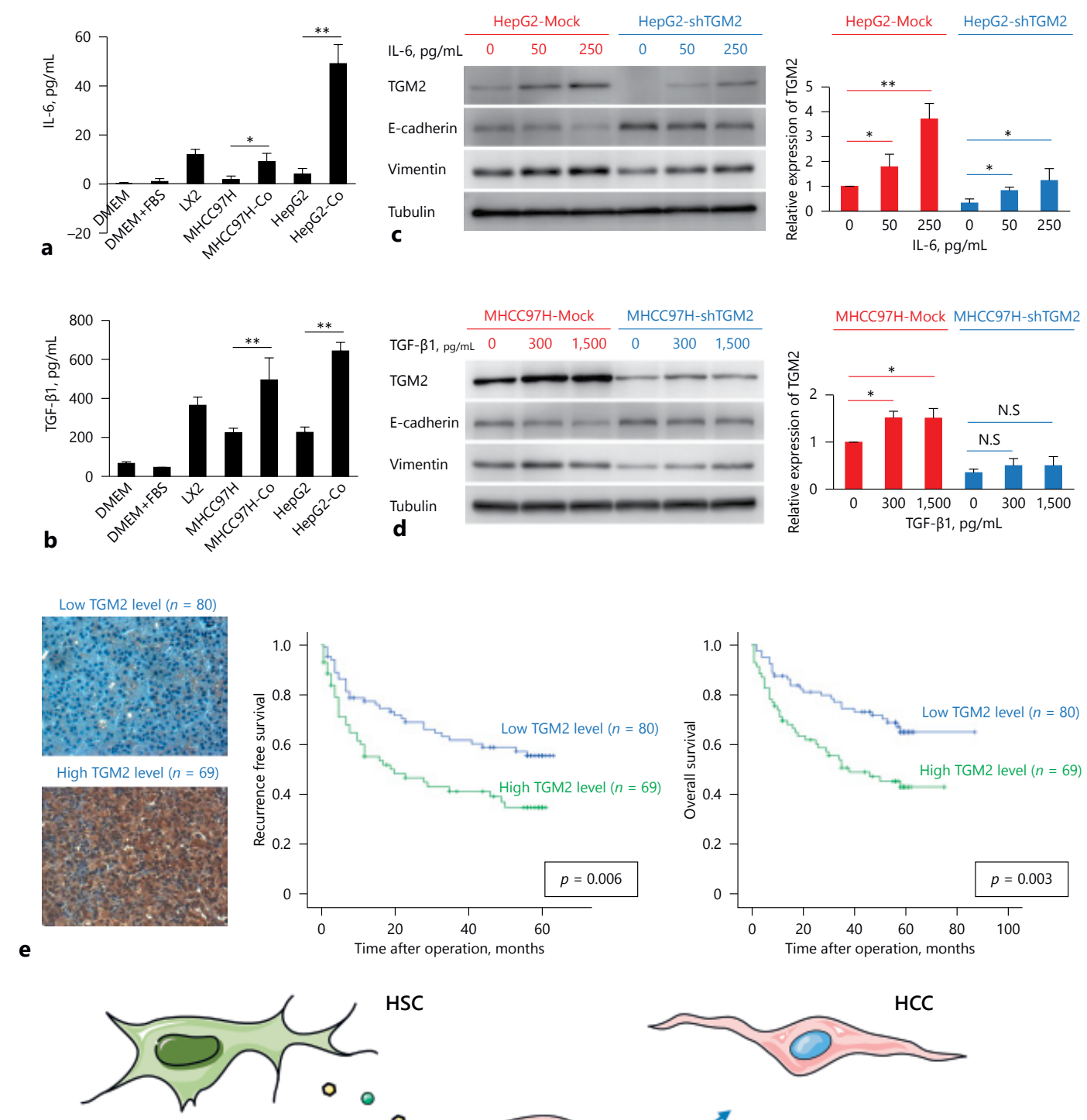

HSC
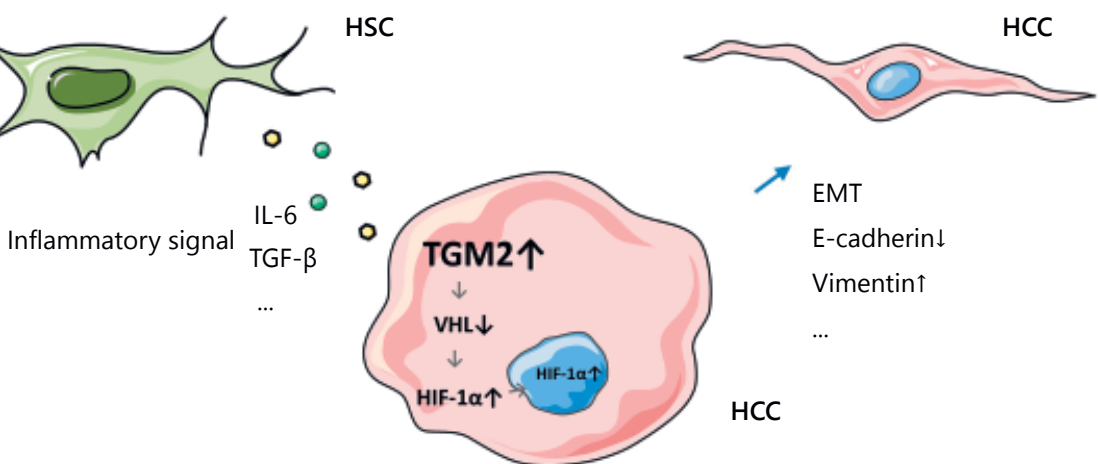

EMT

E-cadherin $\downarrow$

Vimentin

f

$\mathrm{HCC}$

Fig. 3. (for Abstract no PO-16). 


\section{PO-18 \\ EZH2 Regulates Positive Epigenetic Expression of UBE2I in Driving PI3K/ AKT Signaling-Induced Hepatocellular Carcinogenesis}

Zhuo Yu, Hai Feng ${ }^{3}$, Xuehua Sun ${ }^{1}$, Man $L i^{2}$, Lingying Huang ${ }^{1}$, Xiaojun Zhu' ${ }^{1}$, Fan Le ${ }^{1}$, Yueqiu Gao ${ }^{1,2}$

${ }^{1}$ Liver Disease Department, Shuguang Hospital

Affiliated to Shanghai University of Traditional Chinese Medicine, Shanghai, P.R.China; ${ }^{2}$ Laboratory of Cellular Immunity, Shuguang Hospital Affiliated to Shanghai University of Traditional Chinese Medicine, Shanghai, P.R. China; ${ }^{3}$ Department of pharmacology, School of Pharmacy, Harbin Medical University, Harbin, P.R. China

Aims: Overexpression of EZH2 has shown to be the hallmark of HCC development with poor prognosis, but the carcinogenic mechanism has yet to be fully investigated. Of note, we found that EZH2 functions as a transcriptional activator to promote cell proliferation and tumorigenesis independent of PRC2-mediated repressive epigenetic modification. Therefore, in the present study, we investigated positive epigenetic regulation of EZH2 targeting potential oncogenes in HCC. Methods: Epigenome-wide RNA interference-based screening was performed to identify potential oncogenes upregulated by EZH2 activation. Gene regulation in vitro and in vivo was assessed by chromatin immunoprecipitation, luciferase reporter assay, quantitative RT-PCR, immunoblotting and immunohistochemistry. Cellular proliferation and tumorigenicity concerning ectopic gene expression and RNA interference were determined by colony formation and soft agar assay, xenograft mouse model. Results: Ubiquitin conjugating enzyme E2I (UBE2I) was identified to be a candidate EZH2 epigenetic regulated effector which stimulated PI3K/AKT signaling in HCC cells. EZH2 was activated through phosphorylation at Ser21 to upregulate UBE2I transcription and expression by recruiting with histone H3 lysine4 trimethylation (H3K4me3) on UBE2I promoter. UBE2I upregulation increased the activity of PI3K/AKT signaling through AKT phosphorylation at Ser473, which could be abolished by suppression of EZH2 or ectopic expression of phosphorylation-deficient Ser21 Ala (EZH2 S21A) mutant. In functional analysis, UBE2I was characterized with oncogenic ability to promote cell proliferation and transformation in vitro and tumorigenesis in vivo. Ablation of UBE2I inactivated PI3K/ AKT signaling and inhibited EZH2-mediated HCC tumor growth in mice, as did EZH2 S21A mutant. Increased EZH2 expression and phosphorylation correlated with the expression of H3K4me3 and UBE2I, PI3K/AKT signaling activity in tumor compared with non-tumor tissues of mice model. Conclusions: Our findings revealed the new mechanism that EZH2 serves as a transcription activator to stimulate UBE2I expression, which promotes HCC tumorigenesis through the activation of PI3K/AKT signaling. Keywords: EZH2, HCC, UBE2I, PI3K/AKT signaling, Epigenetics.

\section{PO-19 \\ Risk Assessment in Patients Treated with TACE Due to Recurrent Hepatocellular Carcinoma after Curative Resection: A Retrospective Multicenter Study}

Mi Young Jeon 1,2, Beom Kyung Kim 1,2, Jun Yong Park ${ }^{1,2}$, Do Young Kim ${ }^{1,2}$, Sang Hoon Ahn ${ }^{1,2}$, Kwang-Hyub $\mathrm{Han}^{1,2}$, Jeong-Hoon Lee ${ }^{3}$, Su Jong Yu ${ }^{3}$, Yoon Jun Kim ${ }^{3}$, Jung-Hwan Yoon ${ }^{3}$, Eun Ju Cho ${ }^{3}$, Seung Up Kim ${ }^{1,2}$

${ }^{1}$ Department of Internal Medicine, Institute of Gastroenterology, Yonsei University College of Medicine, ${ }^{2}$ Yonsei Liver Center, Severance Hospital, Seoul, South Korea; ${ }^{3}$ Department of Internal Medicine and Liver Research Institute, Seoul National University College of Medicine, Seoul, South Korea

Aims: Few prognostic models are available for patients with recurrent hepatocellular carcinoma (HCC) following curative resection. A new postoperative hepatoma arterialembolization prognostic (HAP) score optimized for patients treated with trans-arterial chemoembolization (TACE) due to recurrent HCC after curative resection was developed and validated. Methods: A total of 448 (training cohort) and 350 (validation cohort) patients with recurrent HCC after curative resection treated with TACE between 2003 and 2016 were enrolled. Cox regression and area under the curve (AUC) analysis were used to identify risk factors and to calculate the predictive performance of risk scores, respectively. Results: The median age of the study population (378 men, 70 women) was 59.4 years. The median time from resection to recurrence was 17.7 (interquartile range, 7.3-37.1) months. Alpha-fetoprotein $>400 \mathrm{ng} / \mathrm{mL}$ (hazard ratio $[\mathrm{HR}]=2.358$ ), serum albumin $\leq 3.5 \mathrm{~g} / \mathrm{dL}(\mathrm{HR}=1.979)$, tumor number $\geq 2$ ( $\mathrm{HR}=$ $1.655)$, segmental portal vein invasion $(H R=2.541)$, and time from resection to recurrence $\leq 2$ years $(H R=1.905)$ independently predicted survival (all $P<0.05$ ). The postoperative HAP (pHAP) model established based on the rounded HR of each variable showed an AUC to predict survival at 3-years of 0.671 significantly higher than those of other HAP-based models including HAP, modified HAP, and modified HAP-II scores (0.578-0.624) (all $P<0.05$ ). The accuracy of pHAP was maintained in the validation cohort $(n=350$; $A U C=0.638$ at 3-year). Conclusions: A new pHAP score optimized for patients treated with TACE due to recurrent HCC after curative resection showed acceptable accuracy and was externally validated. Further studies should investigate methods to select treatment options other than TACE for high-risk patients according to pHAP scores. Keywords: Hepatocellular carcinoma, TACE, Resection, Risk assessment. 


\section{PO-20 \\ PDL1CD47CD73 Score Emerges as a Novel Independent Prognostic Factor in Patients with Hepatocellular Carcinoma after Curative Resection}

Jin-Long Huang, Yi-Peng Fu, Yong Yi, Wei Gan, Liu Gao, Shuang-Jian Qiu

Department of Liver Surgery and Transplantation, Liver Cancer Institute, Zhongshan Hospital, Fudan University; Key Laboratory of Carcinogenesis and Cancer Invasion of Ministry of Education, Shanghai, China

Aims: The aberrant expression of immune checkpoints on tumor cells dampens antitumor immunity, resulting in tumor immune evasion. However, conflicting results on the association between the expression of immune checkpoints and prognosis still confuse us. In this study, we combined the expression of PD-L1, CD47 and CD73 (namely PDL1CD47CD73 score) in human hepatocellular carcinoma (HCC) to define their prognostic significance after curative surgery. Moreover, we formulated prognostic nomograms for OS and RFS comprehensively considering the immune status and tumor burden for HCC after hepatectomy. Methods: Immunohistochemistry was applied to explore the expression of PD-L1, CD47 and CD73 as well as CD8+CTLs and CD4+FOXP3+ regulatory T cells (Tregs) infiltration in tissue microarrays in training cohort comprising 348 randomly selected HCC patients who underwent curative resection. The results were further verified in an independent validation cohort of 346 HCC patients. Nomograms were established based on the results of multivariate analysis. The predictive accuracy of the nomograms were measured by concordance index and calibration. The decision curve analysis was used to evaluate the clinical benefit of the nomogram. Results: Patients with higher PDL1CD47CD73 score had a significantly poorer prognosis than patients with lower score. PDL1CD47CD73 score and the ratio of CD8+/ CD4+Foxp3+ were also identified as independent prognostic factors for OS and RFS in multivariate analysis. A significant positive correlation was found between PDL1CD47CD73 score and the ratio of CD8+/CD4+Foxp3+. The nomogram comprising PDL1CD47CD73 score, the ratio of CD8+/CD4+Foxp3+ and tumor burden had better predictive accuracy when compared with BCLC stage and CLIP score for OS and RFS. These results were further validated in the validation cohort. Conclusions: Our data suggest for the first time that PDL1CD47CD73 score emerged as a novel independent prognostic factor for OS and RFS, which should be incorporated into nomograms to generate more accurate predictive models. Keywords: Immune checkpoints, Hepatocellular carcinoma, Prognosis, Nomogram.

\section{Treatment:Systemic, Targeted, Novel TargetorExperimental Therapy}

\author{
PO-21 \\ How May We Harness the Immune \\ Modulatory Effects of Molecular Targeted \\ Therapy for Hepatocellular Carcinoma \\ (HCC)?: A Systematic Review
}

Yu-Yang Lin', Chia-Wei Chen ${ }^{2}$, Ching-Ting Tan ${ }^{3,4}$, Da-Liang Ou 2,3, Ann-Lii Cheng 2,3,5, Chiun Hsu 2,3,5

${ }^{1}$ School of Medicine, National Taiwan University College of Medicine, ${ }^{2}$ Graduate Institute of Oncology, National Taiwan University College of Medicine, ${ }^{3}$ National Taiwan University Cancer Center, ${ }^{4}$ Department of Otolaryngology, National Taiwan University Hospital, ${ }^{5}$ Department of Oncology, National Taiwan University Hospital, Taiwan

Aims: Four multi-kinase inhibitors (sorafenib, regorafenib, lenvatinib, and cabozantinib) have demonstrated antitumor activity for advanced HCC. This study reviewed comprehensively the pre-clinical evidence of their immune modulatory effects to see how they may be incorporated into future combination regimens with immunotherapy. Methods: PUBMED was searched and 404 abstracts were identified using the following strategy: combination of individual drug name with any one of the following terms: T cell, natural killer cell, macrophage, myeloid-derived suppressive cells (MDSC), cytokine, or microenvironment. Original research articles were independently reviewed by 2 researchers (Y.Y.L. and C.W.C., equal contribution). In vitro and in vivo research methodologies were reviewed, and the immune modulatory effects were categorized as direct effects on immune cells or indirect effects on the cancer cells or the microenvironment. Results: Studies of sorafenib comprised 64 of the 72 research articles reviewed (41 studies using in vivo models; 24 studies of in vitro tests only). Potentially beneficial effects on anti-tumor immunity of sorafenib may result from increased M1 polarization of macrophages and suppression of regulatory T cells. However, hypoxia associated with sorafenib treatment may activate stromal cellderived factor $\alpha / \mathrm{C}-\mathrm{X}-\mathrm{C}$ receptor type 4 signaling and contribute to immune suppression in the tumor microenvironment. Effects of sorafenib on CD8/CD4 T cells, MDSC, and natural killer cells were more contradictory. Confounding factors may include the experimental dosage (lower dosage was more likely to exert beneficial immune modulatory effects) and difference in vivo models (orthotopic vs. heterotopic). Increased M1 polarization of macrophages and stimulation of CD8 T cell function may contribute to the anti-tumor immunity of regorafenib/cabozantinib/lenvatinib. Conclusions: Sorafenib and other multikinase inhibitors may promote anti-tumor immunity through modulation of multiple immune cell types as well as the tumor 
The 9th Asia-Pacific Primary Liver Cancer Expert Meeting (APPLE 2018)

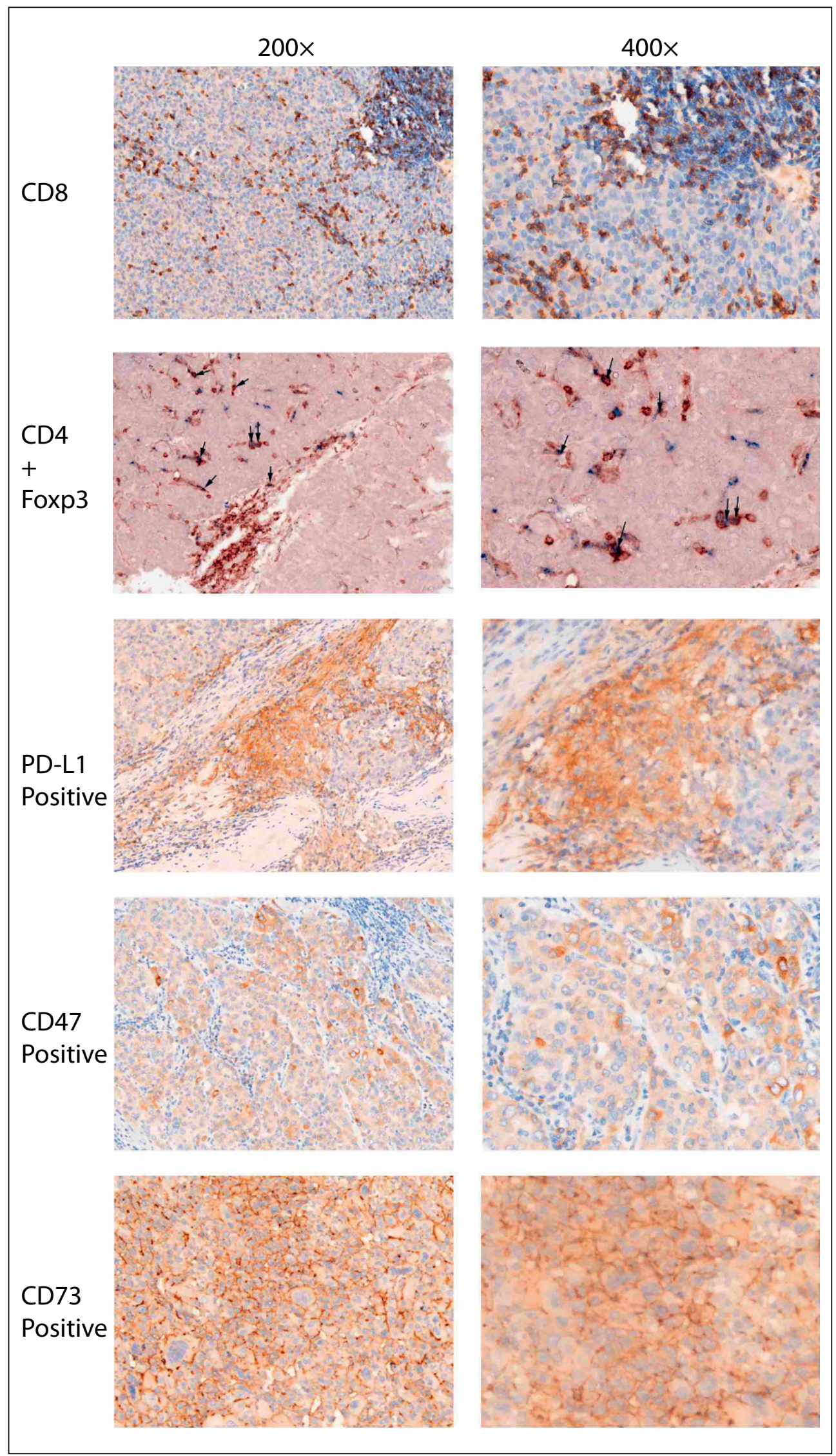

Fig. 1. (for Abstract no PO20). 
microenvironment. The optimal immune modulatory dosage should be defined to facilitate design of future combination regimens. Keywords: Molecular targeted therapy, Immunotherapy, Tumor micro-environment, Tumor-associated macrophages, Regulatory T cells.

\section{PO-22 \\ Hepatic Safety and Biomarker Assessments in Sorafenib-Experienced Patients with Advanced Hepatocellular Carcinoma Treated with Nivolumab in the Checkmate-040 Study}

Tim Meyer ${ }^{1}$, Ignacio Melero ${ }^{2,3}$, Thomas $\mathrm{Yau}^{4}$, Chiun Hsu ${ }^{5}$, Masatoshi Kudo ${ }^{6}$, Su-Pin Choo ${ }^{7}$, Jörg Trojan ${ }^{8}$, Theodore H. Welling ${ }^{9}$, Yoon-Koo Kang ${ }^{10}$, Winnie Yeo ${ }^{11}$, Akhil Chopra ${ }^{12}$, Adyb Baakili' ${ }^{13}$, Christine dela Cruz ${ }^{13}$, Huanyu Zhao ${ }^{13}$, Jaclyn Neely ${ }^{13}$, Todd S. Crocenzi ${ }^{14}$, Anthony B. El-Khoueiry ${ }^{15}$, Bruno Sangro ${ }^{16}$

${ }^{1}$ Royal Free Hospital, London, UK; ${ }^{2}$ Clinica Universidad de Navarra and CIBERONC, Pamplona, Spain; ${ }^{3}$ Center for Applied Medical Research (CIMA), Pamplona, Spain; ${ }^{4}$ University of Hong Kong, Hong Kong, China; ${ }^{5}$ National Taiwan University Hospital, Taipei, Taiwan; ${ }^{6}$ Kindai University Faculty of Medicine, Osaka, Japan; ${ }^{7}$ National Cancer Centre, Singapore, ${ }^{8}$ Goethe University Hospital and Cancer Center, Frankfurt, Germany; ${ }^{9} \mathrm{New}$ York University Langone Health, New York, NY, USA;

${ }^{10}$ Asan Medical Center, University of Ulsan, Seoul, Korea; ${ }^{11}$ Chinese University of Hong Kong, Hong Kong, China; ${ }^{12}$ Johns Hopkins Singapore International Medical Centre, Singapore, ${ }^{13}$ Bristol-Myers Squibb, Princeton, NJ, USA; ${ }^{14}$ Providence Cancer Center, Portland, OR, USA; ${ }^{15}$ USC Norris Comprehensive Cancer Center, Los Angeles, CA, USA; ${ }^{16}$ Clinica Universidad de Navarra and CIBEREHD, Pamplona, Spain

Aims: Nivolumab (NIVO) is a fully human anti-PD-1 IgG4 mAb that demonstrated durable responses, manageable safety, and long-term survival in pts with advanced HCC (aHCC) in CheckMate-040 (El-Khoueiry AB, Sangro B, et al. Lancet 2017). Here we present updated hepatic safety and biomarker analyses in sorafenib-experienced (sor-exp) pts in CheckMate-040. Methods: Sor-exp pts with or without chronic viral hepatitis received NIVO $3 \mathrm{mg} / \mathrm{kg}$ Q2W. Primary endpoint was objective response rate (ORR) reported by blinded independent central review using RECIST v1.1. Secondary endpoints included overall survival (OS), disease control rate (DCR), and safety. Exploratory analyses of on-treatment HCV and HBV viral kinetics and alpha-fetoprotein (AFP) levels were performed. Results: Median duration of follow-up was 14.9 mo. Baseline Child-Pugh scores of 5 or 6 and extrahepatic metastases were observed in $99 \%$ and $71 \%$ of pts, respectively. The ORR with NIVO was $14 \%$; the DCR was 56\%; median OS was 15.6 mo. Any-grade and grade 3-4 hepatic treatment-related AEs (TRAEs) occurred in $12(8 \%)$ and $5(3 \%)$ pts, respectively; $100 \%$ of grade 3-4 hepatic TRAEs resolved. Frequencies of grade 3-4 treatment-related ALT/AST elevations were 2\%-3\%. No drug-related deaths due to hepatic AEs occurred, and no new safety signals were observed. AFP levels at baseline were not associated with response; however, AFP levels in responders appeared to decrease on treatment. Updated data will be presented. Conclusions: NIVO demonstrated long-term survival and objective responses across etiologies and manageable overall and hepatic safety profile in aHCC. Responses occurred irrespective of baseline AFP levels, and AFP declines were associated with response. Keywords: Hepatocellular carcinoma, Nivolumab, Immune checkpoint.

\section{PO-23 \\ Clinical Characteristics of Long Term Survivors Following Sorafenib Treatment for Unresectable Hepatocellular Carcinoma: Korean National Multi-Center Retrospective Cohort Study}

Young Youn Cho ${ }^{1,2}$, Su Jong Yu', Hye Won Lee ${ }^{3}$, Do Young Kim ${ }^{3}$, Wonseok Kang ${ }^{4}$, Yong-Han Paik', Pil Soo Sung ${ }^{5}$, Si Hyun Bae ${ }^{5}$, Su Cheol Park ${ }^{6}$, Young Seok Doh ${ }^{7,8}$, Kang Mo Kim ${ }^{8}$, Eun Sun Jang ${ }^{9}$, In Hee Kim ${ }^{10}$, Won Kim ${ }^{11}$, Yoon Jun Kim ${ }^{1 *}$

${ }^{1}$ Department of Internal Medicine and Liver Research Institute, Seoul National University College of Medicine, Seoul, South Korea; ${ }^{2}$ Department of Internal Medicine, Chung-Ang University Hospital, Seoul, South Korea; ${ }^{3}$ Department of Internal Medicine, Yonsei University College of Medicine, Seoul, South Korea; ${ }^{4}$ Department of Medicine, Samsung Medical Center, Sungkyunkwan University School of Medicine, Seoul, South Korea; ${ }^{5}$ Department of Internal Medicine, Kangnam St. Mary's Hospital, The Catholic University of Korea College of Medicine, Seoul, South Korea; ${ }^{6}$ Department of Internal Medicine, Korea Cancer Center Hospital, Korea Institute of Radiological and Medical Sciences, Seoul, South Korea; ${ }^{7}$ Department of Internal Medicine, Eulji University Hospital, Daejeon, Korea; ${ }^{8}$ Department of Gastroenterology, Asan Liver Center, Asan Medical Center, University of Ulsan College of Medicine, Seoul, South Korea; ${ }^{9}$ Departments of Internal Medicine, Seoul National University Bundang Hospital, Seoul National University College of Medicine, Seoul, South Korea; ${ }^{10}$ Department of Internal Medicine, Chonbuk National University Hospital, Chonbuk National University Medical School, Cheongju-si, South Korea; ${ }^{11}$ Department of Internal Medicine, Seoul Metropolitan Government Seoul National University Boramae Medical Center, Seoul, South Korea

Aims: Sorafenib is the standard systemic therapy for treatment of advanced stage hepatocellular carcinoma 
(HCC), and progressive HCC after locoregional therapy. The efficacy and safety of the drug in clinical practice have been addressed by several real-world studies. However, little data are available regarding clinical factors of good responders following sorafenib treatment. This study aimed to evaluate prognostic factors of long term survivors. Methods: This multi-center retrospective cohort study included 1,605 unresectable HCC patients who received sorafenib treatment between 2007 and 2014 in 9 tertiary centers in Korea. The patients were classified into long-term survivor group (survival longer than 2 years, $n=246$ ) or control group ( $\mathrm{n}=1,359)$. The primary endpoint was prognostic factors on survival for overall patients. Secondary endpoints included time-to-progression and other safety profiles. Results: The patients were predominantly male (83.8\%), chronic hepatitis B (77.3\%), and Barcelona Clinic of Liver Cancer stage C (78.3\%). The median overall survival was 9.0 months. After treatment, 12 patients (0.7\%) achieved complete response, and 146 patients (9.1\%) achieved partial response by mRECIST criteria. The prognostic factors predicting long-term survival were metformin use (hazard ratio $[\mathrm{HR}]=2.44 ; P=0.004$ ), hand foot skin reaction $(\mathrm{HR}=1.77 ; \mathrm{P}=0.001)$, and concomitant treatment with chemoembolization or radiotherapy $(\mathrm{HR}=2.59 ; \mathrm{P}<$ 0.001). Prognostic factors against long-term survival were Child-Pugh score B (HR $=0.375 ; P<0.001)$, presence of extrahepatic metastasis ( $\mathrm{HR}=0.625 ; \mathrm{P}=0.002)$, main portal vein invasion ( $\mathrm{HR}=0.625 ; \mathrm{P}=0.002)$, and elevated AFP $(>1,000 \mathrm{ng} / \mathrm{mL} ; \mathrm{HR}=0.393 ; \mathrm{P}<0.001)$. Conclusions: This large multi-center retrospective study showed that the objective response was $9.8 \%$, and the proportion of longterm survivors was $15.3 \%$ in Korean patients. The prognostic factors predicting long-term survival after sorafenib treatment were hand foot skin reaction, metformin use, concomitant treatment, good liver function, absence of extrahepatic metastasis, low degree of portal vein thrombosis, and low AFP. Keywords: Sorafenib, Survival, Hepatocellular carcinoma, Chemotherapy.

\section{PO-24 \\ Understanding Recurrence Related Oncogenic Kinases and Metabolites in Liver Cancer}

Hongping Xia ${ }^{1,2}$, Kam M. Hui ${ }^{1,2}$

${ }^{1}$ Laboratory of Cancer Genomics, National Cancer Center, Singapore; ${ }^{2}$ Department of Pathology, School of Basic Medical Sciences \& The Affiliated Sir Run Run Hospital, Nanjing Medical University, Nanjing, China

Aims: Hepatocellular carcinoma (HCC) is the most common type of primary liver cancer and is the second leading cause of cancer-related death worldwide. Once diagnosed with HCC, only $30 \%$ of patients are eligible for curative treatments. Even after curative resection in patients with early-stage disease, tumor recurrence is estimated to occur in $70 \%$ of patients. Hence, there is an unmet need for understanding of the mechanism contributed to recurrence of HCC. Methods: Through comprehensive whole kinome expression analysis and global metabolomics profiling of HCC patients' tumor and matched normal samples with recurrence and non-recurrence information. The significantly dysregulated kinases and metabolites were further validated and characterized in HCC cells and xenograft models. Results: We have identified a panel of HCC overexpressed oncogenic kinases and metabolites. The retinal and retinol metabolites were significantly downregulated in our HCC tissues compared with matched normal liver tissues. The expression of some kinases and metabolites is significantly associated with recurrence and patients' survival of HCC patients. HCC recurrence is characterized by increasing glycolysis with increasing expression of PRKAA2 kinase and $\alpha$-ketoglutarate and NAD+/NADH metabolites. Overexpression of PRKAA2 promotes glycolysis in different HCC cells by seahorse assay analysis. Meanwhile, stable overexpression of PRKAA2 also promotes cell growth and tumorigenicity of HCC cells with increasing of $\alpha$-ketoglutarate and $\mathrm{NAD+}$ NADH metabolites. Conclusions: The results from this study will understand the important role of recurrence related oncogenic kinases and metabolites in hepatocarcinogenesis and recurrence. It is promising to develop novel kinase inhibitors as personalized targeted therapeutic strategies for advanced HCC. Keywords: Recurrence, Oncogenic kinases, Metabolites, Liver cancer.

\section{PO-25 \\ Eukaryotic Elongation Factor 2 Kinase Promotes Tumor Growth, Metastasis and Angiogenesis in Hepatocellular Carcinoma Kaikai Shen*, Ying Zhou, Yan Shen, Yaoting Li \\ School of Basic Medical Sciences, Shanghai University of Traditional Chinese Medicine, Shanghai, China}

Aims: Hepatocellular carcinoma (HCC) has a high incidence in China, Australia, Europe and United States. Alarmingly, of all cancers in China, liver cancer has the fastest growing death rate. HCC is highly malignant with considerable metastatic potential, which underlines the necessity for developing novel potential effective therapeutics. Eukaryotic elongation factor 2 kinase (eEF2K) negatively regulates the elongation phase of mRNA translation and hence protein synthesis. Previous study indicates that expression of eEF2K was observed significantly high in HCC tissues. The present study is to determine whether or not eEF2K play an important role in liver cancer progression. Methods: Cancer cell proliferation was determined by cell viability assay and colony formation assay. Cancer cell 


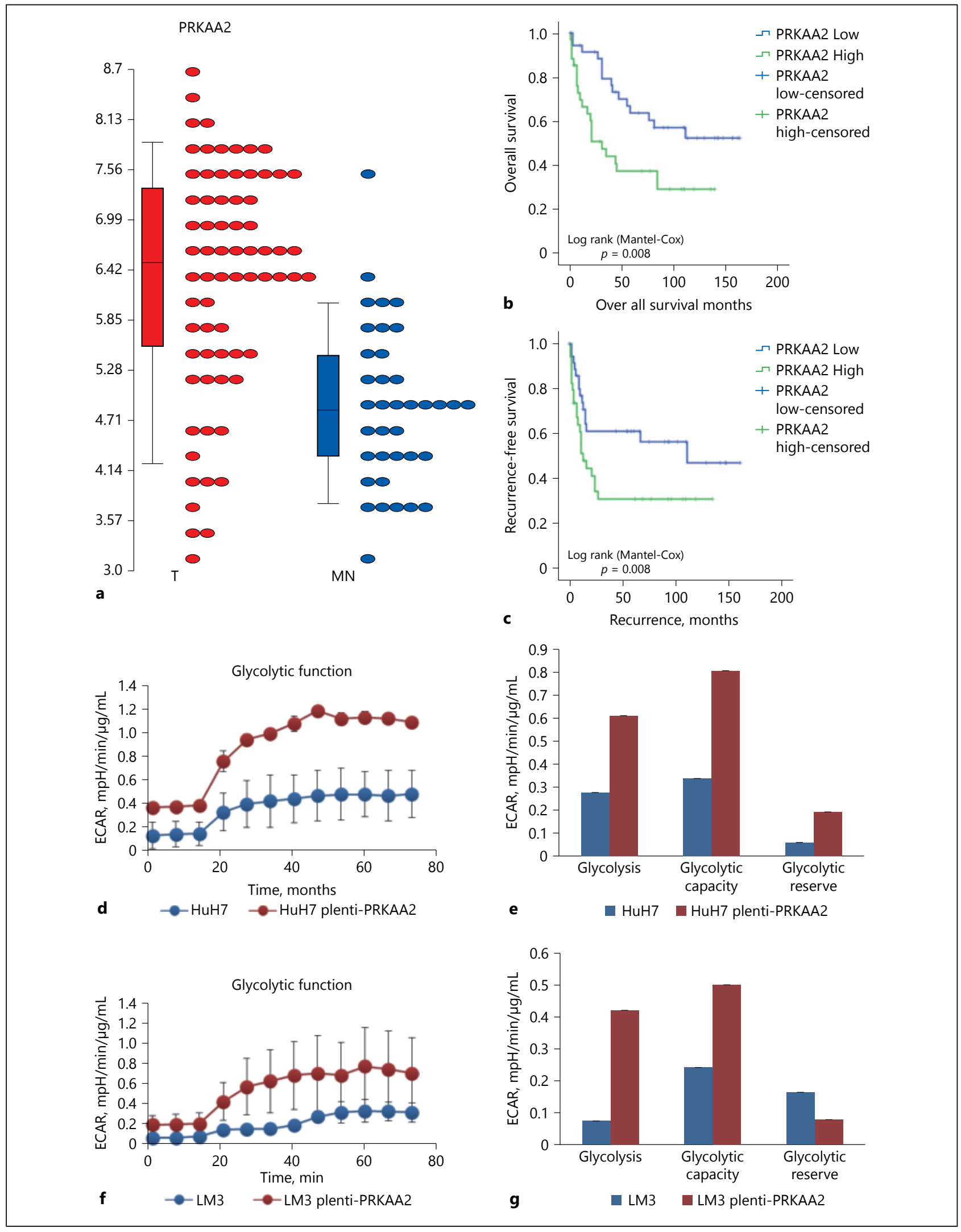

Fig. 1. (for Abstract no PO-24). 


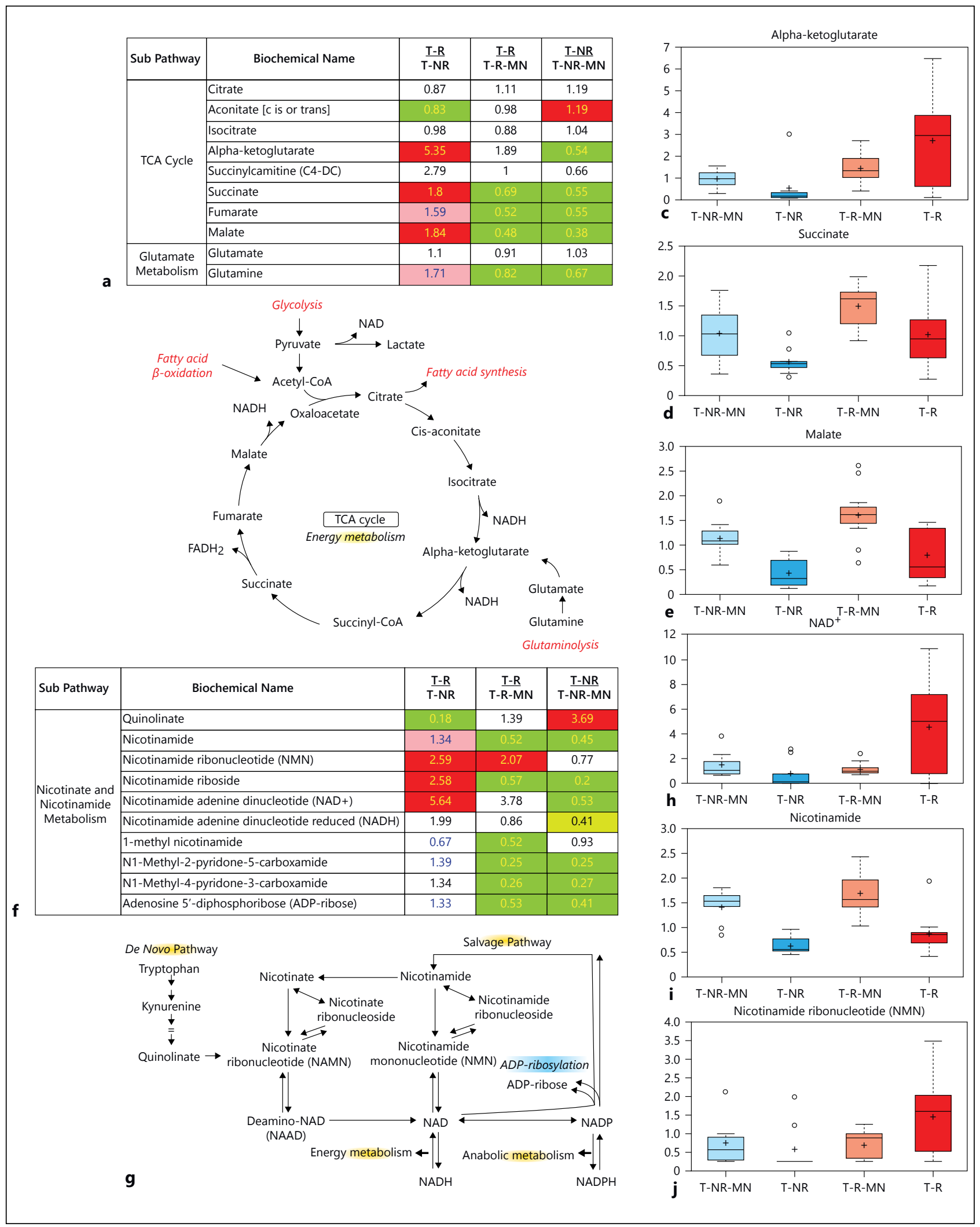

Fig. 2. (for Abstract no PO-24). 
migration and invasion were determined by two-dimensional wound-healing assay and three-dimensional trans well invasion assay, respectively. The mRNA expression of VEGF and VEGFR2 was determined by real time PCR. The presence of CD-31 was confirmed by immunohistochemistry in MHCC97H liver cancer xenografts. Results: Knocking down or inhibiting eEF2K in high metastatic liver cancer cells (MHCC97Hs) inhibited cell colony formation for 15 days and impaired migration and invasion of cancer cells for $24 \mathrm{~h}$ and $48 \mathrm{~h}$. Conversely, exogenously expressing eEF2K accelerated migration and invasion in MHCC97Hs. Importantly, inhibition of eEF2K expression either in MHCC97Hs or in endothelial cells (HUVECs) significantly diminished migration of endothelial cells in co-culture assay. Besides, incubation of eEF2K siRNA transfected HUVECs with conditioned media of MHCC97Hs reduced tube formation. Importantly, knocking down eEF2K inhibited VEGF expression on MHCC97Hs and VEGFR2 expression on HUVECs and thus restricted crosstalk between liver cancer cells and endothelial cells. Notably, pharmacological or genetic inhibition of eEF2K almost completely blocked tumor growth and effectively prevented the metastasis to lung in nude mice. The presence of CD-31, as the number of blood vessels in tumors, was decreased in sheEF2K-treated group as compared with that in control group. High levels of eEF2K expression were associated with invasive carcinoma and metastatic liver cancer. Conclusions: These data provide the evidence that eEF2K promotes metastasis and angiogenesis in HCC and hence it may be considered as a novel potential therapeutic target for preventing tumor progression of HCC. Keywords: Hepatocellular carcinoma, Eukaryotic elongation factor 2 kinase, Tumor progression.

\section{Treatment: Clinical Trials}

\section{PO-26 \\ Cabozantinib versus Placebo in Patients with Previously Treated Advanced Hepatocellular Carcinoma (HCC): Results from the Randomized Phase 3 CELESTIAL Trial}

\author{
Ann-Lii Cheng ${ }^{1}$, Robin Kate Kelley ${ }^{2}$, Tim Meyer ${ }^{3}$, \\ Lorenza Rimassa ${ }^{4}$, Baek-Yeol Ryoo ${ }^{5}$, Irfan Cicin ${ }^{6}$, \\ Philippe Merle ${ }^{7}$, Joong-Won Park ${ }^{8}$, Jean-Frederic Blanc ${ }^{9}$, \\ Heinz-Josef Klümpen ${ }^{10}$, Stephen L. Chan ${ }^{11}$, \\ Vittorina Zagonel ${ }^{12}$, Colin Hessel ${ }^{13}$, \\ Anne E. Borgman-Hagey ${ }^{13}$, Gisela Schwab ${ }^{13}$, \\ Anthony B. El-Khoueiry ${ }^{14}$, Ghassan K. Abou-Alfa ${ }^{15}$ \\ ${ }^{1}$ National Taiwan University Hospital, Taipei, Taiwan; \\ 2UCSF Helen Diller Family Comprehensive Cancer \\ Center, San Francisco, CA, USA; ${ }^{3}$ Royal Free Hospital, \\ London, UK; ${ }^{4}$ Humanitas Cancer Center, Humanitas \\ Clinical and Research Center, Rozzano (Milan), Italy; \\ ${ }^{5}$ Asan Medical Center, University of Ulsan College of \\ Medicine, Seoul, Republic of Korea; ${ }^{6}$ Trakya University \\ School of Medicine, Edirne, Turkey; ${ }^{7}$ Groupement \\ Hospitalier Lyon Nord, Lyon, France; ${ }^{8}$ National Cancer \\ Center, Goyang, South Korea; ${ }^{9}$ Hôpital Haut-Lévêque, \\ CHU Bordeaux, France; ${ }^{10}$ Department of Medical \\ Oncology, Academic Medical Center, Amsterdam, The \\ Netherlands; ${ }^{11}$ The Chinese University of Hong Kong, \\ State Key Laboratory in Oncology in South China; \\ ${ }^{12}$ Medical Oncology Unit 1, Istituto Oncologico Veneto, \\ IRCCS, Padova, Italy; ${ }^{13}$ Exelixis Inc., South San Francisco, \\ CA, USA; ${ }^{14}$ USC Norris Comprehensive Cancer Center, \\ Los Angeles, CA, USA; ${ }^{15}$ Memorial Sloan Kettering \\ Cancer Center, New York, NY, USA
}

Aims: Cabozantinib, an inhibitor of MET, VEGF receptors, and AXL, has previously shown clinical activity in patients with advanced HCC. The phase 3 CELESTIAL trial (NCT01908426) evaluated cabozantinib versus placebo in patients with previously treated advanced HCC. Methods: In this double-blind, international, phase 3 trial, patients were randomized 2:1 to cabozantinib (60 mg qd) or matched placebo. Eligible patients had pathologic diagnosis of HCC, Child-Pugh score A, and ECOG PS $\leq 1$. Patients must have received prior sorafenib, were allowed to receive up to two lines of prior systemic therapy for HCC, and must have progressed following at least one. Stratification was by disease etiology, geographic region, and extent of disease. The primary endpoint was overall survival (OS). Secondary endpoints were investigator-assessed progressionfree survival (PFS) and objective response rate (ORR) per RECIST 1.1. Results: As of June 1, 2017, 707 patients were randomized, and 484 deaths had occurred. Baseline characteristics were balanced between the two arms: median age was 64 years, $82 \%$ were male, $38 \%$ had HBV, $24 \%$ had HCV, and $25 \%$ 
enrolled in Asia. The study met the primary endpoint at the second planned interim analysis; median OS was 10.2 mo with cabozantinib versus 8.0 mo with placebo (HR 0.76, 95\% CI $0.63-0.92 ; \mathrm{p}=0.0049$ ). Median PFS was 5.2 mo with cabozantinib versus 1.9 mo with placebo (HR $0.44,95 \%$ CI $0.36-0.52$; $\mathrm{p}<0.0001)$, and ORR was $4 \%$ versus $0.4 \%(\mathrm{p}=0.0086)$. Grade 3 or 4 adverse events occurred in $68 \%$ of cabozantinib-treated patients and $36 \%$ of placebo-treated patients; the most common high-grade events with cabozantinib were palmarplantar erythrodysesthesia (17\% of patients) and hypertension (16\% of patients). Conclusions: Cabozantinib significantly improved OS and PFS versus placebo in patients with previously treated advanced HCC. Adverse events were consistent with the known safety profile of cabozantinib.

\section{PO-27 \\ Randomized, Open Label, Multicenter, Phase II Trial of Transcatheter Arterial Chemoembolization (TACE) Therapy in Combination with Sorafenib as Compared with TACE Alone in Patients with Hepatocellular Carcinoma: TACTICS Trial}

Kazuomi Ueshima*,1, Masatoshi Kudo', Masafumi Ikeda ${ }^{2}$, Takuji Torimura ${ }^{3}$, Nobukazu Tanabe ${ }^{4}$, Hiroshi Aikata ${ }^{5}$, Namiki Izumi ${ }^{6}$, Takahiro Yamasaki ${ }^{7}$, Shunsuke Nojiri ${ }^{8}$, Keisuke Hino ${ }^{9}$, Hidetaka Tsumura ${ }^{10}$, Teiji Kuzuya ${ }^{11}$, Norio Isoda ${ }^{12}$, Kohichiroh Yasui ${ }^{13}$, Kenichi Yoshimura ${ }^{14}$, Takuji Okusaka ${ }^{15}$, Junji Furuse ${ }^{16}$, Norihiro Kokudo ${ }^{17}$, Kiwamu Okita ${ }^{18}$, Yasuaki Arai ${ }^{15}$ TACTICS study group

${ }^{1}$ Gastroenterology and Hepatology, Kindai University Faculty of Medicine, Osaka-Sayama, ${ }^{2}$ National Cancer Center Hospital East, Kashiwa Chiba, ${ }^{3}$ Kurume University Hospital, Kurume Fukuoka, ${ }^{4}$ Sendai Medical Center, Sendai Miyagi, ${ }^{5}$ Hiroshima University Hospital, Hiroshima, ${ }^{6}$ Musashino Red Cross Hospital, Musashino Tokyo, ${ }^{7}$ Yamaguchi University Hospital, Yamaguchi, ${ }^{8}$ Nagoya City University Graduate School of Medical Sciences, Nagoya, ${ }^{9}$ Kawasaki Medical University Hospital, Kurashiki Okayama, ${ }^{10}$ Hyogo Cancer Center, Akashi Hyogo, ${ }^{11}$ Nagoya University Hospital, Nagoya Aichi, ${ }^{12}$ Jichi University Hospital, Tochigi, ${ }^{13}$ Kyoto Prefectural University of Medicine, Kyoto, ${ }^{14}$ Kanazawa University Hospital, Kanazawa Ishikawa, ${ }^{15}$ National Cancer Center Hospital, ${ }^{16}$ Kyorin University Hospital,

${ }^{17}$ National Center for Global Health and Medicine,

Tokyo, ${ }^{18}$ Shunan Memorial Hospital, Yamaguchi, Japan

Aims: There is no proven evidence that combination therapy of TACE with sorafenib (TS group) prolong progression-free survival (PFS) and/or overall survival (OS) compared to TACE alone (T group) in patients with unresectable HCC. Methods: In this randomized, open label, multi- center, comparative trial (NCT01217034), patients with unresectable HCC, Child-Pugh score $\leq 7$, ECOG performance status $0-1$, no vascular invasion (VI), no extrahepatic spread (EHS), size $\leq 10 \mathrm{~cm}$ and number $\leq 10$ and adequate organ function were randomized 1:1 (stratification by institution, Milan criteria in or out, and number of previous TACE 0 or 1-2) to T or TS. In TS group, sorafenib $400 \mathrm{mg}$ once daily was pretreated for 2-3 weeks prior to TACE followed by $800 \mathrm{mg}$ once daily during on-demand conventional TACE sessions until the time to untreatable progression (TTUP), which was defined as the time to the date of a state when TACE continuation is not possible due to untreatable tumor progression, deterioration to Child-Pugh C or appearance of VI/EHS. Co-primary endpoints are PFS and OS. Multiplicity is adjusted using a gatekeeping hierarchical testing. PFS event in this trial was defined as death or time to TTUP. Key secondary endpoints were time to progression and safety. PFS is expected to $40 \%$ extension from 18 months (control arm) to 25 months, target HR was 0.71 , with a power of 0.80 . Results: The trial was conducted in 33 institutions and a total of 156 patients were randomized to $\mathrm{T}(\mathrm{n}=76)$ or TS $(\mathrm{n}=80)$. Median PFS in the T group and TS group was 13.5 and 25.2 months (HR $=0.59,95 \%$ CI 0.41-0.87; $\mathrm{p}=0.006)$, respectively. The number of OS events has not reached. Median TTP was 13.5 and 24.1 months in the T and TS groups (HR $=0.56,95 \%$ CI $0.38-0.83 ; \mathrm{p}=0.004$ ). Median TTUP was 20.6 and 26.7 months in the $\mathrm{T}$ and $\mathrm{TS}$ groups (HR = $0.57,95 \%$ CI $0.35-0.92 ; p=0.02$ ), respectively. There was no unexpected toxicity. Conclusions: Sorafenib in combination with TACE significantly improved PFS over TACE alone in patients with unresectable HCC. Adverse events were consistent with the known safety profile with previous TACE combination trials. Clinical trial information: NCT01217034. Keywords: Hepatocellular carcinoma, TACE, Sorafenib, Clinical trial. 


\section{PO-28 \\ Efficacy of Sorafenib Monotherapy versus Transarterial Chemoembolization (TACE)- Sorafenib Sequential Therapy in HCC Patients with Extrahepatic Metastasis - An Interim Analysis of Randomized Controlled Trial}

Hyung Joon Yim ${ }^{1}$, Sang Jun Suh ${ }^{1}$, Young Kul Jung ${ }^{1}$ Sung-Bum Cho ${ }^{2}$, Woo Jin Chung ${ }^{3}$, Young Seok Kim ${ }^{4}$, Si-Hyun Bae ${ }^{5}$, Jun Young Park

${ }^{1}$ Department of Internal Medicine, Korea University College of Medicine, Seoul, South Korea; ${ }^{2}$ Department of Internal Medicine, Chonnam National University Medical School, Gwangju, South Korea; ${ }^{3}$ Department of Internal Medicine, Keimyung University College of Medicine, Daegu, South Korea; ${ }^{4}$ Department of Internal Medicine, Soonchunghyang University College of Medicine, Bucheon, South Korea; ${ }^{5}$ Department of Internal Medicine, College of Medicine, The Catholic University of Korea, Seoul, South Korea; ${ }^{6}$ Department of Internal Medicine, Yonsei University College of Medicine, Seoul, South Korea

Aims: Sorafenib is the standard therapy for hepatocellular carcinoma (HCC) with extrahepatic metastasis (EHM) However, transarterial chemoembolization (TACE) which is a standard therapy for intermediate stage may be beneficial for controlling intrahepatic tumor, thereby providing chance of improving survival in HCC patients with EHM. We aimed to compare the efficacy between the sorafenib monotherapy and TACE-sorafenib sequential therapy in HCC patients with EHM. Methods: This study is a prospective randomized controlled study conducted at 6 tertiary hospitals in South Korea. HCC patients with EHM were enrolled and randomized into sorafenib monotherapy or TACE-sorafenib sequential therapy group. Patients with main portal vein invasion, Child-Pugh class B or C, and history of TACE or previous systemic therapy were excluded. The sorafenib monotherapy group received sorafenib immediately after randomization while the TACEsorafenib group received 2 4 times of TACE before starting sorafenib. Response evaluation was performed every 2 months, and overall survival (OS), time to progression (TTP), and progression free survival (PFS) were compared. We initially planned 130 patients for the present study, and the results of interim analysis are presented. Results: A total of 65 patients were enrolled currently: 33 patients into the monotherapy and 32 into the sequential therapy group. Baseline characteristics of the patients such as gender, age, aetiology of liver disease, Child-Pugh score, HCC stage, and tumor burden were not significantly different between two groups. Median OS were $6.4(0.4-30.6)$ months and median TTP were 3.5 (0.9-25.7) months in all patients. The probability of survival rates were plotted by Kaplan-Meier curve and compared by log-rank test. Median OS were not different in both groups: monotherapy $4.3(0.4-30.6)$ months and sequential therapy $7.4(1.8-25.7)$ months ( $p=0.364)$. However, median TTP were longer in sequential therapy group: monotherapy 2.6 (0.99.3) months and sequential therapy $4.6(1.0-25.7)$ months ( $p=0.003)$, and the median PFS were also better in the sequential therapy group: monotherapy $2.6(0.4-9.3)$ months and sequential therapy $4.6(1.0-25.7)$ months $(p=0.015)$. The disease control rate were better in sequential therapy group: monotherapy $40.7 \%$ and sequential therapy $71.0 \%(p=$ 0.020 ). Conclusions: The TACE-sorafenib sequential therapy would be a better strategy than sorafenib monotherapy for the treatment of HCC patients with EHM, especially, in controlling tumor progression. Keywords: Hepatocellular carcinoma, Sorafenib, Transarterial chemoembolization, Metastasis.

\section{PO-29 \\ Survival Outcomes and Adverse Events in Clinical Trials for Advanced Hepatocellular Carcinoma (HCC)}

Rui Xin Ng, Sean Lim Zi Qing, Su Pin Choo

Duke-NUS Medical School, Singapore

Aims: Enrolling advanced HCC patients into clinical trials can be challenging because of their limited prognoses. There is little data on outcome of HCC patients who participate in trials compared to non-trial patients. This study aims to investigate the effects of trial participation for hepatocellular carcinoma patients at National Cancer Centre Singapore (NCCS) in terms of survival outcomes and adverse events. Methods: Clinical course and treatment outcomes of patients with Hepatocellular Carcinoma treated between 2007 and 2017 at NCCS Department of Medical Oncology (DMO) were recorded. Overall survival and adverse events were compared between 136 trial and 123 non-trial participants. The study was conducted between August 2017 to July 2018. Results: The study included 136 trial participants who were treated in 30 trials, and 123 non-trial participants who received standard of care, involving chemotherapy, small molecule therapy or best supportive care. Median overall survival was comparable in trial vs. non-trial participants (11.87 vs 9.27 months ( $\mathrm{p}=$ 0.519)). Multivariate analysis indicated that prior primary liver resection, BCLC stage and Child Pugh score remained prognostic of OS. Trial participation did not have a statistically significant effect on patients' overall survival (hazard ratio = $1.102(p=0.519)$ ). Difference in OS was statistically significant across trials of different drug classes $(p=0.022)$ in univariate and multivariate analysis, most significantly in small molecule vs immunotherapy, with hazard ratio 1.996 (95\% CI 1.119-3.561). Statistically significant difference was found between the two groups in adverse event occurrence (63.24\% vs. $33.33 \%$. ( $<<0.001))$ and frequency $(34.56 \%$ vs. $7.32 \%$. $(\mathrm{p}<0.001))$. Conclusions: Trial participation neither improved nor worsened overall survival of advanced HCC patients but is associated with increased adverse event occur- 
rence and frequency. Developing a trial participation prognostic score may be helpful in identifying patients who would benefit from trial participation in terms of increased overall survival and minimized adverse events.

\section{PO-30 \\ Practice Patterns and Deterioration of Liver Function after Transarterial Chemoembolization (TACE): Final Analysis of Optimis in Asian Regions}

\begin{abstract}
Han Chu Lee ${ }^{1}$, Ann-Lii Cheng' ${ }^{2}$, Jean-Luc Raoul', Markus Peck-Radosavljevic ${ }^{4,5}$, Jeong Heo ${ }^{6}$, Shi-Ming Lin ${ }^{7}$, Hong Shan ${ }^{8}$, Yefa Yang ${ }^{9}$, Inga Bayh ${ }^{10}$, Keiko Nakajima ${ }^{11}$, Masatoshi Kudo ${ }^{12}$

${ }^{1}$ Asan Medical Center, Seoul, South Korea; ${ }^{2}$ National Taiwan University Hospital, Taipei, Taiwan; ${ }^{3}$ Digestive Oncology, Institut de Cancérologie de l'Ouest, Nantes, France; ${ }^{4}$ Medical University of Vienna, Vienna, Austria; ${ }^{5}$ Klinikum Klagenfurt am Wörthersee, Klagenfurt, Austria; ${ }^{6}$ College of Medicine, Pusan National University and Medical Research Institute, Pusan National University Hospital, Busan, Republic of Korea; ${ }^{7}$ Chang Gung University and Chang Gung Memorial Hospital, Taoyuan, Taiwan; ${ }^{8}$ Department of Radiology, The Third Affiliated Hospital, Sun Yat-sen University, Guangzhou, Guangdong, China; ${ }^{9}$ Eastern Hepatobiliary Surgery Hospital, Second Military Medical University, Shanghai, China; ${ }^{10}$ Bayer AG, SBU Oncology, Pharmaceuticals, Wuppertal, Germany; ${ }^{11}$ Bayer HealthCare Pharmaceuticals, Whippany, NJ, USA; ${ }^{12}$ Kindai University Faculty of Medicine, Osaka, Japan
\end{abstract}

Aims: TACE is often used to treat unresectable HCC (uHCC). However, there is a lack of global consensus on the definition of TACE ineligibility. It is critical to evaluate the risk of TACE because it may impact patients' ability to receive subsequent effective treatments. Methods: OPTIMIS, an international, prospective, non-interventional study, enrolled patients with uHCC for whom a decision of TACE treatment was made prior to enrollment. Here we describe practice patterns, subsequent treatments, and liver deterioration data from Asian subgroups. TACE ineligibility was defined and consistent with international and regional guidelines. Results: Overall, 1650 enrolled patients received TACE including 1134 from Asia. After exclusion of patients with prior sorafenib treatment, $40 \%, 13 \%, 37 \%$, and $22 \%$ of patients from Japan, China, Korea, and the rest of Asia (ROA) became TACE ineligible during the study. Of those, only $21 \%, 16 \%, 6 \%$, and $3 \%$ of patients in Japan, China, Korea, and ROA received sorafenib early following TACE ineligibility. At inclusion, patients in most regions were BCLC stage $\mathrm{B}$. The presence of extrahepatic spread and portal vein thrombosis was highest in China and lowest in Japan, and the proportion of patients receiving TACE despite ineligibility was highest in China and lowest in Japan (Table). After first TACE, chronic liver function deterioration (worsening in CTCAE grade 30-90 days post TACE) varied between regions. In patients with available laboratory values, chronic bilirubin deterioration was evident in 9/176, 12/68, $11 / 94$, and 13/53 patients in Japan, China, Korea, and ROA, respectively. Conclusions: The heterogeneity of TACE use across Asia highlights a need for consensus on appropriate TACE use. Japan appears to be the most adherent to international TACE eligibility guidelines, compared with other Asian regions and the global population. These results also suggest that following TACE ineligibility, systemic therapies are not commonly used in the real-world setting in Asia. Keywords: Hepatocellular carcinoma, TACE, Sorafenib, Liver deterioration.

Table 1. Disease characteristics and TACE ineligibility at inclusion (for Abstract no P0-30)

\begin{tabular}{|c|c|c|c|c|c|}
\hline \multirow[b]{2}{*}{ n (\%) } & \multicolumn{4}{|l|}{ Asia } & \multirow{2}{*}{$\begin{array}{l}\text { Global total (North } \\
\text {-America/Europe, Central/ } \\
\text { South America, Asia) } \\
(\mathrm{n}=1650)\end{array}$} \\
\hline & $\begin{array}{l}\text { Japan } \\
(n=233)\end{array}$ & $\begin{array}{l}\text { China } \\
(n=150)\end{array}$ & $\begin{array}{l}\text { Korea } \\
(n=292)\end{array}$ & $\begin{array}{l}\text { Rest of Asia } \\
(n=459\}\end{array}$ & \\
\hline \multicolumn{6}{|l|}{ Disease status } \\
\hline Extra hepatic spread & $5(2)$ & $36(24)$ & 25 (9) & $33(7)$ & $118(7)$ \\
\hline Portal vein thrombosis & $3(1)$ & $53(35)$ & $30(10)$ & $22(5)$ & $123(7)$ \\
\hline \multicolumn{6}{|l|}{ BCLC stage } \\
\hline B & $199(85)$ & $35(23)$ & $204(70)$ & $284(62)$ & $1034(63)$ \\
\hline $\mathrm{C}$ & 31 (13) & $112(75)$ & 79 (27) & $154(34)$ & $529(32)$ \\
\hline $\mathrm{D}$ & $1(<1)$ & $2(1)$ & $3(1)$ & $10(2)$ & $19(1)$ \\
\hline Missing & $2(1)$ & $1(1)$ & $6(2)$ & $11(2)$ & $68(4)$ \\
\hline Ineligible for TACE & $38(16)$ & $114(76)$ & 85 (29) & $193(42)$ & $636(39)$ \\
\hline
\end{tabular}




\section{Treatment: Surgical Resection and Transplantation}

\author{
PO-31 \\ Hepatic Resection for Hepatocellular \\ Carcinoma in the Octogenarian: Is It \\ Justified? \\ Chao-Wei Lee ${ }^{1,2,3}$, Hsin-I Tsai, ${ }^{3,4}$, Ming-Chin Yu ${ }^{1,2,3 *}$, \\ Wei-Chen Lee ${ }^{1,2}$, Miin-Fu Chen ${ }^{1,2}$ \\ ${ }^{1}$ Department of Surgery, Chang Gung Memorial \\ Hospital, Linkou, ${ }^{2}$ College of Medicine, Chang Gung \\ University, ${ }^{3}$ Graduate Institute of Clinical Medical \\ Sciences, Chang Gung University, ${ }^{4}$ Department of \\ Anesthesiology, Chang Gung Memorial Hospital, \\ Linkou, Guishan, Taoyuan, Taiwan
}

Aims: Liver resection has been regarded as a standard treatment for primary hepatocellular carcinoma (HCC). However, few studies have dealt with the safety profile and long term results of this major operation in the octogenarian. The purpose of this study was to investigate the clinicopathological characteristics of hepatocellular carcinoma and longterm outcome of liver resection in the octogenarian. Methods: We retrospectively reviewed records of patients undergoing liver resection for primary HCC at our hospital between 1983 and 2015. Patient demographics, laboratory data, treatment, pathologic characteristics, and survival outcome were collected. Statistical analyses were conducted between patients older and younger than the age of 80. Survival was analyzed and compared by the Kaplan-Meier method and the log-rank test. Cox regression method was used to identify prognostic factors. Results: A total of 3,386 patients with HCC underwent surgery during this study period. There were 77 patients at or older the age of 80 (O-HCC group) and 3,309 patients younger than 80-year-old (Y-HCC group). The mean age of diagnosis was 83 and 57-year-old, respectively. 0-HCC group had significantly more co-morbidity $(p<0.001)$ but less HBV surface antigen positivity $(p<0.001)$ and cirrhosis $(p=$ 0.007). The O-HCC group had comparable perioperative morbidity and mortality rates to the Y-HCC group ( $p=0.147$ and 0.162 , respectively). Survival analyses showed that both the disease-free survival and overall survival were similar between the two groups ( $p=0.888$ and 0.371 , respectively). Multivariate analysis revealed that $\alpha$-fetoprotein $\geq 400 \mathrm{ng} /$ $\mathrm{mL}$, tumor size $\geq 10 \mathrm{~cm}$, and vascular invasion were independent prognostic factors for overall survival. Conclusions: This study demonstrated that liver resection in the octogenarian is a safe and justified surgical procedure for the treatment of HCC. Large tumor, high $\alpha$-fetoprotein, and vascular invasion are significant predictors of worse survival. The less incidence of HBV infection and cirrhosis may indicate that HCC in the octogenarian may have different carcinogenesis from that of younger patients. Keywords: Hepatectomy, Hepatocellular carcinoma, Hepatoma, Elderly, Octogenarian, Liver resection.

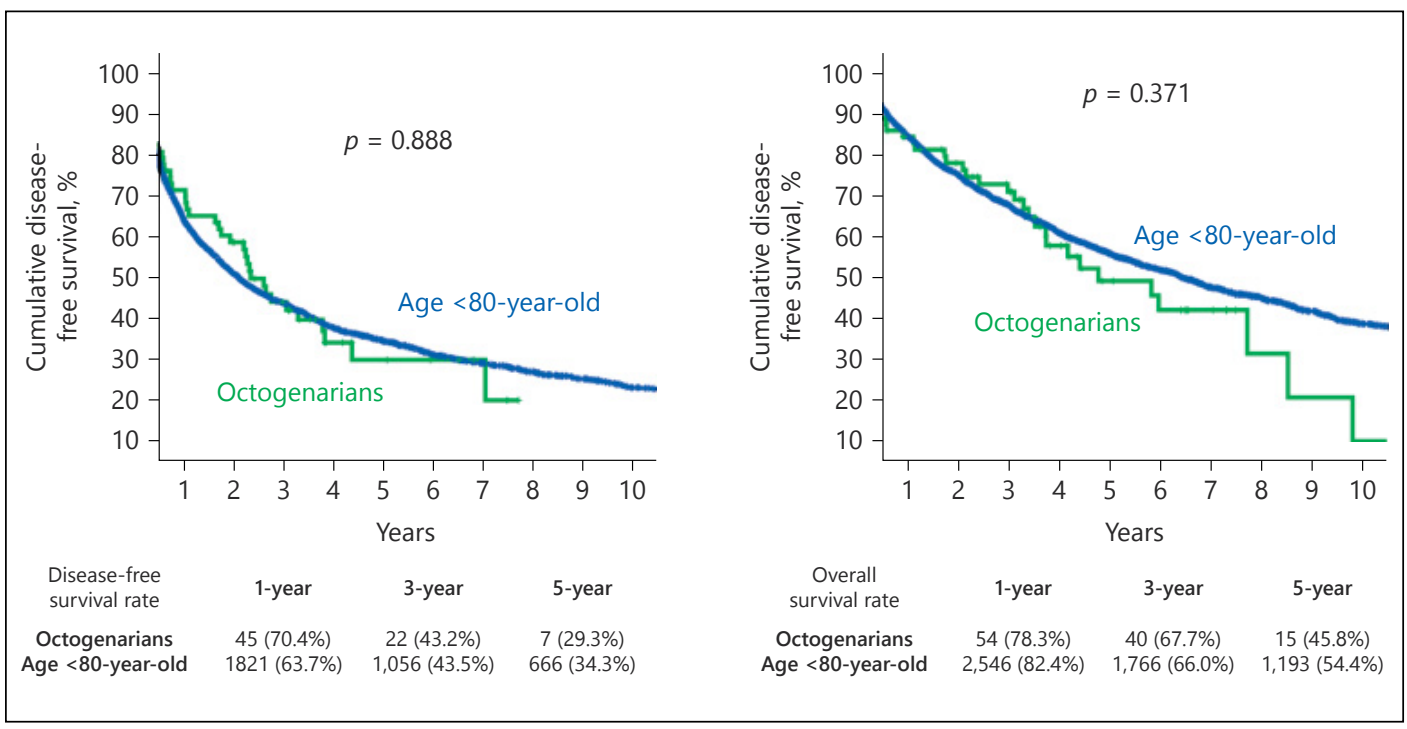

Fig. 1. (for Abstract no PO-31). 


\section{PO-32}

Is Surgical Resection Justified for Hepatocellular Carcinoma with Portal Vein Tumor Thrombus? A Systematic Review and Meta-Analysis

Lei Liang ${ }^{1}$, Chao Li ${ }^{1}$, Hao Xing ${ }^{1}$, Wan Yee Lau ${ }^{1,2}$, Meng Chao Wu ${ }^{1}$, Feng Shen ${ }^{1}$, Tian Yang ${ }^{1}$

${ }^{1}$ Department of Hepatobiliary Surgery, Eastern Hepatobiliary Surgery Hospital, Second Military Medical University, Shanghai, China; ${ }^{2}$ Faculty of Medicine, the Chinese University of Hong Kong, Shatin, New Territories, Hong Kong SAR, China

Aims: The prognosis of hepatocellular carcinoma (HCC) with portal vein tumor thrombus (PVTT) is very poor. According to most of authoritative HCC guidelines worldwide, sorafenib, transarterial chemoembolization (TACE) or other non-surgical treatments are recommended as the first-line therapy for these patients. In real world, however, a significant number of selected patients with HCC and PVTT may have undergone surgical resection (SR). This meta-analysis summarized evidence of the effectiveness of SR compared with Non-SR. Methods: PubMed, Embase, Medline and Cochrane library were searched for studies comparing SR with non-surgical resection treatments (Non-SR) for HCC with PVTT, which were published before December 2017. Results: 4,810 patients from 7 studies were enrolled in this metaanalysis, which were divided into the SR group $(n=2,344$, $48.5 \%$ ) and the Non-SR group ( $\mathrm{n}=2,476,51.5 \%)$. When compared with the Non-SR group, the pooled hazard ratios (HRs) for the 1, 3 and 5-year OS rates of the SR group were 0.57 (95\% CI 0.48-0.67, $P<0.001$ ), 0.66 (95\% CI 0.56-0.77, $P<$ 0.001 ) and 0.68 (95\% CI $0.57-0.81, P<0.001)$, respectively. For subgroup analysis, when compared with the mere TACE group, the pooled HRs for the 1, 3 and 5-year OS rates of the SR group were 0.62 (95\% CI 0.54-0.71, $P<0.001), 0.74$ (95\% CI $0.66-0.83, P<0.001)$ and 0.78 (95\% CI $0.70-0.87, P<0.001)$, respectively. Conclusions: This meta-analysis shows that SR has a better OS than TACE or other Non-SR treatments for HCC with PVTT. SR is certainly worth considering for selected patients with resectable HCC and removable PVTT. Keywords: Hepatocellular carcinoma, Portal vein tumor thrombus, Surgical resection, Survival.

\section{PO-33 \\ Laparoscopic Right Hepatectomy is Feasible and Safe in Solitary Hepatocellular Carcinoma}

Jong Man Kim, Sang Jin Kim, Jong Wook Oh, Young Jae Jung, Jinsoo Rhu, Kyung-Sik Kim, Jisoo Lee, Sun Jae Kim, Eun Young Lee, Gyu-Seong Choi, Choon Hyuck David Kwon, Jae-Won Joh

Department of Surgery, Samsung Medical Center, Sungkyunkwan University School of Medicine, Seoul, Republic of Korea

Aims: Laparoscopic liver resection has been reported as a safe and effective approach for the management of hepatocellular carcinoma (HCC). However, its perioperative and oncological outcomes have not been evaluated in right hepatectomy (RH) patients. Aim of present study is to compare the outcomes between laparoscopic RH (LRH) and open RH (ORH) in HCC patients. Methods: From January 2013 to August 2017, 345 patients with HCC underwent RH. Patients with portal vein tumor thrombosis, history of preoperative locoregional therapies, multiple tumors, bile duct tumor thrombosis, the history of abdominal operation, and serosal involvement were excluded. 189 patients were selected because of Child-Pugh class A and solitary HCC. Results: The numbers of ORH and LRH groups were 134 and 55 patients. Among LRH group, four patients (7.3\%) converted open conversion due to bleeding $(n=3)$ and close to the tumor $(n=$ 1). Median tumor size of ORH and LRH was $4.0 \mathrm{~cm}$ and $3.5 \mathrm{~cm}$, respectively $(\mathrm{P}=0.086)$. Preoperative factors, preoperative AFP levels, and pathologic factors were not different between the two groups. Operation time in ORH group was shorter than in the LRH group (243 min vs. $271 \mathrm{~min}$; $\mathrm{P}=0.032$ ) but amounts of blood loss in the ORH group was more than in the LRH ( $300 \mathrm{~mL}$ vs. $200 \mathrm{~mL}$; $<0.001)$. Two patients in the $\mathrm{ORH}$ group received red blood cells transfusion, but none in the LRH group were not transfused. Ten patients in the $\mathrm{ORH}$ group developed postoperative complications (Clavien grade I and II), but three patients in the LRH group had postoperative complications (Clavien grade 1 and II). Median hospitalization in the ORH group was shorter than in the LRH group (10 days vs. 7 days; $\mathrm{P}<0.001$ ). Disease-free survival (DFS) rates and patient survival (PS) rates at 1-, 2-, and 3-year were $92.1 \%, 89.2 \%, 87.4 \%$ and $95.3 \%, 92.4 \%, 90.2 \%$ in the ORH group and $100 \%, 94.1 \%, 94.1 \%$ and $100 \%, 100 \%, 100 \%$ in the LRH group, respectively. The DFS and PS in the LRH group were better than in the ORH group, but the difference did not reach significant levels. Conclusions: $\mathrm{LRH}$ is feasible and safe for solitary HCC patients in experienced center. However, the oncologic outcome of LRH should be needed in further investigations. Keywords: Minimal invasive surgery, Complications, Hepatectomy, Hepatocellular carcinoma. 


\section{PO-34 \\ The Role of Normothermic Perfusion in Liver Transplant (TRaNsIT Study): A Systematic Review of Preliminary Studies}

\section{Kumar Jayant, Isabella Reccia}

Department of Surgery and Cancer, Imperial College London, London, UK

Aims: The current organ preservation technique i.e static cold storage (SCS) is not suitable for marginal organs. Alternatively, normothermic machine perfusion (NMP) promises to recreate the physiological environment and thus provide better organ preservation. The objective of this systematic review, is to provide an overview on the safety, benefits and other potential useful parameters of NMP device. Methods: We searched the current literature following registration in with the International Prospective Register of Systematic Reviews (PROSPERO) with registration number CRD42018086034 for prospective trials comparing the role of NMP device to SCS in liver transplant by searching the PubMed, EMBASE, Cochrane, BIOSIS, Crossref, Scopus databases and clinical trial registry. Results: The literature search identified five prospective clinical trials (four early phase single institutional and single randomized multi-institutional) comparing 187 donor liver on NMP device to 275 donor livers on SCS. The primary outcome of interest was to assess the safety and graft survival at day 30 after transplant following NMP of the donor liver. Secondary outcomes included were early allograft dysfunction (EAD) in the first seven days; serum measures of liver functions as (bilirubin, aspartate aminotransferase (AST), alanine amino transferase (ALT), alkaline phosphatase (ALP), international normalized ratio (INR) on days 1-7; major complications as defined by a Clavien-Dindo score $\geq 3$; patient \& graft survival and biliary complications at six months. The peaked median AST level between day 1-7 in the five trials was from 417-1252 U/L (range 84-15009 U/L) while on NMP and 839-1474 U/L (range 153-8786 U/L) in SCS group. The median bilirubin level on day 7 was ranged from 25-79 $\mu \mathrm{mol} / \mathrm{L}$ (range 8-344 $\mu \mathrm{mol} / \mathrm{l}$ ) and 30-47.53 $\mu \mathrm{mol} / \mathrm{l}$ (range 9-340 $\mu \mathrm{mol} / \mathrm{l}$ ) in NMP and SCS groups respectively. There was no reported PNF in either groups in the three trials. There was inter trial variability in EAD which ranged from $15-56 \%$ in NMP group while 23-30 in SCS group. Biliary complications in form of 6 months' biliary stricture were observed in NMP group ranged from $0-20 \%$. None of the three trials reported any machine failure, although inadvertent events of catheter occlusion (four events) in hepatic vein and bile duct or unrecognized twist in portal vein (single event) were reported (Table 1,2). Conclusions: This review outlines that NMP not only demonstrated safety and efficacy but also provides the favourable environment of organ preservation, repair, and viability assessment to donor liver with low rate of complication as PNF, EAD and biliary complication following transplantation, however further studies are needed to broaden our horizon.

Table 1. Pre-transplant and perioperative characteristics of included studies (for Abstract no P0-34)

\begin{tabular}{|c|c|c|c|c|c|}
\hline Study & $\begin{array}{l}\text { Sample Size } \\
\text { (NMP vs Control }\end{array}$ & $\begin{array}{l}\text { Donor Age (years) } \\
\text { (NMP vs Control) }\end{array}$ & $\begin{array}{l}\text { MELD Score } \\
\text { (NMP vs Control) }\end{array}$ & $\begin{array}{l}\text { NMP Time vs Control } \\
\text { SCS in minutes } \\
\text { [median (range)] }\end{array}$ & $\begin{array}{l}\text { DCD } \\
\text { (NMP vs Control) }\end{array}$ \\
\hline $\begin{array}{l}\text { Ravikumar et al. } \\
\text { (May 2016) }\end{array}$ & 20 vs 40 & $\begin{array}{l}58.0(21-85) \text { vs } \\
58.5(21-82) \\
(p=0.93)\end{array}$ & $\begin{array}{l}12.0(7-27) \text { vs } \\
14.0(6-25) \\
(p=0.55)\end{array}$ & $\begin{array}{l}558(210-1170) v s \\
534(242-684) \\
(p=0.63)\end{array}$ & 4 vs 4 \\
\hline $\begin{array}{l}\text { Selzner et al. } \\
\text { (October 2016) }\end{array}$ & 10 vs 30 & $\begin{array}{l}48.0(17-75) \text { vs } \\
46.0(22-68) \\
(p=0.56)\end{array}$ & $\begin{array}{l}21.0(8-40) v s \\
23.0(7-37) \\
(p=0.85)\end{array}$ & $\begin{array}{l}586(221-731) \text { vs } \\
634(523-783) \\
(p=0.11)\end{array}$ & 2 vs 6 \\
\hline $\begin{array}{l}\text { Bral et al. } \\
\text { (Nov 2016) }\end{array}$ & 10 vs 30 & $\begin{array}{l}56.0(14-71) \text { vs } \\
52.0(20-77)(p= \\
0.91)\end{array}$ & $\begin{array}{l}13.0(9-32) \text { vs } \\
19.0(7-34) \\
(p=0.37)\end{array}$ & $\begin{array}{l}786(304-1631) \mathrm{vs} \\
235(64-890) \\
(p=0.001)\end{array}$ & 4 vs 8 \\
\hline $\begin{array}{l}\text { Liu et al. } \\
\text { (May 2017) }\end{array}$ & 10 vs 40 & NA $(p>0.05)$ & $\begin{array}{l}\text { NA } \\
(p>0.05)\end{array}$ & $\begin{array}{l}(240-472) \text { vs } \\
\text { NA } \\
(p>0.05)\end{array}$ & 2 vs 8 \\
\hline $\begin{array}{l}\text { Nasralla et al. } \\
\text { (May 2017) }\end{array}$ & 137 vs 135 & NA & NA & $\begin{array}{l}700 \text { vs } 429 \\
(p<0.01)\end{array}$ & NA \\
\hline
\end{tabular}

MELD, Model for End-stage Liver Disease; DCD, Donation after circulatory death; NMP, Normothermic machine perfusion; WIT, Warm ischemia time; SCS, Static cold storage; NA, Not Available; AST, Aspartate aminotransferase; ALT, Alanine amino transferase; ALP, Alkaline phosphatase. 
Table 2. Post-transplant outcomes of included studies (for Abstract no PO-34)

\begin{tabular}{|c|c|c|c|c|c|c|}
\hline Study & $\begin{array}{l}\text { PNF n (\%) } \\
\text { (NMP vs } \\
\text { Control) }\end{array}$ & $\begin{array}{l}\text { EAD n (\%) } \\
\text { (NMP vs } \\
\text { Control) }\end{array}$ & $\begin{array}{l}\text { ITU stay days } \\
\text { [median (range)] } \\
\text { (NMP vs Control) }\end{array}$ & $\begin{array}{l}\text { Hospital stay days } \\
\text { [median (range)] } \\
\text { (NMP vs Control) }\end{array}$ & $\begin{array}{l}\text { Major } \\
\text { Complications } \\
\text { (Clavien-Dindo } \\
\geq 3 \mathrm{~b} \text { ) n (\%) } \\
\text { (NMP vs Control) }\end{array}$ & $\begin{array}{l}\text { Biliary } \\
\text { Complications } \\
\text { (6months) } \\
\text { NMP n (\%) }\end{array}$ \\
\hline $\begin{array}{l}\text { Ravikumar } \\
\text { et al. }\end{array}$ & $\begin{array}{l}0(0) \mathrm{vs} \\
0(0) \\
(p=1.0)\end{array}$ & $\begin{array}{l}3(15) \text { vs } \\
9(22.5) \\
(p=0.73)\end{array}$ & $\begin{array}{l}3.0(1-8) \text { vs } \\
3(1-41) \\
(p=0.45)\end{array}$ & $\begin{array}{l}12.0(6-34) \text { vs } \\
14.0(8-88) \\
(p=0.10)\end{array}$ & NA & $4(20)$ \\
\hline $\begin{array}{l}\text { Selzner } \\
\text { et al. }\end{array}$ & $\begin{array}{l}0(0) \text { vs } \\
0(0) \\
(p=1.0)\end{array}$ & NA & $\begin{array}{l}1.0(0-8) \text { vs } \\
2(0-23) \\
(p=0.54)\end{array}$ & $\begin{array}{l}11.0(8-17) \text { vs } \\
13.0(7-89) \\
(p=0.23)\end{array}$ & $\begin{array}{l}1(10) \text { vs } \\
7(23) \\
(p=0.5)\end{array}$ & $0(0)$ \\
\hline Bral et al. & $\begin{array}{l}0(0) \text { vs } \\
0(0) \\
(p=1.0)\end{array}$ & $\begin{array}{l}5(55.5) \text { vs } \\
8(29.6) \\
(p=0.23)\end{array}$ & $\begin{array}{l}16.0(2-65) \text { vs } \\
4(1-29) \\
(p=0.004)\end{array}$ & $\begin{array}{l}11.0(8-17) \text { vs } \\
13.0(7-89) \\
(p=0.23)\end{array}$ & $\begin{array}{l}2(22) \mathrm{vs} \\
10(37) \\
(\mathrm{p}=0.6)\end{array}$ & $0(0)$ \\
\hline Liu et al. & NA & $\begin{array}{l}1(10 \%) \mathrm{vs} \\
15(36.8 \%) \\
(p=0.13)\end{array}$ & NA & NA & NA & NA \\
\hline $\begin{array}{l}\text { Nasralla } \\
\text { et al. }\end{array}$ & NA & $\begin{array}{l}15(12.6 \%) \text { vs } \\
30(29.9 \%) \\
(p=0.002)\end{array}$ & NA & NA & NA & NA \\
\hline
\end{tabular}

PNF, Primary Nonfunction; EAD, Early graft dysfunction; NA: Not Available.

\section{PO-35 \\ Postoperative Portal Vein Perfusion Chemotherapy Improved Clinical Outcome for Advanced Hepatocellular Carcinoma with Portal Vein Tumor Thrombus: A Propensity Score Matching Analysis}

Xin-Rong Yang, Yang Gao, Jian Zhou, Jia Fan

Department of Liver Surgery and transplantation, Liver Cancer Institute, Zhongshan Hospital, Fudan University, Shanghai, P.R. China

Aims: Portal vein tumor thrombosis (PVTT) is a common complication in hepatocellular carcinoma (HCC) and indicates advanced condition, difficult treatment and dismal outcomes. Surgical resection provided a potentially curative approach and yielded acceptable clinical outcomes in selected patients. However, improvement in therapeutic outcome is difficult with surgical treatment alone. The aim of this study was to evaluate the survival benefit of postoperative portal vein perfusion chemotherapy (PVC) for HCC patients with PVTT after resection. Methods: From 2009 to 2015, 401 consecutive HCC patients with PVTT who underwent hepatic resection were retrospectively reviewed, including 67 patients with adjuvant PVC and 334 patients without PVC. We determined the control group ( $\mathrm{n}=67$ ) and PVC group ( $\mathrm{n}=67$ ) through propensity score matching (PSM). The clinical outcomes were compared before and after propensity score matching. During the course of PVC, cisplatin $(40 \mathrm{mg} / \mathrm{m} 2 / \mathrm{d})$ and Doxorubicin $(30 \mathrm{mg} / \mathrm{m} 2 / \mathrm{d})$ at day $15-\mathrm{FU}(650 \mathrm{mg} / \mathrm{m} 2 / \mathrm{d})$ at day 1 and 2 , were administrated through portal vein pump every 4 weeks with 4-6 course according to the patient's tolerance Results: Before PSM, no statistical significance was observed between PVC group and no PVC group for overall survival (OS) or relapse-free survival (RFS). After PSM, the median OS and RFS were significantly longer in PVC group compared with no PVC group (19.0 vs. 13.4 months, $\mathrm{P}=0.034 ; 12.3$ vs. 6.2 months, $\mathrm{P}=0.008$, respectively). The 1-, 3-, 5-year OS and RFS rates were $83.6 \%, 49.8 \%$ and 7.5, and $48.4 \%, 5.6 \%$ and $1.5 \%$ in PVC group, while the 1-, 3-, 5-year OS and RFS rates were $55.5 \%, 6.0 \%$ and $3.0 \%, 23.9 \%, 3.2 \%$ and $1.5 \%$ in no PVC group. Multivariate analysis identified postoperative PVC as a significant predictor for RFS [hazard ratio (HR) $=0.577, \mathrm{P}=0.006]$ and $\mathrm{OS}(\mathrm{HR}=0.608, \mathrm{P}=0.013)$. Postoperative PVC could reduce early recurrence $(\leq 1$ year) rate after tumor resection $(40.3 \%$ vs. $64.2 \%, P=0.006)$. Subgroup analysis revealed that postoperative PVC tended to provide a potential clinical benefit, especially in those with type PVTT (tumor thrombi involving the main portal vein trunk), multiple tumor, and HBVDNA $\leq 104 \mathrm{IU} / \mathrm{ml}$. Conclusions: Compared with surgical resection alone, postoperative adjuvant PVC treatment could provide survival benefits and deceased tumor early recurrence after HCC patients receiving tumor resection and PVTT removal, especially in those with type PVTT, multiple tumor, HBVDNA $\leq 104 \mathrm{IU} / \mathrm{ml}$. Keywords: Hepatocellular carcinoma, Portal vein tumor thrombosis, Portal vein perfusion chemotherapy, Propensity score matching. 


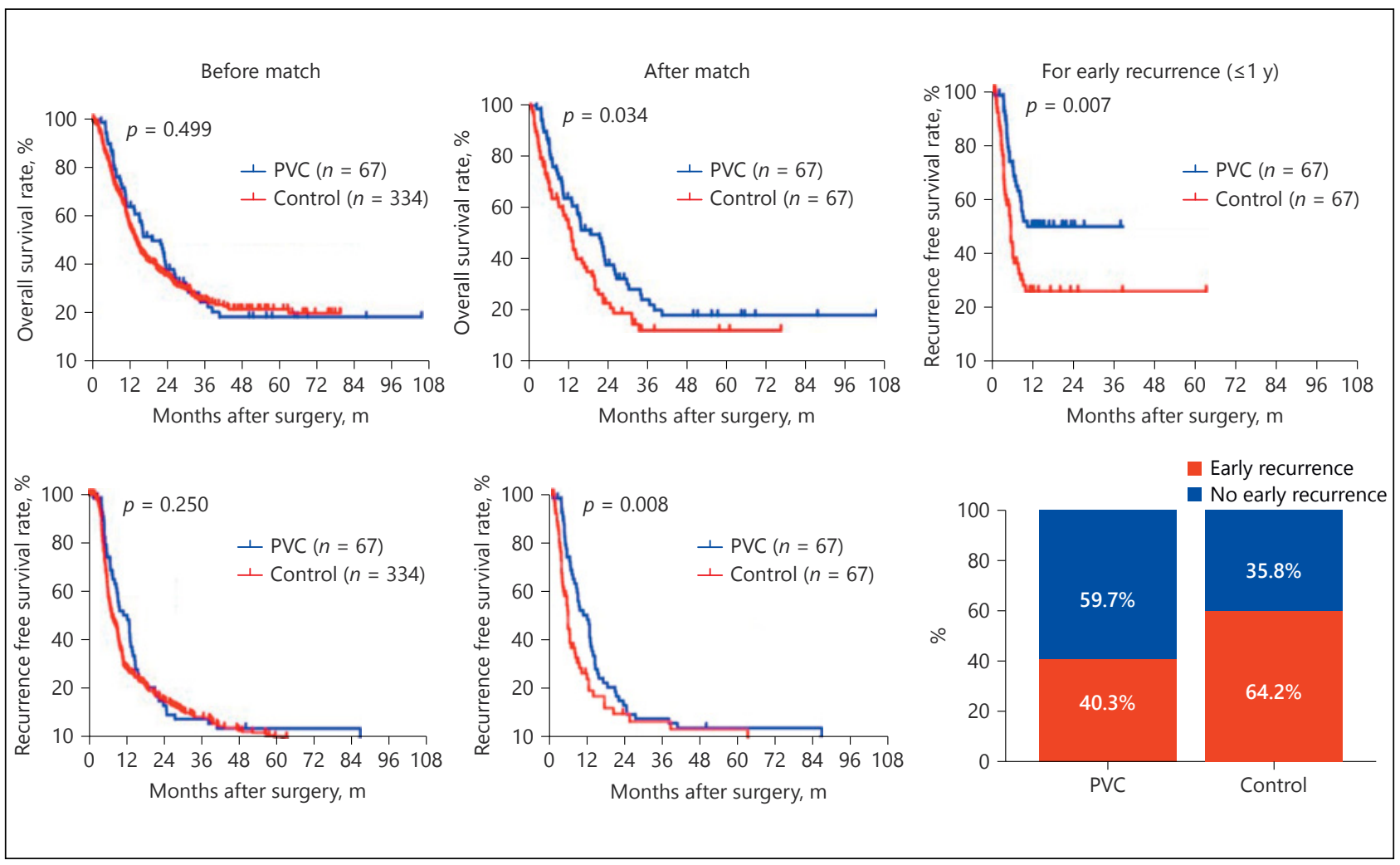

Fig. 1. (for Abstract no PO-35).

\section{Treatment: Transarterial Approach / Percutaneous Ablative Therapy / Radiation Therapy}

\section{PO-36 \\ Different Survival Outcomes between Patients with Treatment-Naïve HCC versus Recurrent HCC after Curative Resection Undergoing TACE}

David S. Kim ${ }^{1}$, Beom Kyung Kim ${ }^{1,2}$, Jun Yong Park ${ }^{1,2}$, Do Young Kim ${ }^{1,2}$, Sang Hoon Ahn ${ }^{1,2}$, Kwang Hyub Han ${ }^{1,2}$, Seung Up Kim ${ }^{1,2}$

${ }^{1}$ Department of Internal Medicine, Institute of Gastroenterology, Yonsei University College of Medicine, Seoul, South Korea; ${ }^{2}$ Yonsei Liver Center, Severance Hospital, Seoul, South Korea

Background/Aims: Trans-arterial chemoembolization (TACE) improves survival of patients with hepatocellular carcinoma (HCC). However, the treatment outcomes of TACE in patients with treatment-naïve HCC versus recurrent HCC after curative resection has not been compared. Methods: A total of 448 patients with treatmentnaïve HCC and 275 patients with recurrent HCC after curative resection treated with TACE as the first-line anticancer treatment were recruited. Cox regression analysis was used to identify independent factors affecting overall mortality. Results: Patients with treatment-naïve HCC at the time of TACE showed a significantly higher proportion of liver cirrhosis (61.9\% vs. $49.3 \%$ ), higher aspartate aminotransferase level (median 48 vs. 31 IU/L), higher alanine aminotransferase level (median 38 vs. 26 IU/L), higher alpha-fetoprotein level (median 96.6 vs. $7.7 \mathrm{ng} / \mathrm{mL}$ ), higher total bilirubin level (mean 0.97 vs. $0.84 \mathrm{mg} / \mathrm{dL}$ ), longer prothrombin time (1.05 vs. 1.01 INR), higher tumor number (mean 2.1 vs. 1.7), larger tumor size (3.1 vs. 1.6 $\mathrm{cm}$ ), and lower proportion of BCLC stage 0-B (vs stage C) (55.6\% vs. $71.9 \%$ ) (all $\mathrm{P}<0.05$ ). Multivariate analysis showed that TACE for treatment-naïve HCC (vs. recurrent HCC after curative resection) was one of independent risk factors of mortality (hazard ratio, 1.328; 95\% confidence interval, 1.038-1.700; $\mathrm{P}=0.024$ ), together with higher alpha-fetoprotein level and higher tumor number (all $\mathrm{P}<$ 0.05). Conclusions: Patients with treatment-naïve HCC showed poorer clinical characteristics than those with recurrent HCC after curative resection at the time of TACE 
and TACE for treatment-naïve HCC (vs. TACE for recurrent HCC after curative resection) was independently associated with the increased risk of mortality. Keywords: Hepatocellular carcinoma, Curative resection, Transarterial chemoembolization, Outcome.

\section{PO-37 \\ Nomogram and Artificial Neural Network for Prognostic Performance on the Albumin-Bilirubin Grade for Hepatocellular Carcinoma Undergoing Transarterial Chemoembolization}

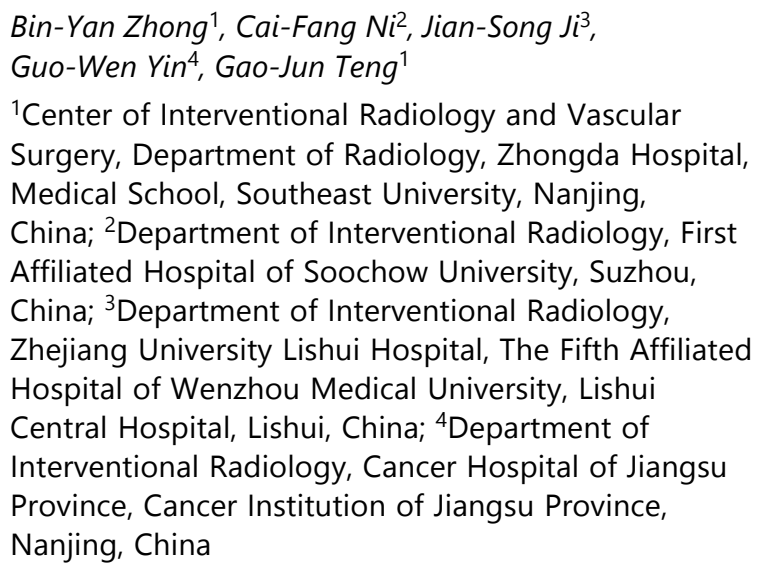

Aims: The albumin-bilirubin (ALBI) grade is a newly raised objective liver function assessment tool and an emerging alternative of the Child-Turcotte-Pugh (CTP) grade in hepatocellular carcinoma (HCC). We aimed to construct ALBI and CTP grades-based nomograms as well as develop an artificial neural network (ANN) to compare the prognostic performance of these two grades for HCC underwent transarterial chemoembolization (TACE). Methods: This multicentric retrospective study included patients with HCC who underwent TACE monotherapy as initial treatment between January 2008 and December 2016 at four institutions. In training cohort, independent risk factors associated with overall survival (OS) were identified by univariate and multivariate analyses. The prognostic nomograms and ANN were established and then validated in two validation cohorts. Results: Totally, 838 patients $(548,115$, and 175 in training cohort and validation cohorts 1 and 2, respectively) were included. In training cohort, both ALBI and CTP grades were identified as independent risk factors. The ALBI and CTP gradesbased nomograms were established separately and showed comparable prognostic performance when assessed externally in validation cohorts ( $\mathrm{C}$-index in validation cohort 1 : 0.823 vs. $0.802, \mathrm{P}=0.417$; in validation cohort 2: 0.716 vs. $0.729, \mathrm{P}=0.793$ ). ANN showed that ALBI grade had higher importance on survival prediction than CTP grade. Conclusions: The ALBI grade outperforms the CTP grade on survival prediction for HCC patients who undergo TACE. Considering the easy application, the ALBI grade should be regarded as an alternative to CTP grade. Keywords: Hepatocellular carcinoma, Transarterial chemoembolization, ALBI grade, Nomogram, Artificial neural network.

\section{PO-38 \\ Hepatocellular Carcinoma with Extrahepatic Metastasis: Who Are Still Candidates for Locoregional Therapy?}

Jihye Kim, Dong-Hyun Sinn, Wonseok Kang, Geum-Youn Gwak, Moon Seok Choi, Yong-Han Paik, Joon Hyeok Lee, Kwang Cheol Koh, Seung Woon Paik

Department of Medicine, Samsung Medical Center, Sungkyunkwan University School of Medicine, Seoul, South Korea

Aims: Nowadays, sorafenib is available for hepatocellular carcinoma (HCC) patients with extrahepatic metastasis (EHM). We aimed to find out whether locoregional treatment is still valid for patients with EHM, and if so, which subgroup may benefit from locoregional treatment. Methods: A total of 187 consecutive HCC patients (median: 55 years, male: $86.1 \%$, hepatitis B virus: $81.2 \%$, Child-Pugh Class A: 82.9\%) with EHM between 2010 and 2014 were analyzed. We categorized patients according to initial treatment modality: sorafenib or locoregional treatment. Overall survival was compared between two groups. Results: During a median follow-up of 6.6 months (range: 0.2-94.6 months), mortality was 90.4\% (169/187). Type of EHM was nodal metastasis in 114 patients $(61.0 \%)$ and distant metastasis with/without nodal metastasis in 73 patients (39.0\%). Tumor morphology was nodular in 156 patients $(83.4 \%)$ and diffuse in 31 patients (16.6\%). Initial treatment modality was locoregional for 118 patients (63.1\%), of which 116 patients underwent transarterial chemoembolization, and was systemic for 69 patients (36.9\%). Median survival was better for patients who received locoregional treatment than systemic treatment (8.9 months vs. 4.6 months, $\mathrm{p}<0.001$ ). However, once the different baseline characteristics of the two groups were adjusted, treatment modality was no longer a risk factor for overall survival (hazard ratio: 1.09, 95\% confidence interval: $0.75-1.57, \mathrm{p}=0.63$ ). When stratified, there was no difference in survival according to treatment modality for those with distant metastasis or diffuse tumor (6.6 vs. 3.9 months for locoregional vs. systemic, $p=0.63$ ). However, for nodular tumor with nodal metastasis, the survival was better in locoregional treatment group than systemic treatment group (11.7 vs. 5.2 months, $p=0.027$ ). Conclusions: Locoregional treatment offered survival 


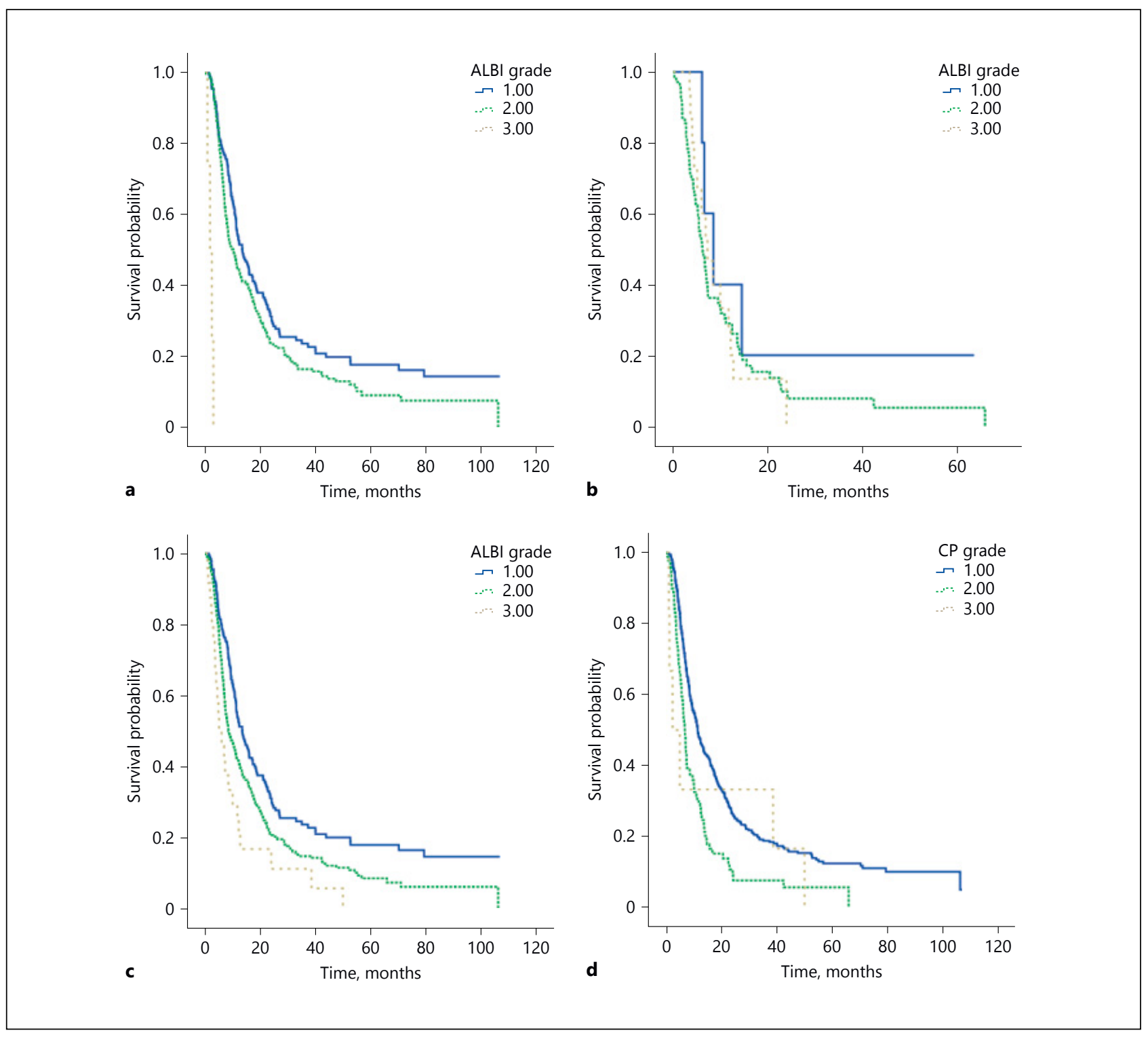

Fig. 1. (for Abstract no PO-37).

benefit for HCC patients with EHM compared to systemic treatment when tumor showed nodular morphology without distant metastasis. Our data indicate that nodal or distant metastasis should be differentiated, which may have therapeutic implication on initial treatment. Keywords: Hepatocellular carcinoma, Extrahepatic metastasis, Locoregional treatment, Systemic treatment, Sorafenib. 


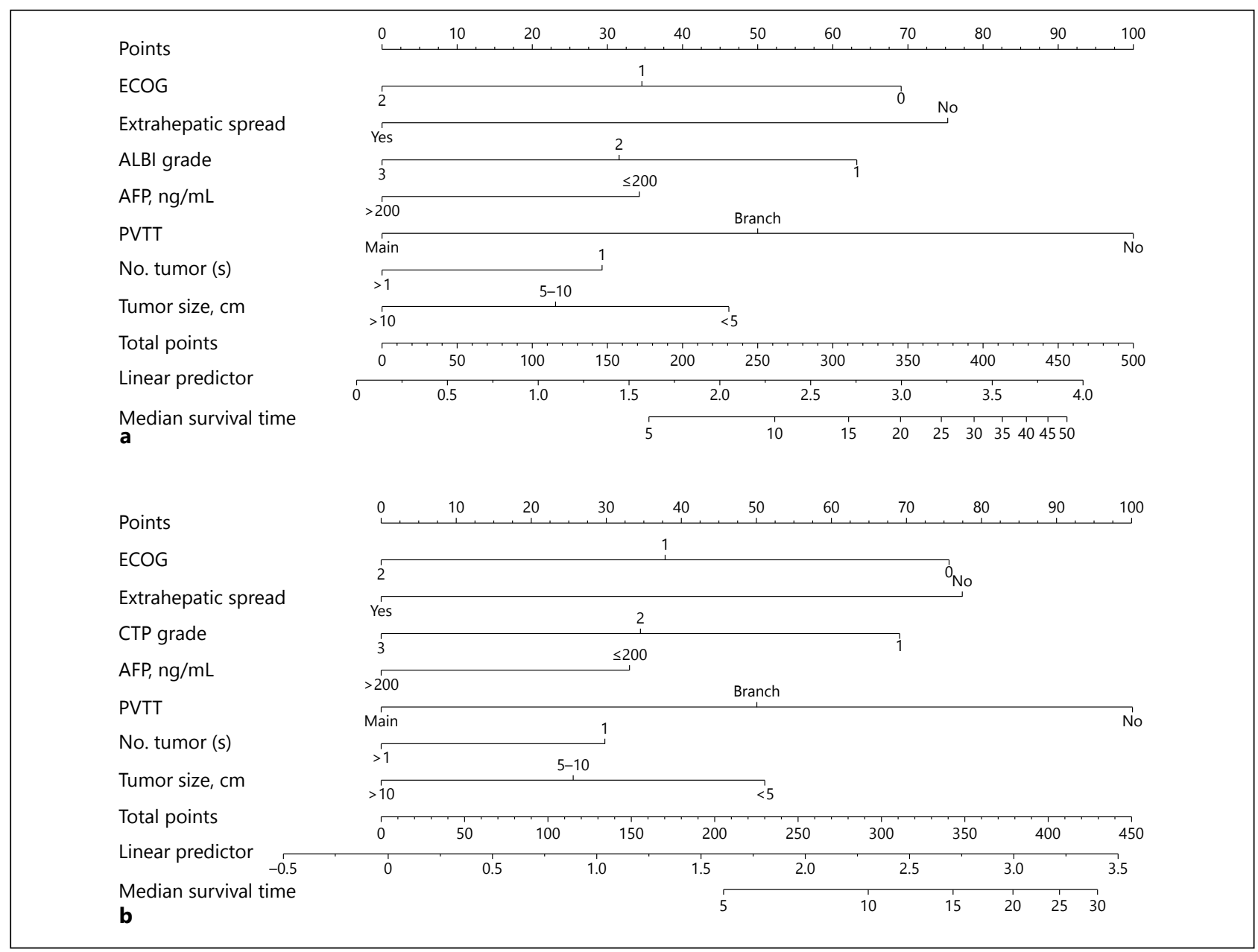

Fig. 2. (for Abstract no PO-37).

\section{PO-39}

Microwave versus Radiofrequency Ablation for Hepatocellular Carcinoma: A Prospective Randomized Controlled Trial

Charing C.N. Chong ${ }^{1}$, Kit Fai Lee ${ }^{1}$, Anthony K.W. Fong ${ }^{1}$, Clement C.M. Chu ${ }^{2}$, Simon C.H.Yu ${ }^{2}$, Andrew K.Y. Fung ${ }^{1}$, Hon Ting Lok ${ }^{1}$, Sunny Y.S. Cheung ${ }^{1}$, John Wong ${ }^{1}$, Paul B.S. Lai

${ }^{1}$ Division of Hepatobiliary and Pancreatic Surgery, Department of Surgery, Prince of Wales Hospital, Chinese University of Hong Kong, Shatin, Hong Kong SAR, ${ }^{2}$ Department of Imaging \& Interventional Radiology, Prince of Wales Hospital, the Chinese University of Hong Kong, Shatin, Hong Kong SAR

Aims: Radiofrequency ablation (RFA) and microwave ablation (MWA) are the most commonly used local ablation therapies for HCC. Studies comparing the both techniques are scarce. This study aimed to compare the efficacy of MWA versus RFA as a treatment for HCC. Methods: Patients diagnosed with HCC who were suitable for local ablation were randomized into MWA or RFA group. All patients will be followed up regularly with triphasic contrast enhanced CT scan performed at 1,3,6,12 months after ablation. Both patients and the radiologists who interpret the post-procedure CT scans were blinded to the type of ablation done. Treatment related morbidity, overall and disease-free survival were analyzed. Results: A total of 93 patients was recruited between April 2011 to March 2017. Among them, 47 and 46 patients were randomized to MWA and RFA respectively. Patients in two groups were comparable in terms of age, child's grading, tumor size and number, as well as operative approach. With a median follow up of around 30 months in both groups, there was no significant difference in the treatment related morbidity, overall and disease-free survival. MWA had a significantly shorter overall ablation time when compared with RFA (12 mins vs 24 mins, $\mathrm{P}<0.001$ ). Conclusions: MWA is as safe and as effective as RFA in treating small HCC. With additional benefits of shorter ablation time and elimination of 


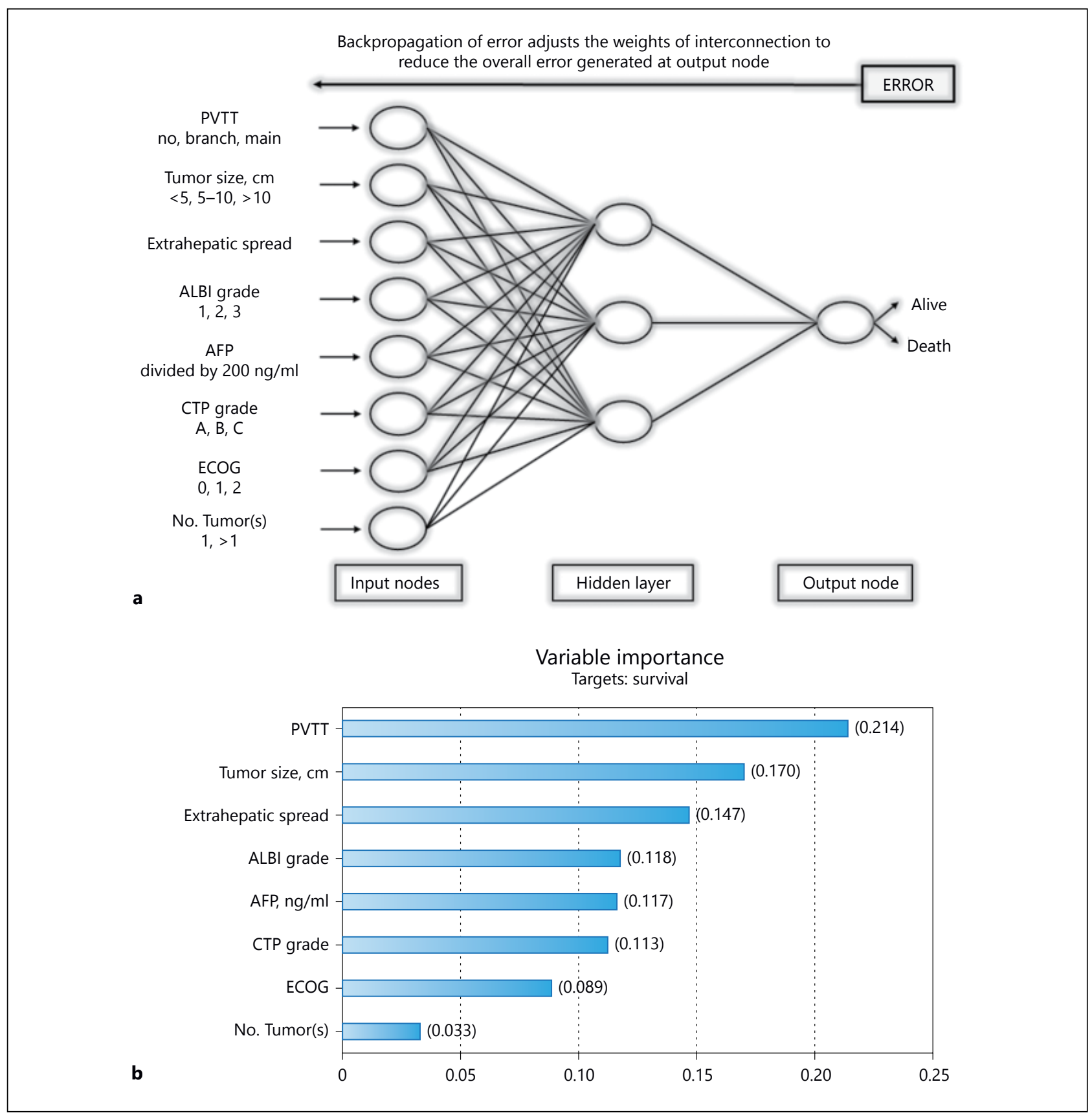

Fig. 3. (for Abstract no PO-37).

burn injury from earth plate, it could be considered as an alternative to RFA in treating HCC. Keywords: Hepatocellular carcinoma, Radiofrequency ablation, Microwave ablation, Local ablation therapy. 


\section{PO-40 \\ Outcomes and Prognostic Factors after Carbon-Ion Radiotherapy for Hepatocellular Carcinoma}

Kei Shibuya ${ }^{1,2}$, Hiroyuki Katoh ${ }^{1}$, Yoshinori Koyama ${ }^{5}$, Shintaro Shiba ${ }^{2}$, Masahiko Okamoto ${ }^{1,2}$, Kenichiro Araki ${ }^{3}$, Satoru Kakizaki ${ }^{4}$, Tatsuya Ohno ${ }^{1}$, Ken Shirabe ${ }^{3}$, Takashi Nakano ${ }^{1,2}$

${ }^{1}$ Gunma University Heavy-ion Medical Center (GHMC), ${ }^{2}$ Department of Radiation Oncology, ${ }^{3}$ Department of Hepatobiliary and pancreatic surgery, ${ }^{4}$ Department of Gastroenterology and Hepatology, Gunma University hospital, ${ }^{5}$ Department of Radiology, Shibukawa Medical Center, National Hospital Organization, Japan

Aims: Carbon ion radiotherapy (C-ion RT) is a promising technique for hepatocellular carcinoma (HCC). We started C-ion RT for HCC using 52.8 Gy (RBE) in four fractions, and escalated dose up to 60 Gy (RBE) for peripheral HCCs and extended indication to the lesions adjacent to alimentary tract or porta hepatis by using 12 fractions. Methods: A total of 111 patients between September 2010 and June 2017 were analyzed. Median age was 76 (range: 45-90) years. ChildPugh class A, B, and C were 96 (86.5\%), 14 (12.6\%), and 1
$(0.9 \%)$ patients, respectively. UICC stage I, II, III, and IVa were $16,72,21$, and 2 patients, respectively. Mean Maximum tumor diameter was 4.0 (range; 0.9-10.4) $\mathrm{cm}$. Dose fractionation schedule was 4-fraction for 93 patients (83.7\%) [52.8 Gy (RBE): 44 patients, and 60 Gy (RBE): 49 patients], and 12-fraction for 18 patients (16.3\%) [60.0 Gy (RBE): 15 patients, and 64.8 Gy (RBE): 3 patients]. Results: The median follow-up period was 28.7 (range 4.0-80.5) months. The 1-, 2-, and 3 -year local control rates were $97.8 \%, 88.0 \%$, and $79.3 \%$, respectively. The 1-, 2-, and 3-year overall survival rates were $94.2 \%, 79.1 \%$, and $68.7 \%$, respectively. Grade 3 adverse events were observed in four patients (3.6\%) including encephalopathy and/or cognitive disorder: three patients, and acute cholecystitis: one patient. Grade 4 or 5 toxicities were not observed. Multivariate analysis showed that PS1-2 ( $p=0.002)$, Child $B / C(p<0.001)$, and prothrombin induced by the absence of vitamin $\mathrm{K}$ or antagonist-II (PIVKA-II) $>200$ $(\mathrm{p}=0.003)$ were significant prognostic factor for overall survival. There was no significance for local control in maximum tumor diameter greater than $3 \mathrm{~cm}$ or not. Conclusions: C-ion RT were safe and effective treatment for HCC. Tumor diameter is not a significant factor for local control, and PIVKAII is a significant prognostic factor for overall survival. Keywords: Radiotherapy, Carbon-ion radiotherapy, Hepatocellular carcinoma. 


\section{Poster Exhibition}

\section{Biomarkers / Cell Biology and Translational Research}

\section{PE-001 \\ PD-1 and PD-L1 Levels Do Not Predict Prognosis in Patients with Hepatocellular Carcinoma}

Hye Won Lee ${ }^{1,2 *}$, Kyung Joo Cho ${ }^{1,2 *}$, Soon Young Shin ${ }^{1,2}$, Beom Kyung Kim ${ }^{1,2}$, Seung Up Kim ${ }^{1,2}$, Jun Yong Park ${ }^{1,2}$, Do Young Kim ${ }^{1,2}$, Sang Hoon Ahn ${ }^{1,2}$, Kwang-Hyub Han ${ }^{1,2}$ ${ }^{1}$ Department of Internal Medicine, Yonsei University College of Medicine, Seoul, Korea, ${ }^{2}$ Yonsei Liver Center, Severance Hospital, Seoul, South Korea

Aims: Programmed death receptor 1 (PD-1) is a promising drug target for patients with hepatocellular carcinoma (HCC). Expression of programmed death ligand 1 (PD-L1) is mainly induced by inflammatory cytokines, and activation of PD-l by its ligands suppresses activation of T cells. A high level of PD-L1 is a possible prognostic indicator for a poor outcome in patients with HCC. Here we investigated the clinical significance of PD-1 and PD-L1 in patients with HCC. Methods: We enrolled patients with HCC who underwent curative surgical resection at Severance Hospital, Seoul, Republic of Korea. We investigated PD-L1 expression in a series of 78 HCC and non-HCC tissues and correlated the results with clinical and histological features (hematoxylin and eosin) as well as immunohistochemical markers. Circulating PD-1 expression levels were estimated by enzyme-linked immunosorbent assay. The associations between the expression levels of PD-L1 and PD-1, and clinicopathological prognosis in a cohort consisting of tumor tissues and matching samples were analyzed statistically. Results: A total of 78 patients were finally analyzed. The median SPD-L1 and sPD-1 levels were $25.72 \mathrm{pg} / \mathrm{ml}$ [interquartile range (IQR 18.51-67.02)] and $341.44 \mathrm{pg} / \mathrm{ml}$ (IQR 152.59-584.51), respectively. The numbers of patients with each tPD-L1 score were 2 (2.6\%) with score 1, 16 (20.5\%) with score 2, 27 (34.6\%) with score 3, and 26 (33.3\%) with score 4 . A positive correlation was detected between tPD-L1 and SPD-1 $\left(R^{2}=0.426, P<0.001\right)$. The median SPD-1 level for each tPD-L1 score increased linearly $(P=0.002)$. During the follow-up (median 16.1 months), HCC recurred in eight $(10.3 \%)$ patients and liver-related mortality occurred in eight (10.3\%) patients, respectively. The tPD-L1 scores in patients who died were significantly higher compared with those who survived (tPD-L1 score $\geq 3,100 \%$ vs. $71.9 \%, P=0.0348$ ). A multivariate analysis showed that higher sPD-L1 (cut-off values, $\geq 19.2 \mathrm{pg} / \mathrm{ml}$ ) tended to be associated with liverrelated mortality [hazard ratio $6.866,95 \%$ confidence interval 0.804--58.659), but no statistical significance $(P=0.078)$. No significant association was observed between PD-L1/PD-1 and recurrence of HCC. Conclusions: The results demonstrate that PD-1 and PD-L1 levels were not significantly associated with clinical outcomes in patients with HCC. Keywords: PD1, PDL1, Prognosis, Hepatocellular carcinoma.

\section{PE-002 \\ Alpha-Fetoprotein and Poorly Differentiated Histology Predict Hepatocellular Carcinoma Recurrence after Liver Transplant}

Q.D. Huang ${ }^{1 *}$, M. Muthiah ${ }^{1 *}$, W.R.B. Tay ${ }^{1}$, G.S. Kew ${ }^{1}$, G.H. Lee ${ }^{1}$, S.G. Lim 1 , Y.M. Lee', B.L.K. Lim ${ }^{1}$, H.C. Low ${ }^{1}$, W.C.A. Kow', S.G. lyer', G.K. Bonney2, K. Madhavan', A. Wee ${ }^{3}$, Y.H. Pang ${ }^{4}$, G. Soon ${ }^{4}$, P.S. Tan ${ }^{1 *}$, Y.Y. Dan ${ }^{1 *}$

${ }^{1}$ Yong Loo Lin School of Medicine, National University of Singapore, Singapore; Division of Gastroenterology and Hepatology, National University Health System, Singapore; National University Centre for Organ Transplantation, National University Health System, Singapore; ${ }^{2}$ Yong Loo Lin School of Medicine, National University of Singapore, Singapore; Division of Hepatobiliary and Pancreatic Surgery, National University Health System, Singapore; National University Centre for Organ Transplantation, National University Health System, Singapore; ${ }^{3}$ Department of Pathology, Yong Loo Lin School of Medicine, National University of Singapore, National University Hospital, National University Health System, Singapore; ${ }^{4}$ Department of Pathology, National University Hospital, National University Health System, Singapore

Aims: Liver transplantation is plagued by the recurrence of disease which makes transplant futile. We report the longterm outcomes of HCC cases transplanted in an Asian setting and the role of alpha-fetoprotein (AFP) and tumor differentiation in predicting survival and recurrence. Methods: Clinical data from all adult liver transplant cases at our center from 1996 to 2015 were recorded. Survival analysis was estimated with Kaplan-Meier curve, and outcome predictors were determined via Cox regression. Between December 1996 and 
August 2015, 170 adult transplants were performed, of which 133 cases had sufficient data for analysis. Mean age was 51.2 years old and 41 cases (30.8\%) were living donor liver transplants (LDLT). 54 cases (40.3\%) had HCC, of which 47 (87.0\%) were within Milan criteria. Results: For non-HCC cases, 1 and 5-year overall survival (OS) were $95.9 \%$ and $93.6 \%$, respectively. HCC cases within Milan criteria had significantly poorer 1 - and 5-year OS of $91.6 \%$ and $74.2 \%(p=0.026)$. Similarly, HCC within University of California San Francisco (UCSF) criteria demonstrated poorer 1- and 5-year OS compared to non-HCC $(\mathrm{p}=0.023)$. The only variable affecting OS in multivariate analysis was tumor grade (HR 10.92, CI 1.08-110.38, $\mathrm{p}=0.043$ ). In multivariate analysis for HCC recurrence, AFP and tumor grade were significant. HCC with AFP level $>100$ $\mathrm{ng} / \mathrm{ml}$ had a recurrence rate of $23.1 \%(\mathrm{p}=0.049)$ while AFP $>400 \mathrm{ng} / \mathrm{ml}$ saw a recurrence rate of $50.0 \%$ ( $<<0.001)$. In patients with AFP $>100 \mathrm{ng} / \mathrm{ml}$, poorly differentiated histology was associated with a $100 \%$ recurrence rate compared to $14.3 \%$ recurrence for those with better differentiated histology ( $p=0.014)$. Conclusions: Patients transplanted for HCC had markedly poorer outcomes compared to non-HCC transplants. The combination of AFP and tumor grade allows a more refined risk prediction, which has significance for the adoption of transplantation criteria. When AFP levels exceed $400 \mathrm{ng} / \mathrm{ml}$, recurrence rates are 50 percent and LDLT should be advocated. Keywords: Hepatocellular carcinoma, Alphafetoprotein, Transplant.

\section{PE-003 \\ 18F-FDG PET CT as an Independent Predictor for Patients with Hepatocellular Carcinoma Combined with Major Portal Vein Tumor Thrombus}

Xu-Guang Hu${ }^{1}$, In-Gyu Kim ${ }^{1}$, Hee-Jung Wang ${ }^{1}$, Bong-Wan Kim ${ }^{1}$, Joon-Kee Yoon ${ }^{2}$, Sung-Yeon Hong ${ }^{1}$, Xue-yin Shen ${ }^{1}$

${ }^{1}$ Division of Hepatobiliary Surgery and Liver Transplantation, Department of Surgery, Ajou University School of Medicine, Suwon, South Korea; ${ }^{2}$ Department of Nuclear Medicine and Molecular Imaging, Ajou University School of Medicine, Suwon, South Korea

Aims: Hepatocellular carcinoma (HCC) patients with major portal vein tumor thrombosis (mPVTT) have an extremely poor prognosis. The aim of the present study was to clarify the role of $18 \mathrm{~F}$-fluorodeoxyglucose positron emission tomography/computed tomography (18F-FDG-PET/CT) imaging as an independent predictor for HCC patients with mPVTT. Methods: From Nov. 2008 to Dec. 2014, a total of 501 cases of HCC patients were operated in our department. Among them, 32 (6.4\%) HCC patients complicated with mPVTT. Six cases were excluded: Two cases of salvage liver transplantation, two cases of re-resection, one case of mPVTT combined with IVC tumor thrombosis and one case of residual
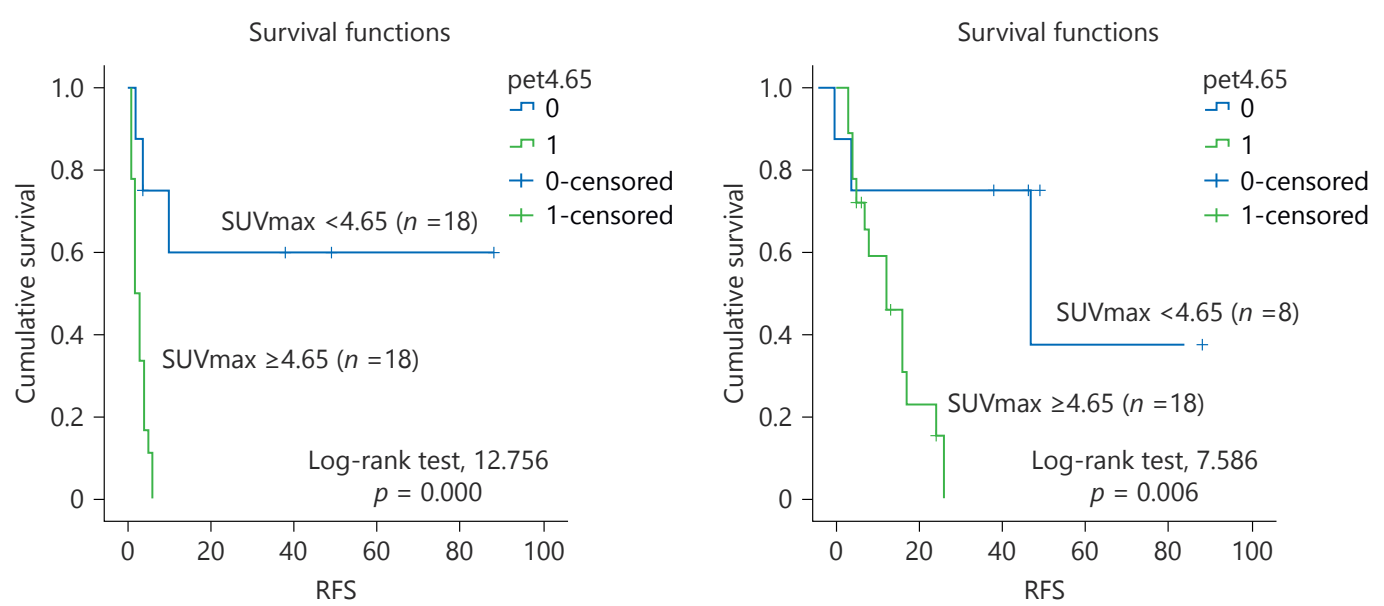

\begin{tabular}{rrrrr}
\hline RFS & \multicolumn{4}{c}{ Number at risk, $\mathrm{n} / \%$} \\
\hline Years & 0 & 1 & 3 & 5 \\
SUVmax $<4.65$ & 8 & 4 & 4 & 1 \\
SUVmax $\geq 4.65$ & & 60 & 60 & 60 \\
& 18 & 0 & 0 & 0 \\
& & 0 & 0 & 0 \\
\hline
\end{tabular}

\begin{tabular}{|c|c|c|c|c|}
\hline \multicolumn{2}{|c|}{ RFS } & \multicolumn{3}{|c|}{ Number at risk, n/\% } \\
\hline \multirow{2}{*}{$\begin{array}{r}\text { Years } \\
\text { SUVmax }<4.65\end{array}$} & 0 & 1 & 3 & 5 \\
\hline & 8 & 6 & 5 & 1 \\
\hline \multirow{3}{*}{ SUVmax $\geq 4.65$} & & 75 & 75 & 37.5 \\
\hline & 18 & 7 & 0 & 0 \\
\hline & & 46 & 0 & 0 \\
\hline
\end{tabular}

Fig. 1. (for Abstract no PE-003). 
Table 1. Cox proportional hazards model of risk factors for OS in HCC patients with mPVTT (for Abstract no PE-003)

\begin{tabular}{|c|c|c|c|c|c|c|}
\hline \multirow[b]{2}{*}{ Factors } & \multicolumn{3}{|c|}{ Univariate Analysis } & \multicolumn{3}{|c|}{ Multivariate Analysis } \\
\hline & OR & $95 \% \mathrm{CI}$ & P value & OR & $95 \mathrm{CI}$ & P value \\
\hline Serum AFP( $\geq 400 \mathrm{ng} / \mathrm{ml})$ & 0.978 & $0.361-2.649$ & 0.965 & & & \\
\hline ICG-R15 (\%) ( $\geq 15 \%)$ & 1.224 & $0.453-3.303$ & 0.690 & & & \\
\hline Tumor size $(>6.5 \mathrm{~cm})$ & 4.503 & $1.022-19.841$ & 0.047 & 3.733 & $0.851-16.720$ & 0.080 \\
\hline Growth type (Ig) & 1.493 & $0.424-5.250$ & 0.532 & & & \\
\hline Micro-Vascular Invasion & 0.753 & $0.097-5.833$ & 0.786 & & & \\
\hline Multiple Tumor & 1.193 & $0.381-3.734$ & 0.762 & & & \\
\hline Liver Cirrhosis & 0.737 & $0.251-2.167$ & 0.579 & & & \\
\hline Resection Margin postitive & 1.927 & $0.703-5.285$ & 0.202 & 5.638 & $1.215-26.161$ & 0.027 \\
\hline Vp4 & 1.246 & $0.401-3.873$ & 0.704 & & & \\
\hline Introhepatic Metastasis & 0.792 & $0.254-2.465$ & 0.687 & & & \\
\hline Postop PVTT recurrence & 2.333 & $0.675-8.061$ & 0.181 & & & \\
\hline PET-SUVmax $(\geq 4.65)$ & 6.717 & $1.419-31.796$ & 0.016 & & & \\
\hline PreopTACE & 1.753 & $0.478-6.433$ & 0.398 & & & \\
\hline
\end{tabular}

AFP: Alpha-Fetoprotein, DFS: Disease Free Survival, HCC: Hepatocellular Carcinoma, ICG-R15, Indocyanine Green Retention Rate at 15 min, Ig: Infiltrate Growth Type, PET-Sm max: Positron Emission Tomography-The Maximum Standard Uptake Value, Preop: Preoperative, Postop: Postoperative, mPVTT: Major Ponal Vein Tumor Thrombosis, TACE: Transcatheter Arterial Chemoembolization, $\mathrm{Vp} 4$ : presence of a tumor thrombus in the main trwik of the portal vein or a ponal vein branch contra lteral to theprimarily im olved lobe (or both).

PVTT. Finally, 26 cases were enrolled in the present study. The maximal tumor standardized uptake value (SUVmax) was evaluated as a predictive factor. The cutoff values of continues variables were decided by receiver operating characteristic (ROC) curve. Univariate and multivariate regression analysis was performed to identify prognostic factors of recurrencefree survival (RFS) and overall survival (OS). Results: The median overall survival time was 16 months in the present study. Most of the operative patients preserve the good liver function (Child-Pugh stage A, 26/27). The 1-, 3-, and 5-year cumulative OS was $55.6 \%, 31.7 \%$, and $31.7 \%$, respectively. The SUVmax more than 4.65 was found to be the only independent risk factor for RFS and OS by multivariate regression analysis. Conclusions: In the present study, the SUVmax was an independent predictor of DFS and OS for the HCC patients with mPVTT. Low SUVmax is an effective factor to select candidates who have a low risk of recurrence and achieve a long-term survival for surgical resection. Keywords: Hepatocellular carcinoma, portal vein tumor thrombus, PET/CT,

\section{PE-004 \\ MicroRNAs Associated with CD44/TGF- $\beta 1$ Regulate the EMT and Migration of Hepatocellular carcinoma}

Na Ri Park', Jung Hoon Cha', Sung Woo Cho', Jung-Hee Kim ${ }^{1}$, Wonhee Hur ${ }^{1}$, Pil Soo Sung ${ }^{1,2}$, Jeong Won Jang ${ }^{1,2}$, Jong Young Choi ${ }^{1,2}$, Seung Kew Yoon ${ }^{1,2}$, Si Hyun Bae ${ }^{1,2}$

${ }^{1}$ The Catholic University Liver Research Center, College of Medicine, The Catholic University of Korea, Seoul, South Korea. ${ }^{2}$ Department of Internal Medicine, College of Medicine, The Catholic University of Korea, Seoul, South Korea

Aims: CD44 have known as important modulators of epithelial-mesenchymal transition (EMT) together with transforming growth factor beta1 (TGF- $\beta 1$ ). Moreover, CD44 and TGF- $\beta 1$ double positive more enhanced cancer stem cell characteristics acquisition, EMT, and metastasis. In cancer, miRNAs functions as tumor suppressors or oncogenes. This study aimed to investigate the identify microRNAs regulating the EMT with CD44/TGF- $\beta 1$ in HCC cells. Methods: We sorted CD44- and CD44+ liver cancer stem cells by fluorescence-activated cell sorting (FACs) in TGF- $\beta 1$-positive SNU-368 cells and TGF- $\beta 1$ negative SNU-354 cells. The miRNA profiles of CD44- and CD44+ HCC cells were analyzed by next-generation sequencing (NGS). To investigate synergy effect of CD44 and TGF- $\beta 1$, we induced EMT by TGF- $\beta 1$ treatment or inhibited EMT by TGF- $\beta 1$ inhibitors. The expression level of mRNA and protein were detected by quantitative real-time PCR (qRT-PCR) and western 
blot. Results: miRNA NGS array data were compared among CD44 or TGF- $\beta 1$ single expression HCC cells and CD44/TGF- $\beta 1$ double positive HCC cells. The results showed that four miRNAs (miR-96-5p, miR-221-5p, miR-186-3p and miR-152-3p) were up-regulated and two miRNAs (miR-296-3p, miR-10a-5p) was down-regulated in CD44+/TGF- $\beta 1+$ cells than an expression of either one alone. These results were confirmed by qRT-PCR. TGF- $\beta 1$-stimulated SNU-354 (CD44+/TGF- $\beta 1+$ ) cells up-regulated three miRNAs (miR-96-5p, miR-221-5p, miR-186-3p) than SNU-354 (CD44+/TGF- $\beta 1-$ ) cells and also, down-regulated the two miRNAs (miR-296-3p, miR-10a-5p). Inhibition of TGF- $\beta 1$ in SNU-368 cells reduced miR-221-5p and increased miR-296-3p. Furthermore, TGF- $\beta 1$-stimulated SNU-354 $(\mathrm{CD} 44+/ \mathrm{TGF}-\beta 1+)$ cells induced EMT and TGF- $\beta 1$ inhibitortreated SNU-368 (CD44+/TGF- $\beta 1$-) cells inhibited EMT. Overexpression of miR-221-5p in SNU-354 (CD44+/TGF- $\beta 1-$ ) cells exhibited lower E-cadherin and higher $\beta$-catenin. Next, the loss of miR-221-5p in SNU-368 (CD44+/TGF- $\beta 1+$ ) cells showed increased E-cadherin. Conclusions: We identified CD44/ TGF- $\beta 1$-related miRNAs and confirmed regulation of EMT by miRNAs in HCC cells. The results would suggest a possible application that CD44/TGF- $\beta 1$-regulated miRNAs may serve as specific biomarkers and therapeutic targets for hepatocellular carcinoma. Keywords: MicroRNA, CD44, TGF- $\beta 1$, HCC.

\section{PE-005 \\ Prognostic Role of 18F-FDG Positron Emission Tomography in Patients with Resectable Hepatocellular Carcinoma}

Felix Che-lok Chow, Tiffany Cho-lam Wong, Tan To Cheung, Albert Chi-yan Chan, Kenneth Siu-ho Chok, Kwok Chai Ng, Wing Chiu Dai, Chung Mau Lo

Division of Hepatobiliary Surgery, Department of Surgery, Queen Mary Hospital, The University of Hong Kong

Aims: 18F-fluorodeoxyglucose positron emission tomography (18F-FDG PET) could predict tumour biology and aggressiveness of hepatocellular carcinoma (HCC). We hypothesized

Table 1. Characteristics and recurrence of PET positive vs. PET negative HCC (for Abstract no PE-005)

\begin{tabular}{|c|c|c|c|}
\hline & PET+ve HCC $(n=83)$ & PET-ve HCC $(n=60)$ & $\mathrm{p}$ value \\
\hline Age, median & $62(19-84)$ & $59(36-83)$ & 0.437 \\
\hline $\operatorname{Sex}(M: F)$ & 67 (80.7\%):16 (19.3\%) & 50 (83.3\%):10 (16.7\%) & 0.690 \\
\hline HBsAg positive (\%) & $67(80.7 \%)$ & $43(71.7 \%)$ & 0.205 \\
\hline n, \% Child-Pugh Grade A & $79(95.2 \%)$ & $55(91.7 \%)$ & 0.614 \\
\hline Bilirubin (umol/L) & $10.0(4-37)$ & $10.0(5-39)$ & 0.948 \\
\hline Creatinine (umol/l) & $85.0(50-131)$ & $80.0(41-131)$ & 0.181 \\
\hline $\operatorname{Albumin}(\mathrm{g} / \mathrm{L})$ & $42.0(20-51)$ & $41.0(32-48)$ & 0.063 \\
\hline IHR & $1.1(0.9-1.2)$ & $1.1(09-1.3)$ & 0.229 \\
\hline $\operatorname{AFP}(\mathrm{ng} / \mathrm{ml})$ & $24.0(1-8,703)$ & $71.0\left(2->1 \times 10^{6}\right)$ & 0.020 \\
\hline ICG $(\%)$ at $15 \mathrm{mins}$ & $9.95(4.2-57.1)$ & $10.4(5.3-24.1)$ & 0.895 \\
\hline Major vs. minor resection & $\begin{array}{l}32 \text { (38.6\%): } \\
51(61.4 \%)\end{array}$ & $\begin{array}{l}46 \text { (76.7\%): } \\
14(23.3 \%)\end{array}$ & $<0.001$ \\
\hline Operative time (min) & $277(76-753)$ & $398(120-1666)$ & 0.002 \\
\hline Hospital stay (days) & $6.0(3-33)$ & $9.0(3-64)$ & 0.004 \\
\hline Major complications (\%) & $10(12.0 \%)$ & $17(28.3 \%)$ & 0.014 \\
\hline Tumour size $(\mathrm{cm})$ & $3.0(1.0-18.0)$ & $9.0(1.9-20.0)$ & $<0.001$ \\
\hline n, \% Solitary tumours & $64(77.1 \%)$ & $36(60 \%)$ & 0.03 \\
\hline Micravascular invasion & $29(35.4 \%)$ & $43(71.7 \%)$ & 0.008 \\
\hline Invasion to adjacent organs & $0(0 \%)$ & $4(6.7 \%)$ & 0.061 \\
\hline Bilobar disease & $9(10.8 \%)$ & $16(26.7 \%)$ & 0.014 \\
\hline Well differentiated & $14(17.3 \%)$ & $0(0 \%)$ & \\
\hline Moderately differentiated & $61(75.3 \%)$ & $37(61.7 \%)$ & \\
\hline Poorly differentiated & $6(7.4 \%)$ & $23(38.3 \%)$ & $<0.001$ \\
\hline \multirow[t]{2}{*}{ Non-cirrhotic vs. cirrhotic liver } & 29 (34.9\%): & $24(40.0 \%):$ & \\
\hline & $54(65.1 \%)$ & $36(60.0 \%)$ & 0.696 \\
\hline Time to recurrence (months) & $31.7(0.9-135.5)$ & $8.6(1.2-163.2)$ & $<0.001$ \\
\hline \multicolumn{4}{|l|}{ Pattern of recurrence } \\
\hline$-\mathrm{No}$ & $54(65.1 \%)$ & $24(40.0 \%)$ & \\
\hline - Intra-hepatic recurrence & $19(22.9 \%)$ & $10(16.7 \%)$ & \\
\hline - Extra-hepatic recurrence & $2(2.4 \%)$ & $5(8.3 \%)$ & $<0.001$ \\
\hline - Both & $8(9.6 \%)$ & $21(35.0 \%)$ & \\
\hline
\end{tabular}


Table 2. Significant predictors of Overall Survival on multivariate analysis (for Abstract no PE-005)

\section{HR $(95 \% \mathrm{Cl}) \quad \mathrm{P}$ value}

T/L ratio $>1.49$ (i.e. PETpositivity) $2.53(1.32-4.84) 0.005$

Serum AFP $>100 \mathrm{ng} / \mathrm{ml}$

$2.22(1.19-4.14) 0.012$

Major vascular invasion

$4.04(1.74-9.36) 0.001$

Bilobar disease

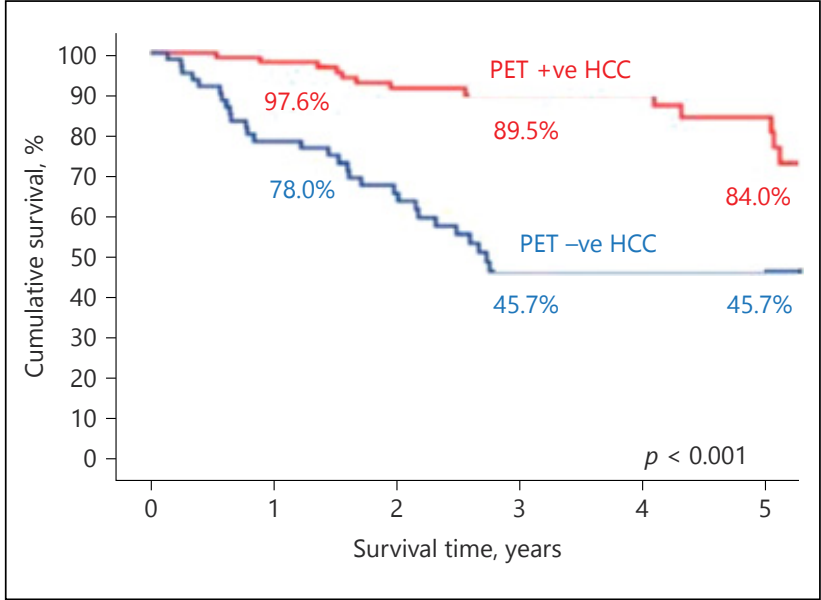

Fig. 1. Overall survival (for Abstract no PE-005).

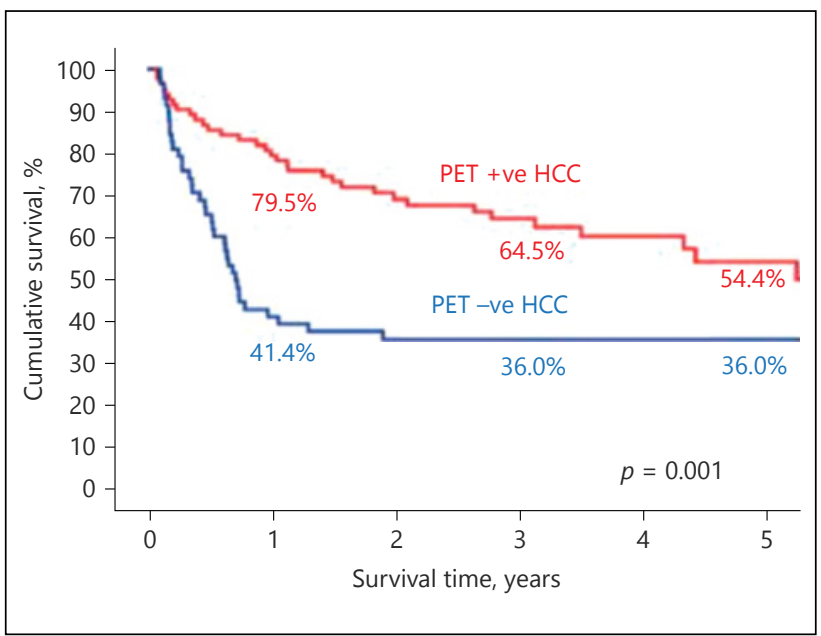

Fig. 2. Disease free survival (for Abstract no PE-005)

that 18F-FDG PET predicted survival of patients with HCC who underwent hepatectomy. Methods: This was a retrospective study at a university center in Hong Kong. Data was retrieved from a prospectively entered database. Maximum standard uptake value (SUVmax) of tumor/ normal liver uptake (T/L ratio) was recorded. The PET positivity for overall survival (OS)

was determined using $\mathrm{T} / \mathrm{L}$ ratio by c-statistics using receiver operating characteristic curve. Multivariate analyses with preoperative, pathological variables and PET positivity were performed to determine the prognostic significance for post hepatectomy OS and recurrence. Results: From 2004 to 2016, there were 143 patients who had 18F-FDG PET before hepatectomy. The 5-year OS and disease-free survival (DFS) were $67.8 \%$ and $47.2 \%$ respectively. The median follow-up was 35 months. Table 1 showed the baseline demographics, operative and pathological characteristics of all patients. Using a T/L ratio cutoff at 1.49, it was found to be an independent predictor of OS [hazard ratio (HR) 2.53, (1.32-4.84), $\mathrm{p}=0.005$ ] (Table2) and was associated with a better OS (Figure1). PET positive HCC were more likely to recur ( 60 vs. $34.9 \%$, p < 0.001) and had a shorter DFS (Figure 2). Conclusions: 18F-FDG PET positivity using $\mathrm{T} / \mathrm{L}$ ratio was an independent factor for OS in HCC patients who underwent hepatectomy. 18F-FDG PET could be explored as a potential radiological biomarker for HCC aggressiveness. Keywords: Positron Emission Tomography, 18F-Fluorodeoxyglucose, Hepatocellular carcinoma, Prognosis, Hepatectomy.

\section{PE-006 \\ Development of Mouse Models for Hepatocellular Carcinoma and Intrahepatic Cholangiocarcinoma Induced by Activated TAZ}

\author{
Hyuk Moon 1,2, Kyungjoo Cho, ${ }^{1,2}$, Do Young Kim²,3, \\ Simon W. Ro ${ }^{1,2}$, Kwang-Hyub Han ${ }^{1-3}$ \\ ${ }^{1}$ Brain Korea 21 PLUS project for Medical Science \\ College of medicine, ${ }^{2}$ Yonsei Liver Center, Severance \\ Hospital, ${ }^{3}$ Department of Internal Medicine, Institute \\ of Gastroenterology, Yonsei University College of \\ Medicine, Seoul, South Korea
}

Aims: Hepatocellular carcinoma (HCC) is the second most common cause of cancer-related deaths worldwide. The Hippo signaling pathway is tumor suppressive, as its inactivation leads to tissue overgrowth and tumor formation via YAP- or TAZ-mediated transcriptional activation. YAP is overexpressed in $62 \%$ of patients with HCC and in almost $90 \%$ of human cholangiocarcinoma (CC). High YAP/TAZ levels correlate with poor prognosis for both types of liver cancer. In this study, we have developed transgenic mouse models for HCC and CC induced by an activated form of TAZ (TAZ $\left.{ }^{\mathrm{S} 89 \mathrm{~A}}\right)$ combined with an activated form of RAS or PI3K, respectively. Methods: Transposons were constructed encoding $\mathrm{TAZ}^{\mathrm{S} 89 \mathrm{~A}}$, an activated from of human H-RAS (HRAS ${ }^{\mathrm{G} 12 \mathrm{~V}}$ ), and an activated form of human $\mathrm{PI} 3 \mathrm{~K} \quad\left(\mathrm{PI}_{3} \mathrm{~K}^{\mathrm{E} 545 \mathrm{~K}}\right)$. Transposons were hydrodynamically delivered to livers of 6 -week-old C57BL/6 mice. Mice were monitored at least twice per week and sacrificed when moribund. Tumor-bearing livers were formalin fixed for hematoxylin-eosin staining and immunohistochemistry. Results: HCCs were induced by the stable expression of $\mathrm{TAZ}^{\mathrm{S} 89 \mathrm{~A}}$ and 
HRAS $^{\mathrm{G} 12 \mathrm{~V}}$, while CCs developed in livers expressing $\mathrm{TAZ}^{\mathrm{S} 89 \mathrm{~A}}$ and $\mathrm{PI}^{\mathrm{E}} \mathrm{E}^{\mathrm{E} 45 \mathrm{~K}}$. Nuclear accumulation of TAZ was confirmed by immunohistochemistry in both types of tumors. There were lung metastases found in $20 \%$ mice bearing HCCs induced by $\mathrm{TAZ}^{\mathrm{S} 89 \mathrm{~A}}$ and HRAS ${ }^{\mathrm{G} 12 \mathrm{~V}}$. Conclusions: TAZ can induce tumorigenesis toward either HCC or CC depending on collaborating oncogene. HCCs induced by $\mathrm{TAZ}^{\mathrm{S} 89 \mathrm{~A}}$ and HRAS $\mathrm{H}^{\mathrm{G} 12 \mathrm{~V}}$ were highly invasive and led to metastasis to the lung in $20 \%$ of mice. Keywords: Liver cancer, TAZ, Mouse model.

\section{PE-007 \\ NR4A2 Facilitate Immune Escape of Intrahepatic Cholangiocarcinoma through Upregulation of CD47 and IDO1}

Chu-Yu Jing, Yi-Peng Fu, Bo-Heng Zhang, Shuang-Jian Qiu

Liver Cancer Institute, Zhongshan Hospital, Fudan

University 180 Fenglin Road, Shanghai, P.R. China

Aims: To investigate the roles of NR4A2 in facilitating immune escape of ICC NR4A2 in facilitating immune escape of ICC. Methods: IHC was performed to study the infiltration of
T cells, cytotoxic T cells (CTLs) and regulatory T cells (Tregs) and the expression pattern of CD47 and ID01 The altered immune-related genes were detected through qRT-PCR and verified by western blot. ChIP assay was used to validate transacivate function of NR4A2 towards the promoter of CD47 and ID01. DNA-binding-deficient NR4A2 overexpression plasmids was used to study the regulatory pattern of NR4A2 towards the target genes. Results: In ICC tissues, overexpression of NR4A2 was significantly correlated with less CTL counts and higher Tregs/CTLs ratio within tumor. In NR4A2 upregulated ICC cells, the expression levels of CD47 and ID01 were significantly upregulated. ChIP assays validated that NR4A2 can bind to the promoter regions of CD47 and ID01. DNA-binding-deficient NR4A2 still promoted the expression of ID01 whereas it failed to upregulate CD47. Moreover, in ICC tissues, NR4A2 overexpression was significantly correlated with CD47 and ID01 expression. Conclusions: NR4A2 facilitates the immune suppression of ICC through upregulating CD47 and ID01. Keywords: Intrahepatic cholangiocarcinoma, ID01, CD47, NR4A2.

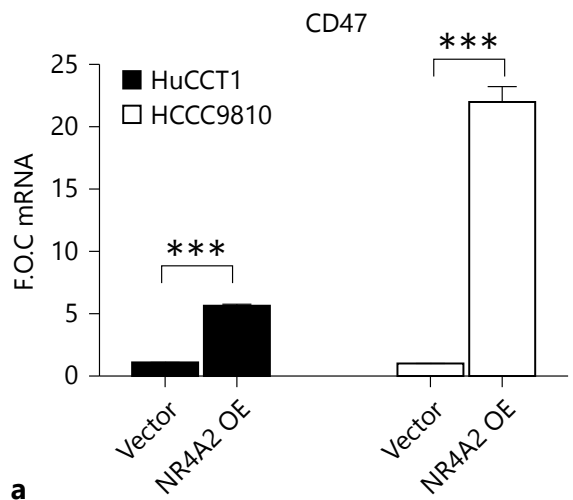

IDO1

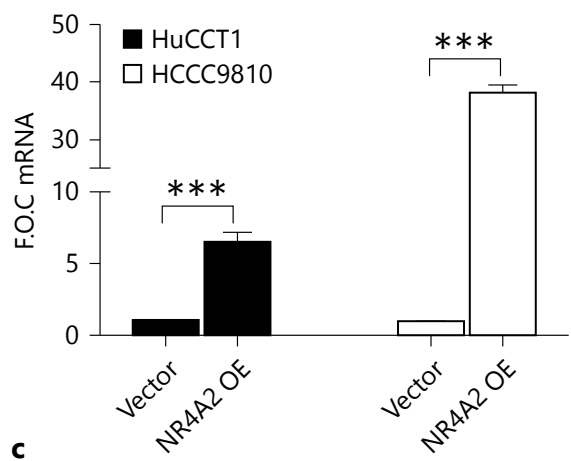

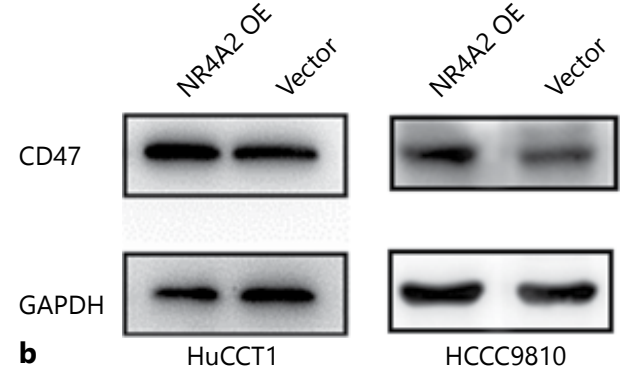

b

HCCC9810

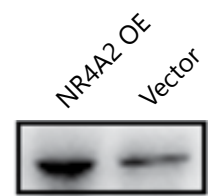

GAPDH

d
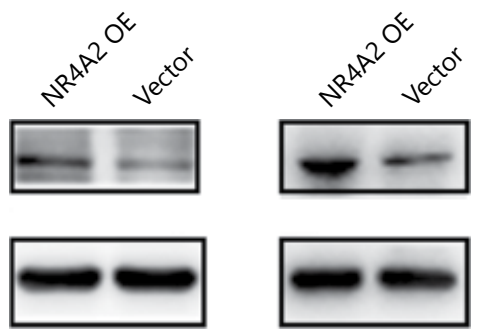

HCCC9810

Fig. 1. (for Abstract no PE-007). 


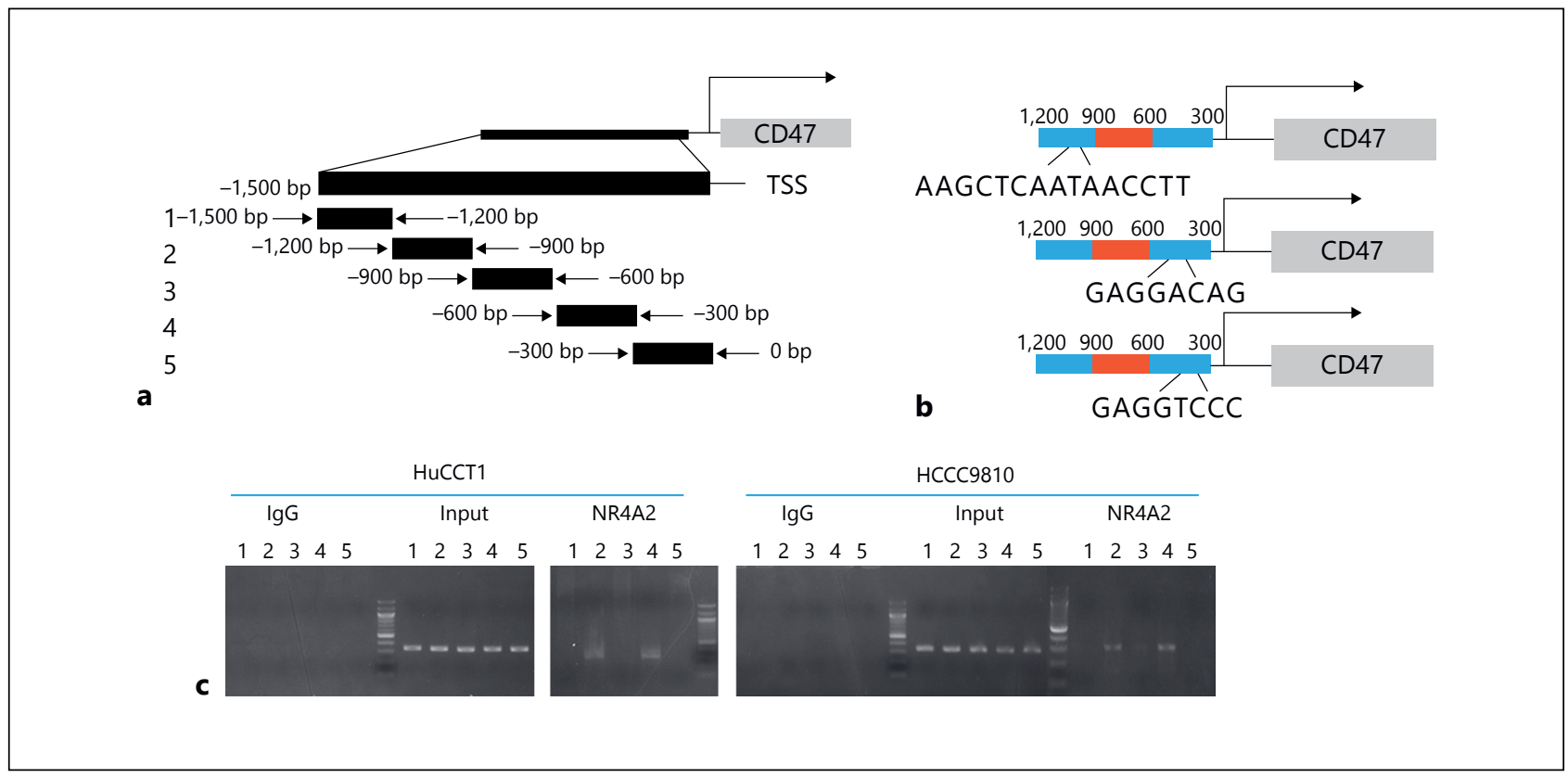

Fig. 2. (for Abstract no PE-007).

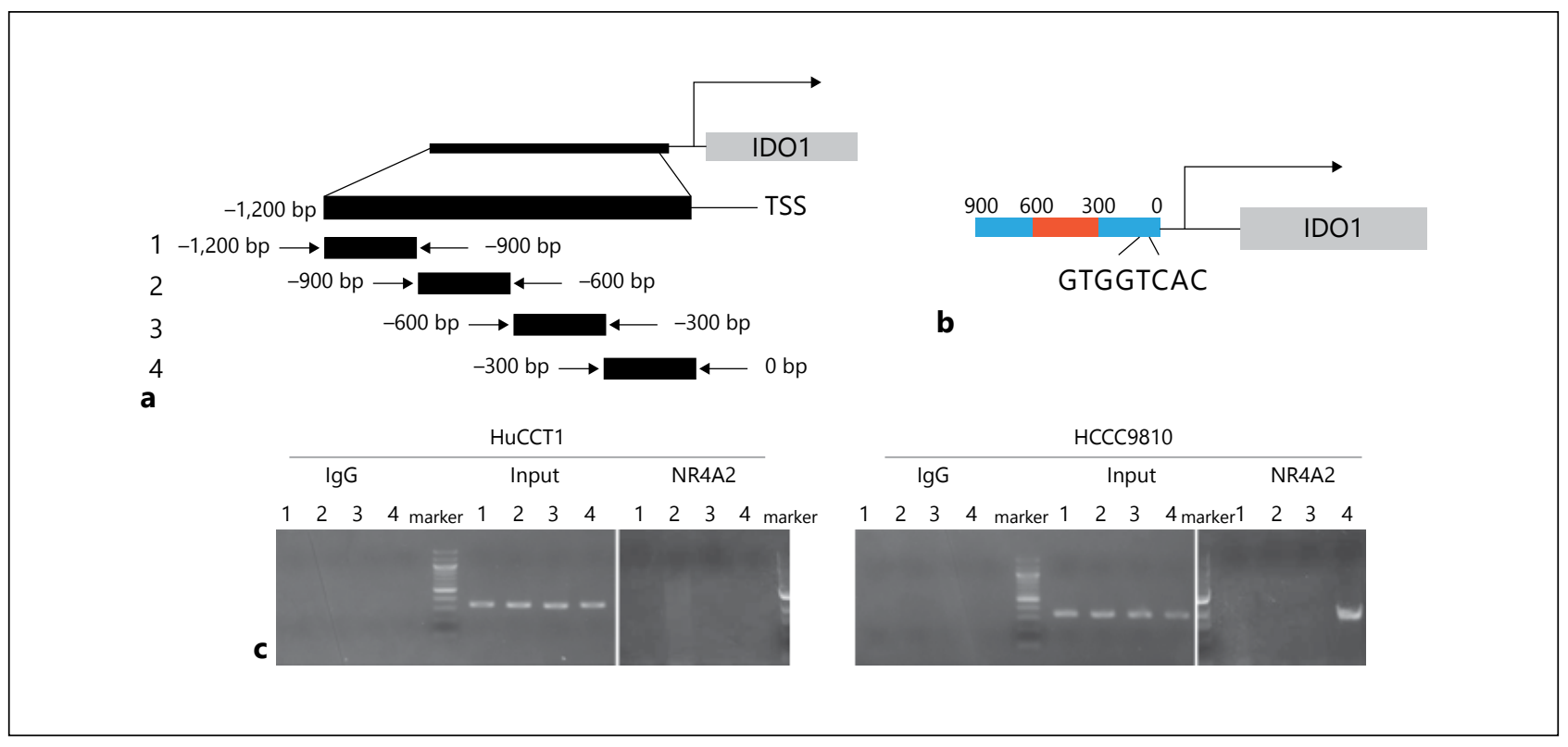

Fig. 3. (for Abstract no PE-007). 


\section{PE-008 \\ Proteomic Approaches to Investigate the Regulation of Human Telomerase Reverse Transcriptase in Hepatocellular Carcinoma Cells}

Sung Hoon Choi ${ }^{1,2}$, Bora Jin ${ }^{2,3}$, Sung Ho Yoon ${ }^{4}$, Hye Won Lee ${ }^{1,2}$, Do Young Kim ${ }^{1,2}$, Kwang-Hyub Han ${ }^{1,2,3}$, Jun Yong Park ${ }^{1,2}$

${ }^{1}$ Department of Internal Medicine, Yonsei University College of Medicine ${ }^{2}$ Yonsei Liver Center, Severance Hospital ${ }^{3}$ BK21 plus project for medical science, Yonsei University College of Medicine ${ }^{4}$ Division of Bioconvergence Analysis, Drug \& Disease Target Group, Korea Basic Science Institute (KBSI)

Aims: Human telomere transferase (hTERT) is expressed during early fetal development and then turned off in most adult tissues, but it becomes reactivated in almost all human cancers, such as hepatocellular carcinoma (HCC). However, the exact mechanism regulating these changes in expression remains unknown. We evaluated whether knockdown of hTERT in HCC cell lines inhibits cell cycle and proliferation and analyzed hTERT-regulating proteins using proteomic analyses. Methods: HCC cell lines were transfected with hTERT small interfering RNA (siTERT) and cultured under normal conditions. Following transfection, the expression levels of hTERT, steroidogenic acute regulatory protein (StAR), and human Kruppel-related 3 (HKR3) were further investigated in the proteomic analysis of hTERT through western blotting, and tumor growth was measured by cell proliferation and cell cycle (PI staining) assays. Results: The upregulation of StAR expression and downregulation of HKR3 expression led to increased hTERT expression and telomerase activity. Inhibition of hTERT by siRNA led to the inhibition of tumor growth. Conclusions: Our results suggested that StAR is positively correlated with telomerase activity, whereas HKR3 may be a negative regulator. Our results facilitate further exploration of the pathways regulating human telomerase activity. Keywords: HCC, TERT, HKR3, StAR.

\section{PE-009 \\ B4GALT1 Gene Knockdown Promotes Hepatocellular Carcinoma Malignancy \\ Po-Da Chen ${ }^{1}$, Yao-Ming Wu ${ }^{1}$, Min-Chuan Huang ${ }^{2}$ \\ ${ }^{1}$ Department of Surgery, National Taiwan University Hospital, Taiwan; ${ }^{2}$ Graduate Institute of Anatomy and Cell Biology, National Taiwan University College of Medicine, Taiwan}

Aims: Hepatocellular carcinoma (HCC) is bound with low eradication rate and poor prognosis inconsideration of cancer cell invasion and metastasis. Abnormal Beta-1,4-galactosyltransferase 1 (B4GALT1) expression is associated with tumor progress in many cancers, as it adds galactose to $\mathrm{N}$-acetylglucosamine residues of glycoprotein carbohydrate chains. However, functional roles of B4GALT1 in HCC remain unclear. Methods: The association of the expression of B4GALT1 of 70 clinical HCC samples with standard pathologic features and prognosis were evaluated. Knockdown and overexpression of B4GALT1 were conducted on HCC cell lines, and malignant phenotypes were verified. Further, Flow cytometry was performed to screen lectin affinity of the regulated glycosylation of HCC cells, and individual lectin pull-down was performed for pathway digging. Results: Our data confirmed that the lower expression of B4GALT1 in cancer tissue is correlated to the higher ratio of vascular invasion and worse overall survival. The malignant phenotype of HCC is regulated with functioning B4GALT1, of which the stable knockdown of B4GALT1 promoted cell migration, and invasion, vice versa in regard to the overexpressed B4GALT1 gene. In addition, LEL lection is modified through regulating B4GALT1, and RTK assay suggested the pathway is associated with interfered hepatocyte growth factor receptor (HGFR), Epidermal growth factor receptor (EGFR), and Axl signaling. Conclusions: B4GALT1 gene expression is correlated with clinical prognosis and malignant phenotypes of HCC. Our finding helped unravel the role of glycosylation in HCC pathogenesis and find a potential predicting factor for HCC. Keywords: HCC, Glycosylation, B4GALT1. 


\section{Viral Hepatitis / Cirrhosis and Related Complications}

\author{
PE-010 \\ Lipidogram in Patients with Type 2 Diabetes \\ Mellitus Infected with Hepatitis B and C \\ Viruses \\ S.N. Lukmonov', U.M. Kurbankulov', O.O. Usmonov', \\ M.U. Ismailov'2, U.A. Azzalarov², K.A. Madatov², \\ A.A. Ismatov ${ }^{3}$ \\ ${ }^{1}$ Department of Oncology and Radiology, Tashkent \\ Medical Academy, ${ }^{2}$ Department of Oncology, \\ Tashkent State Pediatric Institute, ${ }^{3}$ Department of \\ Surgery, Republican Clinical Hospital №1, Tashkent, \\ Uzbekistan
}

Aims: To study lipid metabolism in patients with type 2 diabetes mellitus (DM) depending on the infection with hepatotropic viruses (HBV, HCV). Methods: 99 patients with diabetes mellitus (men - $24(24,2 \%)$, average age $(58,0 \pm$ $11,7)$ years) were examined. The standard markers of viral hepatitis B and C (EIA), the main lipidogram indices (total cholesterol, VLDL, LDL, HDL, triglycerides - TG, the coefficient of atherogenity - CA) were studied. Results: Based on the results of testing for markers of viral hepatitis B and C, all patients were divided into two groups: infected with HBV/ HCV (group 1, 32.3\%) and uninfected (group 2, 67.7\%). In a comparative analysis, it was found that in HBV/HCV infected patients with diabetes, the levels of almost all parameters were statistically significantly lower than those of uninfected patients. Thus, the median of total cholesterol in the first group was $5.5 \mathrm{mmol} / \mathrm{l}$, in the second $-6.0 \mathrm{mmol} / \mathrm{L}(\mathrm{p}=0.007)$, median TG content -1.5 and $2.5 \mathrm{mmol} / \mathrm{l}(\mathrm{p}=0.002)$; LDL-2.5 and $3.6 \mathrm{mmol} / \mathrm{l}(\mathrm{p}=0.002)$; VLDL -0.7 and $1.1 \mathrm{mmol} / \mathrm{l}(\mathrm{p}=$ $0.044) ; \mathrm{KA}-2.7$ and $4.2(p=0.018)$. The level of HDL was the same in patients of both groups and was $1.2(1.0-1.4) \mathrm{mmol} / \mathrm{l}$, respectively. Further, patients of the 1st group (HBV/HCVinfected) were divided into two subgroups. The first group included patients with a normal level of aminotransferases, the second - with an increased level of aminotransferases. The same trend was observed: in patients with normal transaminases, the majority of lipidogram indices were lower than in patients with cytolysis, although the differences in most cases did not reach statistical significance. Conclusions: The obtained results testify to the possible role of chronic hepatotropic viral infection in the violation of the functional state of the liver and lipid metabolism in patients with type 2 diabetes mellitus. Keywords: Lipidogram, 2 Diabetes mellitus, Hepatitis B, C viruses.

\section{PE-011 \\ Comparison of Prognosis of Spontaneous Bacterial Peritonitis between in HCC Patients and Non-HCC Patients}

\author{
Jeong Han Kim, Won Hyeok Choe, So Young Kwon, \\ Byung-chul Yoo
}

Department of Internal Medicine, Konkuk University School of Medicine, Seoul, South Korea

Aims: Spontaneous bacterial peritonitis (SBP) is a serious infectious complication in patients with liver cirrhosis. SBP occur frequently also in hepatocellular carcinoma (HCC) patients. However, information about prognosis of SBP in HCC patients is limited. We aimed to review clinical course of SBP in HCC patients. Methods: This study enrolled patients diagnosed with SBP from year 2005 to year 2015. The medical records of patients were reviewed. Clinical course was compared between non-HCC and HCC groups. Results: Total 123 SBP cases including 49 HCC cases were enrolled. Male patients were predominant $(48 / 74,64.9 \%$ vs. $34 / 49,69.4 \%$, $\mathrm{p}=0.697$ ), and mean age was 58.4 years and 58.2 years in non-HCC and HCC groups ( $\mathrm{p}=0.929)$. The most common etiology was alcohol $(32 / 74,43.2 \%)$ in non-HCC group and hepatitis B $(30 / 49,61.2 \%)$ in HCC group ( $p=0.009)$. Platelet count $\left(\mathrm{x} 10^{3} / \mathrm{mm}^{3}\right)$ was lower (73.8 vs. 103.6, $\left.\mathrm{p}=0.005\right)$ and Prothrombin time (INR) was more prolonged (2.1 vs. 1.8, $\mathrm{p}=$ 0.041 ) in non-HCC group than HCC group. Antibiotics resistance rate was higher in non-HCC group than HCC group $(29.7 \%$ vs. $12.2 \%, p=0.028)$ In hospital mortality rate was not different between two groups $(25 / 74,33.8 \%$ vs. $13 / 49$, $26.5 \%, \mathrm{p}=0.431$ ), but recurrence rate was lower in HCC group $(29 / 74,39.2 \%$ vs. $10 / 49,20.4 \%, p=0.031)$. Development rate of hepatorenal syndrome was not different $(25 / 74,33.8 \%$ vs. $13 / 49,26.5 \% . p=0.431$ ), hepatic encephalopathy was less common in HCC group $(26 / 74,35.2 \%$ vs. $9 / 49,18.3 \%$, $p=$ 0.008). Conclusions: Prognosis of SBP in HCC patients seems relatively less severe. This result may be related with antibiotics resistance rate and development rate of other complication such as hepatic encephalopathy. This may be also related with presence of regular follow up management. Prospective investigation is required about this point of view. 


\section{PE-012 \\ Possibilities of Ultrasound Method in Diagnosis of Cirrhosis in People Who Work with Polyamides for a Long Time}

S.N. Lukmonov', U.M. Kurbankulov', O.O. Usmonov', M.U. Ismailov'2, U.A. Azzalarov², K.A. Madatov²,

A.A. Ismatov ${ }^{3}$

${ }^{1}$ Department of Oncology and Radiology, Tashkent Medical Academy, ${ }^{2}$ Department of Oncology, Tashkent State Pediatric Institute, ${ }^{3}$ Department of Surgery, Republican Clinical Hospital №1, Tashkent, Uzbekistan

Aims: The aim of the study was to determine the value of the ultrasound of the method in the diagnosis of liver cirrhosis (LC) in persons who work with polyamides for a long time. Methods: During periodic medical examinations with ultrasound, the liver status was studied in 412 people, working with polyamides for 2-12 years, for the detection of early signs of liver cirrhosis (LC). According to the indications, in addition to the two-dimensional scanning, ultrasound dopplerography), CT, MRI, liver scintigraphy. The obtained data were compared with the results of liver biopsy, operative and sectional observations. The study was conducted according to a single program. Results: Symptoms of LC by ultrasound data were increased (53\%) or decreased (13\%) in liver size. Since false positive results are observed with fatty liver dystrophy, and false negative - in the initial stage of the disease, it is possible to reliably distinguish LC from other chronic liver diseases only in dynamics with a retrospective analysis of the obtained data of radiation studies. With the development of LC, an increase in liver size was observed in $56 \%$ of cases, a decrease in liver size was $14 \%$, and in $30 \%$ of patients the liver size remained within normal limits. In the evaluation of fibrous tissue with ultrasound, sensitivity in the definition of mild fibrosis was $41 \%$. Carrying out the UD made it possible to judge the state of the vascular bed, the functional state of the blood flow. However, this analysis is objective only when dynamically observing the diameter of the portal vein and the linear velocity of blood flow in it in the same patient, from the time of admission to work and to the detection of signs of the LC. Conclusions: Detection of early ultrasound signs of liver LC in people who work with polyamides for a long time is possible only if they are monitored dynamically during the passage of medical examinations. Keywords: Polyamides, Liver cirrhosis, Ultrasound, Ultrasound dopplerography.

\section{PE-013 \\ Utility of Shear Wave Elastography (SWE) in Assessment of Hepatic Fibrosis Improvement after Direct-Acting Antiviral Arugs (DAAs) \\ Mohamed Kohla, Mohamed Akl Rady, \\ Mohamed El-Fayoumi, Mervat Abdel-Kareem, Mahmoud EL-Sakhawy}

National Liver Institute, Menoufia University, Egypt

Aims: Assessment of liver fibrosis by SWE before and after treatment with direct antiviral drugs in chronic HCV infected patients. Methods: In this prospective study, we enrolled 161 patients with chronic hepatitis $C$ recruited from the outpatient clinic at National Liver Institute (NLI), Menoufia University who received DAAs. All patients' demographics, laboratory characteristics, and abdominal ultrasound were evaluated at baseline and the end of treatment (at 12 weeks). Liver stiffness measurements by SWE were performed before and after treatment. Results: Serum alanine aminotransferase levels (ALT) were significantly lower at the end of treatment $(18 \pm 4.3 \mathrm{IU} / \mathrm{L})$ compared to those at base line $(46.23 \pm 30.92$ IU/L) with a P-value <0.001. Similarly, Serum aspartate aminotransferase levels (AST) were significantly lower at the EOT $(19.7 \pm 4.9 \mathrm{IU} / \mathrm{L})$ compared to those baseline $(45.8 \pm 30.3$ IU/L) with a P-value $<0.001$. Liver stiffness value showed marked improvement at the end of treatment from the baseline $(7.43 \pm 4 \mathrm{KPas})$ compared to the EOT $(6.25 \pm 3.6$ KPas) with a P-value $<0.001$. Advanced fibrosis (F3\&F4) was detected in 35 out of 161 patients at baseline (21\%) while that at the end of treatment was detected in 20 out of 161 patients (12\%), showing an improvement in liver fibrosis after DAAs assessed by Shear Wave Elastography Conclusions: These results showed early improvement of liver stiffness scores after administration of DAAs. SWE is an easy and reliable method for assessment of fibrosis improvement after DAAs. Keywords: Shear wave elastography, Liver fibrosis, Viral hepatitis, DAAs. 


\section{PE-014 \\ The Incidence of Portal Hypertensive Gastropathy in Patients with Liver Cirrhosis}

S.N. Lukmonov', U.M. Kurbankulov', O.O. Usmonov', M.U. Ismailov², U.A. Azzalarov², K.A. Madatov², A.A. Ismatov ${ }^{3}$

${ }^{1}$ Department of Oncology and Radiology, Tashkent Medical Academy, ${ }^{2}$ Department of Oncology, Tashkent State Pediatric Institute, ${ }^{3}$ Department of Surgery, Republican Clinical Hospital №1, Tashkent, Uzbekistan

Aims: The aim of the study was to evaluate the incidence and risk factors for portal hypertensive gastropathy (PHG) in patients with cirrhosis of the liver in the city of Tashkent. Methods: A total of 251 patients with LC with different etiology were examined. The patients were divided into 2 groups, depending on the presence of the PHG. The first group consisted of 56 patients with LC who had PHG (men - 46.2\%, mean age ( $47.9 \pm 11.3$ ) years); the second -195 patients without signs of PHG (men 48.7\%, mean age (48.9 \pm 12.7 ) years). The groups did not differ in terms of sex, age, etiology of LC, degree of compensation and severity of portal hypertension (PH). Results: The incidence of PHG in patients with LC in this study was $22.3 \%$. In patients who underwent bleeding from varicose-dilated esophagus veins (VDEV), the incidence of PHG was 2 times lower than in those who did not have a history of bleeding, but the differences did not reach statistical significance $(11.1 \%$ vs. $23.2 \%, \chi 2=1.4, p=0.24)$. The development of PHG did not depend on sex, age, the presence of hepatic-cell insufficiency, the degree of expansion of the VDEV, the presence of ascites, splenomegaly, diameter v.portae. In the case of class C CPC, Childe-Pugh PHG was twice as frequent as in class C CIs (31.3\% vs. $15.7 \%, \chi 2=6.85, \mathrm{p}=$ 0.009 ). The incidence of $H$. pylori gastric contamination in patients with PHG was $36.5 \%$ and was comparable to the incidence of contamination in patients with $\mathrm{CP}$ without PHG $(26.7 \%, \chi 2=1.62, p=0.21)$. At the same time, in patients with LC, infected with $\mathrm{H}$. pylori, PHG was almost 2 times less common than in uninfected $(16.3 \%$ vs. $30.0 \%, \chi 2=6.68, p=$ $0.01)$. Conclusions: The frequency of development of PHG in patients with LC in this study was $22.3 \%$. The development of the PHG did not depend on the etiology of LC, the severity of PHG, and the history of bleeding from the VDEV. In the case of grade $\mathrm{B}$ PHG, it was 2 times more common than with $\mathrm{C}$ class $\mathrm{C}$ class. In the N. pylori infected, the PHG was 2 times less frequent than in the uninfected, which confirms the existing point of view about the non-involvement of $\mathrm{H}$. pylori in the pathogenesis of the PHG. Keywords: Portal hypertensive gastropathy, Portal hypertension, H. pylori, Varicose-dilated esophagus veins.

\section{PE-015 \\ Comparison of Clinical Outcomes in Chronic Hepatitis B between Patients with Untreated Immune-Tolerant Phase vs. Those with Virological Response by Antiviral Therapy}

Hye Won Lee ${ }^{1-3}$, Seung Up Kim ${ }^{1-3}$, Jun Yong Park ${ }^{1-3}$, Do Young Kim ${ }^{1-3}$, Sang Hoon Ahn ${ }^{1-3}$, Kwang-Hyub Han ${ }^{1-3}$, Beom Kyung Kim ${ }^{1-3}$

${ }^{1}$ Department of Internal Medicine, ${ }^{2}$ Institute of Gastroenterology, Yonsei University College of medicine, ${ }^{3}$ Yonsei Liver Center, Severance Hospital, Seoul, Republic of Korea

Aims: Currently, routine nucleos(t)ide analogs (NUCs) therapy has not yet been recommended for patients with immune-tolerant (IT) phase in chronic hepatitis B virus (HBV) infection. We aimed to compare prognosis of patients in untreated IT-phase (UIT group), compared to those in immune-active phase who achieved virological response (VR; serum HBV-DNA $<2000 \mathrm{IU} / \mathrm{mL}$ ) by NUCs according to guidelines (VR group). Methods: Consecutive patients with chronic HBV infection between 2006 and 2012 were considered eligible. Those with cirrhosis or hepatocellular carcinoma (HCC) at enrollment were excluded. Cumulative risks of HCC and liver-related events (LREs) development were assessed using Kaplan-Meier method. Furthermore, to adjust for imbalances between two groups, propensity-score (PS) matching with 1:1 ratio was performed based upon 4 variables; age, gender, presence of diabetes, liver stiffness (LS) by transient elastography. Results: UIT group $(n=126)$ showed younger age, lower proportion of male gender and lower LS than VR group ( $\mathrm{n}=641$ ). UIT group had similar 10-year cumulative risks of HCC (2.7\% vs. $1.9 \%$, respectively; $p=0.704)$ and LRE ( $4.6 \%$ vs. $6.1 \%$, respectively; $p=0.903)$ development, compared to VR group. When we re-defined UIT group by the lower serum alanine aminotransferase levels (males, < $30 \mathrm{IU} /$ $\mathrm{mL}$; females, < $19 \mathrm{IU} / \mathrm{mL}$ ), 10-year cumulative risks of HCC and LRE development were $2.9 \%$ and $4.8 \%$, respectively, with no significant differences, compared to VR group. Using PS matching, similar results were also reproduced (both $\mathrm{p}>$ 0.05). Conclusions: UIT group consistently showed similar prognosis compared to VR group. Therefore, in general, earlier antiviral therapy should be reserved only for selected IT-phase patients. Keywords: Hepatitis B, Immune tolerant, Virolgoical response, Hepatocellular carcinoma. 


\section{PE-016 \\ Treatment of Patients with Bleeding in Portal Hypertension due to Liver Cirrhosis}

Sh.A. Eshmurodov, S.N. Lukmonov, M.M. Salakhidinov

Department of Faculty Surgery, Tashkent Medical

Academy

Aims: Treatment of patients with bleeding from varicose veins with portal hypertension due to liver cirrhosis is an actual and complex task. Therefore, the introduction of new methods of stopping bleeding against the background of hepatotropic therapy is relevant for liver cirrhosis. Methods: The treatment of 280 patients with bleeding from varicose veins of the esophagus has been analyzed. The age of the patients varied from 26 to 79 years, men were 68\%, women $32 \%$. Patients with cirrhosis of stage A in the Child-Pugh had 40 (14.3\%), stage B - 140 (50.0\%), stage C - 100 (35.7\%). Severe bleeding was observed in $76(27.1 \%)$ patients, mean - in 141 (50.4\%), and in mild - in 63 (22.5\%). Out of 280 Endoscopic hemostasis was performed in 196 (70.0\%) patients. Endoscopic sclerotherapy was performed in 108 patients, endoscopic ligation in 68, endoscopic filling with fibrin glue in 20. U 107 (38.2) patients underwent endovascular embolization of the splenic artery in various variants of blood flow reduction. In $42(15.0 \%)$ patients performed laparoscopic devascularization of the proximal part of the stomach and abdominal part of the esophagus, consisting in flushing the vessels of the abdominal part of the esophagus, crossing and coagulation of the short vessels of the stomach, clipping of the left jaw In 15 (5.4\%) cases after bleeding stopped, we performed laparoscopic implantation of plateletrich plasma (IPP) in liver sites with the most pronounced cirrhosis. This technique was tested in 87 rats and showed high efficiency in reverse development of the liver fibrosis process. Results: Analysis of the data obtained showed that the use of endoscopic local hemostasis in 196 patients allowed to stop bleeding in $81 \%$. Lethality due to ineffectiveness of local hemostasis was $19 \%$. The most effective method of stopping bleeding was endoscopic ligation of the vesico-dilated esophagus veins. Over the course of three years, 143 patients were observed who had only endoscopic hemostasis and conservative therapy. The recurrence of bleeding in this group was in 63 patients (44\%), of which 21 (33\%) died. Over the course of three years, 26 of 48 patients who underwent laparoscopic ligation of the esophagus and cardiac stomach, had no recurrence of bleeding. One patient died from liver failure. X-ray endovascular embolization was performed in 67 patients. In this group, 5 patients died because of recurrence of bleeding. In 15 patients who underwent IPP implantation in liver sites with the most severe cirrhosis, there was no long-term mortality. Stabilization and improvement of functional liver samples were noted. Conclusions: 1. Endoscopic local hemostasis effectively stops bleeding from varicose veins of the esophagus in patients with cirrhosis. X-ray endovascular embolization of the splenic vein, laparoscopic devascularization of the abdominal part of the esophagus and ligation of the left ventricular artery and vein, and short veins of the stomach can reliably reduce the risk of bleeding recurrence and mortality in the long-term period. 3. The use of IPP in patients with cirrhosis of the liver can improve functional tests of the liver, which indicates a slowing down of the process of liver fibrosis. Keywords: Portal hypertension, Hepatotropic therapy, Liver cirrhosis, Endoscopic sclerotherapy.

\section{PE-017 \\ Features of Clinical Picture, Diagnosis and Treatment of Acute Cholecystitis in Patients with Acute and Chronic Viral Hepatitis}

\author{
Javohir Toshnazarov, S.N. Lukmonov, K.A. Madatov, \\ U.A. Allazarov
}

Faculty surgery department, Tashkent Medical Academy

Aims: The aim of the study was to study the frequency and structure of acute cholecystitis in patients with acute and chronic viral hepatitis (CVH). Methods: The data of 31 medical records of patients operated on for OX in the infectious surgical department on the basis of faculty surgery of the Tashkent Medical Academy in the period from 2006 to 2017 were analyzed. In all patients, AC developed against the background of the current SH. Among the hospitalized sick men there were $58 \%$ (18 people), women $-42 \%$ (13 people). The average age was 45 years. Results: When analyzing the data of the disease, it was established that all patients were hospitalized with suspicion of VH. On examination, acute viral hepatitis A was detected in 2 (6.5\%) patients, exacerbation of $\mathrm{CVH}$ - in 29 (93.5\%) people. Among patients with chronic hepatitis, CVH was diagnosed in 11 (35.5\%) patients, CVH C - in 9 (29\%), combination of CVH B and C in 5 (16.1\%), unverified hepatitis in $4(20 \%)$ patients. At the stage of the admission department, AC was diagnosed in 15 (48.3\%) patients. In 16 (51.7\%) patients the diagnosis of AC was established in the period from 1 to 7 days from the moment of hospitalization. The leading symptoms in most patients were nausea and multiple vomiting (16 (51.6\%) patients), jaundice (11 (35.5\%) patients), marked weakness and decreased appetite (24 (77.4\%) patients). Pain abdominal syndrome in varying degrees of severity was found in all cases - 31 (100\%). All patients received moderate and severe conditions, which was probably due to endogenous intoxication against the background of the current infectious disease. All patients were operated on: in emergency - 20 (64.5\%), in delayed - 11 (35.5\%) patients. Attempt of endovideosurgical intervention was undertaken in 12 (38.7\%) patients. However, local peritonitis in $6(19.3 \%)$ cases and cirrhosis in $3(9.6 \%)$ patients required access conversion. In 10 (32.3\%) patients during operative intervention, increased bleeding of the tissues was 
observed, caused by the phenomena of portal hypertension and hypocoagulation against a background of hepatic insufficiency. The presence of biliary hypertension required drainage of the bile ducts in 7 (22.6\%) patients. In 8 (25.8\%) patients, the operation was completed by plugging the bed of the gallbladder. In the early postoperative period complications developed in 2 patients: bleeding from the bed of the gallbladder and bile flow with the development of biliary peritonitis. Conclusions: 1. Diagnosis of AC on the background of $\mathrm{VH}$ is difficult due to the fact that the clinical picture is masked by a background disease. In connection with the development of the syndrome of mutual fatigue, a differentiated approach is required when choosing the method of surgical intervention in this category of patients. Keywords: Chronic viral hepatitis, Acute cholecystitis, Bile flow, Biliary peritonitis.

\section{PE-018 \\ Treatment of Esophageal-Gastric Bleeding in Patients with Portal Hypertension Syndrome in Liver Cirrhosis}

\author{
Sh.A. Eshmurodov', S.N. Lukmonov', M.M. Salakhidinov', \\ O.O. Usmonov ${ }^{1}$, A.A. Ismatov ${ }^{1}$, M.U. Ismailov ${ }^{2}$, \\ S.N. Petrosov ${ }^{2}$ \\ ${ }^{1}$ Department of Faculty Surgery, Tashkent Medical \\ Academy, ${ }^{2}$ Department of Surgery, Tashkent State \\ Pediatric Institute
}

Aims: The aim of the study was to improve the results of treatment of patients with bleeding from varicose veins (BVV) of the esophagus and stomach. Methods: Over the past 12 years we have experience in treating 110 patients with esophageal and gastric bleeding from the esophagus and cardiac stomach. The age of the patients ranged from 19 to 79 years. The men were 68 (61.8\%), women - 42 (38.2\%). The cause of cirrhosis of the liver in $90 \%$ of patients was viral hepatitis. Clinical laboratory and instrumental methods of investigation, including endoscopic, ultrasound (ultrasound) and magnetic resonance imaging (MRI), have been performed to diagnose esophageal-gastric bleeding in patients with cirrhosis. Results: According to the methods of treatment, patients were divided into two groups: treatment was started with conservative hemostatic therapy with the use of drugs that reduce portal pressure (ednit, nitroglycerin, octreotide); To stop bleeding from the BVV of the esophageal-gastric zone, the Sengstacken-Blackmore probe was used in combination with hemostatic therapy. The results of treatment showed the effectiveness of conservative hemostatic therapy in combination with drugs that reduce portal pressure in $37.3 \%$ of patients. A lethal outcome occurred in $10 \%$ of cases. After using the Sengstacken-Blackmore probe in combination with conservative methods, a lethal outcome was noted in $7.3 \%$ of observations. It should be noted that against a back- ground of esophageal-gastric bleeding, a mixed form of hypoxia is observed due to disruption of transport and utilization of oxygen by tissues, and therefore studied the state of homeostasis in the posthemorrhagic period in 27 patients with esophageal-gastric bleeding of various severity. It turned out that the leading factor in the development of disturbance of homeostasis is the posthemorrhagic hypoxia of tissues, especially the liver, due to a decrease in the oxygen tension in the mixed venous blood and in the blood of the portal system. Based on this, in 14 patients, a hypoxic drug (oxygen carrier perfluorane). The positive effect of perfluorane on the gas transport function of blood was detected, a significant increase in oxygen tension was observed not only in the arterial blood, but also in mixed venous and portal blood. The favorable effect of perfluorane on ischemic liver tissue is due to its positive effect on hepatocytes. In recent years, in the treatment of esophageal-gastric bleeding, along with the stitching of bleeding perforated varicose veins and in order to reduce portal pressure, stem vagotomy with piloroplasty at the height of esophageal-gastric bleeding with good immediate results was performed in 7 patients. To the above, it should be added that the haemostatic effect of stem vagotomy is due not to hypotensive effect on portal pressure, but also the suppression of peptic activity of gastric juice, which is one of the leading factors in the development of esophageal-gastric bleeding. Conclusions: Diagnosis of esophageal-gastric bleeding should be complex (ultrasound, endoscopy and MRI). In the complex of conservative therapy of esophageal-gastric bleeding it is expedient to include preparations - carriers of oxygen. A promising method of surgical treatment of esophageal-gastric bleeding is the flushing of the bleeding BVV of the esophagus and cardia in combination with stem vagotomy and pyloroplasty. Keywords: Esophageal-gastric bleeding, Viral hepatitis, Sengstaken-Blackmore probe, Piloroplasty.

\section{PE-019 \\ Insulin Resistance in Patients with Chronic Hepatitis C Virus Infection \\ Safaa Khalaf, Asmaa Naser, Ali Kassem', Elham Hamed ${ }^{2}$, Ahmed Roshdy ${ }^{3}$ \\ Departments of ${ }^{1}$ Tropical Medicine and Gastroenterology, Internal Medicine, ${ }^{2}$ Clinical Pathology and ${ }^{3}$ Pathology, Faculty of Medicine, Sohag University, Egypt}

Aims: In the last few years factors such as insulin resistance (IR) and hepatic steatosis, have been linked to progression of hepatic fibrosis. Patients with chronic liver disease and cirrhosis in particular, are known to be prone to IR. However, chronic hepatitis $\mathrm{C}$ virus (HCV) infection may induce IR, regardless of the presence of liver cirrhosis. Our aims are to study insulin resistance (IR) assessed by HOMA-IR 
as a possible risk factor for disease progression in patients with HCV infection and to evaluate the role of IR in hepatic fibrosis progression. The correlations of HOMA-IR values to laboratory, virological and histopathological parameters of chronic HCV are also examined. Methods: The study included 50 individuals classified into 30 chronic HCV patients diagnosed by PCR within the previous 6 months and 20 healthy controls. The functional and morphological status of the liver were evaluated by ultrasonography and laboratory investigations including liver function tests and by liver biopsy. Fasting blood glucose and fasting insulin levels were measured, and body mass index and insulin resistance were calculated. Patients having HOMA-IR $>2.5$ were labeled as insulin resistant. Results: Chronic hepatitis $C$ patients with IR showed significantly higher mean values of BMI and fasting insulin than those without IR $(\mathrm{P}<0.000)$. Patients with IR were more likely to have steatosis $(p=0.006)$, higher necroinflammatory activity as they had higher scores of HAI ( $p=$ 0.05). No significant differences were found between the two groups regarding hepatic fibrosis. Conclusions: HOMA-IR measurement could represent a novel marker to identify the cirrhotic patients at greater risk for the progression of liver disease. As IR is a potentially modifiable risk factor, these findings may have important prognostic and therapeutic implications. Assessment of IR by HOMA-IR and improving insulin sensitivity are recommended in patients with HCV and related chronic liver disease. Keywords: Hepatic steatosis, Hepatitis C virus infection, Insulin resistance, Liver cirrhosis.

\section{PE-020 \\ Hemodynamic Response to Dopamine and Prognosis of the Development of Refractory Ascites in Patients with Liver Cirrhosis}

\author{
Sh.A. Eshmurodov, S.N. Lukmonov, M.M. Salakhidinov, \\ O.O. Usmonov, A.A. Ismatov \\ Department of Faculty Syrgery, Tashkent Medical \\ Academy
}

Aims: Evaluate the effect of dopamine on systemic hemodynamics, renal function and predicting the course of ascites in patients with LC. Methods: The study included 51 patients with normal serum creatinine level (mean age (54 \pm 3) years, men $90.3 \%$, women $9.7 \%$ ). All the patients were examined: daily urine volume (DUV), urinary sodium excretion (USE), glomerular filtration rate (GFR), 24-hour BP monitoring before and after dopamine administration at a dose of $2-3 \mu \mathrm{g} / \mathrm{kg} / \mathrm{min}$ for 4 hours. An increase in GFR of less than $5 \%$ is a reliable indicator of severe VCG and the development of refractory ascites (an increase in GFR of more than $10-15 \%$ is the norm, GFR less than $6-9 \%$ is the initial sign of DUV). Results: In 25 patients (48.5\%), with the introduction of dopamine, there was a significant increase in DUV ( $p=$
0.03 ), USE ( $p=0.04$ ), GFR (more than $10 \%, p=0.035$ ), mean BP $(p=0.01)$. This group had a low risk of developing refractory ascites $(\mathrm{p}=0.001)$. In 20 patients $(30.9 \%)$ ( 9 patients - class B in Child-Pugh, 8 - class C in Child-Pugh) a significant increase in coma and mean blood pressure was detected, but a slight increase in GFR (less than 6-9\%, p = 0.05 ). In 6 patients (11.6\%) (2 patients - Child-Pugh class B, 4 patients - Child-Pugh class C): GFR $<5 \%$, absence of reliable increase in DUV and USE, which means a high risk of developing refractory ascites in this group. Conclusions: The hemodynamic response to dopamine is an indicator predicting the course of ascites and the risk of complications (refractory ascites, hepatorenal syndrome). Keywords: Dopamine, Glomerular filtration rate, Daily urine volume, Urinary sodium excretion.

\section{PE-021 \\ Management of Hydrothorax in Cirrhotic Patients: Is Surgical Management Safe And Efficacious?}

\author{
Jae Hyun Yoon ${ }^{1}$, Dong Hyun Kim ${ }^{1}$, Jiyun Hong ${ }^{2}$, \\ Chung Hwan Jun ${ }^{1}$, Sung Bum Cho' ${ }^{2}$ Yo Chun Jung ${ }^{3}$, \\ Sung Kyu Choi ${ }^{1}$ \\ ${ }^{1}$ Department of Internal Medicine, Chonnam National \\ University Hospital and Medical School, ${ }^{2}$ Department \\ of Internal Medicine, Hwasun Chonnam National \\ University Hospital and Medical School, ${ }^{3}$ Department \\ of thoracic and cardiovascular surgery, Chonnam \\ National University Hospital and Medical School
}

Aims: Hepatic hydrothorax is a complication of decompensated liver cirrhosis and its management is very difficult and complex. Due to refractoriness of pleural effusion and low reserved liver function which cannot be improved without liver transplantation, there are many limitations managing hydrothorax only with diuretics or thoracentesis. Therefore, we analyzed clinical features and management of hepatic hydrothorax and compared outcomes between different treatment modalities especially surgical management. Methods: From January of 2013 to December of 2017, patients who were diagnosed as hepatic hydrothorax by pleural fluid analysis were enrolled. Patients who has possibility of pleural effusion owing to other etiologies other than liver cirrhosis such as heart failure, acute kidney injury or pneumonia were excluded. Results: 46 patients were enrolled and 33 patients $(71.7 \%)$ were male and mean age was 60.11 . The etiology of liver cirrhosis was alcohol (45.7\%), hepatitis B virus (26.1\%), hepatitis $\mathrm{C}$ virus $(8.7 \%)$ and non-alcoholic fatty liver (4.3\%). 10 patients $(21.7 \%)$ underwent surgery, 2 patients had liver transplantation, 16 patients $(35.6 \%)$ were treated with diuretics and pigtail drainage and 18 patients (40.0\%) were treated with diuretics only. The mean CPT (Child-PughTourette) score was 10.2 and mean MELD (Model for end- 
stage liver disease) score was 18.8. The mean follow-up duration was 310 days and overall mortality was $47.8 \%$. On Cox-regression analysis, CPT score, body weight, body mass index and treatment modalities were related to mortality $(\mathrm{p}<$ $0.05)$. Mean survival duration was 252.3 days on surgical management group, 129.9 days on diuretics and pigtail drainage group, 351 days on liver transplantation group and 512.1 days on diuretics only group. There was no statistically significant difference between surgical management group and pigtail drainage group, but survival duration was significantly higher in diuretics only group compared to surgical management group and pigtail drainage group with statistic significance. Due to small sample size, liver transplantation group comparison was not possible. Among 10 patients who underwent surgery, 6 patients had aggravation of ascites and 2 patients had recurrence of hepatic hydrothorax after operation. Conclusions: Surgical management of hepatic hydrothorax showed safe and high success rate. Also, there was no statistically significant difference in survival duration compared with pigtail drainage group. However aggravation of ascites was frequently observed and careful monitoring is needed to those who undergo surgery of hepatic hydrothorax. Keywords: Hydrothorax, Liver cirrhosis, Surgery, Clinical outcome.

\section{PE-022 \\ Possibilities of Endoscopic Ligation and Sclerotherapy with Bleeding from Esophageal Varices in Liver Cirrhosis}

\author{
J.F. Toshnazarov, S.N. Lukmonov, K.A. Mamatkulov \\ Department of Faculty Surgery, Tashkent Medical \\ Academy
}

Aims: The aim of the study was to study the results of treatment of patients with bleeding from varicose veins of the esophagus and stomach (VVES) with the use of endoscopic ligation. Methods: The results of treatment of 178 patients with bleeding from VVES are analyzed. The age of patients ranged from 17 to 68 years (an average of $42.50 \pm$ 2.34 years). There were 124 men (69.7\%), women - 54 (30.3\%). To assess the state of the esophagus and stomach, the classification of AG was used. Scherzinger (1983). In accordance with it, I degree of varicose veins was found in 54 (30.3\%), II - in 66 (37.1\%), III - in $58(32.6 \%)$ patients. The trunk type of varicose veins was detected in 154 (86.5\%), mixed - in 24 (13.5\%) patients. Localization of varicose veins in the distal third of the esophagus was present in 45 (25.3\%), in the lower and middle third in 81 (45.5\%), veins in the esophagus and cardiac stomach were found in 49 (27.5\%), local interest cardiac part of the stomach took place in 3 $(1.7 \%)$ patients. The presence of "red markers" was noted in 55 (30.9\%), the phenomena of erosive esophagitis were diagnosed in $27(15.2 \%)$ cases. Active blood flow from the defect in the vein wall was found in 59 (33.1\%) patients, signs of bleeding were detected in 119 (66.9\%) patients. Results: Ligation of VVES was performed in 51 (28.7\%) patients (at the height of bleeding in 9 patients and against the background of stopped bleeding to prevent its recurrence in 42 cases). Simultaneously, 4-8 endoligatures were applied to the veins. Endoscopic sclerotherapy was performed by $26(14.6 \%)$ patients: in order to prevent recurrence after primary stop bleeding - 20 (76.9\%) and in the late postoperative period due to the recurrence of varicose veins- 6 (23.1\%). As a sclerosing agent, fibroven was used. Usually, no more than 2 injections were performed during one session, affecting 1-2 venous trunks. 8 (12.9\%) patients had a recurrence of bleeding before discharge from endoscopic hemostasis (in 5 patients after endoscopic ligation, 3 - after endoscopic sclerotherapy). Patients were urgently subjected to surgical intervention (Operation Patricia). In the group of patients who underwent endoscopic sclerotherapy, 2 $(18.2 \%)$ deaths were noted. After endoscopic ligation, 4 (7.8\%) of the patient died. The cause of death in 3 patients was posthemorrhagic anemia, in 3 - progressive liver failure. Conclusions: Endoscopic hemostasis is a highly effective method of stopping and preventing the recurrence of bleeding and VVES, especially in patients with a high degree of operational risk. The use of endoscopic methods of hemostasis allows to reduce the lethality in this severe group of patients. Keywords: Endoscopic ligation, Sclerotherapy, Edoligatures, Operation Patricia

\section{PE-023 \\ Increased Intraoperative Fluid Administration Prolongs ICU Stay after Liver Resections and other HPB Surgeries}

\section{Muhammad Zakria}

Department of Hepatobiliary, Masood Hospital, Lahore, Pakistan

Aims: In the past so much has been written on the amount of fluids administration during hepatobiliary surgery. Massive administration of fluids during surgery has their own complications starting from electrolytes disturbances to disseminated intravascular coagulation. Many things have improved over the years but still complications rate of hepatobiliary surgeries are high even in best perioperative care and patients selection. We do not have sufficient evidences about the effects of massive fluid administrations during the surgery and its effects in the initial few days. Methods: We have record of 45 patients who underwent different hepatobiliary and pancreatic surgeries over a period of 5 years. These patients had benign, malignant diseases. Trauma patients were also part of this group. Perioperative fluid administration, intake output record, duration of ICU stay with any other morbidity were also noted down. We had 
divided these patients into two groups. Group 1 high fluid volume $>9 \mathrm{ml} / \mathrm{kg}, \mathrm{n}=25$ and Group 2 low fluid volume $<9 \mathrm{ml} /$ $\mathrm{kg} \mathrm{n}=20$. In HPB trauma patients' blood loss was replaced with blood. Results: Considering the colloids, Group 1 received more colloids both during and first 12 hours after the surgery $(\mathrm{p}<0.003$ and $\mathrm{p}<0.006)$ respectively). Bile leak, drainage fluid, pancreatic fistula, respiratory complications were higher in group 1 as compared to group 2 and in the same way ICU stay was also prolonged in group 1 patients. Conclusions: Increased fluid administration during surgery is associated with increased postoperative morbidity. There is no benefit in long term survival of patients after increased fluid administration. There should be strict monitoring of fluid administration during and after the surgery.

\section{PE-024 \\ Stress Ulcers as Complications after Liver Resection and Their Prevention \\ K.A. Madatov, U.A. Allazarov, S.N. Lukmonov \\ Department of Faculty Surgery, Tashkent Medical \\ Academy}

Aims: Evaluate the results of the developed measures to prevent stress ulcers after liver resection. Methods: The study included 83 patients on the basis of faculty surgery of Tashkent Medical Academy, which performed radical and palliative surgery for liver alveococcosis. Patients were divided into two groups. Group I (investigational) - 31 (37.3\%) patients, who intraoperatively and in the early postoperative period used a set of measures aimed at preventing the occurrence of stress ulcers. Prevention was as follows: at the end of the operation, a nasogastric tube was injected, and gastric irrigation was performed through it, followed by aspiration with an ozonized solution of common salt with an ozone concentration of 8-10 $\mu \mathrm{g} / \mathrm{mg}$ starting from the end of the operation and for 1-2 days, and from the second days began fractional enteral nutrition, in the interval between meals, the stomach was again irrigated with an ozonized solution. From the first day after the operation, a squamel of $20 \mathrm{mg}$ per $400 \mathrm{ml}$ of physiological saline solution was administered intravenously twice a day for 7-10 days. To reduce oxygen deficiency, oxygen inhalations were prescribed for 20-40 minutes 2-3 times a day. In addition, intravenous ozonized solutions of $400 \mathrm{ml}$ with an ozone concentration of $3-4 \mu \mathrm{g} / \mathrm{ml}$ were administered daily for 4-5 days. Group II (control) - 52 (62.7\%) patients were observed, for which specific measures aimed to prevent the occurrence of stress ulcers, did not apply. Results: In group I, the selected measures reduced aggression and increased the protective function of mucous membranes. Gastroduodenal bleeding did not occur in any patient. With gastroscopy performed in 21 patients, no stress ulcers were found. The parameters of the general blood test and the functional state of the liver and kidneys quickly came to normal. In the second group, in the postoperative period, gastroduodenal hemorrhage on the basis of stress ulcers occurred on day 3-4. Hemostatic, substitutive, anti-inflammatory therapy was performed, 2 bleeding was stopped, and 2 died from increasing anemia, the lethality was $3.8 \%$. An analysis of the case histories showed that this complication arose in patients whose blood loss was greatest, and the surgical intervention was more prolonged. Conclusions: Proposed prevention measures can prevent the development of gastroduodenal bleeding in the postoperative period in patients who underwent radical and palliative surgery for liver alveococcosis. These preventive measures can also be used after liver resections performed for other diseases Keywords: Alveococcosis, Gastroduodenal hemorrhages, Nasogastric tube, Gastric irrigation.

\section{Diagnosis and Liver Imaging / Epidemiology and Surveillance / Miscellaneous}

\author{
PE-025 \\ Imaging Subclassification of Intrahepatic \\ Cholangiocarcinoma: Correlation with \\ Pathological Findings and Surgical Outcomes \\ Chih-Horng Wu ${ }^{1}$, Yung-Ming Jeng ${ }^{2}$, Po-Chin Liang ${ }^{1}$, \\ $\mathrm{Yu}-\mathrm{H}$ sin Lee $\mathrm{Le}^{3}$ Ming-Chih $\mathrm{Ho}^{3}$ \\ ${ }^{1}$ Departments of Medical Imaging and Radiology, \\ National Taiwan University Hospital and College \\ of Medicine, ${ }^{2}$ Department of Pathology, National \\ Taiwan University Hospital and College of Medicine, \\ ${ }^{3}$ Department of Surgery, National Taiwan University \\ Hospital and College of Medicine
}

Aims: Using dynamic computed tomography (CT) and magnetic resonance imaging (MRI), we subclassified intrahepatic cholangiocarcinoma (ICC) into hepatocellular carcinoma (HCC)-like ICC and ICC-like ICC according to growth and enhancement patterns, which may reflect microscopic findings and prognoses. Methods: This is a singlecenter, retrospective study. From 2008 to 2012, 70 patients undergoing liver resection received preoperative dynamic CT, MRI, or both. We analyzed the imaging findings in terms of growth and enhancement patterns and correlated the likelihood of ICC versus HCC. The difference of the imaging subclassification of ICC in clinical parameters, imaging findings, microscopic results and prognosis were compared. Results: Thirty-three patients had HCC-like ICC (Fig.1) and 37 had ICC-like ICC (Fig.2). HCC-like ICC was significantly 


\section{Liver Cancer}

\begin{tabular}{l|l}
\hline Liver Cancer 2018;7(suppl 1):1-220 \\
\hline DOI 10.1159/000490877 & $\begin{array}{l}\text { @ 2018 S. Karger AG, Basel } \\
\text { www.karger.com/lic }\end{array}$ \\
\hline
\end{tabular}

The 9th Asia-Pacific Primary Liver Cancer Expert Meeting (APPLE 2018)
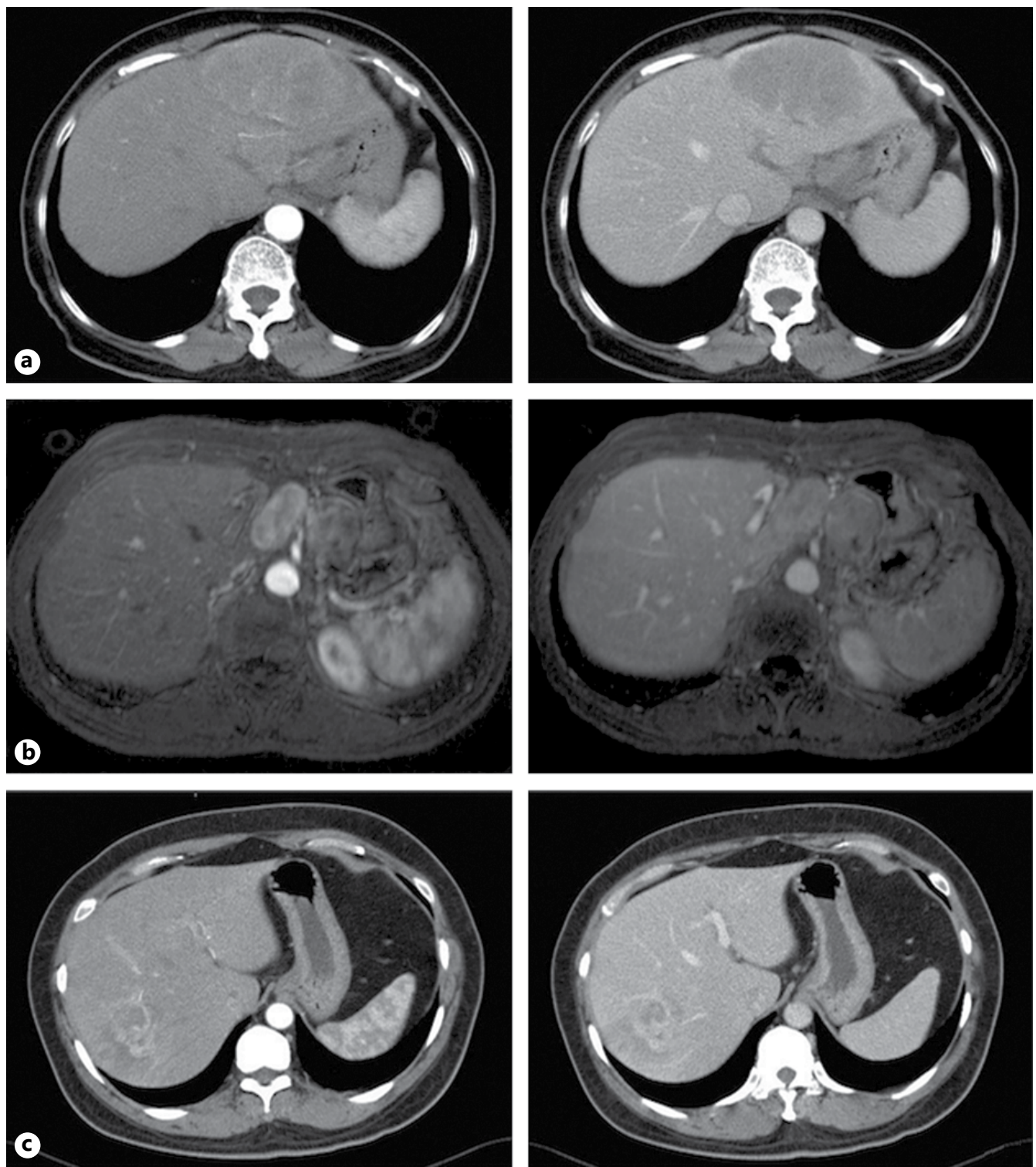

Fig. 1. (for Abstract no PE-025).

associated with peripheral location, larger size, surface retraction, and cholangiolar-type ICC. ICC-like ICC was significantly associated with bile-duct-type ICC (97.3\% [36/37] vs. $18.2 \%$ [6/33], $\mathrm{p}<0.001$ ), positive lymph node metastasis (29.7\% [11/37] vs. 0.0\% [0/33], p = 0.001), and microscopic vascular invasion $(64.9 \%$ [24/37] vs. $39.4 \%$ [13/33], p = 0.033 ) and was an unfavorable prognostic factor for time to recurrence $(p=0.004)$, overall survival in univariate (hazard ratio $[\mathrm{HR}]=2.485,95 \%$ confidence interval $[\mathrm{CI}]: 1.229-$ 5.027, $\mathrm{p}=0.011$ ) and multivariate ( $\mathrm{HR}=2.461,95 \% \mathrm{CI}$ : 1.209-5.010, $\mathrm{p}=0.013$ ) analyses (Fig.3). Conclusions: Our study demonstrated that subclassifying ICC based on combined gross growth and enhancement patterns by using dynamic CT or MRI provides effective pathological and surgical outcome prediction capability. 


\section{Liver Cancer}

\begin{tabular}{l|l}
\hline Liver Cancer 2018;7(suppl 1):1-220 \\
\hline DOI 10.1159/000490877 & $\begin{array}{l}\text { @ 2018 S. Karger AG, Basel } \\
\text { www.karger.com/lic }\end{array}$ \\
\hline
\end{tabular}

The 9th Asia-Pacific Primary Liver Cancer Expert Meeting (APPLE 2018)
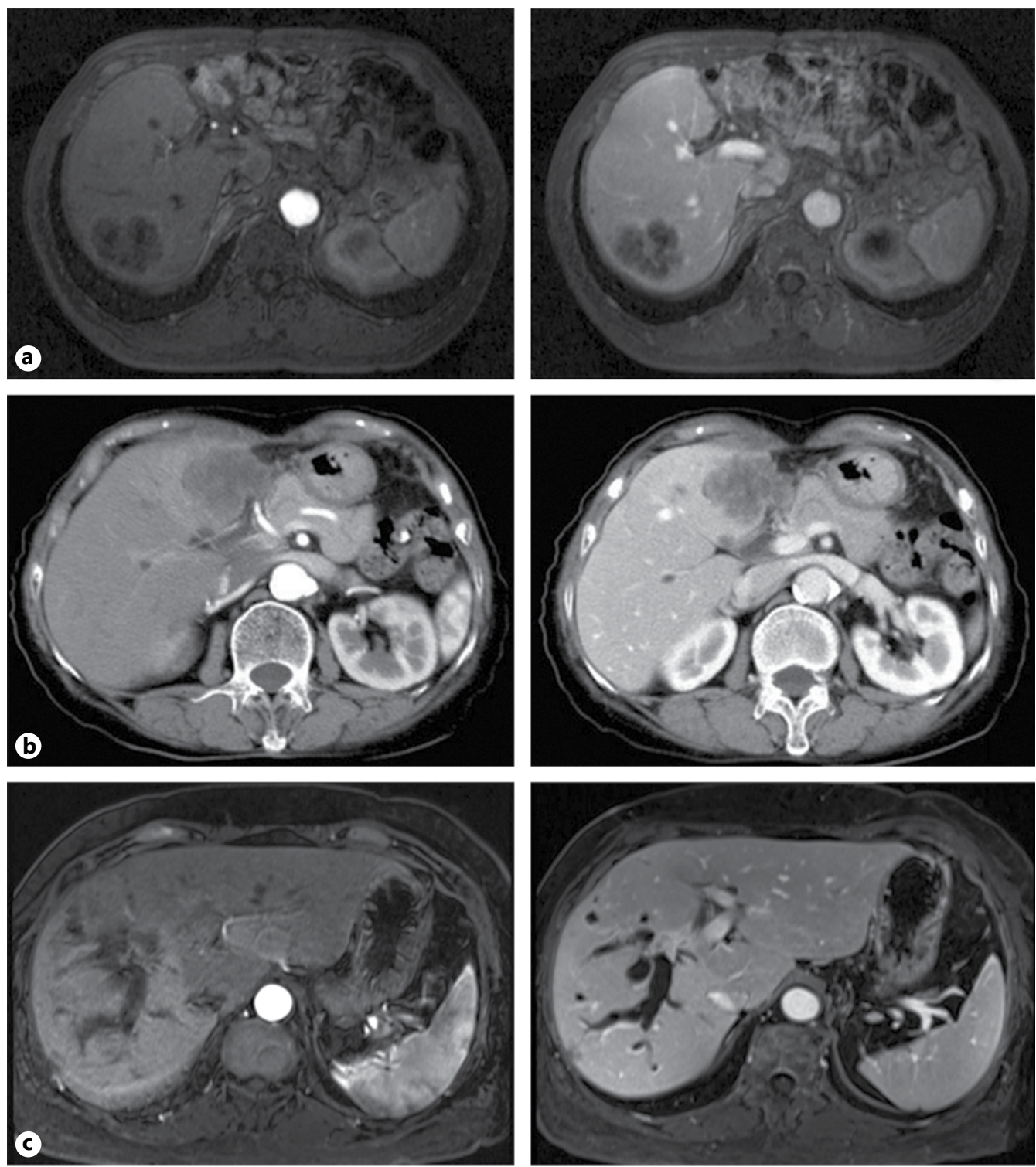

Fig. 2, 3. (for Abstract no PE-025).

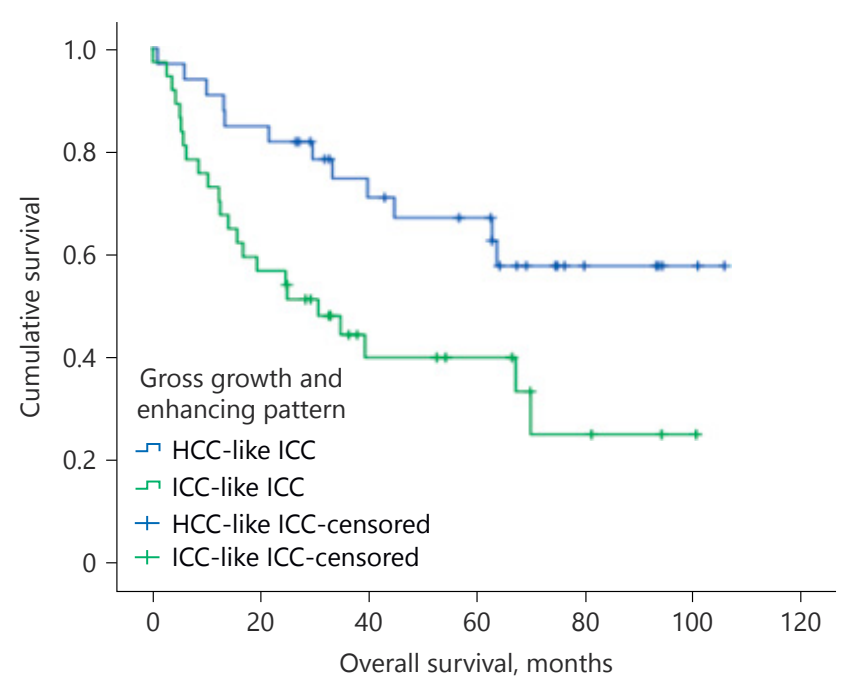




\section{PE-026 \\ Role of the GALAD and a New Comparable Serologic Model in Diagnosis of Hepatocellular Carcinoma in Chinese Patients}

Miaoxia Liu, Ruihong Wu, Xu Liu, Qinglong Jin, Xiaoyu Wen, Junqi Niu

Department of Hepatology, The First Hospital of Jilin University, Changchun, China

Aims: We aimed to evaluate the effectiveness of GALAD model and explore new models in diagnosis of hepatocellular carcinoma (HCC) in Chinese patients. Methods: A total of 641 cases were enrolled in the First Hospital of Jilin University from 2012 to 2015, including 254 with HCC (untreated and diagnosed for the first time), 334 with chronic liver disease (CLD), and 53 healthy Controls (HC). Serum biomarkers AFP, AFP-L3 and PIVKA-II were measured. We established a model called GALAD-C ("C" means China) using the same variables (Gender, Age, AFP-L3, AFP and DCP) as GALAD and a new model called GAAP (consisting of Gender, Age, AFP, and PIVKA-II) by logistic regression. Another 308 patients (including 169 HCC patients and 139 CLD patients) enrolled from 2015 to 2016 were used to validate the GAAP model. Serum AFP was measured by Roche electrochemiluminescence immunoassay, Serum AFP-L3 was measured by Rejing Biotect company, Serum PIVKA-II was measured by
ARCHITECT immunoassay. Results: In all analysis, the area under the receiver operating characteristic curve (AUROC) of GALAD-C model was greatest compared with single markers or other combinations of markers, ranging from 0.817 to 0.985. But we found that the performance of GAAP model was comparable with GALAD-C when we discriminate HCC patients from others (AUROC, 0.899 vs. $0.905, \mathrm{P}=0.73$ in HCC vs CLD; 0.906 vs $0.914, \mathrm{P}=0.68$ in HCC vs Cirrhosis; 0.817 vs $0.817, \mathrm{P}=1$ in HCC with Milan criteria vs CLD; 0.868 vs 0.863 , $\mathrm{P}=0.84$ in HCC with max diameter $<5 \mathrm{~cm}$ vs CLD; 0.974 vs $0.977, \mathrm{P}=0.81$ in HCC with max diameter $\geq 5 \mathrm{~cm}$ vs CLD; 0.984 vs $0.985, \mathrm{P}=0.91$ in HCC vs $\mathrm{HC}$ ). In the validation data set, the AUROC of GAAP model was 0.924 , with a sensitivity of $86 \%$ and a specificity of $86 \%$. Conclusions: GAAP model, using only four variables, showed comparable performance with GALAD-C model in diagnosis of HCC in Chinese patients. GAAP model is more economic and suitable for Chinese people. Keywords: GALAD model, AFP, PIVKA-II, AFP-L3, Hepatocellular carcinoma.

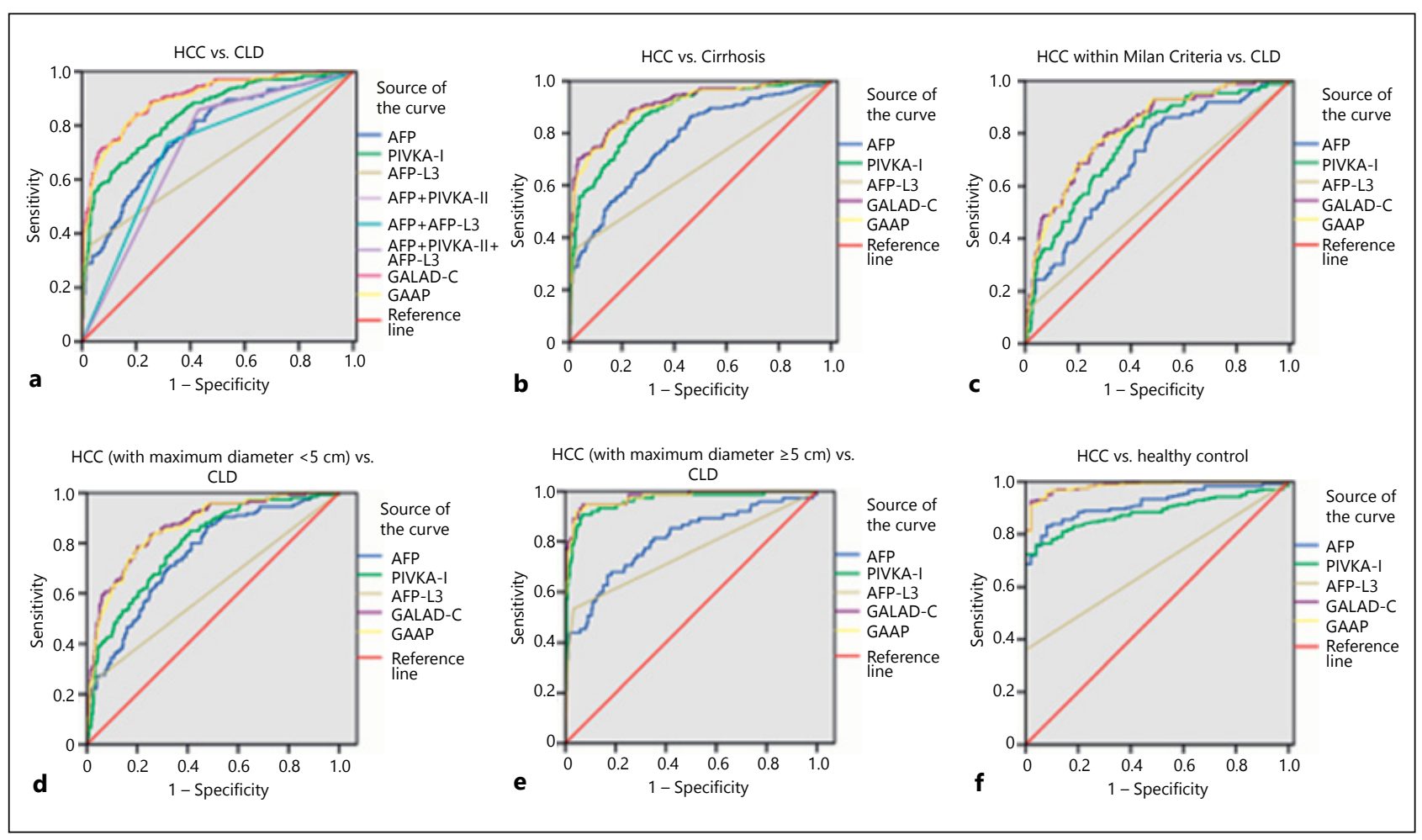

Fig. 1. ROC for biomarkers of models to discriminate HCC from others "+" means "OR" (for Abstract no PE-026). 


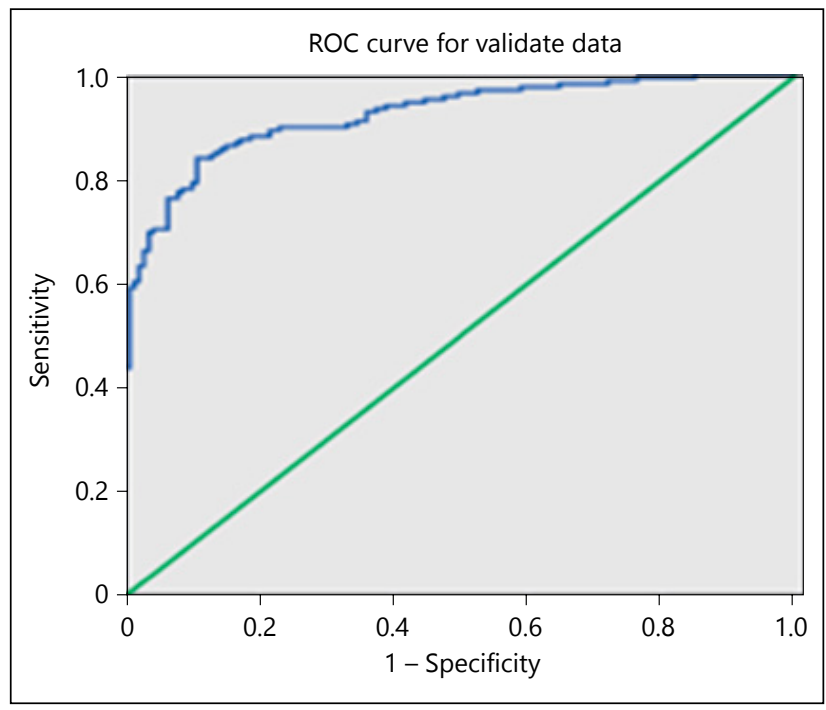

Fig. 2. ROC of GAAP model in the validation data set by discriminating HCC from CLD AUC: $0.924(\mathrm{~N}=308)$ (for Abstract no PE-026).

\section{PE-027 \\ Usefulness of Synapse 3D in Preoperative Planning of Liver Surgery, Concerning Anatomical Variations of Third-Order Branches of Portal Vein in Couinaud's Eight-Segment Scheme}

Sung Yeon Hong, Hee-Jung Wang, Bong-Wan Kim, In-Gyu Kim, Xu-Guang Hu

Division of Liver Transplantation and Hepatobiliary Surgery, Department of surgery, Ajou University School of Medicine, Suwon, South Korea

Aims: To stratify usefulness of Synapse 3D (Fujifilm) in planning of systematic inflow-oriented liver resection in living-donor liver transplantation (LDLT) donors. Methods: 107 liver donors of LDLTs performed from 2008 to 2017 were enrolled in the current study. Anatomical variations of PV 3rd-order branches were analyzed using Fujifilm Synapse 3D. First, the 3D reconstruction images were used to determine the right and left portal branch (1st order). The right anterior and posterior branches were defined as 2nd-order branches. The number of 3rd order branches in the right hemiliver and the branches of the left portal vein (corresponding 3rd-order branches in left hemiliver) were recorded. The intrahepatic anatomical variations according to the Couinaud's eightsegment scheme were analyzed. Results: Among the 107 donors, 93 (86.9\%), 3 (2.8\%), 11 (10.3\%) had portal vein type 1,2 and 3, respectively. Anatomical variation entities of third order branches of portal vein are sliding of origin (3rd order branches from other 2nd order branches in Couinaud scheme) and superficial hypertrophies (superficially crossing the inter-sectional plane). There were considerable number of cases in which variations of 3rd order branch territories were present. Accuracy of the Synapse 3D volumetry compared to the conventional (manual) CT volumetry: During the donor hepatectomy, the portal pedicle to the graft were temporarily clampled, and the stained area was resected. The actual graft weight after donor hepatectomy was measured and compared with manual volumetry and the Synapse 3D volumetry. There were no significant difference in accuracy. Conclusions: Current study showed usefulness of 3D imaging system (Synapse 3D) in overcoming difficulties generated by inconsistencies between the 8-segment scheme of Couinaud and the actual segmental liver anatomy. Keywords: Anatomy, 3DVolumetry, Couinaud Scheme

\section{PE-028 Prognosis of Additional Hypointense
Nodules detected by EOB-MRI in a
Resectable Hepatocellular Carcinoma}

Seoung yoon Rho', Dae hoon Han ${ }^{1}$, Gi hong Choi', Kyoung sik Kim ${ }^{1}$, Jin sub Choi ${ }^{1}$

${ }^{1}$ Division of Hepatobiliary and Pancreas, Department of Surgery, Yonsei University College of Medicine, Seoul, South Korea

Aims: It is known that Gadolinium Ethoxybenzyl Diethylenetriamine Pentaacetic Acid (Gd-EOB-ETPA, EOB) enhanced magnetic resonance imaging (EOB-MRI) improved the detection and diagnosis rate of hepatocellular carcinoma (HCC). In preoperative MRI, additional hypointense nodule (AHIN) is found sometimes, however there are only few studies about prognosis of curative treatments of AHIN and primary HCC lesion together. We aimed to confirm prognosis after surgical treatment in patients with these additional hypointense nodule in EOB-MRI. Methods: We retrospectively reviewed medical records and preoperative images of 522 HCC patients who underwent surgical curative treatment at Hepatoma Clinic, Yonsei Cancer Center, Seoul, South Korea from January 2008 to December 2012. We analyzed general characteristics and conducted propensity score matching analysis of patients. Results: Among 522 patients, we excluded MRI only or CT only and multiple HCC patients. Eventually, 403 patients included and 340 patients with single overt HCC and 63 patients with additional lesion in EOB-MRI were analyzed. Of these 63 patients, 19 patients were confirmed HCC pathologically and 44 patients were not confirmed because of treated RFA or non-HCC lesion pathologically. Among 19 patients, non-hypervascular hypointense nodule were 7 (36.8\%). Overall survival and disease-free survival analysis showed significant difference between single overt HCC patients and single overt HCC with additional hypointense nodule patients ( $p=0.027, p<0.001$, respectively). After propensity score match analysis, there were no statisti- 
Table 1. (for Abstract no PE-028)

\begin{tabular}{|c|c|c|c|}
\hline Variables (Mean \pm SD) & Single overt HCC $(N=63)$ & AHIN (N = 63) & $\mathrm{p}$ \\
\hline Sex & & & 1.000 \\
\hline Male & $53(84.1 \%)$ & $52(82.5 \%)$ & \\
\hline Female & $10(15.9 \%)$ & $11(17.5 \%)$ & \\
\hline Age (years) & $58.1 \pm 8.1$ & $57.4 \pm 8.9$ & 0.660 \\
\hline Body mass index $\left(\mathrm{kg} / \mathrm{m}^{2}\right)$ & $24.9 \pm 3.3$ & $24.3 \pm 3.0$ & 0.327 \\
\hline Serum Total bilirubin (mg/dL) & $1.0 \pm 0.7$ & $1.0 \pm 0.6$ & 0.912 \\
\hline Serum Albumin $(\mathrm{g} / \mathrm{dL})$ & $4.1 \pm 0.6$ & $4.1 \pm 0.5$ & 0.872 \\
\hline INR & $1.1 \pm 0.2$ & $1.1 \pm 0.1$ & 0.704 \\
\hline MELD score & $8.3 \pm 1.8$ & $8.1 \pm 1.8$ & 0.687 \\
\hline Platelet count & $142127.0 \pm 48992.6$ & $144381.0 \pm 54279.6$ & 0.807 \\
\hline AST (lU/mL) & $54.6 \pm 46.2$ & $65.4 \pm 58.0$ & 0.252 \\
\hline ALT (lU/mL) & $49.2 \pm 37.3$ & $54.6 \pm 32.3$ & 0.391 \\
\hline AFP (ng/mL) & $719.4 \pm 3636.4$ & $1091.4 \pm 3596.5$ & 0.565 \\
\hline PIVKA (mAU/mL) & $321.7 \pm 687.2$ & $569.3 \pm 2435.1$ & 0.440 \\
\hline Etiology & & & 0.313 \\
\hline Hepatitis B & $48(76.2 \%)$ & $50(79.4 \%)$ & \\
\hline Hepatitis C & $6(9.5 \%)$ & 7 (11.1\%) & \\
\hline Alcoholics & $2(3.2 \%)$ & $4(6.3 \%)$ & \\
\hline Non-B, Non-C & $7(11.1 \%)$ & $2(3.2 \%)$ & \\
\hline Cirrhosis & & & 1.000 \\
\hline No & $20(31.7 \%)$ & $20(31.7 \%)$ & \\
\hline Yes & $43(68.3 \%)$ & $43(68.3 \%)$ & \\
\hline
\end{tabular}

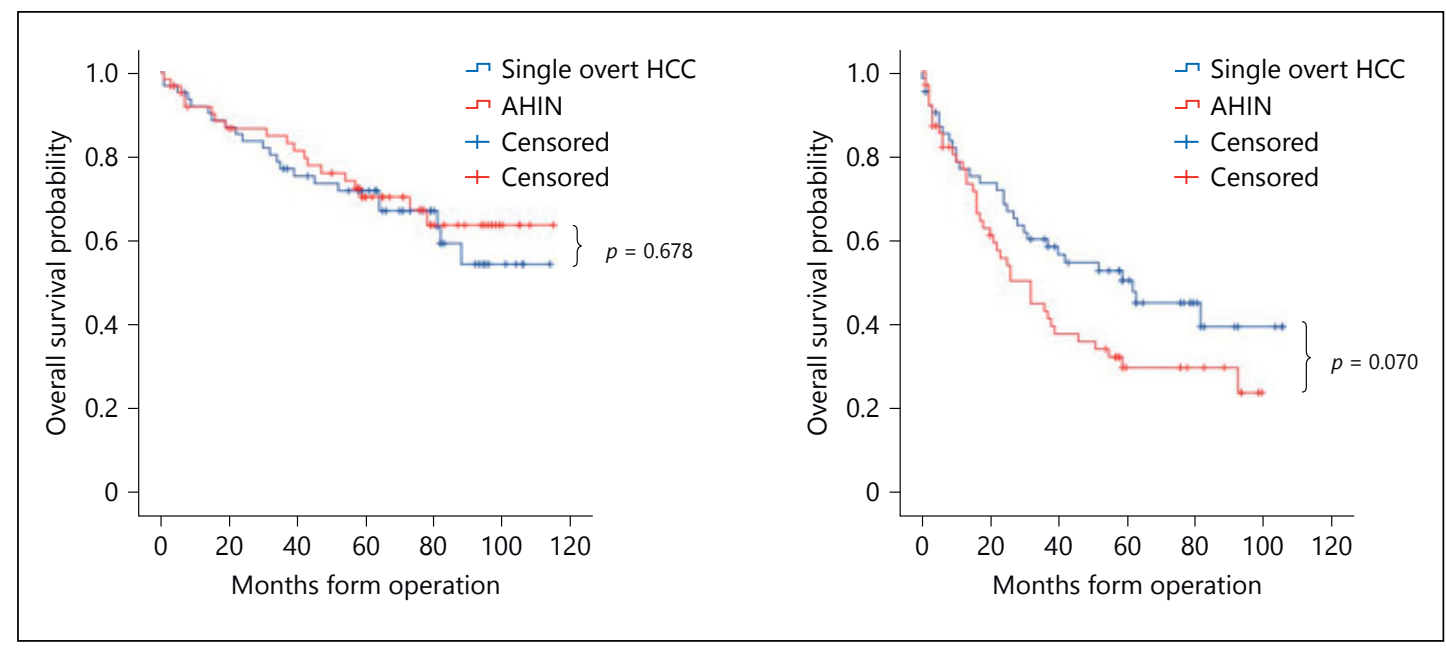

Fig. 1. (for Abstract no PE-028).

cally significant differences between single overt HCC patients and single overt HCC with additional hypointense nodule patients in overall survival analysis (mean 82.272 months, 95\% CI [71.603-92.942] vs. mean 87.101 months, 95\% CI [76.553-97.650], $\mathrm{p}=0.678$ ) and disease-free survival analysis (median 62 months, 95\% CI [29.164-94.836] vs. 32 months, 95\% CI [21.240-42.760], p = 0.070). Conclusions: In general, survival probabilities of single overt HCC with AHIN patients are inferior to single overt HCC group. After propensity score matching, there were no significant difference between single overt HCC and single overt HCC with AHIN in overall survival and disease-free survival. Therefore, single overt HCC with AHIN should be considered a surgical candidate and combined active treatment is recommended if their liver function is allowed Keywords: Hepatocellular carcinoma, Gadolinium ethoxybenzyl diethylenetriamine pentaacetic Acid, Magnetic resonance imaging, EOB MRI 


\section{PE-029 \\ Clinical Applicable Near-Infrared Fluorescent Probe for In Vivo Imaging and Tracking of Transplanted Human Pluripotent Stem Cells-Derived Hepatocytes \\ Jiyou Han 1,2, Jiyou Park', Jiwon Park', Eugeine Jung ${ }^{1}$, Jong Seung Kim ${ }^{3}$, Jong-Hoon Kim ${ }^{2}$ \\ ${ }^{1}$ Department of Biological Sciences, Laboratory of Stem Cell Research and Biotechnology, Hyupsung University, Republic of Korea ${ }^{2}$ Laboratory of Stem Cells \&Tissue Regeneration, Department of Biotechnology, College of Life Sciences \& Biotechnology, Korea University, Republic of Korea ${ }^{3}$ Department of Chemistry, Korea University, Republic of Korea}

Aims: In vivo imaging and tracking of transplanted somatic cells derived from human embryonic stem cells (hESCs) and induced pluripotent stem cells (hiPSCs) is an important issue when the cells were used in clinical purposes as cell therapy. Previously, we reported that functional hepatocytes can be efficiently obtained from hESCs and hiPSCs in high purity. Therefore, a high-resolution cell tracking system using a sensitive probe should be developed before stem cellreplacement therapies becomes a reality in near future. Methods: Here, we introduce an accurate and safe method for in vivo delivering and tracing stem cell-derived hepatocytes. We designed and synthesized a novel near-infrared (NIR) fluorescent probe, which can be applied for cell tracking in vivo, particularly for hepatocytes differentiated from hESCs. Together with the NIR fluorescence dye indocyanine green (ICG), clinically proven compound A is used as a guiding unit in this system to enhance the selective uptake and to improve the biocompatibility of this probe. Results: Our in vitro and in vivo results clearly showed that compound A-appended ICG (A-ICG) enabled in vivo long-term monitoring of transplanted hepatocytes in a mouse model with acute liver injury. Additionally, our immunocytochemistry data (ALB and CD31)

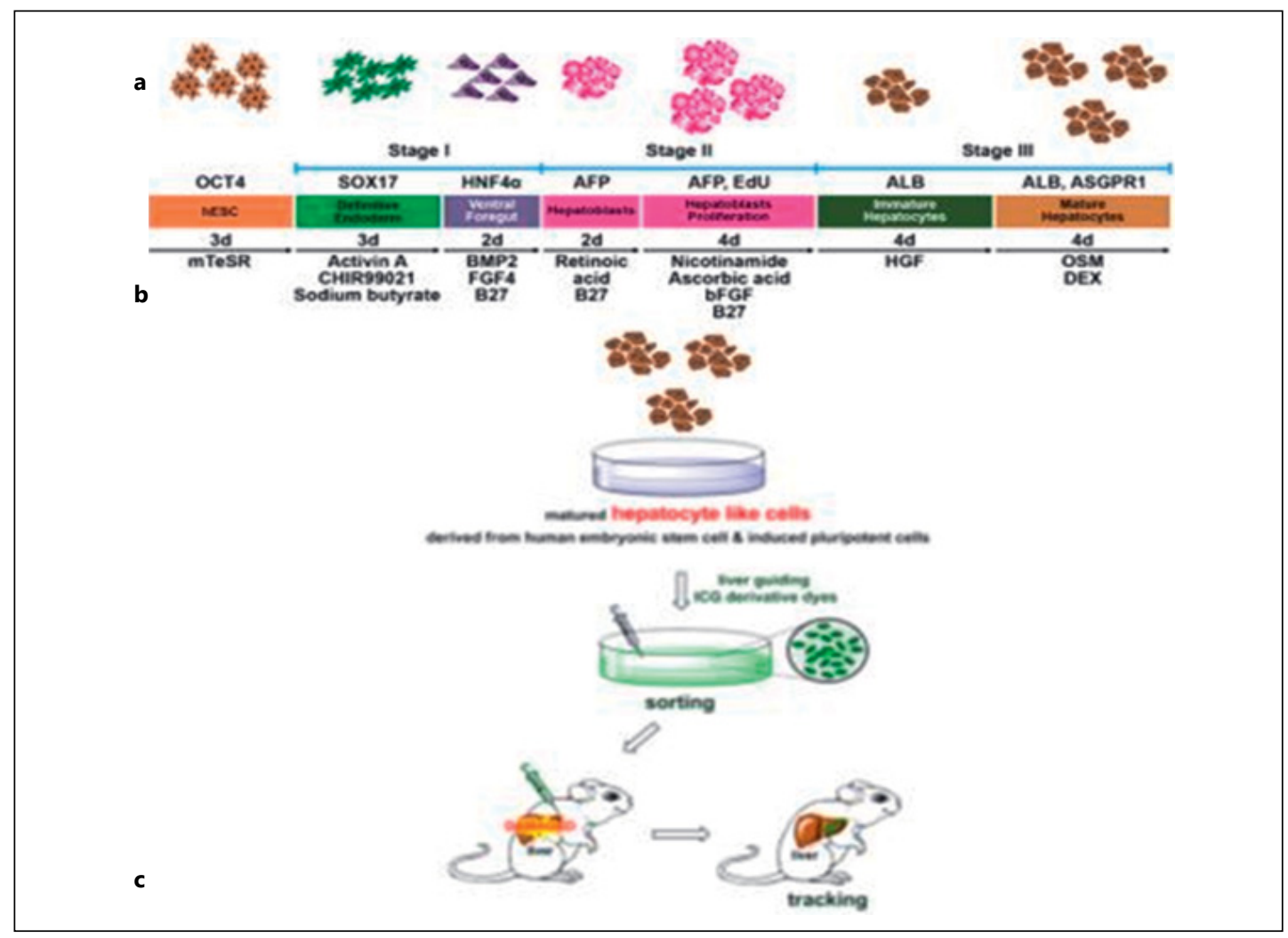

Fig. 1. Schematic depiction and analysis of hepatic differentiation and transplantation into Cdj-üver injured mice. Human ESCs were differentiated into early hepatocytes according to our protocol previously reported (Fig 1A). After 11 days of hepatic differentiation, hESC-derived hepatoblasts were dissociated into single cells using trypLE, and the single cell suspension was split into 2D and 3D culture groups separately. For 2D culture condition, dishes were coated with collagentype I, whereas ultra-low-attachment dishes were used for 3D culture condition. ITS medium containing $20 \mathrm{ng} / \mathrm{ml}$ hepatocyte growth factor, $10 \mathrm{ng} / \mathrm{ml}$ ojicostatin $\mathrm{M}$, and $1 \mu \mathrm{M}$ dexamethasone was used for further hepatic differentiation. Obtained matured hESC-derived hepatocytes were incubated with A-ICG for $24 \mathrm{~h}$. Then the cells were transplanted into CCI4-liver injured mouse spleen (splenic transplantation). Time series in vivo images of transplanted hepatocytes were monitored up to $96 \mathrm{~h}$. (for Abstract no PE-029). 


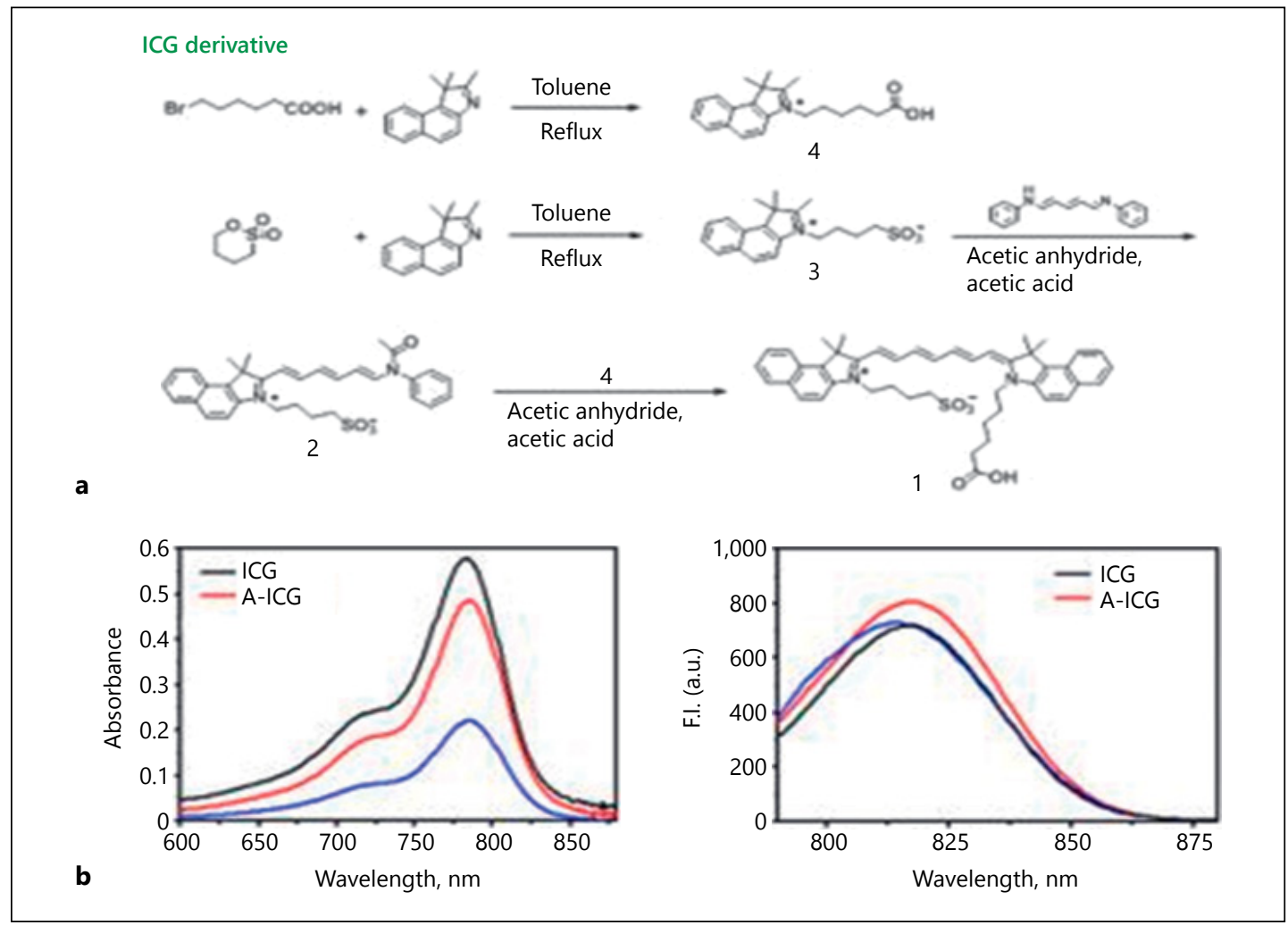

Fig. 2. Schematic flow chart of synthesizing the ICG derivative compound 1 . The entire scheme can be divided into three parts - synthesis of ICG derivative compound 1_ Then A-ICG was modified based on compound 1_ First, near-infrared fluorophore compound 1 was synthesized as shown in Scheme. After the synthesis of compound 1 has done, liver cell guiding units bl and gl were prepared and attached to compound 1 respectively. For the modification of a, the synthesis was started from the reaction of $\mathrm{D}$-A with $\mathrm{N}$-hydroxvsuccjnímide in the presence of EDCI and DMF. After A was obtained in $60 \%$ yield, it was reacted with ethyjenedjamjne and triethylamine to give bl in 52\% yield. Finally. A was then reacted with compound 1 by HATU coupling to afford the novel stem cell tracker A-ICG

showed that liver injury was significantly recovered in A-ICG tagged cells transplanted liver, compared to the sham and control group. It might be the compound A prevent the transplanted cells to reactive oxygen damages. Conclusions: Our NIR cell tracking probe can provide a useful information for evaluating the efficacy and safety of transplanted stem cellderived hepatocytes for clinical application. Keywords: Hepatic cancer, Theranostic, Cancer stem cell, 3D tumor spheroid. in $16 \%$ yield. The structural identities of each of the compounds were confirmed by $1 \mathrm{H}, 13 \mathrm{C}$ NMR spectra, ESI-MS and HPLC data in Supporting Information. b After synthesizing the desired final compounds A-ICG, their absorption and emission properties were measured and compared with the commercially available ICG. As shown in Figure 2, A-ICG and Gal-ICG showed absorption spectra at $785 \mathrm{~nm}$, which was similar to that of ICG. Upon excitation at $785 \mathrm{~nm}$, the fluorescence spectra of B-ICG and Gal-ICG exhibited a maximum at $817 \mathrm{~nm}$ and $815 \mathrm{~nm}$ respectively. Both ICG derivatives showed comparable fluorescence intensities with ICG, which reveals their potential to be used as effective NIR fluorophores (for Abstract no PE-029).

\section{PE-030 \\ Dynamic Multi-phasic CT-Based Model for Predicting Local Responses and Overall Survival for HCC Patients Receiving SBRT: Combination of Radiomics and Machine Learning}

Yang-Hong Dai', Wen-Yen Huang ${ }^{2}$

Department of Radiation Oncology, Tri-Service General Hospital, Taipei, Taiwan

Aims: Stereotactic body radiotherapy (SBRT) is usually used for the advanced stage hepatocellular carcinoma (HCC) when serial locoregional therapies fail to provide therapeutic 


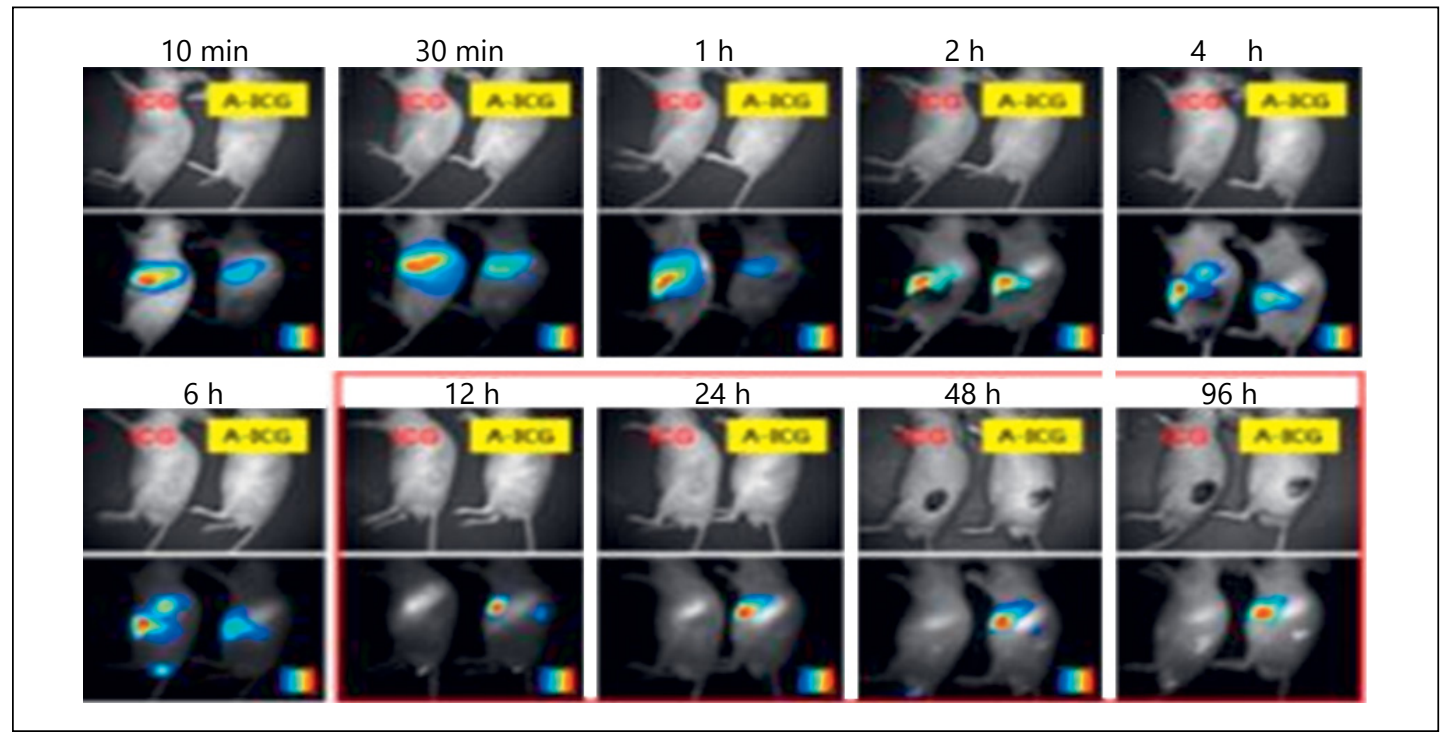

Fig. 3. Cytotxxicity of A-ICG and time course imaging and biodistributjon of in vivo mouse model after tail intravenous injection of ICG or A-ICG. a Effect of A-ICG on cell viability in HeLa cells and NIH3T3 cells. b In an effort to assess extended retention time of A-ICG in vivo, time course imaging and biodistribution of mouse models were measured. We intravenously injected mice tails with either ICG or A-ICG and ob- served fluorescence intensity changes in time. Strong fluorescence signal in the A-ICG injected mouse has lasted for $96 \mathrm{~h}$ in contrast to that of ICG which has fully disappeared after $6 \mathrm{~h}$. These results support our hypothesis that both ICG and A-ICG were localized in liver, exhibiting their hepatocyte targeting ability (for Abstract no PE-029).

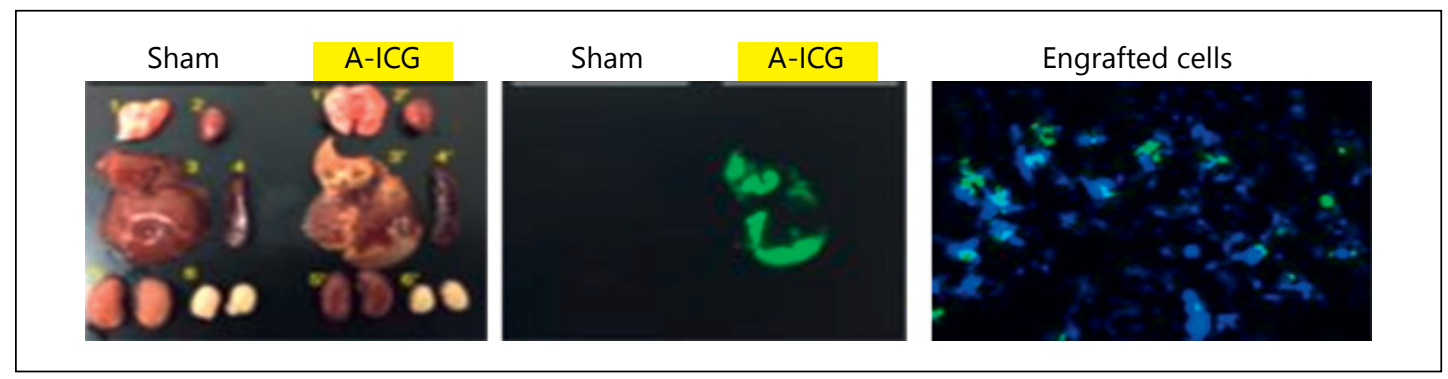

Fig. 4. Ex vivo-dissected organs and optical fluorescent images of the Sham- and A-ICG labeled hESC-derived hepatocytes transplanted mice. Lung (1 and 1), heart (2 and 2'), liver ( 3 and $3^{\prime}$ ), lung ( 3 and $3^{\prime}$ ), spleen (4 and $4^{\prime}$ ), kidney (5 and $\delta^{\prime}$ ) and testis (6 and 6). (Excitation at $785 \mathrm{~nm}$ ). Engrafted hepatocytes were successfully detected in the CCI4-injured mouse liver at 4 days after splenic transplantation. DAPI (blue) and hepatocytes (green) (for Abstract no PE-029). effects. However, the effect of such high-energy radiation on HCC has not been well elucidated. Therefore, we aimed to construct predictive models to identify predictors of local responses and overall survival for HCC treated with SBRT, using radiomics and machine learning. Methods: Radiomic analyses were performed by MATLAB to extract potential features from the multi-phasic computed tomography scans. Predictive models that combined radiomics and clinical factors were constructed by using machine leaning to obtain optimal performance for classification of local responses and overall survival. The events evaluated were the tumor sizes that achieved the mRECIST (modified response evaluation criteria in solid tumor) within 1-year (W1), in the end of the follow-up (End) and in-field failure free (IFFF) responses. Kaplan-Meier analysis was performed for overall survival evaluation. Results: A total of 33 patients with 41 tumors receiving SBRT were included in our study. Overall 19 machine learning algorithms were used to identify the optimal classifiers. The best predictive models were constructed by Ensemble bagged tree (Accuracy $=90.2 \%$ ) for W1 response, cubic Support Vector Machine (SVM) (Accuracy $=82.9 \%$ ) for End response, and medium SVM (Accuracy $=85.4 \%$ ) for IFFF 
response. For the overall survival, the best predictors were EQD2 (Equivalent dose in 2Gy fraction), BDA (Biaxial deviating gradient), RNC (Ratio of necrotic content), SZHGE (Short Zone High Gray-Level Emphasis), and tumor size (importance by learning vector quantization $=0.8113,0.75,0.7279,0.7181$ and 0.7132 , respectively). For overall survival, EQD2 $<71.25$ Gy and tumor size $>8.2 \mathrm{~cm}$ were associated with shorter survival months ( $p=0.0387$ and 0.0422 ). Conclusions: Radiomics and machine learning provide great platform for the construction of models for predicting the effect of SBRT on HCC. Keywords: Radiomics, Machine learning, SBRT, HCC

\section{PE-031 \\ Liver Cancer Mortality in Korean Workers Occupationally Exposed to Methanol}

Young-Sun Min ${ }^{1}$, Hongsuk Choi ${ }^{1}$, Cheol-In Yoo ${ }^{2}$, Yeon-Soon $\mathrm{Ahn}^{3}$

1Department of Occupational and Environmental Medicine, Dongguk University Gyeongju Hospital, Korea ${ }^{2}$ Department of Occupational and Environmental Medicine, Ulsan University Hospital, University of Ulsan College of Medicine, Korea; ${ }^{3}$ Department of Preventive Medicine, Yonsei University Wonju College of Medicine, Korea

Aims: The comprehensive conclusion of liver cancer development of methanol in studies on animals is currently controversial, and it is difficult to apply findings from animal studies directly to humans. Therefore, the epidemiologic study of humans was conducted to evaluate the relationship between methanol exposure and liver cancer mortality among Korean workers occupationally exposed to methanol. Methods: The study cohort was derived from the Korea Occupational Safety and Health Agency data on methanol-exposed male worker who underwent methanol-associated medical check-ups at least once between January, 2000 and December, 2004. Workplace methanol exposure levels for each worker were divided into three grades: $<10 \%$ of Threshold Limit Value-Time-Weighted Average (TLV-TWA), 10-50\% of TLVTWA, and $\geq 50 \%$ of TLV-TWA. External analyses compared cancer mortality rates of methanol-exposed workers with those of the general population. In terms of intra-cohort analyses, hazard ratios were estimated with a Cox proportional hazards model. Results: No statistically significant differences were shown between the entire methanol-exposed workers and reference population. When the data were stratified by each cancer code (including liver cancer) and workplace methanol exposure levels, no significant differences were shown between group with $<10 \%$ of TLV-TWA and $10-50 \%$ or $\geq 50 \%$. Conclusions: Although this study did not reveal significant correlation between methanol exposure and liver cancer mortality, we believe it is meaningful, being the first large-scale epidemiological study done in humans.
However, the carcinogenic potential of methanol remains an open question, and further studies with longer follow-up of methanol-exposed workers are needed.

\section{PE-032 \\ Impact of Surveillance on Long-Term Survival and Recurrence after Curative Liver Resection for Hepatitis B Related Hepatocellular Carcinoma: A Study on 1,075 Patients \\ Zhen Li Li ${ }^{1}$, Jun Han ${ }^{1}$, Hao Xing ${ }^{1}$, Han Wu', Wen Tao Yan 1,2, Li Yang Sun 1,2, Jia He Wang 1,2, Bing Quan ${ }^{1,2}$, Chao Li ${ }^{1}$, Ming Da Wang ${ }^{1}$, Han Zhang ${ }^{1}$, Wan Yee Lau ${ }^{1,3}$, Meng Chao Wu ${ }^{1}$, Feng Shen ${ }^{1}$, Tian Yang ${ }^{1}$ \\ ${ }^{1}$ Department of Hepatobiliary Surgery, Eastern Hepatobiliary Surgery Hospital, Second Military Medical University, Shanghai, China; ${ }^{2}$ Department of Clinical Medicine, Second Military Medical University, Shanghai 200433, China; ${ }^{3}$ Faculty of Medicine, the Chinese University of Hong Kong, Shatin, New Territories, Hong Kong SAR, China}

Aims: Clinical guidelines recommend surveillance in highrisk populations to detect hepatocellular carcinoma (HCC) early, when curative treatment such as liver resection can be applied. However, it is largely unknown whether surveillance brings survival benefit to patients after curative treatment for HCC. Methods: A prospectively maintained database on patients with hepatitis B virus (HBV)-related HCC who underwent curative liver resection from 2003 to 2014 was reviewed. Patients' overall survival (OS) and recurrence-free survival (RFS) were compared between the groups of patients whose HCCs were diagnosed by surveillance and by symptoms, as well as between the groups of patients operated in the first (2003-2008) and second (20092014) 6-year periods. Results: Of 1,075 patients, 452 (42.0\%) patients were diagnosed by surveillance. Compared with the symptomatic group, the OS and RFS rates were significantly better in the surveillancegroup (both $P<0.001$ ). Surveillancewas associated with a $55 \%$ decrease in the overall survival risk and a $48 \%$ decrease in the recurrence risk (HR 0.45, 95\% CI 0.38-0.53, and HR $0.52,95 \% \mathrm{CI} 0.44-0.61$ ). The proportion of patients with HCC diagnosed by surveillance increased from $37.3 \%$ during 2003-2008 to 44.8\% during 2009-2014. Compared with the first period, significant reduction of $12 \%$ and $19 \%$ in the overall death and recurrence risks were observed in the second period (HR $0.88,95 \%$ CI $0.78-0.97$, and HR $0.81,95 \%$ CI 0.70-0.95). Conclusions: Surveillance of HCC was associated with $55 \%$ and $48 \%$ reduction in overall death and recurrence risks in patients with HBV-related HCC who underwent curative liver resection. With increasing use of surveillance, the overall prognosis of patients after curative liver resection for HCC has improved. Keywords: Hepatocellular carcinoma, Hepatitis B virus, Surveillance, Liver resection, Recurrence, Survival, Effectiveness. 
The 9th Asia-Pacific Primary Liver Cancer Expert Meeting (APPLE 2018)

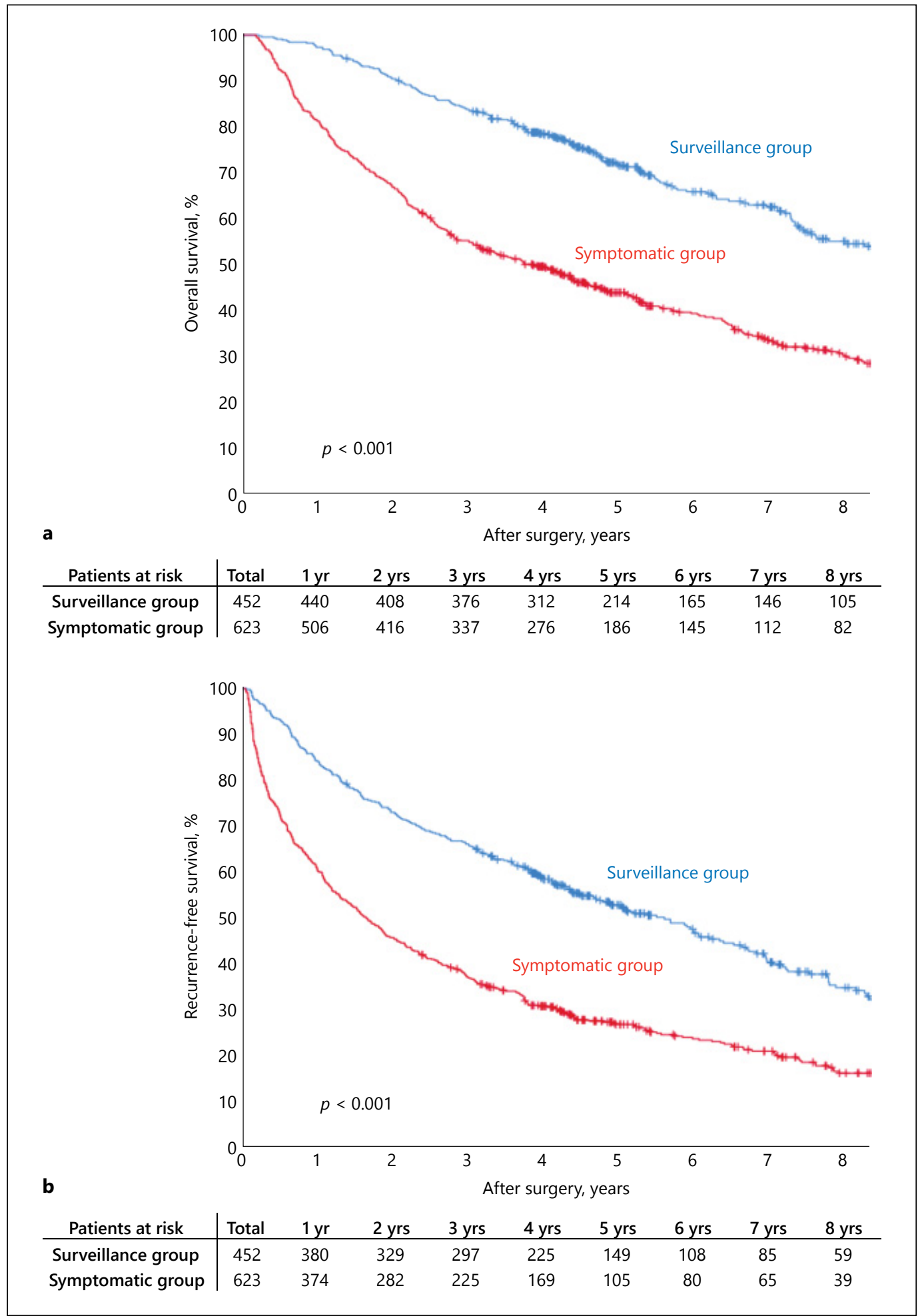

Fig. 1. Cumulative incidence of overall survival (a) and recurrence-free survival (b) curves. Comparisons between patients with hepatocellular carcinoma detected by surveillance (the surveillance group) and symptoms (the symptomatic group) (for Abstract no PE-032). 


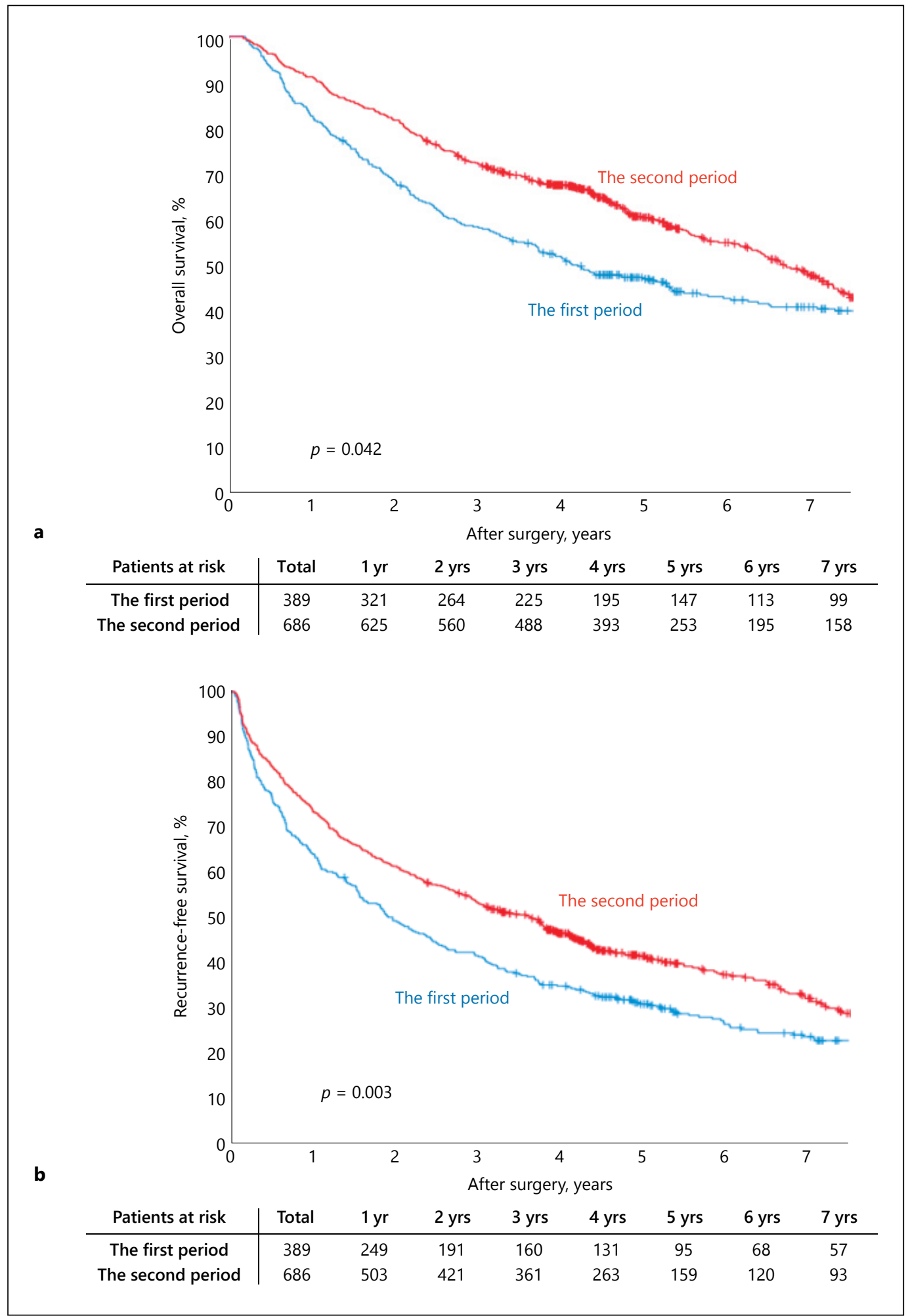

Fig. 2. Cumulative incidence of overall survival (a) and recurrence-free survival (b) curves. Comparisons between the first period (from 2003 to 2008) and the second period (from 2009 to 2014) (for Abstract no PE-032). 
a

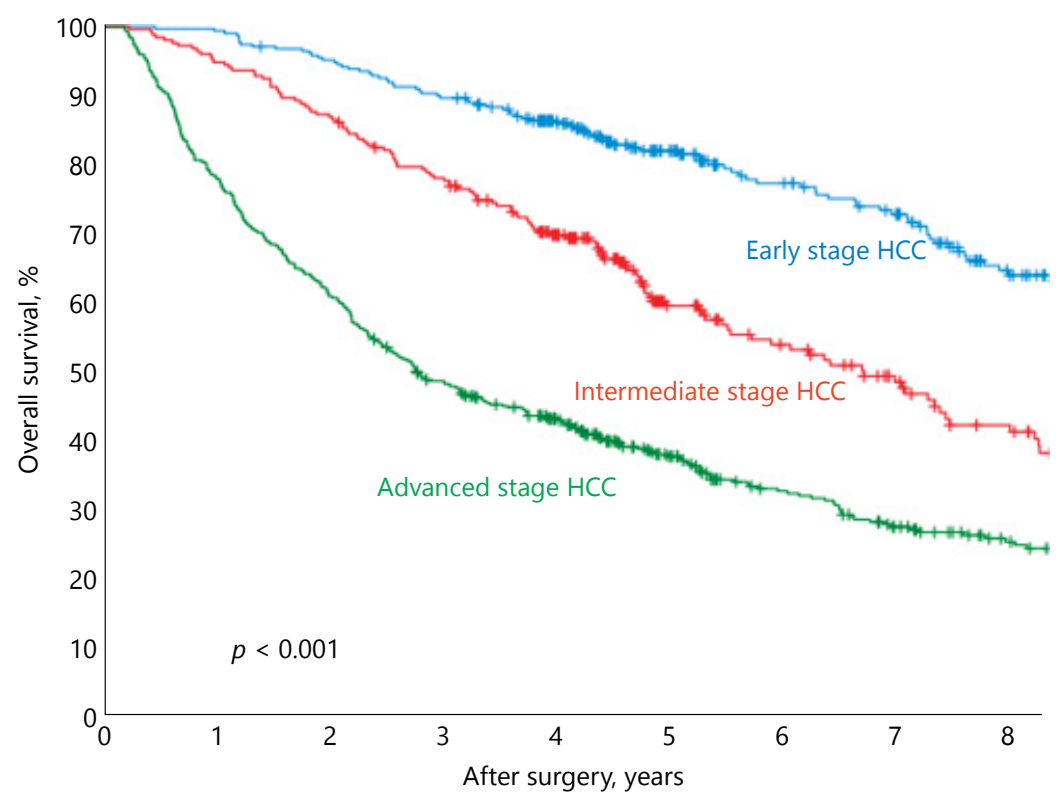

Patients at risk

Early HCC

Intermediate HCC

Advanced HCC

\begin{tabular}{|ccccccccc} 
Total & $\mathbf{1}$ yr & $\mathbf{2}$ yrs & $\mathbf{3}$ yrs & $\mathbf{4}$ yrs & $\mathbf{5}$ yrs & $\mathbf{6}$ yrs & $\mathbf{7 y r s}$ & $\mathbf{8}$ yrs \\
\hline 309 & 307 & 293 & 276 & 238 & 178 & 142 & 128 & 90 \\
251 & 238 & 218 & 194 & 155 & 92 & 73 & 57 & 44 \\
515 & 401 & 313 & 244 & 195 & 130 & 95 & 73 & 53
\end{tabular}

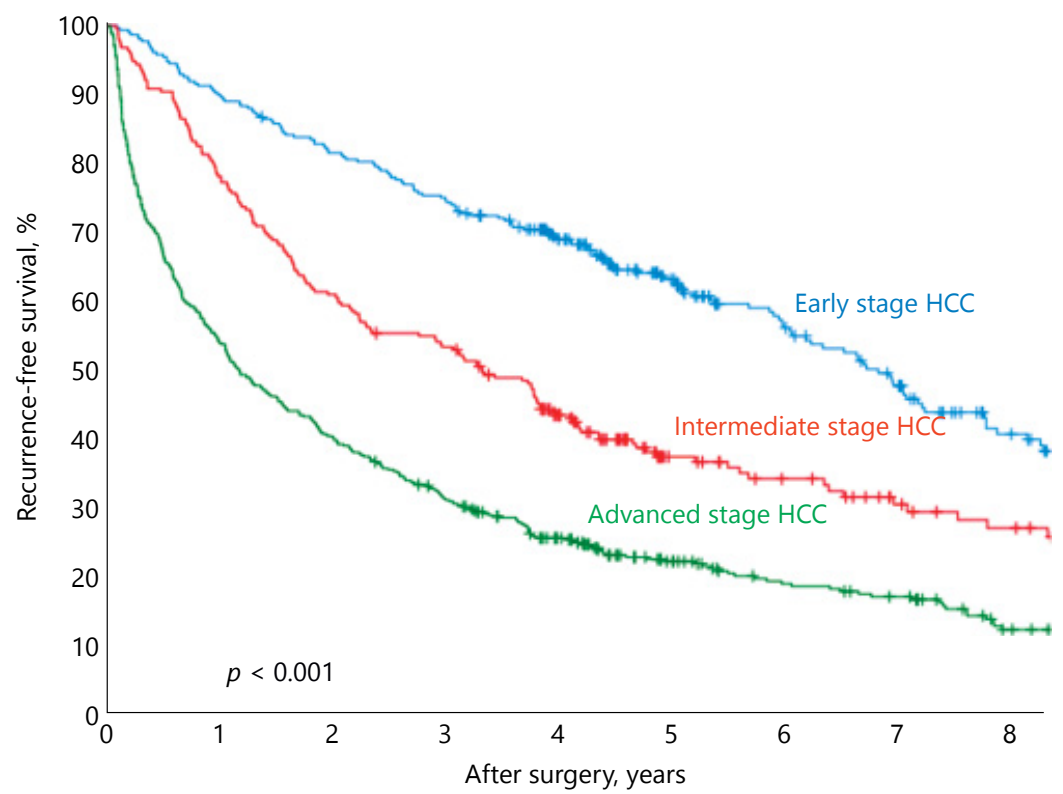

b

After surgery, years

\begin{tabular}{c|ccccccccc} 
Patients at risk & Total & $\mathbf{1}$ yr & $\mathbf{2}$ yrs & $\mathbf{3}$ yrs & $\mathbf{4}$ yrs & $\mathbf{5}$ yrs & $\mathbf{6}$ yrs & $\mathbf{7}$ yrs & $\mathbf{8}$ yrs \\
\hline Early HCC & 309 & 278 & 251 & 231 & 187 & 133 & 97 & 78 & 52 \\
Intermediate HCC & 251 & 196 & 153 & 133 & 93 & 50 & 40 & 29 & 23 \\
Advanced HCC & 515 & 278 & 208 & 159 & 114 & 71 & 51 & 43 & 23
\end{tabular}

Fig. 3. Cumulative incidence of overall survival (a) and recurrence-free survival (b) curves. Comparisons among patients with early (BCLC A stage), intermediate (BCLC B stage), and advanced (BCLC C stage) hepatocellular carcinoma (HCC) (for Abstract no PE-032). 


\section{PE-033 \\ Addition of Albumin-Bilirubin Grade to PAGE-B Score Enhances Risk Assessment for Hepatocellular Carcinoma Development among Patients on Entecavir Therapy \\ Jungyoon Kim, Dong Hyun Sinn, Wonseok Kang, Geum-Youn Gwak, Yong-Han Paik, Moon Seok Choi, Joon Hyeok Lee, Kwang Cheol Koh, Seung Woon Paik \\ Department of Medicine, Samsung Medical Center, Sungkyunkwan University School of Medicine, Seoul, South Korea}

Aims: PAGE-B is a validated simple score, made of age, sex and platelet count, that can help predict the risk of developing hepatocellular carcinoma (HCC) among chronic hepatitis B patients on antiviral therapy. Yet, PAGE-B score encompass patients at intermediate risk, and requires crossvalidation from Asian countries. We tested usefulness of PAGE-B score among chronic hepatitis B patients on entecavir therapy. We also tested whether performance of PAGE-B score can be improved by adding albumin-bilirubin (ALBI) grade. Methods: A total of 894 treatment-naïve, chronic hepatitis B patients, who started entecavir therapy between January 2007 and June 2012 were analyzed. The virological parameters and biochemical laboratory data including platelet, albumin, bilirubin for calculation of ALBI and PAGE scores were measured at baseline and at 1 year of entecavir therapy. Results: During a median 5.6 years of follow-up (range: 1.0-10.2 years), HCC was diagnosed in 100 patients (11.2\%). The ALBI and PAGE score both at baseline and at year 1, respectively, were associated with development of HCC. After 1 year of entecavir therapy, the ALBI score improved (median: -2.69 to $-2.96, \mathrm{p}<0.001$ ), whereas PAGE-B score did not (median: 14 to $14.5, \mathrm{p}=0.619$ ). Combination of ALBI and PAGE-B score showed the highest areas under the receiver operating characteristic curves (AUROCs) for the prediction of HCC development at 3 and 5 years, at baseline and at year 1, respectively, compared with ALBI and PAGE-B alone. Especially, the ALBI grade was able to further classify patient with intermediate risk according to PAGE grade at baseline and year 1, respectively. Conclusions: The use of ALBI score and PAGE-B score in combination, compared to their solitary use, was more effective in predicting HCC risk for chronic hepatitis B patients on entecavir therapy. Performance of PAGE-B score can be improved by simply adding ALBI score. Keywords: Hepatocellular carcinoma, Albumin-bilirubin grade, PAGE-B grade, Entecavir, Hepatitis B.

\section{PE-034 \\ Effect of Obesity and Statins on Liver Cancer Mortality in Patients with Chronic Hepatitis B}

Jae-Jun Shim ${ }^{1}$, Minah Jon ${ }^{1}$, Chi Hyuck Oh ${ }^{1}$, Ji Sung Lee ${ }^{3}$, Byung-Ho Kim ${ }^{1}$, In-Hwan $\mathrm{Oh}^{2}$

${ }^{1}$ Department of Internal Medicine, ${ }^{2}$ Department of Preventive Medicine, ${ }^{4}$ Department of Medical Education and Humanities, Kyung Hee University School of Medicine, Seoul, Republic of Korea, ${ }^{3}$ Clinical Research Center, Asan Medical Center, Seoul, Republic of Korea

Aims: Statins are associated with a lower risk of developing hepatocellular carcinoma in patients with chronic hepatitis B (CHB). In many population studies, being overweight or mildly obese was associated with lower overall mortality in healthy subjects. We hypothesized that this protective effect of statins might be associated with mild obesity or hypercholesterolemia. Methods: We analyzed the data of the Health Examination Cohort of the National Health Insurance Service of Korea from 2002 to 2013. In total, 13,063 patients aged 40-79 years with CHB and no other concurrent liver disease, such as liver cirrhosis, were identified. Statin use was defined as $\geq 28$ cumulative defined daily doses. Results: In total, 3 of 980 statin users and 190 of 12,066 non-users died from liver cancer during the mean 10.6-year follow-up period. Mortality was 0.29 per 1,000 person-years [ $95 \%$ confidence interval (CI) 0.09-0.89] for statin users and 1.48 per 1,000 personyears (95\% CI 1.28-1.71) for non-statin users ( $\mathrm{P}<0.001)$. After adjusting for age, sex, BMI, smoking, alcohol consumption, plasma levels of glucose and cholesterol, and liver enzymes, statin use was independently associated with a lower risk of liver cancer mortality [hazard ratio (HR) $0.17,95 \%$ CI $0.04-$ 0.68 ]. Only the overtly obese patients (BMI $\geq 30 \mathrm{~kg} / \mathrm{m}^{2}, \mathrm{n}=$ 340 ) had a higher risk of death due to liver cancer (HR 2.42, 95\% CI 1.18-4.96) compared with normal patients. Being overweight or mildly obese (BMI $25.0-29.9 \mathrm{~kg} / \mathrm{m}^{2}$ ) was not associated with mortality (HR $0.95,95 \%$ CI $0.64-1.40$ ). A cholesterol level $>240 \mathrm{mg} / \mathrm{dL}$ was independently associated with a lower risk of mortality (HR 0.45 , 95\% CI 0.23-0.86). Liver cancer mortality in patients with relatively low cholesterol levels at baseline $(<200 \mathrm{mg} / \mathrm{dL}, \mathrm{n}=7,380)$ was 1.84 per 1,000 person-years (95\% CI 1.56-2.17). Conclusions: Statin use was clearly associated with lower liver cancer mortality in patients with CHB. The effect of statins on mortality was not dependent on BMI. However, a relatively low baseline cholesterol level was significantly associated with a higher risk of death due to liver cancer. The effects of statins in these patients need to be clarified. Keywords: Chronic hepatitis B, Mortality, Hepatocellular carcinoma, Hydroxymethylglutaryl-CoA Reductase Inhibitors, Obesity. 


\section{PE-035 \\ Prediction of Hepatocellular Carcinoma Using Decision Tree Classification in Treatment Naïve Patients for Chronic Hepatitis B}

Sang Bong Ahn', Jae Yoon Jeong ${ }^{2}$, Dae Won Jun ${ }^{3}$, Eileen L. Yoon ${ }^{4}$, Sung Eun Kim ${ }^{5}$, Jae-Jun Shim ${ }^{6}$, Yong Kyun $\mathrm{Cho}^{7}$, Soung Won Jeong ${ }^{8}$, Hyoung Su Kim ${ }^{9}$, Jun Choi ${ }^{10}$

${ }^{1}$ Department of Internal Medicine, Nowon Eulji Medical Center, Seoul Hospital, Eulji University, Seoul, ${ }^{2}$ Department of Internal Medicine, Hanyang University Guri Hospital, Hanyang University College of Medicine, Guri, ${ }^{3}$ Department of Internal Medicine, Hanyang University Hospital, Hanyang University College of Medicine, Seoul, ${ }^{4}$ Department of Internal Medicine, Sanggye Paik Hospital, Inje University Seoul, ${ }^{5}$ Department of Internal Medicine, Hallym University Sacred Heart Hospital, Hallym University College of Medicine, Anyang, ${ }^{6}$ Department of Internal Medicine, Kyung Hee University Hospital, Kyung Hee University School of Medicine, Seoul, ${ }^{7}$ Department of Internal Medicine, Kangbuk Samsung Hospital, Sungkyunkwan University School of Medicine, Seoul, ${ }^{8}$ Department of Internal Medicine, Soonchunhyang University Hospital, Soonchunhyang University College of Medicine, Seoul, ${ }^{9}$ Department of Internal Medicine, Hallym University Kangdong Sacred Heart Hospital, Hallym University College of Medicine, ${ }^{10}$ Department of Fusion Data Analytics, School of Industrial Management Engineering, Korea University

Aims: This study aimed to develop prediction model for the development of hepatocellular carcinoma (HCC) in treatment-naïve patients receiving entecavir or tenofovir for chronic hepatitis B (CHB). Methods: We enrolled 3184 patients treated with chronic hepatitis B. We analyzed 1350 patients who were treated with entecavir or tenofovir as initial treatment, those with initial clinical records, and those without cancer within oneyear after treatment. HCC prediction decision tree was constructed through logistic regression using 90 variables including demographic, laboratory data, and cirrhosis. We also analysis with support vector machine (SVM) analysis and random forest analysis. Results: Eightyfour patients were diagnosed with hepatocellular carcinoma. Age $(\mathrm{OR}=12.3, \mathrm{p}=0.001)$, family history of $\mathrm{HBV}(\mathrm{OR}=7.2, \mathrm{p}=$ $0.006)$, cirrhosis $(\mathrm{OR}=35.1, \mathrm{p}=0.001)$, diuretics use $(\mathrm{OR}=4.1$, $\mathrm{p}=0.04)$, WBC count $(\mathrm{OR}=4.1, \mathrm{p}=0.04)$, hemoglobin $(\mathrm{OR}=$ 4.6, $\mathrm{p}=0.03)$, admission history ( $\mathrm{OR}=6.2, \mathrm{p}=0.01)$, and ascites (OR $=10.7, p=0.001$ ) were the significant predictors of hepatocellular carcinoma development. Cirrhosis, family history of HBV, serum albumin, serum alkaline phosphatase, smoking history, platelet count, and WBC count were selected to set up a decision tree as the prediction model. Decision tree algorithm showed high HCC prediction [96\% (95\% CI, 93.098.0)]. Decision tree algorithm show better area under the curve (AUC) than other analysis. Conclusions: Proposed decision tree algorithm produces high accuracy for prediction of hepatocellular carcinoma in chronic hepatitis B patients. Keywords: Hepatocellular carcinoma, Hepatitis B.

\section{PE-036 \\ Peri-Transplant Change in AFP Level Is a Useful Predictor of Hepatocellular Carcinoma Recurrence Following Liver Transplantation \\ Tae Yoo ${ }^{1,2}$, Kwang-Woong Lee ${ }^{1}$, Nam-Joon Yi ${ }^{1}$, YoungRok Choi ${ }^{1}$, Hyeyoung Kim ${ }^{1}$, SukWon Suh ${ }^{1}$, JaeHong Jeong ${ }^{1}$, JeongMoo Lee ${ }^{1}$, Kyung-Suk Suh ${ }^{1}$ \\ ${ }^{1}$ Department of Surgery, Seoul National University College of Medicine 101 Daehak-no, Jongno-gu, Seoul, Republic of Korea; ${ }^{2}$ Department of Surgery, Hallym University College of Medicine, Hwaseong, Republic of Korea}

Aims: Pretransplant alpha-fetoprotein (AFP) is a useful tumor marker predicting recurrence of HCC. Little is known, however, about the relationship between changes in AFP concentration and prognosis. This study investigated the clinical significance of change in peri-transplant AFP level as a predictor of HCC recurrence. Methods: Data from 125 HCC patients with elevated pretransplant AFP level who underwent LT between February 2000 and December 2010 were retrospectively reviewed. Results: Patients with AFP normalization within 1 month after LT were classified into the rapid normalization group $(\mathrm{n}=97)$, with all other patients classified into the non-rapid normalization group $(\mathrm{n}=28)$. Tumor recurrence was observed in 17 of the 25 patients (17.5\%) with rapid normalization; of these, 11 had high and six had normal AFP levels at recurrence. In contrast, tumor recurrence was observed in 24 of the 28 patients (85.7\%) without rapid normalization, with all 24 having high AFP levels at recurrence. Multivariate analysis showed that non-rapid normalization (HR $=4.41, p<0.001)$, $\operatorname{sex}(\mathrm{HR}=3.26, p=0.03)$, tumor size ( $\mathrm{HR}=1.15, p=0.001)$, and microvascular invasion (HR = 2.65, $p=0.005$ ) were independent risk factors for recurrencefree survival. Conclusions: Rapid normalization of post-LT AFP level at 1 month is a useful clinical marker for HCC recurrence. Special strategies are needed for patients who do not show rapid normalization. 


\section{PE-037 \\ Challenges in Using Web-Based Survey Systems for Asian American Cancer Survivors}

Soo Jin Lee 1 , Yun $\mathrm{Hu}^{2}$, Hyeoung Park ${ }^{3}$, Eunji Cho ${ }^{3}$, Ayako Inohara, Eunice Chee ${ }^{3}$, Wonshik Chee ${ }^{3}$, Eun-Ok $\mathrm{Im}^{3}$

${ }^{1}$ Korea National Open University, Seoul, South Korea; ${ }^{2}$ Shanghai Jiaotong University, China; ${ }^{3}$ Duke University, USA

Aims: With an increasing number of racial/ethnic minorities in the cancer research field, using of multiple languages in web-based survey system become essential and frequently adopted to generate multilingual version of web-based questionnaires. However, little is still known about the technological challenges in implementing the web-based survey systems in multilingual cancer research. The purpose of this study is to explore challenges in using multiple language versions (English, Mandarin Chinese, Korean, and Japanese) of web-based survey system in Asian Americans cancer survivorship research. Methods: For the web-based survey system, the REDCap system supported by the university to which the study team belongs was used. Throughout the study, team members kept written records of the challenges. The challenges were discussed in the team meeting. Then, the written records and team meeting memos were analyzed using the content analysis. Results: The five challenges were categorized: (a) necessities to modify the instructions given by original instruments; (b) necessities to manage different versions across institutes; (c) limitations of system usage other languages than English; (d) difficulties in ensuring consistencies across different languages; (e) lack of IT team members with experience in multilingual survey. Therefore, the future researcher need to (a) check the instructions in existing instruments, (b) be aware of technological limitations and strategize the methods to deal with the limitations, (c) set strategies to ensure consistencies across different languages and different versions of Web-based survey systems. Conclusions: The use of Web-based system in the cancer research is highly feasible for multiple languages. However, we need various strategies for unexpected challenges due to cultural and industrial differences. Keywords: Web-based Survey System, Asian American Cancer Survivors, Multilingual cancer research,

\section{PE-038 \\ Association of Non-Alcoholic Fatty Liver Disease with Hepatocellular Carcinoma}

Jung Hwan Yu, Young-Joo Jin, Jin-Woo Lee

Inha University Hospital, Inha University School of Medicine

Aims: We intended to evaluate the association between non-alcoholic fatty liver disease (NAFLD) and hepatocellular carcinoma (HCC) development between 2005 and 2015 in South Korea. Methods: We retrospectively analyzed 1,327 patients initially diagnosed with HCC at our institution between January 2005 and December 2015. Patients with other malignancies in addition to HCC were excluded. During these period, changes in the proportion of NAFLD-associated HCC among all HCCs were evaluated. Furthermore, the clinical characteristics of NAFLD-associated HCC were assessed. Results: Among the 1,327 subjects, HBV was the most common (65.5\%) cause of HCC, and the overall rate of NAFLD-associated HCC was $4.7 \%$. As compared with HBV-associated HCC patients, NAFLD-associated HCC patients were older, had a higher median body mass index, and a larger median tumor size ( $p$-values for all <0.05). Liver cirrhosis was less frequent in NAFLD-associated than in HBV-associated HCC patients $(\mathrm{p}<0.05)$. The annual proportions of NAFLD-associated HCC patients were $3.4 \%$ in $2005,3.6 \%$ in $2006,3.5 \%$ in $2007,3.2 \%$ in $2008,4.2 \%$ in $2009,4.4 \%$ in $2010,5.6 \%$ in $2011,5.2 \%$ in $2012,5.8 \%$ in $2013,7.0 \%$ in 2014 , and $6.7 \%$ in 2015 . From 2008 to 2015, the percentage of them steadily increased. Conclusions: Annual proportion of NAFLD-associated HCC patients among all HCC patients ranged from 3.2\% to $3.5 \%$ before 2008, but thereafter, it increased gradually and had doubled to $7.0 \%$ by 2014 . In addition, NAFLD-associated HCC was found to develop more commonly in non-cirrhotic liver and to present with larger tumor sizes than those of HCCs associated to other causes. Keywords: Hepatocellular carcinoma, Non-alcoholic fatty liver disease. 


\section{Molecular Pathogenesis and Pathology / Staging and Prognosis}

\author{
PE-039 \\ Establishment of 3D Multicellular Tumor \\ Spheroids (MCTS) using Hepatocellular \\ Carcinoma Cell Lines and Stromal Cells for \\ Screening Anti-Cancer Therapeutic Agents \\ Kyungjoo Cho ${ }^{1,2}$, Hyuk Moon 1,2, Soonyoung Shin ${ }^{1,2}$, \\ Simon W. Ro ${ }^{2}$, Hye Won Lee ${ }^{2,3}$, Beom Kyung Kim ${ }^{2,3}$, \\ Do Young Kim ${ }^{2,3}$, Kwang-Hyub Han ${ }^{1-3}$ \\ ${ }^{1}$ Brain Korea 21 PLUS project for Medical Science \\ College of medicine, ${ }^{2}$ Yonsei Liver Center, Severance \\ Hospital, ${ }^{3}$ Department of Internal Medicine, Yonsei \\ University College of Medicine, Seoul, South Korea
}

compactness of tumor spheroids, while Hep3B MCTS had the highest compactness. Of note, YAP/TAZ levels in HCC MCTS were significantly different. SNU449 MCTS model had a low level of YAP/TAZ, while Hep3B MCTS model had the highest level of YAP/TAZ expression. Drug penetration into tumor spheroids was significantly retarded due to the multi-cellular components within HCC MCTS. HCC MCTS with higher YAP/ TAZ levels increased the compactness and inhibited drug penetration. Conclusions: In this study, diverse MCTS models have been developed using HCC of different degrees of differentiation and stromal cells such as HSCs, fibroblasts, and endothelial cells. MCTS with poorly differentiated HCC showed an increased compactness of spheroids, an elevated level of YAP/ TAZ and a limited drug penetration. Reducing tumor compactness or stromal activation should be considered to improve a response to chemotherapy in patients with advanced HCC. Keywords: MCTS (multicellular tumor spheroid), 3D cell culture, Tumor microenvironment, YAP/TAZ, Drug screening.

\section{PE-040 \\ Activated HSCs Upregulate NR4A2 Expression in ICC and Thereby Promote the Aggressiveness of ICC \\ Yi-Peng Fu, Chu-Yu Jing, Yong Yi, Jin-Long Huang, Wei Gan, Shuang-Jian Qiu \\ Liver Cancer Institute, Zhongshan Hospital and Shanghai Medical School, Fudan University, Key Laboratory for Carcinogenesis and Cancer Invasion, The Chinese Ministry of Education, Shanghai 200032, People's Republic of China}

Aims: To investigate the expression pattern and prognostic significance of NR4A2 in ICC patients and the role of NR4A2 on malignant behaviors of ICC cells. Methods: Expession levels of NR4A2 and its downstream target genes in ICC cells were analyzed by western blot and qRT-PCR. The expression pattern of NR4A2 in ICC tissues was analyzed through IHC. The function profiles of NR4A2 overexpressed ICC cells were studied by CCK-8 assay, wound healing assay and transwell assays. The cell culture supernatant was analyzed by human magnetic luminex assay. Results: The expression of NR4A2 was significantly upregulated in aHSCsprimed ICC cell lines. Overexpression of NR4A2 was identified as an independent risk factor in ICC patients underwent curative resection. Upregulation of NR4A2 promoted the proliferation, migration and invasion ability of ICC cells. Upregulation of NR4A2 enhanced the expression level of OPN, and downregulation of NR4A2 also inhibited the expression of OPN. Moreover, NR4A2 promoted EMT of ICC through upregulating vimentin and $\beta$-catenin and downregulating E-cadherin. Conclusions: aHSCs may promote ICC progression through NR4A2 mediated upregulation of OPN and EMT.

Keywords: ICC, NR4A2, OPN, EMT.
MCTS were heterogeneous depending on the differentiation degree. Well differentiated SNU449 MCTS showed the least 

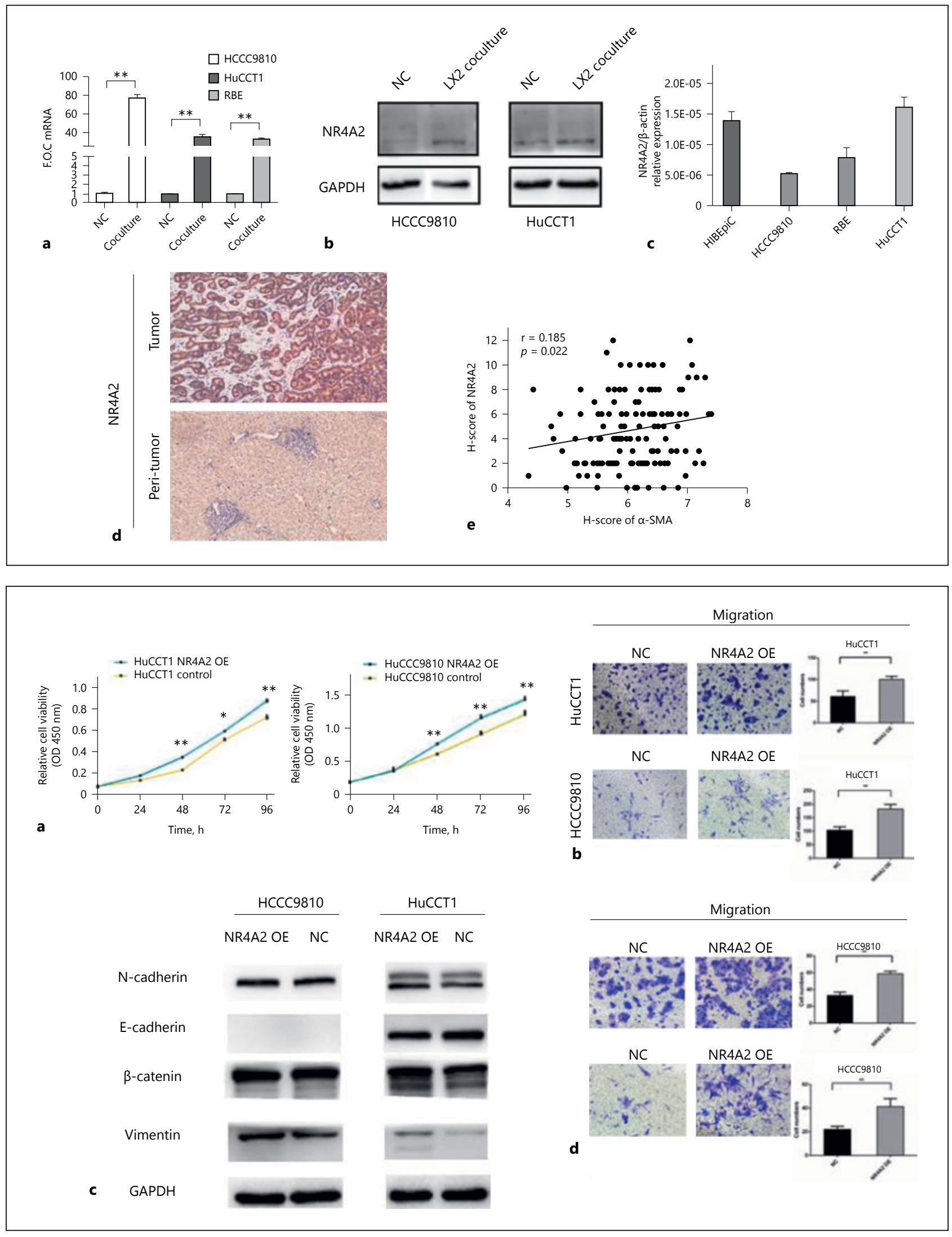

Fig. 1, 2. (for Abstract no PE-040). 


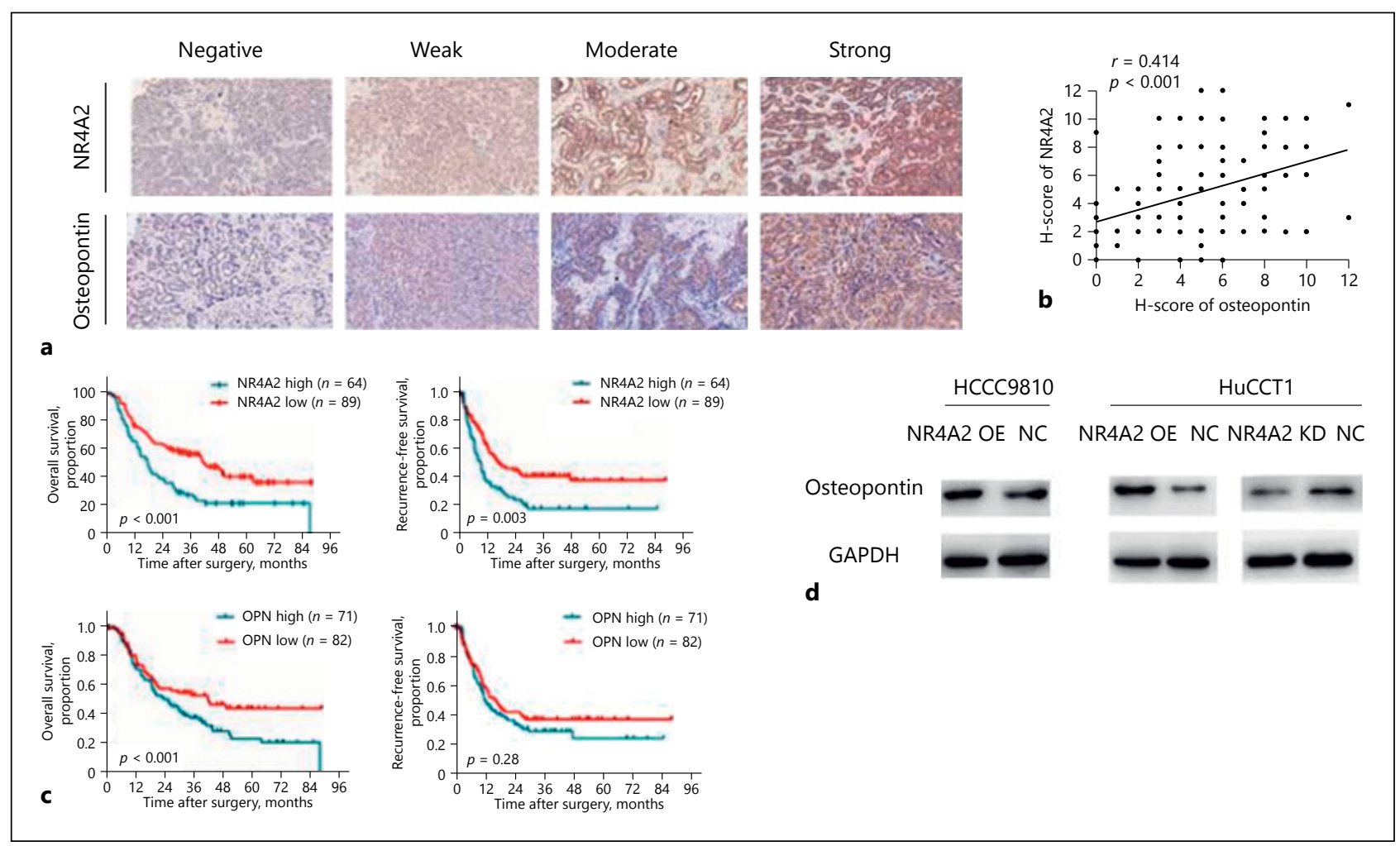

Fig. 3. (for Abstract no PE-040).

\section{PE-041 \\ Autophagy Inhibitor, Chloroquine Did Not Affect Tumor Development in a Transgenic Mouse Model of HCC}

Soonyoung Shin ${ }^{1,2}$, Hyuk Moon 1,2, Kyungjoo Cho ${ }^{1,2}$, Simon W. Ro, Beom Kyung Kim 2,3, Seung Up Kim ${ }^{2,3}$ Do Young Kim ${ }^{2,3}$, Kwang-Hyub Han ${ }^{1-3}$

${ }^{1}$ Brain Korea 21 PLUS project for Medical Science College of medicine, ${ }^{2}$ Yonsei Liver Center, Severance Hospital, ${ }^{3}$ Department of Internal Medicine, Institute of Gastroenterology, Yonsei University College of Medicine, Seoul, South Korea

Aims: Autophagy is an intracellular recycling process by which damaged or superfluous proteins are delivered to lysosomes for degradation, and then utilized as energy resources and macromolecular precursors. Autophagy in cancer is a highly debated subject. Research has shown that autophagy can become either tumor-promoting or tumor-suppressive depending on cellular or genetic context. Here, we investigated the role of autophagy in hepatocellular carcinoma (HCC) by applying an autophagy inhibitor, chloroquine to a transgenic mouse model of HCC. Methods: Transposons were constructed encoding an activated from of RAS (HRAS ${ }^{\mathrm{G} 12 \mathrm{~V}}$ ) and short hairpin suppressing P53 (shp53). Transposons were hydrodynamically delivered to livers of 6-week-old C57BL/6 mice. Mice were administered intraperitoneally with chloroquine at a daily dose of $60 \mathrm{mg} / \mathrm{kg}$ for five weeks. Control mice were given a phosphate buffered saline (PBS). Mice were monitored at least twice per week. Results: The sizes and numbers of tumor nodules were similar between chloroquine group and control when livers were harvested at 5 weeks after the delivery of oncogenes. Animal survivals were not significantly different between the two groups, suggesting that the treatment with chloroquine does not affect liver tumorigenesis induced by HRAS ${ }^{\mathrm{G} 12 \mathrm{~V}}$ plus shp53. Conclusions: Our study suggests that autophagy inhibition has a minimal role in HCC under the genetic context of RAS signaling activation and P53 downregulation. Keywords: Autophagy, Chloroquine, Transgenic mouse model, HCC. 


\section{PE-042 \\ Control of Intracranial Disease Is Associated with Improved Survival for Patients with Brain Metastases from Hepatocellular Carcinoma}

Pil Soo Sung ${ }^{1}$, Dong Jin Yoon ${ }^{1}$, Do Seon Song ${ }^{1}$, Jung Hyun Kwon', Soon Woo Nam'1, Jeong Won Jang', Jong Young Choi', Seung Kew Yoon', Seok Whan Moon ${ }^{2}$, Hong Seok Jang ${ }^{3}$, Si Hyun Bae

${ }^{1}$ Liver Research Center, Department of Internal Medicine, The Catholic University of Korea, Seoul, South Korea; ${ }^{2}$ Department of Thoracic and Cardiovascular Surgery, Seoul St. Mary's Hospital, Seoul St. Mary's Hospital, The Catholic University of Korea College of Medicine, Seoul, South Korea; ${ }^{3}$ Department of Radiation oncology, Seoul St. Mary's Hospital, Seoul St. Mary's Hospital, The Catholic University of Korea College of Medicine, Seoul, South Korea.

Aims: We performed a retrospective study to identify prognostic factors and determine outcomes for patients with brain metastases from hepatocellular carcinoma (HCC). Methods: A total of 72 patients with brain metastases from HCC were identified from two institutions; 26 of 72 patients received tumor-removing surgery or stereotactic radiosurgery with or without whole-brain radiotherapy (WBRT), 23 of 72 received WBRT only, and 23 of 72 received conservative treatment. Estimates for overall survival (OS) after brain metastases were determined and clinical prognostic factors were identified by Cox proportional hazards modeling. Results: Median OS after development of brain metastases was 48 days (one day to 536 days). The median age at the time patients were diagnosed with brain metastasis was 54 years.
Fifty-one patients (71\%) were male, and 62 patients (86\%) were infected with hepatitis B virus. Median time from diagnosis of HCC to brain metastasis was 17.2 months, and 4 patients had brain metastases at the time diagnosis. Intracranial hemorrhage was frequently associated $(42 \%)$ with brain metastasis. The most common presenting symptoms were motor weakness, mental change, and headache. Sixtythree patients (88\%) had lung metastases, 25 patients (35\%) had bone metastases, 18 patients (25\%) had metastatic lymphadenopathy, and $7(10 \%)$ patients had peritoneal seeding when diagnosed with brain involvement, and there was only one patient without metastasis to other organs. Univariate analyses showed that treatment with curative intent (surgery or SRS) and serum AFP levels were associated with improved survival $(\mathrm{P}<0.001$, and 0.045 , respectively), whereas a single brain metastasis, size of metastatic tumor, or intracranial hemorrhage were not. We further divided patients with three groups by the treatment modality; surgery or radiosurgery (group1), WBRT (group 2), or conservative treatment (group 3). Group 1 showed best survival, followed by group 2 and group 3 , sequentially $(\mathrm{P}<0.001)$. Subgroup analysis with patients with single brain metastasis showed similar results. Multivariate analysis showed that treatment modality was the only factor that is associated with improved OS $(P=0.001)$ Conclusions: When patients with lung metastases from HCC present neurologic symptoms, brain imaging is required to confirm intracranial metastases. Although the overall prognosis of patients with brain metastases from HCC is extremely poor, patients actively treated with surgery or radiosurgery have prolonged survival, suggesting that interventions to control intracranial disease are important treatment modalities. Keywords: Hepatocellular carcinoma, Brain metastasis, Radiation treatment,

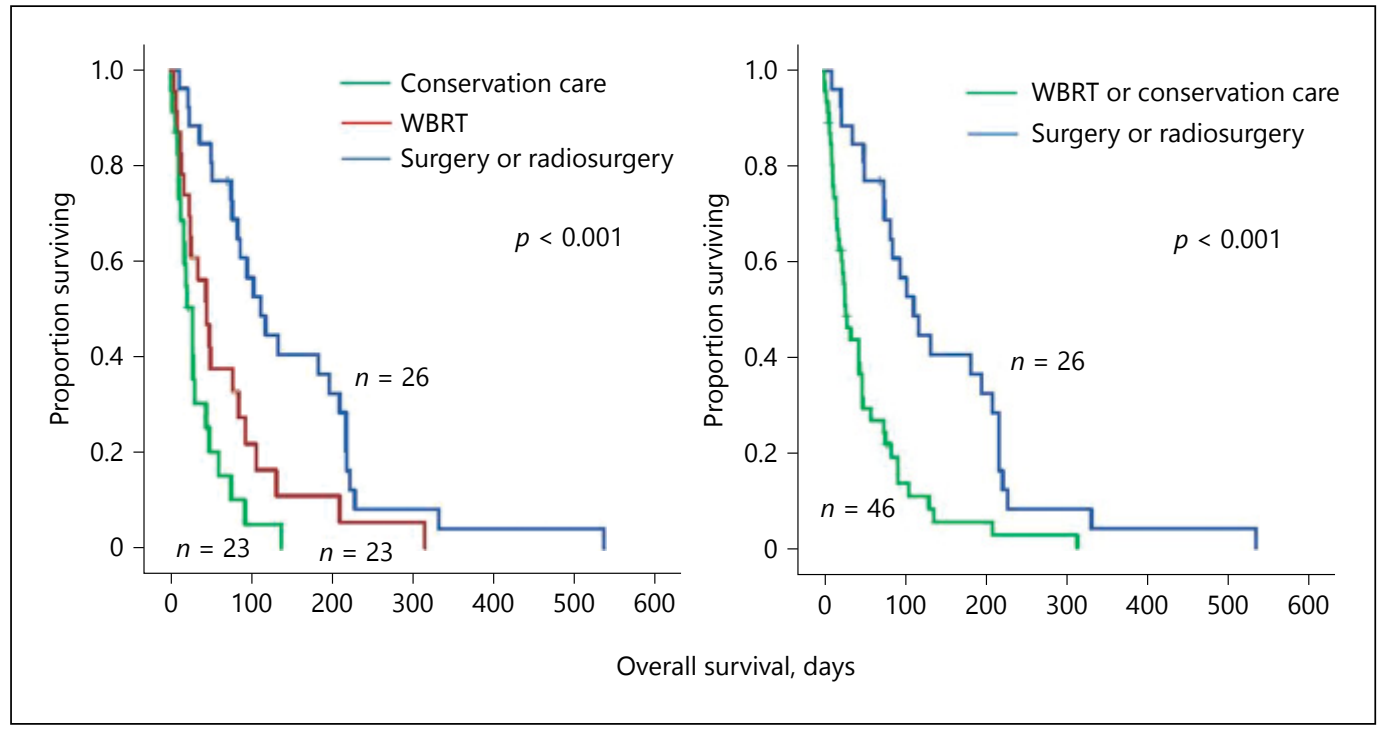

Fig. 1. (for Abstract no PE-042). 


\section{PE-043 \\ Differences in Presentation and Management Patterns in Patients with Hepatocellular Carcinoma (HCC) between China and Singapore: Initial Data from the HCC Registry in Asia}

Pierce K. H. Chow', Lequn Li $i^{2}$, Jiangtao $L i^{3}$, Poh-Seng Tan ${ }^{4}$, Fan Jia ${ }^{5}$, Shukui Qin ${ }^{6}$, Yvanka Marie Gilliam ${ }^{7}$, Joanna Zhi Jie Ling ${ }^{8}$, Mihir Gandhi, on behalf of Asia-Pacific Hepatocellular Carcinoma (AHCC) Trials Group

${ }^{1}$ National Cancer Centre Singapore ${ }^{2}$ Guangxi Medical University Cancer Center ${ }^{3}$ Second Affiliated Hospital Zhejiang University School of Medicine ${ }^{4}$ National University Hospital ${ }^{5}$ Zhongshan Hospital, Fudan University ${ }^{6}$ Nanjing Bayi Hospital ${ }^{7}$ IQVIA ${ }^{8}$ Singapore

Clinical Research Institute Pte Ltd

Aims: To identify and elucidate the differences in the manifestation and patient management of HCC in patients from China and Singapore. Methods: The HCC Registry in Asia (AHCC08) is an investigator-initiated multi-country longitudinal cohort study of patients newly diagnosed with HCC between 2013 and 2018 in Asia-Pacific (ClinicalTrials.gov identifier: NCT03233360). Planned sample size is 2500 patients from 9 countries. We present initial data on diagnosis, etiology, stage at presentation, and treatment modalities of HCC from China [CN] (4 centers) and Singapore [SG] (2 centers). Results: 174 patients (132 CN; 42 SG) were enrolled till December, 2017. Patients from China were younger (mean age 52 years $\mathrm{CN}$; 67 years SG), with a higher proportion of regular alcohol drinkers ( $46 \% \mathrm{CN} ; 21 \% \mathrm{SG}$ ). $93 \%$ and $41 \%$ of patients had hepatitis B in China and Singapore, respectively. Most Singapore patients were diagnosed by imaging criteria [AASLD/APASL] (98\% SG; 14\% CN). Patients in China present at later Barcelona clinic liver cancer [BCLC] stages (stage A: $32 \%$ CN, $48 \%$ SG; stage B: $11 \%$ CN, 21\% SG). Few radiofrequency ablation [RFA] were carried out as the first-line therapy in China $(<2 \% \mathrm{CN} ; 12 \% \mathrm{SG})$. Liver resection was preferred first-line HCC treatment in China $(61 \% \mathrm{CN} ; 17 \%$ SG), followed by loco-regional therapy (TACE/TAE/TAI 30\% CN; TACE/TAE/Y90 38\% SG) and systemic therapy $(10 \% \mathrm{CN}$; $19 \%$ SG). Conclusions: There is considerable variation in presentation and management patterns between China and Singapore. These data on real world practice will be useful to health policymakers, pharmaceutical companies and clinicians in improving public health policies and developing treatment strategies for HCC. Keywords: Hepatocellular carcinoma, Registry, Liver, Management.

\section{PE-044 \\ Pathological Response, Rather Than Radiological Response, to Transarterial Chemoembolisation for HCC Predicts Survival after Surgery}

Pil Soo Sung ${ }^{1 *}$, Keungmo Yang ${ }^{1 *}$, Young Kyoung You', Dong Goo Kim², Jung Suk Oh${ }^{3}$, Ho Jong Chun ${ }^{3}$, Seawon Hwang ${ }^{1}$, Jeong Won Jang ${ }^{1}$, Si Hyun Bae ${ }^{1}$, Jong Young Choi', Seung Kew Yoon

${ }^{1}$ Department of Internal Medicine, Seoul St. Mary's Hospital, The Catholic University Liver Research Center, The Catholic University of Korea, Seoul, Republic of Korea; ${ }^{2}$ Department of Surgery, Seoul St. Mary's Hospital, The Catholic University of Korea, Seoul, Republic of Korea; ${ }^{3}$ Department of Radiology, Seoul St. Mary's Hospital, The Catholic University of Korea, Seoul, Republic of Korea

Aims: In this study, we aimed to confirm the prognostic significance of a pathological response (PR) achieved by preoperative transarterial chemoembolisation (TACE) for hepatocellular carcinoma (HCC) preceding liver resection (LR) or liver transplantation (LT). Methods: Between 2005 and 2016, 124 patients underwent preoperative TACE before LR, and 166 patients underwent preoperative TACE before LT. The PR was defined as the mean percentage of necrotic tumor area within each tumor. A complete PR (CPR) was defined as the absence of viable tumor in the surgical specimens. Results: A total of $34(27 \%)$ and $38(23 \%)$ patients had CPR before LR and LT, respectively. Five-year overall survival (OS) was higher in patients with CPR compared to those without, after LR ( $87 \%$ vs $63 \%, P=0.005)$ and LT ( $91 \%$ vs $75 \%, P=0.021)$. The 5-year recurrence-free survival (RFS) rates were also significantly higher in patients with CPR $(71 \%$ vs $38 \%$ after LR, $P=0.001$ and $97 \%$ vs $75 \%$ after LT, $P=0.008$ ). Among patients with complete radiological remission, patients with CPR had better prognosis than those without CPR. Subgroup analyses showed that PR $\geq 90 \%$ predicted OS and RFS in patients with single resected tumor. On multivariate analyses, $\mathrm{PR} \geq 90 \%$ remained an independent factor for better OS and RFS in both groups. Independent factors associated with CPR were a preoperative alpha-fetoprotein (AFP) level $<100 \mathrm{ng} /$ $\mathrm{mL}$ and single tumor. Conclusions: Overall, PR rather than radiological response after TACE reflects the biological nature of the tumor. CPR or nearly CPR improves long-term survival after LR and LT independently of other pathological and clinical variables. Keywords: Hepatocellular carcinoma, Transarterial chemoembolization, Liver resection, Liver transplantation. 


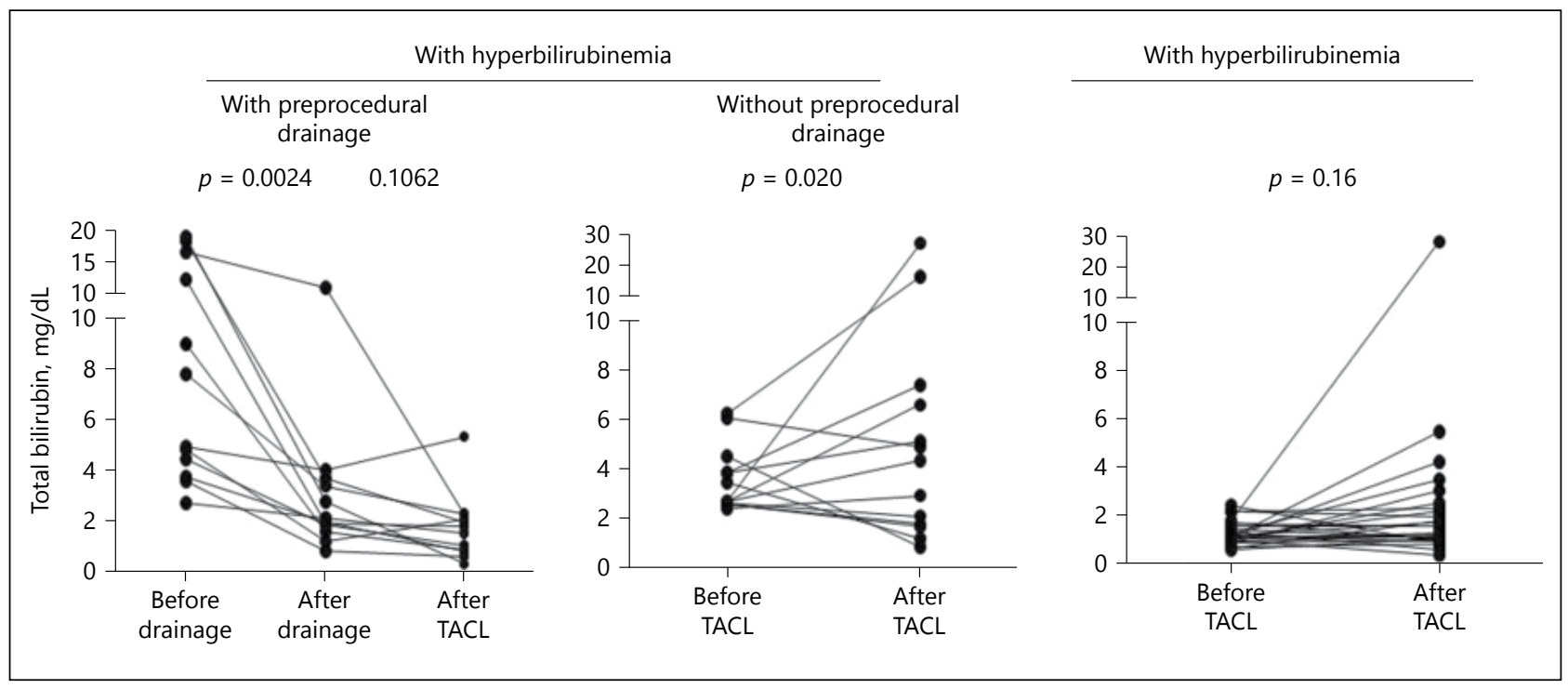

Fig. 1. (for Abstract no PE-044).

\section{PE-045 \\ Comparison of Clinical Outcomes Following Hepatic Resection in NAFLD-Related Hepatocellular Carcinoma and HBV-Related Hepatocellular Carcinoma: Propensity Score-Matched Analysis}

Yoon Bin Jung ${ }^{1}$, Dai Hoon Han ${ }^{1}$, Kyung Sik Kim 1 , Jin Sub Choi', Do Young Kim², Jeong Eun Yoo', Young Nyun Park ${ }^{3}$, Gi Hong Choi ${ }^{1}$

${ }^{1}$ Departments of General Surgery, Yonsei University College of Medicine, Seoul, South Korea; ${ }^{2}$ Department of Internal Medicine, Institute of Gastroenterology, Yonsei University College of Medicine, Seoul, South Korea; ${ }^{3}$ Department of Pathology, Yonsei University College of Medicine, Seoul, South Korea

Aims: Since it was first reported in 1990, NAFLD-related HCC has increased with the increase in metabolic syndrome. In the prior study, the prognosis for NAFLD-related HCC is controversial, and a comparison with HBV-HCC is insufficient. Clinical characteristics of HCC caused by NAFLD or HBV were compared in a retrospective analysis. Methods: A total of 338 patients underwent hepatic resection for HCC in Severance Hospital between 2005 and 2015 were retrospectively reviewed. Metabolic syndrome is defined according to the NCEP-ATP III report. Occult HBV infection was identified by nested PCR analysis using known primers. Propensity analysis was carried out using logistic regression in order to minimize lead-time bias caused by lack of surveillance in NAFLD patients. Surgical and oncologic outcomes were compared between two groups. Results: Among 338 patients underwent hepatectomy for HCC, 23 (6.9\%) had NAFLD-HCC. Before matched analysis, cirrhosis was more detected in HBV-HCC patient. (30.4 vs $61.3 \%, p=0.004$ ). Surgical outcomes were similar between the two groups, especially in morbidity rates ( 22.0 vs $26.1 \%, \mathrm{p}=0.795)$, in hospital stay ( 12.0 vs 13.4 days, $\mathrm{p}=0.379$ ) and in intraoperative bleeding ( $495 \mathrm{vs} 442 \mathrm{~mL}, \mathrm{p}=$ $0.659)$. Tumor size is larger in the NALFD group than in the HBV group ( 4.6 vs $3.3 \mathrm{~cm}, \mathrm{p}=0.004$ ). After a median follow-up of 63.0 months (range 0-139 months), although not statistically significant, the survival analyses after propensity scorematching showed similar 5-year overall survival (44.3 vs $44.3 \%, \mathrm{p}=0.317$ ) and recurrent-free survival rates (67.4 vs $65.1 \%, p=0.483$ ) between the two groups. Conclusions: In NAFLD group, the tumor size is larger than HBV group at the time of diagnosis, but there were no statistically significant differences in the long-term outcomes between the two groups after matched analysis for tumor size. These results might result from a delayed diagnosis in patients with a more advanced tumor stage rather than to a more aggressive biology of tumor. Keywords: NAFLD, NASH, Metabolic syndrome, HCC. 


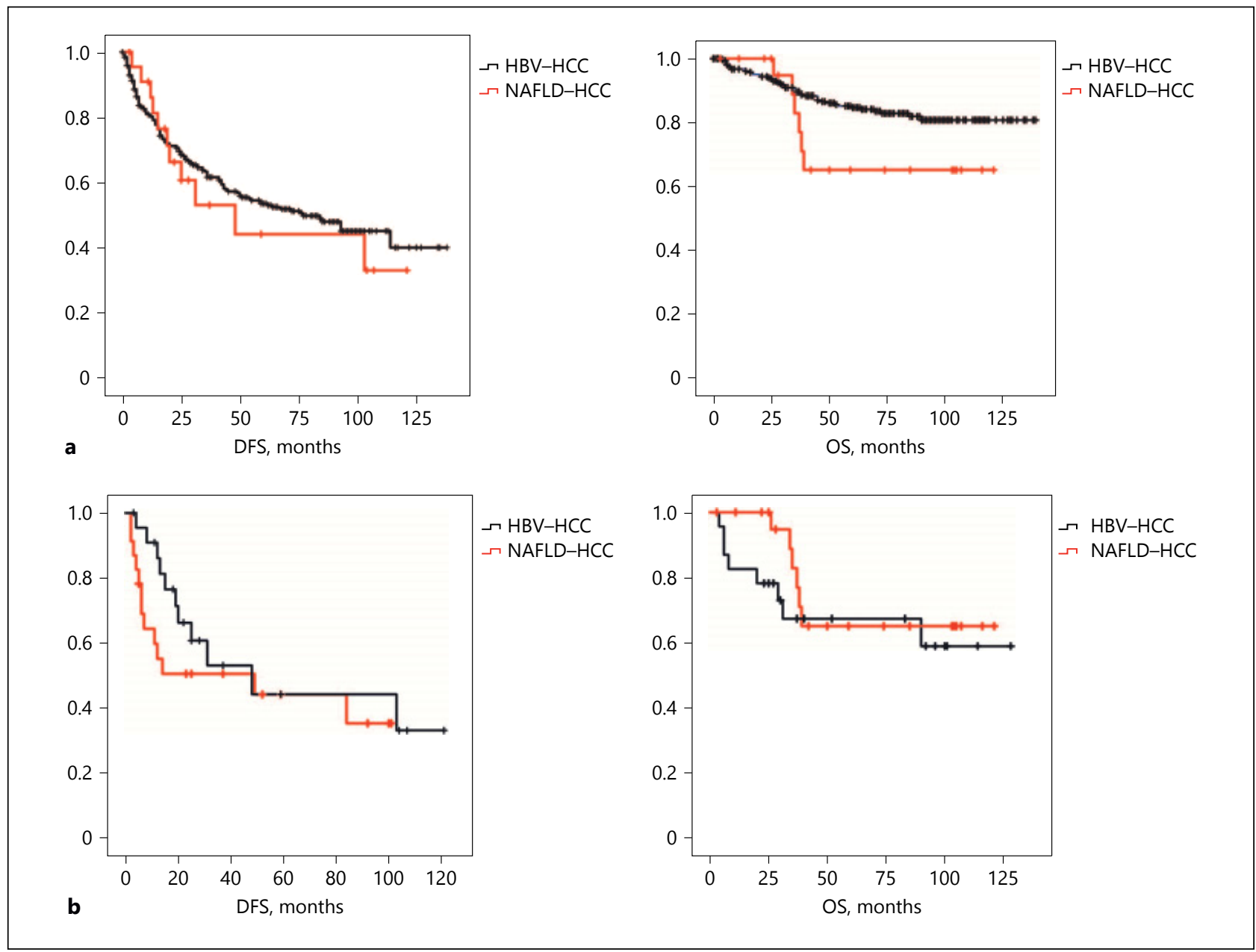

Fig. 1. Survival analysis after hepatectomy for HCC in the HBV and NAFLD group. a Disease-free survival (5YR DFS 53.7 vs $44.3 \%, \mathrm{p}=0.651$ ) and overall survival (5YR surv ival $84.7 \mathrm{vs}$ $65.1 \%, p=0.108$ ) before matched analysis; b Disease-free

\section{PE-046}

A Comparative Study between PlateletAlbumin-Bilirubin (PALBI) and AlbuminBilirubin (ALBI) Grade in Predicting Overall Survival for Hepatocellular Carcinoma

Soon Kyu Lee ${ }^{1}$, Seok Hwan Kim¹, Myeong Jun Song ${ }^{123 *}$

${ }^{1}$ The Catholic University Liver Research Center, Department of Internal Medicine, Daejeon St. Mary's Hospital, College of Medicine College of Medicine, The Catholic University of Korea, Seoul, Korea, ${ }^{2}$ Korean Liver Cancer Study Group, ${ }^{3}$ Ministry of Health and Welfare, Korea Central Cancer Registry

Aims: Child-Turcotte-Pugh (CTP) class is well-known grade to reflect the liver function in hepatocellular carcinoma (HCC). Recently, albumin-bilirubin (ALBI) and platelet- survival (5YR DFS 44.3 vs $44.3 \%, \mathrm{p}=0.317$ ) and overall survival (5YR survival 67.4 vs $65.1 \%, p=0.483$ ) after matched analysis. (for Abstract no PE-045).

albumin-bilirubin (PALBI) was introduced to access the liver function and predict survival. We aimed to validate the PALBI grade and compare with ALBI grade on overall survival (OS) and its applicability according to treatment modalities. Methods: The 6,669 patients were randomly extracted from the national cohort of Korean Central Cancer Registry during 2008-2012. Of them, the 6,507 patients were evaluated on OS with CTP class, model for end stage liver disease (MELD), ALBI and PALBI grade. Moreover, OS according to treatment modality was evaluated with PALBI grade and compared with ALBI grade, MELD, and CTP class. Results: The mean age was 59.7 years and $79.1 \%$ were male. The major cause of HCC was hepatitis B (63.4\%) and majority was CTP class A $(71.8 \%)$. Median survival time of PALBI grade 1 (38.4\%), grade 2 (33.2\%) and grade 3 (28.4\%) were 81, 30 and 5 months, respectively $(\mathrm{P}<0.001)$. PALBI grade had higher area under receiver-operator-characteristic curve (AUC) at 1, 3, 5 years- 
mortality than CTP class, MELD grade and ALBI grade (overall AUC; 0.722 vs. $0.633,0.645,0.642$, respectively, $\mathrm{P}<0.001$ ). In multivariate analysis, PALBI and ALBI grade were also significant risk factor for overall survival. Comparing ALBI and PALBI grade according to treatment modalities, PALBI could predict survival in treatment of trans-arterial chemoembolization and supportive care. Meanwhile, ALBI grade could predict survival in treatment of surgical resection, radiofrequency ablation. Conclusions: This study showed PALBI and ALBI grade was reliable model to access liver function and survival. According to treatment modalities, ALBI may predict OS better in curative treatment. On the other hand, PALBI showed more applicable to the locoregional therapy. Keywords: Hepatocellular carcinoma, Survival, PALBI, ALBI.

\section{PE-047 \\ Refractoriness to Transarterial Chemoembolization in Patients with Recurrent Hepatocellular Carcinoma after Curative Resection}

Mi Young Jeon ${ }^{1,2}$, Hye Soo Kim, ${ }^{1}$ Beom Kyung Kim, ${ }^{1,2}$ Jun Yong Park, 1,2 Do Young Kim, 1,2 Sang Hoon Ahn, ${ }^{1,2}$ Kwang-Hyub Han ${ }^{1,2}$, Seung Up Kim ${ }^{1,2}$

${ }^{1}$ Department of Internal Medicine, Institute of Gastroenterology, Yonsei University College of Medicine, Seoul, South Korea, ${ }^{2}$ Yonsei Liver Center, Severance Hospital, Seoul, South Korea

Aims: Identification of patients who are refractory to transarterial chemoembolization (TACE) used to treat hepatocellular carcinoma (HCC) is important. We investigated predictors of poor treatment outcomes in patients with recurrent HCC treated with TACE after curative resection. Methods: A total of 428 patients with recurrent HCC after curative resection who were treated with TACE between 2006 and 2015 were enrolled (median follow-up, 59.2 months). Results: The median age of the study population (362 males and 66 females) was 59.2 years, and the median time from resection to the first TACE was 17.8 months. On multivariate analysis, more than two TACE procedures within the first 6 months ( $\mathrm{HR}=1.898)$, and the des-gamma carboxyprothrombin level (hazard ratio $[\mathrm{HR}]=1.000$ ) were independently predictors of the progression to BCLC stage $C$ in patients with BCLC stage 0 -B (both $P<0.05$ ). In addition, more than two TACE procedures within the first 6 months $(\mathrm{HR}=1.863)$, the serum albumin level, an alpha-fetoprotein $>100 \mathrm{ng} / \mathrm{mL}$, and multiple tumors, were identified as an independent predictor of mortality in the entire study population (all $P<0.05$ ). The cumulative incidence of stage progression to BCLC stage $C$ of patients with BCLC stage 0 -B and that of mortality of the entire study population were significantly higher in patients undergoing more than two TACE procedures within the first 6 months compared with those undergoing fewer procedures (log-rank, $P=0.003$, and $P<0.001$, respectively). Conclusions: More than two TACE procedures within the first 6 months may indicate refractoriness to TACE in patients with recurrent HCC after curative resection. Thus, if patients meet this criterion, a switch to other treatments should be considered. Keywords: TACE, Hepatocellular carcinoma, Refractoriness, Curative resection

\section{PE-048 \\ Hepatocellular Carcinoma Risk in Chronic Hepatitis B Patients Receiving Entecavir or Tenofovir}

Jung Hwan Yu, Young-Joo Jin, Jin-Woo Lee

Digestive Disease Center, Department of Internal

Medicine, Inha University Hospital, Inha University

School of Medicine, Incheon, South Korea

Aims: We intended to analyze the incidence rate of hepatocellular carcinoma (HCC) in chronic hepatitis B (CHB) patients treated with entecavir or tenofovir in South Korea, and to identify predictors of HCC development in these patients. Methods: We retrospectively 582 CHB patients initially received entecavir $(\mathrm{n}=406,69.8 \%)$ or tenofovir $(\mathrm{n}=$ 176, 30.2\%) for CHB between January, 2007 and December, 2015 Results: During a median follow-up of 57.1 months, HCC developed in 38 (6.5\%) of the 582 patients, regardless of antiviral agent type. Entecavir and tenofovir treated patients had similar HCC development rates $(\mathrm{p}=0.471)$. For the 582 patients, 2-, 4- and 6-year cumulative HCC development rates were $2.6 \%, 4.4 \%$, and $8.3 \%$, respectively, and the $2-$, $4-$, and 6-year cumulative HCC development rates of patients with liver cirrhosis were significantly greater than those of patients without liver cirrhosis $(6.2 \%, 9.8 \%$, and $18.4 \%$ vs. $0.3 \%, 1.1 \%$, and $2.2 \%$, respectively, $\mathrm{p}<0.001$ ). Older ( $\geq 60$ years) patients regardless of the presence of cirrhosis and cirrhotic patients with age of $\geq 40$ years showed significantly higher risk of HCC development compared to others ( $p<0.05$, respectively). Multivariate analysis showed that an older age ( $\geq 50$ years; hazard ratio [HR] 5.02, $\mathrm{p}=0.009$ ), and the presence of cirrhosis (HR 4.95, p = 0.002) independently predicted HCC development. Conclusions: We found that the HCC development rate was $6.5 \%$ in CHB patients treated with ETV or TDF over a median follow-up of 6 year in South Korea. An age of $\geq 50$ and liver cirrhosis were predictors of HCC development in these patients. Keywords: Chronic hepatitis B, Hepatocellular carcinoma, Entecavir, Tenofovir. 


\section{PE-049 \\ Hepatocellular Carcinoma in Mongolia: A Multicenter Study \\ M.Batzaya ${ }^{1,4}$, Kh. Ariunaa ${ }^{2,4}$, R.Sanduijav $^{2,3}$, O. Baatarkhuu ${ }^{3,4}$ \\ ${ }^{1}$ Proliance LLC, Ulaanbaatar, Mongolia ${ }^{2}$ National Cancer Center of Mongolia ${ }^{3}$ Mongolian National University of Medical Sciences, Ulaanbaatar, Mongolia \\ ${ }^{4}$ Mongolian Association for the Study of Liver Diseases, Ulaanbaatar, Mongolia}

Aims: Hepatocellular carcinoma (HCC) is the most common cancer in Mongolia, with an occurrence of 54.1 cases per 100,000 people. We aimed to investigate the clinical features, therapeutic modalities, overall survival, and prognostic factors for Mongolian patients with HCC. Methods: 195 patients with HCC were consecutively enrolled in our study. Diagnosis of HCC was made according to the EASL guidelines Results: The mean age (108 males and 87 females) was 61.7 years. A large proportion of patients ( $n=165,84.6 \%)$ had underlying liver cirrhosis. The most common etiology for HCC was HBV infection ( $\mathrm{n}=67,34.4 \%$ ), followed by HCV infection $(\mathrm{n}=89,45.6 \%)$. The mean tumor diameter was $6.0 \pm 2.6 \mathrm{~cm}$. Only $29(14.9 \%)$ patients had a single lesion, while $39(20.0 \%)$ had $>3$ lesions. Extra hepatic metastasis to the lung $(n=23)$, bone $(\mathrm{n}=10)$, and lymph node $(\mathrm{n}=3)$ was detected in 36 (18.5\%) patients. The mean serum AFP level was $196.0 \mathrm{ng} / \mathrm{ml}$. Most patients had advanced HCC; 88 (45.1\%) in stage III and $57(29.2 \%)$ in stage IV. Surgical resection was performed in 27 (13.8\%) patients, RFA in 23 (11.8\%), and TACE in 107 (54.9\%). In $38(19.5 \%)$ patients with distant metastasis or poor liver function, the best supportive care was provided. When all the patients were categorized as 'treated' $(n=156)$ and 'not treated' $(n=39)$, the 3 years survival was significantly lower in the 'not treated' group than in the 'treated' group (11\% vs $0 \%, P<0.001)$. Tumor diameter $(<3 \mathrm{~cm}$ vs $\geq 3 \mathrm{~cm})$, extra hepatic metastasis, TNM stage (I/II $v s$. III/IV), and treatment (or supportive care) were selected as independent predictors for survival. Conclusions: The number of patients diagnosed with an advanced stage of HCC in Mongolia is relatively high, and the survival rate of these patients is lower compared to other countries due tolimited treatment modalities. Keywords: HCC, Prognosis, Mongolia, Stage.

\section{PE-050 \\ Recurrence after Curative Resection of Small Hepatocellular Carcinoma: Histology as Potent Prognostic Factors}

${ }^{1}$ Yoo Ra Lee, ${ }^{2}$ Yoo Jin Lee, ${ }^{1}$ Tae Hyung Kim, ${ }^{1}$ Sun Young Yim, ${ }^{1}$ Yeon Sok Seo, ${ }^{1}$ Soon Ho Um

${ }^{1}$ Division of Gastroenterology and Hepatology, Department of Internal Medicine, Korea University College of Medicine, Seoul, South Korea; ${ }^{2}$ Department of Pathology, Korea University College of Medicine, Seoul, South Korea

Aims: Hepatocellular carcinoma (HCC) is one of the leading causes of cancer-related death worldwide despite the improvement in diagnostic methods and treatment modality. Curative treatment is recommended for patients with small HCCs and good liver function based on Milan criteria, but nevertheless the risk of tumor recurrence is still high in these selected patients. Therefore, we aimed to identify the factors that could predict HCC recurrence after resection focusing on the histologic findings. Methods: A total of 99 HCC patients who had undergone resection from June 2003 to December 2015 with HCCs $\leq 3 \mathrm{~cm}$ and total number of tumors $\leq 3$ were retrospectively reviewed. Patients with child-Pugh grade A were included in the study. Histologic features including gross pattern, Edmonson grade, microvascular invasion, capsule invasion, free resection margin for tumor while presence of cirrhosis in non-tumor lesion were analyzed. Predictive efficacy of HCC recurrence was also analyzed using clinical variables and lab findings including tumor markers. Results: Forty patients (40.4\%) exhibited HCC recurrence following resection. In univariate analysis, factors associated with recurrence were presence of DM, increased baseline PIVKA level, prolonged INR and histological microvascular invasion, high Edmonson grade (grade4), cirrhosis. In multivariate analysis, presence of DM, increased PIVKA level and high Edmonson grade were associated with HCC recurrence. In subgroup analysis, presence of microvascular invasion and increased PIVKA level were associated with early recurrence ( $\leq 2$ years) (both, $\mathrm{p}<0.05$ ) while presence of DM and low albumin level tended to be associated with late recurrence $(>2$ years). When recurrence free survival was analyzed, presence of DM and microvascular invasion (both, $p<0.05$ ) remained as associated factors. Conclusions: Our findings show that in addition to tumor marker, histologic findings and presence of DM are important prognostic factors that could help in identifying patients who will recur from resection. Keywords: Hepatocellular carcinoma, Histology, Prognosis. 


\section{PE-051 \\ Prognostic Impact of Lactic Dehydrogenase to Albumin Ratio in Hepatocellular Carcinoma Patients with Child-Pugh I Who Underwent Curative Resection: A Prognostic Nomogram Study}

Wei Gan; Yong Yi; Jinlong Huang; Shuangjian Qiu

Department of Liver Surgery and Liver transplantation, Liver Cancer Institute, Zhongshan Hospital and Shanghai Medical School, Fudan University, Key Laboratory for Carcinogenesis \& Cancer Invasion, The Chinese Ministry of Education, Shanghai, China

Aims: Radical resection is the treatment of choice for patients with hepatocellular carcinoma (HCC). However, the prognosis of HCC remains poor, and the efficacy of current predictive models is unsatisfactory. Here, we developed an accurate and easy-to-use prognostic index for patients with HCC who underwent curative resection. Methods: The study population comprised 1,041 patients with HCC whounderwent curative resection in Zhongshan Hospital, including 768 patients in 2012 as the training cohort and 273 patients treated in 2007 as the validation cohort. Results: The optimal cutoff value for the lactic dehydrogenase to albumin ratio (LAR) was 5.5, and the LAR was identified as a significant prognostic index for both overall survival (OS) and recurrence-free survival (RFS) in two independent cohorts. The C-index of the LAR was superior to other inflammatory scores and serum parameters and was a stable predictive index in the validation cohort. The new nomogram combining the LAR with the Barcelona Clinic Liver Cancer (BCLC) staging system showed an improved ability to discriminate OS and RFS. Nomogram predictions were consistent with actual observations based on the calibration curve and decisive curve analysis in the two independent cohorts. Conclusions: The LAR is a novel, convenient, reliable, and accurate prognostic predictor in patients with HCC undergoing curative resection. The use of the LAR in routine clinical practice is recommended. Keywords: Liver cancer, Nomogram, Lar, Surgical.

\section{PE-052 \\ Prognostic Characteristics and Grade Prognostic Assessment in Patients with Lung Metastasis from Hepatocellular Carcinoma \\ Kangpyo Kim ${ }^{1}$, Jinsil Seong \\ ${ }^{1}$ Department of Radiation Oncology, Yonsei University \\ College of Medicine, Seoul, South Korea}

Aims: To find out the prognostic characteristics of patients and treatment approaches in HCC with lung metastasis, and furtherly, to group the patients by grade prognostic assessment (GPA) score who can benefit from local treatment to metastatic lesions. Methods: The characteristics of patients and treatment options for viable liver tumor and metastatic lesion were reviewed along with survival analysis. Overall survival is calculated from the time oflung metastasis development. Factors for survival ( $2 \mathrm{yr}$ ) are analyzed via multivariate cox regression analysis and weighted by the magnitude of their regression coefficients to define GPA score. Results: 157 patients of HCC with lung single organ metastasis were selected. In multivariate analysis, CP grade A at the time of lung metastasis, limited number and metachronous development of lung metastasis, local treatment application for lung metastasis were shown as significant prognostic factors. We divided the patients into 3 subgroups according to the GPA scores which are made by 3 significant prognostic factors. For the most promising group (GPA score 0), 2 yr survival of $73.7 \%$ was shown in patients with local treatment application group compared to $28.6 \%$ for systemic treatment group ( $\mathrm{p}=0.004)$. There were only 42 yr survivors for CP grade B or C group (GPA score 2 through 4) as CP grade was most weighted factor. In intermediate risk group (GPA score 1) of the patients, who are characterized by having limited number of lung metastasis or metachronous metastasis, local treatmentapplication group showed better 2 yr survival than systemic treatment one $(40.0 \%$ vs $18.4 \%, p=0.027)$. Conclusions: Liver function CP grade A at the time of metastasis, limited number (1 4) of metastasis, metachronous lung metastasis pattern were requirements to take advantage from local treatment and significant prognostic factors for better survival. This study plays a role in that adding an evidence of oligometastases status in HCC. Keywords: HCC, Lung metastasis, GPA, Local treatment.

\section{Treatment: Systemic, Targeted, Novel Target or Experimental Therapy}

\author{
PE-053 \\ Sorafenib-Related Adverse Events Predict \\ Early Therapy Response of Hepatocellular \\ Carcinoma: Experience at a Single Center in \\ Taiwan \\ Shao-Wu Lee, Teng-Yu Lee, Sheng-Shun Yang, \\ Hong-Zen Yeh, Chi-Sen Chang \\ Division of Gastroenterology, Department of Internal \\ Medicine, Taichung Veterans General Hospital, \\ Taichung, Taiwan
}

Aims: Hepatocellular carcinoma (HCC) is associated with a poor prognosis and low chemotherapeutic efficiency except sorafenib. Previous studies reported adverse events (AEs) of 


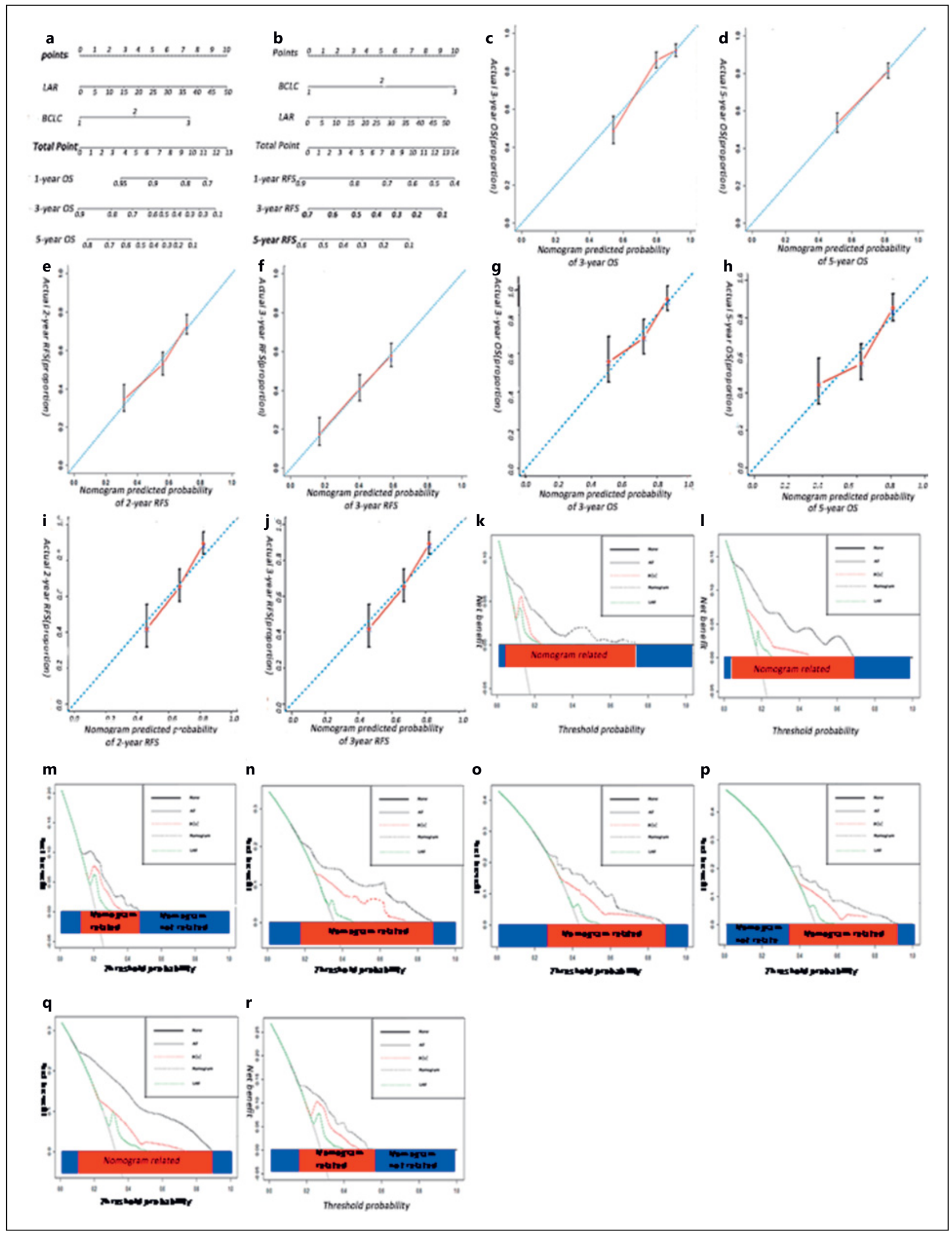

Fig. 1. (for Abstract no PE-051). 

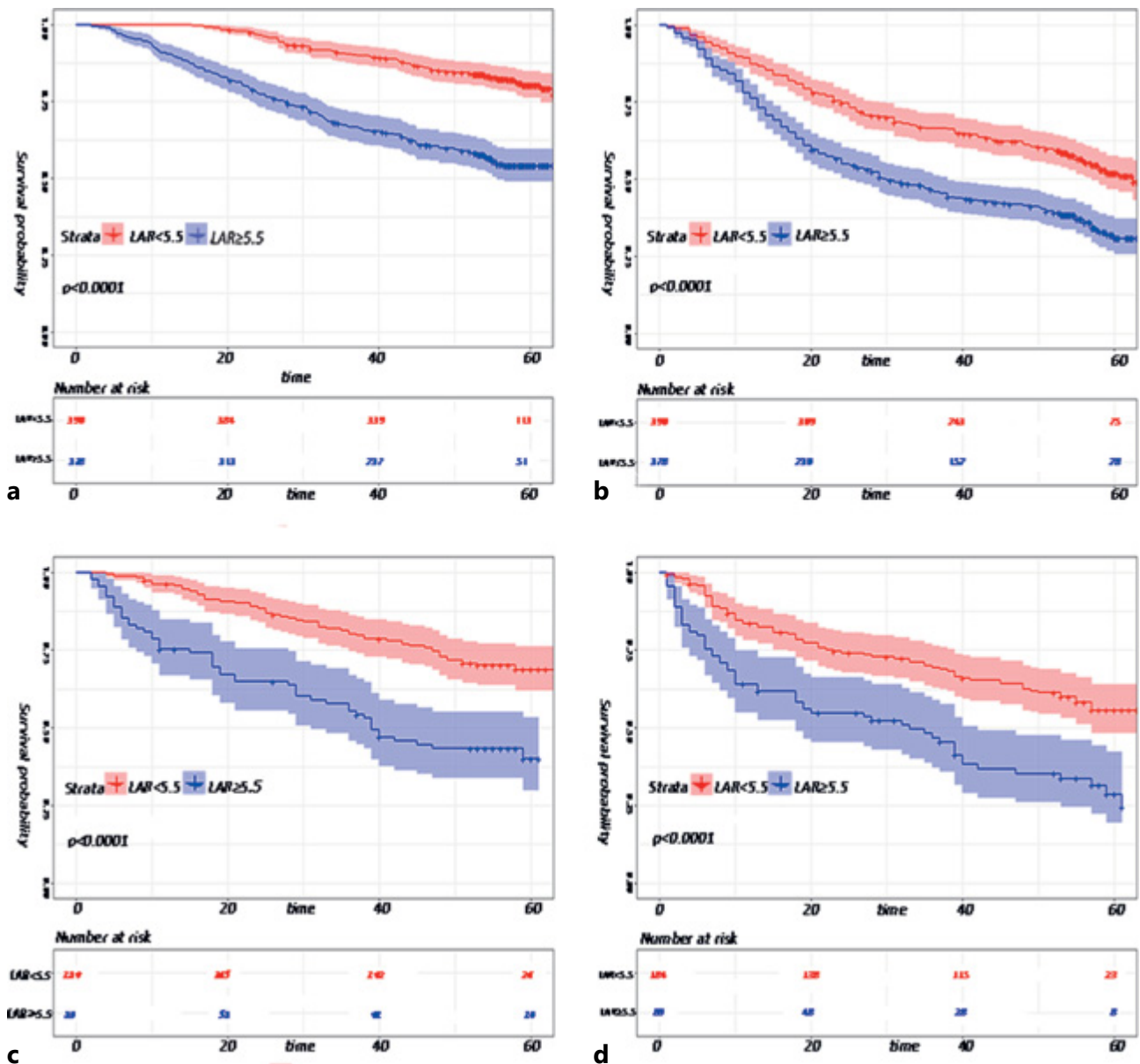

Fig. 2. (for Abstract no PE-051).

sorafenib predict therapy efficacy to HCC. The aim of the study is to evaluate the early efficacy and AEs of sorafenib therapy at one Taiwan's center. Methods: Data for the subjects with HCC receiving sorafenib at Taichung Veterans General Hospital from June 2012 to October 2016 were evaluated. All enrolled cases belonged to HCC BCLC classification stage C and cirrhosis Child-Pugh classification stage A. The early efficacy of sorafenib was classified as partial response (PR), stable disease (SD) and progressive disease (PD) according to the mRECIST criteria, within 6 weeks after sorafenib started. The AEs were defined as $\geq$ grade 2 appearances of hand-foot skin reaction (HFSR), hypertension, hair loss or diarrhea. The exclusion criteria included poor performance status, lack of compliance to drug, loss follow-up or mortality within the following day. The associations of AEs and efficacy of sorafenib were analyzed. Results: Among a total of 222 subjects enrolled, there were 8 (3.6\%), 82 (36.9\%), 132 (59.5\%) cases belong to the group of PR, SD and PD. The PD group owned the significant highest serum level of AFP. The PS group had a signif- icant highest ratio of HFSR (62.4\%), hypertension (37.5\%) and hair loss (25\%), than the other two groups did. The associations between AEs and therapeutic response were significant in HFSR (OR 2.50, 95\% CI 1.36-4.61), hypertension (OR 5.50, 95\% CI 1.81-16.43) and hair loss (OR 16.5, 95\% CI 2.66102.39), but insignificant in diarrhea (OR 1.57, 95\% CI 0.455.56). Conclusions: The appearances of sorafenib AEs, including HFSR, hypertension and hair loss, predict good early therapy efficacy to HCC. 


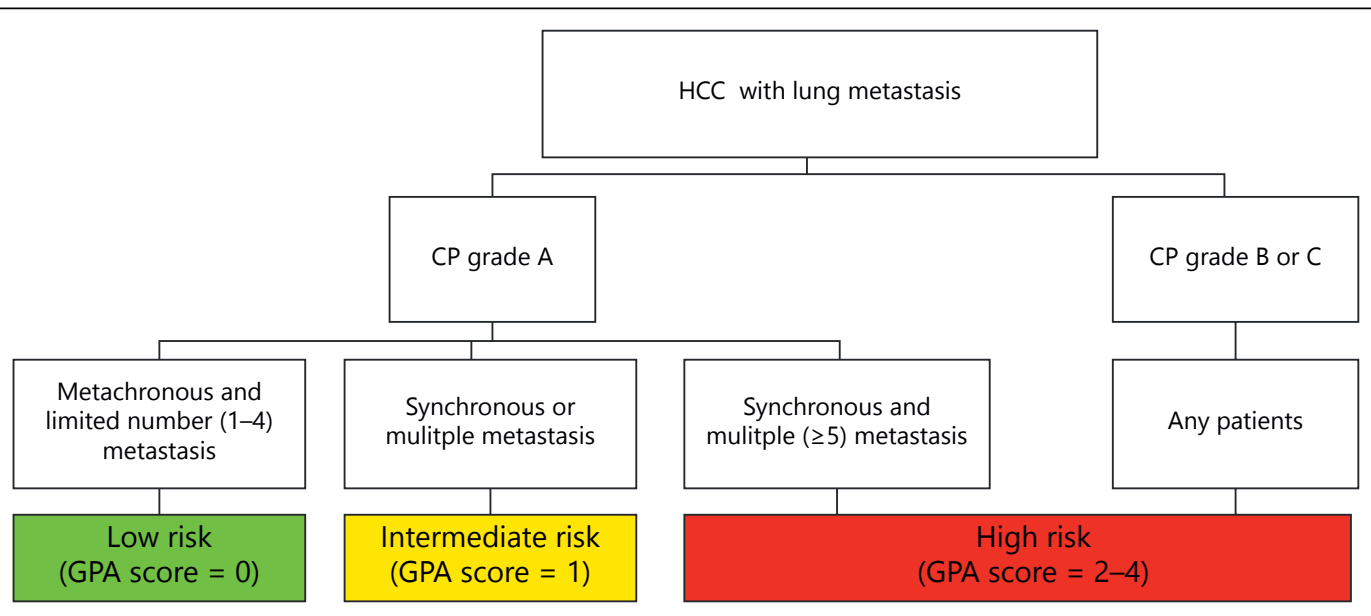

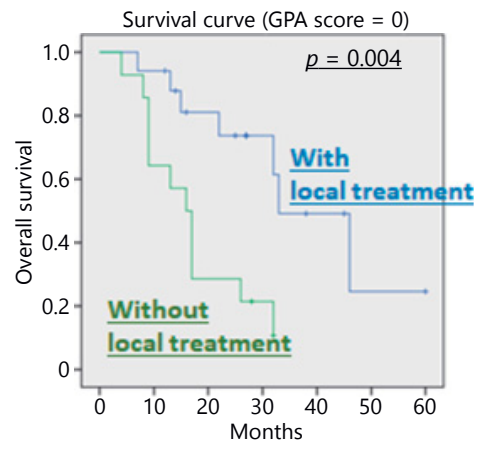

- Low risk group (GPA score $=0$ )

- With local treatment $(n=17)$

. 2 years survival: $73.7 \%$

- Without local treatment $(n=15)$

- 2 year survival: $28.6 \%$

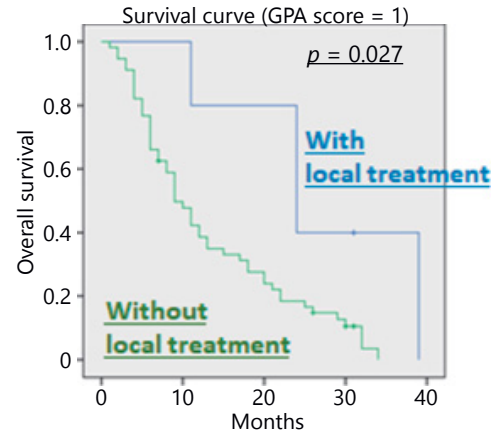

- Intermediate risk group (GPA score $=1$ )

- With local treatment $(n=5)$

- 2 years survival: $40.0 \%$

- Without local treatment $(n=56)$

- 2 year survival: $18.4 \%$

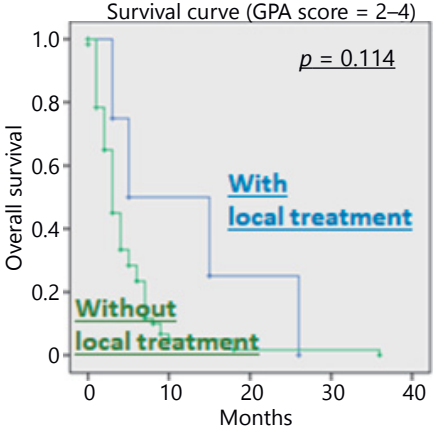

- High risk group (GPA score $=2-4$ )

- With local treatment $(n=4)$ - 2 years survival: $25.0 \%$

- Without local treatment $(n=60)$ - 2 year survival: $1.7 \%$

Fig. 1. (for Abstract no PE-052).

\section{PE-054}

Albumin-Bilirubin Grade Predicts Survival of Advanced HCC Patients Treated with Sorafenib in a Hepatitis B-virus Endemic Area

Hee Yeon Kim ${ }^{1,2}$, Chang Wook Kim ${ }^{1,2}$,

Sang Wook Choi ${ }^{1,2}$, Do Seon Song 1,2, U Im Chang 1,2, Jin Mo Yang 1,2, Sung Won Lee 1,2, Hae Lim Lee 1,2,

Nam Ik Han ${ }^{1,2}$, Sun Hong Yoo ${ }^{1,2}$, Jung Hyun Kwon 1,2, Soon Woo Nam ${ }^{1,2}$, Seawon Hwang ${ }^{1,2}$, Pil Soo Sung ${ }^{1,2}$, Jeong Won Jang 1,2, Si Hyun Bae 1,2, Jong Young Choi ${ }^{1,2}$, Seung Kew Yoon ${ }^{1,2}$

${ }^{1}$ Department of Internal Medicine, College of Medicine, The Catholic University of Korea, Seoul, Republic of Korea ${ }^{2}$ The Catholic University Liver Research Center

Aims: Hepatocellular carcinoma (HCC) patients often have cirrhosis, and the severity of liver dysfunction influence the prognosis of HCC. Albumin-Bilirubin (ALBI) grade is a recently introduced measurement for hepatic reserve in HCC patients. We aimed to investigate the prognostic value of ALBI grade in advanced HCC patients in a hepatitis B-virus endemic area. Methods: A total of 411 consecutive advanced HCC patients in Child-Pugh A receiving sorafenib monotherapy from September 2008 to October 2017 were evaluated. Overall survival (OS) was assessed using the Kaplan-Meier method and a Cox proportional hazard model. Results: Hepatitis B virus-related HCCs comprised 73.9\% (304/411) of enrolled patients. Among 411 enrolled patients, 113 patients (27.5\%) were classified as ALBI grade 1 and 298 patients $(72.5 \%)$ were classified as ALBI grade 2 in baseline. Majority of the patients with ALBI grade $1(110 / 113,97.3 \%)$ had a Child-Pugh score of 5 . Among patients with ALBI grade 2, $60.7 \%$ (181 patients) had a Child-Pugh score of 6 . The median OS was 24.5 and 10.8 months for ALBI grade 1 and 2, respectively $(\mathrm{P}<0.001)$. Hazard ratio was 2.29 (95\% CI: 1.60-3.27, $\mathrm{P}<0.001$ ) for grade 2 vs 1 . Conclusions: ALBI grade could predict the overall survival of advanced HCC patients in Child- 


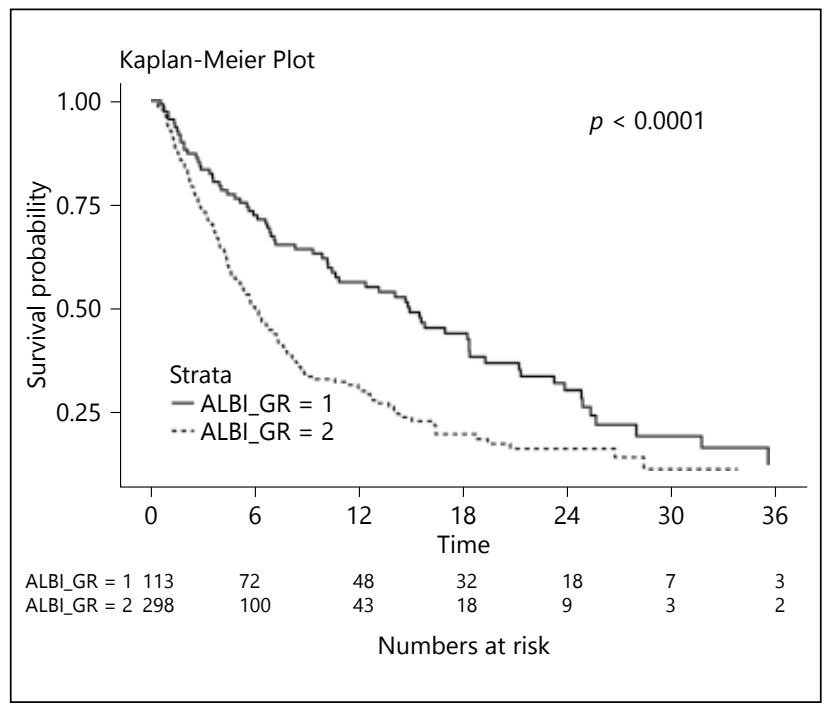

Fig. 1. (for Abstract no PE-054).

Pugh A treated with sorafenib in a hepatitis B virus-endemic area. Keywords: Hepatocellular carcinoma, Sorafenib, Albumin-bilirubin grade, Prognosis.

\section{PE-055 \\ Treatment Outcomes of Immune Checkpoint Inhibitor in Advanced Hepatocellular Carcinoma Patients Including Child Pugh Class B Cirrhosis \\ Jooho Lee ${ }^{1 *}$, Hong Jae Jeon ${ }^{2}$, Hana Park', Young Eun Chon ${ }^{1}$, Yun Bin Lee ${ }^{1}$, Seong Gyu Hwang ${ }^{1}$, Kyu Sung $\operatorname{Rim}^{1}$ \\ Department of Internal Medicine, ${ }^{1}$ Division of Hepatology and ${ }^{2}$ Division of Oncology, CHA Bundang Medical Center, CHA University, Seongnam, Korea}

Aims: Hepatocellular carcinoma (HCC) has extremely poor prognosis. Immunotherapy has emerged as a new treatment for a number of cancers. Programmed death 1 (PD-1) and its ligand (PD-L1) play pivotal roles in regulating host immune responses. In this study, we investigated the pre-treatment and on-treatment factors associated with treatment outcomes of the PD-1 inhibitor therapy in advanced HCC patients. Methods: A total of 8 patients with advanced HCC [75\% male, mean age 53.4 yr, Etiology (hepatitis B/ hepatitis C/other, $63 \% / 25 \% / 12 \%$ ), Child-Pugh class (A/B, $63 \% / 37 \%$ ), BCLC stage (B/C, 13\%/87\%)] had been administered nivolumab (the PD-1 inhibitor). We started to administer the nivolumab $2-3 \mathrm{mg} / \mathrm{kg}$ with 2 weeks interval after the confirmation of disease progression (PD) on sorafenib therapy. Results: At 6 months following the nivolumab therapy, number of partial response (PR)/stable disease
(SD)/ PD was $1 / 1 / 6$, respectively. The 6-month overall survival rate was $50 \%$ and the 60 -month progression free survival rate was $25 \%$. For the baseline characteristics of the HCC patients, Child-Pugh class B and major vascular invasion were significantly associated with unfavorable treatment outcome (PD or death). Liver disorder including hyperbilirubinemia after nivolumab administration was observed in $50 \%$ of patients. Grade 3/4 adverse events (AE) were noted in $37 \%$ of patients and these grade $3 / 4 \mathrm{AE}$ revealed to be the risk factors for the unfavorable treatment outcome (PD or death). Conclusions: Our data indicate that pre-treatment liver function such as Child-Pugh class can be associated with unfavorable treatment outcome of PD-1 inhibitor therapy in advanced HCC patients. Further studies will be needed for the efficacy and safety of PD-1 inhibitor therapy in advanced HCC, especially in patients with Child-Pugh class B. Keywords: Hepatocellular carcinoma, Immunotherapy, Programmed cell death 1, Liver cirrhosis.

\section{PE-056 \\ Liquid Crystalline Nanoparticles (LCNPS) based delivery of an Anticancer bioactive, Methotrexate \\ Vishal Bhargava', Saurabh Bhargava ${ }^{2}$ \\ ${ }^{1}$ GTB Hospital, ${ }^{2}$ Himalayan University}

Aims: Liver cancer is a disease of uncontrolled cell growth, which may invade adjacent tissue and cause infiltration beyond the liver. Most of the potent and effective anticancer drugs used in liver cancer therapy shows poor bioavailability at desired site as well as toxic in nature. The aim of the study was to investigate mannose modified Liquid Crystalline Nanoparticle (LCNPs) carrier for efficient and site specific delivery of potent anticancer drug (Methotrexate) used in hepatic carcinoma therapy. Methods: MTX loaded LCNPs were prepared by lipid cast film method and sonication method. The nanoparticles were characterized in-vitro for their shape, size, percent drug entrapment and stability by Optical Microscopy, Cross Polarized Light Microscpy (CPLM), Transmission Electron Microscopy (TEM), X-ray diffraction (XRD) and Atomic Force Microscopy (AFM). Results: In-vitro stability studies reveal that LCNPs formulations are stable for 120 days at room temperature. Ex-vivo cell cytotoxicity was performed on Human hepatoma cell line. In-vivo studies included fluorescence microscopy and organ distribution studies which show the Mannose modified LCNPs exhibit better accumulation in liver as compared to unmodified system. The results of the present study indicate, this system is more stable as compared to other system. Conclusions: Eventually it may be concluded that incorporation of MTX in mannose modified LCNPs increases the residing time of drug in the body by altering of pharmacokinetics and biodistribution pattern, and the drug primarily 
concentrates in the liver. This system showed excellent cytotoxicity towards cancer cells. From the present investigation it is evident that this system may be used for liver cancer and other liver disease.

\section{PE-057 \\ Efficacy and Safety of Regorafenib after Progression on Sorafenib in Practice of Korean Patients with Advanced Hepatocellular Carcinoma (HCC)}

\begin{abstract}
Changhoon Yoo ${ }^{1}$, Joong-Won Park ${ }^{2}$, Yoon Jun Kim³, Do Young Kim ${ }^{4}$, Tae Seop Lim ${ }^{4}$, Baek-Yeol Ryoo ${ }^{1}$, Sujin Lee ${ }^{5}$, Ho Yeong Lim $^{5}$

${ }^{1}$ Department of Oncology, Asan Medical Center, University of Ulsan College of Medicine, Seoul, South Korea; ${ }^{2}$ National Cancer Center, Goyang, South Korea; ${ }^{3}$ Department of Internal Medicine, Seoul National University College of Medicine, Seoul, South Korea; ${ }^{4}$ Department of Internal Medicine, Yonsei University College of Medicine, Seoul, South Korea; ${ }^{5}$ Division of Hematology and Oncology, Department of Medicine, Samsung Medical Center, Sungkyunkwan University School of Medicine, Seoul, South Korea
\end{abstract}

Aims: Regorafenib has proven the efficacy as $2^{\text {nd }}$-line therapy for patients with advanced HCC who progressed on $1^{\text {st }}$-line sorafenib in the pivotal RESORCE trial. Real-world data are needed to measure the clinical outcomes and adverse events of regorafenib in a daily practice setting. Methods: Between April 2017 and August 2017, the Named Patient Program (NPP) was activated to provide controlled, preapproval access of regorafenib in Korea. This analysis is multicenter retrospective study for patients who received regorafenib under the NPP. Results: A total of 49 patients entered into this NPP and 40 patients received regorafenib in 5 Korean institutions. All but one patient received regorafenib as $2^{\text {nd }}$-line therapy after progression on sorafenib, and $36(90 \%)$ and $34(85 \%)$ patients were classified as ChildPugh A and BCLC stage C, respectively. Best response with regorafenib was partial response $(n=4,10 \%)$, stable disease $(19,48 \%)$, and progressive disease $(15,37 \%)$. Median progression-free survival (PFS) was 3.7 months (95\% CI, 2.5-4.9). Median overall survival (OS) was not reached and 10 -month OS rate was $52.5 \%$. The TTP on prior sorafenib (<median [4.8 months] vs $\geq$ median) was significantly associated PFS (median 8.0 vs 2.5 months; $p=0.002$ ) and OS (not reached vs 8.2 months; $\mathrm{p}=0.02$ ). Most common grade $3-4$ toxicities were hand-foot skin reaction $(n=3,8 \%)$, followed by hypertension $(2,5 \%)$ and aspartate aminotransferase increase $(2,5 \%)$. Conclusions: Regorafenib was well tolerated and effective for patients with advanced HCC progressed on $1^{\text {st }}$-line sorafenib, as our efficacy and safety data are consistent with the previous RESORCE trial. TTP on $1^{\text {st }}$-line sorafenib may predict the efficacy outcomes with subsequent regorafenib. Keywords: Hepatocellular carcinoma, Regorafenib, Targeted therapy.

\section{PE-058 \\ Complete Response of Hepatocellular Carcinoma with Right Atrium and Pulmonary Metastases Treated by Combined treatments: A Possible Relationship between Anaphylaxis and Treatment Efficacy?}

Dong Hyun Kim ${ }^{1}$, Eunae Cho', Sung Bum Cho ${ }^{1}$, Sung Kyu Choi ${ }^{1}$, Jieun Yu${ }^{2}$, Young-ll Koh ${ }^{2}$, Da Woon Sim*2, Chung Hwan Jun*1

${ }^{1}$ Division of Gastroenterology, Department of Internal Medicine, Chonnam National University Hospital and Medical School, Gwangju, South Korea; ${ }^{2}$ Division of Allergy, Asthma, and Clinical Immunology, Department of Internal Medicine, Chonnam National University Hospital and Medical School, Gwangju, South Korea

Aims: Hepatocellular carcinomas (HCCs) with metastases to the right atrium and lungs are rare but have a poor prognosis. Furthermore, the treatment outcomes of patients with advanced HCCs remain unsatisfactory. Methods: A 46-year-old man was diagnosed with HCC and extra-hepatic metastases, including multiple lung metastases, in the right atrium. He received multidisciplinary treatment including radiotherapy (RT), trans-arterial chemoembolization (TACE), and sorafenib. After the initial treatment, follow-up computed tomography using radio-contrast media was performed to evaluate the tumoral response. During the procedure, he experienced radio-contrast-induced anaphylaxis. Results: Following the anaphylaxis event accompanied by additional treatment such as RT, TACE, and sorafenib, the patient's tumor burden decreased, and he eventually achieved a complete response as per the modified Response Evaluation Criteria in Solid Tumors. Subsequent blood tests showed increased natural killer (NK) cell activity, which was significantly higher than that seen in other HCC patients of the same age, receiving sorafenib and with an identical stage of the tumor. This suggests that the antitumor effect of the NK cells induced by anaphylaxis influenced the tumor burden and contributed to the long-term survival of the patient. The patient is still alive, 30 months after the episode. We report here a rare case of long-term survival of a HCC patient with multiple metastases, who achieved complete response after multidisciplinary treatment and an anaphylactic episode. Conclusions: we report a rare case of long-term survival in a HCC patient with multiple metastases, who was treated with multidisciplinary modalities and experienced anaphylaxis. Keywords: Antitumor effect, Anaphylaxis, Natural killer cell, Hepatocellular carcinoma 


\section{PE-059 \\ Effects of Dental Scaling on the Admission Outcomes of Liver Cancer: A Nationwide Matched Study \\ Yi-Chun Chou', Chien-Chang Liao' ${ }^{2}$,Ta-Liang Chen ${ }^{2}$ \\ ${ }^{1}$ Department of Physical Medicine and Rehabilitation, China Medical University Hospital, Taiwan; ${ }^{2}$ Department of Anesthesiology, Taipei Medical University Hospital, Taiwan}

Aims: Little was known about the association between dental scaling (DS) and cancer outcomes. The purpose of this study is to evaluate the effects of DS on the outcomes of liver cancer. Methods: Using reimbursement claims data of National Health Insurance in Taiwan in 2008-2013, we conducted a matched cohort study including 32330 patients aged $\geq 20$ years underwent inpatient care of liver cancer. Using propensity score matching procedure, we selected 4068 patients with preoperative DS and 4068 patients without DS for comparison. Logistic regressions were used to calculate odds ratios (ORs) with 95\% confidence intervals (CIs) of DS associated with complications and in-hospital mortality during admission of liver cancer. Results: Patients with liver cancer receiving DS showed lower risks of septicemia (OR $0.68,95 \%$ CI $0.54-0.85$ ), acute renal failure (OR $0.45,95 \%$ CI 0.31-0.64), and urinary tract infection (OR 60, 95\% CI 0.440.82 ) compared with those without DS. In addition, DS was associated with decreased risks of intensive care (OR 0.84, 95\% CI 0.74-0.94) and in-hospital mortality (OR 0.59, 95\% CI 0.41-0.85) during the cancer admission after adjustment. Shorter length of hospital stays $(\mathrm{p}<0.05)$ and less medical expenditure $(\mathrm{p}<0.05)$ were also noted in DS patients than in non-DS group. Conclusions: Among patients with admission of liver cancer, patients with DS showed lower risks of complications and in-hospital mortality compared with those had no DS. Further studies are needed to explain how DS improves outcomes of liver cancer. Keywords: Liver cancer, Dental scaling, Admission, Outcome

\section{PE-060 \\ Disruption of CD47-SIRPa Axis as a Novel Potent Immunotherapy in Hepatocellular Carcinoma \\ Wonseok Kang, Sera Yang, Sohee Kang, Ji-Young Kim, Yong-Han Paik \\ Department of Medicine, Samsung Medical Center, Sungkyunkwan University School of Medicine, Seoul, South Korea}

Aims: CD47 is an innate immune checkpoint molecule known as 'don't-eat me' signal, which plays a critical role in macrophage activation and phagocytosis. In the current study, we investigated the anti-tumor effect of CD47 inhibition in a syngeneic preclinical model of hepatocellular carcinoma (HCC). Methods: We examined the effect of anti-CD47 blockade on macrophage-mediated phagocytosis and tumor growth in both in vitro and in vivo. The therapeutic efficacy of anti-CD47 blockade was examined in the syngeneic preclinical model of HCC. Results: Blockade of CD47 induced macrophage-mediated phagocytosis of tumor cells. Using a syngeneic preclinical model of HCC, we found that anti-CD47 blockade exerted significant tumor suppression and improved survival. Conclusions: Anti-CD47 blockade may be a promising immunotherapeutic strategy for the treatment of HCC patients. Keywords: HCC, CD47, SIRPa, Immunotherapy

\section{Treatment: Clinical Trials}

\section{PE-061 \\ Benefit of Local Treatment Including External Beam Radiation Therapy in BCLC C Patients: Korean Nationwide Cohort Analysis}

Chai Hong Rim ${ }^{1}$, Jeongshim Lee ${ }^{2}$, Korean Liver Cancer Study group, Korea Central Cancer Registry,

${ }^{1}$ Department of Radiation Oncology, Ansan Hospital, Korea University Medical College, Ansan, Gyeonggi-do, Republic of Korea; ${ }^{2}$ Inha University Medical College, Incheon, Republic of Korea

Aims: The current standard treatment recommended for Barcelona Clinic of Liver Cancer (BCLC) C patients is sorafenib. However, the appliance of local treatment including radiation therapy (RT) is not uncommon. This study is to find the role of local treatment including RT (LRT) among BCLC C patients. Methods: From 2008 to 2014, 10,580 patients with hepatocellular carcinoma were enrolled in the Korea Central Cancer Registry. Of these patients, 3401 (32.1\%) were of BCLC C stage. The BCLC C Patients who were treated with sorafenib, LRT, and no treatment as the initial treatments (the first treatment and the second treatment within 60 days after the first treatment) were included in the study. As clinical factors, gender, age, hepatitis B, hepatitis C, Eastern Cooperative Group performance score (ECOG), tumor size $>10 \mathrm{~cm}$, portal invasion (PI), lymph node metastasis (LNM), distant metastasis (DM), Child-Pugh score (CPS) were analyzed. Results: A total of 1530 patients were included in this study. The number of patients treated with sorafenib, LRT, and no treatment as the initial treatments were 306 (20\%), 319 (20.8\%), and 905 (59.2\%), respectively. Between sorafenib and LRT groups, DM and tumor size $>10 \mathrm{~cm}$ out of all clinical factors were significantly different (DM: $50.8 \%$ Vs. $33.3 \%$, p < 0.001; tumor size $>10 \mathrm{~cm}: 54.9 \%$ Vs. $42.8 \%, \mathrm{p}=0.003$ ). Survival analyses were 
performed for patients of all BCLC C, with PI, LN metastasis, without DM, and with DM. No treatment group showed most inferior results among 3 groups in all 5 subgroup analyses. Comparing sorafenib and LRT groups, survival curves were significantly different in all 5 subgroups analyses favoring LRT group. In multivariate analysis, initial treatment of sorafenib Vs. LRT was significant factor affecting survival of All BCLC C patients [Hazard ratio (HR), 95\% confidence interval (CI): $1.49,1.22-1.83, \mathrm{p}<0.001]$, with PI $(1.54,1.22-$ $1.94, \mathrm{p}<0.001)$, with $\operatorname{LNM}(2.42,1.53-3.83, \mathrm{p}<0.001)$, without DM $(1.42,1.09-1.85, \mathrm{p}=0.009$,) and with DM $(1.57,1.13-2.19$, $\mathrm{p}=0.007)$. Conclusions: Initial LRT has shown survival benefit as compared to sorafenib in BCLC C patients. Keywords: Hepatocellular carcinoma, BCLC C, Sorafenib, Radiotherapy

\section{PE-062 \\ Complex Treatment of Primary and Metastatic Liver Cancer in Conditions of the Republican Clinical Hospital № 1 of Tashkent City \\ S.N. Lukmonov', U.M. Kurbankulov', O.O. Usmonov', M.U. Ismailov², U.A. Azzalarov³, K.A. Madatov'3, A.A. Ismatov ${ }^{1}$ \\ ${ }^{1}$ Department of Oncology and Radiology, Tashkent Medical Academy, Tashkent, Uzbekistan ${ }^{2}$ Department of Oncology, Tashkent State Pediatric Institute, Tashkent, Uzbekistan ${ }^{3}$ Department of Surgery, Republican Clinical Hospital №1, Tashkent, Uzbekistan}

Aims: In recent years, there has been an increase in the number of patients with liver tumors, including those with malignant tumors. Among the latter prevail metastatic, rarely - primary, in a ratio of approximately 10: 1 . Methods: During the period from 2004 to 2017,353 patients with malignant neoplasms of the liver were treated on the basis of faculty surgery and the Scientific Research Center for the Study of Liver and Biliary Disease in the RCH No. 1 in Tashkent, including 28 (7.9\%) with primary, 325 (92.1\%) with metastatic cancer, aged 20 to 76 years. They performed both surgical and x-ray endovascular methods, both as monotherapy and in the complex. Results: 151 patients were surgically resected $(n=233,66.0 \%)$ in the liver, 82 patients were palliative. Among the resection methods, we performed the removal of 1-2 segments ( $n=23)$; left-sided hemihepatectomy (HGE, $n=40)$, including the expanded $(n=17)$; righthand HGE $(n=88)$, including expanded $(n=39)$. X-ray endovascular interventions as monotherapy were performed in 99 (28.0\%) patients with metastatic colorectal $(n=89)$ and primary $(\mathrm{n}=10)$ liver cancer. Complex treatment (surgical + HEPA) was performed by $21(6.0 \%)$ patients. Of these, in 4 cases, 1-2 cycles of HEPA were performed as neoadjuvant chemotherapy, followed by liver resection. Adjuvant HEPA was performed in 17 cases after liver resection $(n=5)$, or after cytoreductive operations $(n=12)$ with removal of the primary focus (mainly - resection of the large intestine). Including in 3 patients after 3-5 cycles of HEPA it was possible to translate a metastatic liver tumor into a resectable one, and to perform GGE with the second stage after resection of the large intestine. Conclusions: Extensive liver resections for primary and metastatic lesions are the only radical method in the treatment of cancerous liver tumors. The most preferred method for the treatment of multiple metastases in the liver (with their nonresectability) is chemoinfusion with selective chemoembolization of the hepatic artery. Complex application of these methods increases the possibilities of treating this category of patients. Keywords: Primary liver cancer, Metastatic liver cancer, Hemihepatectomy, Neoadjuvant chemotherapy.

\section{PE-063 \\ Global Phase 3 Study of Tislelizumab Versus Sorafenib as First-Line Treatment in Patients with Advanced Hepatocellular Carcinoma: A Trial-in-Progress}

\begin{abstract}
Shukui Qin ${ }^{1}$, Richard S. Finn², Masatoshi Kudo ${ }^{3}$, Tim Meyer ${ }^{4}$, Arndt Vogel ${ }^{5}$, Michel Ducreux ${ }^{6}$, Teresa Mercade Macarulla ${ }^{7}$, Gianluca Tomasello ${ }^{8}$, Frederic Boisserie ${ }^{9}$, Jeannie Hou ${ }^{10}$, Cindy $\mathrm{Li}^{11}$, James Song ${ }^{9}$, Andrew X. Zhu ${ }^{12}$

${ }^{1}$ People's Liberation Army (PLA) 81 Hospital, Nanjing, China; ${ }^{2}$ University of California Los Angeles, Los Angeles, CA; ${ }^{3}$ Department of Gastroenterology and Hepatology, Kindai University School of Medicine, Osaka, Japan; ${ }^{4}$ University College London Cancer Institute, London, United Kingdom; ${ }^{5}$ Medizinische Hochschule Hannover, Hannover, Germany; ${ }^{6}$ Gustave Roussy, Villejuif, France; ${ }^{7}$ Vall d'Hebron Institute of Oncology, Barcelona, Spain; ${ }^{8}$ Azienda Istituti Ospitalieri di Cremona, Cremona, Italy; ${ }^{9}$ BeiGene USA, Inc., Fort Lee, NJ; ${ }^{10}$ BeiGene USA, Inc., Emeryville, CA; ${ }^{11}$ BeiGene (Beijing) Co., Ltd., Beijing, China; ${ }^{12}$ Harvard Medical School, Massachusetts General Hospital Cancer Center, Boston, MA
\end{abstract}

Aims: Unresectable hepatocellular carcinoma (HCC) accounts for $70 \%$ of diagnosed HCC. Tislelizumab (BGB-A317) is a humanized IgG4 monoclonal antibody with high affinity and specificity for programmed cell death receptor-1 (PD-1). Tislelizumab was specifically engineered to minimize $F_{c} g R$ binding on macrophages that, based on preclinical evidence, is believed to minimize potentially negative interactions with other immune cells. A first-in-human, phase 1A/1B study (NCT02407990) demonstrated that single-agent tislelizumab was generally well tolerated and showed preliminary evidence of antitumor activity in patients with advanced solid tumors, including HCC. A recommended phase 3 dose of $200 \mathrm{mg}$ administered intravenously (IV) every 3 weeks (Q3W) has been established for tislelizumab. Methods: This global, phase 
3, randomized, multicenter study (NCT03412773) was designed to evaluate the efficacy and safety of tislelizumab compared with sorafenib as a potential first-line treatment of unresectable HCC. Adult patients, aged $\geq 18$ years, with unresectable, histologically confirmed HCC, an ECOG score $\leq 1$, Child-Pugh A classification, BCLC Stage C disease or BCLC Stage B disease that has relapsed after loco-regional therapy, and who have not received prior systemic therapy, are being enrolled. Approximately 640 patients from 100 international centers are planned to be randomized (1:1) to receive tislelizumab $200 \mathrm{mg}$ IV Q3W or sorafenib $400 \mathrm{mg}$ orally BID. The primary outcome of this study is overall survival (OS) of patients treated with tislelizumab compared with OS of patients treated with sorafenib; secondary outcomes include objective response rate, progression-free survival, duration of response, time to progression, and quality-of-life outcomes. Safety/tolerability assessments include monitoring adverse events (AEs), including immune-related AEs, as well as physical examinations, vital signs, and electrocardiograms. Exploratory endpoints include assessment of potential biomarkers, characterization of the tislelizumab pharmacokinetic profile in patients with HCC, and assessment of host immunogenicity to tislelizumab. Keywords: Tislelizumab, Phase 3, Advanced hepatocellular carcinoma.

\section{PE-064 \\ Phase 2 Study of Tislelizumab, an Anti-Programmed Cell Death Receptor-1 (PD-1) Antibody, in Patients with Unresectable Hepatocellular Carcinoma (HCC): Trial-in-Progress}

Ann-Lii Cheng ${ }^{1}$, Ghassan K. Abou-Alfa ${ }^{2}$, Zhenggang Ren ${ }^{3}$, Eric Assenat ${ }^{4}$, Antonio Cubillo5, Stefan Pluntke ${ }^{6}$, Lorenza Rimassa ${ }^{7}$, Paul J. Ross ${ }^{8}$, Lucjan Wyrwicz ${ }^{9}$, Jeannie Hou ${ }^{10}$, Cindy $L i^{11}$, John Wu ${ }^{12}$, Michel Ducreux ${ }^{13}$

${ }^{1}$ National Taiwan University Hospital, Taipei, Taiwan; ${ }^{2}$ Memorial Sloan Kettering Cancer Center, New York, NY; ${ }^{3}$ Zhongshan Hospital, Fudan University, Shanghai, China; ${ }^{4} \mathrm{CHRU}$ Saint Eloi, Montpellier, France; ${ }^{5} \mathrm{HM}$ Universitario Sanchinarro, Centro Integral Oncológico Clara Campal (ClOCC), Madrid, Spain; ${ }^{6}$ Kliniken Essen-Mitte, Essen, Germany; ${ }^{7}$ Humanitas Clinical and Research Center, Rozzano, Italy; ${ }^{8}$ Guy's and St Thomas' NHS Foundation Trust, London, United Kingdom; ${ }^{9}$ Centrum OnkologiiInstytut im. M. Sklodowskiej Curie, Warszawa, Poland; ${ }^{10}$ BeiGene USA, Inc., Emeryville, CA; ${ }^{11}$ BeiGene (Beijing) Co., Ltd., Beijing, China; ${ }^{12}$ BeiGene USA, Inc., Fort Lee, NJ; ${ }^{13}$ Gustave Roussy, Villejuif, France

Aims: Tislelizumab is a humanized IgG4 monoclonal antibody with high affinity and specificity for PD-1. Tislelizumab was specifically engineered to minimize $\mathrm{F}_{\mathrm{c}} \mathrm{gR}$ binding on macrophages that, based on preclinical evidence, is believed to minimize potentially negative interactions with other immune cells. A first-in-human, phase 1A/1B study (NCT02407990) demonstrated that single-agent tislelizumab was generally well tolerated and showed preliminary evidence of antitumor activity in patients with advanced solid tumors, including HCC. A recommended dose of tislelizumab administered at $200 \mathrm{mg}$ intravenously (IV) every 3 weeks (Q3W) has been established.Methods:This phase 2 study (NCT03419897) is designed to evaluate the efficacy, safety/tolerability, and pharmacokinetics of tislelizumab in patients with previously treated, unresectable HCC. Patients who are $\geq 18$ years of age with histologically confirmed, locally advanced or unresectable HCC, including patients who are not amenable to, or who have relapsed after, a curative treatment approach or locoregional therapy, are eligible. Patients must also have a Child-Pugh score A, ECOG performance status $\leq 1$, and must have experienced $\leq 2$ lines of prior systemic therapy. Patients will be excluded if they have received prior PD-1 or PD-L1 treatment or have received sorafenib, regorafenib, or any systemic therapy within 14 days of the first tislelizumab infusion. A total of 228 patients worldwide are planned to be treated with tislelizumab $200 \mathrm{mg}$ IV Q3W. Objective response rate, evaluated by Independent Review Committee per RECIST $\mathrm{v} 1.1$, is the primary endpoint; radiological assessment of tumor-response status will be performed every 6 weeks in the first 18 weeks and then every 9 weeks thereafter. Secondary endpoints include duration of response, progression-free survival, disease control rate, clinical benefit rate, overall survival, quality-of-life outcomes, and assessment of the pharmacokinetic and safety/tolerability profiles of tislelizumab. Safety/tolerability assessments will include monitoring of adverse events (AEs), including immune-related AEs. Keywords: 14152978774, Phase 2, Hepatocellular Carcinoma. 


\section{PE-065 \\ IMbrave150: a Randomized Phase III Study of $1 \mathrm{~L}$ Atezolizumab Plus Bevacizumab versus Sorafenib in Locally Advanced or Metastatic Hepatocellular Carcinoma}

\author{
Tae-You Kim ${ }^{1}$, Ann-Lii Cheng ${ }^{2}$, Masafumi Ikeda ${ }^{3}$, \\ Michel Ducreux 4 , Peter R. Galle ${ }^{5}$, Andrew X. Zhu', \\ Derek-Zhen $X u^{7}$, Wendy Verret ${ }^{8}$, Juan Liu ${ }^{7}$, \\ Richard S. Finn ${ }^{9}$, Shukui Qin ${ }^{10}$ \\ ${ }^{1}$ Seoul National University College of Medicine, Seoul, \\ Korea; ${ }^{2}$ National Taiwan University Hospital, Taipei, \\ Taiwan; ${ }^{3}$ National Cancer Center Hospital East, Kashiwa, \\ Japan; ${ }^{4}$ Gustave Roussy, Villejuif, France; ${ }^{5}$ University \\ Medical Center Mainz, Mainz, Germany; ${ }^{6}$ Harvard \\ Medical School, Massachusetts General Hospital \\ Cancer Center, Boston, MA, USA; ${ }^{7}$ Roche Product \\ Development, Shanghai, China; ${ }^{8}$ Genentech, Inc., South \\ San Francisco, CA, USA; ${ }^{9}$ Geffen School of Medicine \\ at UCLA, Los Angeles, CA, USA; ${ }^{10}$ People's Liberation \\ Army Cancer Center, Nanjing, People's Republic of \\ China
}

Aims: Patients with hepatocellular carcinoma (HCC) have a high unmet medical need with a high mortality-to-incidence ratio. The current standard of care for $1 \mathrm{~L}$ treatment of patients with locally advanced or metastatic HCC is the multikinase inhibitor sorafenib. While single-agent inhibition of PD-L1/PD-1 signaling or VEGF has shown modest activity in HCC, evidence from a Phase I study (Stein ASCO 2018, submitted) supports combining atezolizumab (anti-PD-L1) with bevacizumab (anti-VEGF) to achieve greater clinical benefit in HCC. In addition to its anti-angiogenic activity, bevacizumab may have immunomodulatory effects (increased DC maturation, enhanced T-cell infiltration, reduced MDSCs and Tregs in tumors) that have the potential to increase the efficacy of atezolizumab in the tumor microenvironment, thereby contributing to reinvigorating anti-tumor immunity. Methods: IMbrave150 (NCT03434379) is a global, multicenter, randomized, open-label Phase III study to evaluate atezolizumab plus bevacizumab versus sorafenib in patients with locally advanced or metastatic and/or unresectable HCC. Eligible patients will be naive to prior systemic therapy for HCC, have $\geq 1$ measurable untreated lesion (per RECIST v1.1), Child-Pugh class A liver function and ECOG PS 0/1. Patients with bleeding or high risk for bleeding with untreated varices will be excluded. $\approx 480$ patients will be randomized $2: 1$ to receive atezolizumab (1200 $\mathrm{mg}$ ) plus bevacizumab (15 mg/ $\mathrm{kg}$ ) IV Q3W or sorafenib (400 mg) PO BID until loss of clinical benefit or unacceptable toxicity. No crossover will be allowed. Stratification factors are region (Asia excluding Japan versus rest of world), macrovascular invasion and/or extrahepatic spread (presence versus absence), baseline alpha fetoprotein level ( $<400$ versus $\geq 400 \mathrm{ng} / \mathrm{mL}$ ) and ECOG PS ( 0 versus 1 ). Co-primary endpoints are investigator-assessed ORR per RECIST v1.1 and OS. Secondary endpoints include safety, investigator- and independent review facility (IRF)-assessed PFS, DOR and time to progression (TTP) per RECIST v1.1, as well as IRF-assessed efficacy endpoints per HCC modified RECIST criteria. Keywords: Atezolizumab, Anti-PD-L1, Bevacizumab, 1 L Treatment, Cancer immunotherapy.

\section{PE-066 \\ Outcomes of Patients with Hepatocellular Carcinoma (HCC) Treated with Transarterial Chemoembolization (TACE): Global OPTIMIS Final Analysis}

Ann-Lii Cheng ${ }^{1}$, Markus Peck-Radosavljevic ${ }^{2,3}$, Jean-Luc Raoul', Han Chu Lee ${ }^{5}$, Thomas Decaens ${ }^{6,7,8}$, Jeong Heo ${ }^{9}$, Shi-Ming Lin ${ }^{10}$, Hong Shan ${ }^{11}$, Yefa Yang ${ }^{12}$, Inga Bayh ${ }^{13}$, Keiko Nakajima ${ }^{14}$, Masatoshi Kudo ${ }^{15}$

${ }^{1}$ National Taiwan University Hospital, Taipei, Taiwan; ${ }^{2}$ Medical University of Vienna, Vienna, Austria; ${ }^{3}$ Klinikum Klagenfurt am Wörthersee, Klagenfurt, Austria; ${ }^{4}$ Digestive Oncology, Institut de Cancérologie de l'Ouest, Nantes, France; ${ }^{5}$ Asan Medical Center, Seoul, South Korea; ${ }^{6}$ Clinique Universitaire d'Hépatogastroentérologie, CHU Grenoble Alpes, Grenoble, France; ${ }^{7}$ Université Grenoble Alpes, Grenoble, France; ${ }^{8}$ Institute for Advanced Biosciences, Research Center Inserm U1209 / CNRS 5309 / UGA, Grenoble, France; ${ }^{9}$ College of Medicine, Pusan National University and Medical Research Institute, Pusan National University Hospital, Busan, Republic of Korea; ${ }^{10}$ Chang Gung University and Chang Gung Memorial Hospital, Taoyuan, Taiwan; ${ }^{11}$ Department of Radiology, The Third Affiliated Hospital, Sun Yat-sen University, Guangzhou, Guangdong, China; ${ }^{12}$ Eastern Hepatobiliary Surgery Hospital, Second Military Medical University, Shanghai, China; ${ }^{13}$ Bayer AG, SBU Oncology, Pharmaceuticals, Wuppertal, Germany; ${ }^{14}$ Bayer HealthCare Pharmaceuticals, Whippany, NJ, USA; ${ }^{15}$ Kindai University Faculty of Medicine, Osaka, Japan

Aims: TACE is often used to treat unresectable HCC (UHCC); however, there is no globally accepted consensus on TACE ineligibility. It is critical to reassess the risk of TACE as it may delay or prevent subsequent treatment options. Methods: OPTIMIS, an international, prospective, non-interventional study, enrolled patients with uHCC for whom TACE treatment was decided at study entry. Practice patterns, safety, subsequent treatments, and outcomes data were collected. TACE ineligibility was defined and consistent with international and regional guidelines. Results: Overall, 1650 patients received TACE; 529 patients (32\%) were BCLC stage C, 118 (7\%) had extrahepatic spread, and 123 (7\%) had portal vein thrombosis. At inclusion visit, 636 patients (39\%) received TACE despite being TACE ineligible. In patients with available laboratory values, chronic liver function deterioration (worsening CTCAE grade 30-90 days post TACE) after 
first TACE ranged from $11 \%$ to $29 \%$ across assessed liver parameters. Complete and partial response rates to first TACE ( $\mathrm{N}=1650$ ) were $14 \%$ and $26 \%$, respectively, which decreased by second $(10 \%$ and $16 \% ; n=1002)$, third $(10 \%$ and $15 \% ; n=$ $580)$, and fourth $(8 \%$ and $17 \% ; n=338)$ TACE. Progressive disease rate increased by number of TACE procedures: $18 \%$, $21 \%, 25 \%$, and $27 \%$ for first, second, third, and fourth TACE, respectively. After excluding patients with prior sorafenib treatment, only 507 patients (31\%) became TACE ineligible during the study. Of those, 47 (9\%) received sorafenib early following TACE ineligibility and 460 (91\%) received sorafenib later or not at all. Considerable imbalances between these cohorts were observed; a propensity score analysis is planned analyzing overall survival from TACE ineligibility. Conclusions: These results indicate that real-world TACE use appears to deviate from treatment guidelines highlighting the need for a globally accepted consensus on the indication and definition of TACE failure. These observations also indicate the importance of monitoring liver function in patients receiving TACE. Keywords: Hepatocellular carcinoma, TACE, Sorafenib, Liver deterioration.

\section{PE-067 \\ A Phase 3 Study of Durvalumab Tremelimumab as First-Line Treatment in Patients With Unresectable Hepatocellular Carcinoma: HIMALAYA Study}

Junji Furuse, Stephen L. Chan, Bruno Sangro,

Peter R. Galle, Robin Kate Kelley, Shukui Qin,

Jon Armstrong, Annie Darilay, Gordana Vlahovic, Yanhua Xu, Alejandra Negro, Ghassan K. Abou-Alfa

Department of Medical Oncology, Kyorin University School of Medicine, Tokyo, Japan; Department of Clinical Oncology, The Chinese University of Hong Kong; Hong Kong; Liver Unit, Clínica Universidad de Navarra and CIBEREHD, Pamplona, Spain; First Department of Internal Medicine, University Medical Center Mainz, I. Medizinische Klinik und Poliklinik Mainz, Germany; Department of Medicine, Helen Diller Family Comprehensive Cancer Center, University of California, San Francisco, CA, USA; Department of Medical Oncology, PLA Cancer Center \& Bayi Clinical Trial Institute, Nanjing, China; Biostatistics \& Informatics Department, AstraZeneca UK Limited; AstraZeneca, Gaithersburg, MD, USA; Department of Gastrointestinal Oncology, Memorial Sloan Kettering Cancer Center, New York, NY, USA

Aims: Thus far, sorafenib remains the standard of care for first-line systemic therapy in patients with advanced hepatocellular carcinoma (HCC) but patient prognosis and quality of life (QOL) continues to be poor. HCC may be responsive to immunotherapy due to higher expression of immunosuppressive cells and upregulation of CTLA- 4 and PD- 1 immune checkpoints (Gao et al 2009, Hato et al 2014, Pardee \& Butterfield 2012). Hepatitis B virus (HBV) and hepatitis C virus (HCV) infection are also associated with upregulation of regulatory T cells and PD-L1/PD-1 expression (Miroux et al 2010, Pardee \& Butterfield 2012). Blockade of PD-L1/PD-1 or CTLA4 immune checkpoints demonstrated clinical benefit in HCC as monotherapy. In preclinical studies, combination of anti-PDL1 and anti-CTLA-4 antibodies enhanced anti-tumor activity compared with monotherapy, indicating that the 2 pathways are non-redundant (Stewart, et al 2015). Early clinical data from a Phase I/II trial combining anti-PD-L1 and CTLA-4 (NCT02519348) demonstrated safety and a durable objective response rate (ORR) of $18 \%$, prompting study expansion. Methods: HIMALAYA (NCT03298451) is the first randomized, open-label, multicenter, Phase 3 study to assess the efficacy and safety of durvalumab (D) + tremelimumab (T) combination therapy versus sorafenib in the first-line treatment of patients with unresectable, histologically-confirmed HCC. Patients will be randomized to arms evaluating D monotherapy, D+T combination therapy, or sorafenib monotherapy. Patients will be stratified according to macrovascular invasion (yes vs no), etiology of liver disease (HBV versus HCV vs others), and performance status (ECOG 0 vs 1 ). The primary endpoint for this study is overall survival (OS). Secondary endpoints include ORR, duration of response, disease control rate, progression-free survival, and time to progression according to RECIST v1.1 using investigator assessments. Safety and health-related quality of life will also be assessed. Keywords: Immuno-oncology, PD-L1, CTLA-4, unresectable.

\section{PE-068 \\ Safety and Clinical Activity Results from a Ph lb Study of Atezolizumab + Bevacizumab Treatment in 1L Hepatocellular Carcinoma (HCC)}

Baek-Yeol Ryoo', Michael S. Lee ${ }^{2}$, Aiwu Ruth $\mathrm{He}^{3}$, Stacey Stein ${ }^{4}$, Sairy Hernandez ${ }^{5}$, Wendy Verret ${ }^{5}$, Antonia Kwan ${ }^{5}$, Bo Liu' ${ }^{5}$,Koho lizuka ${ }^{5}$, Kyung-Hun Lee

${ }^{1}$ Asan Medical Center, University of Ulsan College of Medicine, Seoul, South Korea; ${ }^{2}$ UNC Lineberger Comprehensive Cancer Center, University of North Carolina at Chapel Hill, Chapel Hill, NC, USA; ${ }^{3}$ Georgetown University Medical Center, Washington, DC, USA; ${ }^{4}$ Yale School of Medicine, New Haven, CT, USA; ${ }^{5}$ Genentech, Inc., South San Francisco, CA, USA; ${ }^{6}$ Seoul National University Hospital, Seoul, South Korea

Aims: Patients with advanced HCC represent a population with unmet medical need. Treatment with a singleagent PD-L1/PD-1 inhibitor or bevacizumab (anti-VEGF) has modest clinical activity in HCC. In addition to its anti-angiogenic activity, bevacizumab has immunomodulatory effects (increased DC maturation, enhanced T-cell infiltration, 
Table 1. Safety overview $(n=26)$ (for Abstract no PE-068)

\begin{tabular}{ll}
\hline Treatment-related AEs, $\mathrm{n}(\%)$ & \\
All grades & $21(81 \%)$ \\
Grade 3-4 & $9(35 \%)$ \\
Grade 5 & 0 \\
Serious, all grades & $2(8 \%)$ \\
Immune-related AEs requiring & $3(12 \%)$ \\
$\quad$ corticosteroid treatment, n (\%) & \\
\hline
\end{tabular}

reduced MDSCs and Tregs in tumors), which create a favorable tumor microenvironment to potentiate the efficacy atezolizumab (anti-PD-L1). Here we evaluate the safety and clinical activity of atezolizumab + bevacizumab in HCC. Methods: Treatment-naive patients with unresectable or metastatic HCC were enrolled in the Ph Ib (NCT02715531) cohort. Atezolizumab was dosed at $1200 \mathrm{mg}$ and bevacizumab at 15 $\mathrm{mg} / \mathrm{kg}$ IV q3w until loss of clinical benefit or unacceptable toxicity. The primary endpoints were safety and tolerability. Secondary efficacy endpoints included ORR, PFS, DOR and time to disease progression (TTP) per RECIST v1.1, and OS. Results: 26 patients were safety evaluable (October 24, 2017). See table for safety overview. Hypertension was the most common treatment-related grade 3-4 AE ( $n=5 ; 19 \%)$. No treatment-related Grade 5 AEs were observed. Treatmentrelated SAEs (autoimmune encephalitis, mental status change and intra-abdominal hemorrhage) occurred in 2 patients $(8 \%$; all grade 3 ). No new safety signals were identified beyond the established safety profile for each agent. 21 patients were efficacy evaluable (minimum follow-up, 16 weeks; median survival follow-up, 8.3 months). 13 patients (62\%) had confirmed PRs regardless of HCC etiology, region (Asia/US), baseline a-fetoprotein levels $(\geq /<400 \mathrm{ng} / \mathrm{mL})$ or extrahepatic tumor spread. Medians for PFS, DOR, TTP and OS have not yet been reached. Conclusions: Atezolizumab + bevacizumab is well tolerated. The confirmed ORR of $62 \%$ suggests this combination has synergistic clinical activity in HCC. Based on these encouraging results, this cohort has been expanded and extended follow-up data will be presented. Evaluation of atezolizumab + bevacizumab in a Ph III study is also ongoing. Keywords: Atezolizumab, Anti-PD-L1, Immunotherapy, Bevacizumab, Hepatocellular carcinoma.

\section{PE-069 \\ Adverse Events Correlation between (SIRT) versus Sorafenib in Locally Advanced Hepatocellular Carcinoma in Mongolia: A Subgroup Analysis of Sirvenib Study Khasbazar Ariunaa ${ }^{1}$, R. Sanduijav², Ya. Bolormaa ${ }^{1}$, A. Tuyatsetseg ${ }^{1}$, M. Adilsaikhan ${ }^{2}$, Pierce Kah-Hoe Chow ${ }^{3}$, Mihir Gandhi', O. Baatarkhuu ${ }^{2}$ \\ ${ }^{1}$ Department of Chemotherapy, National Cancer Center of Mongolia, ${ }^{2}$ Mongolian National University of Medical Sciences, ${ }^{3}$ National Cancer Center of Singapore ${ }^{4}$ Singapore Clinical Research Institute}

Aims: This study will evaluate the efficacy of selective internal radiation therapy (SIRT) using SIR-Spheres ${ }^{\circledR}$ yttrium-90 resin microspheres compared to sorafenib in the treatment of patients with locally advanced hepatocellular carcinoma (HCC). Methods: Total 360 patients were enrolled to the study from 27 sites of Asia-Pacific 11 countries. The National Cancer Center of Mongolia was one of the sites this study and we were recruited a total 39 patients into the study from March 2011 to June 2016 in Mongolia. In this multicenter, phase III, randomized-controlled clinic trial, we randomly assigned patients with advanced hepatocellular carcinoma who had not received surgical therapy for HCC to receive sorafenib (at a dose of $400 \mathrm{mg}$ twice daily) or yttrium-90 resin microspheres by the transarterial route. Results: Among the 39 patients recruited in Mongolia, 19 received at least one dose sorafenib and 20 received SIRT treatment in Singapore; these patients were included in the safety analysis. Adverse events that were reported for patients receiving sorafenib were predominantly grade 1 or 2 in gastrointestinal, constitutional and dermatologic in nature. The most frequently reported drug-related adverse events in patients treated with sorafenib were hypertension, hand-foot skin reaction, diarrhea, alopecia, fatigue. Common procedurerelated adverse events were usually mild (grade 1/2) and included nausea and vomiting $(27.7 \%$ all grades $)$ and abdominal pain $(22.1 \%$ all grades), with very few grade 3 . Conclusions: In conclusion, we found that patients treated SIRT had significantly fewer total numbers of adverse events (p 0.0964) when compared with those treated with sorafenib. Keywords: HCC, Adverse event, SIRT. 


\section{PE-070 \\ Phase I Dose-Finding Study of OPB-111077, a Novel STAT3 Inhibitor, in Patients with Advanced Hepatocellular Carcinoma}

Chanhoon Yoo ${ }^{1}$, Jihoon Kang 1, Ho Yeong Lim², Jee Hyun Kim ${ }^{3}$, Myung-Ah Lee ${ }^{4}$, Kyung-Hun Lee ${ }^{5}$, Tae-You Kim ${ }^{5}$, Baek-Yeol Ryoo ${ }^{1}$

${ }^{1}$ Department of Oncology, Asan Medical Center, University of Ulsan College of Medicine, Seoul, South Korea; ${ }^{2}$ Division of Hematology-Oncology, Department of Medicine, Samsung Medical Center, Sungkyunkwan University School of Medicine, Seoul, South Korea; ${ }^{3}$ Department of Internal Medicine, Seoul National University Bundang Hospital, Seoul National University College of Medicine, Seongnam, South Korea; ${ }^{4}$ Division of Medical Oncology, Department of Internal Medicine, Seoul St. Mary's Hospital, The Catholic University of Korea College of Medicine, Seoul, South Korea; ${ }^{5}$ Department of Internal Medicine, Seoul National University Hospital, Seoul National University College of Medicine, Seoul, South Korea

Aims: The signal transducer and activator of transcription 3 (STAT3) signaling pathway might be a promising therapeutic target for hepatocellular carcinoma (HCC). Methods: This study was a multicenter, open-label, noncomparative, dose escalating phase I study of OPB-111077, an oral STAT3 inhibitor, in patients with advanced HCC who failed on sorafenib. Continuous dosing (daily administration: $50 \mathrm{mg}$ to $400 \mathrm{mg}$ ) and intermittent dosing (4-days on/3-days off administration: $300 \mathrm{mg}$ to $900 \mathrm{mg}$ ) regimens were evaluated and the dose-limiting toxicities (DLTs), maximal tolerated dose (MTD), and recommended dose (RD) were the primary endpoints. Results: A total of 33 patients (19 for continuous dosing and 14 for intermittent dosing) were enrolled. One patient experienced a DLT with grade 3 dizziness, but the MTD was identified in neither the continuous nor the intermittent dosing cohorts. The RDs were determined to be $250 \mathrm{mg}$ for the continuous dosing regimen and $600 \mathrm{mg}$ for the intermittent dosing regimen. There was no treatment-related death; 6 patients $(18 \%)$ had grade $3-4$ toxicities including thrombocytopenia (6\%), fatigue (3\%), and dizziness $(3 \%)$. No patients achieved complete or partial responses and the median progression-free survival was 1.4 months (95\% confidence interval, 0.8-2.8). Conclusions: OPB-11107 was well tolerated in patients with advanced HCC after sorafenib failure, but only showed limited preliminary efficacy outcomes. Further investigation of the role of the STAT3 signaling pathway in HCC and the development of biomarkers for STAT3 inhibitors are warranted. Keywords: Phase 1 trial, STAT3, Hepatocellular carcinoma.

\section{PE-071 \\ Safety and Effectiveness of Regorafenib in Patients with Unresectable Hepatocellular Carcinoma in Routine Clinical Practice: REFINE, a Prospective, Observational Study}

Richard S. Finn ${ }^{1}$, Catherine Frenette ${ }^{2}$, Alessandro Granito ${ }^{3}$, Masafumi Ikeda ${ }^{4}$, Ho Yeong Lim ${ }^{5}$ Philippe Merle ${ }^{6}$, Kirhan Ozgurdal ${ }^{7}$, Masatoshi Kudo $^{8}$

${ }^{1}$ David Geffen School of Medicine at UCLA, Los Angeles, CA, USA; ${ }^{2}$ Scripps Green Hospital, La Jolla, CA, USA; Department of Medical and Surgical Sciences, University of Bologna, S. Orsola-Malpighi Hospital, Bologna, Italy; ${ }^{4}$ National Cancer Center Hospital East, Kashiwa, Japan; ${ }^{5}$ Samsung Medical Center, Sungkyunkwan University School of Medicine, Seoul, South Korea; ${ }^{6}$ Groupement Hospitalier Lyon Nord, Hepatology Unit, Lyon, France; ${ }^{7}$ Bayer HealthCare, Istanbul, Turkey; ${ }^{8}$ Kindai University Faculty of Medicine, Osaka, Japan

Aims: The oral multikinase inhibitor regorafenib significantly improved overall survival versus placebo in patients with unresectable hepatocellular carcinoma (uHCC) who progressed on prior sorafenib in the phase 3 RESORCE trial (Bruix et al. Lancet 2017). REFINE will describe the safety and effectiveness of regorafenib and will also provide information about treatment patterns and management of uHCC in the real-world setting. Methods: This prospective, multicenter, observational trial (NCT03289273) will be conducted in routine clinical practice settings in approximately 30 countries across Europe, North America, Asia, Latin America, the Eastern Mediterranean, the Middle East, and North Africa. The trial aims to recruit 1000 patients with uHCC previously treated with sorafenib, and for whom a decision to treat with regorafenib has been made by the treating physician prior to enrollment according to the local health authority approved label. Dose modifications will be permitted for the management of adverse events (AEs). The primary endpoint is the incidence of treatment-emergent AEs (TEAEs) and dose modifications due to TEAEs (NCI-CTCAE v4.03). Secondary endpoints are overall survival, progression-free survival, time to progression, best overall tumor response, and duration of regorafenib treatment. Investigator-assessed data, according to local standard, will be used to evaluate tumor response and radiologic progression. Data sources will include medical records and routine clinical measurements. All variables will be analyzed descriptively with appropriate statistical methods. Patients receiving $\geq 1$ dose of regorafenib will be included in the final analysis. An interim analysis for safety monitoring is planned after 500 patients have been observed for $\geq 4$ months. The final analysis will be performed after the final patient remaining on study has been observed for 24 months or is no longer under observation due to consent withdrawal or death. Recruitment is underway, with 52 patients enrolled as of January 31, 2018. The estimated 
primary completion date is July 2022. Keywords: Regorafenib, Safety and effectiveness, Hepatocellular carcinoma, Observational.

\section{Treatment: Surgical Resection and Transplantation}

\section{PE-072 \\ Regular Postoperative Surveillance Improves Prognosis in Patients with Late Recurrence after Hepatic Resection of Hepatocellular Carcinoma}

Xin-Fei $X u^{1,2}$, Hao Xing ${ }^{1}$, Jun Han ${ }^{1}$, Jiong-Jie $Y u^{1,2}$, Ju-Dong Li,2, Li-Yang Sun 1,2, Han Zhang ${ }^{1}$, Ming-Da Wang ${ }^{1}$, Zhen-Li Li ${ }^{1}$, Ya-Hao Zhou ${ }^{3}$, Wei-Min Gu ${ }^{4}$, Hong Wang ${ }^{5}$, Ting-Hao Chen ${ }^{6}$, Yong-Yi Zeng ${ }^{7}$, Feng Shen ${ }^{1}$, Tian Yang ${ }^{1}$

${ }^{1}$ Department of Hepatobiliary Surgery, Eastern Hepatobiliary Surgery Hospital, Second Military Medical University, Shanghai, China; ${ }^{2}$ Department of Clinical Medicine, Second Military Medical University, Shanghai, China; ${ }^{3}$ Department of Hepatobiliary Surgery, Pu'er People's Hospital, Yunnan, China; ${ }^{4}$ The First Department of General Surgery, the Fourth Hospital of Harbin, Heilongjiang, China; ${ }^{5}$ Department of General Surgery, Liuyang People's Hospital, Hunan, China; ${ }^{6}$ Department of General Surgery, Ziyang First People's Hospital, Sichuan, China; ${ }^{7}$ Department of Hepatobiliary Surgery, Mengchao Hepatobiliary Hospital, Fujian Medical University, Fujian, China

Aims: Late recurrence ( $>2$ years) after hepatic resection of hepatocellular carcinoma (HCC) is considered as multicentric tumors or de novo cancer formation. We aimed to investigate patterns and prognosis of patients with late recurrence after HCC resection. Methods: 734 patients from a multicenter database who were alive and recurrence-free at 2 years after curative resection of initial HCC were enrolled into this retrospective study. Patterns, treatments and long-term prognosis were investigated and analyzed. Factors associated with overall survival of patients with late recurrence were analyzed by multivariate Cox-regression analysis. Results: Among 303 patients who developed late recurrence, 273 $(90.1 \%)$ were sole intrahepatic recurrence, 30 (9.9\%) were concurrent intrahepatic and extrahepatic recurrence, while none of them was sole extrahepatic recurrence. Curative treatments were performed in 165 (54.4\%) patients with late recurrence, including re-resection, transplantation and local ablation. Multivariate analysis showed regular postoperative surveillance (HR 0.515, 95\% CI 0.343-0.772, $P=0.001$ ) was independently associated with increased overall survival in patients with late recurrence after curative resection of HCC. Conclusions: The patterns of late recurrence suggest that postoperative surveillance after 2 years of surgery should be more targeted. Regular postoperative surveillance improves the probability to receive curative treatments for recurrent HCC, yielding to better prognosis for patients with late recurrence. Keywords: Hepatocellular carcinoma, Hepatic resection, Late recurrence, Postoperative surveillance.

\section{PE-073 \\ Central-located Massive Tumor with Inflow and Outflow Reconstruction For Massive Hepatectomy - A Case Report \\ Xue-Yin Shen, Xu-Guang Hu, Sung-Yeon Hong, Bong-Wan Kim, Hee-Jung Wang \\ Department of surgery, Ajou university medical center, Suwon, South Korea}

Aims: Right trisectionectomy was the most effective curative treatment with massive tumor located in the right lobe especially central location of liver. Due to the compressing or even invasion from huge tumor to main branches of portal vein, hepatic vein and bile duct, adequate consideration of inflow and outflow reconstruction during surgery was needed and vessels acquired from cadaveric donor or artificial should be prepared preoperatively. Methods: We present the case of a 59-year-old man who found a $10 \mathrm{~cm}$ malignant mass in the right lobe with the invasion of right anterior portal vein, right hepatic vein and middle hepatic vein. By performing right portal vein embolization, left lateral section volume was increased from $26.5 \%$ to $37.3 \%$, satisfy the condition of right trisectionectomy. Outflow reconstruction was performed using pericardium, which was acquired from previous cadaveric donor. Results: The recovery process was smooth, patient was discharged in POD 58 without any complication, Conclusions: Preoperatively, sufficient consideration of using allograft to perform inflow or outflow reconstruction is needed in patient with vascular invasion. Postoperatively, regular hepatic mesenteric doppler $\mathrm{F} / \mathrm{U}$ is needed. Usage of allograft is limited in the hospital where cadaveric donor liver transplantation is feasible. Thus, artificial graft should be prepared in the vascular invasion cases. Keywords: Massiver tumor, Hepatectomy, Inflow and outflow reconstruction, 


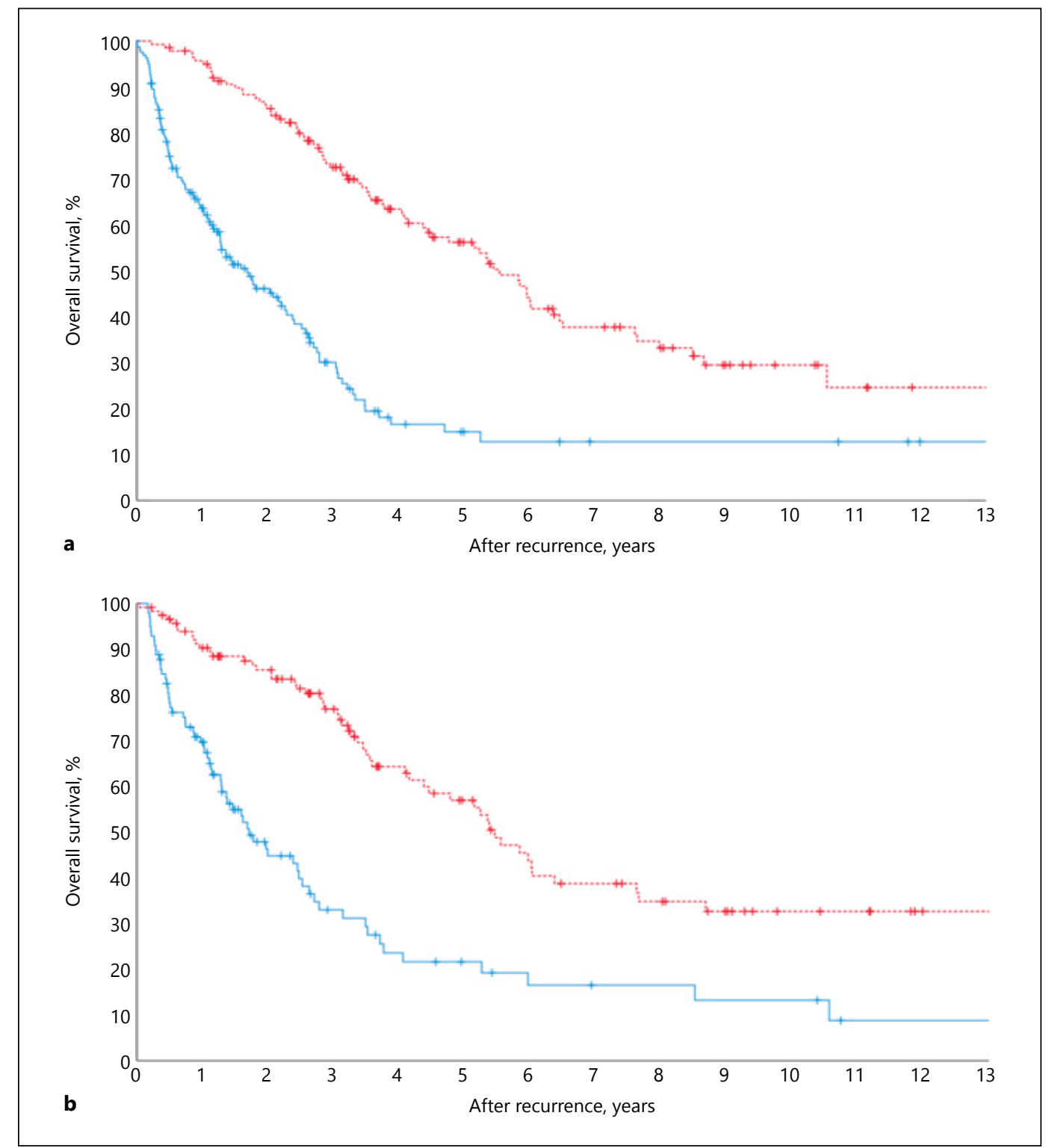

Fig. 1. Overall survival curves of patients with late recurrence after curative resection of hepatocellular carcinoma from the diagnosis of late recurrence (a) Comparisons between patients with regular and irregular/no surveillance $(p<0.001$

\section{PE-074}

\section{Short-Term Outcomes of Patients with} Hepatocellular Carcinoma Who Underwent Liver Resection in Mandalay General Hospital

A Mi Mi Kyaw, Kyaw Soe, Shein Myint

Department of Surgery, Mandalay General Hospital

Aims: Liver Cancer is currently the second most common cause of cancer-related death worldwide and hepatocellular carcinoma (HCC) accounts for more than $90 \%$ of liver cancers. by log-rank test). (b) Comparisons between patients receiving curative and non-curative/no treatment ( $\mathrm{p}<0.001$ by logrank test) (for Abstract no PE-072).

The surgical indications for HCC are decided not only according to the conditions of the tumor, but also according to the liver function (Child-Pugh score and MELD). This study evaluates the short-term outcomes following resection. Methods: Patients who underwent surgical resection for HCC from January 2016 to December 2017 at Surgical Unit (1), Mandalay General Hospital were retrospectively identified. Patients with macro vascular invasion and extra hepatic invasion were excluded. Results: We identified a total of 96 HCC patients who underwent surgical resection $77.08 \%$ male and $22.92 \%$ female. Cirrhosis was present in 80 patients (83.33\%) and the 
proportion of Child-Pugh A and B were $95.83 \%$ and $4.17 \%$ respectively. The risks of HCC were Hepatitis B (61.46\%), Hepatitis C (19.79\%), Hepatitis B and C co-infection (1.04\%) and about $17.71 \%$ in Non-B Non-C Hepatitis cases. The right lobe of liver was more affected. Patients with tumor size larger than $5 \mathrm{~cm}$ were $70.83 \%$ and AFP level above $1000 \mathrm{ng} / \mathrm{dl}$ were $22.92 \%$ and MELD $>8$ were $33.3 \%$ respectively. Patients with BCLC A and B stages were $10.42 \%$ and $89.58 \%$ respectively. The morbidity and in hospital mortality were $11.2 \%$ and $4.17 \%$ respectively. Conclusions: Most of the cases are presented in late stages in Myanmar. According to our study, hepatic resection can be performed in patients with late stage with acceptable morbidity and mortality. A nationwide regular surveillance program is needed in high-risk patients. Keywords: Short-term outcomes, Hepatocellular carcinoma, Liver resection, Mandalay General Hospital.

\section{PE-075 \\ Gender Differences in Recurrence and Mortality after Liver Resection of Hepatocellular Carcinoma: A Multicenter Study}

Han Zhang ${ }^{1}$, Jun Han ${ }^{1}$, Hao Xing ${ }^{1}$, Jiong Jie $\mathrm{Yu}^{1,7}$, Zhen Li Li ${ }^{1}$, Ya Hao Zhou ${ }^{2}$, Ting Hao Chen ${ }^{3}$, Hong Wang ${ }^{4}$, Wei Min Gu ${ }^{5}$, Wan Yee Lau ${ }^{1,6}$, Han Wu ${ }^{1}$, Lei Liang ${ }^{1}$, Chao Li ${ }^{1}$, Ming Da Wang ${ }^{1}$, Xin Fei X $u^{1,7}$, Ju Dong Li $i^{1,7}$, Tian Yi Zhang ${ }^{8}$, Meng Chao Wu ${ }^{1}$, Feng Shen ${ }^{1}$, Myron E. Schwartz ${ }^{9}$, Tian Yang ${ }^{1,9}$

${ }^{1}$ Department of Hepatobiliary Surgery, Eastern Hepatobiliary Surgery Hospital, Second Military Medical University, Shanghai, China; ${ }^{2}$ Department of Hepatobiliary Surgery, Pu'er People's Hospital, Yunnan, China; ${ }^{3}$ Department of General Surgery, Ziyang First People's Hospital, Sichuan, China; ${ }^{4}$ Department of General Surgery, Liuyang People's Hospital, Hunan, China; ${ }^{5}$ The First Department of General Surgery, the Fourth Hospital of Harbin, Heilongjiang, China; ${ }^{6}$ Faculty of Medicine, the Chinese University of Hong Kong, Shatin, New Territories, Hong Kong SAR, China; ${ }^{7}$ Department of Clinical Medicine, Second Military Medical University, Shanghai, China; ${ }^{8}$ Department of Health Statistics, Second Military Medical University, Shanghai, China; ${ }^{9}$ Liver Cancer Program, Recanati/Miller Transplantation Institute, Icahn School of Medicine at Mount Sinai, New York, NY, the United States

Aims: There is a striking male predominance in hepatocellular carcinoma (HCC). The impact of gender on the incidence of recurrence after curative resection of HCC remains unknown. The aim of this study was to assess the sex differences in the rates of recurrence and mortality in patients after curative resection of HCC. Methods: Data from 1,435 HCC patients (1,228 males and 207 females) treated with curative resection between 2004 and 2014 at five institutions in China were retrospectively analyzed. Patients' clinicopathological and operative variables, and rates of early recurrence $(\leq 2$ years after resection), late recurrence ( $>2$ years) and cancerspecific mortality (CSM) were compared. To clarify the true oncologic impact of gender, multivariable competing-risks regression analyses were performed to identify predictors of early and late recurrences, and CSM. Results: The early recurrence rates in males and females were similar $(43.3 \%$ vs. $42.0 \%, P=0.728)$. The late recurrence and CSM rates in males were significantly higher than females $(17.2 \%$ vs. $11.2 \%, P=$ 0.044 ; and $42.8 \%$ vs. $34.3 \%, P=0.022$, respectively). Multivariable competing-risks regression analyses revealed no gender difference in early recurrence; however, males had a significantly higher late recurrence rate [hazard ratio (HR), 1.752; 95\% CI, 1.145-2.682; $P=0.010$ ] and CSM (HR, 1.307; 95\% CI, 1.015-1.683; $P=0.038$ ) than females. Conclusions: Males had significantly higher late recurrence and CSM rates after curative resection of HCC than females. More researches are needed on the underlying role of sex hormone-related therapy for anti-recurrence of HCC. Keywords: Hepatocellular carcinoma, Hepatectomy, Sex, Recurrence, Cancer-specific mortality.

\section{PE-076 \\ Advanced Intrahepatic Cholangiocarcinoma with Long-Term Survival: A Case Report}

Akihisa Nagatsu, Toshiya Kamiyama, Takayoshi Ohata, Shingo Shimada, Kenji Wakayama, Tatsuya Orimo, Hideki Yokoo, Hirofumi Kamachi, Akinobu Taketomi

Department of Gastroeterological Surgery, Graduate School of Medicine, Hokkaido University, Japan

Aims: Lymph node metastasis is widely known as a prognostic factor in intrahepatic cholangiocarcinoma. However, there is no consensus about the area of lymph node dissection or adjuvant treatment. Results: The patient is a 76-year-old female who was pointed out a huge nodular lesion in the left lobe of the liver by echo examination for discomfort in the left lower abdomen. It is suspected of intrahepatic cholangiocarcinoma in computed tomography. Furthermore, para-aortic lymph node swelling was recognized. The extended left lobe resection of the liver, lymph node dissection, and cholecystectomy were performed in our department. The operation time was 6 hours 47 minutes, the bleeding was $165 \mathrm{ml}$, and there is no adverse event during operation or hospital course. The pathological diagnosis is intrahepatic cholangiocarcinoma, mass formation type, $11 \times 7.5 \mathrm{~cm}$, T3N1M1 Stage $4 \mathrm{~b}$ (UICC8th). As adjuvant chemotherapy, the administration of Gemcitabine was scheduled. However, in the evaluation CT before chemotherapy about 1 month after surgery, multiple lymph node swelling was found in the peritoneal cavity. Therapeutic Gemcitabine was administered biweekly, and then the 


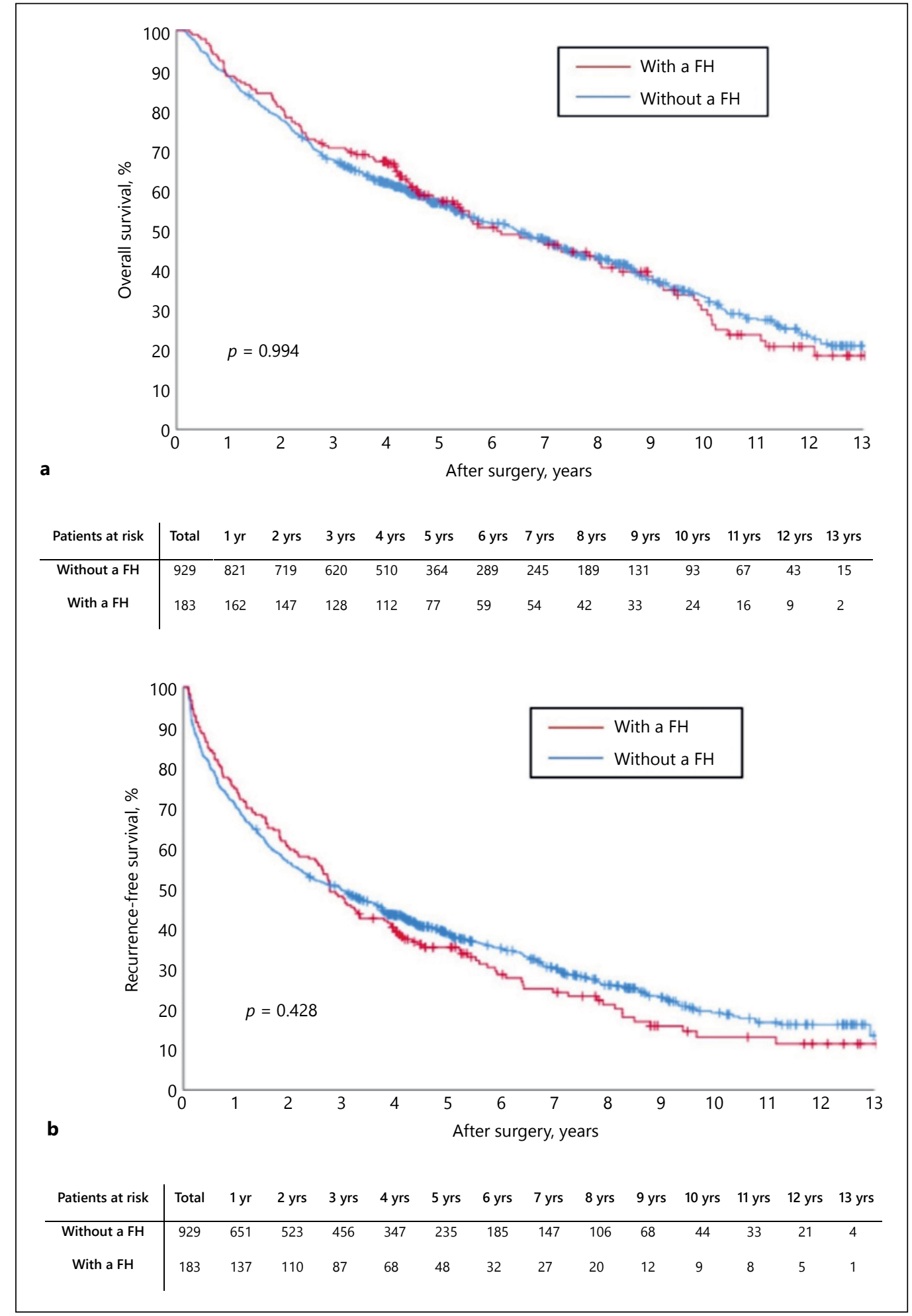

Fig. 1. Cumulative incidence of overall survival (a) and recurrence-free survival (b) curves comparisons between patients with and without a family history (FH) in the entire cohort (for Abstract no PE075). 


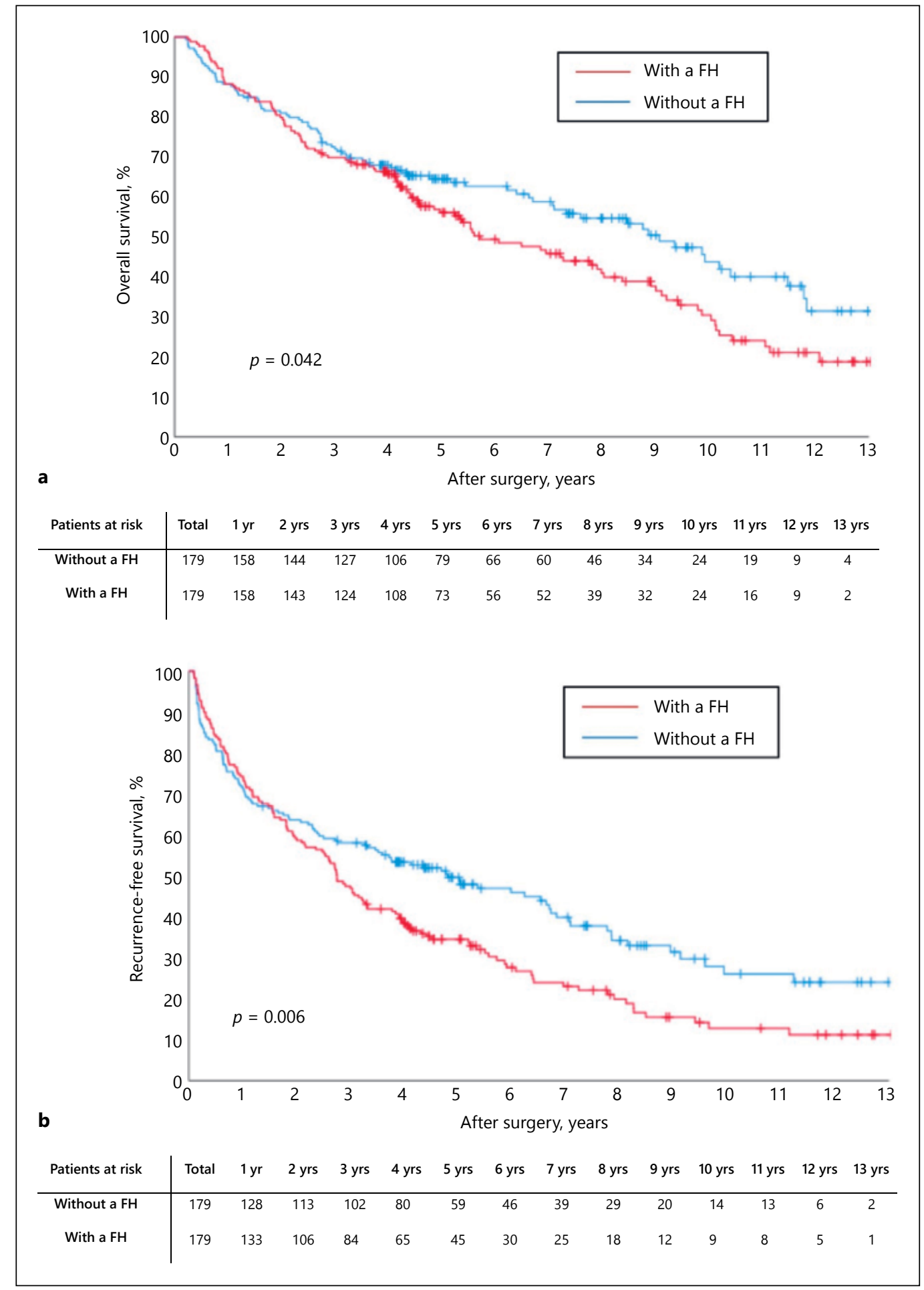

Fig. 2. Cumulative incidence of overall survival (2A) and recurrence-free survival (2B) curves comparisons between patients with and without a family history $(\mathrm{FH})$ in the propensity score matching (PSM) cohort (for Abstract no PE-075). 
lymph node shrank. She is still alive without regrowth of lymph node after 2 years and 4 months. Conclusions: In intrahepatic cholangiocarcinoma with advanced lymph node metastasis, there is a possibility that combination therapy of aggressive surgery and Gemcitabine is effective for intrahepatic cholangiocarcinoma with advanced metastasis of lymph node. Keywords: Intrahepatic cholangiocarcinoma, Lymph node metastasis, Dissection of lymph node, Gemcitabine.

\section{PE-077 \\ Preoperative Low Prealbumin Level Is Associated with Decreased Overall and Recurrence-Free Survivals after Hepatic Resection of Hepatocellular Carcinoma: A Multi-Institutional Study}

Ju-Dong $L i^{1,2}$, Xin-Fei $X u^{1,2}$, Jun Han ${ }^{1}$, Han Wu$u^{1}$, Hao Xing ${ }^{1}$, Chao Li $i^{1}$, Han Zhang ${ }^{1}$, Jiong-Jie $Y u^{1,2}$, Li-Yang Sun 1,2, Ya-Hao Zhou ${ }^{3}$, Wei-Min Gu', Hong Wang ${ }^{5}$, Ting-Hao Chen ${ }^{6}$, Yong-Yi Zeng ${ }^{7}$, Meng-Chao Wu ${ }^{1}$, Feng Shen ${ }^{1}$, Tian Yang ${ }^{1}$

${ }^{1}$ Department of Hepatobiliary Surgery, Eastern Hepatobiliary Surgery Hospital, Second Military Medical University, Shanghai, China; ${ }^{2}$ Department of Clinical Medicine, Second Military Medical University, Shanghai, China; ${ }^{3}$ Department of Hepatobiliary Surgery, Pu'er People's Hospital, Yunnan, China; ${ }^{4}$ The First Department of General Surgery, the Fourth Hospital of Harbin, Heilongjiang, China; ${ }^{5}$ Department of General Surgery, Liuyang People's Hospital, Hunan, China; ${ }^{6}$ Department of General Surgery, Ziyang First People's Hospital, Sichuan, China; ${ }^{7}$ Department of Hepatobiliary Surgery, Mengchao Hepatobiliary Hospital, Fujian Medical University, Fujian, China

Aims: Serum prealbumin is a sensitive and stable marker to profile nutritional status and liver function. This study aims to identify whether preoperative prealbumin level is associated with the long-term prognosis in patients undergoing hepatic resection for hepatocellular carcinoma (HCC). Methods: Patients treated with curative hepatic resection of HCC between 2001 and 2014 at six institutions in China were enrolled. By using $170 \mathrm{mg} / \mathrm{L}$ as cut-off value of serum prealbumin level, these patients were divided into the low and normal preoperative prealbumin groups. The overall survival (OS) and recurrence-free survival (RFS) were analyzed and compared between these two groups. Univariable and multivariable Cox-regression analyses were performed to identify independent predictive factors of OS and RFS. Results: Among 1,483 patients, 437 (29.5\%) had a low prealbumin level within a week before surgery. The 1-, 3-, and 5-year OS and RFS rates of patients in the low prealbumin group were 83.8, 57.0, and $31.1 \%$, and $67.0,39.8$, and $19.9 \%$, which was significantly poorer than those in the normal prealbumin group $(93.0,75.5$, and $42.6 \%$, and $77.0,56.4$, and $28.4 \%$, both $P<0.001$ ). Multivariable Cox-regression analyses revealed that preoperative prealbumin level, but not albumin level, was an independent predictor of OS (HR, 1.454, 95\% CI: 1.241-1.703, $P<0.001$ ) and RFS (HR, 1.277, 95\% CI: 1.102-1.480, $P<0.001)$. Conclusions: Preoperative prealbumin level could be used in predicting long-term prognosis for patients with HCC who underwent hepatic resection. Keywords: Hepatocellular carcinoma, Hepatectomy, Prealbumin, Albumin, Overall survival, Recurrence-free survival.

\section{PE-078 \\ Validation of Risk Factors Affecting The Early Recurrence of Single Lesion Hepatocellular Carcinoma Patients with HBV-Related Liver Cirrhosis After Curative Resection \\ Wan-Joon Kim ${ }^{1}$, Tae-Wan Lim', Pyoung-Jae Park ${ }^{2}$, Sae-Byeol Choi ${ }^{1}$, Wan-Bae Kim ${ }^{1}$ \\ ${ }^{1}$ Division of Hepato-biliary-Pancreas Surgery, Department of Surgery, Korea University Guro Hospital, Korea University Medical College, Seoul, South Korea; ${ }^{2}$ Division of Transplant-vascular Surgery, Department of Surgery, Korea University Guro Hospital, Korea University Medical College, Seoul, South Korea}

Aims: This study was designed to validate the risk factors affecting the early recurrence of single lesion HCC patients with HBV-related liver cirrhosis (LC). Methods: A total of 430 consecutive HCC patients were enrolled in our institution between January 2002 and December 2015. We divided the cohort into three groups: non-recurrence group, the early recurrence (ER) group, which had recurrence within 12 months after resection, and the non-early recurrence (NER) group. Survival rate, univariate, and multivariate analyses were performed to identify the variables associated with recurrence and early recurrence especially. Results: The selected cut-off values with sufficient sensitivity and specificity were 2.0 for Neutrophil-to-Lymphocyte Ratio (NLR) and 200 for PIVKA. The overall survival rate was significantly lower in the ER group than in the NER group ( $p<0.001)$. According to the multivariate analysis, PIVKA greater than $200(p=0.035)$, NLR greater than $2.0(p<$ $0.001)$, elevated Glasgow Prognostic Score (GPS) ( $p=0.003$ ), tumor size greater than $5 \mathrm{~cm}(\mathrm{p}=0.002)$ and presence of lymphovascular invasion ( $p=0.002$ ) were significantly different among the groups and affected the early recurrence of HCC Conclusions: NLR, GPS and serum level of PIVKA offer significant prognostic information associated with early recurrence following single lesion hepatocellular carcinoma patients with HBV-related liver cirrhosis after curative resection. Keywords: Early recurrence, Hepatocellular carcinoma, Neutrophil-to-Lymphocyte ratio, Curative resection 
The 9th Asia-Pacific Primary Liver Cancer Expert Meeting (APPLE 2018)

a

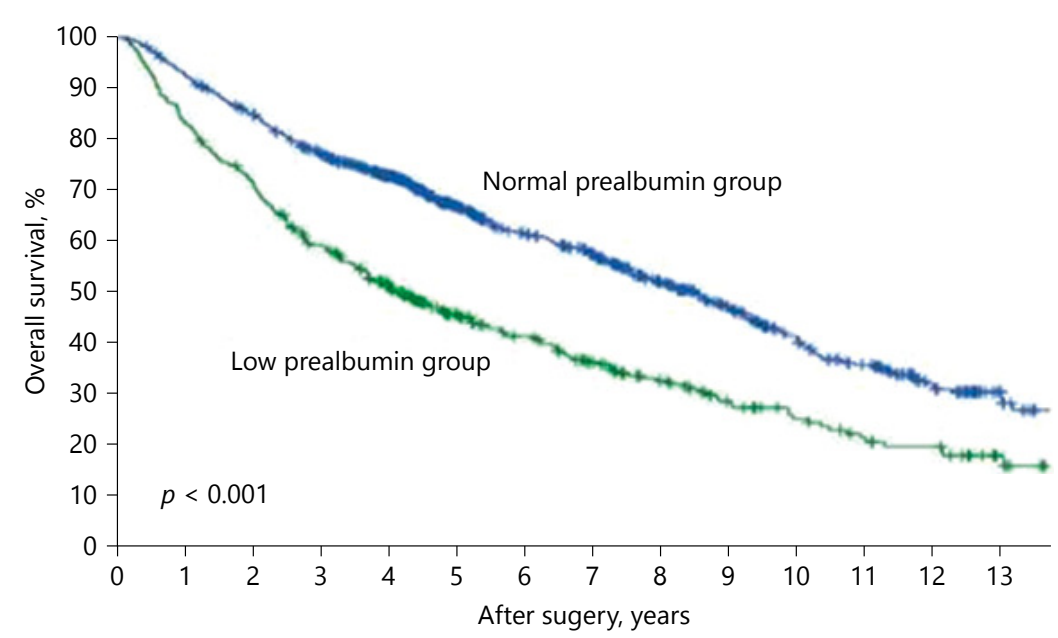

Patients at risk Normal prealbumin group Low prealbumin group

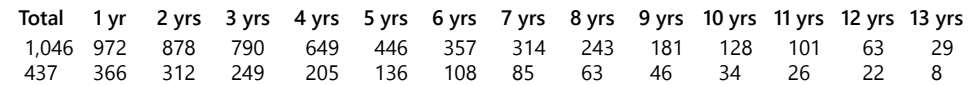

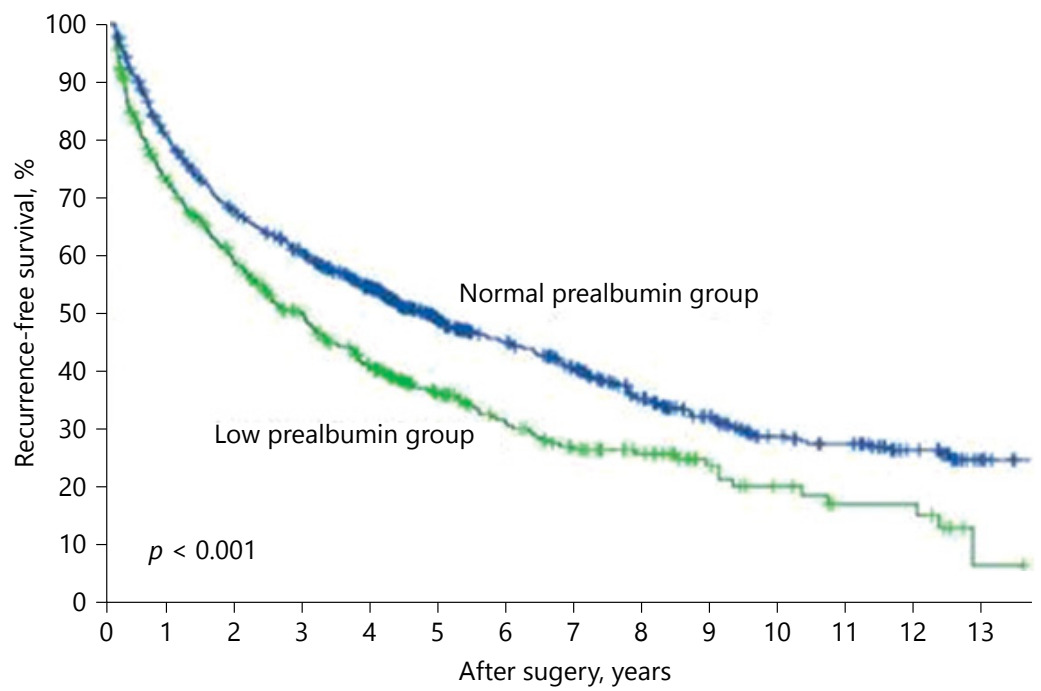

Patients at risk

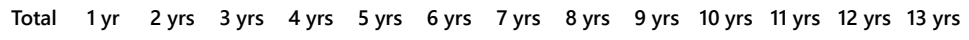
Normal prealbumin group Low prealbumin group

$\begin{array}{llllllllllllll}1,046 & 805 & 665 & 590 & 460 & 297 & 235 & 187 & 138 & 100 & 69 & 61 & 40 & 13\end{array}$

$\begin{array}{llllllllllllll}437 & 293 & 230 & 174 & 128 & 87 & 60 & 45 & 35 & 20 & 13 & 9 & 8 & 1\end{array}$

Fig. 1. (for Abstract no PE-077). 


\section{PE-079 \\ Could Preoperative Downstaging Treatment Decrease Hepatocellular Carcinoma Recurrence after Liver Transplantation? \\ Kuo-Shyang Jeng ${ }^{1}$, Chun-Chieh Huang ${ }^{2}$, Chien-Kuan Lin ${ }^{3}$, Chien-Chu Lin ${ }^{3}$, Chung-Tsui Huang ${ }^{3}$, Chen-Shuan Chung ${ }^{3}$, Kuo-Hsin Chen ${ }^{1}$ \\ ${ }^{1}$ Department of Surgery, Far Eastern Memorial Hospital, Taipei, Taiwan; ${ }^{2}$ Department of Radiology, Far Eastern Memorial Hospital, Taipei, Taiwan; ${ }^{3}$ Division of Gastroenterology, Far Eastern Memorial Hospital, Taipei, Taiwan}

Aims: Downstaging treatment of hepatocellular carcinoma (HCC) prior to liver transplantation (LT) is an accepted strategy. The main aim is to meet Milan or UCSF criteria. Whether the treatment decreases the HCC recurrence after LT remains elucidation. Methods: Twenty-six patients who received downstagings (group A) and 6 patients who did not (group B) were compared in demographics, MELD scores, tumor sizes, tumor numbers, waiting period, pre-LT images, explant pathology and recurrence (time and pattern) were compared. The patients with HCC recurrence after LT were analyzed (recurrent pattern, treatment and outcome). Results: The significant factors may affect recurrence included tumor number before downstaging $(P=0.04)$, tumor size after downstaging $(P=0.01)$ and tumor size in the explant liver $(P=0.02)$. Recurrence developed in 4 patients. The most common site of recurrence is the lung. Conclusions: Some limitation exists because the two groups patients were not completely similar. However, the tumor burden prior to downstagings is a major determinant of HCC recurrence after transplantation. The undetected tiny lesions on preoperative images may affect the outcomes. Downstaging make the HCC to meet Milan or UCSF criteria but probably could not decrease the postoperative recurrence. The individual biological behavior of tumor has to be concerned. Keywords: Downstaging, Hepatocellular carcinoma, Recurrence, Liver transplantation.

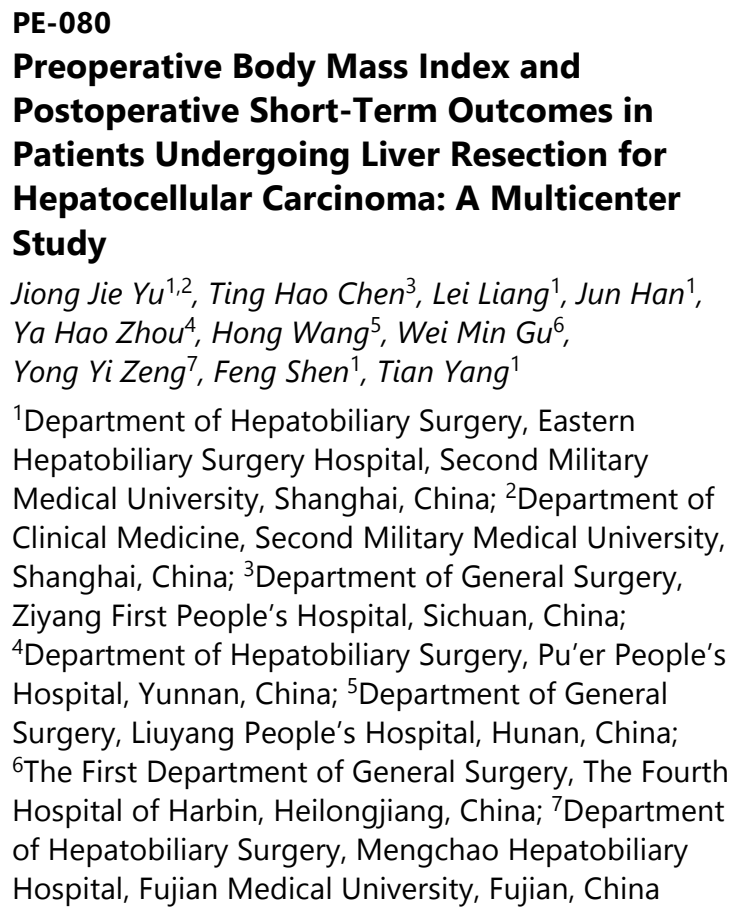

Aims: Whether preoperative body weight is associated with short-term outcomes in patients after liver resection for hepatocellular carcinoma (HCC) is controversial. We aimed to investigate the relationship among groups of patients with under-, normal- or over-weight with short-term mortality and morbidity after curative liver resection for HCC. Methods: The patients with HCC who underwent curative liver resection from 2014 to 2016 in six centers in China were consecutively enrolled in this study and retrospectively analyzed. These patients were divided into 3 groups according to the preoperative body mass index (BMI): underweight $\left(\mathrm{BMI} \leq 18.4 \mathrm{~kg} / \mathrm{m}^{2}\right)$, normal-weight (BMI $18.5-24.9 \mathrm{~kg} / \mathrm{m}^{2}$ ) and overweight (BMI $\geq 25.0 \mathrm{~kg} / \mathrm{m}^{2}$ ). Patients' baseline characteristics, intraoperative variables, and postoperative overall mortality and morbidity were compared. Univariable and multivariable logistic regression analyses were performed to identify the risk factors of postoperative 30-day morbidity after liver resection. Results: Of 1,324 patients, 108 patients (8.2\%) were underweight, 733 (55.4\%) normal-weight, and 483 (36.5\%) overweight. Postoperative overall mortality and morbidity was $1.4 \%$ and $27.1 \%$ in the whole cohort. There were no differences in overall mortality among the three groups. Compared with normal-weight group, both overall and major morbidity were significantly higher in underweight group (33.3\% vs. $22.9 \%, P=0.018$, and $9.3 \%$ vs. $4.9 \%, P=$ $0.028)$ and overweight group $(32.1 \%$ vs. $22.9 \%, P<0.001$, and $8.5 \%$ vs. $4.9 \%, P=0.035$ ). Multivariable analyses identified both underweight and overweight were independent associated with overall morbidity (OR 1.690, 95\% CI 1.058-2.702, $P=0.028$, and OR $1.509,95 \%$ CI $1.146-1.985, P=0.003)$. The similar results were also confirmed for major morbidity and 
the incidence of surgical site infection. Conclusions: Preoperative underweight and overweight were independently associated with postoperative overall and major morbidity in patients undergoing liver resection of HCC. Keywords: Hepatocellular carcinoma, Liver resection, Body mass index, Mortality, Morbidity.

\section{PE-081 \\ How to Treat Single Very Large Hepatocellular Carcinoma without Portal Vein Thrombosis: A Single Center Retrospective Study \\ Soon Kyu Lee ${ }^{1}$, Pil Soo Sung ${ }^{1}$, Jeong Won Jang ${ }^{1}$, Si Hyun Bae ${ }^{1}$, Jong Young Choi ${ }^{1 *}$, Seng Kew Yoon ${ }^{1}$ \\ ${ }^{1}$ The Catholic University Liver Research Center, Department of Internal Medicine, Seoul St. Mary's Hospital, College of Medicine College of Medicine, The Catholic University of Korea, Seoul, South Korea}

Aims: In the treatment of hepatocellular carcinoma (HCC), single very large carcinoma ( $\geq 7 \mathrm{~cm}$ ) is still difficult for clinician to choose treatment modality due to concern of recurrence. In this study, we aimed to evaluate the survival across treatment modalities in single large carcinoma without portal vein thrombosis (PVT). Methods: From 2002 to 2013, 1,685 patients were newly diagnosed HCC patients in Seoul St. Mary's hospital. Of them, 1,628 patients were excluded with following reasons: metastasis (187 patients), less than $7 \mathrm{~cm}$ (1,095 patients), PVT (249 patients) and multiple HCC (93 patients). 57 patients were included and evaluated on overall survival according to treatment mortalities. Moreover, response rate using RECIST 1.1, recurrence free survival and risk factor for survival were also examined. Results: Fiftyseven included patients had mean 59.7 years and hepatitis B (50.9\%) was the main etiology. The majority were CTP class A $(86.0 \%)$ and 18 patients had cirrhosis. The median tumor size and AFP were $10.0 \mathrm{~cm}(7.0-14.9 \mathrm{~cm}), 70.6 \mathrm{ng} / \mathrm{mL}(0.7-$ $200,000)$, respectively. There was no significant difference in baseline characteristics between treatment modalities. Surgery based group $(\mathrm{n}=11)$ had higher survival than other treatment group ( $\mathrm{p}=0.008)$. In subgroup analysis, surgerybased group showed higher survival than TACE only group $(\mathrm{n}=21)$, TACE based group $(\mathrm{n}=6)$ and conservative group $(n=4)$. However, compared with TARE based group $(n=5)$, there was no significant difference. Better response (complete response [CR] rate: $91 \%$ ) was note in surgery-based group than that of TACE only group (CR rate: $41 \%$ ) and TACE based group (CR rate: 19\%). Surgery based group had better recurrence free survival than other treatment group without significance $(p=0.062)$. In multivariate analysis, surgery-based treatment $(p=0.027)$, creatinine $(p=0.016)$ and AFP $(p=$ 0.004 ) were independent prognostic factors for survival. Conclusions: In the treatment of single large $(\geq 7 \mathrm{~cm})$ HCC without PVT, surgery-based treatment was better treatment modality than other treatments. Keywords: Hepatocellular carcinoma, Treatment, Single, Large.
PE-082
Development and Validation of Nomograms to Provide Individualized Predictions of Survival Benefits from Surgery in Patients with Intermediate/Advanced Hepatocellular Carcinoma
Xin-Fei Xu 1,2, Wen-Tao Yan 1,2, Jia-He Wang 1,2, Ming-Da Wang ${ }^{1}$, Zheng Wang ${ }^{1,2}$, Bing Quan ${ }^{1,2}$, Ya-Hao Zhou ${ }^{3}$, Wei-Min Gu', Hong Wang ${ }^{5}$, Ting-Hao Chen ${ }^{6}$, Feng Shen ${ }^{1}$, Tian Yang ${ }^{1}$
${ }^{1}$ Department of Hepatobiliary Surgery, Eastern Hepatobiliary Surgery Hospital, Second Military Medical University, Shanghai, China; ${ }^{2}$ Department of Clinical Medicine, Second Military Medical University, Shanghai, China; ${ }^{3}$ Department of Hepatobiliary Surgery, Pu'er People's Hospital, Yunnan, China; ${ }^{4}$ The First Department of General Surgery, the Fourth Hospital of Harbin, Heilongjiang, China; ${ }^{5}$ Department of General Surgery, Liuyang People's Hospital, Hunan, China; ${ }^{6}$ Department of General Surgery, Ziyang First People's Hospital, Sichuan, China

Aims: According to the BCLC treatment guidelines, surgery is not recommended for intermediate/advanced hepatocellular carcinoma (HCC). In real world, however, liver resections are often performed in patients with intermediate/ advanced but resectable HCC, especially in the East. Methods: We retrospectively evaluated multicentric data of 1,325 patients newly diagnosed with intermediate/advanced HCC who underwent curative resection. We randomly divided the subjects into development $(n=875)$ and validation $(n=450)$ samples. Multivariate Cox proportional hazards models were developed and separately validated on the basis of patients' clinicopathological variables assessed for associations with 1-year recurrence and 3-year mortality. The discriminatory accuracy of these models was compared with conventional tools by analyzing receiver operating characteristic (ROC) curves. Results: The statistical nomograms built based on performance status, Child-Pugh grade, portal hypertension, preoperative alpha-fetoprotein level, tumor rupture, largest tumor diameter, tumor number, macrovascular invasion, and satellites had good calibration and discriminatory abilities, with c-indices of 0.70 (1-year recurrence) and 0.68 (3-year survival), respectively. These models showed satisfactory goodness-of-fit and discrimination abilities in the validation cohort (c-index, 0.68 for 1 -year recurrence and 0.69 for 3 -year survival). The areas under the ROC curve using these nomograms exceeded those of traditional staging systems, indicating superior discriminatory capability (c-indices, 0.60- 
0.63 and $0.56-0.62$, respectively). Conclusions: Our proposed online nomograms, which present graphically postoperative prognostic models for recurrence and survival in patients with intermediate/advanced but resectable HCC, offer valuable guidance to surgeons and hepatologists for individually predicting survival benefits from surgery and planning recurrence surveillance and adjuvant therapy. Keywords: Hepatocellular carcinoma, Nomograms, Curative resection, Survival benefits.

\section{PE-083 \\ No Touch Isolation Technique for The Prevention of Postoperative Recurrence of Hepatocellular Carcinoma after Liver Transplantation-Combined with Transarterial Radioembolization}

Jeong-Moo Lee ${ }^{1}$, Kwang-Woong Lee ${ }^{1}$, Suk Kyun Hong ${ }^{1}$, Kyung Chul Yoon ${ }^{1}$, Jae-Hyung Cho ${ }^{1}$, Nam-Joon Yi ${ }^{1}$, Kyung-Suk Suh ${ }^{1}$

${ }^{1}$ Department of Surgery, Seoul National University College of Medicine, Seoul, Korea

Aims: Recently, trans-arterial radioembolization (TARE) was done in the patients who had advance stage hepatocellular carcinoma. Sometimes totally necrosis of tumor was reported after operation. No touch isolation technique is concept of preventing tumor spread during tumor operation. We expected that if we can use these techniques and control all viable tumors before transplantation. We could get better outcomes in the hepatocellular patients. We performed living donor liver transplantation using no touch isolation technique in the patients who had multinodular hepatocellular carcinoma and high AFP, PIVKA level after TARE and conventional TACE. Methods: 36-year old female patient had liver cirrhosis with hepatitis $\mathrm{B}$ virus infection and multiple hepatocellular carcinoma in both lobe. Alpha-feto protein level was 850,000 PIVKA 136,000. At first, there were high change of recurrence, we did not consider liver transplantation. Hepatologist decided to do TARE and additional conventional TACE. After that treatment, AFP and PIVKA level were dramatically decreased, and there was no viable tumor in follow up CT after 3 weeks. Results: Living donor liver transplantation wad done, donor was 32-year old her sister, there was no variation in the donor side. Total operative time was 4-hour $30 \mathrm{~min}$ and blood loss were $100 \mathrm{cc}$. We did recipient hepatectomy using no touch isolation technique, suprahepatic and infrahepatic IVC were isolated and clamped. Then we clamped hilum and resected using high hilar dissection technique. Immediate postoperative period there was no acute complication, patients transferred to general ward at postoperative 4 days then discharged postoperative 14 days. Postoperative 1 month, patients is alive, there is no recurrence, and AFP level is 11.5 and PIVKA level is 33 Conclusions: TARE (trans- arterial radioembolization) is good modality for pre-transplant treatment for far advanced stage case of hepatocellular carcinoma. No touch isolation technique during recipient hepatectomy might be helpful in advanced stage hepatocellular carcinoma patients. Keywords: Hepatocellular carcinoma, Transarterial Radioembolization, Liver transplantation,

\section{PE-084 \\ Association of Family History with Cancer Recurrence and Survival in Patients with Hepatitis B-related Hepatocellular Carcinoma: A Propensity Matching Analysis}

Jun Han ${ }^{1}$, Zhen Li Li ${ }^{1}$, Hao Xing ${ }^{1}$, Han Zhang ${ }^{1}$, Han Wu${ }^{1}$, Chao Li ${ }^{1}$, Ming Da Wang ${ }^{1}$, Wan Yee Lau ${ }^{1,2}$, Meng-Chao Wu ${ }^{1}$, Feng Shen ${ }^{1}$, Tian Yang ${ }^{1}$

${ }^{1}$ Department of Hepatobiliary Surgery, Eastern Hepatobiliary Surgery Hospital, Second Military Medical University, Shanghai, China; ${ }^{2}$ Faculty of Medicine, the Chinese University of Hong Kong, Shatin, New Territories, Hong Kong SAR, China

Aims: A family history of liver cancer is regarded as a risk factor for hepatocellular carcinoma (HCC) development. In this study, we investigated the association between family history and cancer recurrence and survival in patients with hepatitis B virus (HBV)-related HCC. Methods: Patients who underwent curative resection of HBV-related HCC between 2003 and 2013 from a tertiary hepatobiliary center in China were enrolled in this study. A family history was defined as a self-reported history of primary liver cancer in the firstdegree relatives. Propensity score matching (PSM) and multivariable Cox-regression analyses were performed to compare the overall survival (OS) and recurrence-free survival (RFS) between patients with and without a family history of liver cancer, thus revealing the true oncologic impact of family history. Results: Of 1,112 patients, 183 patients (16.5\%) had a family history of liver cancer. A family history was not associated with OS and RFS ( $\mathrm{P}=0.994$ and 0.428 , respectively) in the entire cohort. Using PSM, 179 pairs of patients with and without a family history but with comparable baseline characteristics and operative variables were created. A family history was associated with decreased OS and RFS (P = 0.042 and 0.006, respectively) in the PSM cohort. On multivariable Coxregression analyses, a family history was significantly associated with decreased OS (HR: 1.574, 95\% CI: 1.171-2.116, P= 0.003 ) and RFS (HR: 1.534, 95\% CI: 1.176-2.002, P = 0.002) after adjusting for other prognostic factors. Conclusions: A family history of liver cancer was associated with decreased OS and RFS rates after curative resection in patients with HBV-related HCC. Keywords: Hepatocellular carcinoma, Family history, Hepatectomy, Overall survival, Recurrencefree survival. 


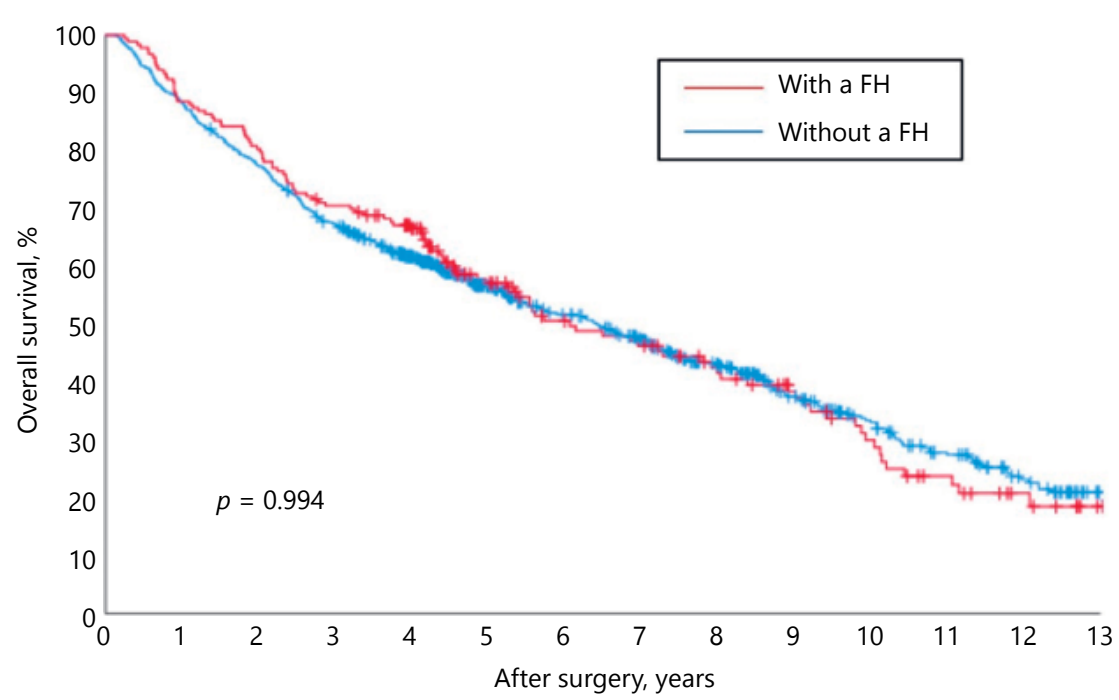

\begin{tabular}{c|cccccccccccccc} 
Patients at risk & Total & $1 \mathrm{yr}$ & $2 \mathrm{yrs}$ & $3 \mathrm{yrs}$ & $4 \mathrm{yrs}$ & $5 \mathrm{yrs}$ & $6 \mathrm{yrs}$ & $\mathbf{7} \mathrm{yrs}$ & $8 \mathrm{yrs}$ & $9 \mathrm{yrs}$ & $10 \mathrm{yrs}$ & $11 \mathrm{yrs}$ & $12 \mathrm{yrs}$ & $13 \mathrm{yrs}$ \\
\hline Without a FH & 929 & 821 & 719 & 620 & 510 & 364 & 289 & 245 & 189 & 131 & 93 & 67 & 43 & 15 \\
With a FH & 183 & 162 & 147 & 128 & 112 & 77 & 59 & 54 & 42 & 33 & 24 & 16 & 9 & 2
\end{tabular}

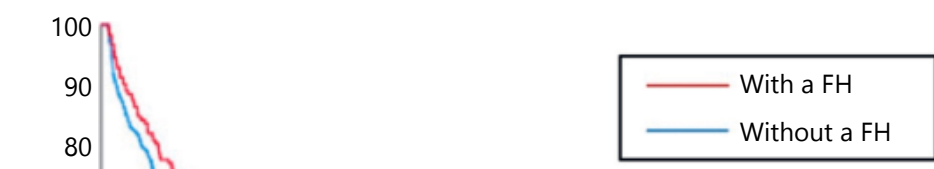

b

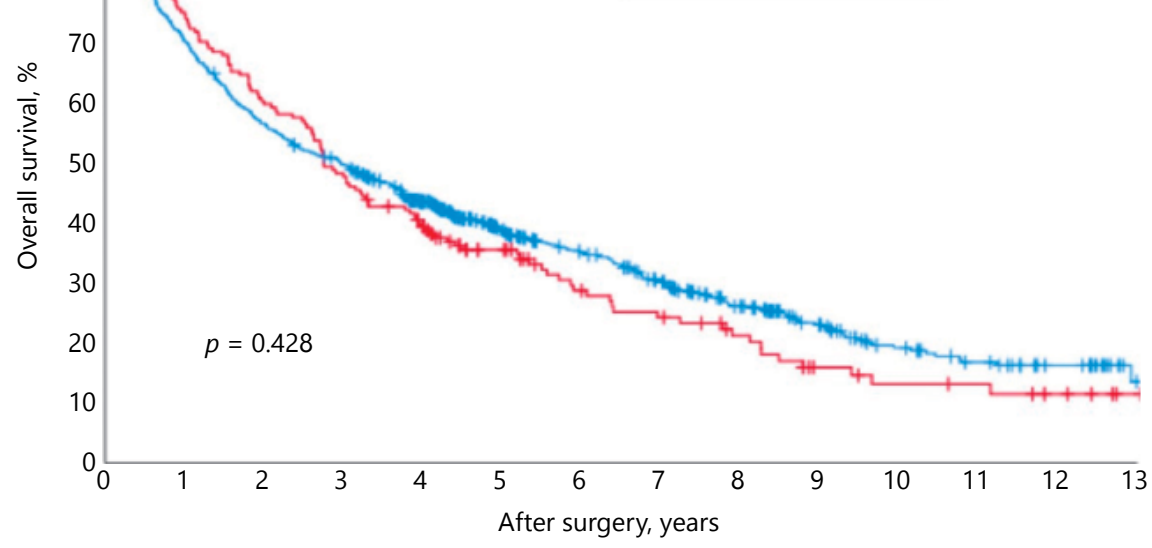

\begin{tabular}{|c|c|c|c|c|c|c|c|c|c|c|c|c|c|c|}
\hline Patients at risk & Total & $1 \mathrm{yr}$ & 2 yrs & 3 yrs & 4 yrs & 5 yrs & 6 yrs & 7 yrs & 8 yrs & 9 yrs & $10 \mathrm{yrs}$ & $11 \mathrm{yrs}$ & 12 yrs & $13 \mathrm{yrs}$ \\
\hline Without a FH & 929 & 651 & 523 & 456 & 347 & 235 & 185 & 147 & 106 & 68 & 44 & 33 & 21 & 4 \\
\hline With a FH & 183 & 137 & 110 & 87 & 68 & 48 & 32 & 27 & 20 & 12 & 9 & 8 & 5 & 1 \\
\hline
\end{tabular}

Fig. 1. Cumulative incidence of overall survival (a) and recurrence-free survival (b) curves comparisons between patients with and without a family history (FH) in the entire cohort (for Abstract no PE-084). 


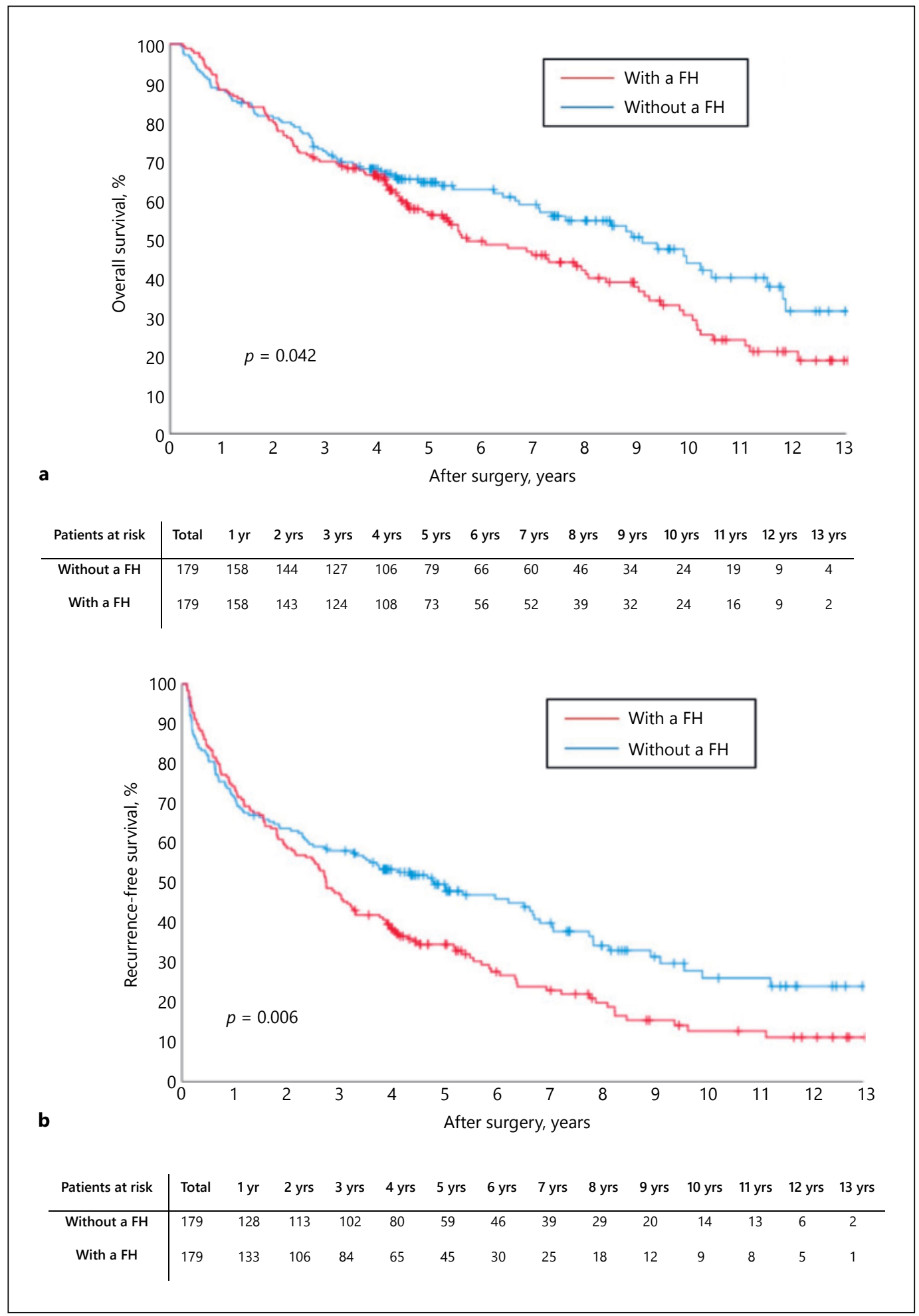

Fig. 2. Cumulative incidence of overall survival (a) and recurrence-free survival (b) curves comparisons between patients with and without a family history $(\mathrm{FH})$ in the propensity score matching (PSM) cohort (for Abstract no PE-084). 


\section{PE-085 \\ Multiple Hepatocellular Carcinoma within the Milan Criteria: When to Consider Surgical Resection?}

Joo Hyun Oh ${ }^{1}$, Dong Hyun Sinn ${ }^{1}$, Gyu-Seong Choi ${ }^{2}$, Jong Man Kim², Jae-Won Joh', Wonseok Kang ${ }^{1}$, Geum-Youn Gwak1, Yong-Han Paik', Joon Hyeok Lee 1 , Kwang Cheol Koh ${ }^{1}$, Seung Woon Paik', Moon Seok Choi ${ }^{1}$

${ }^{1}$ Department of Medicine, ${ }^{2}$ Department of Surgery, Samsung Medical Center, Sungkyunkwan University School of Medicine, Seoul, Korea

Aims: Although surgical resection is usually considered for single tumor, several reports suggested that resection can be considered for multiple tumor and may provide better outcome. We analyzed whether resection can provide better long-term outcome for patients with multiple hepatocellular carcinoma (HCC) within the Milan criteria, and looked for factors that may guide treatment selection. Methods: A total of 314 consecutive patients with multiple HCCs within Milan criteria and had preserved liver function, defined by ChildPugh class $A$, who underwent resection $(n=49)$, radiofrequency ablation (RFA) $(\mathrm{n}=97)$ or transarterial chemoembolization (TACE) $(\mathrm{n}=168)$, as an initial treatment, between January 2009 and December 2013 were analyzed. Results: The 5-year overall survival rates were $91.3 \%, 69.1 \%$, and $61.0 \%$, for patients received resection, RFA and TACE, respectively $(p=0.003)$. Patients who received resection were younger and had more preserved liver function assessed by albumin-bilirubin (ALBI) grade, showed different tumor characteristics (more frequently two tumors and had higher PIVKA-II levels) compared to those received RFA or TACE. Resection was rarely performed for those with ALBI grade 2 $(\mathrm{n}=2)$. In multivariable analysis, initial treatment modality was independent factor associated with overall survival, along with ALBI grade and PIVKA-II levels. When stratified according to ALBI grade and PIVKA-II levels, long-term outcome was significantly different (5-year survival rates: 90.7\%, 72.6\% and $45.7 \%$ for resection, RFA and TACE, respectively, $\mathrm{p}=$ 0.004 ) by initial treatment modality for those with ALBI grade 1 and high PIVKA-II levels ( $>40 \mathrm{mAU} / \mathrm{ml}$ ). For other subgroups, there was no significant different of overall survival by initial treatment modalities. Conclusions: Our findings suggest that resection can provide better long-term outcome than RFA or TACE in selected multiple HCC patients within the Milan criteria. ALBI grade and PIVKA-II levels were factors that can be used to guide initial treatment modalities in this situation, which warrants prospective validation. Keywords: Multiple hepatocellular carcinoma, Resection, Radiofrequency ablation, Transarterial chemoembolization, Albumin-bilirubin grade, PIVKA-II.

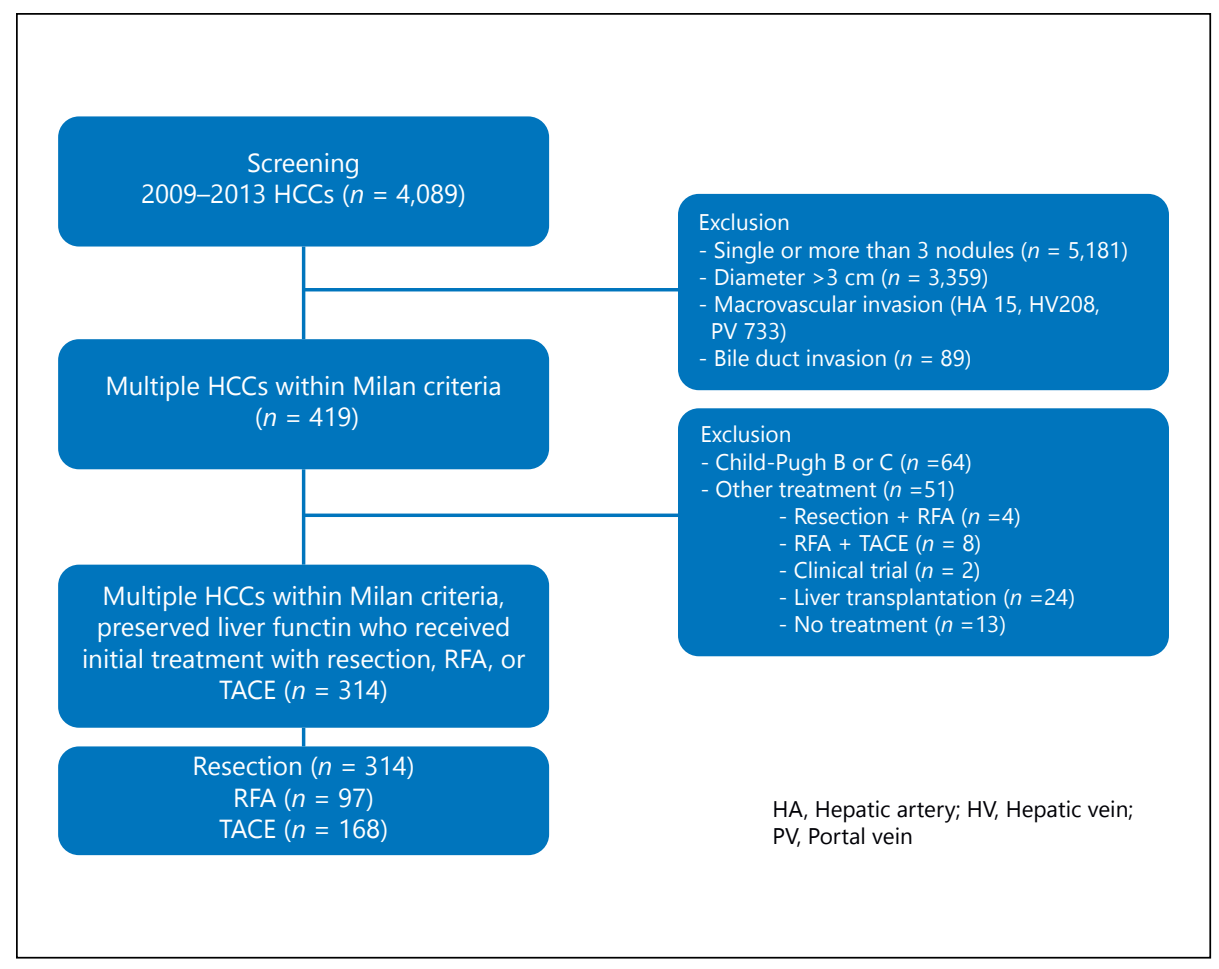

Fig. 1. Participants (for $\mathrm{Ab}$ stract no PE-085). 
The 9th Asia-Pacific Primary Liver Cancer Expert Meeting (APPLE 2018)

Fig. 2. Overall survival by initial treatment modality (for Abstract no PE-085).

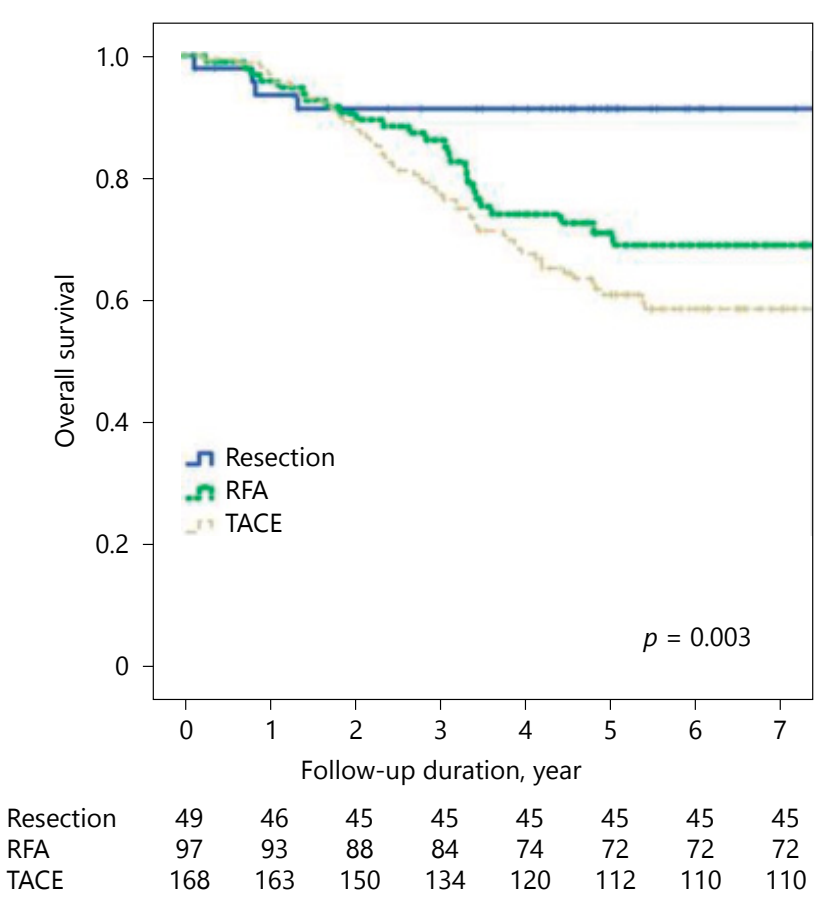

Fig. 3. Overall survival rates based on ALBI grade and PIVKA-II (for Abstract no PE085).

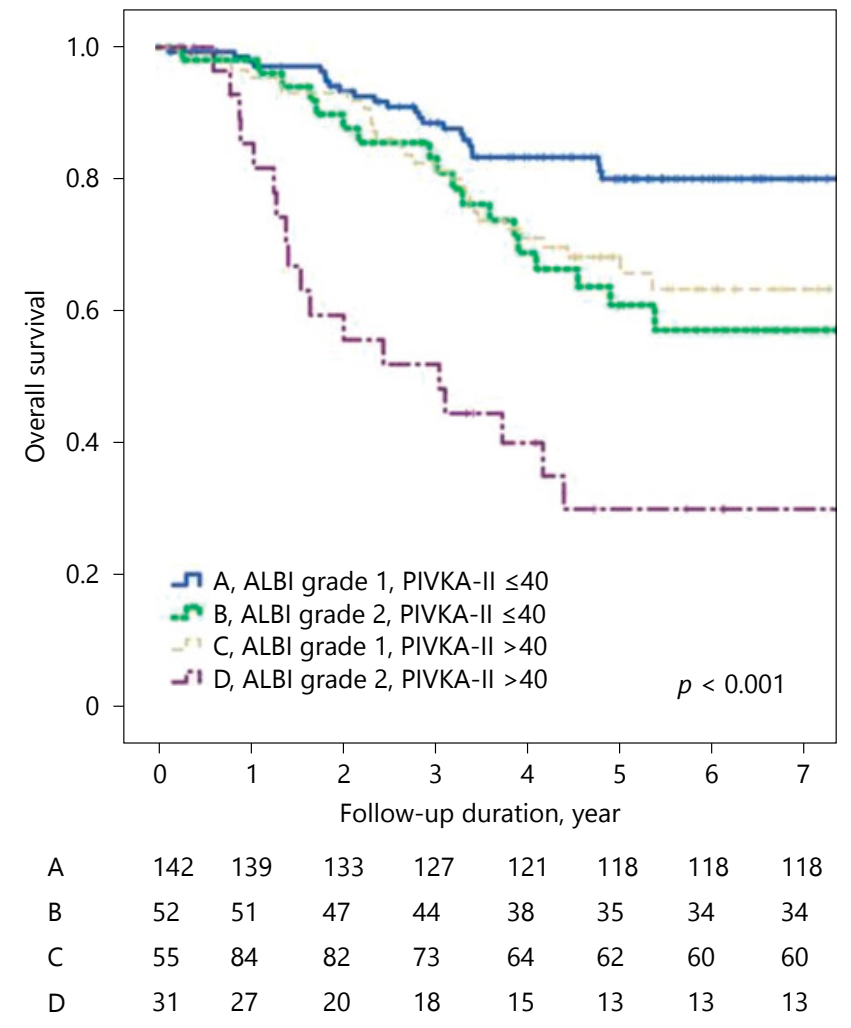

\section{KARGER}




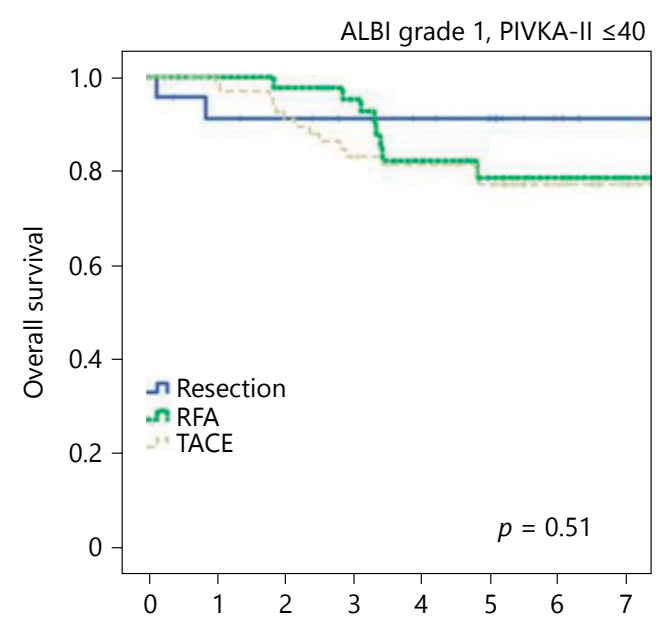

a Follow-up duration, year $\begin{array}{lllllllll}\text { Resection } & 24 & 22 & 22 & 22 & 22 & 22 & 22 & 22\end{array}$ $\begin{array}{lllllllll}\text { RFA } & 47 & 47 & 46 & 45 & 40 & 39 & 39 & 39\end{array}$ $\begin{array}{lllllllll}\text { TACE } & 72 & 71 & 66 & 61 & 60 & 58 & 58 & 58\end{array}$

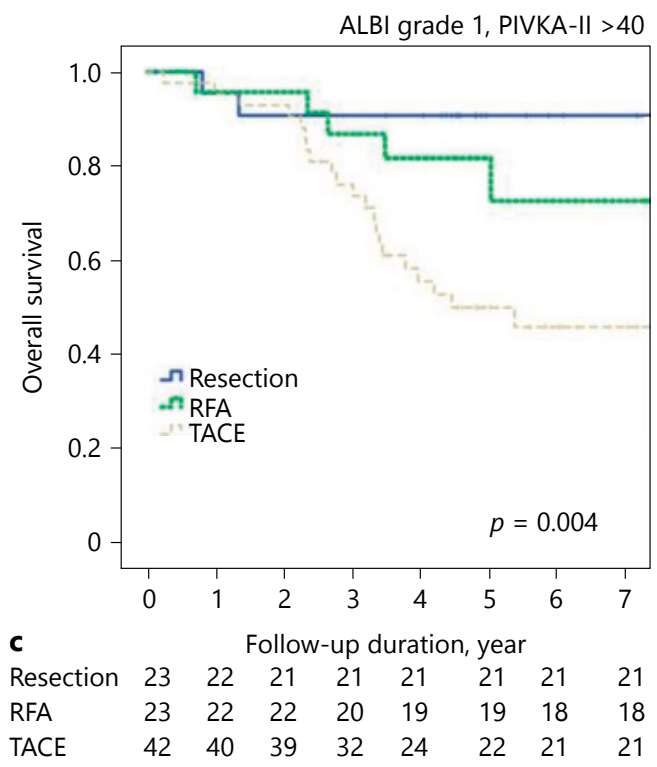

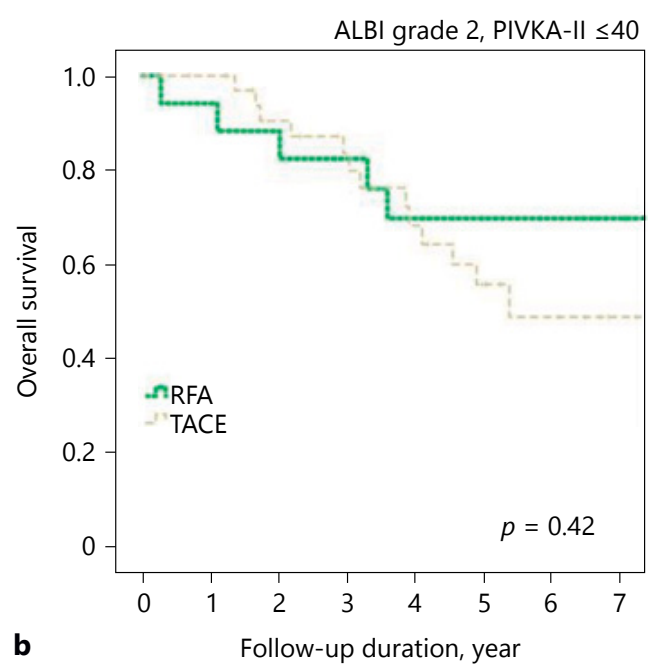

Resection 0

$\begin{array}{lllllllll}\text { RFA } & 17 & 16 & 15 & 14 & 12 & 12 & 12 & 12\end{array}$
$\begin{array}{lllllllll}\text { TACE } & 35 & 35 & 32 & 20 & 17 & 14 & 13 & 13\end{array}$

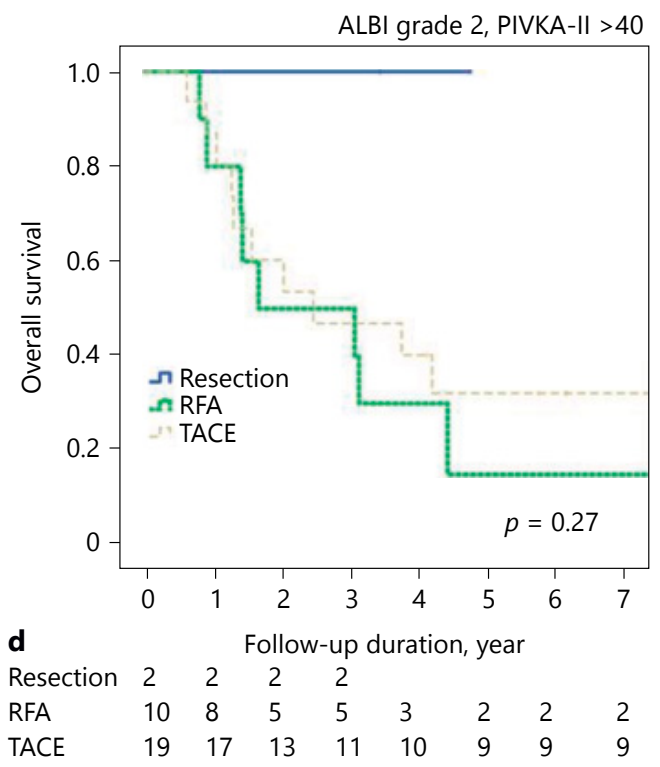

Fig. 4. Subgroup analysis according to ALBI grade and PIVKA-II (for Abstract no PE-085). 


\section{PE-086 \\ Role of Performance Status in Surgical Resection for Hepatocellular Carcinoma: A Lesson from Real-world Clinical Practice} Han Wu', Wen Tao Yan ${ }^{1,2}$, Jun Han ${ }^{1}$, Hao Xing ${ }^{1}$, Zhen Li Li ${ }^{1}$, Ya Hao Zhou ${ }^{3}$, Wei Min Gu ${ }^{4}$, Hong Wang ${ }^{5}$, Ting Hao Chen ${ }^{6}$, Jian Hong Zhong ${ }^{7}$, Yong Yi Zeng ${ }^{8}$, Bing Quan 1,2, Li Yang Sun 1,2, Zheng Wang ${ }^{1}$, Han Zhang ${ }^{1}$, Chao Li ${ }^{1}$, Ming Da Wang ${ }^{1}$, Wan Yee Lau ${ }^{1,9}$, Meng Chao W ${ }^{1}$, Feng Shen ${ }^{1}$, Tian Yang ${ }^{1}$

${ }^{1}$ Department of Hepatobiliary Surgery, Eastern Hepatobiliary Surgery Hospital, Second Military Medical University, Shanghai, China; ${ }^{2}$ Department of Clinical Medicine, Second Military Medical University, Shanghai, China; ${ }^{3}$ Department of Hepatobiliary Surgery, Pu'er People's Hospital, Yunnan, China; ${ }^{4}$ The First Department of General Surgery, the Fourth Hospital of Harbin, Heilongjiang, China; ${ }^{5}$ Department of General Surgery, Liuyang People's Hospital, Hunan, China; ${ }^{6}$ Department of General Surgery, Ziyang First People's Hospital, Sichuan, China; ${ }^{7}$ Department of Hepatobiliary Surgery, Affiliated Tumor Hospital of Guangxi Medical University, Nanning, China; ${ }^{8}$ Department of Hepatobiliary Surgery, Mengchao Hepatobiliary Hospital, Fujian Medical University, Fujian, China; ${ }^{9}$ Faculty of Medicine, the Chinese University of Hong Kong, Shatin, New Territories, Hong Kong SAR, China

Aims: According to the Barcelona Clinic Liver Cancer (BCLC) staging, advanced hepatocellular carcinoma (HCC) could be identified when combining with vascular invasion (VI)/extrahepatic metastasis (ES), or with a poor performance status (PS) (1-2), which was not recommended performing surgical resection. In the real-world clinical practice, however, PS is never a crucial indicator of surgical resection for HCC, and the impact of PS on the long-term prognosis after surgical resection of HCC remains unclear. To investigate the role of PS in surgical resection for HCC. Methods: Multi-institutional data of patients who underwent curative liver resection of HCC between 2005 and 2015 were retrospectively analyzed. PS $\geq 2$ patients $(n=54)$ were excluded from this study, and the remaining patients were divided into PS 0 group $(n=836)$ and PS 1 group $(n=695)$. Perioperative mortality and morbidity, overall survival (OS) and recurrence-free survival (RFS) were compared between the two groups. Univariable and multivariable Cox-regression analyses of OS and RFS were performed. Results: The perioperative mortality and major morbidity rates of PS 0 and 1 patients were similar $(1.4 \%$ vs. $1.6 \%, P=0.525$, and $11.4 \%$ vs. $12.1 \%, P=0.103)$. The median OS and RFS of PS 1 patients were 34.0 and 20.5 months, which was significantly poorer than PS 0 patients (107.6 and 60.6 months, both $P<$ 0.001). Multivariable Cox-regression analysis revealed that PS 1 was an independent risk factor of decreased OS (HR: 1.301, 95\% CI: 1.111-1.523, $P<0.001$ ) and RFS (HR: 1.420,
95\% CI: $1.034-1.358, P=0.007)$. In the subgroup analysis for PS 1 patients, the median OS and RFS of those patients without VI/ES were obviously better than those with VI/ES (54.5 vs. 8.3 months, and 35.3 vs. 2.7 months, both $P<0.001$ ). Conclusions: Surgical resection for PS 1 HCC patients was as safe as for PS 0 HCC patients. Although PS 1 was identified as an independent risk of decreased OS and RFS, surgical resection still was feasible and acceptable for PS 1 patients with HCC, especially when combined without VI/ES. Keywords: Hepatocellular carcinoma, Liver resection, Performance status, Mortality, Morbidity, Survival, Recurrence.

\section{PE-087 \\ Synergistic Anticancer Effect of Metformin in Combination with Immunosuppressant on Hepatocellular Carcinoma Cell Lines}

Suk-Won Suh ${ }^{1}$, Kwang-Woong Lee, Yoo-Shin Choi ${ }^{1}$

${ }^{1}$ Department of Surgery, Chung-Ang University College of Medicine, ${ }^{2}$ Department of Surgery, Seoul National University College of Medicine

Aims: After liver transplantation (LT), immunosuppression is needed to avoid rejection and graft loss, however, it can stimulate hepatocellular carcinoma (HCC) recurrence and progression. Previous studies have shown that metformin had an anticancer effect on several cancers, including HCC. The aim of this study was to evaluate the interactions between metformin and immunosuppressive agents including sirolimus, tacrolimus and mycophenolate mofetil (MMF) for antitumor activity. Methods: Three cell lines (Huh7, HepG2 and Hep3b) were tested. Cell viability was determined using a MTT assay and Western blot analysis for mammalian target of rapamycin (mTOR) pathway related proteins were performed to reveal their mechanism. Results: Metformin and sirolimus had synergistic antiproliferative effect and sirolimus plus metformin supplemented with MMF also showed synergistic antiproliferative effect in specific HCC cells. Synergistic effect of metformin and sirolimus through the inhibition of mTOR and its down-stream, p70S6K, and p-4EBP1 were demonstrated. Metformin and sirolimus also showed synergistic effect for the down-regulation of Livin and Survivin expressions in HepG2 and Hep3b cells. Conclusions: Metformin had synergistic interactions with sirolimus in terms of anticancer effects for HCC cells and the mechanism explaining this synergistic inhibition might be related with mTOR pathway. These results may provide a foundation for further studies for patients with HCC who underwent LT in clinical era. Keywords: Metformin, Anticancer effect, Synergistic, immunosuppressant. 


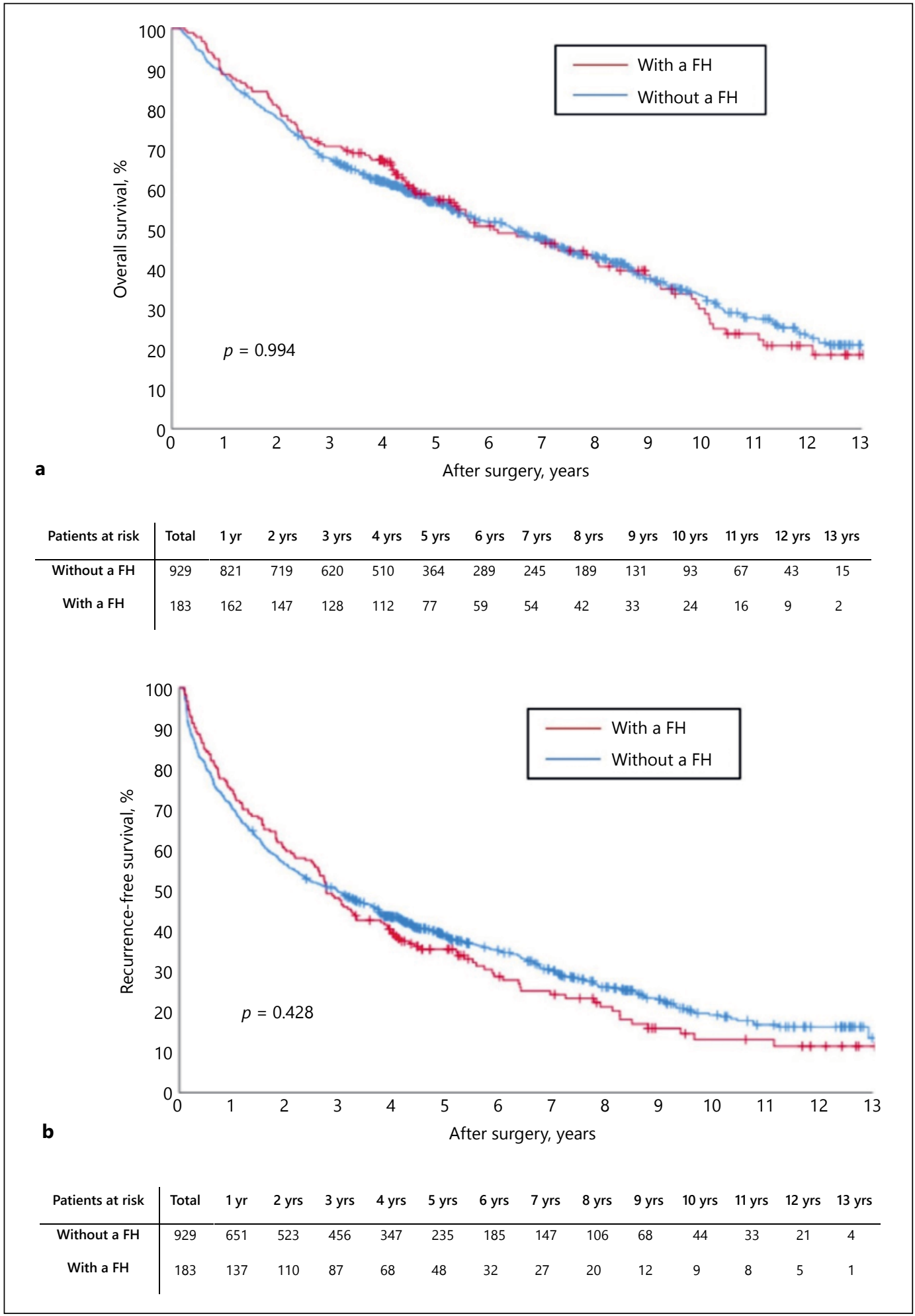

Fig. 1. Cumulative incidence of overall survival (a) and recurrence-free survival (b) curves comparisons between patients with and without a family history (FH) in the entire cohort (for Abstract no PE-086). 


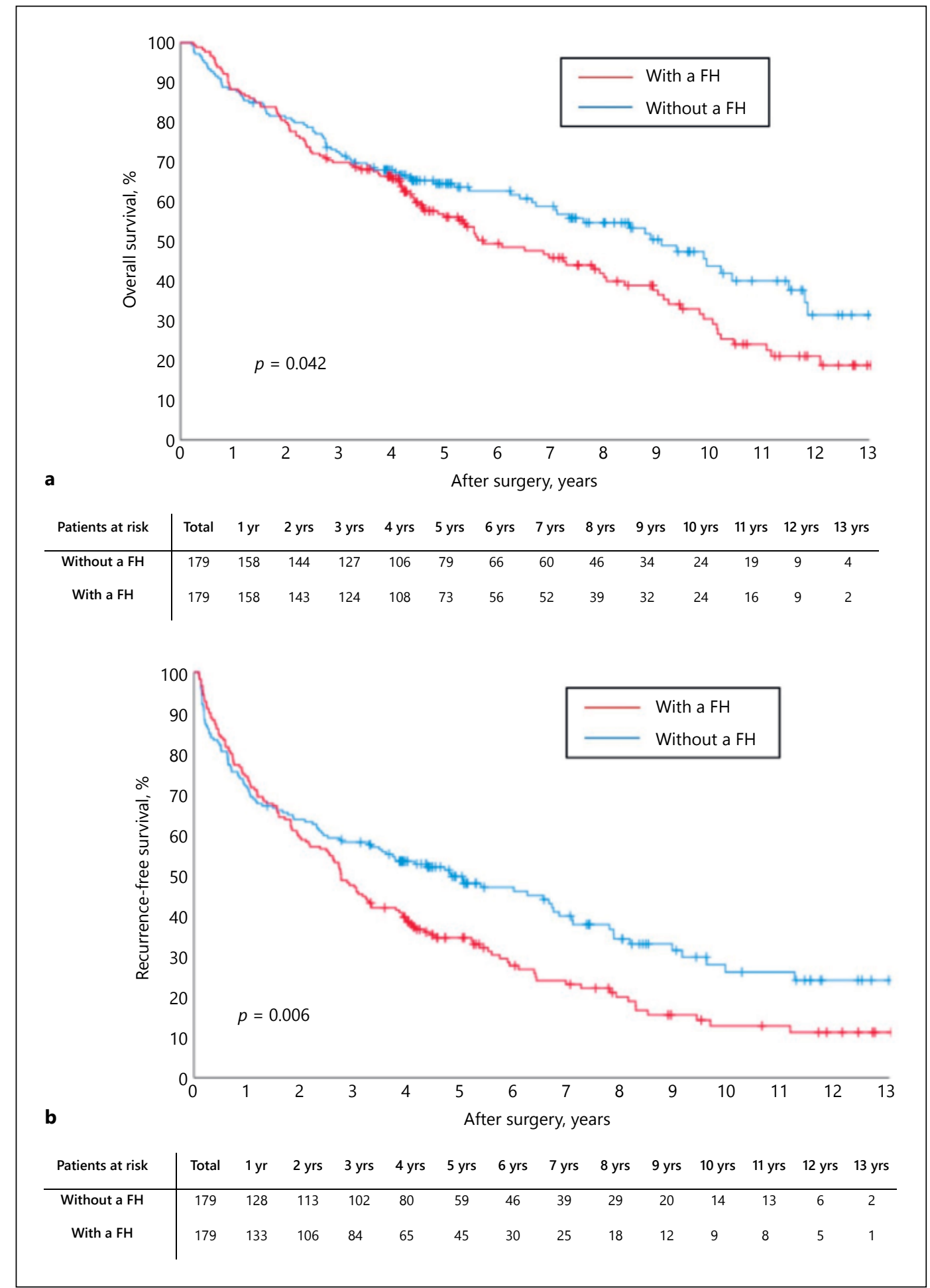

Fig. 2. Cumulative incidence of overall survival (a) and recurrence-free survival (b) curves comparisons between patients with and without a family history (FH) in the propensity score matching (PSM) cohort (for Abstract no PE-086). 


\section{PE-088 \\ Impact of Preoperative Body Weight on Long-Term Prognosis after Liver Resection for Hepatocellular Carcinoma: A Multicenter Study \\ Jiong Jie Yu ${ }^{1,2}$, Ting Hao Chen ${ }^{3}$, Lie Liang ${ }^{1}$, Jun Han ${ }^{1}$, Ya Hao Zhou ${ }^{4}$, Hong Wang ${ }^{5}$, Wei Min Gu, Wan Yee Lau ${ }^{1,7}$, Feng Shen ${ }^{1}$, Tian Yang ${ }^{1}$ \\ ${ }^{1}$ Department of Hepatobiliary Surgery, Eastern Hepatobiliary Surgery Hospital, Second Military Medical University, Shanghai, China; ${ }^{2}$ Department of Clinical Medicine, Second Military Medical University, Shanghai, China; ${ }^{3}$ Department of General Surgery, Ziyang First People's Hospital, Sichuan, China; ${ }^{4}$ Department of Hepatobiliary Surgery, Pu'er People's Hospital, Yunnan, China; ${ }^{5}$ Department of General Surgery, Liuyang People's Hospital, Hunan, China; ${ }^{6}$ The First Department of General Surgery, Fourth Hospital of Harbin, Heilongjiang, China; ${ }^{7}$ Faculty of Medicine, the Chinese University of Hong Kong, Shatin, New Territories, Hong Kong SAR, China}

Aims: Whether preoperative body weight is associated with long-term prognosis in patients after liver resection for hepatocellular carcinoma (HCC) is controversial. We aimed to investigate the relationship among groups of patients with under-, normal- or over-weight with long-term recurrence and overall survival after curative liver resection for HCC. Methods: The data on HCC patients who underwent curative liver resection between 2000 and 2015 in five centers in China were retrospectively analyzed. These patients were divided into 3 groups according to the preoperative body mass index (BMI): underweight (BMI $\leq 18.4 \mathrm{~kg} / \mathrm{m}^{2}$ ), normal-weight (BMI 18.5$24.9 \mathrm{~kg} / \mathrm{m}^{2}$ ) and overweight (BMI $\geq 25.0 \mathrm{~kg} / \mathrm{m}^{2}$ ). Patients' baseline characteristics, operative variables, and long-term survival outcomes were compared. Univariable and multivariable Cox-regression analyses were performed to identify the risk factors of overall survival (OS) and recurrence-free survival (RFS) after resection. Results: Of 1,524 patients, 107 patients $(7.0 \%)$ were underweight, 891 (58.5\%) normalweight, and $526(34.5 \%)$ overweight. Univariable analyses showed underweight and overweight patients had poorer OS (both $P<0.001$ ) and RFS (both $P<0.001$ ) than normalweight patients. Multivariable Cox-regression analysis also identified both underweight and overweight to be independent risk factors of OS (HR 1.217, 95\% CI 1.189-1.561, $P=0.019$, and HR $1.568,95 \%$ CI 1.359-1.810, $P<0.001$, respectively), and RFS (HR 1.278, 95\% CI 1.156-1.533, $P=0.028$, and HR 1.342, 95\% CI 1.171-1.537, $P<0.001$, respectively). Conclusions: Preoperative underweight and overweight were significantly associated with decreased OS and RFS in patients after curative liver resection for HCC. Keywords: Hepatocellular carcinoma, Liver resection, Body mass index, Survival, Recurrence.

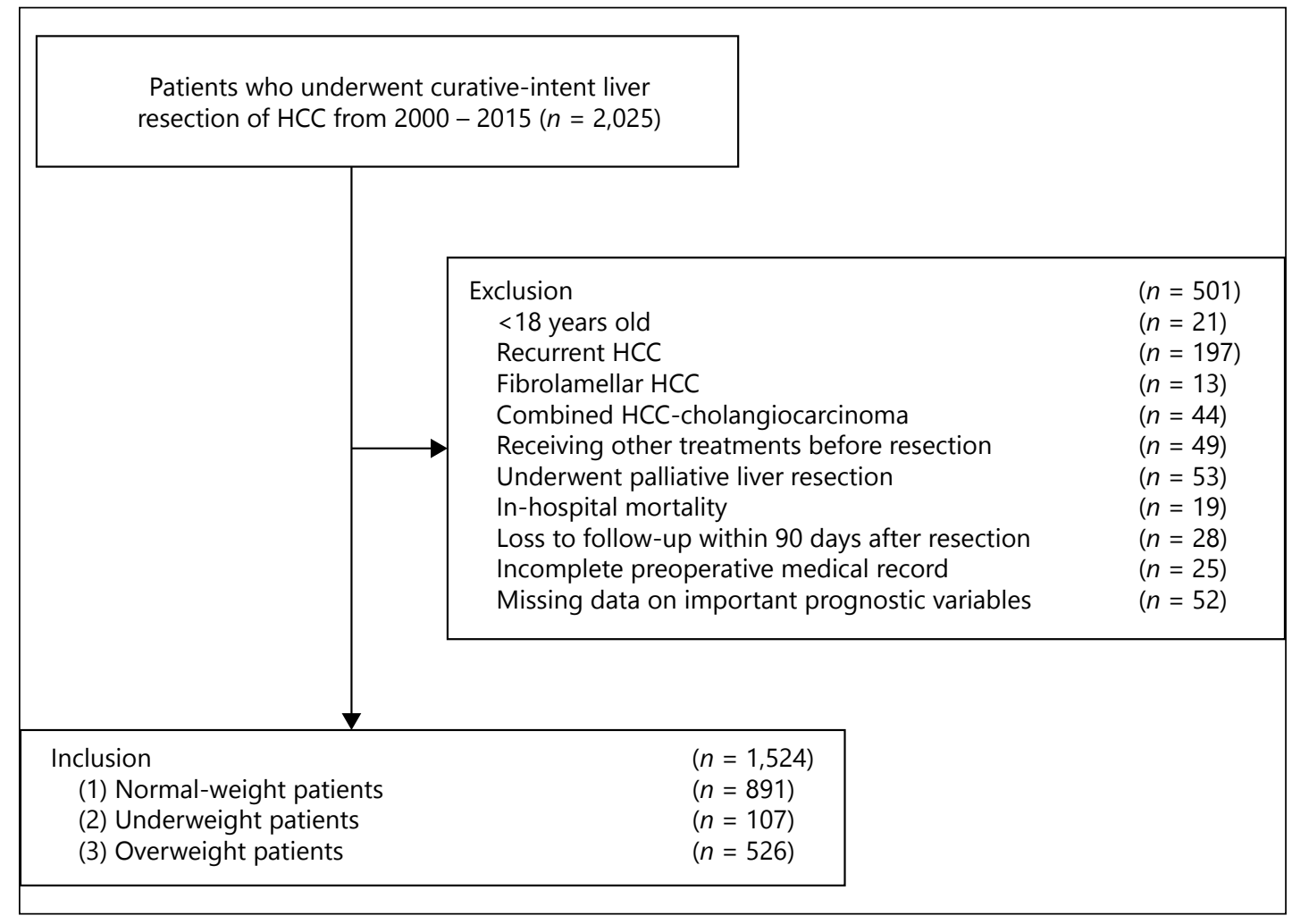

Fig. 1. Selection of the study population (for Abstract no PE-088). 


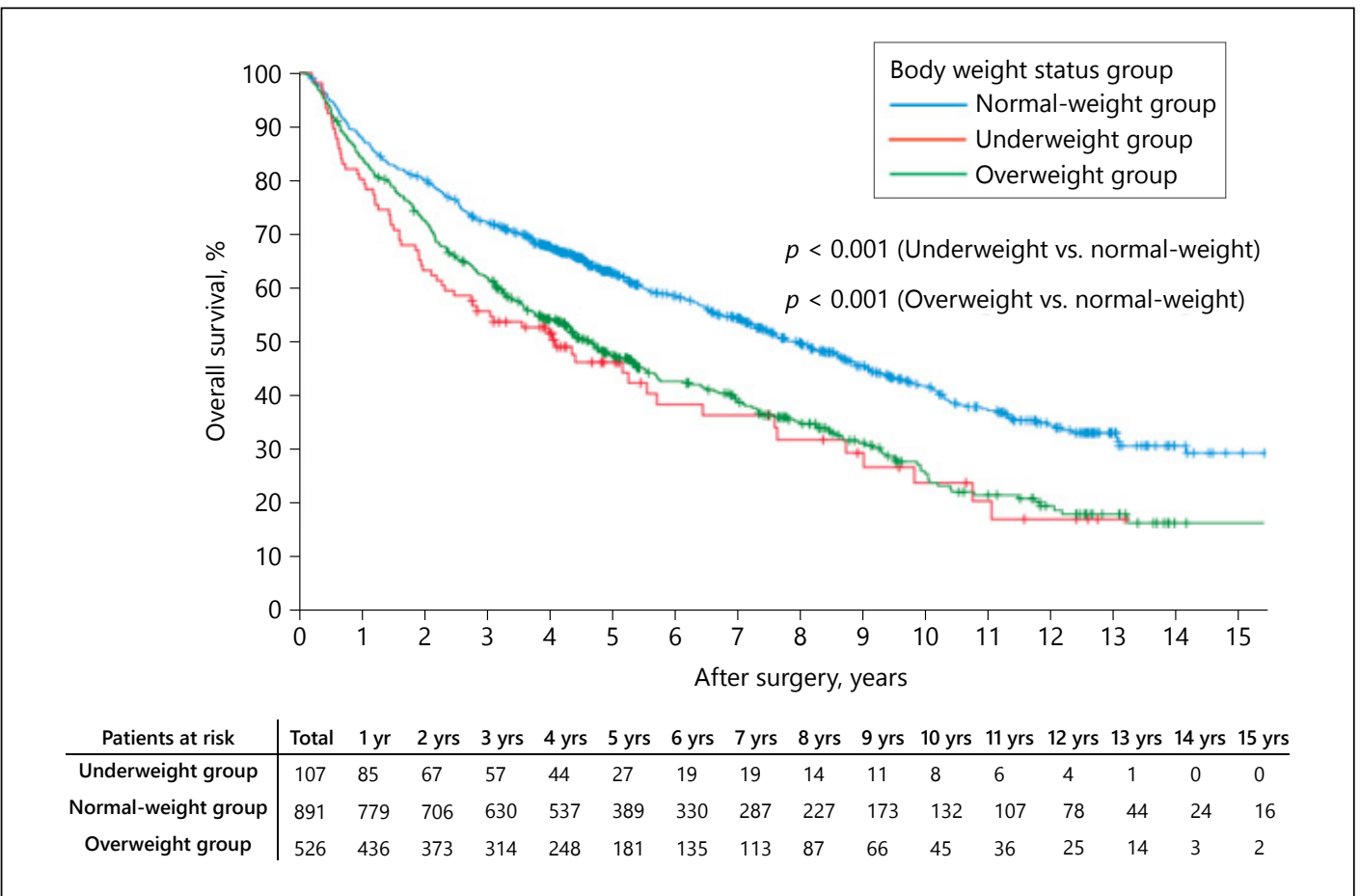

Fig. 2. Overall survival curves comparisons among the noj overweight groups (for Abstract no PE-088).

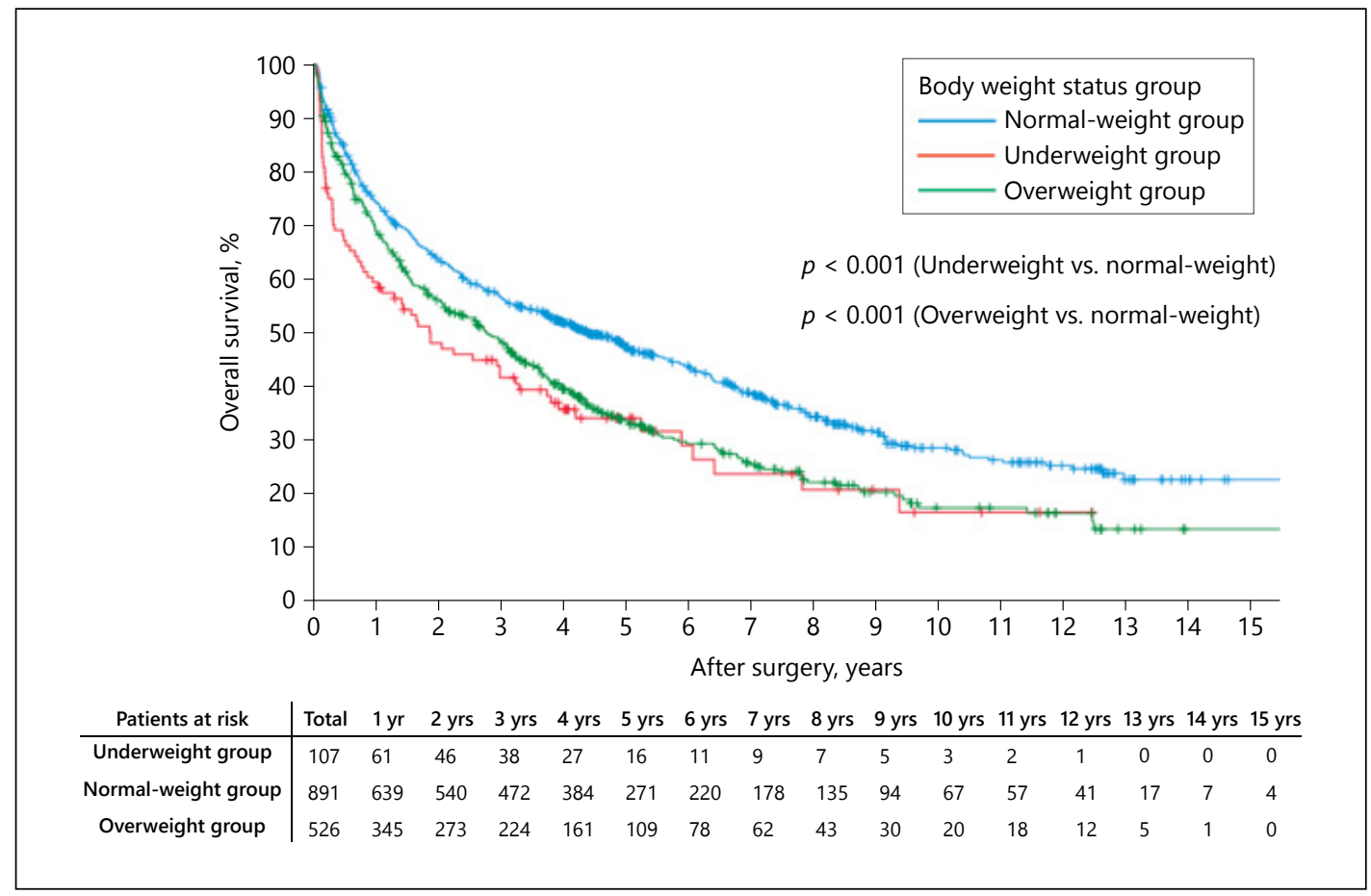

Fig. 3. Recurrence-free survival curves comparisons amon and overweight groups (for Abstract no PE088). 


\section{PE-089}

\section{Single Port Laparoscopic Liver Resection}

Hyung Joon Han' ${ }^{1}$, Tae-Jin Song ${ }^{1}$, Sae Byeol Choi', Wna-Bae Kim ${ }^{1}$, Young Dong Yu' ${ }^{1}$ Dong-Sik Kim ${ }^{1}$

${ }^{1}$ Departmet of Surgery, Korea University College of Medicine

Aims: Laparoscopic surgery has become the standard approach for many abdominal procedures and can be considered one of the major technical advances in surgery over the past 20 years. Since the first laparoscopic liver resection was reported in 1992, there has been an exponential increase in the number of reported laparoscopic liver resection. Recently, due to improved laparoscopic instruments and increasing experience with laparoscopic and liver surgery, the technical difficulty of laparoscopic liver resection is slowly being overcome. More recently, in an attempt to decrease abdominal wall trauma and visible scar, single-port access surgery has been developed, and this field is rapidly evolving. Although single port laparoscopic surgery practice is relatively new, it has already been applied in many surgical procedures by ambitious surgeons who always look for the best option. Many procedures such as cholecystectomy, adrenalectomy, laparoscopic inguinal hernia repair, colectomy, gastrectomy, pancreatectomy, and nephrectomy have been conducted through single port and reported to the world literature. We herein report early initial experience of single port laparoscopic liver resection for hepatic neoplasms. The aim of this study was to evaluate the technical feasibility and limitation of this procedure for hepatic neoplasms. Methods: Patients: In this study, 45 patients underwent laparoscopic liver resection from December 2009 to December 2016 in Korea University Ansan Hospital were enrolled. Surgical technique: The patient was placed in the "French" position, with the first surgeon standing between the patient's legs, with one assistant on each side. A $3 \mathrm{~cm}$-sized incision was made on RUQ region or transumbilical area of the patient. We used a homemade glove port or Glover port (Nelis, South Korea). Homemade glove port was consisted of Alexis wound retractor (small, Applied Medical, CA, USA), a surgical glove, a $12 \mathrm{~mm}$ trocar, an $11 \mathrm{~mm}$ trocar and a $5 \mathrm{~mm}$ trocar (Ethicon, $\mathrm{OH}$, USA). The index, middle, and ring fingers of the glove were partially cut, and three standard laparoscopic trocars were inserted through the hole of each finger and tied. The little finger of the glove was used for carbon dioxide gas insufflations. Intraoperative ultrasonography was used to plan the parenchyma division line when it was needed, and the extent of the resection was outlined with electrocautery marks. A suture on a straight needle (W8400 2-0 prolene: Ethicon, Cincinnati, $\mathrm{OH}$, USA) tagged with cotton was inserted through the $12 \mathrm{~mm}$ trocar, passed through the liver of specimen side and then out through the abdominal wall. Extracorporeal traction of this suture permitted a "puppeteering" of the liver to allow excellent exposure of parenchyma division line. The hepatic parenchyma was transected using a vessel-sealing laparo- scopic device and an endoscopic stapling device as appropriate. The specimen was extracted in a retrieval bag through the single port site. Standard straight laparoscopic instruments were used except the homemade single-port and the extracorporeal traction suture. Portal clamping was not used for all cases. Results: Mean age was $55.9 \pm 9.9$ years old. Male to female ratio was $4.6: 1$. Mean operation time is $154 \pm 94$ minutes and portal clamping was not used for all patients. Mean size of tumor was $2.3 \pm 1.34 \mathrm{~cm}$. The resection margin was free in all cases, however, the mean length from tumor to nearest resection margin was $1.34 \pm 1.28 \mathrm{~cm}$. Twelve patients $(26.7 \%)$ were transfused in perioperative period. Ten patients underwent single port laparoscopic liver resection and 1 patients had conversion to conventional laparoscopic operation. Nonanatomical resection were performed in 29 patients (64.4\%). Tumors were located in segment 2 (5 cases, 11.1\%), segment 3 (10 cases, 22.2\%), segment 4 (3 cases, 6.7\%), segment 5 (10 cases, 22.2\%), segment 6 (15 cases, 33.3\%), and segment 8 ( 2 cases, $4.4 \%$ ). There was no significant difference in gender, operation time, tumor size, tumor location, margin status, presence of transfusion, length of hospital stay, operative type and recurrence. Conclusions: Single port laparoscopic liver resection can be performed safely and effectively in patients with hepatic malignancy. Keywords: Single port, Outcome, Laparoscopy

\section{PE-090 \\ The Effect of Preoperative Treatment for Hepatic Resection in Patients with Hepatocellular Carcinoma: Does Preoperative Treatment Improve Prognosis after Surgery?}

Ho Joong Choi, Dong Goo Kim, Yumi Kim, Bong Jun Kwak, Jae Hyun Han, Tae Ho Hong, Young Kyoung You

Department of Surgery, Seoul St. Mary's Hospital, The Catholic University of Korea, Seoul, South Korea

Aims: The treatment modality of hepatocellular carcinoma (HCC) varies, so several staging systems have been developed to guide management of HCC. This study was performed to review the outcomes of HCC after hepatic resection with or without transarterial chemoembolization (TACE). Methods: From January 2005 to December 2015, 274 patients who underwent hepatic resection with or without TACE for HCC in our center were included. And then, they were divided into hepatic resection only (HRO) group and preoperative TACE (PT) group. To assess the outcomes associated with TACE, we evaluated recurrence, the disease-free survival rate (DFS), the overall survival rate (OS), and various other factors based on the characteristics of patients and tumors Results: Of the 274 patients, 5-year DFS and OS rates were $42.6 \%$ and $69.8 \%$. HRO group was 208 (75.9\%) patients and PT group was $66(24.1 \%)$ patients. We found no statisti- 
cally significant difference in the DFS and OS rates between HRO and PT group. However, when PT group was divided into responder (complete response and partial response) and nonresponder (stable disease and progressive disease) according to modified response evaluation criteria in solid tumors (mRECIST), HRO group and responder group showed no difference between DFS and OS, but non-responder group showed significantly worse in both DFS and OS. Conclusions: This study showed that immediate hepatic resection has similar prognosis as resection after TACE. If resection is possible, there is no need to plan TACE. And if TACE is performed, non-responders to TACE need to be cautious in considering resection later. Keywords: Hepatocellular carcinoma, Hepatic resection, TACE.

\section{Treatment: Transarterial Approach/Percutaneous Ablative Therapy/Radiation Therapy}

\author{
PE-091 \\ High-Dose Radiation in Liver-Directed \\ Concurrent Chemoradiotherapy Is Effective \\ for Locally Advanced BCLC Stage C \\ Hepatocellular Carcinoma \\ Hwa Kyung Byun ${ }^{1}$, Hyun Ju Kim¹, Yoo Ri Im¹, \\ Do Young Kim², Kwang-Hyub Han ${ }^{2}$, Jinsil Seong ${ }^{1}$ \\ ${ }^{1}$ Department of Radiation Oncology, Yonsei Cancer \\ Center, Yonsei University College of Medicine, Seoul, \\ South Korea; ${ }^{2}$ Department of Internal Medicine, \\ Severance Hospital, Yonsei University College of \\ Medicine, Seoul, South Korea
}

Aims: Recent advances in radiotherapy (RT) technologies, such as intensity-modulated radiotherapy (IMRT), have enabled the delivery of tumoricidal doses to intrahepatic malignancies while protecting the normal organs. This study evaluated the effects of high-dose RT among patients with locally advanced Barcelona Clinic Liver Cancer Stage $C$ hepatocellular carcinoma (BCLC-C HCC). Methods: This study retrospectively evaluated data from 637 patients who received RT with concurrent hepatic arterial floxuridine for BCLC-C HCC without distant metastases during 2005-2016. These cases included 514 patients (81\%) who were treatmentnaïve. Receiver operating characteristic curve analysis showed that the optimal cut-off for the biologically effective dose (BED) was $72 \mathrm{~Gy}$, and the patients were divided into $\geq 72$ Gy $(\mathrm{n}=101)$ and BED $<72$ Gy $(\mathrm{n}=536)$. In the BED $\geq 72$ Gy and $<72$ Gy groups, 94 of 101 patients (93\%) and 128 of 536 patients (24\%) used IMRT, respectively. Results: The median overall survival (OS) was 16.3 months, with a median followup of 12.4 months (19.8 months among patients who were alive). The BED $\geq 72$ Gy and $<72$ Gy groups had median OSs of 39.7 months and 14.5 months, and 1-year OS rates of $73 \%$ and $58 \%$, respectively $(P<0.001)$. The 1 -year local control (LC) rate was significantly higher in the high-dose group (95\% vs. $78 \%$; $P<0.001$ ). After propensity score matching, 83 matched pairs were created, and the high-dose group still had significantly better 1 -year LC rate $(95 \%$ vs. $82 \% ; P=0.006)$ and 1 -year OS rate $(71 \%$ vs. $62 \% ; P=0.01)$. In the multivariate model, the BED $\geq 72$ Gy was an independent predictor of LC (hazard ratio [HR], 0.26; 95\% confidence interval [CI], 0.11$0.61 ; P=0.002$ ) and OS (HR 0.50; 95\% CI, 0.33-0.77, $P=$ $0.002)$. The surgical conversion rate was significantly higher in the high-dose group ( $20 \%$ vs. $12 \%, P=0.03$ ), with markedly increased median OS among patients who underwent surgery (103.8 months vs. 13.1 months; $P<0.001$ ). There were no significant differences in gastrointestinal bleeding or radiation-induced liver disease. Conclusions: High-dose RT which was enabled by IMRT increased LC and OS after liver-directed chemoradiotherapy for locally advanced BCLC-C HCC. It also increased the surgical conversion rate, which contributed to the improved OS.

\section{PE-092 \\ Transarterial Chemoembolization Treatment in the Management of BCLC C Stage Hepatocellular Carcinoma: A Large Real- World Observational Study of $\mathbf{8 8 5}$ Cases}

Xin Yin, Bei-Tang Jia Yuan, Miao Li, Yu-hong Gang, Yan-Hong Wang, Ning-lin Ge, Yi Chen, Rong-Xin Chen, Li-xin Li, Bi-Wei Yang, Lan Zhang, Tong-chun Xue, Bo-Heng Zhang, Zheng-Gang Ren 1\#

Liver Cancer Institute, Zhongshan Hospital, Fudan University

Aims: Patients with advanced stage hepatocellular carcinoma (HCC) have a dismal prognosis. The Barcelona Clinic Liver Cancer (BCLC) staging and treatment strategy does not recommend transarterial chemoembolization (TACE) for treating BCLC C HCC. However, numerous AsianPacific institutes still perform TACE for this patient group. There remains a paucity of large real-world data characterizing treatment outcomes of patients with advanced stage HCC following TACE-based monotherapy or combination therapy. We aimed to share experiences and opinions of 885 cases of BCLC C stage HCC patients treated by TACE in realworld practice. Methods: The study was approved by the institutional ethics review boards at Zhongshan Hospital. 885 patients were included in this real-world study who received TACE based monotherapy or combination therapy in liver cancer institute, Fudan University between January 2008 and December 2015. We investigated the clinical outcomes of 

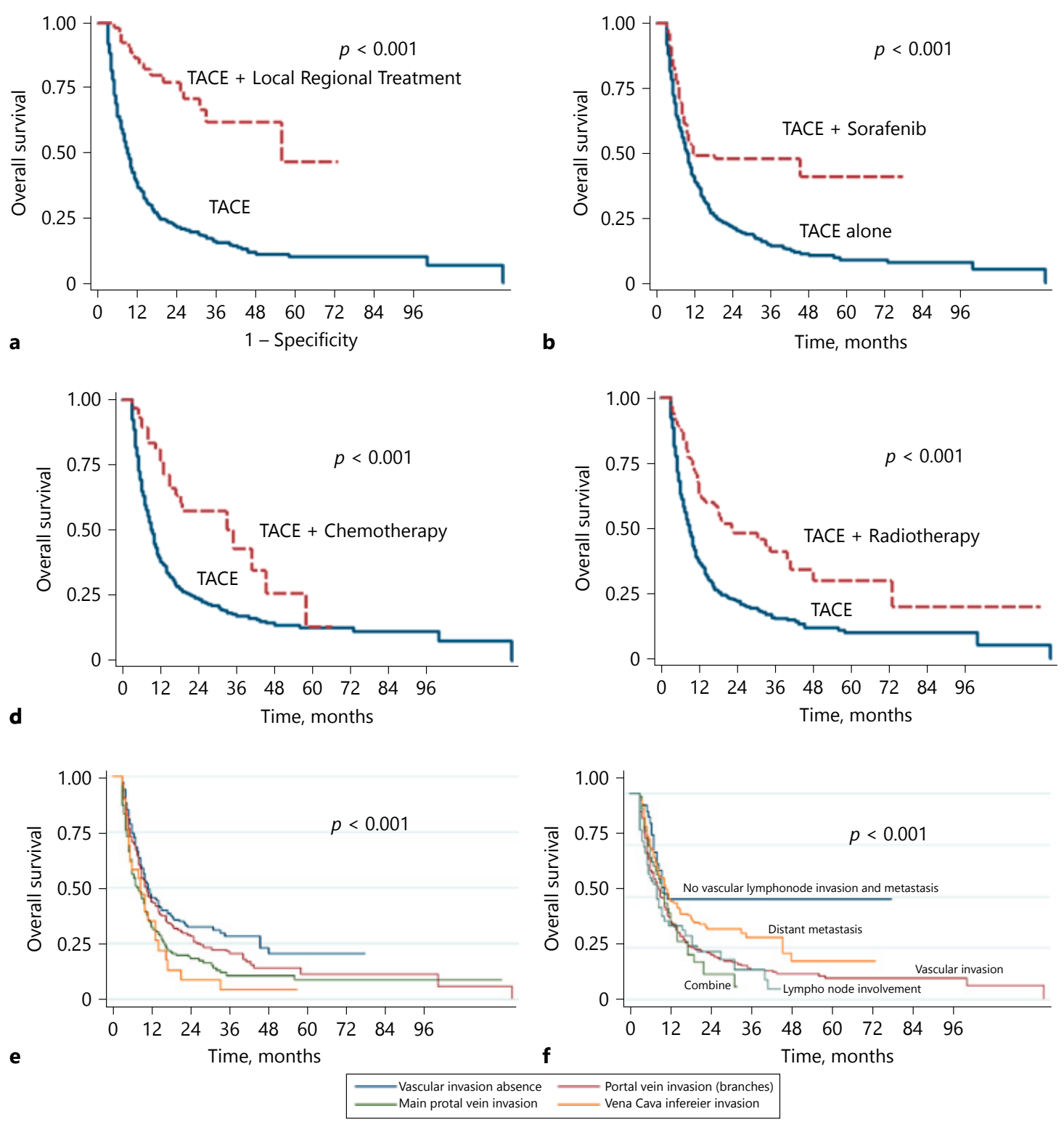

Fig. 1. (for Abstract no PE-092).

TACE-based treatment in BCLC C stage HCC. Results: In this cohort of 885 BCLC C HCC patients, vascular invasion was present in 582 patients $(65.8 \%)$, lymph node involvement was present in 108 patients (12.2\%), extrahepatic metastases were present in 232 patients (26.2\%). Median tumor size was $9.0 \mathrm{~cm}$ (range 1.5-15). The mean number of TACE per patient was $3.0 \pm 1.9$ sessions. Median overall survival was 8.0 months (range 3-37) for the entire study population. Of all the patients, 600 patients received TACE-alone treatment, 137 patients received TACE combined Sorafenib treatment (TACE + Sorafenib), 41 patients received TACE combined local regional treatment (TACE + LRT), 59 patients received TACE with chemotherapy (TACE + chemotherapy), 123 patients received TACE with radiotherapy (TACE + radiotherapy). The median survival time was 6.0 months for TACE-alone group, 11.5 months for TACE + Sorafenib group, 19.8 months for TACE+LRT group, 12.0 month for TACE + chemotherapy group and 12.0 months for TACE + radiotherapy group respectively. These data showed that TACE-based combination therapy can improve overall survival in BCLC C stage HCC, in comparison with TACE alone. After propensity score matching and adjusting for potential confounders, there were still significant OS differences between TACE-alone group and TACE-based combination treatments mentioned above. 
Conclusions: Although the prognosis of BCLC C stage HCC was dismal, the TACE-based combination therapy can improve overall survival in Asian HCC patients.

\section{PE-093 \\ The role of External Beam Radiotherapy for Hepatocellular Carcinoma Patients with Lymph Node Metastasis: A Meta-Analysis and Systematic Review}

\author{
Chai Hong Rim ${ }^{1}$, Chul Yong Kim², Dae Sik Yang ${ }^{3}$, \\ Won Sup Yoon ${ }^{1}$ \\ ${ }^{1}$ Department of Radiation Oncology, Ansan Hospital, \\ Korea University Medical College, Ansan, Gyeonggi-do, \\ Republic of Korea; ${ }^{2}$ Department of Radiation Oncology, \\ Anam Hospital, Korea University Medical College, \\ Seoul, Republic of Korea; ${ }^{3}$ Department of Radiation \\ Oncology, Guro Hospital, Korea University Medical \\ College, Seoul, Republic of Korea
}

Aims: Lymph node metastasis (LNM) of hepatocellular carcinoma (HCC) is a rare condition with poor prognosis and standard treatment is yet to be established. This meta-analysis and systemic review is aimed to evaluate efficacy and safety of EBRT and discuss the optimal treatment strategy. Methods: PubMed, Medline, Cochrane library, and Embase were systemically searched until December 17, 2017. The primary endpoints of analysis were response rate (RR) and 1-year overall survival (OS) rate. Results: A total of 8 studies comprising 521 patients were included in this meta-analysis. The prescribed dose, which was calculated to biologically equivalent dose (BED) using alpha-beta ratio of 10 , ranged from $50 \mathrm{~Gy}_{10}$ to $75 \mathrm{~Gy}_{10}$ with the median of $60 \mathrm{~Gy}_{10}$. The pooled RR was $73.1 \%$ (95\% confidence interval [CI]: 63.6-80.9), and the 1-year OS rate was $41.0 \%$ (95\% CI: 32.9-49.6). High-dose EBRT groups had better RR than the low-dose group $(82.2 \%$ [95\% CI: 74.4-88.1] vs. 51.1\% [95\% CI: 40.3-61.7]; $p=0.001$ ). Six studies assessed the survival benefit according to RR, and $5(83.3 \%)$ of these 6 studies reported statistically significant survival benefit. The most common grade $\geq 3$ toxicities were thrombocytopenia and gastrointestinal complication, and the overall rates were $4.5 \%$ and $2.2 \%$, respectively. Conclusions: EBRT for LNM of HCC showed a favorable pooled RR of 73.1\% and was safely performed. EBRT is an option of treatment which expect to palliate the local symptom of LNM and improve survival. High-dose EBRT should be considered with technical consideration of reducing complication.

\begin{abstract}
PE-094
Safety evaluation after radiotherapy for hepatocellular carcinoma in patients with Child-Pugh classification B: A multicenter analysis in Korea (KROG 16-05)

Sun Hyun Bae ${ }^{1}$, Hee Chul Park², Won Sup Yoon ${ }^{3}$, Sang Min Yoon ${ }^{4}$, Ik Jae Lee ${ }^{5}$, Jun Won Kim ${ }^{5}$, Jinsil Seong ${ }^{6}$, Tae Hyun Kim ${ }^{7}$, Taek-Keun Nam ${ }^{8}$, Young Min Choi ${ }^{9}$, Sun Young Lee ${ }^{10}$, Hong Seok Jang ${ }^{11}$, Dong Soo Lee ${ }^{12}$, Jin Hee Kim ${ }^{13}$

Department of Radiation Oncology, ' ${ }^{1}$ Soonchunhyang University College of Medicine, Bucheon, Korea, ${ }^{2}$ Samsung Medical Center, Sungkyunkwan University School of Medicine, Seoul, Korea, ${ }^{3}$ Ansan Hospital, Korea University Medical Center, Ansan, Korea, ${ }^{4}$ Asan Liver Center, University of Ulsan College of Medicine, Seoul, Korea, ${ }^{5}$ Gangnam Severance Hospital, Yonsei University College of Medicine, Seoul, Korea, ${ }^{6}$ Severance Hospital, Yonsei University College of Medicine, Seoul, Korea, ${ }^{7}$ Center for Liver Cancer, Research Institute and Hospital, National Cancer Center, Goyang, Korea, ${ }^{8}$ Chonnam National University Medical School, Gwangju, Korea, ${ }^{9}$ Dong-A University College of Medicine, Pusan, Korea, ${ }^{10}$ Chonbuk National University Hospital, Jeonju, Korea, ${ }^{11}$ Seoul St. Mary's Hospital, The Catholic University of Korea, Seoul, Korea, ${ }^{12}$ Uijeongbu St. Mary's Hospital, The Catholic University of Korea, Uijeongbu, Korea, ${ }^{13}$ Dongsan Medical Center, Keimyung University School of Medicine, Daegu, Korea
\end{abstract}

Aims: To evaluate the efficacy and toxicity of radiotherapy (RT) for hepatocellular carcinoma (HCC) in patients with Child-Pugh classification B (CP B). Methods: We retrospectively reviewed 184 HCC patients with CP B treated with RT between 2009 and 2014 at 13 institutions in Korea. Initial CP score was 7 in $62.0 \%, 8$ in $31.0 \%$, and 9 in $7.0 \%$. According to the modified International Union Against Cancer staging classification, T1, T2, T3, and T4 was 5.4\%, 11.4\%, 33.2\%, and $50.0 \%$. Portal vein tumor thrombosis (PVTT) was presented in $66.3 \%$. The total dose ranged from 31.5 Gy to $64.0 \mathrm{~Gy}$ (median $44.0 \mathrm{~Gy}$ ). The biologically effective dose (BED), when $\alpha / \beta$ was assumed to be $10.0 \mathrm{~Gy}$, was $40.4 \mathrm{~Gy}_{10}$ to $89.6 \mathrm{~Gy}_{10}$ (median $56.0 \mathrm{~Gy}_{10}$ ). After completion of RT, 48.4\% received additional treatment. For dosimetric parameters, all doses converted into the equivalent dose of 2 Gy per fraction using linear quadratic model when $\alpha / \beta$ for the normal liver was assumed to be 8 Gy. Results: The median overall survival (OS) was 9.4 months. Local progression-free survival, intrahepatic progression-free survival, and OS rates at 1 -year were $58.9 \%$, $22.1 \%$, and $39.8 \%$, respectively. On univariate analysis, hepatitis, HCC size $>5 \mathrm{~cm}$, advanced T stage, nodal involvement, numbers of HCC $\geq 4$, PVTT, pre_RT alpha-fetoprotein (AFP) > $200 \mathrm{IU} / \mathrm{mL}$, AFP reduction $\leq 20 \%$ at 1 month after RT, nonclassic radiation- induced liver disease (RILD), BED $\leq 53 \mathrm{~Gy}_{10}$, and no additional treatment were associated with poor OS. On 
multivariate analysis, non-classic RILD ( $\mathrm{P}=0.000)$ and additional treatment $(\mathrm{P}=0.000)$ were the most significant prognostic factors on OS. In 132 evaluable patients without progressive disease, $19.7 \%$ experienced non-classic RILD, which was defined as a worsening of CP score by $\geq 2$ points or elevated transaminases more than 5 times within 3 months after RT. Of dosimetric parameters, normal liver volume, mean dose of total liver volume and V15Gy, V20Gy, V35Gy and V40Gy of total liver volume were associated with the risk of non-classic RILD. Conclusions: RT is an effective treatment option in HCC patients with CP B, although non-classic RILD occurred with moderate risk. Further studies should be needed to validate the safety of fractionated RT for CP B.

\section{PE-095 \\ Skeletal Muscle Depletion Predicts the Prognosis of Patients with Hepatocellular Carcinoma Treated with Radiotherapy \\ Joongyo Lee, Sangjoon Park, Yeona Cho, Ik Jae Lee \\ Department of Radiation Oncology, Yonsei University College of Medicine, Seoul, Republic of Korea}

Aims: Sarcopenia is gaining attention, being identified as a poor prognostic factor for various types of malignancies. Particularly, sarcopenia occurs frequently in cases of hepatocellular carcinoma (HCC) after treatment. However, the clinical significances of sarcopenia in patients underwent radiotherapy (RT) for HCC is not yet been identified. The purpose of this study is to evaluate the association between sarcopenia and survival in HCC patients underwent RT to the primary site. Methods: Between January 2009 and November 2016, a total number of 156 patients with stage I-IVB primary HCC underwent radiotherapy to the liver. Among these, 64 patients $(41.0 \%)$ received RT with definitive aim, 43 patients (27.6\%) with palliative aim, 49 patients $(31.4 \%)$ with salvage aim. 81 patients $(51.9 \%)$ were treated with concurrent chemotherapy, and 75 patients (48.1\%) were treated with RT only. The median RT dose was 50.4 Gy (range, 30 Gy-70 Gy). The cross-sectional area of muscle at the level of the third lumbar vertebra was measured using baseline CT images (L3 skeletal muscle index; L3-SMI). Sarcopenia was defined as a L3-SMI of less than $49 \mathrm{~cm}^{2} / \mathrm{m}^{2}$ for men and less than $41 \mathrm{~cm}^{2} /$ $\mathrm{m}^{2}$ for women as proposed by Korean-specific cut off value. The sarcopenia was identified at two-time points; pre- and post-RT. Pre-RT sarcopenia was diagnosed with data from the simulation CT prior to RT, while post-RT sarcopenia was diagnosed with data from the follow-up CT taken within 3 months from the end of radiotherapy. Overall survival (OS) and progression-free survival (PFS) were estimated from the start of RT, and the prognostic factors associated with the survival were analyzed. Results: Among total of 156 patients, 3 patients $(1.9 \%)$ were stage I, 15 patients $(9.6 \%)$ were stage II, 48 patients were stage III $(30.8 \%), 90$ patients were stage IV
(57.7\%), respectively. According to Korean-specific cut off standard, 99 patients $(63.5 \%)$ had pre-RT sarcopenia, while 109 patients (69.9\%) had post-RT sarcopenia. Pre-RT sarcopenia group had higher percentage of patients with previous chemotherapy $(14.1 \%$ vs $1.8 \%, \mathrm{p}=0.011)$, compared with those without pre-RT sarcopenia. At a median follow up 9.3 months, median OS were significantly differed according to the presence of pre-RT and post-RT sarcopenia (pre-RT; 7.1 months vs. 15.3 months, $\mathrm{p}<0.001$, post-RT; 7.5 months vs. 16.8 months, $\mathrm{p}<0.001$ ), while median PFS differed according to pre-RT sarcopenia with borderline significance (3.3 months vs. 5.6 months, $\mathrm{p}=0.051$ ). In univariate analysis, total dose $(p<0.001)$, Child-Pugh classification $(p=0.041)$, cancer stage at time of RT ( $p=0.009)$, AFP level $(p=0.015)$, NeutrophilLymphocyte ratio $(\mathrm{P}=0.05)$, Platelet-Lymphocyte ratio $(\mathrm{p}=$ $0.019)$, pre-RT sarcopenia ( $<<0.001)$, and post-RT sarcopenia $(p<0.001)$ have shown to be significant prognostic factors for OS. In multivariate analysis, total dose ( $p=0.027)$ and pre-RT sarcopenia ( $\mathrm{p}=0.012)$ were identified as independent prognostic factors for OS. Conclusions: In our study, pre-RT sarcopenia was associated with a poor prognosis for HCC patients underwent RT to the liver. This result suggests the possibility that the identification of sarcopenia in HCC patients before RT might enable the early intervention to maintain muscle mass and improve prognosis. Keywords: Hepatocellular carcinoma, Sarcopenia, Radiotherapy, Treatment Outcome, Survival

\section{PE-096 \\ Comparison of Long-Term Outcome for Single Small Hepatocellular Carcinoma between Different Treatment Modalities According to the Size and Tumor Marker}

Sang-geul Lee, Dong Hyun Sinn', Gye-Seong Choi², Jong Man Kim², Tae Wook Kang ${ }^{3}$, Min Woo Lee ${ }^{3}$, Dongho Hyun ${ }^{3}$, Wonseok Kang ${ }^{1}$, Geum-Youn Gwak', Yong-Han Paik', Moon Seok Choi', Joon Hyeok Lee', Kwang Cheol Koh', Seung Woon Paik ${ }^{1}$

${ }^{1}$ Department of Medicine, ${ }^{2}$ Department of Surgery, ${ }^{3}$ Department of Radiology and Center for Imaging Science, Samsung Medical Center, Seoul, South Korea

Aims: For single small hepatocellular carcinoma (HCC), different therapeutic modalities can be tried for patients with preserved liver function. We aim to identify prognostic factors associated with overall survival, that can be used to guide treatment selection. Methods: Between 2010 and 2013, we analyzed 896 patients who received resection, radiofrequency (RF) ablation or transarterial chemoembolization (TACE) as a first-line therapy for single, small $(<3 \mathrm{~cm})$ HCC. We first identified risk factors associated overall survival in patients who were treated with RF ablation, and then compared long-term outcome according treatment modalities, stratified based on 
risk factors. Results: Among 425 patients treated with RF ablation, tumor size and PIVKA-II levels were independent factors associated with overall survival. When patients were stratified according to the tumor size and PIVKA-II levels, overall survival of patients treated with RF ablation was significantly different by subgroups (group 1: tumor sized $\leq 2$ $\mathrm{cm}$ with low PIVKA-II levels $(<30 \mathrm{mAU} / \mathrm{ml})$; group 2: tumor sized $2-3 \mathrm{~cm}$ with low PIVKA-II levels $(<30 \mathrm{mAU} / \mathrm{ml}$ ) or tumor sized $\leq 2 \mathrm{~cm}$ with elevated PIVKA-II levels $(\geq 30 \mathrm{mAU} / \mathrm{ml}$ ); group 3: tumor sized 2-3 cm with elevated PIVKA-II levels $(\geq 30 \mathrm{mAU} / \mathrm{ml})$ ). When compared to resection, overall survival of those treated with RF ablation was not different to those who received resection in group 1 or group 2 but was significantly lower in group 3. When compared to TACE, those treated with RF ablation showed better survival in group 1 or group 2 but was not different in group 3 . Conclusions: Tumor size and PIVKA-II levels were associated with overall survival of patients treated with RF ablation. When patients were stratified according to tumor size and PIVKA-II levels, different long-term outcome by treatment modalities was observed. Our data suggests that these two factors can be valuable factors in choosing first-line treatment option for single small HCC. Keywords: Resection, RFA, HCC, Treatment modality

\section{PE-097 \\ Multicentric Assessment of the Hong Kong Liver Cancer Staging System in Chinese Patients following Transarterial Chemoembolization}

\author{
Bin-Yan Zhong ${ }^{1}$, Cai-Fang $\mathrm{Ni}^{2}$, Guo-Wen Yin ${ }^{3}$, \\ Gao-Jun Teng \\ ${ }^{1}$ Center of Interventional Radiology \& Vascular \\ Surgery, Department of Radiology, Zhongda Hospital, \\ Medical School, Southeast University, Nanjing, \\ China; ${ }^{2}$ Department of Interventional Radiology, First \\ Affiliated Hospital of Soochow University, Suzhou, \\ China; ${ }^{3}$ Department of Interventional Radiology, Cancer \\ Hospital of Jiangsu Province, Cancer Institution of \\ Jiangsu Province, Nanjing, China
}

Aims: We aimed to validate the performance of the hepatitis B-based Hong Kong Liver Cancer (HKLC) staging system compared with the Barcelona Clinic Liver Cancer (BCLC) staging system in Chinese hepatocellular carcinoma (HCC) patients treated with conventional transarterial chemoembolization (TACE) as initial treatment. Methods: The study was approved by the Institutional Review Boards at all participating centers. This retrospective study included 715 patients with HCC who underwent TACE as initial treatment between January 2008 and December 2016 at three Chinese institutions. All of the patients were calculated HCC stage using 5-substage HKLC (HKLC-5), 9-substage HKLC (HKLC-9), and the BCLC system. Based on overall survival (OS), these three staging systems' performance on treatment outcome prediction were compared using $\mathrm{C}$ statistic, Akaike information criterion (AIC), area under the receiver operating characteristic curve (AUC), linear trend chi-square, likelihood ratio chi-square, and calibration plots, respectively. Results: The median OS was 10.1 months. Compared with the BCLC system, the HKLC system, especially HKLC- 9 showed better performance on survival prediction (HKLC-9: $\mathrm{C}=0.689$, $\mathrm{AIC}=$ 6646.162; HKLC-5: $\mathrm{C}=0.683$, $\mathrm{AIC}=6662.663$; $\mathrm{BCLC}: \mathrm{C}=0.680$, AIC $=6654.146$ ), homogeneity (likelihood ratio chi-square: HKLC-9 = 232.38, HKLC-5 = 215.87, and BCLC = 224.39, $\mathrm{p}<$ 0.001 ) and calibration ( $\mathrm{R}^{2}$ : HKLC-9 $=0.923$, HKLC-5 $=0.916$, and BCLC $=0.914$ ). HKLC-9 outperformed on AUC at 6, 12, and 24 months' survival prediction than HKLC-5 and BCLC. BCLC showed better performance on monotonicity (linear trend chi-square: HKLC-9 = 121.641, HKLC-5 = 117.389, and BCLC = 125.752; $\mathrm{p}<0.001$ ). Conclusions: Combining survival prediction, discrimination, and calibration, the HKLC, especially HKLC-9 system performed better for Chinese patients treated with TACE than the BCLC system. Keywords: Hepatocellular carcinoma, Transarterial chemoembolization, HKLC, BCLC
PE-098
Effective Local Tumor Control in
Both Small- and Medium- Sized
Hepatocellular Carcinoma with Combined Chemoembolization and Stereotactic Body Radiation Therapy
Baek Gyu Jun1, Young Don Kim¹, Gab Jin Cheon', Jae Young Jang ${ }^{3}$, Sae Hwan Lee ${ }^{4}$, Hong Soo Kim ${ }^{4}$
${ }^{1}$ Department of Internal Medicine, University of Ulsan College of Medicine, Gangneung Asan Hospital, Gangneung, South Korea, ${ }^{2}$ Department of Internal Medicine, Soonchunhyang University South Korea, ${ }^{3}$ Department of Internal Medicine, Soonchunhyang University College of Medicine Seoul Hospital, Seoul, South Korea, ${ }^{4}$ Department of Internal Medicine, Soonchunhyang University College of Medicine Cheonan Hospital, Cheonan, South Korea. Sang Gyune Kim², Young Seok Kim², Soung Won Jeong ${ }^{3}$, College of Medicine Bucheon Hospital, Bucheon,

Aims: We evaluated the efficacy of stereotactic body radiotherapy (SBRT) for hepatocellular carcinoma (HCC) patients treated in combination with transarterial chemoembolization (TACE) Methods: We retrospectively reviewed 104 patients with HCC of $\leq 5 \mathrm{~cm}$ who underwent combined SBRT with TACE from August 2011 to December 2016 in four institutions. We evaluated local control (LC) rate and overall survival (OS) after combined treatment. Outcomes in patients with small $(<3 \mathrm{~cm})$-sized HCC $(\mathrm{n}=77)$ were compared to with outcomes in patients with medium $(3-5 \mathrm{~cm})$-sized HCC $(\mathrm{n}=$ 


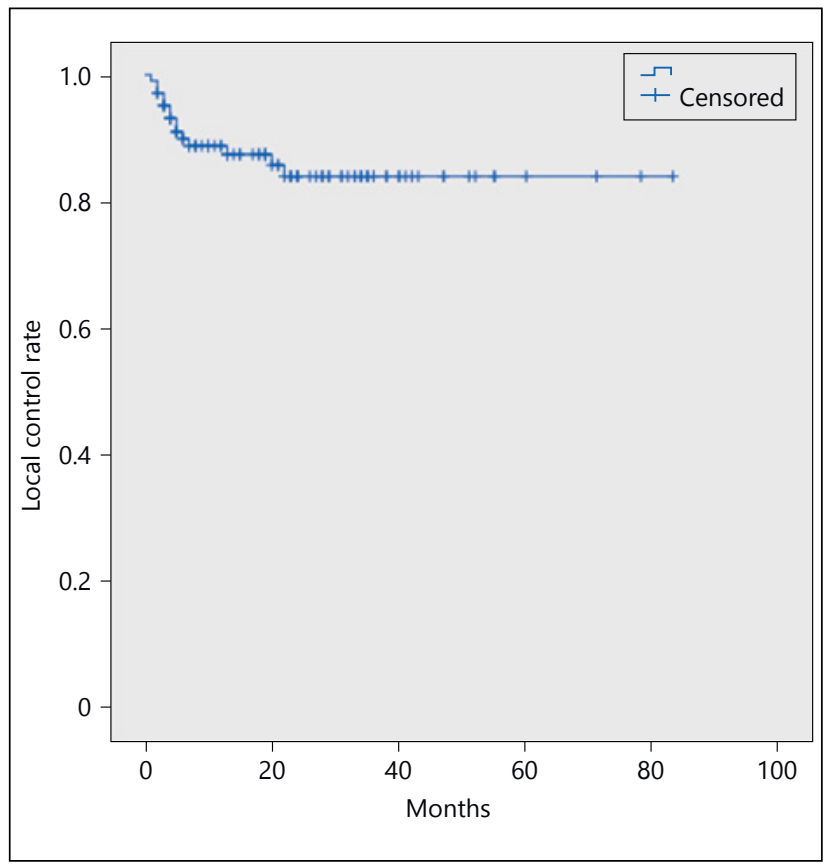

Fig. 1. Local control rate (for Abstract no PE-098).

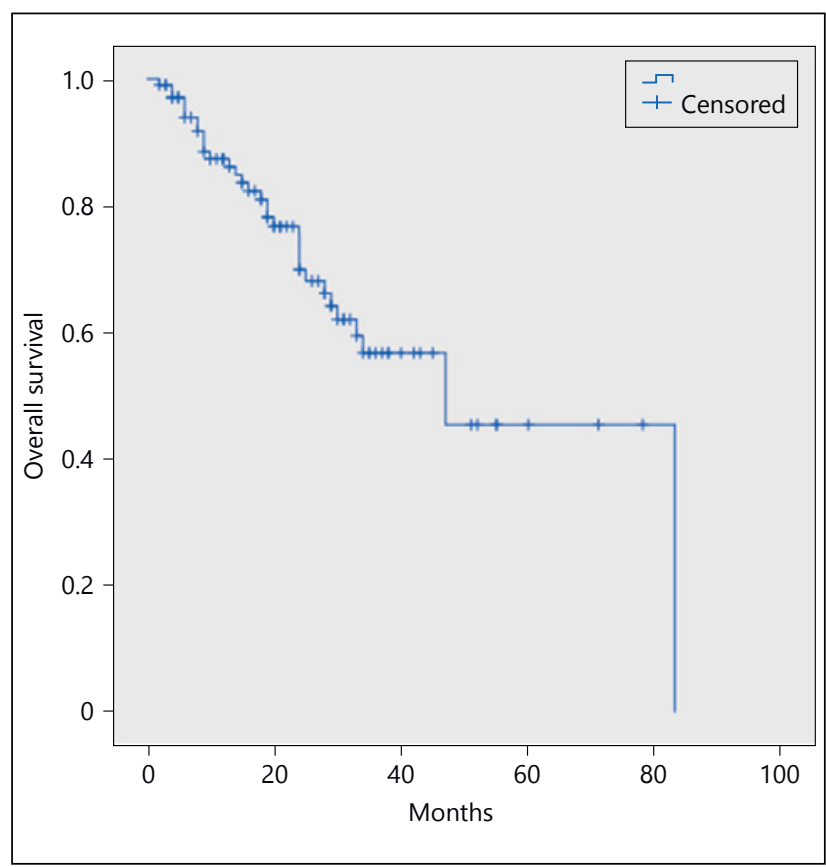

Fig. 3. Overall survival (for Abstract no PE-098).

27). Radiation induced liver disease (RILD) was defined as worsening Child Pugh score by two points.

Results: The median follows up duration after SBRT with TACE was 22.0 months (range, 2-83 months). Mean size of tumors was $2.27 \pm 1.13 \mathrm{~cm}$ Mean $52.1 \pm 10.5 \mathrm{~Gy}$ in

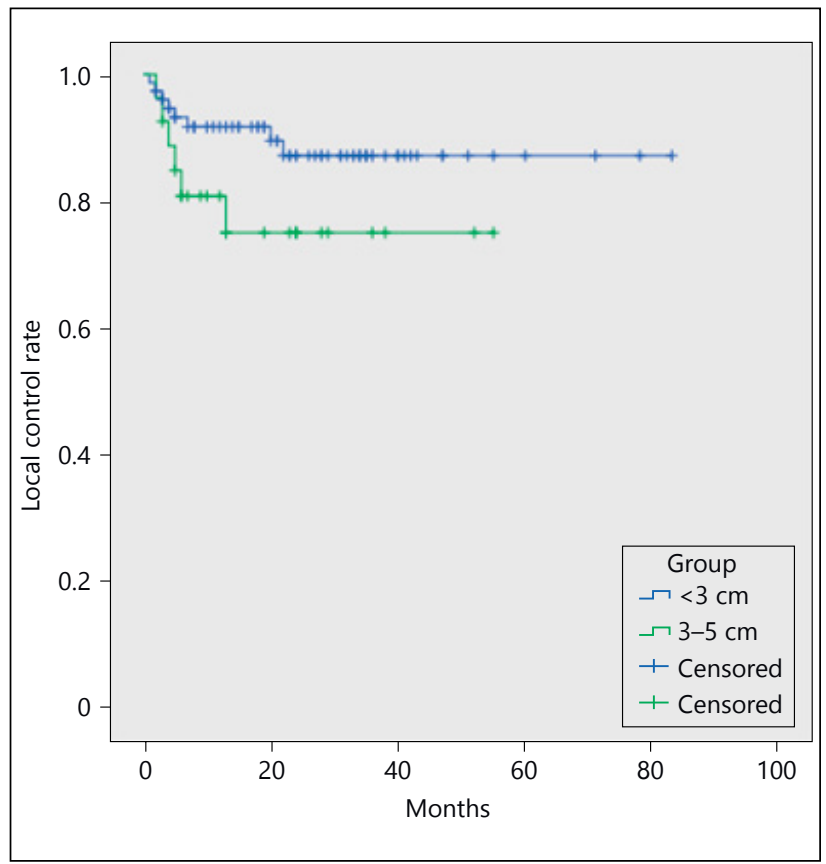

Fig. 2. Local control rate according to tumor size (for Abstract no PE-098).

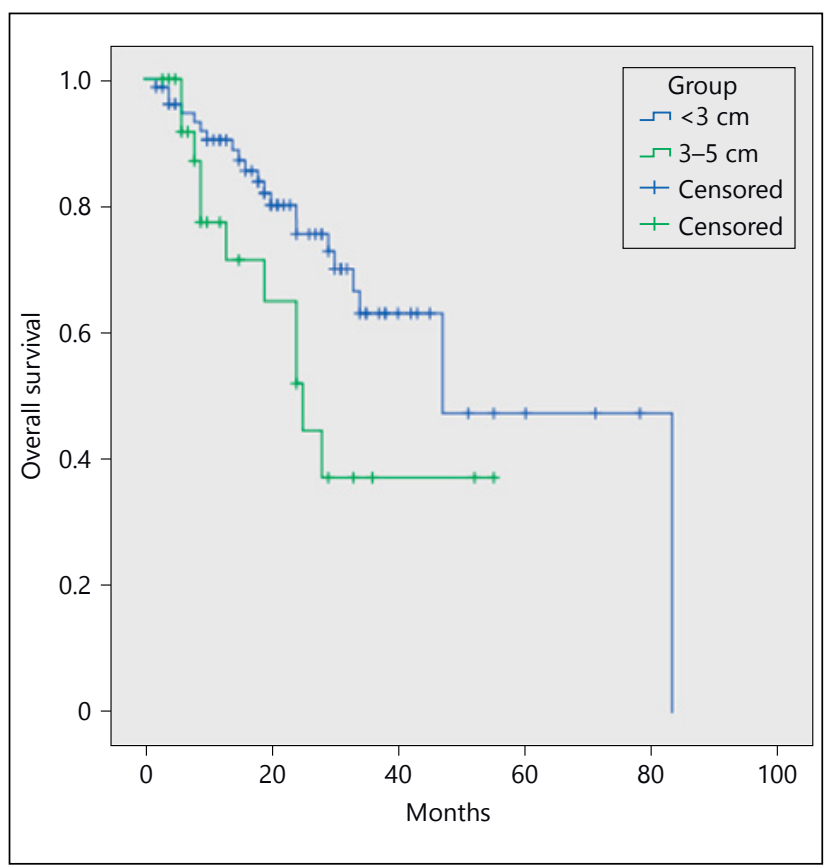

Fig. 4. Overall surivival according to tumor size (for Abstract no PE-098).

3-5 fractions was prescribed. 1-,2-,3-year LC rates for all patients were $93.1 \%, 89.9 \%$ and $88.8 \%$ respectively. 1 -year LC rate did not differ between small- and medium-sized HCC (93.2\% vs $80.8 \%$, log-rank p $=0.109)$. 1-,3-,5-year OS were $87.4 \%, 56.7 \%$ and $45.4 \%$ respectively. 3 -year OS rate 
Table 1. Baseline characteristics in both small- and medium- sized hepatocellular carcinoma (for Abstract no PE-098)

\begin{tabular}{|c|c|c|c|c|}
\hline Variable & $\begin{array}{l}\text { Small HCC } \\
(<3 \mathrm{~cm}) \\
(\mathrm{n}=77)\end{array}$ & $\begin{array}{l}\text { Medium sized HCC } \\
(3-5 \mathrm{~cm}) \\
(\mathrm{n}=27)\end{array}$ & $\begin{array}{l}\text { Total } \\
(\mathrm{n}=114)\end{array}$ & P-value \\
\hline \multicolumn{5}{|l|}{ Sex } \\
\hline Male & 61 & 19 & 80 & 0.348 \\
\hline Female & 16 & 8 & 24 & \\
\hline Mean age $($ mean $\pm S D)$ & $61.7 \pm 10.04$ & $62.78 \pm 11.31$ & $61.98 \pm 10.34$ & 0.644 \\
\hline \multicolumn{5}{|l|}{ Number } \\
\hline 1 & 51 & 16 & 67 & 0.862 \\
\hline 2 & 16 & 8 & 24 & \\
\hline$>3$ & 10 & 3 & 13 & \\
\hline Mean tumor size (mean \pm SD) & $1.71 \pm 0.55$ & $3.86 \pm 0.77$ & $2.27 \pm 1.13$ & $<0.001$ \\
\hline Child-pugh score (mean \pm SD) & $5.65 \pm 0.98$ & $5.52 \pm 0.80$ & $5.62 \pm 0.93$ & 0.535 \\
\hline \multicolumn{4}{|l|}{ BCLC stage on diagnosis (\%) } & 0.013 \\
\hline 0 & $21(27.3)$ & $5(18.5)$ & 26 & \\
\hline A & 43 (55.8) & $9(33.3)$ & 52 & \\
\hline B & $10(13.0)$ & $11(40.7)$ & 21 & \\
\hline $\mathrm{C}$ & $3(3.9)$ & $2(7.4)$ & 5 & \\
\hline \multicolumn{4}{|l|}{ BCLC stage on treatment (\%) } & 0.009 \\
\hline A & $54(70.1)$ & $10(37.0)$ & 64 & \\
\hline B & $14(18.2)$ & $11(40.7)$ & 25 & \\
\hline $\mathrm{C}$ & $9(11.7)$ & $6(22.2)$ & 15 & \\
\hline \multicolumn{4}{|l|}{ The etiology } & 0.357 \\
\hline Alcohol & 18 & 9 & 27 & \\
\hline Hepatitis B virus & 44 & 15 & 59 & \\
\hline Hepatitis C virus & 8 & 3 & 11 & \\
\hline others & 7 & 0 & 7 & \\
\hline Radiation dose & $52.7 \pm 11.3$ & $50.4 \pm 7.9$ & $52.1 \pm 10.5$ & 0.337 \\
\hline ALT & $28.3 \pm 27.1$ & $30.7 \pm 17.8$ & $28.98 \pm 24.99$ & 0.679 \\
\hline Total bilirubin (mg/dl) & $1.01 \pm 0.64$ & $1.03 \pm 0.65$ & $1.01 \pm 0.64$ & 0.878 \\
\hline Platelet count $\left(\mathrm{x} 10^{9} / \mathrm{L}\right)$ & $125.4+65.5$ & $130.3 \pm 68.8$ & $126.7 \pm 65.7$ & 0.7 .9 \\
\hline
\end{tabular}

Values are expressed as n only, mean \pm SD, n (\%), ALT alanine transaminase,

Table 2. Liver toxicity in both small- and medium- sized hepatocellular carcinoma (for Abstract no PE-098)

\begin{tabular}{llll}
\hline Variable & $\begin{array}{l}\text { Small HCC } \\
(<3 \mathrm{~cm}) \\
(\mathrm{n}=77)\end{array}$ & $\begin{array}{l}\text { Medium sized HCC } \\
(3-5 \mathrm{~cm}) \\
(\mathrm{n}=27)\end{array}$ & P-value \\
\hline RILD (\%) & $\begin{array}{c}12(15.6) \\
5(6.5)\end{array}$ & $\begin{array}{l}8(29.6) \\
3(2.1)\end{array}$ & 0.111 \\
Radiation induced hepatitis (\%) & & 0.438 \\
\hline
\end{tabular}

RILD, radiation induced liver disease.

of small-HCC was better than that of medium-sized HCC (63.0\% vs $37.1 \%$, log-rank $\mathrm{p}=0.047)$. RILD was observed in $20(17.5 \%)$ patients. RILD did not differ between smalland medium-sized HCC (15.6\% vs $29.6 \%$, p $=0.111)$. Conclusions: Treatment with SBRT + TACE can be an effective local therapeutic tool for small and medium sized HCC patients, with good local tumor control and low treatment-related toxicity. Keywords: Hepatocellular carcinoma, Stereotactic body radiotherapy, Transarterial chemoembolization. 


\section{PE-099 \\ A case of Repeated Transcatheter Arterial Chemoembolization in a Patient with Hepatocellular Carcinoma Accompanying Incidental Aortic Dissection \\ SungKeun Kim ${ }^{1}$, Hee Yeon Kim¹, Chang Wook Kim¹, Su Lim Lee L $^{2}$ Yoo Dong Won ${ }^{2}$ \\ ${ }^{1}$ Department of Internal Medicine, Uijeongbu St. Mary's Hospital, College of Medicine, The Catholic University of Korea, Kyeonggido, South Korea; ${ }^{2}$ Department of Radiology, Uijeongbu St. Mary's Hospital, College of Medicine, The Catholic University of Korea, Kyeonggido, South Korea}

Aims: Transcatheter arterial chemoembolization (TACE) for hepatocellular carcinoma (HCC) in patients with aortic dissection is a potentially risky and demanding technique. Access to the true aortic lumen might not be allowed by the arterial entry route chosen. Here we report a case of repeated sessions of TACE through true and false lumens in an HCC patient with chronic aortic dissection. Methods: A 56-year old male presented with infiltrative HCC with right portal vein tumor thrombosis. Abdominal computer tomography scan also revealed an incidental asymptomatic aortic dissection involving descending aorta from proximal segment to superior mesenteric artery (SMA) origin level. Celiac trunk was supplied by false lumen, and SMA was supplied by true lumen. Results: On SMA angiography, right hepatic artery was originated from SMA. HCC was supplied by segment 7 and 8 branch of right hepatic artery. Infusion of adriamycin and lipiodol mixture and subsequent embolization was performed to superior branches of right hepatic artery. Catheterization to celiac axis was failed due to its origin from pseudolumen caused by aortic dissection. Complete response was achieved after sequential radiation therapy. After 1-year, nodular HCC recurred at segment 4 . On this session of repeated TACE, catheterization to false lumen was successful. Left hepatic angiography showed tumor stain supplied by segment 4 branch, and embolization was performed. One year later, viable portion was noted in infiltrative HCC at segment 7. Repeated TACE was performed to embolize segment 7 branch supplying infiltrative tumor. Conclusions: In this case, we presented our experience with selective hepatic angiography and SMA angiography in an HCC patient with descending aortic dissection. To our knowledge, this is the first description of hepatic angiography using true and false lumen in a HCC patient with a concomitant aortic dissection. Keywords: Hepatocellular carcinoma, Chemoembolization, Aortic dissection.

\section{PE-100 \\ Comparison of the Safety and Imaging Response in Different Size of Beads on DEB-TACE: 75-150 $\mathrm{\mu m}$ VS 100-300 $\mathrm{\mu m}$}

Kyungmin Park ${ }^{1}$, Hyun Pyo Hong ${ }^{1}$, Byung Ik Kim²

${ }^{1}$ Department of Radiology, Kangbuk Samsung Hospital, Sungkyunkwan University School of Medicine, Seoul, Republic of Korea; ${ }^{2}$ Department of Internal Medicine, Kangbuk Samsung Hospital, Sungkyunkwan University School of Medicine, Seoul, Republic of Korea

Aims: The purpose of this study is to compare the safety and imaging response between different sizes of beads on DEB-TACE, 75-150 $\mu \mathrm{m}$ and 100-300 $\mu \mathrm{m}$. Methods: DEB-TACE using 75-150 $\mu \mathrm{m}(\mathrm{n}=14)$ and $100-300 \mu \mathrm{m}(\mathrm{n}=13)$ were conducted from February 2016 to June 2017. There were 19 tumors in 75-150 um group and 18 tumors in 100-300 um group. DC beads loaded with $50 \mathrm{mg}$ of doxorubicin were used in all patients. Patient demographics such as age, sex, etiology of liver disease, Child-Pugh classification, BCLC staging, performance status, tumor size and number were not significantly different between the two groups. All patients were treated first time in their life for HCC and procedures were performed in a single session. Imaging response was evaluated by two radiologists based on mRECIST criteria with follow up CT or MRI which was performed at 1 month after the procedure. Symptoms related to toxicity and biochemical abnormality grades were available on EMR. Results: There was no significant difference in imaging response between two groups but there was a trend showing higher complete response and objective response (CR and PR) rate in 75-150 um group than 100-300 um group. (CR: $52.6 \%$ vs $22.2 \%, \mathrm{P}=$ 0.108; CR+PR: $84.2 \%$ vs $66.7 \%, \mathrm{P}=0.269$ ) There was no significant difference of post procedure biochemical profile such as total bilirubin, prothrombin time (PT), alkaline phosphatase (ALP), aspartate aminotransferase (AST) and aspartate aminotransferase (AST) change between two groups. One case of bile duct injury on follow up CT occurred in 100-300 um group. Post embolization syndrome (PES) was occurred more in the patients of 75-150 um group (57.1\% vs $23.1 \%, \mathrm{P}=0.072$ ), but there was no significant difference in hospital day between two groups (8.1 days vs 9.0 days, $\mathrm{P}=$ 0.712). Conclusions: In DEB-TACE, 75-150 um beads showed the trend toward good imaging response, compared with 100-300 um beads but there were more PES in the 75-150 um beads group than the 100-300 um beads group. Keywords: DEB-TACE, Bead size. 


\section{PE-101 \\ The Challenge of Advance Hepatocellular Carcinoma: Result of a Single-Institutional Experience on Stereotactic Body Radiation Therapy

\author{
Jenny Que 1,2, Li-Ching Lin', Chia-Hui Lin ${ }^{1}$, \\ Ching-Chieh Yang ${ }^{1}$, Kuei-Li Lin ${ }^{1}$, Yu-Wei Lin ${ }^{1}$ \\ ${ }^{1}$ Department of Radiation Oncology, Chi Mei Medical \\ Center, Tainan, Taiwan; ${ }^{2}$ Department of Hospital and \\ Health Care Administration, Chia Nan University of \\ Pharmacy and Science, Tainan, Taiwan
}

Aims: Sorafenib is recommended as the major standard treatment for advance hepatocellular carcinoma (HCC) in accordance to Barcelona Clinic Liver Cancer (BCLC) treatment guideline. However, the treatment results remain modest. In this study, we evaluated the feasibility and efficacy of Stereotactic Body Radiation Therapy (SBRT) in the treatment of advance hepatocellular carcinoma. Methods: This retrospective study evaluated patients with advance HCC who underwent Cyberknife SBRT between January 2009 and September 2017. All patients had BCLC-C, Child-TurcottePugh (CTP) score A-B. In-field control, overall survival (OS), progression free survival (PFS), prognostic factors were evaluated. Results: In this study, a total of 141 patients were analyzed. Objective response rate was achieved in $81.5 \%$ patients ( $C R=36.2 \%$, PR $=45.3 \%$ ). Median survival was 15.44 months, the 1-yr, 3-yrs., 5 -yrs overall survival rate were $56 \%$, $28 \%$ and $20 \%$, respectively. Median progression-free rate was 6 months, the progression free survival rate at 1-yr, 3-yrs and 5 -yrs were $35 \%, 14 \%$, and $10 \%$, respectively. Local control rate was achieved in $68.3 \%$ of patients. Major pattern of failure was Out-field intrahepatic failure comprising $42.9 \%$. Multivariate analysis revealed CTP score, macrovascular invasion, advance AJCC stage (III-IV), and tumor response rate were independent predictors of OS. Conclusions: SBRT was as effective and safe as sorafenib for advance HCC, and it provides a longer overall survival, higher tumor response rate and a more sustain local tumor control rate. Keywords: Hepatocellular carcinoma, CyberKnife, Stereotactic body radiation therapy.

\section{PE-102 \\ Bridging and Downstaging Role of Transarterial Radioembolization (TARE) for Expected Small Remnant Volume after Liver Resection in Hepatocellular Carcinoma}

\author{
Jeong-Moo Lee ${ }^{1}$, Kyung-Suk Suh ${ }^{1}$, Suk Kyun Hong ${ }^{1}$, \\ Kyung Chul Yoon ${ }^{1}$, Jae-Hyung Cho ${ }^{1}$, Nam-Joon $\mathrm{Yi}^{1}$, \\ Kwang-Woong Lee ${ }^{1}$ \\ ${ }^{1}$ Department of Surgery, Seoul National University \\ College of Medicine, Seoul, South Korea
}

Aims: Hepatectomy is the best treatment to improve survival in liver cancer. However, if the tumor is located in the central portion or the remaining remnant liver volume is small, there is a risk of complications such as hepatic failure after surgical treatment. Transarterial radioembolization (TARE) is a radiosurgical technique using yttrium-90 to induce primary tumor necrosis and hypertrophy of remnant liver to reduce the risk of liver failure and enable safe hepatic resection. In this study, we report a case of patients with hepatic resection after TARE. Methods: Between January 2017 and December 2017, we performed hemihepatectomy in 5 patients who had unresectable causes like central located, small remnant liver volume, vascular invasion to major vessel. We performed TARE 3 month before surgical resection. Then we check tumor volume and expected remnant liver volume after surgical resection. Re-evaluating of liver function test, CT scan 4 weeks after TARE, and ICG test for patient safety, then we performed surgical resection. Results: None of the 5 patients had recurrence during observation period. There was no hepatic failure after surgical resection 4 cases underwent Rt. hemihepatectomy and 1 case underwent extended Rt. hemihepatectomy. The mean operative time was 160 minutes. Mean hospital day was 7.5 days and mean blood loss $120 \mathrm{cc}$. Mean tumor shrinkage rate was $-21.1 \%$ and mean remnant liver hypertrophy rate was $39.5 \%$. Only 1 case had wound complication, but the others had no postoperative complication. Conclusions: Surgical resection after TARE is feasible technique for surgically unresectable cases of hepatocellular carcinoma. Keywords: Hepatocellular carcinoma, Transarterial radioembolization, Hepatectomy, 


\section{PE-103 \\ Initial Treatment Response Is Significantly Associated Survival Outcomes in Patients Treated with TACE for Recurrent HCC after Curative Resection}

Jae Seung Lee ${ }^{1}$, Beom Kyung Kim 1,2,3, Jun Yong Park ${ }^{1,2,3}$ Do Young Kim 1,2,3, Sang Hoon Ahn 1,2,3,

Kwang-Hyub Han ${ }^{1,2,3}$, Seung Up Kim ${ }^{1,2,3}$

${ }^{1}$ Department of Internal Medicine, Yonsei University College of Medicine, Seoul, Republic of Korea;

${ }^{2}$ Institute of Gastroenterology, Yonsei University College of Medicine, Seoul, Republic of Korea; ${ }^{3}$ Yonsei Liver Center, Severance Hospital, Seoul, Republic of Korea

Aims: Trans-arterial chemoembolization (TACE) prolongs the survival of patients with hepatocellular carcinoma (HCC). We evaluated whether the initial treatment response by TACE for recurrent HCC after curative resection is associated with better survival. Methods: Between 2003 and 2015, 385 patients with recurrent HCC after curative resection who were treated with TACE were recruited for this retrospective study. Modified Response Evaluation Criteria in Solid Tumor (mRECIST) was used for response evaluation. Results: After the first TACE, 266 (69.1\%), 75 (19.5\%), 18 (5.7\%), and 26 (6.7\%) showed complete response (CR), partial response (PR), stable disease (SD), and progressive disease (PD), respectively. Patients with CR achievement after the first TACE had significantly better survival than those with PR/SD and PD (median 83.7 vs. 45.6 and 13.7 months; all $P<0.05$, log-rank tests). Similarly, patients with CR achievement as the best response during repeated TACE showed significantly better survival than those with PR/SD and PD (median 78.8 vs. 28.2 and 7.8 months; all $P<0.05$, log-rank tests). On multivariate analysis, CR achievement after the first TACE (hazard ratio $[\mathrm{HR}]=0.629$; 95\% confidence interval [CI], 0.454-0.872; $P=0.005$ ) or during repeated TACE (HR $=0.354 ; 95 \% \mathrm{CI}, 0.237-0.528 ; P<0.001$ ) was independently associated with the reduced risk of mortality, whereas multiple tumors, BCLC stage C (vs. A-B), and Child-Pugh class B liver function (vs. A) were associated with the increased risk of poor outcomes (all $P<0.05$ ). Conclusions: CR achievement after the first TACE or during repeated TACE strongly predicted favorable survival outcome in patients with recurrent HCC after curative resection. Keywords: Recurrence, Complete response, Trans-arterial chemoembolization, Hepatocellular carcinoma.

\section{PE-104 \\ Efficacy and Safety of Transarterial Chemoembolization with New Calibrated Gelatin Sponge Microparticles for Patients with Unresectable Hepatocellular Carcinoma: Single Center Prospective Study}

Su Ho Kim, Ho Jong Chun, Jung Suk Oh, Byung Gil Choi, Hae Giu Lee

Department of Radiology, Seoul St. Mary's Hospital, College of Medicine, The Catholic University of Korea

Aims: To evaluate safety and efficacy of transarterial chemoembolization (TACE) with new calibrated gelatin sponge microparticles (GSM) in patients with unresectable

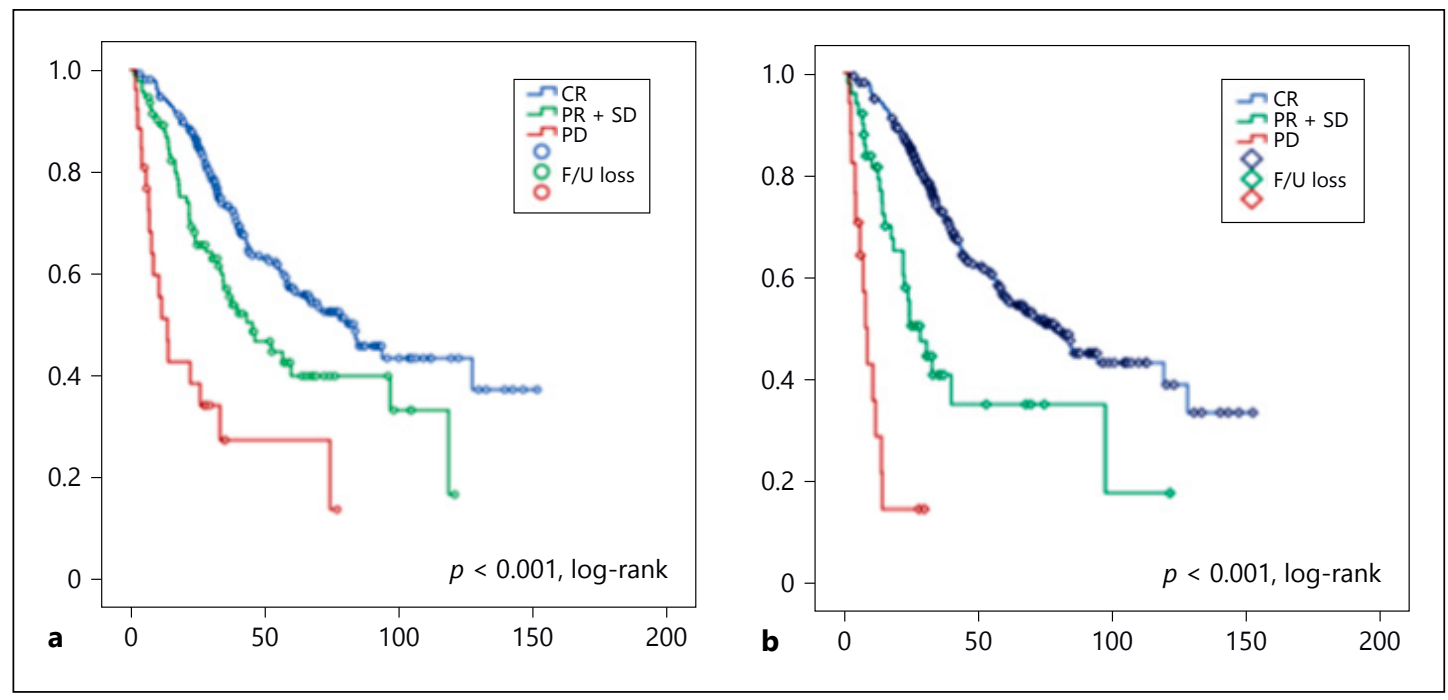

Fig. 1. Kaplan-Meier curves generated among entire population to compare survival, according to die (Left) initial and die (Right) best response. ${ }^{*} \mathrm{X}$-axis means Overall survival (months), and y-axis means cummuiative survival propability (for Abstract no PE-103). 
Table 1. Predictors of TACE outcomes (for Abstract no PE-103)

\begin{tabular}{|c|c|c|c|c|}
\hline \multirow[b]{2}{*}{ Variables } & \multicolumn{2}{|c|}{ Multivariate analysis } & \multicolumn{2}{|c|}{ Multivariate analysis } \\
\hline & $P$ value & HR (95 CI) & $\mathrm{P}$ value & $\operatorname{HR}(95 \% \mathrm{C} \Gamma)$ \\
\hline \multicolumn{5}{|l|}{ Baseline variables } \\
\hline AFP: $n g / m L$ & 0.283 & $1.255(0.829-1.893)$ & 0.203 & $1.303(0.867-1.958)$ \\
\hline PIVKA-II mAlГ mL & 0.382 & $1.275(0.740-2.197)$ & 0.126 & $1.545(0.885-2.696)$ \\
\hline Multiple tumor & 0.022 & $1.465(1.058-2.029)$ & 0.073 & $1.349(0.972-1.373)$ \\
\hline Max. tumor size $>3 \mathrm{~cm}$ & 0.244 & $1.426(0.784-2.594)$ & 0.566 & $1190(0.657-2.157)$ \\
\hline BCLC stage C (vs. 0-B) & 0.002 & $3.007(1.487-6.082)$ & 0.021 & $2.328(1.133-4.780)$ \\
\hline Child-Pugb class B (vs. A) & 0.035 & $3.030(1.081-8.495)$ & 0029 & $3.108(1.123-3.001)$ \\
\hline$<2$ year between resection and the first & 0.425 & $1.170(0.795-1.723)$ & 0.801 & $0.951(0.645-1.403)$ \\
\hline Complete response after the first TACE & 0.005 & $0.629(0.454-0.872)$ & & \\
\hline Complete response as the best response during repeated TACE & - & - & $<0.001$ & $0.354(0.237-0.528)$ \\
\hline
\end{tabular}

TACE, transarterial chemoembolization; HR, hazard ratio; Cl, confidence interval; AFP, alpha-fetoprotein; PIVKA-II, protein induced by vitamin $\mathrm{K}$ absence or antagonist II; BCLC, Barcelona-clinic liver cancer.

hepatocellular carcinoma (HCC) Methods: In this single center prospective study, 48 consecutive patients with unresectable HCC (44 patients with Child-Pugh score A and 4 patients with B) underwent TACE with GSM (150-300 $\mu \mathrm{m}$, EG-gel, Korea) between April 2017 and February 2018. A total of 70 lesions with a mean ( \pm standard deviation) size of $2.2 \pm$ $1.6 \mathrm{~cm}$ were treated with an emulsion of doxorubicin in lipiodol, followed by feeding artery embolization with GSM. Tumor response was assessed with contrast enhanced MR or $\mathrm{CT}$, performed before and 1 month after the treatment using modified RECIST. The recanalization rate was assessed only in patients undergoing FU angiography. Adverse event related to treatment was assessed based on presence of post-embolization syndrome (PES), hospital day and degree of elevation of serum ALT level. Results: TACE with GSM was successfully performed in all 48 sessions. On 1-month postprocedural imaging, objective tumor response was achieved in 37 patients (92.5\%); partial response in 10 patients $(25 \%)$ and complete response in 27 patients (67.5\%). Only 3 patients showed stable disease and no patient had progression disease. Total 28 patients performed follow-up angiography, showing complete recanalization in 18 patients (64.2\%), incomplete recanalization in 7 patients $(25 \%)$ and total occlusion in 3 patients (11\%). Twelve patients (25\%) experienced mild form of PES, and 10 patients (28\%) required prolonged hospitalization. Grade 3 or 4 ALT elevation was seen in two patients when classified as CTCAE grade, but it was normalized after 5 days in all patients. Conclusions: TACE with GSM is safe and effective in achieving local tumor control in patients with unresectable HCC. Keywords: Transarterial chemoembolization, Hepatocellular carcinoma, Gelatin sponge microparticle,

\section{PE-105 \\ Effects of Oral L-Carnitine on Liver Functions after Transarterial Chemoembolization in Patients with Hepatocellular Carcinoma.}

Ali Kassem ${ }^{1}$, Abeer. Hassan 1 , Yasuhiro Tsuda? Akira Asai ${ }^{2}$, Keisuke Yokohama ${ }^{2}$, Hideko Ohama ${ }^{2}$, Ken Nakamura ${ }^{2}$, Tetsuya Sujishi', Yusuke Tsuchimoto ${ }^{2}$, Shinya Fukunishi ${ }^{2}$, Ali. Hassan ${ }^{1}$, Kazuhide Higuchi ${ }^{2}$

${ }^{1}$ Sohag Faculty of Medicine, Sohag University, Egypt and ${ }^{2}$ Oska School of Medicine, Osaka, Japan

Aims: Transarterial chemoembolization (TACE) for hepatocellular carcinoma (HCC) is usually followed by hepatic dysfunction that limits its efficacy. L-carnitine is recently studied as hepatoprotective agent. Our aim is to evaluate the L-carnitine effects against the deterioration of liver functions after TACE. Methods: Fifty-three patients with intermediate stage HCC were assigned into two groups; L-carnitine group (26 patients) who received L-carnitine $300 \mathrm{mg}$ tablet twice daily from $2 \mathrm{wk}$ before to $12 \mathrm{wk}$ after TACE and control group (27 patients) without L-carnitine therapy. 28 of studied patients received branched chain amino acids granules. Results: There were significant differences between L-carnitine Vs control group in mean serum albumin change from baseline to $1 \mathrm{wk}$ and $4 \mathrm{wk}$ after TACE ( $<<0.05$ ). L-Carnitine maintained Child-Pugh score at $1 \mathrm{wk}$ after TACE and exhibited improvement at $4 \mathrm{k}$ w after TACE ( $\mathrm{p}<0.01 \mathrm{Vs} 1$ week after TACE). Control group has significant Child-Pugh score deterioration from baseline to 1 wk after TACE ( $p<$ $0.05)$ and $12 \mathrm{wk}$ after TACE $(\mathrm{p}<0.05)$. There were significant differences between L-carnitine and control groups in mean Child-Pugh score change from baseline to $4 \mathrm{wk}(\mathrm{p}<0.05)$ and 12 wkafterTACE $(\mathrm{p}<0.05)$. L-carnitine displayed improvement in (PT) from baseline to $1 \mathrm{wk}, 4 \mathrm{w}(\mathrm{p}<0.05)$ and $12 \mathrm{wk}$ after 
TACE. PT in control group declined less than baseline along all follow up intervals. Total bilirubin in L-carnitine group decreased at 1 wk post TACE while in control group, it significantly increased at $1 \mathrm{wk}(\mathrm{p}=0.01)$. ALT and C-reactive protein elevation were suppressed at 1 wk after TACE in Lcarnitine group. The hepatoprotective effects of L-carnitine were enhanced by concomitant use of branched chain amino acids. Conclusions: L-carnitine and BCAA combination therapy offer a novel supportive strategy after TACE in HCC patients. Keywords: Hepatocellular carcinoma, L Carnitine, Liver functions, Transarterial chemoembolization.

\section{PE-106 \\ Reduction of Intrahepatic Tumor Burden by Hepatic Arterial Infusion Chemotherapy Prolongs Survival in Advanced Hepatocellular Carcinoma}

\author{
Pil Soo Sung ${ }^{1 *}$, Keungmo Yang ${ }^{1 *}$, Si Hyun Bae ${ }^{1 \#,}$ \\ Jung Suk Oh${ }^{2}$, Ho Jong Chun ${ }^{2}$, Jeong Won Jang ${ }^{1}$, \\ Jong Young Choi ${ }^{1}$, Seung Kew Yoon ${ }^{1}$ \\ ${ }^{1}$ Department of Internal Medicine, Seoul St. Mary's \\ Hospital, The Catholic University Liver Research Center, \\ The Catholic University of Korea, Seoul, Republic of \\ Korea; ${ }^{2}$ Department of Radiology, Seoul St. Mary's \\ Hospital, The Catholic University of Korea, Seoul, \\ Republic of Korea
}

Aims: There are limited studies on the impact of intrahepatic tumor control on patient survival in advanced hepatocellular carcinoma (HCC). The purpose of this study is to confirm survival benefits of reduced intrahepatic tumor burden by hepatic arterial infusion chemotherapy (HAIC) in advanced HCC patients. Methods: Between January 2012 and December 2017, a total of 138 consecutive patients with advanced HCC treated with HAIC were enrolled. Thirty-six patients (26.1\%) had extrahepatic metastasis when HAIC was started. Survival outcomes and tumor response rate (both intrahepatic and extrahepatic) after HAIC were analyzed. Multivariate analysis was performed to estimate clinical factors associated with survival outcomes. Results: Most of the patients (86.2\%) included in the study were on Barcelona Clinic Liver Cancer stage $C$. The presence of extrahepatic metastasis at the start of HAIC had no significant effect on overall survival $(P=0.17)$. The intrahepatic objective response rate (ORR) (complete response + partial response) of all the enrolled patients to HAIC was $17.4 \%$. The intrahepatic OR was $13.8 \%$ for patients with extrahepatic metastasis, and $18.6 \%$ for patients without extrahepatic metastasis. Patients with intrahepatic OR by HAIC showed significantly better survival outcomes than those without OR did, irrespective of the initial distant metastases. On multivariate analysis, the achievement of intrahepatic OR by HAIC and Child-Pugh classification at the time of first response evaluation were two independent factors affecting better overall survivals. Conclusions: HAIC-induced intrahepatic tumor reduction prolongs patient survival irrespective of the status of distant metastasis. Our study demonstrates that it is critical to reduce intrahepatic tumor burden in advanced HCC even when patients have initial extrahepatic metastasis. Keywords: Hepatocellular carcinoma, Hepatic arterial infusion chemotherapy, Objective response,

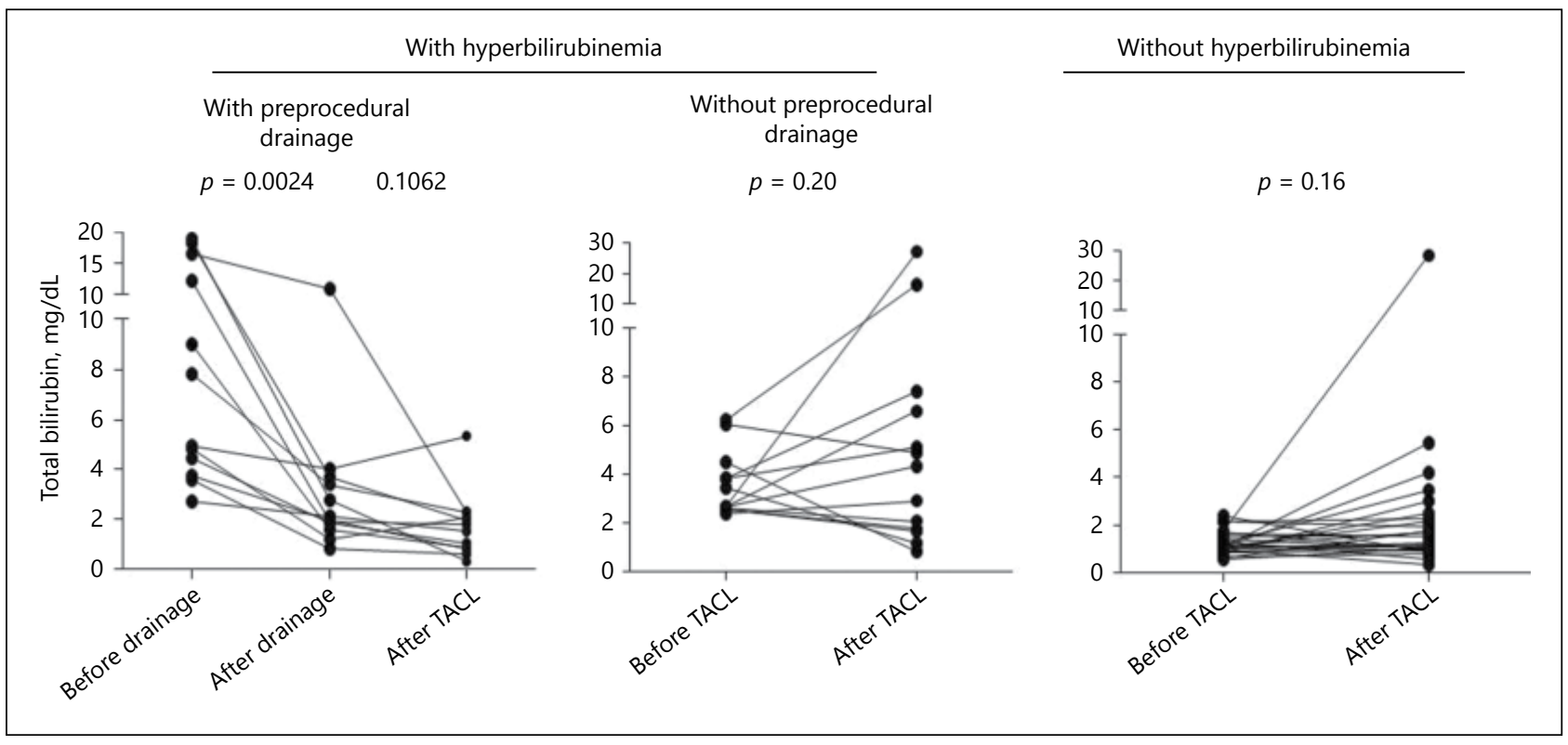

Fig. 1. (for Abstract no PE-106). 


\section{PE-107 \\ Clinical Research Progress of Traditional Chinese Medicine Treatment of Primary Liver Cancer}

Zhong Ying Han, Zheng Han, Jun Feng Zhu

Shanghai Municipal Hospital of Traditional Chinese Medicine

Aims: Primary liver cancer is one of clinical common malignant tumor, Originated from the epithelial tissue of liver, and the pathogeny is still uncertain the main cause is associated with cirrhosis of liver, virus hepatitis, chemical carcinogen like flavacin and environment. High malignant degree of liver cancer, the death rate in malignant tumor in the second place. Nowadays western medicine the main treatment is surgery, chemotherapy and biological treatment. Methods: Using traditional Chinese medicine treatment of liver cancer has certain curative effect, relieve the side effects of chemotherapy, the importance of protecting liver function, reduce jaundice, prolong survival patients with liver cancer and improve the quality of survival. But various professors in the treatment concept of liver cancer are different. Meanwhile, lacking of unified treatment and therapeutic effects evaluation, treats in the often are based on personal experience. The paper summarized the progress in the treatment of primary liver cancer with TCM in recent years.

\section{PE-108 \\ Transarterial Chemolipiodolization for Hepatocellular Carcinoma with Central Bile Duct Invasion Causing Conjugated Hyperbilirubinemia: Safety and Prognostic Factors for Survival \\ Pil Soo Sung ${ }^{1}$, Jung Suk Oh${ }^{2}$, Ho Jong Chun ${ }^{2}$, Jeong Won Jang ${ }^{1}$, Si Hyun Bae ${ }^{1}$, Jong Young Choi ${ }^{1}$, Seung Kew Yoon ${ }^{1, \#}$ \\ ${ }^{1}$ Department of Internal Medicine, Seoul St. Mary's Hospital, The Catholic University Liver Research Center, The Catholic University of Korea, Seoul, 06591, Republic of Korea; ${ }^{2}$ Department of Radiology, Seoul St. Mary's Hospital, The Catholic University of Korea, Seoul, Republic of Korea}

Aims: Bile duct invasion of hepatocellular carcinoma (HCC) is relatively uncommon. For patients with conjugated hyperbilirubinemia caused by HCC with bile duct invasion, it has been reported that effective biliary drainage followed by transarterial chemolipiodolization (TACL) may prolong survival. However, there are few reports comparing the clinical outcomes between tumors with central bile duct invasion causing conjugated hyperbilirubinemia and tumors with central bile duct invasion without hyperbilirubinemia, after TACL. Methods: Between January 2005 and December 2017, a total of 50 patients with HCC invading central bile duct (right or left hepatic duct, common hepatic or bile duct) and treated with TACL were enrolled. Patients were divided into three groups: hyperbilirubinemia (total bilirubin $\geq 2.5 \mathrm{mg} / \mathrm{dL}$ ) with pre-TACL

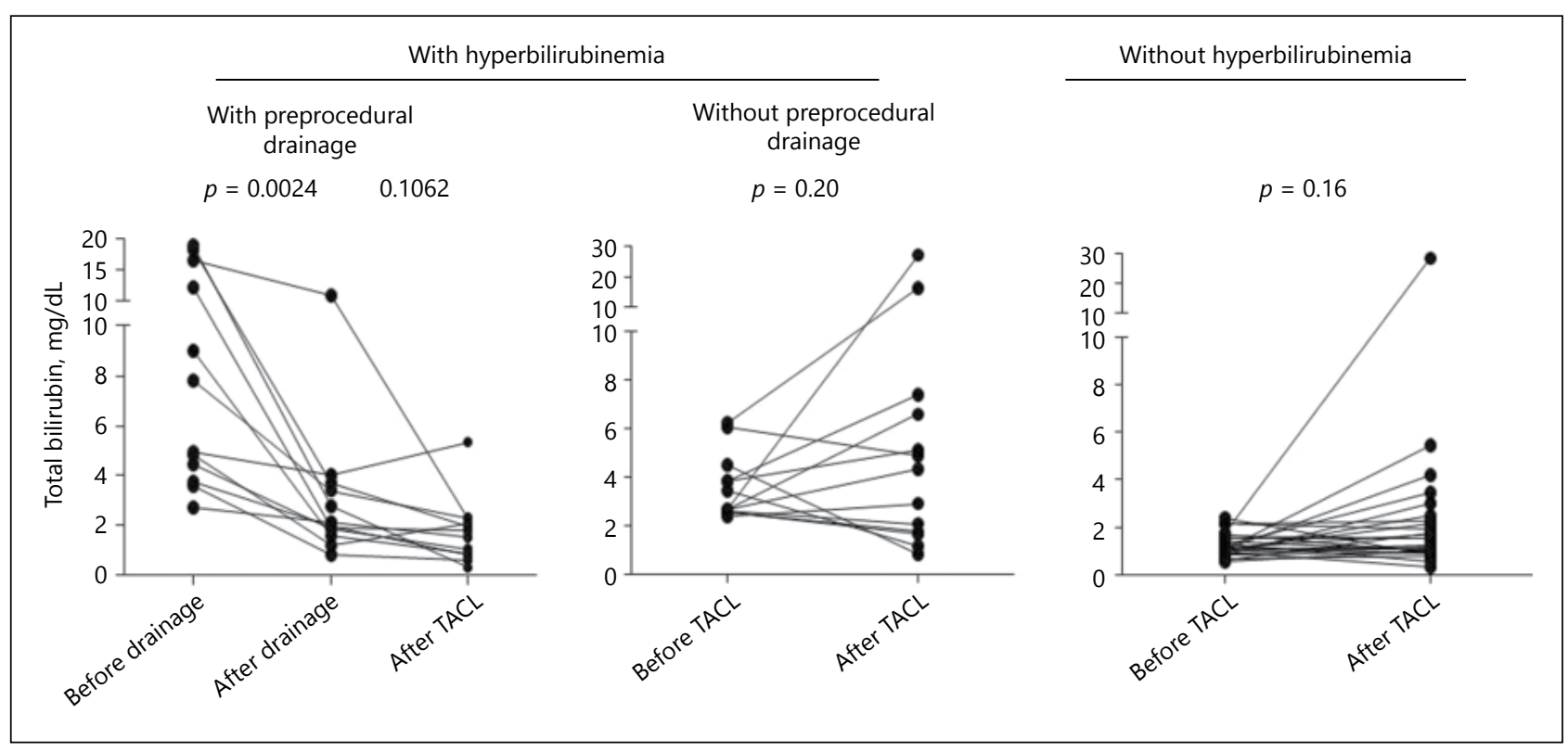

Fig. 1. (for Abstract no PE-108). 
biliary drainage group $(n=12)$, hyperbilirubinemia without biliary drainage group ( $\mathrm{n}=12$ ), and without hyperbilirubinemia group $(\mathrm{n}=26)$. Tumor response to TACL, survival outcomes, length of hospitalization, adverse events recorded using Common Terminology Criteria for Adverse Events (CTCAE), and factors affecting overall survival were compared among three groups. Results: The mean length of hospitalization was shorter in patients without hyperbilirubinemia compared to hyperbilirubinemia (10.2 vs 14.5 days, $P=0.017$ ), although mean CTCAE grade for laboratory parameters were not significantly increased after TACL among three groups. Significant decrease in serum bilirubin level was observed among patients who underwent pre-TACL biliary drainage (mean of differences: 5.860, $P=0.002$ ). However, there were no significant differences in serum bilirubin level between that of the TACL day and highest level within one month after TACL, in all three groups. The tumor response was also not significantly different between patients with hyperbilirubinemia and without hyperbilirubinemia $(P=0.573)$. Survival between patients with hyperbilirubinemia and without hyperbilirubinemia was not significantly different $(P=0.097)$. In multivariate analysis, $\alpha$-fetoprotein less than $400 \mathrm{ng} / \mathrm{dL}(\mathrm{HR}=0.477, P=$ 0.048 ), and highest total bilirubin $<2.5 \mathrm{mg} / \mathrm{dL}$ within one month after TACL (HR $=0.335, P=0.004$ ) were significantly associated with longer survival. Conclusions: TACL can be a safe and effective treatment for patients who have HCCs with central bile duct invasion, irrespective of the presence of conjugated hyperbilirubinemia. Keywords: Hepatocellular carcinoma, Hyperbilirubinemia, Bile duct invasion, Chemolipiodolization.

\section{PE-109 \\ Transarterial Infusion of Epirubicin and Cisplatin Combined with Systemic Infusion of 5-Flurouracil versus Sorafenib for Hepatocellular Carcinoma with Failure of Transarterial Chemoembolization Using Doxorubicin}

Sun Hong Yoo', Jung Hyun Kwon', Soon Woo Nam', Jong Youl Lee ${ }^{1}$, Kyoung Won Baik ${ }^{1}$, Jiyun Jung ${ }^{1}$, Jong Hyun Byeon ${ }^{2}$, Dong Jae Shim², Seung Won Lee ${ }^{3}$, Jeong Won Jang ${ }^{4}$

${ }^{1}$ Department of Internal Medicine, Incheon St. Mary's Hospital, The Catholic University of Korea, ${ }^{2}$ Department of Radiology, Incheon St. Mary's Hospital, The Catholic University of Korea, ${ }^{3}$ Department of Internal Medicine, Bucheon St. Mary's Hospital, The Catholic University of Korea, ${ }^{4}$ Department of Internal Medicine, Seoul St. Mary's Hospital, The Catholic University of Korea

Aims: Transarterial chemoembolization using doxorubicin (TAC-DOX) has been recognized as effective therapy for advanced hepatocellular carcinoma (HCC). However, there are few limited options in the patients with TACE failure. We compared the efficacy between sorafenib and transarterial chemoembolization using epirubicin and cisplatin combined with systemic infusion of 5 -flurouracil (TAC-ECF) in the patients with TAC-DOX failure. Methods: We analyzed the consecutively enrolled 742 patients who received TAC-DOX as the first-line therapy for HCC between January 2007 and December 2017. Among the patients who failed with TAC-DOX, 49 patients were treated with TAC-ECF and 37 patients were treated with sorafenib as a rescue therapy. The regimens of TAC-ECF consisted of transarterial infusion of epirubicin $\left(50 \mathrm{mg} / \mathrm{m}^{2}\right)$ and cisplatin $\left(60 \mathrm{mg} / \mathrm{m}^{2}\right)$ combined with systemic infusion of $5-\mathrm{FU}\left(200 \mathrm{mg} / \mathrm{m}^{2}\right.$ or $\left.1000 \mathrm{mg} / \mathrm{m}^{2}\right)$. Overall survival and progression-free survival were analyzed. Results: Of 86 patients, 22 and 64 patients were in Barcelona Clinic Liver Cancer (BCLC) stage B and C, respectively. The patients with Child-Pugh class A (CPC A) were 70.9\%. There was no difference of baseline stage or CPC in both group. The median duration of sorafenib administration was 2.8 months (1.017.8 months) and the median number of TAC-ECF was 2 times (1-10 times). Overall survival after rescue therapy did not differ between sorafenib group and TAC-ECF group (4.7 vs. 6.4 months, $P=0.730$ ). When we performed subgroup analysis by same CPC or BCLC stage, there is no difference of overall survival after rescue therapy in both groups $(P>0.05)$. In multivariate analysis for predicting poor survival, the patients with CPC B $(P=0.016$, HR 1.863, 95\% CI 1.124-3.087) and BCLC stage C HCC $(P<0.0001$, HR 2.999, 95\% CI 1.653-5.440) showed shorter survival than those with CP A and BCLC stage B HCC. There was no difference in progression-free survival between the sorafenib group and TAC-ECF group (3.0 vs. 3.5 months, $P=0.598)$. In the multivariate analyses, male gender $(P=0.034$, HR 1.991, 95\% CI 1.054-3.762) and BCLC stage C HCC $(P=0.035$, HR 1.822, 95\% CI 1.044-3.180) were proved as unfavorable factors for progression-free survival. Conclusions: The present study showed that the overall survival and progression-free survival in patient with rescue TAC-ECF therapy were consistent with sorafenib therapy among the HCC patients who failed with TAC-DOX. TAC-ECF might be considered as an alternative therapy for the patients with TAC-DOX failure, especially CPC B patients who are not indicated for sorafenib therapy. Keywords: Hepatocellular carcinoma, Transarterial chemoembolization, Failure. 


\section{PE-110 \\ Radiotherapy plus Transarterial Chemoembolization as a First-Line Treatment for Hepatocellular Carcinoma with Vascular Invasion and Suggestion of New Subclassification Models}

Jinhong Jung*1, Sang Min Yoon ${ }^{1}$, Yeon Joo Kim ${ }^{1}$, Ji Hyeon Joo ${ }^{1}$, Jong Hoon Kim ${ }^{1}$

${ }^{1}$ Departments of Radiation Oncology, Asan Medical Center, University of Ulsan College of Medicine, Seoul, Republic of Korea

Aims: Although sorafenib is the standard care for hepatocellular carcinoma (HCC) with major vascular invasion (MVI) according to the Barcelona Clinic Liver Cancer staging system, the survival benefits are modest. Alternatively, transarterial chemoembolization (TACE) plus three-dimensional conventional radiotherapy (3D-CRT) have shown favorable outcomes. We evaluated the clinical outcomes of patients who received respiratory-gated 3D-CRT and TACE for treatment naïve HCC with MVI and proposed a new subclassification

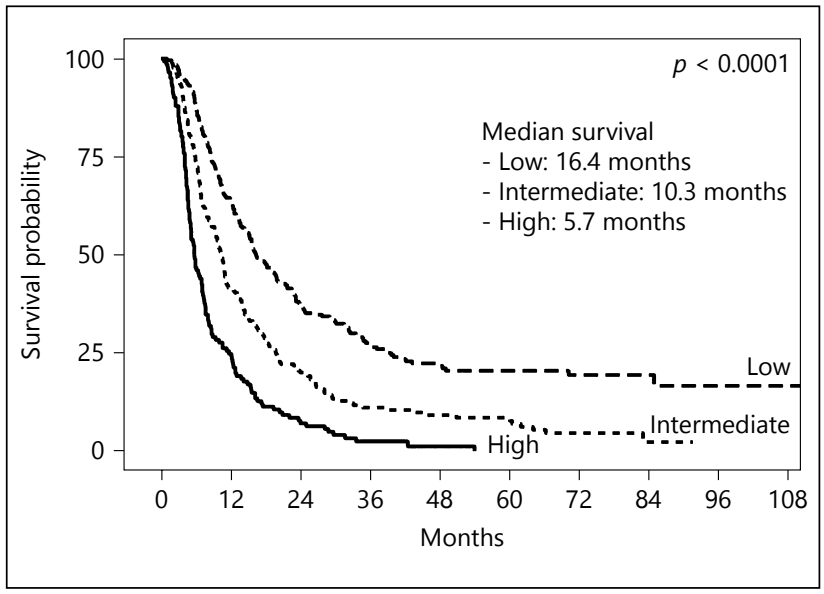

model. Methods: A total of 639 patients were treated with TACE and 3D-CRT for HCC with MVI as a first-line treatment between 2010 and 2013. Four-dimensional computed tomography was used for radiotherapy planning and a respiratorygated beam delivery was used in most patients. Results: Median age of the patients was 58 years. Main or bilateral portal vein tumor thrombus was observed in 313 (49\%) patients and inferior vena cava tumor thrombus was observed in $61(10 \%)$ patients. Median radiation dose was 39 Gy (range, 24-50) with median fraction size of 2.5 Gy (range, 2-5). Median patient survival was 10.7 months, and the 1- and 2 -year survival rates were $47 \%$ and $24 \%$, respectively. Tumor size $\leq 10 \mathrm{~cm}$, nodular type, absence of extrahepatic metastasis, Child-Pugh classification A, AFP $\leq 150000$ and radiation dose $>40$ Gy were good prognostic factors for the patients. Patients were classified according to the liver function, extrahepatic disease, and intrahepatic tumor burden as suggested below. Finally, patients were classified into low $(n=248)$, intermediate $(n=250)$, and high $(n=141)$ risk groups, and the median survivals were $16.4,10.3$, and 5.7 month, respectively ( $\mathrm{p}<$ 0.001). Conclusions: Radiotherapy combined with TACE is effective and safe for treating HCC with MVI and could be considered as a first-line treatment option. Proposed subclassification in the present study predicted prognosis of the study cohort accurately and would be an useful method for treatment decision-making and future studies. Keywords: Hepatocellular carcinoma, Radiotherapy, Transarterial chemoembolization, Subclassification model, Vascular invasion.

Fig. 1. (for Abstract no PE-110).

Table 1. (for Abstract no PE-110)

\begin{tabular}{|c|c|c|c|c|c|c|c|c|c|c|c|c|}
\hline \multirow{3}{*}{$\begin{array}{l}\text { Child-Pugh classification } \\
\text { Extrahepatic metastasis } \\
\text { Number of following risk factors; } \\
\text { Main/bilateral PV or IVC invasion } \\
\text { Size }>10 \mathrm{~cm} \\
\text { Infilitrative/diffuse type }\end{array}$} & \multicolumn{6}{|l|}{ A } & \multicolumn{6}{|l|}{ B } \\
\hline & \multicolumn{3}{|l|}{ No } & \multicolumn{3}{|l|}{ Yes } & \multicolumn{3}{|l|}{ No } & \multicolumn{3}{|c|}{ Yes } \\
\hline & 0 & $1-2$ & 3 & 0 & $1-2$ & 3 & 0 & $1-2$ & 3 & 0 & $1-2$ & 3 \\
\hline Number of patients & 36 & 206 & 55 & 6 & 76 & 14 & 10 & 109 & 54 & 3 & 47 & 23 \\
\hline Median survival (months) & 84.8 & 14.8 & 11 & 15.1 & 9.5 & 6.7 & 9.5 & 10.7 & 5.3 & 7.1 & 5.9 & 4.4 \\
\hline Classification & Low & Inter & liate & Low & Intermediate & High & Inte & nediate & & & High & \\
\hline
\end{tabular}




\section{PE-111 \\ Thermal Ablation Combined with Simultaneous TACE for Treatment of Hepatocellular Carcinoma $\leq 5 \mathrm{~cm}$}

Mengfei Wu ${ }^{1 *}$, Shanshan Gao ${ }^{12 *}$, Huadan Song ${ }^{1}$, Zihan Zhang ${ }^{1}$, Zhiping Yan ${ }^{1}$, Jianhua Wang ${ }^{12}$, Xiaolin Wang ${ }^{12}$, Jiemin Cheng ${ }^{1}$, Lingxiao Liu ${ }^{1}$

${ }^{1}$ Department of Interventional Radiology, Zhongshan Hospital, Fudan University, Shanghai, China; ${ }^{2}$ Shanghai Institute of Medical Imaging, Shanghai, China

Aims: To investigate efficacy of thermal ablation combined with simultaneous TACE and explore prognostic factors for survival of patients with HCC $\leq 5 \mathrm{~cm}$. Methods: From June 2010 to March 2017, a total of 377 patients (male 308 , female 69; age: range $25-85$ years, mean 61.6 years; the follow-up time: range 6.8-88.7 months, median 26 months) who underwent percutaneous thermal ablation combined with simultaneous TACE for HCC with tumors less than $5 \mathrm{~cm}$ were selected, and their clinical data were collected and retrospective analyzed. The procedure-associated mortality, major complication rate, 1,3,5-year overall survival (OS) rate and progression free survival (PFS) rate were evaluated as prognostic factors of survival. Survival results were estimated using Kaplan Meier, and multivariable analysis was carried out using Cox regression model. Results: No treatment related death. The major complications were found in 3 cases (0.8\%). The total ablation rate was $93.9 \%$. The median OS was 30.17 months (95\%CI 20.495-39.839). The OS rates in 1,3,5-years were $96.9 \%, 61.7 \%$ and $48.2 \%$ respectively. The median PFS was 9.20 months (95\%CI 7.168-11.232). The COX multivariate analysis showed that the tumor BCLC stages $(\mathrm{HR}=2.314,95 \% \mathrm{CI}=1.835-2.919, \mathrm{P}=0.000)$, Number of operations $(\mathrm{HR}=0.61695 \% \mathrm{CI}=0.440-0.862, \mathrm{P}=0.005)$, Serum AFP $(\mathrm{HR}=1.39195 \% \mathrm{CI}=1.024-1.889, \mathrm{P}=0.014$ ) were independent prognostic factors for overall survival time. Conclusions: Percutaneous thermal ablation combined with simultaneous TACE is a safe and effective treatment for

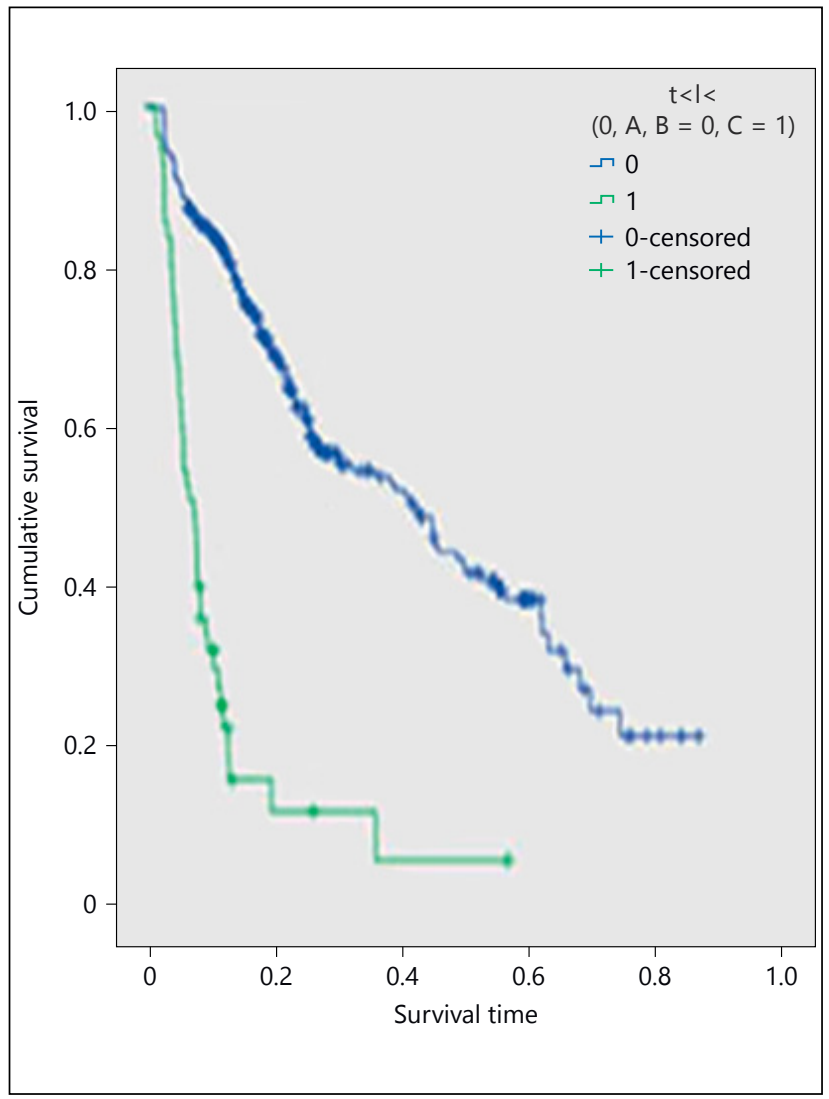

Fig. 1. (for Abstract no PE-111).

Hepatocellular carcinoma (HCC) less than $5 \mathrm{~cm}$ in diameter. Keywords: Hepatocellular carcinoma, TACE, Ablation, Overall survival.

Table 1. (for Abstract no PE-111)

\begin{tabular}{llll}
\hline Variable & Univariate & Multivariate & HR(95\%CI) \\
\hline Sex & 0.776 & & \\
Age $(\geq 65)$ & 0.746 & & \\
Liver tumor surgery & 0.832 & & \\
Bclc & 0.000 & 0.000 & $2.314(1.835-2.919)$ \\
Child-Pugh classication & 0.015 & 0.425 & $1.226(0.743-2.024)$ \\
Serum AFP $(\geq 20 \mathrm{ng} / \mathrm{mL})$ & 0.000 & 0.035 & $1.391(1.024-1.889)$ \\
Number of operations $(<2)$ & 0.017 & 0.005 & $0.616(0.440-0.862)$ \\
Tumor number $(>3)$ & 0.000 & 0.789 & $1.044(0.763-1.429)$ \\
Tumor size $(\mathrm{cm})$ & 0.002 & 0.420 & $1.135(0.835-2.919)$ \\
Serum albumin $(<35 \mathrm{~g} / \mathrm{L})$ & 0.032 & 0.160 & $1.325(0.895-1.960)$ \\
\hline
\end{tabular}




\section{PE-112 \\ Outcome of Radiotherapy for Bone Metastases from Hepatocellular Carcinoma \\ Young Joo Park, Hyun Young Woo, Jeong Heo, Mong Cho \\ Department of Internal Medicine, College of Medicine, Pusan National University, Busan, South Korea}

Aims: Recently, bone metastases from hepatocellular carcinoma (HCC) have been noted much more frequently as survival and imaging technology have been improved. The aim of current study is to investigate the outcome of patients with HCC who underwent radiotherapy for bone metastasis. Methods: 65 patients who received radiotherapy for bone metastases from HCC were retrospectively analyzed between January 2013 and March 2018 at the Pusan National University Hospital. Results: During the study period, 65 patients received 90 sessions of radiotherapy for bone metastasis from HCC. The most common treatment site was spine (46\%), followed by pelvic bone (20\%) and rib (12.2\%). The average number of radiotherapy per patient was 1.4 sessions (range, 1-4) per patients and 30 sessions (33.3\%) were performed on multiple sites in one session. The median total dose per radiotherapy was $30.0 \mathrm{~Gy}$ (range, 3-120 Gy). In 22 (24.4\%) among total session, radiotherapy was prematurely interrupted mostly due to poor general condition No severe adverse event due to radiotherapy was not observed. Pain was the most common symptom before radiotherapy, and overall rate of pain control after radiotherapy was 44 sessions (53.0\%) among 83 sessions. Overall survival rate was 10.8\% (7/65) and median survival time from the beginning of radiotherapy was 3.0 months (95\% CI, 1.75-4.24 months). In univariate analysis, the presence of concurrent metastasis other than bone and multiple session of radiotherapy was significant factor for survival ( $p=0.042, p=0.049$, respectively). In multivariate analysis, multiple session of radiotherapy was significant factor for survival $(p=0.024)$. Conclusions: Radiotherapy for bone metastasis from HCC is relatively safe and effective for pain relief. The greater number of radiotherapy reduced the risk of death. Keywords: Hepatocellular carcinoma, Bone metastases, Radiotherapy,

\section{PE-113 \\ Aggressive Extrahepatic Invasion after Percutaneous Radiofrequency Ablation and Transarterial Chemoembolization for Hepatocellular Carcinoma \\ Ying-Chin Yang \\ Department of Surgery, Buddhist Tzu Chi General \\ Hospital, Hualien, Taiwan.}

Aims: Combination of percutaneous radiofrequency ablation (pRFA) and transarterial embolisation or transarterial chemoembolisation (TAE/TACE) may reduce the risk of recurrence and progression of hepatocellular carcinoma (HCC). However, there were still some cases of aggressive recurrence. Most reports about HCC aggressive recurrence after pRFA were about intrahepatic recurrence or progression. There were few discussions about extrahepatic seeding or extrahepatic direct invasion after pRFA. Here I reported two cases of patients of post-treatment extrahepatic invasion after the combination of pRFA and TACE for early HCC. Methods: The first case was a 55-year-old man with chronic hepatitis B without cirrhosis. He had a $1.8 \mathrm{~cm} \mathrm{S8}$ tumor and a $2.5 \mathrm{~cm} \mathrm{S6}$ tumor. They were treated by pRFA initially. Multiple intrahepatic recurrences inside right hemi-liver happened within one year. After repeat pRFA plus TACE, the intrahepatic response was reasonable in CT and MRI when the tumors and also the whole right hemi-liver shrank. However, extrahepatic recurrence in pleural space and chest wall beside the shrank right hemi-liver happened and progressed in next one year. Sorafenib plus radiotherapy cannot stop such progression. A $2 \mathrm{~cm}$ left lung single metastasis had occurred when he visited my department. The second case was a 58-year-old woman with hepatitis B related cirrhosis in the status of Child-Pugh A5. She had a single $3 \mathrm{~cm}$ S2 tumor. Initial treatment was TACE plus pRFA. Intrahepatic local progression was found two months after initial treatment. Repeat TACE cannot stop tumor progression and this tumor enlarged to $7 \mathrm{~cm}$ one month after repeat TACE. This tumor had invaded her pericardium and pericardial space directly at that time. Results: Surgical resection was used as salvage treatment for both patients. The man of the first case had surgical resection of his right hemiliver with part of his diaphragm and pleura, resection of right chest wall tumor, and resection of left lung metastasis. Pathologic examination confirmed cancer invasion in the chest wall. Sorafenib was used again after these surgical operations. However, bilateral lung recurrent metastases, a right axillary metastasis, left hemi-liver multiple recurrent tumors, and multiple distant subcutaneous metastases at right chest wall happened in the following three months. They were treated by repeat surgical resections and repeat TACE for his liver tumors. He was still alive 11 months after the first surgical operation but was not disease free in lungs. The woman of the second case had an en bloc resection of her S4s/S3/S2 of liver and pericardium. She had bilateral lung metastases 6 months 
later and was treated by surgical resections again. She had Sorafenib after lung tumors resections. She was still alive 11 months after her first surgical resection and was still disease free. Conclusions: Extrahepatic invasion and progression can happen after combined pRFA and TACE for early HCC. Although such extrahepatic invasions may be focal perihepatic problems initially, distant metastases may occur soon after salvage resections. The mechanism of such aggressive local disease progression and subsequently rapid distant metastases was not known. However, seeding in the local area and also seeding in circulation during pRFA may be a key for this situation. Keywords: Aggressive recurrence, Extrahepatic recurrence, Seeding, Hepatocellular carcinoma, Radiofrequency ablation, Transarterial embolization, Extrahepatic invasion.

\section{PE-114 \\ Percutaneous Cryoablation in Early Stage Hepatocellular Carcinoma: Analysis of Local Tumor Progression Factors}

\section{Jong Yun Won, Dong Kyu Kim, Kichang Han, Gyoung Min Kim, Joon Ho Kwon, Man-Deuk Kim, Do Yun Lee \\ Department of Radiology, Severance Hospital, Research Institute of Radiological Science, Yonsei University College of Medicine}

\begin{abstract}
Aims: To assess the efficacy and safety of percutaneous cryoablation for early or very early stage hepatocellular carcinoma and evaluate riskfactors for local tumor progression after percutaneous cryoablation. Methods: In this retrospective study, 34 consecutive treatment-naïve patients were treated with percutaneous cryoablation for three or fewer nodules smaller than $3 \mathrm{~cm}$ in size. The safety of percutaneous cryoablation was evaluated by assessing percutaneous cryoablation-related complications and comparing hepatic function before and after percutaneous cryoablation. The efficacy was evaluated by calculating technical success, local tumor progression rates, and disease progression rates. Furthermore, prognostic factors of percutaneous cryoablation for local tumor progression were analyzed. Results: The technical success rate was $100 \%$, and complete response was achieved in all patients (100\%) by 1 month after percutaneous cryoablation. During a mean $24.5 \pm 12$.0-month followup interval, the incidences of local tumor and disease progression were $11.8 \%$ and $38.2 \%$, respectively. The respective cumulative local tumor progression-free and disease-free survival rates were $93.1 \%$ and $83.6 \%$ at 1 year and $84.0 \%$ and $60.7 \%$ at 2 years. Hepatic function was normalized within 3 months after percutaneous cryoablation. No major complications occurred other than one case of small hematoma. On multivariate analysis, a minimal ablative margin $<5 \mathrm{~mm}$ was a significant risk factor associated with
\end{abstract}

local tumor progression. Conclusions: Percutaneous cryoablation is an effective and safe therapy for patients with early or very early stage hepatocellular carcinoma. A minimal ablative margin $<5 \mathrm{~mm}$ was a significant prognostic factor for local tumor progression. Keywords: Hepatocellular carcinoma, Cryoablation, Percutaneous.

\section{PE-115 \\ Risk Factor of the Recurrence for Hepatocellular Carcinoma after Intra-Operative Radiofrequency Ablation \\ Myung Hee Kim ${ }^{1}$, Hyuk Soo Eun ${ }^{1,2}$, Min Seong Kim ${ }^{1}$, Min Kyung Baek ${ }^{1}$, Deok Yeong Kim ${ }^{1}$, Jae Ho Park ${ }^{1,2}$, Woo Sun Rou 1,2, Jong Seok Joo 1,2, Eaum Seok Lee ${ }^{1,2}$, Seok Hwan Kim 2,3, In Sang Song 2,3, Byung Seok Lee ${ }^{1,2}$, Kwangsik Chun ${ }^{2,3}$, Seok Hyun Kim ${ }^{1,2}$ \\ ${ }^{1}$ Division of Hepatology, Department of Internal Medicine, Chungnam National University Hospital, ${ }^{2}$ Department of Internal Medicine, Chungnam National University, College of Medicine, ${ }^{3}$ Department of Surgery, Chungnam National University Hospital}

Aims: Despite the highly complete tumor necrosis rate of radiofrequency ablation (RFA), tumor recurrence, either locoregional or newly developing lesion, was regarded as significant issue. Recurrence reduction through appropriate procedure selection as well as patients' selection could improve survival of patients. Therefore, we analyzed the conditional factor for recurrence after intra-operative RFA treatment on hepatocellular carcinoma (HCC). Methods: We investigated 98 patients who were treated with intra-operative RFA as initial treatment for HCC. The mean follow-up period was $33 \pm 14.5$ months. We evaluated the disease-free survival of recurred patients, including local tumor progression (LTP) and intrahepatic distant recurrence (IDR). For these patients, multiple factors were assessed to their significance for recurrence and survival. Results: Almost baseline characteristics were not shown statistically significant difference, except for few factors between the groups. The incidence of overall recurrence was $47.9 \%$. LTP was found in 20 of 47 patients (20.4\%) and occurred 4-33 months [median period: 14 months] after RFA. IDR was found in 27 of 47 patients (27.5\%) and occurred 6-88 months [median period: 22 months] after RFA. Interestingly, on multivariate analysis for whole recurred patients, serum $\alpha$-fetoprotein (AFP) was significantly associated factor with recurrence of the tumor $[p=0.006$, 95\% C.I.: 1.001-1.007, HR = 1.004]. In addition, INR and Child-Pugh scores, factors representing severity of underlying liver disease status, were significantly associated with survival of the patients $(\mathrm{p}=0.039$ and $\mathrm{p}=0.046)$. Especially, AFP was also significantly associated factor with patients' survival. $[\mathrm{p}=0.016$, 95\% C.I.: 1.001-1.006, HR = 1.004]. After 
subgroup analysis for recurred patients, we found that patients with higher AFP levels had more recurrence patterns of LTP rather than IDR. Conclusions: Patients with high AFP level received intraoperative RFA for HCC should be carefully followed-up and considered more active radical treatment modality because of higher risk of recurrence and mortality. Keywords: Recurrence, Intra-operative radiofrequency ablation, Alpha-fetoprotein, Local tumor progression, Survival.

\section{PE-116 \\ A Model to Predict Outcome of Transarterial Chemoembolization in Patients with Treatment-Naïve Hepatocellular Carcinoma}

Ya-Wen Hung ${ }^{1}$, Chen-Ta Chi ${ }^{1,2}$, Rheun-Chuan Lee ${ }^{3}$, Ming-Chih Hou', Yi-Hsiang Huang 1,2,4*

${ }^{1}$ Department of Medicine, Taipei Veterans General Hospital, Taipei, Taiwan; ${ }^{2}$ Division of Gastroenterology and Hepatology, Department of Medicine, Taipei Veterans General Hospital, Taipei, Taiwan.; ${ }^{3}$ Department of Radiology, Taipei Veterans General Hospital, Taipei, Taiwan; ${ }^{4}$ Institute of Clinical Medicine, National Yang-Ming University, Taipei, Taiwan.

Aims: Transarterial chemoembolization (TACE) is a standard treatment for intermediate stage hepatocellular carcinoma (HCC), but the outcome varied. Factors associated with survival should be determined. The study aims to stablish a model to predict the outcome of TACE in treatmentnaïve HCC patients. Methods: Consecutive 982 treatmentnaïve patients undergoing TACE as the initial treatment for HCC from 2007 to 2016 in Taipei Veterans General Hospital were retrospective reviewed. Detailed tumor characteristics and liver function were carefully recorded. Factors associated with survival were analyzed. Results: The mean age was 67.6 years old with male predominant $(75.6 \%)$. The distribution of tumor stages were 190 (19.3\%) in BCLC A, $570(58 \%)$ in BCLC B, and 222 (22.6\%) in BCLC C/D. A total of 2,759 TACE were performed for 982 patients. In the multivariate analysis, ALBI grade, APRI, TACE after 2012, AFP level, tumor size, tumor number, post-TACE surgery or transplantation, serum creatinine, ECOG status, BCLC stage were significantly associated with overall survival. Of the 570 BCLC B cases, ALBI grade, AFP $\leq 200 \mathrm{ng} / \mathrm{ml}$, tumor size $£ 10 \mathrm{~cm}$ were significant factors associated with survival. An ALBI-TA embolization (ALBI-TAE) model based on tumor size $(>10 \mathrm{~cm}$ or not), AFP ( $>200 \mathrm{ng} / \mathrm{ml}$ or not), and ALBI grade was established to classify TACE patients into 4 subgroups (0,1, 2 and 3$)$. The performance of ALBI-TAE was superior to Hepatoma arterial-embolization prognostic (HAP) score with a higher homogeneity and a lower AICc value (4049 for ALBI-TAE vs 4060 for HAP score). Conclusions: ALBI grade is an important factor associated with survival in patients undergoing TACE. ALBI-TAE can be applied to select patients who can get the benefit from TACE. Keywords: HCC, TACE, ALBI grade.

\section{PE-117 \\ Hypofractionated Proton Beam Therapy for Hepatocellular Carcinoma \\ Tae Hyun Kim ${ }^{1,2}$, Joong-Won Park ${ }^{1}$, Bo Hyun Kim', Dae Yong Kim², Sung Ho Moon', Sang Soo Kim², Ju Hee Lee ${ }^{1}$, Sang Myung Woo', Young-Hwan Koh', Woo Jin Lee ${ }^{1}$, Chang-Min Kim ${ }^{1}$ \\ ${ }^{1}$ Center for Liver Cancer, Research Institute and Hospital, National Cancer Center, ${ }^{2}$ Center for Liver Cancer, Research Institute and Hospital, National Cancer Center}

Aims: Technological innovations in the field of radiotherapy (RT), such as three-dimensional conformal RT, intensity-modulated RT, stereotactic body RT, and proton beam therapy (PBT), could potentially deliver radiation more precisely to tumors while sparing the normal tissues. We retrospectively analyzed the clinical outcomes of hypofractionated PBT in inoperable or recurrent hepatocellular carcinoma (HCC) patients to evaluate its clinical effectiveness. Methods: Between May 2013 and February 2015, 71 inoperable or recurrent HCC patients who had received hypofractionated PBT using 66 GyE in 10 fractions were included in this study. HCC was diagnosed by pathologic confirmation $(n=17)$ or on the basis of radiologic findings plus serum alpha-fetoprotein (AFP) concentrations $\geq 200 \mathrm{ng} / \mathrm{mL}$ ( $\mathrm{n}=54$ ), in accordance with the guidelines of the Korean Liver Cancer Study Group and the National Cancer Center The tumor responses were defined as the maximal tumor response observed during the follow-up period using the modified Response Evaluation Criteria in Solid Tumors criteria. Results: At time of analysis, 49 of 71 (69\%) patients developed disease recurrence, including 6 (8.5\%) with local progression, 49 (69\%) with intrahepatic recurrence, and 11 (15.5\%) with distant metastases of all patients, 66 patients (93\%) eventually reached complete response (CR) after PBT: 93.9\% (62 of 66) of patients who reached $\mathrm{CR}$ within 12 months, and the remaining 4 patients $(6.1 \%)$ reached $C R$ at $12.5,16.2,19.1$ and 21.7 months, respectively. The three-year local progressionfree survival (LPFS), relapse-free survival (RFS) and OS rates were $89.9 \%, 26.8 \%$, and $74.4 \%$, respectively. Multivariate analysis revealed that the tumor response was an independent prognostic factor for LPFS, RFS, and OS. Overall, treatment was well tolerated with no grade toxicity $\geq 3$. Conclusions: Hypofractionated PBT could be one of good alternatives for HCC patients who are unsuitable for surgical or invasive treatments with curative intent. Keywords: Hepatocellular Carcinoma, Proton Beam Therapy, Tumor response, 


\section{PE-118}

Stereotactic Body Radiotherapy versus Transarterial Chemoembolization for Medium-Sized Hepatocellular Carcinoma: A Propensity Score Matching Analysis

Wen-Yen Huang, Po-Chien Shen, Yang-Hong Dai, Jen-Fu Yang, Cheng-Hsiang Lo

Department of Radiation Oncology, Tri-Service General Hospital, National Defense Medical Center, Taiwan

Aims: Because the data that guide selection of optimal therapy for the unresectable medium-sized (3-8 cm) hepatocellular carcinoma (HCC) are lacking, we aim to compare stereotactic body radiotherapy (SBRT) with transarterial chemoembolization (TACE) using a propensity score matching analysis. Methods: We enrolled patients who were diagnosed with HCC, with tumors sized 3-8 cm, and were treated with SBRT or TACE. The endpoints of the study were overall survival (OS) and infield failure-free survival (IFFS). They were compared using 1:2 propensity score matching analysis based on sex, age, types of hepatitis, tumor size, AJCC stage, BCLC stage, Child-Pugh classification, ECOG performance status, and new diagnosis/recurrence. Kaplan-Meier method and Cox regression analyses were used to evaluate the outcome and prognostic factors. Results: After matching, there were 44 patients in the SBRT group and 88 patients in the TACE group. No statistically different parameters existed between the two groups. The 1, 2-year IFFS rates were $66.0 \%$, $53.5 \%$ in the SBRT group and $40.4 \%, 20.1 \%$ in the TACE groups $(\mathrm{p}=0.001)$. The 1, 2-year OS rates were $76.6 \%, 73.2 \%$ in the SBRT and $52.9 \%, 24.5 \%$ in the TACE groups ( $<<0.001$, fig. 1). In multivariate analysis, tumor size (hazard ratio $[\mathrm{HR}]=$ $1.24, p=0.013)$, SBRT vs TACE $(H R=0.32, p<0.001)$, ECOG

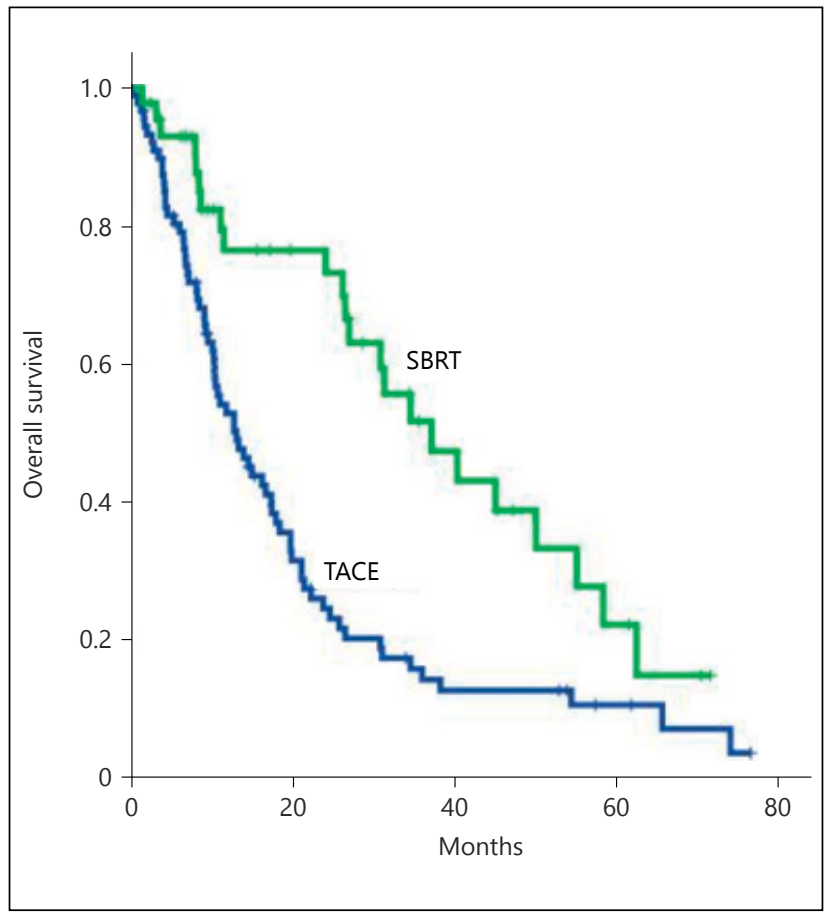

Fig. 1. (for Abstract no PE-118).

$(\mathrm{HR}=2.93, \mathrm{p}=0.006)$, Child B vs A $(\mathrm{HR}=2.16, \mathrm{p}=0.010)$, and recurrence vs new diagnosis ( $\mathrm{HR}=1.67, \mathrm{p}=0.026)$ were associated with OS. Conclusions: For 3-8 cm HCC, SBRT generates superior IFFS and OS when compared with TACE. Further prospective randomized trials to compare SBRT with TACE were warranted. Keywords: Stereotactic body radiotherapy, Transarterial chemoembolization, Hepatocellular carcinoma, Radiation therapy. 


\section{Liver Cancer}

Abdel-Kareem, M. 130

Abdul Fattah, A. 90

Abodeya, S. 92

Abou-Alfa, G. 39

Abou-Alfa, G.K. 107, 169, 171

Adilsaikhan, M. 172

Aggan, H.E. 85

Ahn, S.B. 150

Ahn, S.H. 90, 100, 115, 121, 131, 159, 204

Ahn, Y.-S. 145

Aikata, H. 108

Akimoto, T. 46

Akl Rady, M. 130

Albani, S. 66

Allam, M.H. 91

Allazarov, U.A. 132, 136

An, C. 95

Arai, Y. 108

Araki, K. 120

Ariunaa, K. 160, 172

Armstrong, J. 171

Asai, A. 205

Assenat, E. 169

Azzalarov, U.A. 129, 130, 131, 168

Baakili, A. 103

Baatarkhuu, 0. 160, 172

Bae, S.H. 103, 123, 155, 156, 164, 182, 197, 206, 207

Bae, S.-H. 109

Baek, M.K. 212

Baik, K.W. 208

Batzaya, M. 160

Bayh, I. 110, 170

Bhargava, S. 165

Bhargava, V. 165

Blanc, J.-F. 107

Boisserie, F. 168

Bolormaa, Y. 172

Bonney, G.K. 121

Borgman-Hagey, A.E. 107

Byeon, J.H. 208

Byun, H.K. 195

Byun, K.S. 95

Cha, J.H. 123

Cha, Y.H. 75

Chan, A. 59

Chan, A.C. 124

\begin{tabular}{l|l}
\hline Liver Cancer 2018;7(suppl 1):1-220 \\
\hline DOI 10.1159/000490877 & $\begin{array}{l}\text { ○ 2018 S. Karger AG, Basel } \\
\text { www.karger.com/lic }\end{array}$ \\
\hline
\end{tabular}

The 9th Asia-Pacific Primary Liver Cancer Expert Meeting (APPLE 2018)

\section{Author Index}

Chan, L.-K. 87, 97

Chan, S.L. 107, 171

Chang, C.-S. 161

Chang, U.I. 164

Chee, E. 151

Chee, W. 151

Chen, C.-W. 101

Chen, K.-H. 181

Chen, M.-F. 111

Chen, P.-D. 128

Chen, R. 96

Chen, R.X. 195

Chen, T.-H. 174, 179, 182

Chen, T.H. 176, 181, 189, 192

Chen, T.-L. 96, 167

Chen, Y. 195

Cheng, A.-L. 8, 38, 101, 107, 110, 169, 170

Cheng, J. 33, 210

Cheng, L. 97

Cheon, G.J. 199

Cheung, S.Y.S. 118

Cheung, T.-T. 87, 97

Cheung, T.T. 124

Chew, V. 66

Chi, C.-T. 213

Chiu, E.Y.-T. 87

Cho, E. 151, 166

Cho, E.J. 100

Cho, J.-H. 183, 203

Cho, K. 125, 152, 154

Cho, K.J. 121

Cho, M. 211

Cho, S.-B. 109

Cho, S.B. 134,166

Cho, S.W. 123

Cho, Y. 198

Cho, Y.K. 150

Cho, Y.Y. 103

Chock, K. 58

Choe, W.H. 129

Choi, B.G. 204

Choi, E.J. 75

Choi, G. 56, 140

Choi, G.H. 57, 157

Choi, G.-S. 112, 186, 198

Choi, H. 145

Choi, H.J. 194

Choi, J. 140, 150

Choi, J.S. 157
Choi, J.W. 75

Choi, J.-Y. 95

Choi, J.Y. 123, 155, 156, 164, 182, 206, 207

Choi, M.S. 116, 149, 186, 198

Choi, S.-B. 179

Choi, S.B. 194

Choi, S.H. 128

Choi, S.K. 134, 166

Choi, S.W. 164

Choi, Y. 150

Choi, Y.M. 197

Choi, Y.-S. 189

Chok, K.S. 124

Chon, H.Y. 90

Chon, Y.E. 165

Chong, C.C.N. 118

Choo, S.-P. 103

Choo, S.P. 109

Chopra, A. 103

Chou, Y.-C. 167

Chow, F.C. 124

Chow, P.K.H. 66, 156

Chow, P.K.-H. 172

Chu, C.C.M. 118

Chua, C. 66

Chun, H. 27

Chun, H.J. 156, 204, 206, 207

Chun, J.M. 86

Chun, K. 212

Chung, A. 66

Chung, C.-S. 181

Chung, W.J. 109

Cicin, I. 107

Crocenzi, T.S. 103

Cubillo, A. 169

Dai, W.C. 124

Dai, Y.-H. 143, 214

Dan, Y.Y. 121

Darilay, A. 171

Decaens, T. 170

dela Cruz, C. 103

Doh, Y.S. 103

Ducreux, M. 168, 169, 170

Ebata, T. 63

El Aggan, H. 92

El-Fayoumi, M. 130

El-Khoueiry, A.B. 103, 107 
Elsabaawy, M.M. 91

EL-Sakhawy, M. 130

Eshmurodov, S.A. 132, 133, 134

Eun, H.S. 212

Fan, J. 114

Feng, H. 100

Filarca, R.L.F. 66

Finn, R.S. 168, 170, 173

Fong, A.K.W. 118

Frenette, C. 173

Fu, Y.-P. 87, 101, 126, 152

Fukunishi, S. 205

Fung, A.K.Y. 118

Furuse, J. 108, 171

Galle, P.R. 7, 30, 170, 171

Gan, W. 101, 152, 161

Gandhi, M. 156, 172

Gang, Y. 195

Gao, L. 101

Gao, S. 210

Gao, Y. 100, 114

Ge, N. 195

Ghweil, A. 90

Gilliam, Y.M. 156

Goh, B.K.P. 66

Granito, A. 173

Greten, T. 43, 73

Gu, W.-M. 174, 179, 182

Gu, W.M. 176, 181, 189, 192

Gwak, G.-Y. 116, 149, 186, 198

Ha, H.T. 86

Ha, S.Y. 93

Hamed, E. 133

Han, D. 140

Han, D.H. 157

Han, H.J. 194

Han, J. 142, 145, 174, 176, 179, 181, $183,189,192$

Han, J.H. 194

Han, K. 212

Han, K.-H. 82, 90, 95, 100, 121, 125, $128,131,152,154,159,195,204$

Han, K.H. 115

Han, M.-H. 86

Han, N.I. 164

Han, Y.S. 86

Han, Z. 207

Han, Z.Y. 207

Hashimoto, E. 52

Hassan, A. 205

Hassona, E. 85

Hazirah, S.N. 66

He, A.R. 171

\begin{tabular}{l|l}
\hline Liver Cancer 2018;7(suppl 1):1-220 \\
\hline DOI 10.1159/000490877 & $\begin{array}{l}\text { @ 2018 S. Karger AG, Basel } \\
\text { www.karger.com/lic }\end{array}$ \\
\hline
\end{tabular}

The 9th Asia-Pacific Primary Liver Cancer Expert Meeting (APPLE 2018)

Helal, A. 91

Helmy, M. 85

Heo, J. 110, 170, 211

Hernandez, S. 171

Hessel, C. 107

Higuchi, K. 205

Hino, K. 108

Ho, D.W.-H. 87, 97

Ho, M.-C. 136

Hong, H.P. 202

Hong, J. 134

Hong, S.K. 183, 203

Hong, S.-Y. 122, 174

Hong, S.Y. 140

Hong, T.H. 194

Hou, J. 168, 169

Hou, M.-C. 213

Hsu, C. 65, 101, 103

Hu, X.-G. 122, 140, 174

Hu, Y. 151

Huang, C.-C. 181

Huang, C.-T. 181

Huang, J. 161

Huang, J.-L. 101, 152

Huang, L. 100

Huang, M.-C. 128

Huang, Q.D. 121

Huang, W.-Y. 143, 214

Huang, Y.-H. 213

Hui, K.M. 104

Hung, Y.-W. 213

Hur, K. 86

Hur, W. 123

Hwang, J.A. 93

Hwang, S. 37, 156, 164

Hwang, S.G. 165

Hyun, D. 198

Iizuka, K. 171

Ikeda, M. 108, 170, 173

Ikejima, K. 54

Im, E.-O. 151

Im, Y.R. 195

Inohara, A. 151

Ismailov, M.U. 129, 130, 131, 133, 168

Ismatov, A.A. 129, 130, 131, 133, 134, 168

Isoda, N. 108

Iyer, S.G. 121

Izumi, N. 44, 108

Jang, E.S. 103

Jang, H.S. 155, 197

Jang, J.W. 18, 123, 155, 156, 164, 182, 206, 207, 208

Jang, J.Y. 199
Jang, S.Y. 86

Jayant, K. 113

Jeng, K.-S. 181

Jeng, Y.-M. 136

Jeon, H.J. 165

Jeon, M.Y. 90, 100, 159

Jeong, B. 86

Jeong, J.H. 150

Jeong, J.Y. 150

Jeong, S.W. 150, 199

Jeong, Y.S. 85

Ji, J.-S. 116

Jia, F. 156

Jiang, H. 96

Jin, B. 128

Jin, Q. 139

Jin, Y.-J. 151, 159

Jing, C.-Y. 87, 126, 152

Joh, J.-W. 112, 186

Jon, M. 149

Joo, J.H. 209

Joo, J.S. 212

Joo, Y. 75

Jun, B.G. 199

Jun, C.H. 134, 166

Jun, D.W. 150

Jung, E. 142

Jung, J. 208, 209

Jung, Y.B. 157

Jung, Y.C. 134

Jung, Y.J. 112

Jung, Y.K. 95, 109

Kakizaki, S. 120

Kam, C.S. 87

Kamachi, H. 176

Kamiyama, T. 176

Kang, G.-E. 86

Kang, M. 75

Kang, S. 167

Kang, T.W. 23, 198

Kang, W. 103, 116, 149, 167, 186, 198

Kang, Y.-K. 103

Kang, J. 173

Kassem, A. 90, 133, 205

Katoh, H. 120

Kelley, R.K. 107, 171

Kew, G.S. 121

Khalaf, S. 133

Kim, B.-H. 149

Kim, B.H. 213

Kim, B.I. 202

Kim, B.K. 90, 100, 115, 121, 131, 152, $154,159,204$

Kim, B.-W. 122, 140, 174

Kim, C.-M. 213 
Kim, C.W. 164, 202

Kim, C.Y. 197

Kim, D.G. 156, 194

Kim, D.H. 134, 166

Kim, D.K. 212

Kim, D.-S. 22, 194

Kim, D.S. 115

Kim, D.Y. 1, 90, 100, 103, 115, 121, $125,128,131,152,154,157,159$, $166,195,204,212,213$

Kim, D.-Y. 95

Kim, G. 86

Kim, G.M. 35, 212

Kim, H. 75, 150

Kim, H.J. 195

Kim, H.S. 75, 150, 159, 199

Kim, H.Y. 164, 202

Kim, I.-G. 122, 140

Kim, I.H. 103

Kim, J. 116, 149

Kim, J.H. 95, 129

Kim, J.-H. 123, 142

Kim, J.H. 173, 197, 209

Kim, J.M. 112, 186, 198

Kim, J.S. 142

Kim, J.-W. 55, 197

Kim, J.-Y. 167

Kim, K. 161

Kim, K.M. 103

Kim, K.-S. 112

Kim, K.S. 157

Kim, M.-D. 212

Kim, M.H. 212

Kim, M.-J. 95

Kim, M.S. 212

Kim, S. 202

Kim, S.E. 150

Kim, S.G. 199

Kim, S.H. 93, 158, 204, 212

Kim, S.J. 112

Kim, S.S. 213

Kim, S.U. 90, 100, 115, 121, 131, 159, 204

Kim, T.H. 95, 160, 197, 213

Kim, T.-Y. 45, 170, 173

Kim, W. 75, 103

Kim, W.-B. 179, 194

Kim, W.-J. 179

Kim, Y. 194

Kim, Y.D. 199

Kim, Y.-H. 75

Kim, Y.J. 34, 100, 103, 166, 209

Kim, Y.-S. 75

Kim, Y.S. 109, 199

Kim, K. 140

Kim, S.U. 154
Liver Cancer 2018;7(suppl 1):1-220

(c) 2018 S. Karger AG, Basel www.karger.com/lic

The 9th Asia-Pacific Primary Liver Cancer Expert Meeting (APPLE 2018)

Kimura, T. 25

Klümpen, H.-J. 107

Koh, K.C. 116, 149, 186, 198

Koh, Y.-H. 213

Koh, Y.-I. 166

Kohla, M. 91, 130

Kokudo, N. 108

Korea Central Cancer Registry 167

Korean Liver Cancer Study group 167

Kow, W.C.A. 121

Koyama, Y. 120

Kubo, A. 91

Kudo, M. 14, 29, 40, 91, 103, 108, 110, $168,170,173$

Kurbankulov, U.M. 129, 130, 131, 168

Kuzuya, T. 108

Kwak, B.J. 194

Kwan, A. 171

Kweon, Y.-0. 86

Kwon, C.H.D. 112

Kwon, J.H. 19, 155, 164, 208, 212

Kwon, S.Y. 129

Kyaw, A.M.M. 175

Lai, L. 66

Lai, P.B.S. 118

Lashen, S. 85

Lau, W.Y. 112, 145, 176, 183, 189, 192

Le, F. 100

Lee, B.S. 212

Lee, C.-W. 111

Lee, D.S. 197

Lee, D.Y. 212

Lee, E. 97

Lee, E.-S. 75

Lee, E.S. 212

Lee, E.W. 24

Lee, E.Y. 112

Lee, G.H. 121

Lee, H.A. 95

Lee, H.C. 110, 170

Lee, H.G. 204

Lee, H.L. 164

Lee, H.W. 90, 103, 121, 128, 131, 152

Lee, I.J. 197, 198

Lee, J. 75, 112, 165, 167, 198

Lee, J.E. 93

Lee, J.-H. 16, 100

Lee, J.H. 116, 149, 186, 198, 213

Lee, J.M. 13, 150

Lee, J.-M. 183, 203

Lee, J.-S. 85

Lee, J.S. 149, 204

Lee, J.-W. 151, 159

Lee, J.Y. 208

Lee, K.F. 118
Lee, K.-H. 171, 173

Lee, K.-W. 32, 150, 183, 189, 203

Lee, M.-A. 173

Lee, M.G. 75

Lee, M.S. 171

Lee, M.W. 198

Lee, R.-C. 213

Lee, S. 75, 93, 166, 198

Lee, S.H. 199

Lee, S.J. 151

Lee, S.K. 158, 182

Lee, S.L. 202

Lee, S.-W. 161

Lee, S.W. 164, 208

Lee, S.Y. 197

Lee, T.K.-W. 97

Lee, T.-Y. 161

Lee, W.-C. 111

Lee, W.G. 86

Lee, W.J. 213

Lee, Y.B. 165

Lee, Y.H. 66

Lee, Y.-H. 136

Lee, Y.J. 160

Lee, Y.M. 121

Lee, Y.R. 86, 160

Lee, Y.-S. 95

Lei, J. 93

Li, C. $112,145,168,169,176,179,183$, 189

Li, J. 156

Li, J.-D. 174,179

Li, J.D. 176

Li, L. 156, 195

Li, M. 100, 195

Li, Y. 104

Li, Z.L. $145,176,183,189$

Li, Z.-L. 174

Liang, L. 112, 176, 181, 192

Liang, P.-C. 136

Liao, C.-C. 96, 167

Lim, B.L.K. 121

Lim, C.J. 66

Lim, H.Y. 166, 173

Lim, K. 21

Lim, S.G. 121

Lim, T.K.H. 66

Lim, T.S. 90, 166

Lim, T.-W. 179

Lim, Y.-S. 11

Lin, C.-C. 181

Lin, C.-H. 203

Lin, C.-K. 181

Lin, K.-L. 203

Lin, L.-C. 203

Lin, S.-M. 110, 170 
Lin, Y.-Y. 101

Lin, Y.-W. 203

Ling, J.Z.J. 156

Liu, B. 171

Liu, J. 170

Liu, L. 210

Liu, M. 139

Liu, X. 139

Lo, C.-H. 214

Lo, C.M. 124

Lo, I.L.O. 87

Lo, R.H.G. 66

Lok, H.T. 118

Low, H.C. 121

Lu, H. 96

Lukmonov, S.N. 129, 130, 131, 132, $133,134,135,136,168$

Ma, H. 96

Macarulla, T.M. 168

Madatov, K.A. 129, 130, 131, 132, 136, 168

Madhavan, K. 121

Mahmoud, S. 92

Mamatkulov, K.A. 135

Matsui, 0. 83

Matsunaka, T. 91

Melero, I. 103

Merle, P. 107, 173

Meyer, T. $103,107,168$

Min, Y.-S. 145

Moon, H. 125, 152, 154

Moon, S.H. 213

Moon, S.W. 155

Morita, M. 91

Moustafa, E. 90

Muthiah, M. 121

Myint, S. 175

Nagatsu, A. 176

Nakajima, K. 110, 170

Nakamura, K. 205

Nakano, T. 120

Nam, S.W. 72, 155, 164, 208

Nam, T.-K. 197

Naser, A. 133

Nasir, N.J.M. 66

Neely, J. 103

Negro, A. 171

Ng, D. 66

Ng, I.O.-L. 4, 76, 78, 87, 97

Ng, K.C. 124

Ng, R.X. 109

Ni, C.-F. 116, 199

Niu, J. 139

Noda, T. 91

\begin{tabular}{l|l}
\hline \multicolumn{2}{l|}{ Liver Cancer 2018;7(suppl 1):1-220 } \\
\hline DOI 10.1159/000490877 & $\begin{array}{l}\text { @ 2018 S. Karger AG, Basel } \\
\text { www.karger.com/lic }\end{array}$ \\
\hline
\end{tabular}

The 9th Asia-Pacific Primary Liver Cancer Expert Meeting (APPLE 2018)

Nojiri, S. 108

Ogawa, C. 91

Oh, C.H. 149

Oh, I.-H. 149

Oh, J.H. 186

Oh, J.S. 156, 204, 206, 207

Oh, J.W. 112

Ohama, H. 205

Ohata, T. 176

Ohno, T. 120

Okamoto, M. 120

Okita, K. 108

Okusaka, T. 108

Omura, A. 91

Orimo, T. 176

Ou, D.-L. 101

Ozgurdal, K. 173

Paik, S. 75

Paik, S.W. 116, 149, 186, 198

Paik, Y.-H. 67, 103, 116, 149, 167, 186, 198

Pan, L. 66

Pang, Y.H. 121

Park, H. 151, 165

Park, H.C. 197

Park, J. 142

Park, J.G. 86

Park, J.H. 212

Park, J.-W. 9, 107, 166, 213

Park, J.Y. 90, 100, 109, 115, 121, 128, $131,159,204$

Park, K. 202

Park, N.R. 123

Park, P.-J. 179

Park, S. 198

Park, S.C. 103

Park, S.Y. 86

Park, W.-Y. 71

Park, Y.J. 211

Park, Y.N. 157

Peck-Radosavljevic, M. 110, 170

Petrosov, S.N. 133

Pluntke, S. 169

Qin, S. 156, 168, 170, 171

Qing, S.L.Z. 109

Qiu, S. 161

Qiu, S.-J. 87, 101, 126, 152

Quan, B. 145, 182, 189

Que, J. 203

Rady, M.A. 91

Raoul, J.-L. 110, 170

Reccia, I. 113
Ren, Z. 96, 169

Ren, Z.-G. 195

Rhee, H. 95

Rho, S. 140

Rhu, J. 112

Rim, C.H. 167, 197

Rim, K.S. 165

Rimassa, L. 107, 169

Ro, S.W. 125, 152, 154

Roberts, L.R. 6

Roshdy, A. 133

Ross, P.J. 169

Rou, W.S. 212

Ryoo, B.-Y. 41, 107, 166, 171, 173

Salah-Eldein, E. 90

Salakhidinov, M.M. 132, 133, 134

Sanduijav, R. 160, 172

Sangro, B. 5, 103, 171

Schwab, G. 107

Schwartz, M.E. 176

Seo, S. 86

Seo, Y.S. 95, 160

Seong, J. 77, 161, 195, 197

Sham, P.-C. 97

Shan, H. 110, 170

Shao, J. 93

Shen, F. $112,145,174,176,179,181$, 182, 183, 189, 192

Shen, K. 104

Shen, P.-C. 214

Shen, X. 122

Shen, X.-Y. 174

Shen, Y. 104

Sherman, M. 10

Shiba, S. 120

Shibatoge, M. 91

Shibuya, K. 120

Shim, D.J. 208

Shim, J.-J. 85, 149, 150

Shim, S.Y. 85

Shimada, S. 176

Shin, E.-C. 42

Shin, J. 75

Shin, J.-H. 85

Shin, S. 152, 154

Shin, S.Y. 121

Shirabe, K. 120

Sim, D.W. 166

Sinn, D.-H. 116

Sinn, D.H. 149, 186, 198

Soe, K. 175

Sohn, B.H. 85

Song, D.S. 155, 164

Song, H. 210

Song, I.S. 212 


\begin{tabular}{l|l}
\hline DOI 10.1159/000490877 & $\begin{array}{l}\text { @ 2018 S. Karger AG, Basel } \\
\text { www.karger.com/lic }\end{array}$ \\
\hline
\end{tabular}

The 9th Asia-Pacific Primary Liver Cancer Expert Meeting (APPLE 2018)

Song, J. 168

Song, J.J. 75

Song, M. 75

Song, M.J. 158

Song, T.-J. 194

Soon, G. 121

Stein, S. 171

Suh, K.-S. 79, 150, 183, 203

Suh, S.J. 95, 109

Suh, S.W. 150

Suh, S.-W. 189

Sujishi, T. 205

Sun, L.Y. 145, 189

Sun, L.-Y. 174,179

Sun, X. 100

Sung, P.S. $103,123,155,156,164,182$, 206, 207

Sze, K.M.-F. 97

TACTICS study group 108

Tak, W.Y. 86

Taketomi, A. 176

Tamaki, H. 91

Tan, C.-T. 101

Tan, P.S. 121

Tan, P.-S. 156

Tanabe, N. 108

Tang, C.N. 87

Tang, V.W.L. 87

Tay, W.R.B. 121

Teng, G.-J. 116, 199

Tomasello, G. 168

Torimura, T. 108

Toshnazarov, J. 132

Toshnazarov, J.F. 135

Trojan, J. 103

Tsai, H.-I. 111

Tsuchimoto, Y. 205

Tsuda, Y. 205

Tsui, S.K.-W. 97

Tsui, Y.-M. 97

Tsumura, H. 108

Tuyatsetseg, A. 172

Ueshima, K. 108

Um, S.H. 95, 160

Usmonov, 0.0. 129, 130, 131, 133, 134, 168

Verret, W. 170, 171

Vlahovic, G. 171

Vogel, A. 168

Wakayama, K. 176

Wang, H. 174, 176, 179, 181, 182, 189, 192
Wang, H.-J. 122, 140, 174

Wang, J. 210

Wang, J.H. 145

Wang, J.-H. 182

Wang, K. 93

Wang, M.D. 145, 176, 183, 189

Wang, M.-D. 174, 182

Wang, X. 210

wang, Y. 93

Wang, Y.-H. 195

Wang, Z. 182, 189

Wee, A. 121

Welling, T.H. 103

Wen, X. 139

Won, J.Y. 212

Won, Y.D. 202

Wong, C.-M. 87

Wong, J. 118

Wong, T.C. 124

Wong, V. 51

Woo, H.Y. 211

Woo, S.M. 213

Wu, A.R. 97

Wu, C.-H. 136

Wu, H. 145, 176, 179, 183, 189

Wu, J. 169

Wu, L. 93

Wu, M. 210

Wu, M.C. $112,145,176,189$

Wu, M.-C. 179, 183

Wu, R. 139

Wu, Y.-M. 128

Wyrwicz, L. 169

Xia, H. 104

Xie, L. 96

Xie, X. 96

Xing, H. 112, 145, 174, 176, 179, 183, 189

Xu, D.-Z. 170

Xu, X.-F. 174, 179, 182

Xu, X.F. 176

Xu, Y. 171

Xue, T. 195

Yamasaki, T. 108

Yan, W.T. 145

Yan, W.-T. 182

Yan, W.T. 189

Yan, Z. 210

Yang, B.-W. 195

Yang, C.-C. 203

Yang, D.S. 197

Yang, J.-F. 214

Yang, J.M. 164

Yang, K. 156, 206
Yang, S. 167

Yang, S.-S. 161

Yang, T. 112, 145, 174, 176, 179, 181, 182, 183, 189, 192

Yang, X.-R. 114

Yang, Y. 110, 170

Yang, Y.-C. 211

Yano, H. 2

Yasui, K. 108

Yau, D.T.W. 87

Yau, T. 103

Yeh, H.-Z. 161

Yeo, W. 103

Yeon, J.E. 95

Yi, N.-J. 150, 183, 203

Yi, Y. 87, 101, 152, 161

Yim, H.J. 95, 109

Yim, S.Y. 95, 160

Yin, G.-W. 116, 199

Yin, X. 96, 195

Yokohama, K. 205

Yokoo, H. 176

Yoo, B. 129

Yoo, C. 166, 173

Yoo, C.-I. 145

Yoo, J.E. 157

Yoo, S.H. 164, 208

Yoo, T. 150

Yook, J.I. 75

Yoon, D.J. 155

Yoon, E.L. 150

Yoon, J.-H. 48, 100

Yoon, J.H. 134

Yoon, J.-K. 122

Yoon, K.C. 183, 203

Yoon, S.H. 128

Yoon, S.K. 49, 123, 155, 156, 164, 182, 207

Yoon, S.M. 36, 197, 209

Yoon, W.S. 197

Yoon, S.K. 206

Yoshimura, K. 108

You, Y.K. 156, 194

Younis, L. 85

Yu, J. 166

Yu, J.H. 151, 159

Yu, J.-J. 174, 179

Yu, J.J. 176

Yu, J.J. 181, 192

Yu, M.-C. 111

Yu, S.C.H. 118

Yu, S.J. 26, 100, 103

Yu, Y.D. 194

Yu, Z. 100

Yuan, B.-T.J. 195

Yuan, R. 93 


\section{Liver \\ Cancer}

Zagonel, V. 107

Zakria, M. 135

Zen, Y. 61

Zeng, Y.-Y. 174, 179

Zeng, Y.Y. 181, 189

Zhang, B.-H. 87, 126, 195

Zhang, H. 145, 174, 176, 179, 183, 189

Liver Cancer 2018;7(suppl 1):1-220

DOI 10.1159/000490877

c 2018 S. Karger AG, Base

www.karger.com/lic

The 9th Asia-Pacific Primary Liver Cancer Expert Meeting (APPLE 2018)

Zhang, L. 96, 195

Zhang, T.Y. 176

Zhang, Z. 210

Zhao, H. 103

Zhong, B.-Y. 116, 199

Zhong, J.H. 189

Zhou, J. 69, 114
Zhou, Y. 104

Zhou, Y.-H. 174, 179, 182

Zhou, Y.H. 176, 181, 189, 192

Zhu, A.X. 168, 170

Zhu, B. 93

Zhu, J.F. 207

Zhu, X. 100 